Alex Miyamoto Mussi

\title{
Performance, Efficiency and Complexity in Multiple Access Large-Scale MIMO Systems
}

Doctoral Thesis presented to the Escola Politécnica da Universidade de São Paulo for obtaining the degree of Doctor of Science. 
Alex Miyamoto Mussi

\section{Performance, Efficiency and Complexity in Multiple Access Large-Scale MIMO Systems}

Doctoral Thesis presented to the Escola Politécnica da Universidade de São Paulo for obtaining the degree of Doctor of Science.

Concentration Area:

3142 - Electronic Systems

Advisor:

Prof. Dr. Taufik Abrão 
Autorizo a reprodução e divulgação total ou parcial deste trabalho, por qualquer meio convencional ou eletrônico, para fins de estudo e pesquisa, desde que citada a fonte.

Este exemplar foi revisado e corrigido em relação à versão original, sob responsabilidade única do autor e com a anuência de seu orientador.

São Paulo, de de

Assinatura do autor:

Assinatura do orientador:

\section{Catalogação-na-publicação}

Mussi, Alex

Performance, Efficiency and Complexity in Multiple Access Large-Scale MIMO Systems / A. Mussi -- versão corr. -- São Paulo, 2019.

$223 \mathrm{p}$.

Tese (Doutorado) - Escola Politécnica da Universidade de São Paulo. Departamento de Engenharia de Telecomunicações e Controle.

1.Telecomunicações 2.Wireless 3.Detecção de dados 4.MIMO massivo I.Universidade de São Paulo. Escola Politécnica. Departamento de Engenharia de Telecomunicações e Controle II.t. 
"Every victory hides an abdication". 


\section{Acknowledgements}

First, I would like to thank God for giving me health and opportunity to develop this work. I express my gratitude to my advisor, Prof. Taufik Abrão, for his continuous willingness to meet the demands of this work, sharing his knowledge and experience. His guidance in this process has given me professional and personal growth. I would like to thank my family for the love and encouragement always dedicated to me: my parents Jorge and Nair, my girlfriend Marcela and my sister Tatiana. Last but not least, I thank my friends and colleagues at the State University of Londrina for their support throughout this work: Ricardo, Edno, Álvaro, José Carlos and Aislan; besides Jair and Luiz by the partnership. 


\section{Abstract}

Systems with multiple transmitting and receiving antennas in large-scale (LS-MIMO - large-scale multipleinput multiple-output) enable high spectral and energy efficiency gains, which results in an increase in the data transmission rate in the same band, without increasing the transmitted power per user. In addition, with the increase of the number of antennas in the base station (BS) it is possible to attend to a larger number of users per cell, in the same occupied band. Furthermore, it has been found in the literature that the reported advantages of LS-MIMO systems can be obtained with a large number of antennas on at least one side of the communication, usually in BS due to physical restriction in user equipments. However, such advantages have their cost: the use of a large number of antennas also difficult tasks involving signal processing, such as estimation of channel coefficients, precoding and signal detection. It is at this juncture that this Doctoral Thesis is developed, in which the computational complexity of performing efficient detection methods in LSMIMO communication systems is explored through the analysis of algorithms and optimization techniques in the solution of specific problems and still open. More precisely, this Thesis discusses and proposes promising detection techniques in LS-MIMO systems, aiming to improve performance metrics - in terms of error rate - and computational complexity - in terms of the number of mathematical operations. Initially, the problem is introduced through a conventional MIMO system model, where channels with imperfect estimates and correlation between transmitter $(T x)$ and receiver $(R x)$ antennas are considered. Preprocessing techniques based on lattice reduction (LR) are applied in linear detectors, in addition to the sphere decoder (SD), which proposes a lookup table procedure in order to provide a reduction in computational complexity. It is shown that the LR method in the pre-detection results in a significant performance gain in both the condition of uncorrelated and correlated channels, and in the latter scenario the improvement is even more remarkable due to the diversity gain provided. On the other hand, the complexity involved in the application of LR in high correlation scenarios becomes preponderant in linear detectors. In the LR-SD using the lookup table procedure, the optimum gain was reached in all scenarios, as expected, and resulted in a lower complexity than maximum likelihood (ML) detector, even with maximum correlation between antennas, which represents the most complex scenario for the LR technique. Next, the message passing (MP) detector is investigated, which makes use of Markov random fields (MRF) and factor graph (FG) graphical models. Moreover, it is shown in the literature that the message damping (MD) method applied to the MRF detector brings relevant performance gain without increasing computational complexity. On the other hand, the DF value is specified for only a restricted range of scenarios. Numerical results are extensively generated, in order to obtain a range of analysis of the MRF with MD, which resulted in the proposition of an optimal value for the DF, based on numerical curve fitting. Finally, in the face of the MGS detector, two approaches are proposed to reduce the negative impact caused by the random solution when high modulation orders are employed. The first is based on an average between multiple samples, called aMGS (averaged MGS). The second approach deploys a direct restriction on the range of the random solution, limiting in $d$ the neighborhood of symbols that can be sorted, being called $d$-sMGS. Numerical simulation results show that both approaches result in gain of convergence in relation to MGS, especially: in regions of high system loading, $d$-sMGS detection demonstrated significant gain in both performance and complexity compared to aMGS and MGS; although in low-medium loading, the aMGS strategy showed less complexity, with performance marginally similar to the others. Furthermore, it is concluded that increasing the dimensions of the system favors a smaller restriction in the neighborhood.

Keywords: LS-MIMO; low complexity detectors; performance-complexity tradeoff; lattice reduction; message passing detector; mixed Gibbs sampling detector. 


\section{Resumo}

Sistemas com múltiplas antenas transmissoras e múltiplas antenas receptoras em larga escala (LS-MIMO large-scale multiple-input multiple-output) possibilitam altos ganhos em eficiência espectral e energética, o que resulta em aumento da taxa de transmissão de dados numa mesma banda ocupada, sem acréscimo da potência transmitida por usuário. Além disso, com o aumento do número de antenas na estação rádio-base (BS - base station) possibilita-se o atendimento de maior número de usuários por célula, em uma mesma banda ocupada. Ademais, comprovou-se na literatura que as vantagens relatadas dos sistemas LS-MIMO podem ser obtidas com um grande número de antenas em, pelo menos, um dos lados da comunicação, geralmente na BS devido à restrição física nos dispositivos móveis. Contudo, tais vantagens têm seu custo: a utilização de um grande número de antenas também dificulta tarefas que envolvem processamento de sinais, como estimação dos coeficientes de canal, precodificação e detecção de sinais. É nessa conjuntura em que se desenvolve esta Tese de Doutorado, na qual se explora o compromisso desempenho versus complexidade computacional de métodos eficientes de detecção em sistemas de comunicações LS-MIMO através da análise de algoritmos e técnicas de otimização na solução de problemas específicos e ainda em aberto. Mais precisamente, a presente Tese discute e propõe técnicas promissoras de detecção em sistemas LS-MIMO, visando a melhoria de métricas de desempenho - em termos de taxa de erro - e complexidade computacional - em termos de quantidade de operações matemáticas. Inicialmente, o problema é introduzido através de um modelo de sistema MIMO convencional, em que são considerados canais com estimativas imperfeitas e com correlação entre as antenas transmissoras $(\mathrm{Tx})$ e entre as receptoras $(\mathrm{Rx})$. Aplicam-se técnicas de pré-processamanto baseadas na redução treliça (LR - lattice reduction) em detectores lineares, além do detector esférico (SD - sphere decoder), o qual é proposto um procedimento de tabela de pesquisa a fim de prover redução na complexidade computacional. Mostra-se que o método LR na pré-detecção resulta em ganho de desempenho significante tanto na condição de canais descorrelacionados quanto fortemente correlacionados, sendo que, neste último cenário a melhoria é ainda mais notável, devido ao ganho de diversidade proporcionado. Por outro lado, a complexidade envolvida na aplicação da LR em alta correlação torna-se preponderante em detectores lineares. No LR-SD utilizando o procedimento de tabela de pesquisa, o ganho ótimo foi alcançado em todos os cenários, como esperado, e resultou em complexidade inferior ao detector de máxima verossimilhança ( $\mathrm{ML}$ - maximum likelihood), mesmo com máxima correlação entre antenas, a qual representa o cenário de maior complexidade a técnica LR. Em seguida, o detector por troca de mensagens (MP - message passing) é investigado, o qual faz uso de modelos grafos do tipo MRF (Markov random fields) e FG (factor graph). Além disso, mostra-se na literatura que o método de amortecimento de mensagens (MD - message damping) aplicado ao detector MRF traz relevante ganho de desempenho sem aumento na complexidade computacional. Por outro lado, o valor do DF (damping factor) é especificado para somente uma variedade restrita de cenários. Resultados numéricos são extensivamente gerados, de forma a dispor de uma gama de análises de comportamento do MRF com MD, resultando na proposição de um valor ótimo para o DF, baseando-se em ajuste de curva numérico. Finalmente, em face ao detector MGS (mixed Gibbs sampling), são propostas duas abordagens visando a redução do impacto negativo causado pela solução aleatória quando altas ordens de modulação são empregadas. A primeira é baseada em uma média entre múltiplas amostras, chamada aMGS (averaged MGS). A segunda abordagem realiza uma restrição direta no alcance da solução aleatória, limitando em até $d$ a vizinhança de símbolos que podem ser sorteados, sendo chamada de $d$-sMGS ( $d$-simplificado MGS). Resultados de simulação numérica demonstram que ambas abordagens resultam em ganho de convergência em relação ao MGS, destacando-se: em regiões de alto carregamento, a detecção $d$-sMGS demonstrou ganho expressivo tanto em desempenho quanto em complexidade se comparada à aMGS e MGS; já em baixo-médio carregamentos, a estratégia aMGS demonstrou menor complexidade, com desempenho marginalmente semelhante às demais. Além disso, conclui-se que o aumento do número de dimensões do sistema favorece uma menor restrição na vizinhança.

Palavras-Chaves: Sistemas de múltiplas antenas em larga-escala; detecção de dados; compromisso desempenho-complexidade; redução treliça; detector por troca de mensagens; amostragem mista de Gibbs. 


\section{List of Figures}

1.1 Projections regarding the number of communications devices and the demand for global mobile traffic; a) Number of electronic communication devices; b) Consumer global mobile internet traffic and c) Average mobile network connection speeds. Source: [1] . . . . . . . . . . 25

1.2 Comparative performance between SISO $1 \times 1$ 64-QAM, SIMO $1 \times 6$ 64-QAM and MIMO $6 \times 6$ BPSK, with spectral efficiency of $6 \mathrm{bps} / \mathrm{Hz}$ in fading channel.

2.1 Introdutory conventional MIMO point-to-point communication system with $N_{t}$ transmitting

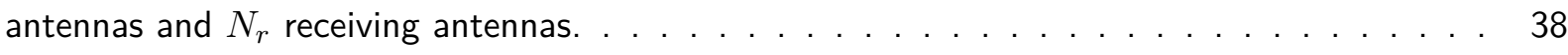

2.2 Example of the tree search expansion of the LR-SD $\ldots \ldots \ldots \ldots$

2.3 BER performance for the $4 \times 4$ MIMO detectors under 4-QAM, pCSI and: a) uncorrelated channels, $\rho=0$; b) highly correlated channels, $\rho=0.9$.

2.4 Performance for the $4 \times 4 \mathrm{MIMO}$ detectors considering iCSI, uncorrelated channels $(\rho=0)$ and:

a) low iCSI, $n_{\varepsilon}=3 ;$ b) highly iCSI, $n_{\varepsilon}=10 \ldots \ldots \ldots \ldots$

2.5 Flop complexities for the analysed MIMO detectors with $4 \times 4$ antennas and pCSI in two scenarios: a) uncorrelated channels, $\rho=0$; b) highly correlated channels, $\rho=0.9 \ldots \ldots \ldots$

2.6 Performance of linear detectors MF, ZF and MMSE, in QPSK modulation, considering several antenna configurations: a) low system loading and b) high system loading scenarios.

3.1 Single-cell uplink LS-MIMO communication system with $K$ single antenna UEs and a BS

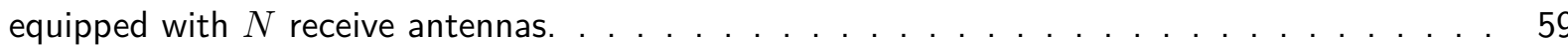

3.2 Temporal structure of transmission/estimation in a MU-LS-MIMO system in TDD mode. . . . 61

3.3 Temporal structure of transmission/estimation in a MU-LS-MIMO system in FDD mode. . . . 63

4.1 Diagram of MRF-BP, based on Markov random field graph model, where $m_{1, k}$ is the message from node 1 to node $k$ of Eq. (4.9).

4.2 The graph model diagram of FG-BP, where $\mathrm{p}_{i}^{k+,(t)}$ is the conditional probability of Eq. (4.16)

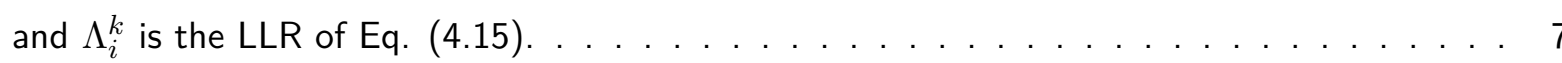

4.3 Influence of the number of iterations $\mathcal{I}$ on the BER performance; both detectors operate under $\gamma=10 \mathrm{~dB}$ and the scenarios are split in: a.1) MRF-BP without MD $\left(\alpha_{\mathrm{MRF}}=0\right)$; b.1) FG-BP without MD ( $\left.\alpha_{\mathrm{FG}}=\beta_{\mathrm{FG}}=0\right)$; a.2) MRF-BP with optimal MD, from Eq. (4.21); b.2) FG-BP with best results found of MD. 
4.4 BER performance of a MRF-BP LS-MIMO system with $\mathcal{I}_{\mathrm{MRF}}=5$ as a function of different SNR scenarios and MRF-BP damping factors, $\alpha_{\mathrm{MRF}}$ : a) $20 \times 20$; b) $100 \times 100$ antennas $\ldots$.

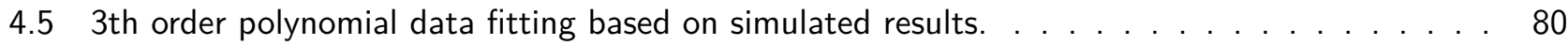

4.6 BER performance of the implemented MRF-BP detector with the best DF value and FG-BP detector without MD, split in two scenarios: a) low to medium number of antennas, b) high number of antennas.

4.7 Analysis of computational complexity, in terms of average number of flops, operating under large-scale MIMO scenarios, $K=N$ antennas and different number of algorithm iterations, $\mathcal{I} \in\{3,4,5\}$, carried out in two aspects: a) percent increment of complexity of MD method applied in MRF-BP detector; b) comparison of complexity and respective BER among MRF-BP and FG-BP detectors, $\gamma_{\mathrm{dB}}=10 \mathrm{~dB}$.

5.1 ML cost function evolution during the aMGS algorithm's iterations and the optimal result (sphere decoder); $K=M=16, \gamma=10 \mathrm{~dB}, \mathcal{I}=250$ iterations, and 4-QAM modulation. . . .

5.2 A brief description of the coordinate update process on the aMGS and the proposed $d$-sMGS detectors.

5.3 An illustrative example showing the stalling effect of conventional GS and denoting that the proposed simplification of the target function of aMGS does not degrade the LS-MIMO performance: $K=N=16$ antennas, $L_{e}=1$ sample, $\mathcal{I}=512$ iterations, $R_{\max }=50,4$-QAM and 16-QAM.

5.4 BER performance convergence of different mixing ratios, $q$, of aMGS in medium number of antennas scenario $(K=96, N=128)$ at $\gamma_{\mathrm{dB}}=25 \mathrm{~dB}, 64-\mathrm{QAM}, R_{\max }=\{1,5,10\}, \mathcal{I}=6000$ and low number of $L_{e}$ samples: a) $\left.L_{e}=1, \mathbf{b}\right) L_{e}=2$.

5.5 BER performance convergence of different mixing ratios, $q$, of aMGS in medium number of antennas scenario $(K=96, N=128)$ at $\gamma_{\mathrm{dB}}=25 \mathrm{~dB}, 64$-QAM, $R_{\max }=\{1,5,10\}, \mathcal{I}=6000$ and high number of $L_{e}$ samples: a) $L_{e}=4$, b) $L_{e}=8$.

5.6 BER performance and complexity vs. convergence for the aMGS algorithm considering 64QAM modulation, best mixing ratio $q$ (curves $[A]$ to $[D]$ ) from Table 5.3 and optimal value as proposed in [2], i.e., $q=1 / 2 K$ and $L_{e}=1$ (curve [E]).

5.7 Performance convergence against the maximum number of iterations $\mathcal{I}$ of the proposed $d$ sMGS detector, with $16 \times 16$ antennas in 64 -QAM modulation among different NL distance values. The number of iterations is related to the parameter $a$, were $\mathcal{I}=a K \sqrt{M}$.

5.8 SNR vs. BER performance/Average number of rops in $d$-sMGS-MR detector against aMGS-MR approach and MGS-MR. Parameters: $K=58, N=64,64-\mathrm{QAM}, \beta \approx 0.9 \ldots \ldots \ldots$

5.9 Performance and complexity versus system loading, considering $\gamma_{\mathrm{dB}}=25 \mathrm{~dB}, 64-\mathrm{QAM}$ : a) Performance for $N=64 ; \mathbf{b})$ Average rop complexity for $N=64$. 
5.10 Performance and complexity versus system loading, considering $\gamma_{\mathrm{dB}}=25 \mathrm{~dB}, 64-\mathrm{QAM}$ : a) Performance for $N=128$; b) Average rop complexity for $N=128 \ldots \ldots \ldots$. . . . . . . 109 


\section{List of Tables}

1.1 Reliability and capacity in SISO, SIMO and MIMO in fading channels. . . . . . . . . 26

1.2 Framework of the most relevant LS-MIMO detection techniques found in literature $\ldots \ldots 30$

2.1 Acronyms and descriptions of the investigated detectors $\ldots \ldots \ldots \ldots$

2.2 Detectors Complexity . . . . . . . . . . . . . . . . . . . . 55

3.1 Computational complexity of the detection techniques investigated in this work $\ldots \ldots 64$

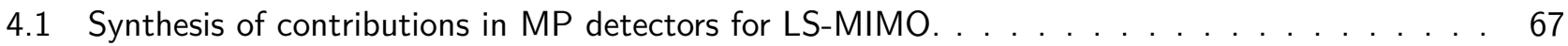

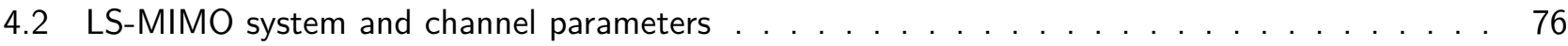

4.3 Convergence comparison between MRF-BP and FG-BP, according to Figure $4.3 \ldots 77$

4.4 Parameters of data fitting function: $\alpha_{\mathrm{MRF}}^{\text {best }}(\gamma, N)$, eq. $(4.21) \ldots \ldots \ldots \ldots \ldots$

4.5 Per-symbol Computational Complexity of MRF-BP and FG-BP Algorithms. . . . . . . . . . 83

5.1 Per-symbol Computational Complexity of aMGS, conventional MGS and MMSE Algorithms. . 99

5.2 LS-MIMO system and channel parameters. . . . . . . . . . . . . . . . . . 100

5.3 Best parameters for aMGS-MR detector, presented in $[3] \ldots \ldots \ldots 10 \ldots$ 


\section{List of Acronyms}

\begin{tabular}{|c|c|}
\hline aMGS & Averaged MGS \\
\hline AWGN & Additive White Gaussian Noise \\
\hline BER & Bit Error Rate \\
\hline BP & Belief Propagation \\
\hline BPSK & Binary Phase Shift Keying \\
\hline BS & Base Station \\
\hline CAGR & Compound Annual Growth Rate \\
\hline CDMA & Code Division Multiple Access \\
\hline CSI & Channel State Information \\
\hline$d$-sMGS & $d$-simplified MGS \\
\hline DF & Damping Factor \\
\hline DL & Downlink \\
\hline ENI & Effective Number of Iterations \\
\hline EXIT & Extrinsic Information Transfer \\
\hline FD-MIMO & Full-Dimension MIMO \\
\hline FG & Factor Graph \\
\hline FG-BP & Factor Graph-Belief Propagation \\
\hline flops & Floating-point Operations \\
\hline FSD & Fixed Sphere Decoder \\
\hline GM & Graphical Model \\
\hline GMPID & Gaussian Message Passing Iterative Detection \\
\hline GS & Gibbs Sampling \\
\hline iCSI & Imperfect Channel State Information \\
\hline LAS & Likelihood Ascent Search \\
\hline LLL & Lenstra - Lenstra - Lovász \\
\hline LLR & Log-likelihood Ratio \\
\hline LR & Lattice Reduction \\
\hline LR-SD & SD aided by $L R$ \\
\hline LR-MMSE & MMSE aided by LR \\
\hline \multicolumn{2}{|c|}{ LR-MMSE-OSIC MMSE with OSIC aided by LR } \\
\hline LR-ZF & ZF aided by LR \\
\hline LS-MIMO & Large-Scale MIMO \\
\hline LS-MIMO-ISI & LS-MIMO Inter-symbol Interference \\
\hline LTE & Long Term Evolution \\
\hline LTS & Layered Tabu Search \\
\hline
\end{tabular}




\begin{tabular}{|c|c|}
\hline MAP & Maximum a posteriori Probability \\
\hline MCMC & Markov Chain Monte Carlo \\
\hline MCS & Monte Carlo Simulation \\
\hline MD & Message Damping \\
\hline MF & Matched Filter \\
\hline MGS & Mixed Gibbs Sampling \\
\hline MIMO & Multiple-Input Multiple-Output \\
\hline ML & Maximum Likelihood \\
\hline MRF & Markov Random Field \\
\hline MRF-BP & Markov Random Field-Belief Propagation \\
\hline MMSE & Minimum Mean Squared Error \\
\hline MMSE-SIC & MMSE with SIC \\
\hline MMSE-OSIC & MMSE with OSIC \\
\hline $\mathbf{m m W}$ & Millimeter Wave \\
\hline MR & Multiple Restarts \\
\hline MSE & Mean Squared Error \\
\hline MP & Message Passing \\
\hline MS & Multiple Sampling \\
\hline MuD & Multiuser Detection \\
\hline Mux-MIMO & Multiplexing gain mode MIMO \\
\hline MU-LS-MIMO & Multiuser LS-MIMO \\
\hline MU-MIMO & Multiuser MIMO \\
\hline NL & Neighborhood Limitation \\
\hline NLOS & Non-line-of-sight \\
\hline OSIC & Ordered SIC \\
\hline PAM & Pulse Amplitude Modulation \\
\hline PED & Partial Euclidean Distance \\
\hline pCSI & Perfect Channel State Information \\
\hline PS & Pilot Sequences \\
\hline QAM & Quadrature Amplitude Modulation \\
\hline QoS & Quality of Service \\
\hline RF & Radio-Frequency \\
\hline RID & Relaxation Iterative Detection \\
\hline RID-MMSE & Relaxation Iterative Detection approximating MMSE detection \\
\hline rmse & Root Mean Squared Error \\
\hline rops & Real Number Operations \\
\hline RTS & Reative Tabu Search \\
\hline $\mathbf{R x}$ & Receiving Antennas \\
\hline SA-GMPID & Scale-and-add GMPID \\
\hline SER & Symbol Error Rate \\
\hline SIC & Successive Interference Cancellation \\
\hline
\end{tabular}




$\begin{array}{ll}\text { SIMO } & \text { Single-Input Multiple-Output } \\ \text { SISO } & \text { Single-Input Single-Output } \\ \text { SD } & \text { Sphere Decoder } \\ \text { SM } & \text { Spatial Modulation } \\ \text { SNR } & \text { Signal-to-noise Ratio } \\ \text { SQRD } & \text { Sorted QR Decomposition } \\ \text { SOCA } & \text { Smart Ordering and Candidate Adding } \\ \text { TDD } & \text { Time-Division Duplexing } \\ \text { TS } & \text { Tabu Search } \\ \text { TX } & \text { Transmitting Antennas } \\ \text { UE } & \text { User Equipment } \\ \text { UL } & \text { Uplink } \\ \text { ULA } & \text { Uniform Linear Array } \\ \text { ZF } & \text { Zero Forcing }\end{array}$




\section{Notations}

\section{Variables definitions}

$k, \gamma, M$

a

A

$\mathcal{A}$

\section{Operators}

$\begin{array}{ll}\underset{a}{\max (\cdot)} & \text { Returns the maximum element of the argument } \\ \min (\cdot) & \text { Returns the minimum element of the argument } \\ \underset{a \in \mathcal{A}}{\arg \max [\cdot]} & \text { Element of the set } \mathcal{A} \text { that maximizes the argument } \\ \underset{a \in \mathcal{A}}{\arg \min [\cdot]} & \text { Element of the set } \mathcal{A} \text { that minimizes the argument } \\ \mathbb{E}\{\cdot\} & \text { Statistical Expectation Operator } \\ \operatorname{Var}\{\cdot\} & \text { Statistical Variance Operator } \\ |a| & \text { Magnitude Operator (absolute value) } \\ |\mathbf{a}| & \text { Cardinality of vector a } \\ |\mathcal{A}| & \text { Cardinality of set } \mathcal{A} \\ \log a & \text { Logarithm of } a \text { in base } 10 \\ \sum_{a=1}^{N}(\cdot) & \text { Summation considering } a=1 \text { to } a=N, \text { with unitary increment } \\ p(a) & \text { Probability of } a \\ p(a \mid b) & \text { Conditional Probability of } a \text { given } b \\ \Re\{\cdot\} & \text { Real part of a complex number } \\ \Im\{\cdot\} & \text { Imaginary part of a complex number } \\ \exp (a) & \text { Exponential of } a \\ \operatorname{Tr}(\cdot) & \text { Trace operator } \\ \operatorname{det}(\cdot) & \text { Determinant operator } \\ \operatorname{sign}(\cdot) & \text { Signal operator } \\ \lfloor a\rfloor & \text { Floor function, i.e., the greatest integer less than or equal to } a \\ \lceil a\rceil & \text { Ceil function, i.e., the least integer greater than or equal to } a \\ \lfloor a\rceil & \\ \mathcal{C N}\left(\mathbf{0}_{M}, \mathbf{I}_{M}\right) & \text { Complex normal distribution with mean } \mathbf{0}_{M} \text { and covariance } \mathbf{I}_{M} \\ & \end{array}$

Scalar, italic character

Vector, lower case bold character

Matrix, upper case bold character

Set, upper case calligraphic character 
$\mathbf{A}^{H} \quad$ Conjugate transpose, or Hermitian transpose, of matrix $\mathbf{A}$

$\mathbf{A}^{-1} \quad$ Inverse of matrix $\mathbf{A}$

$\mathbf{A}^{\dagger} \quad$ Moore-Penrose pseudo-inverse of $\mathbf{A}$

\section{Subscript indexes}

$a_{i} \quad i$-th elcment of vector a

$a_{i, j} \quad$ Element of $i$-th row and $j$-th column of matrix $\mathbf{A}$

$\mathbf{a}_{k} \quad$ Vector represented by the $k$-th column of matrix $\mathbf{A}$

$\hat{\mathbf{s}}_{i, j} \quad$ Chapter 5: denotes the vector $\hat{\mathbf{s}}^{(t)}$ with its $i$-th position changed to the symbol $a_{j}$ 


\section{List of Symbols}

$\Theta_{s}$
Chapter 2: Parameter for updating the radius on the LR-SD algorithm Chapter 5: Mixing time parameter of MGS and MGS-based detectors Damping factor of MRF-BP algorithm Damping factor at observation nodes of FG-BP algorithm Temperature parameter, used by MGS algorithm Damping factor at variable nodes of FG-BP algorithm Loading factor Random variable employing mixture, used to symbol estimation in $d$-sMGS algorithm

Performance-complexity quality metric

Real-valued vector of AWGN samples in uplink channel Complex-valued vector of AWGN samples in uplink channel Transformed vector of AWGN samples, referring to the SD algorithm Phase of a uniform distribution in the interval $(0,2 \pi]$ Average SNR value at each receiving antenna

Denotes the symbol distance function in the real-valued constellation adopted

Variance of the noise, $\boldsymbol{\eta}$

Variance of the term interference-plus-noise, $\lambda_{i l}$, used in the algorithm FG$\mathrm{BP}$

Chapter 2: Edge potential of the MRF-BP algorithm

Chapter 5: Target function, related to mixing time $\alpha$, of MGS algorithm

Chapter 2: Self potential of the MRF-BP algorithm

Chapter 2: Quality metric of $\hat{\mathbf{s}}^{(t)}$

Carrier wavelength

Interference-plus-noise term, used in the algorithm FG-BP

Logarithmic likelihood ratio, used in FG-BP algorithm

Mean of the term interference-plus-noise, $\lambda_{i l}$, used in the algorithm FG-BP

Distribution of interest, for a given temperature parameter $\alpha_{\text {MGS }}$, used in the MGS algorithm

Random variable employing mixture, used to symbol estimation in aMGS algorithm

Stalling limit for iterations of MGS and MGS-based algorithms 


\begin{tabular}{|c|c|}
\hline$\Theta_{r}\left(\phi\left(\hat{\mathbf{s}}^{(t)}\right)\right)$ & Stalling limit for restarts of MGS and MGS-based algorithms \\
\hline$v\left(\hat{s}_{i}^{(t-1)}, d\right)$ & $\begin{array}{l}\text { Denotes an uniform sorted symbol in the constellation neighborhood of } \\
\hat{s}_{i}^{(t-1)} \text {, with distance } d\end{array}$ \\
\hline$\varsigma$ & $\begin{array}{l}\text { Term that references the channel conditions in calculating the complexity } \\
\text { of the SD algorithm }\end{array}$ \\
\hline$\xi$ & Simplified target function employed in aMGS and $d$-sMGS algorithms \\
\hline $\mathcal{A}_{c}$ & Complex valued QAM constellation \\
\hline $\mathcal{A}$ & $\begin{array}{l}\text { Real valued QAM constellation, i.e., values from the underlying PAM } \\
\text { alphabet }\end{array}$ \\
\hline$\tilde{\mathcal{A}}$ & Alphabet $\mathcal{A}$ in LR reduced basis \\
\hline$b_{i}\left(s_{i}\right)$ & Final belief, referring to the symbol $s_{i}$, of the algorithm MRF-BP \\
\hline $\mathcal{B}(i)$ & $\begin{array}{l}\text { Set of all the neighboring nodes to the node } i \text {, referring to the algorithm } \\
\text { MRF-BP }\end{array}$ \\
\hline$c_{1}$ & Constant of iteration criteria of MGS and MGS-based algorithms \\
\hline$c_{2}$ & Constant of iteration criteria of MGS and MGS-based algorithms \\
\hline$c_{\min }$ & Constant of iteration criteria of MGS and MGS-based algorithms \\
\hline $\mathcal{C}_{I}$ & $\begin{array}{l}\text { Computation complexity of initial solution, from MGS and MGS-based } \\
\text { algorithms }\end{array}$ \\
\hline $\mathcal{C}_{T}$ & Total computation complexity, from MGS and MGS-based algorithms \\
\hline$d$ & Neighborhood distance of $d$-sMGS algorithm \\
\hline $\mathcal{D}\left(\hat{s}_{i}^{(t-1)}, d\right)$ & Set of neighborhood symbols of $\hat{s}_{i}^{(t-1)}$ with distance $d$ \\
\hline$E_{\mathbf{s}}$ & Average energy of symbols transmitted in uplink channel \\
\hline ENI & $\begin{array}{l}\text { Mean value of } \mathcal{I}_{\text {eff }} \text { during a MCS method of } T \text { number of realizations } \\
\text { (symbol periods) }\end{array}$ \\
\hline$f_{i}^{\text {ord }}$ & Represents the $i$ th position of $\mathbf{f}$ in descending order, for $i=1, \ldots,|\mathcal{A}|$ \\
\hline $\mathbf{H}_{c}$ & Complex matrix containing the channel coefficients with Rayleigh flat fading \\
\hline $\mathbf{H}$ & $\begin{array}{l}\text { Matrix, in real-form, containing the channel coefficients with Rayleigh flat } \\
\text { fading }\end{array}$ \\
\hline $\mathbf{I}_{K}$ & Identity matrix with dimension $K \times K$ \\
\hline $\mathcal{I}$ & Maximum number of iterations of the MGS and MGS-based algorithms \\
\hline $\mathcal{I}_{\mathrm{MRF}}$ & Maximum number of iterations of the MRF-BP algorithm \\
\hline $\mathcal{I}_{\mathrm{FG}}$ & Maximum number of iterations of the FG-BP algorithm \\
\hline $\mathcal{I}_{\text {eff }}$ & $\begin{array}{l}\text { Number of iterations effective consumed in a symbol period of MGS and } \\
\text { MGS-based algorithms }\end{array}$ \\
\hline$K$ & Number of active single-antenna users \\
\hline$L_{e}$ & Number of samples, from aMGS algorithm \\
\hline$m_{i, j}^{(t)}$ & $\begin{array}{l}\text { Normalized message, after MD technique, from the } i \text { th node to the } j \text { th } \\
\text { node in the } t \text {-th iteration referring to the MRF-BP algorithm }\end{array}$ \\
\hline
\end{tabular}




\begin{tabular}{|c|c|}
\hline$m_{i, j}^{\prime}(t)$ & $\begin{array}{l}\text { Message, after MD technique, from the } i \text { th node to the } j \text { th node in the } t \text {-th } \\
\text { iteration referring to the MRF-BP algorithm }\end{array}$ \\
\hline$\tilde{m}_{i, j}^{(t)}$ & $\begin{array}{l}\text { Message from the } i \text { th node to the } j \text { th node in the } t \text {-th iteration referring to } \\
\text { the MRF-BP algorithm }\end{array}$ \\
\hline$N$ & Number of receiving antennas in base station \\
\hline$N_{r}$ & Number of receiving antennas in conventional MIMO scenario, Chapter 2 \\
\hline$N_{t}$ & $\begin{array}{l}\text { Number of transmitting antennas in conventional MIMO scenario, Chapter } \\
2\end{array}$ \\
\hline $\mathrm{p}_{i}^{k+}$ & $\begin{array}{l}\text { Conditional probability of } s_{k}=+1 \text { given } \mathbf{y} \text {, calculated on } k \text { th variable node } \\
\text { based on information from } i \text { th observation node, referring to algorithm FG- } \\
\text { BP }\end{array}$ \\
\hline$P_{e}$ & Error probability \\
\hline$q$ & Mixing ratio, referring to MGS algorithm \\
\hline Q & Orthogonal matrix referring to $Q R$ decomposition of $\mathbf{H}$ \\
\hline$r$ & SD algorithm search radius \\
\hline $\mathbf{R}$ & Upper triangular matrix relating to $\mathrm{QR}$ decomposition of $\mathbf{H}$ \\
\hline $\mathbf{R}_{c}$ & Correlation matrix used for simplification in the MRF-BP algorithm \\
\hline$R_{\max }$ & Maximum number of restarts, from MGS and MGS-based algorithms \\
\hline $\mathbf{s}_{c}$ & $\begin{array}{l}\text { Transmitted symbols vector in uplink channel, complex-valued from QAM } \\
\text { constellation }\end{array}$ \\
\hline $\mathbf{s}$ & $\begin{array}{l}\text { Transmitted symbols vector in uplink channel, real-valued from PAM } \\
\text { constellation }\end{array}$ \\
\hline$\hat{\mathbf{s}}$ & Estimated transmitted signal vector \\
\hline$\hat{\mathbf{s}}_{i, j}$ & $\begin{array}{l}\text { Chapter 5: Denotes the vector } \hat{\mathbf{s}}^{(t)} \text { with its } i \text { th position changed to the } \\
\text { symbol } a_{j} \text { from the constellation adopted }\end{array}$ \\
\hline $\mathbf{T}$ & Chapter 2: Unimodular matrix from LR technique \\
\hline $\mathbf{T}$ & $\begin{array}{l}\text { Chapter 5: Number of realizations (symbol periods) in a Monte-Carlo } \\
\text { Simulation }\end{array}$ \\
\hline $\mathbf{U}$ & Unimodular matrix from LR technique \\
\hline $\mathbf{W}$ & Generic linear detection matrix \\
\hline $\mathbf{W}_{\mathrm{MF}}$ & Linear detection matrix from MF detector \\
\hline $\mathbf{W}_{\mathrm{ZF}}$ & Linear detection matrix from ZF detector \\
\hline $\mathbf{W}_{\text {MMSE }}$ & Linear detection matrix from MMSE detector \\
\hline $\mathrm{x}$ & Transformed received signal vector, $\mathbf{x}=\mathbf{Q}^{H} \mathbf{y}$ \\
\hline $\mathbf{y}_{c}$ & Vector, in complex form, of received signals by base station in uplink channel \\
\hline $\mathbf{y}$ & Vector, in real form, of received signals at base station in uplink channel \\
\hline$\tilde{\mathbf{y}}$ & $\begin{array}{l}\text { Transformed vector of received signals at the base station, referring to the } \\
\text { SD algorithm }\end{array}$ \\
\hline
\end{tabular}




\section{Contents}

1 Introduction $\quad 24$

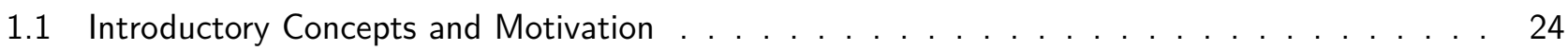

1.2 Framework of main contributions in LS-MIMO signal detection . . . . . . . . . . . . . 29

1.3 Problem Formulation $\ldots \ldots \ldots \ldots \ldots \ldots \ldots$

1.4 Scope and Objectives . . . . . . . . . . . . . . . . . . . . . . . . 32

1.4 .1 Scope . . . . . . . . . . . . . . . . . . . . . . 32

1.4 .2 Objetives . . . . . . . . . . . . . . . . . . . . 32

1.5 Organization of the Doctoral Thesis $\ldots \ldots \ldots \ldots \ldots \ldots$

1.6 Summary of contributions and generated publications . . . . . . . . . . . 34

1.6.1 Contributions of this Thesis . . . . . . . . . . . . . . . . . . 34

1.6.2 Publications directly associated to the theme . . . . . . . . . . . 34

1.6.3 Publications indirectly associated to the theme $\ldots \ldots \ldots \ldots$

2 Introductory investigation: conventional MIMO to large-scale MIMO 36

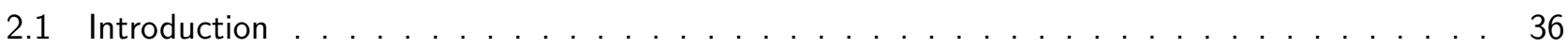

2.2 Conventional MIMO System Model . . . . . . . . . . . . . . . . . . . . . . 37

2.2.1 Imperfect Channel State Information . . . . . . . . . . . . . . . . 38

2.2 .2 Antenna Correlation . . . . . . . . . . . . . . . . . . . . . . . . . . 39

2.3 Conventional MIMO Detectors $\ldots \ldots \ldots \ldots \ldots$

$2.3 .1 \quad$ Linear MIMO Detection . . . . . . . . . . . . . . . . . . . . . . . . 40

2.3.2 MMSE-SIC MIMO Detection . . . . . . . . . . . . . . . . . . . 42

2.3 .3 MMSE-OSIC MIMO Detection . . . . . . . . . . . . . . . . . . . 43

2.3.4 Maximum Likelihood Detector . . . . . . . . . . . . . . . . . . . . . . . 43

2.4 Lattice Reduction Based MIMO Detection . . . . . . . . . . . . . . . . . . . . 44

2.5 Lattice Reduction aided MIMO Detectors in Correlated Channels with iCSI . . . . . . . . . 46 
2.6 LR-aided Sphere Decoder . . . . . . . . . . . . . . . . . . . . . . . 46

2.6.1 Proposed Lookup Table Procedure . . . . . . . . . . . . . . . . . . . . . . . 48

2.7 Numerical Results . . . . . . . . . . . . . . . . . . . . . . . . . . . . . 51

2.8 Complexity Analysis . . . . . . . . . . . . . . . . . . . . . . . . 54

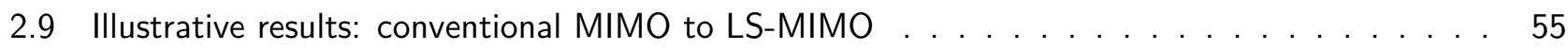

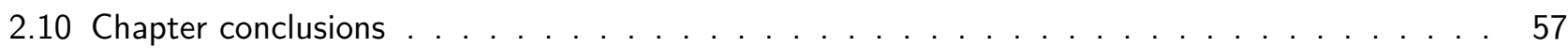

3 System Model for LS-MIMO Systems $\quad 59$

3.1 System Model . . . . . . . . . . . . . . . . . . . . . . . . . 59

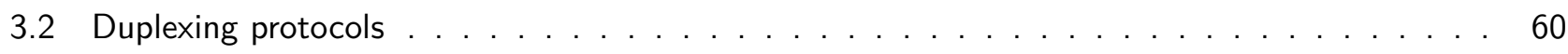

3.2 .1 Time Division Duplexing . . . . . . . . . . . . . . . . 61

3.2 .2 Frequency Division Duplexing . . . . . . . . . . . . . . . . . 62

3.2.3 Computational Complexities of Detection Techniques . . . . . . . . . . . . . . . . . 64

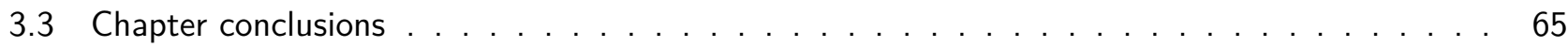

4 Message Passing Detectors for Large Scale MIMO Systems: Damping Factor Analysis 66

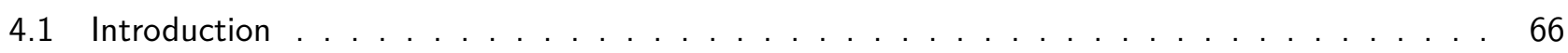

4.2 System Model and Basic Concepts . . . . . . . . . . . . . . . . . . . . . 67

4.2 .1 Graphical Models . . . . . . . . . . . . . . . . . . . . . . . . . 68

4.2 .2 Belief Propagation . . . . . . . . . . . . . . . . . . . 68

4.3 Message Passing via MRF for Large Scale MIMO Detection . . . . . . . . . . . . . . . . . 69

4.3.1 Message Damping on MRF-BP Detector . . . . . . . . . . . . . . 70

4.4 Message Passing via FG for Large Scale MIMO Detection . . . . . . . . . . . . . . . . 72

4.4.1 Message Damping on FG-BP Detector . . . . . . . . . . . . . . . 74

4.5 Numerical Results . . . . . . . . . . . . . . . . . . . . . . 75

4.5.1 Optimal Damping Factor Determination $\left(\alpha^{\text {best }}\right) \ldots \ldots \ldots \ldots$

4.5 .2 Computational Complexity . . . . . . . . . . . . . . . . . . 81

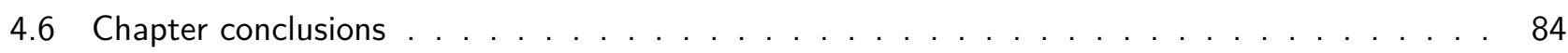

5 Reducing the Noisy Solution Impact of Mixed Gibbs Sampling Detector in High-Order Modulation Large-Scale MIMO Systems 


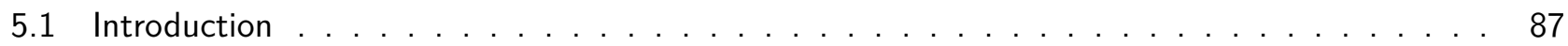

5.2 Review of Mixed Gibbs Sampling detection . . . . . . . . . . . . . . . . . 88

5.2 .1 Multiple restarts . . . . . . . . . . . . . . . . . . 90

5.2 .2 Stopping criterion . . . . . . . . . . . . . . . . . . . 90

5.3 Reducing the Impact of Noisy Solution . . . . . . . . . . . . . . . . . . 92

5.3.1 Approach \#1: Averaged MGS LS-MIMO detector . . . . . . . . . . . . . . . 93

5.3.2 Approach \#2: Simplified MGS with Neighborhood Limitation LS-MIMO detector . . . 94

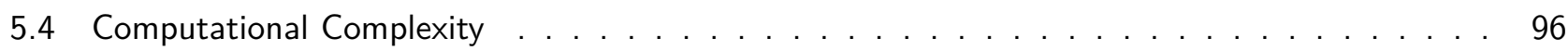

5.4 .1 Quality Metric . . . . . . . . . . . . . . . . . . . . 98

5.5 Numerical results and discussion $\ldots \ldots \ldots \ldots \ldots \ldots$

5.5 .1 Parameters discussion . . . . . . . . . . . . . . . . . . . 100

5.5.2 Analysis on the proposed approaches . . . . . . . . . . . . . 104

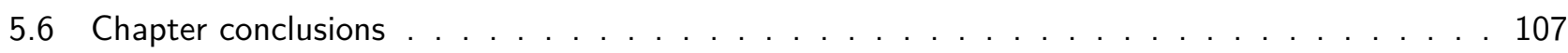

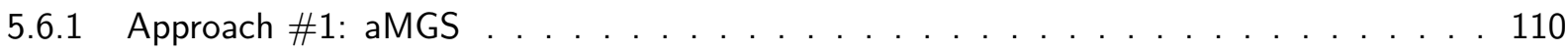

5.6 .2 Approach \#2: $d$-sMGS . . . . . . . . . . . . . . . . . . . 110

6 Conclusions $\quad 111$

6.1 Future Research Directions . . . . . . . . . . . . . . . . . . . . . 112

$\begin{array}{ll}\text { Bibliography } & 114\end{array}$

Appendix A - Full paper published in the journal "Wireless Personal Communications (WIRE)",

October $2016 \quad 122$

Appendix B - Full paper published in conference “XXXIV Simpósio Brasileiro de Telecomunicações" (SBrT 2016), August 2016

Appendix C - Full paper published in the journal “IET Signal Processing”, July $2017 \quad 161$

Appendix D - Full paper published in the journal “IET Signal Processing”, November 2018175

Appendix E - Full paper under R1 review in the journal "EURASIP Journal on Advances in Signal

Processing", January 2019 


\section{Introduction}

This chapter aims to introduce the basic concepts and a literature review regarding signal detection in large scale multiple-input multiple-output (LS-MIMO) systems. In addition, the objectives of the research are listed, as well as the main contributions achieved. Finally, the structure of this PhD Thesis is briefly described.

\subsection{Introductory Concepts and Motivation}

Digital communications are the basis of a whole new conception of multimedia electronic communication, being able to provide integration of voice, data and video in a single channel of wireless communication; this generation of systems also increasingly requires voice quality and high rate of data transmission in the services provided. In the last decade, studies related to wireless and cellular communications have allowed progressive reductions in the size and power consumption of mobile terminals. At the same time, the availability of these multimedia services has been meeting an increasing demand of users and coverage of regions, and it is expected that this growing trend should continue in the coming years [1, 4]. Fig. 1.1 shows the projection, presented in [1], of the growth in the number of electronic communication devices, consumer global mobile internet traffic and average mobile network connection speeds. It can be clearly understood that a significant growth is foreseen in all the presented questions. With regard to the communication devices, Fig. 1.1.a, a growth of approximately 10 billion devices is expected in the period 2017 to 2022, and for the year 2019 it already is expected a little more of 20 billion devices. Relative to consumer internet traffic, Fig. 1.1.b, and average speeds of the mobile network, Fig. 1.1.c, there is a stronger compound annual growth rate (CAGR) in the mobile compared to fixed data traffic. Also, a CAGR of $20 \%$ to $30 \%$ is expected in the average mobile network connection speeds, in the period from 2017 to 2022. Thus, it can be concluded that both the number of mobile devices as the required throughput tend to grow significantly in the coming years.

On the other hand, the physical resources (for example, band of frequencies, number of time slots, user spatial distribution, among others) required for wireless data transmission will remain the same. Therefore, wireless communication systems need to be innovative in order to combine coverage area, efficiency in both bandwidth and power, and also being able to operate in different scenarios: urban, suburban and rural, interior and exterior, with maintenance of quality of service (QoS).

At the end of the 1990s, Alamouti and Foschini proposed innovative communication schemes from the perspective of increasing reliability and capacity respectively, using the concept of multiple transmitting and receiving antennas, sharing the same physical environment $[5,6]$. These transmission schemes, called MIMO, inaugurated a new era in wireless communications subject to fading. In point-to-point communication using multiple antennas, it has been demonstrated in $[6,7]$ that the channel capacity grows linearly with the minimum 


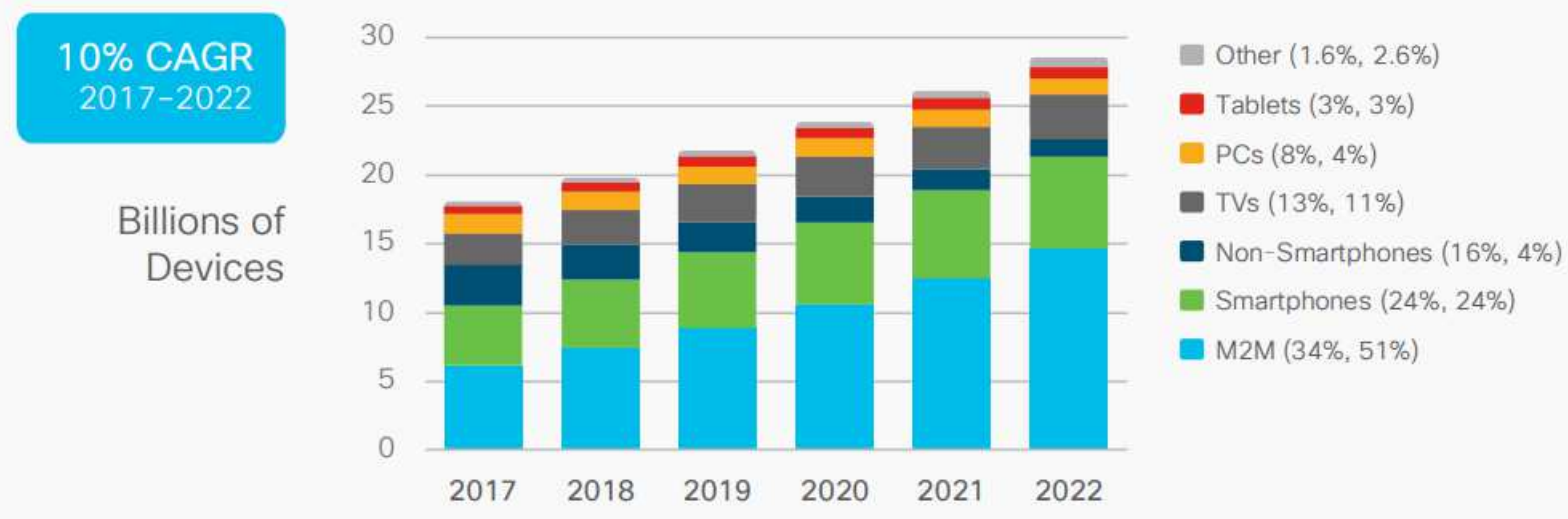

(a)

\begin{tabular}{|l|l|l|l|l|l|l|l|}
\hline $\begin{array}{l}\text { Consumer Internet } \\
\text { Traffic, 2017-2022 }\end{array}$ & 2017 & 2018 & 2019 & 2020 & 2021 & 2022 & $\begin{array}{l}\text { CAGR } \\
\text { (2017-2022) }\end{array}$ \\
\hline $\begin{array}{llllllll}\text { By Type (EB per Month) } \\
\text { Fixed }\end{array}$ & 67 & 86 & 111 & 141 & 179 & 225 & $27 \%$ \\
Mobile & 10 & 16 & 25 & 36 & 50 & 68 & $47 \%$ \\
\hline
\end{tabular}

(b)

\begin{tabular}{|c|c|c|c|c|c|c|c|}
\hline & 2017 & 2018 & 2019 & 2020 & 2021 & 2022 & $\begin{array}{l}\text { CAGR } \\
(2017-2022)\end{array}$ \\
\hline \multicolumn{8}{|l|}{ Global } \\
\hline Global speed: All handsets & 8.7 & 13.2 & 17.7 & 21.0 & 24.8 & 28.5 & $27 \%$ \\
\hline Western Europe & 16.0 & 23.6 & 31.2 & 37.2 & 43.8 & 50.5 & $26 \%$ \\
\hline Central and Eastern Europe & 10.1 & 12.9 & 15.7 & 19.5 & 22.8 & 26.2 & $21 \%$ \\
\hline Middle East and Africa & 4.4 & 6.9 & 9.4 & 11.2 & 13.2 & 15.3 & $28 \%$ \\
\hline North America & 16.3 & 21.6 & 27.0 & 31.9 & 36.9 & 42.0 & $21 \%$ \\
\hline Asia Pacific & 10.6 & 14.3 & 18.0 & 21.7 & 25.3 & 28.8 & $22 \%$ \\
\hline Latin America & 4.9 & 8.0 & 11.2 & 13.0 & 15.3 & 17.7 & $30 \%$ \\
\hline
\end{tabular}

(c)

Figure 1.1: Projections regarding the number of communications devices and the demand for global mobile traffic; a) Number of electronic communication devices; b) Consumer global mobile internet traffic and c) Average mobile network connection speeds. Source: [1].

number of transmitting and receiving antennas. Thus, the tendency is to use a large number of antennas in base stations (BS) and, where possible, also in user equipment (UE).

Later in [8], communication structures have been proposed and analysed using tens to hundreds of antennas in transmitting and receiving the signals, which have been termed Massive MIMO or LS-MIMO structures. The main advantage obtained with the use of a large number of antennas is in the increased throughput, since the system channel capacity with multiple antennas grows linearly with the minimum number of transmitting and receiving antennas [9]. More appropriately, LS-MIMO is defined as a transmit/receive system which typically utilizes several dozen or even hundreds of antennas in at least one of the communication terminals, generally in BS due to the reduced physical space within the UEs $[10,11]$, causing a single antenna scheme to be adopted in the UEs; on the other hand, a high number of antennas is installed in each BS. Generally speaking, the main benefits of LS-MIMO systems are: 
1. High reliability and high spectral efficiency: considering the reliability, in terms of error probability $\left(P_{e}\right)$, and system capacity, in bps $/ \mathrm{Hz}$, one can compare SISO (single-input single-output), SIMO (singleinput multiple-output) and MIMO. Consider a unicellular system with $N$ antennas in BS and $K$ singleantenna users, in reverse channel with perfect knowledge of channel conditions in BS. In the case of SISO ( $K=N=1$ ), the error probability decreases linearly with the increase of the signal-to-noise ratio (SNR), $\gamma$, effect resulting from fading channel. With the increase in the number of receiver antennas, i.e., SIMO ( $K=1$ and $N>1$ ), there is a spatial diversity gain at the receiver, with that, the error probability decreases at a rate of $\gamma^{-N}$ with increasing SNR [12]. Although the SIMO showed a performance improvement in relation to SISO, regarding channel capacity, both presented a logarithmic growth with increase of the SNR. Consequently, they require a significant increase in power to increase capacity. In the MIMO systems $(K>1$ and $N>1$ ), the probability of error decreases with the SNR on a scale of $\gamma^{-K N}$. In addition, with MIMO, a proportionally linear increase in the channel capacity is obtained by the minimum amount between transmitting and receiving antennas, which exceeds the logarithmic condition obtained in SISO and SIMO [12] cases. With the use of a large number of transmitting and receiving antennas, such as LS-MIMO $(K \gg 1$ e $N \gg 1)$, an approximately exponential decay can be obtained for the error probability, as seen that the probability of error decays in $\gamma^{-K N}$ [12]. As for the capacity of the LS-MIMO channel, it is demonstrated in [13] that in the case $K \gg N, K \rightarrow \infty$, capacity can be approximated as $\mathcal{C} \approx N \log _{2}(1+\gamma)$; while in the case $N \gg K, N \rightarrow \infty$, it is obtained $\mathcal{C} \approx K \log _{2}\left(1+\frac{\gamma N}{K}\right)$. In both cases, the upper bound of the MIMO channel capacity is limited by $[11,13]:$

$$
\log _{2}(1+\gamma N) \leq \mathcal{C} \leq \min (K, N) \log _{2}\left(1+\frac{\gamma \max (K, N)}{K}\right)
$$

One can notice that, in the case of LS-MIMO, it is possible to obtain a large improvement in capacity by only increasing the quantity of antennas $N$ and $K$, which also represents a large increase in spectral efficiency, since it increases throughput in the same frequency band. Analogously, the throughput is increased without raising the transmitted power per user, representing high energy efficiency. Therefore, MIMO systems, especially LS-MIMO, are attractive in both reliability and channel capacity attainable. The table 1.1 summarizes, in a simplified way, the gains in channel reliability and capacity obtained in SISO, SIMO and MIMO systems [12].

Table 1.1: Reliability and capacity in SISO, SIMO and MIMO in fading channels.

\begin{tabular}{ccc}
\hline \hline System type & Error probability $\left(\boldsymbol{P}_{\boldsymbol{e}}\right)$ & Capacity $(\mathcal{C})$, in bps/Hz \\
\hline SISO $(K=N=1)$ & $P_{e} \propto \gamma^{-1}$ & $\mathcal{C}=\log _{2}(1+\gamma)$ \\
SIMO $(K=1$ e $N>1)$ & $P_{e} \propto \gamma^{-N}$ & $\mathcal{C}=\log _{2}(1+\gamma)$ \\
MIMO $(K>1$ e $N>1)$ & & \\
Full-diversity gain & $P_{e} \propto \gamma^{-K N}$ & $\mathcal{C}=\log _{2}(1+\gamma)$ \\
Full-multiplexing gain & $P_{e} \propto \gamma^{-N}$ & $\mathcal{C}=\min (K, N) \log _{2}(1+\gamma)$ \\
\hline \hline
\end{tabular}

2. High energy efficiency: in general, the spectral efficiency in a communication system can be enhanced by increasing the modulation order (e.g., 16-QAM to 64-QAM, QAM - quadrature amplitude modulation) 
or increasing the number of spatial dimensions for signaling (i.e., increasing $K$ ), or even by combining the two conditions. However, a question may arise: it would be more advantageous to use a single antenna system (SISO) with high modulation order (e.g., 64-QAM) or a MIMO system with loworder modulation, e.g., binary phase shift keying (BPSK)? In order to increase spectral efficiency in a given communication system, the augmentation in the number of transmitting antennas, even with low modulation order, is more energy efficient than increasing the modulation order with low number of antennas [12]. An example is shown in Fig. 1.2, where three scenarios were simulated, so that all achieve the same spectral efficiency of $6 \mathrm{bps} / \mathrm{Hz}$ : SISO $(K=M=1)$ in 64-QAM modulation, SIMO ( $K=1$ and $M=6)$ in 64-QAM and MIMO $(K=M=6)$ with BPSK modulation. It is possible to notice that, in the case of SISO 64-QAM, the performance is quite poor and only decreases linearly with the increase of the SNR, condition already predicted according to Table 1.1. By only increasing the number of receiver antennas in SIMO 64-QAM, it is possible to identify a significant performance improvement, caused by spatial diversity gain at the receiver, where the error probability decays in $\gamma^{-M}$ with the SNR. In the performance of the MIMO BPSK system, the spatial diversity gain is similar to the SIMO case, however, it is noted that, for a $10^{-3}$ bit error rate (BER), the MIMO system outperforms the SIMO by about $7 \mathrm{~dB}$, thus presenting better energy efficiency. Therefore, it can be stated that for a given spectral efficiency to be achieved, the option of increasing the number of spatial dimensions for signaling is more advantageous than increasing the order of modulation. This is another condition that favors the use of large-scale antennas, i.e., LS-MIMO.

3. Channel hardening condition: the channel matrix, $\mathbf{H} \in \mathbb{C}^{M \times K}$, follows an i.i.d. (independently identically distributed) distribution. With the increase of its dimensions and keeping fixed the relation $\frac{K}{M}$, the distribution of its singular values tend to become less related to the values of the channel matrix distribution. This is a result of the Marčenko-Pastur Law which states that if the $M \times K$ entries of a matrix $\mathbf{H}$ are i.i.d. of zero mean and variance $\frac{1}{M}$, then the empirical distribution of the eigenvalues of $\mathbf{H}^{H} \mathbf{H}$ and $\mathbf{H H}^{H}$ converge, with $K, M \rightarrow \infty$ and fixed ratio $\frac{K}{M}$, for a deterministic density function [14]. In this sense, it is said that the channel "hardens", becoming progressively deterministic with the increase of the number of antennas. In addition, it is noticed that in the channel hardening condition, the elements of the $\mathbf{H}^{H} \mathbf{H}$ main diagonal become more and more predominant in relation to the other elements of the matrix, allowing a diagonal matrix approximation. The channel hardening condition also results in a number of advantages in large signal processing, such as, for example, the operation of the matrix inversion required by some detection methods can be approximated using series expansion techniques, resulting in a decrease in detection complexity. In addition, channel hardening may imply that simple LS-MIMO detection methods result in quasi-optimal performance [12].

In the work of [8], it is shown that in an asymptotic model of the LS-MIMO scheme in which an infinite number of antennas are assumed in the BS, the following conditions can be obtained: the effects of background noise and fast fading of the channel disappear, the transmission rate and number of UEs become independent of cell size, spectral efficiency becomes independent of bandwidth and the energy required by bit transmission tends to zero. However, these are fully achieved as long as this unlimited number of antennas in BS meets a fixed number of UEs. In the work of [15] a communication system using LS-MIMO is proposed capable 


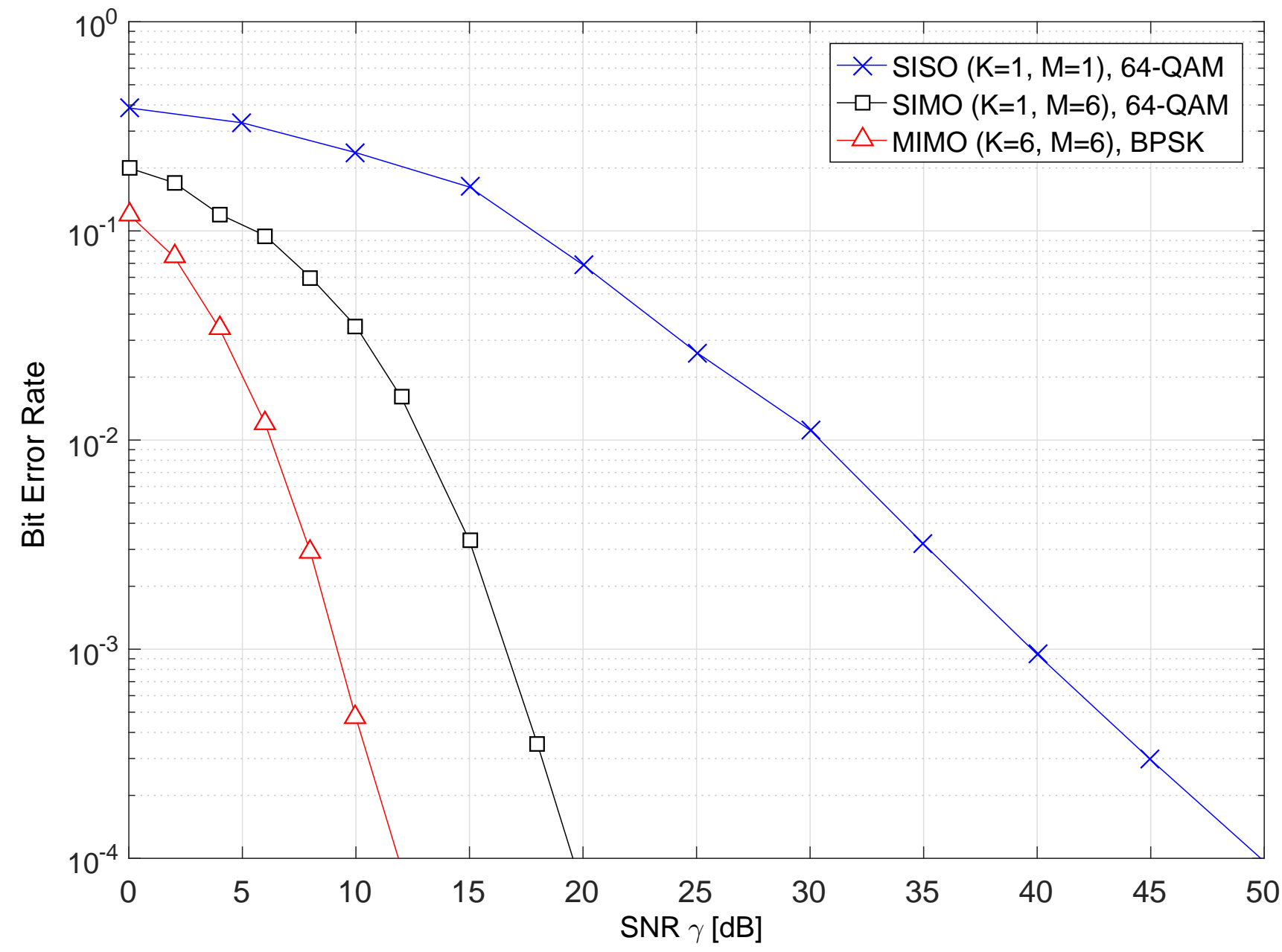

Figure 1.2: Comparative performance between SISO $1 \times 1$ 64-QAM, SIMO $1 \times 6$ 64-QAM and MIMO $6 \times 6$ BPSK, with spectral efficiency of $6 \mathrm{bps} / \mathrm{Hz}$ in fading channel. 
of achieving a transmission rate of up to 4 times compared to current 4G LTE (long term evolution). On the other hand, with an increasing number of antennas in BSs and UEs, spectral efficiency can become quite compromised by the interference of simultaneous transmissions from neighboring areas [16]. In addition, the implementation of large numbers of antennas in the UEs becomes a difficult task to be feasible due to the reduced space found inside these devices, even considering carrier frequencies of third and fourth generation systems (3G, 4G, respectively) keeping in mind that the ultimate goal of the LS-MIMO scheme is to offer all the benefits of conventional MIMO, but on a large scale. In this way, it is noticed that there are still open questions regarding the implementation of LS-MIMO systems.

In addition, multiuser schemes can be combined with MIMO systems, creating the so-called MU-MIMO (multiuser-MIMO) in which the BS communicates with several UEs simultaneously within its spatial domain. As an added benefit, total capacity can be increased by pre-decoding and decoding in the BS, even when the UE has a small number of antennas [17]. Therefore, the multi-user schema combined with LS-MIMO, called MU-LS-MIMO, is a very promising proposal, and currently intense efforts are being made in the literature, such as [15], in which a MU-LS-MIMO system is proposed, called FD-MIMO (Full-Dimension MIMO). In this transmission-reception scheme, 32 antennas were employed, achieving a mean transmission rate gain in the cell from 2 to 3.6 times compared to the conventional 4G-LTE system.

As in LS-MIMO, the main advantage reached with the MU-LS-MIMO scheme is the high transmission rate achieved, however, the estimation of the channel parameters is a key point in this process. A large number of antennas also generate a high number of wireless channels to be estimated. In order to do this, some of the most promising works use the transmission of the channel's current state parameters as part of the transmitted information, usually called a pilot signal [8]. In this way, the problem of channel estimates is bypassed. In addition, it has been found that a sufficient increase in the number of BS antennas in an LS-MIMO system is able to mitigate the damaging effect of the error in channel estimates due to the condition of channel hardening, and these results become very interesting in scenarios with imprecise channel estimates due to high noise power, ie, reduced SNR. On the other hand, a major problem of inter-cellular interference in LS-MIMO TDD (time division duplexing) systems has also been reported due to the use of the same spreading pilots sequences (PS) in different cells. The problem is called pilot contamination, persisting even in scenarios with infinite number of antennas in BS.

\subsection{Framework of main contributions in LS-MIMO signal detection}

This section discusses the relevant techniques currently found in the literature for signal detection in LS-MIMO systems. A summary framework containing the contributions related to the techniques is presented in Table 1.2. In Subsection 3.2.3, the computational complexities of those detection strategies are compared.

\subsection{Problem Formulation}

It is noted that LS-MIMO systems offer a number of advantages that meet the needs of modern wireless communication systems. On the other hand, there are technical challenges that must be solved for the 
Table 1.2: Framework of the most relevant LS-MIMO detection techniques found in literature

\begin{tabular}{|c|c|c|c|}
\hline Year & Authors & Detector & Main contributions \\
\hline 2008 & Vardhan et al. [18] & $\begin{array}{l}\text { Likelihood Ascent } \\
\text { Search (LAS) }\end{array}$ & $\begin{array}{l}\text { It proposes the LAS heuristic detection technique in } \\
\text { code division multiple access (CDMA) multiuser detec- } \\
\text { tion (MuD) systems. The technique demonstrates low } \\
\text { complexity and good performance results and is also } \\
\text { applicable for LS-MIMO systems, however, it does not } \\
\text { escape from local minima, which compromises its per- } \\
\text { formance under certain conditions. }\end{array}$ \\
\hline 2009 & Rajan et al. [19] & $\begin{array}{c}\text { Reactive Tabu Search } \\
\text { (RTS) }\end{array}$ & $\begin{array}{l}\text { It proposes the RTS heuristic detection technique in } \\
\text { LS-MIMO systems. It is demonstrated that the RTS } \\
\text { technique has quasi-optimum performance with large } \\
\text { number of antennas and presents an improved strat- } \\
\text { egy for local minima escape, however its performance } \\
\text { is greatly degraded at high modulation orders. }\end{array}$ \\
\hline 2010 & Som et al. [20] & FG-BP & $\begin{array}{l}\text { It proposes a message passing (MP) algorithm through } \\
\text { BP applied in Factor Graph (FG) graphical model, } \\
\text { called FG-BP detector. The FG-BP demonstrates } \\
\text { quasi-optimal performance results with increase of an- } \\
\text { tennas, which is favorable to LS-MIMO systems. Lower } \\
\text { complexity order compared to MRF-BP. }\end{array}$ \\
\hline 2011 & Srinidhi et al. [21] & $\begin{array}{c}\text { Layered Tabu Search } \\
\text { (LTS) }\end{array}$ & $\begin{array}{l}\text { It proposes the technique of LTS heuristic detection in } \\
\text { LS-MIMO systems that applies the Tabu Search (TS) } \\
\text { technique in layers, in order to solve the performance } \\
\text { degradation in high modulation orders presented by } \\
\text { RTS. }\end{array}$ \\
\hline 2013 & Datta et al. [2] & $\begin{array}{l}\text { Mixed Gibbs Sampling } \\
\text { (MGS) }\end{array}$ & $\begin{array}{l}\text { It proposes the MGS detector to solve the performance } \\
\text { problem (called stalling problem) presented by the con- } \\
\text { ventional GS technique. It is demonstrated that MGS } \\
\text { solves the proposed problem and presents near-optimal } \\
\text { performance under low modulation orders. }\end{array}$ \\
\hline 2015 & Guo et al. [22] & $\begin{array}{l}\text { Relaxation Iteration } \\
\text { Method (RID), called } \\
\text { RID-MMSE }\end{array}$ & $\begin{array}{l}\text { It proposes the RID-MMSE technique to calculate } \\
\text { MMSE detection through iterative convergence, in LS- } \\
\text { MIMO scenarios, with reduced complexity when com- } \\
\text { pared to MMSE and marginally similar performance. } \\
\text { Like MMSE, it performs poorly with high system load- } \\
\text { ing. }\end{array}$ \\
\hline
\end{tabular}


implementation of such systems, among which we can highlight:

- Allocation of a large number of antennas and RF (radio-frequency) chains: the physical allocation of a large number of antennas is a challenging task, even when it comes to BS. In the case of the UEs, the difficulty becomes even greater, since many of the mobile devices have small size (of the order of centimeters). Given a certain available physical space, increasing the number of antennas reduces the distance between them, which can increase spatial correlation, and consequently reduce channel capacity. As a general rule, it is assumed that a minimum distance of $\frac{\lambda}{2}$ (where $\lambda$ is the carrier wavelength) between the antennas is sufficient to guarantee a spatial correlation approximately null between the antennas. Although it does not represent the central topic of this Doctoral work, it is one of the LS-MIMO problems that have been explored in the literature, and the proposed solutions are divided essentially in the following areas:

- High frequency carriers: using carriers operating at high frequencies (i.e., above $10 \mathrm{GHz}$ ) allows the allocation of a larger number of antennas in a given physical space, since the wavelength is inversely proportional to the frequency (e.g., $\frac{\lambda}{2}=3 \mathrm{~cm}$ in $5 \mathrm{GHz}$ ), which allows the allocation of a larger number of antennas while maintaining the minimum inter-antenna spacing that ensures the decorrelation. In this sense, it is possible to obtain wavelengths in the order of millimeters, called in millimeter wave $(\mathrm{mmW})$ in literature [23-27].

- Array of antennas using 3 dimensions (volume): in addition to the one-dimensional or twodimensional antenna arrays, it is possible to use the third dimension, in order to explore the volume of these arrangements. This approach is called in the literature of MIMO cubes in which the multiple antennas are arranged in cubic-shaped structures [28-30].

- Compact antenna arrays: in this approach, the antenna spacing less than $\frac{\lambda}{2}$ is used in order to explore solutions that bypass the degradation caused [31-34].

- Spatial modulation: spatial modulation (SM) is a modulation technique in which the selection of the active antennas relates to the transmitted information, so that the amount of RF chains can be reduced without compromising the spectral efficiency. In addition, SM can reduce the complexity, size and cost of implementing the system [24, 35-37].

- Problems in multicellular operation: the LS-MIMO operation in multicellular scenario brings with it a series of operational challenges to be solved. As already mentioned, one of the main problems related to the multicellular LS-MIMO operation is the intercellular interference caused by the pilot contamination. In addition to this central problem, it may be mentioned the following studies fields: cell size, management of resource allocation between cells, techniques to mitigate inter-user and intercellular interference. Currently, one of the most promising approaches to solving most of these problems is through cooperative networks, also known as BS cooperation, in which there is exchange of information among BSs, for example, to combat system intercellular interference [38-41].

- Signal processing techniques with low computational complexity: due to the high dimension of the LS-MIMO problem, low complexity algorithms for signal detection, precoding, channel estimation, among others, become essential for the feasibility of implementing such systems [42-46]. This aspect 
represents the central scope of this Doctoral work, more precisely the efficient signal detection with very low computational complexity. Further details on the contributions achieved with this work, in addition to the resulting publications are found in the Sec. 1.6.

\subsection{Scope and Objectives}

\subsubsection{Scope}

From the fundamental aspects and considerations presented in Section 1.1, the main concepts considered in this work can be highlighted:

- LS-MIMO: communication systems employing transmitting and receiving antennas in large-scale are part of the central theme of this work;

- System performance: main metric of analysis and optimization described in this work, measured through the error rate;

- Efficient signal detection: the search for detection techniques that result in improved computational complexity versus performance is one of the main scopes of this work;

- Low complexity techniques: signal processing techniques that employ few mathematical operations to solve the problems of interest in the data detection are targeted in this study, in view of the considerations given in relation to LS-MIMO systems.

\subsubsection{Objetives}

This Doctoral Thesis has the objetives listed below:

1. General: Systematic analysis of promising methods of detection in multiple access LS-MIMO systems, including the analysis of the following metrics: (a) performance (b) computational complexity (c) performance it versus complexity tradeoff as well as the (d) diversity gain analysis of the proposed schemes, seeking the construction of a viability framework for the implementation of these transmission/detection schemes in large-scale MIMO systems.

2. Specific:

(a) Numerical analysis (simulation) of multiuser systems under LS-MIMO baseband channels through promising techniques for signal detection;

(b) Proposition of strategies that aim at the improvement of the performance $\times$ complexity tradeoff, considering promising detection techniques found in the literature;

(c) Analysis of performance $\times$ computational complexity relative to each transmission scheme analysed or proposed; 


\subsection{Organization of the Doctoral Thesis}

This Thesis is organized as follows: Chapter 2 makes an introduction to the main scope of the Thesis by adopting a conventional MIMO system in order to establish a relationship with LS-MIMO schemes. The introductory system model is presented, in addition to modeling for errors in channel estimates and correlation between antennas. Then, the main conventional detectors arranged in the literature are presented and the pre-processing lattice reduction (LR) technique is explained. Besides that, the main results of the performance-complexity analysis of known MIMO detectors assisted by the LR technique, considering correlated channels and imperfect estimates of channel conditions are discussed. These results portray the first problem investigated in this work and are part of published work, which is in its full version in the Appendix A.

Chapter 3 defines the LS-MIMO system model adopted, which refers to the background for the main research of this Thesis. In addition, a summary table is presented, relating the computational complexities of the detection techniques studied in this research, in order to subsidize the choice of strategies investigated and contributions achieved during this Doctoral Thesis.

In Chapter 4 is presented the main contributions regarding the analysis of the MP LS-MIMO detector. Such detector is based on BP algorithm through MRF and FG graphical models. The message damping (MD) method, which is known to increase the performance of BP-based detectors with no extra computational complexity [20], is applied on both MRF-BP and FG-BP approaches. A numerical analysis is made in an extensive range of LS-MIMO scenarios and an optimal value for damping factor (DF) is proposed. The results of this chapter are from the published paper, which is available in the Appendix C.

Chapter 5 brings the main results and contributions regarding the proposed multiple sampling in the MGS detector, named averaged MGS (aMGS). The aMGS aims to reduce the impact caused by the noisy solution from the mixture used in the MGS, especially when a high order of modulation is considered. Numerical results showed an algorithm convergence improvement, which resulted in a better performance $\times$ complexity tradeoff, especially when the system loading is high. This proposed scheme is part of the published work in the Appendix D. In addition, a second noisy solution mitigation approach was proposed, called $d$-sMGS ( $d$-simplified MGS). The $d$-sMGS detector is based on the neighborhood restriction of the noisy solution at a distance $d$, considering the constellation adopted. Regarding aMGS and MGS, the $d$-sMGS detector presented better performance $\times$ complexity tradeoff in high SNR regions and the increase in distance $d$ showed to be an interesting choice with increasing problem dimensions. The proposition of the $d$-sMGS detector is part of the paper currently under R1 revision process and a copy of this work is presented in the Appendix $\mathrm{E}$.

Finally, in Chapter 6 the main conclusions delineated by the development of the works investigated during this $\mathrm{PhD}$ Thesis are placed. The main results obtained are summarized, highlighting the contributions.

Furthermore, it is worth noting that Appendices $A$ to $E$ refer the main results obtained in the form of scientific papers. The three first papers are already published in specialized journals, while the fourth is under R1 review. 


\subsection{Summary of contributions and generated publications}

In the following, the main contributions achieved during this Doctoral work are listed. Also, it is summarized the scientific production directly and indirectly related to this Thesis, since the student admission in the Doctorate Program of the EPUSP (March 2014) until the present moment.

\subsubsection{Contributions of this Thesis}

1. Lookup table procedure on Sphere Decoder aided by Lattice Reduction: it is proposed a lookup table procedure during the tree search on a MIMO sphere decoder (SD) aided by LR. Since the symbols constellation in the LR reduced basis has a different structure from the original one, the conventional SD tree search cannot be performed. Thus, the proposed procedure stores previously calculated values during the tree search, avoiding that they are recalculated, which results in computational complexity reduction.

2. Damping factor analysis on Message Passing detectors: an optimal DF is proposed, which is a function of the number of antennas and SNR value, based on an extensive analysis of the message damping method impact on the BER performance. The proposed optimal DF has demonstrated a promising performance versus complexity tradeoff for MRF-BP detector, especially in LS-MIMO scenarios.

3. Multiple sampling scheme on Mixed Gibbs Sampling detector: it is proposed a procedure that considers the output of each detected position in iterative symbol detection process, called coordinate updating process, as an average between multiple samples, which if called multiple sampling (MS) strategy. The proposed scheme demonstrated to enhance the LS-MIMO MGS algorithm convergence, since the negative impact of the random solutions, which is used in the MGS detection, is alleviated.

4. Neighborhood limitation strategy on Mixed Gibbs Sampling detector: with the same goal of multiple sampling scheme, it is proposed a strategy that limits the range of the MGS random solutions, called neighborhood limitation (NL) scheme, and also result in convergence gain of the MGS algorithm. Compared to the MS strategy, the NL resulted in better performance-complexity tradeoff when a high LS-MIMO system loading is considered.

\subsubsection{Publications directly associated to the theme}

1. A. M. Mussi, B. F. Costa, T. Abrão. Efficient Lattice Reduction Aided Detectors Under Realistic MIMO Channels. Full paper published in the Wireless Personal Communications journal $(\mathrm{IF}=1.200$, B1-Eng. IV in QUALIS-CAPES), in October 2016. Copy of the published article is presented in Appendix A.

2. A. M. Mussi, T. Abrão. Analysis of Message Passing Detectors for Large Scale MIMO Systems. Full paper published in the IET Signal Processing journal (IF=1.250, A2-Eng. IV in QUALIS-CAPES), in July 2017. Copy of the published article is presented in Appendix C. 
3. A. M. Mussi, T. Abrão. Multiple restarts mixed Gibbs sampling detector for large-scale antenna systems. Full paper published in the IET Signal Processing journal (IF=1.250, A2-Eng. IV in QUALISCAPES), in November 2018. Copy of the published article is presented in Appendix D.

4. A. M. Mussi, T. Abrão. Mitigating the noisy solution impact of mixed Gibbs sampling detector in high-order modulation large-scale MIMO systems. Full paper under R1 review in the EURASIP Journal on Advances in Signal Processing journal (IF=1.639, A2-Eng. IV in QUALIS-CAPES), resubmitted in January 2019. Copy of the R1 submitted version in Appendix E.

5. A. M. Mussi, T. Abrão. Damping Factor Analysis on Large Scale MIMO Detector Based on Message Passing. Full paper published in the "XXXIV Simpósio Brasileiro de Telecomunicações" (SBrT2016), in August 2016. Copy of the published conference article is presented in Appendix B.

\subsubsection{Publications indirectly associated to the theme}

1. R. T. Kobayashi, A. M. Mussi, T. Abrão. BER minimisation via optimal power allocation and eigenbeamforming in MIMO systems. Full paper published in the Telecommunication Systems journal (IF=1.527, A2-Eng. IV in QUALIS-CAPES), in July 2018.

2. R. T. Kobayashi, A. M. Mussi, T. Abrão. Near-Perfect Reconstruction Short Length Pulses for FBMC systems: Re-optimizing OFDP design via Semi-Definite Programming. Full paper accepted for publication in the IET Signal Processing journal (IF=1.250, A2-Eng. IV in QUALISCAPES), accepted in February 2019.

3. J. L. Negrão, A. M. Mussi, T. Abrão. Semidefinite Relaxation for Large Scale MIMO Detection. Full paper published in the "XXXIV Simpósio Brasileiro de Telecomunicações" (SBrT2016), in August 2016. 


\section{Introductory investigation: conventional MIMO to large-scale MIMO}

This chapter introduces the central problem of LS-MIMO signal detection systems by adopting a MIMO conventional point-to-point system. This choice aims to investigate the basic characteristics of conventional MIMO in order to establish a relationship with large-scale antennas systems.

In addition, conventional MIMO detectors are presented, ranging from linear to exhaustive and tree searching. In addition, we discuss the numerical results related to the first work developed in this Thesis dealing with the performance of conventional detectors and sphere decoder (SD) aided by the LR preprocessing technique in hostile channels.

Initially, a brief bibliographic review is contextualized. Next, the adopted point-to-point MIMO system is described, in addition to the model for channel estimation error and antenna correlation. Then, conventional detectors are presented, besides a detailed demonstration of the proposed detection procedure for the LR-aided SD detector. At the end, the main numerical results obtained are presented and discussed, followed by the main conclusions.

\subsection{Introduction}

In MIMO signal detection, the LR acts as a preprocessing technique which results in improvement in the system performance-complexity tradeoff due to the quasi-orthogonalization of the MIMO channel achieved with the LR technique, which, in turn, reduces considerably the detector processing task. The LR is a mathematical concept deployed to solve many problems involving lattice points. For instance, in the MIMO signal detection problem, the LR can be used to improve the channel matrix conditioning, thus allowing the use of simpler detector structures [47]; in other words, reduction in computational complexity can be achieved with the LR technique aggregation while maintain overall system performance acceptable [48], [49].

The SD detector can achieve the exact ML detection performance, but requires considerable amount of computations compared with various suboptimal detections. Furthermore, in scenarios with large number of transmit and receive antennas with high order modulation (e.g., 64-QAM, 128-QAM, and so on) the SD becomes impracticable. To reduce the overall SD complexity, a lattice-reduction-aided sphere decoding (LRSD) was proposed during this Doctoral work. Since the SD already achieves optimal performance, the LR-SD would not result in performance improvement; nevertheless the LR preprocessing technique would aim in the SD complexity reduction, specially under large dimensions of detection, e.g., considering MIMO channels with high order modulations and number of transmitting-receiving antennas. 
The concept of LR-SD for MIMO detections has been mentioned in recent literature, particularly in [5054]. In [50,51], lattice reduction is considered not being useful for the sphere decoding for MIMO detections, which is a closest lattice vector problem over a (finite) subset of the infinite lattice. The reason given is that the lattice transforming matrix often destroys the properties of the subset, and the result of LR-SD, which is the closest lattice point to the received signal point, is not necessarily the ML detection result, which is the closest lattice point to the received signal point with the finite subset as the search space. The authors of [52] believe that although the LR-aided SD for MIMO detections can achieve the ML detection BER performance, it can not offer the complexity reduction because the search may be repeated many times to exclude the undesired lattice points. However, recent theoretical analysis in [53] confirmed that the LR-SD for MIMO detections is able to reduce the computational complexity significantly, but the authors admit it assumes a performance gap to the $M L$ detection because sometimes its search result is not equal to the $M L$ detection result. The computational complexity reduction achieved by the LR-SD detector compared to the conventional SD is also pointed out in $[55,56]$; the reason is that the preprocessing technique is able to reduce the total number of search paths. Also, the LR-SD is used in [54], but the exact ML performance can not be achieved essentially owing to the same reason as presented in [53]. Notice that in most of the studies involving LR-SD MIMO detector perfect channel condition scenarios (pCSI and uncorrelated channels) have been considered. Specifically in the actual research, the LR-SD MIMO detector is analyzed under unfavorable channel conditions.

\subsection{Conventional MIMO System Model}

In this chapter, a baseband linear non-line-of-sight (NLOS) transmission system with $N_{t}$ inputs and $N_{r}$ outputs is adopted, which is called conventional point-to-point MIMO and is described in Fig. 2.1. The disposition of antennas in both the transmitter and the receiver is through an uniform linear array (ULA). The ULA in the transmitter can either represent a single device with multiple antennas or, from a multiuser point of view, as a cluster of users confined in an environment (for example, a corridor).

The matrix model for the system under investigation is

$$
\mathbf{y}_{c}=\mathbf{H}_{c} \mathbf{s}_{c}+\boldsymbol{\eta}_{c}
$$

where $\mathbf{H}_{c}$ represents an $N_{r} \times N_{t}$ fading coefficients matrix following a Rayleigh distribution (for amplitudes) representing NLOS point-to-point communication, $\mathbf{s}_{c}$ is the vector $N_{t} \times 1$ of transmitted symbols and $\boldsymbol{\eta}_{c}$ is the independent additive white Gaussian noise (AWGN). The AWGN vector $\boldsymbol{\eta}_{c}$ is assumed to be a complex Gaussian random variable with zero mean and variance given by:

$$
\mathbb{E}\left[\boldsymbol{\eta}_{c} \boldsymbol{\eta}_{c}^{H}\right]=\sigma^{2} \mathbf{I}_{N_{r}}
$$

where $\sigma^{2}$ is the noise variance at each receive antenna.

The availability of channel status information in BS is considered in two modes: perfect channel state information $(\mathrm{pCSI})$ or imperfect channel state information (iCSI). The estimation errors related to the iCSI are described in Subsec. 2.2.1. The correlation between antennas is depicted in Subsec. 2.2.2. The average 


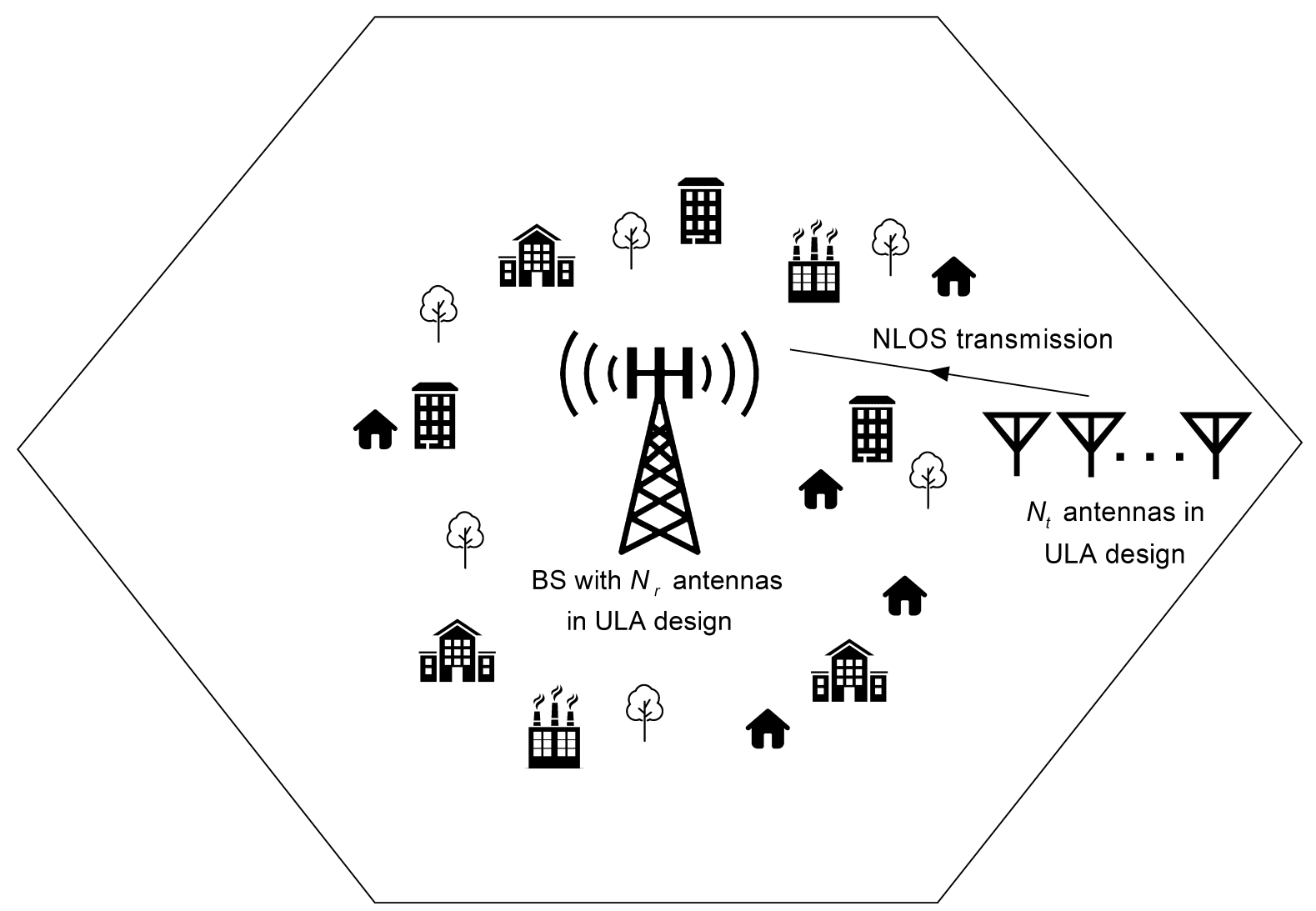

Figure 2.1: Introdutory conventional MIMO point-to-point communication system with $N_{t}$ transmitting antennas and $N_{r}$ receiving antennas.

received SNR at each receive antenna can be modeled as:

$$
\gamma=\frac{N_{t} P_{\mathrm{s}}}{\sigma^{2}}
$$

where $P_{\mathrm{s}}$ is the average power of the received symbols.

The equivalent real-form [57] received signal can be written as:

$$
\mathbf{y}=\mathbf{H s}+\boldsymbol{\eta}
$$

where $\mathbf{y} \in \mathbb{R}^{2 N_{r} \times 1}, \mathbf{H} \in \mathbb{R}^{2 N_{r} \times 2 N_{t}}, \mathbf{s} \in \mathbb{R}^{2 N_{t} \times 1}, \boldsymbol{\eta} \in \mathbb{R}^{2 N_{r} \times 1}$, and defined as:

$$
\begin{aligned}
\mathbf{H}= & {\left[\begin{array}{rr}
\Re\left\{\mathbf{H}_{c}\right\} & -\Im\left\{\mathbf{H}_{c}\right\} \\
\Im\left\{\mathbf{H}_{c}\right\} & \Re\left\{\mathbf{H}_{c}\right\}
\end{array}\right] } \\
\mathbf{s}= & {\left[\begin{array}{c}
\Re\left\{\mathbf{s}_{c}\right\} \\
\Im\left\{\mathbf{s}_{c}\right\}
\end{array}\right], \quad \boldsymbol{\eta}=\left[\begin{array}{c}
\Re\left\{\boldsymbol{\eta}_{c}\right\} \\
\Im\left\{\boldsymbol{\eta}_{c}\right\}
\end{array}\right], \quad \mathbf{y}=\left[\begin{array}{c}
\Re\left\{\mathbf{y}_{c}\right\} \\
\Im\left\{\mathbf{y}_{c}\right\}
\end{array}\right] }
\end{aligned}
$$

where $\Re\{\cdot\}$ and $\Im\{\cdot\}$ denotes the real and imaginary parts of a complex number, respectively.

\subsubsection{Imperfect Channel State Information}

The model adopted in this study incorporates statistical errors to the estimate received in BS, which is called iCSI. It is important to emphasize that this channel error estimation model comprises both module and phase, being the last more harmful for the detection process. Thus, the estimated channel matrix can be modeled 
as:

$$
\widehat{\mathbf{H}}_{c}^{\prime}=\mathbf{H}_{c}+\varepsilon
$$

where $\varepsilon=\varepsilon_{\mathrm{I}}+j \varepsilon_{\mathrm{Q}}$ is a stochastic complex error, with in-phase and quadrature components assumed i.i.d. Gaussian variables, with $\varepsilon_{\mathrm{I}}$ and $\boldsymbol{\varepsilon}_{\mathrm{Q}} \sim \mathcal{N}\left(0, \sigma_{\epsilon}^{2} / 2\right)$. Also, this estimation must be normalized by $\left(1+\sigma_{\epsilon}^{2}\right)^{-1}$, as suggested by [58]; therefore the estimated MIMO channel matrix $\widehat{\mathbf{H}}$ becomes:

$$
\widehat{\mathbf{H}}_{c}=\frac{\mathbf{H}_{c}+\varepsilon}{1+\sigma_{\varepsilon}^{2}}
$$

This normalization is necessary aiming to not change the original average power channel characteristics. Furthermore, in this modeling, the variance $\sigma_{\varepsilon}^{2}$ is assumed inversely proportional to the SNR:

$$
\sigma_{\varepsilon}^{2}=\frac{n_{\varepsilon}}{\gamma}
$$

where $n_{\varepsilon}$ represents the channel error factor.

\subsubsection{Antenna Correlation}

One important class of MIMO channel model assumes that the correlation between the transmitting antennas $(T x)$ is independent of the correlation among receiving antennas $(R x)$; hence, admitting a Rayleigh flat-fading MIMO channel, [59], one can express the fading coefficients matrix:

$$
\mathbf{H}_{c}=\sqrt{\mathbf{R}_{\mathrm{H}, \mathrm{Rx}}} \mathbf{G} \sqrt{\mathbf{R}_{\mathrm{H}, \mathrm{Tx}}}
$$

where $\mathbf{G} \in \mathbb{C}^{\mathrm{N}_{\mathrm{r}} \times \mathrm{N}_{\mathrm{t}}}$ is an i.i.d. complex Gaussian zero-mean and unit variance elements. The correlation matrices $\mathbf{R}_{\mathrm{H}, \mathrm{Tx}} \in \mathbb{R}_{\mathrm{N}_{\mathrm{t}} \mathrm{N}_{\mathrm{t}}}$ and $\mathbf{R}_{\mathrm{H}, \mathrm{Rx}} \in \mathbb{R}_{\mathrm{N}_{\mathrm{r}} \mathrm{N}_{\mathrm{r}}}$ denote correlation observed among the transmitter antennas and receiver antennas, respectively. Considering an ULA on the transmitter and receiver, the matrices $\mathbf{R}_{\mathrm{H}, \mathrm{Tx}}$ and $\mathbf{R}_{\mathrm{H}, \mathrm{Rx}}$ can be written as:

$$
\begin{aligned}
\mathbf{R}_{\mathrm{H}, \mathrm{Tx}} & =\left[\begin{array}{ccccc}
1 & \rho_{\mathrm{Tx}} & \rho_{\mathrm{Tx}}^{4} & \cdots & \rho_{\mathrm{Tx}}^{\left(N_{t}-1\right)^{2}} \\
\rho_{\mathrm{Tx}} & 1 & \rho_{\mathrm{Tx}} & \cdots & \vdots \\
\rho_{\mathrm{Tx}}^{4} & \rho_{\mathrm{Tx}} & 1 & \cdots & \rho_{\mathrm{Tx}}^{4} \\
\vdots & \vdots & \vdots & \ddots & \rho_{\mathrm{Tx}} \\
\rho_{\mathrm{Tx}}^{\left(N_{t}-1\right)^{2}} & \cdots & \rho_{\mathrm{Tx}}^{4} & \rho_{\mathrm{Tx}} & 1
\end{array}\right], \\
\mathbf{R}_{\mathrm{H}, \mathrm{Rx}} & =\left[\begin{array}{ccccc}
1 & \rho_{\mathrm{Rx}} & \rho_{\mathrm{Rx}}^{4} & \cdots & \rho_{\mathrm{Rx}}^{(N-1)^{2}} \\
\rho_{\mathrm{Rx}} & 1 & \rho_{\mathrm{Rx}} & \cdots & \vdots \\
\rho_{\mathrm{Rx}}^{4} & \rho_{\mathrm{Rx}} & 1 & \cdots & \rho_{\mathrm{Rx}}^{4} \\
\vdots & \vdots & \vdots & \ddots & \rho_{\mathrm{Rx}} \\
\rho_{\mathrm{Rx}}^{(N-1)^{2}} & \cdots & \rho_{\mathrm{Rx}}^{4} & \rho_{\mathrm{Rx}} & 1
\end{array}\right],
\end{aligned}
$$

where $\rho_{\mathrm{Tx}}$ and $\rho_{\mathrm{Rx}}$ are the normalized correlation index in $\mathrm{Tx}$ and $\mathrm{Rx}$, respectively. Note that a totally uncorrelated scenario means $\rho_{\mathrm{Tx}}=\rho_{\mathrm{Rx}}=0$, while a fully correlated scenario implies $\rho_{\mathrm{Tx}}=\rho_{\mathrm{Rx}}=1$.

In this chapter, an equivalent antenna correlation in $\mathrm{Tx}$ and $\mathrm{Rx}$ is considered, thus, for simplicity, $\rho_{\mathrm{Tx}}=$ 
$\rho_{\mathrm{Rx}}=\rho$.

\subsection{Conventional MIMO Detectors}

In the sequel, classical MIMO detectors found in the literature are revisited, including linear MIMO detectors: matched filter (MF), zero forcing (ZF), minimum mean squared error (MMSE); besides the successive interference cancellation (SIC), maximum likelihood (ML), sphere decoder (SD), and the sorted QR decompositionbased MIMO detectors are also presented.

The techniques presented in this section can be either calculated in the complex or real domain. In order to simplify the variables nomenclature, the equivalent real-valued system model will be considered.

\subsubsection{Linear MIMO Detection}

The linear detection scheme in BS is given by multiplying the received signal, $\mathbf{y} \in \mathbb{Z}^{2 N_{r} \times 1}$, by a linear detection matrix, $\mathbf{W} \in \mathbb{Z}^{2 N_{r} \times 2 N_{t}}$. Considering the real-valued system model for UL transmission, Eq. (2.4), the estimated symbols vector in the linear detection can be written as:

$$
\hat{\mathbf{s}}=\mathbf{W}^{H} \mathbf{y}=\mathbf{W}^{H} \mathbf{H} \mathbf{s}+\mathbf{W}^{H} \boldsymbol{\eta}
$$

From Eq. (2.12), the estimated symbol from the $k$ th user, $s_{k}$, can be given by:

$$
\hat{s}_{k}=\underbrace{\mathbf{w}_{k}^{H} \mathbf{h}_{k} s_{k}}_{\text {desired signal }}+\underbrace{\sum_{m \neq k}^{N_{t}} \mathbf{w}_{m}^{H} \mathbf{h}_{m} s_{m}}_{\text {inter-user interference }}+\underbrace{\mathbf{w}_{k}^{H} \boldsymbol{\eta}}_{\text {modified background noise }}
$$

where $\mathbf{w}_{k}$ denotes the $k$ th column of the linear detection matrix $\mathbf{W}$. Below, the linear detectors MF, ZF and MMSE are described.

\section{Matched Filter Detector}

The MF represents the simplest linear detection technique. In the detection of a given user symbol, interference from other users is treated simply as noise. The linear detection matrix MF is given by:

$$
\mathbf{W}_{\mathrm{MF}}^{H}=\mathbf{H}^{H}
$$

The Fig. 2.6 exemplifies the BER performance results of linear detectors in BPSK modulation. It can be observed that the simple MF detection technique is not sufficient to solve the MIMO detection problem in heavily loaded scenarios, e.g., for $N_{t}=N_{r}$ antennas.

\section{ZF Detector}

The ZF detector is a linear detector based on the cancellation of inter-user interference, however, the background noise effect is ignored. Through the pseudo-inverse of the channel matrix, $\mathbf{H}^{\dagger}$, the ZF detector 
completely cancels multiuser interference by projecting each inter-user interference into the orthogonal complement of the desired signal. In this case, the linear detection matrix is given by the pseudo-inverse of the channel:

$$
\mathbf{W}_{\mathrm{ZF}}^{H}=\left(\mathbf{H}^{H} \mathbf{H}\right)^{-1} \mathbf{H}^{H}
$$

And the respective detected symbols by the detector ZF can be simply obtained as:

$$
\begin{aligned}
& \hat{\mathbf{s}}_{\mathrm{ZF}}=\mathbf{W}_{\mathrm{ZF}}^{H} \mathbf{y}=\left(\mathbf{H}^{H} \mathbf{H}\right)^{-1} \mathbf{H}^{H} \mathbf{H} \mathbf{s}+\left(\mathbf{H}^{H} \mathbf{H}\right)^{-1} \mathbf{H}^{H} \boldsymbol{\eta} \\
& \hat{\mathbf{s}}_{\mathrm{ZF}}=\mathbf{s}+\left(\mathbf{H}^{H} \mathbf{H}\right)^{-1} \mathbf{H}^{H} \boldsymbol{\eta}
\end{aligned}
$$

Since $\mathbf{W}_{\mathrm{ZF}}^{H} \mathbf{H}=\mathbf{I}_{2 N_{t}}$, the transformation $\mathbf{W}_{\mathrm{ZF}}^{H} \mathbf{y}$ provided the complete cancellation of inter-user interference in UL. On the other hand, it is noted by Eq. (2.16) that background noise was amplified during the interference elimination process, which causes significant degradation of performance, especially in low SNR scenarios. The per-symbol complexity of the ZF detection algorithm is quadratic with the number of transmitting antennas, i.e. $\mathcal{O}\left(N_{t}^{2}\right)$, due to the matrix inversion operation. Despite acceptable computational complexity, its performance is degraded in scenarios with increasing number of Tx antennas and high system loading, as seen in Fig. 2.6, where the ZF detector is presented with BPSK modulation, attesting not to be an appropriate technique for LS-MIMO scenarios.

\section{MMSE Detector}

The MMSE detector is a detector in which its linear detection matrix is obtained from the criterion of mean squared error (MSE) between the transmitted symbols vector, $\mathbf{s}$, and the transformed received signal vector, $\mathbf{W}_{\text {MMSE }}^{H} \mathbf{y}$. More precisely,

$$
\mathbf{W}_{\mathrm{MMSE}}^{H}=\min _{\mathbf{W} \in \mathbb{Z}^{2 N_{r} \times 2 N_{t}}} \mathbb{E}\left\{\left\|\mathbf{W}^{H} \mathbf{y}-\mathbf{s}\right\|\right\}
$$

where $\mathbb{E}\{\cdot\}$ is the statistical expectation operator.

The solution for Eq (2.17) is given by [60]:

$$
\mathbf{W}_{\text {MMSE }}^{H}=\left(\mathbf{H}^{H} \mathbf{H}+\sigma^{2} \mathbf{I}_{2 N_{t}}\right)^{-1} \mathbf{H}^{H}
$$

And the detected signal by the MMSE detector is given by:

$$
\hat{\mathbf{s}}_{\mathrm{MMSE}}=\mathbf{W}_{\mathrm{MMSE}}^{H} \mathbf{y}
$$

The MMSE detector combines the performance qualities of the MF and ZF detectors [12]. According to Eq. (2.19), in high SNR scenarios the MMSE detector performs as the ZF, since the second term inside the parentheses becomes negligible. At low SNR (unfavorable scenario to the ZF detector), the MMSE detector operates as MF (surpassing the ZF performance). Its per-symbol complexity, like the ZF, is quadratic with the number of $\operatorname{Tx}\left(\mathcal{O}\left(N_{t}^{2}\right)\right)$, due to inverse matrix operation. However, like $Z F$, its performance is also degraded in medium to high system loading LS-MIMO scenarios [12]. 


\subsubsection{MMSE-SIC MIMO Detection}

The MMSE-SIC MIMO detector is performed from the decomposition of the channel matrix $\mathbf{H}$ and assuming that $\mathbf{H}$ is square or tall, i.e. $N_{t} \leq N_{r}$. Hence, applying for instance the QR factorization on the channel matrix H:

$$
\mathbf{H}=\mathbf{Q R}
$$

where $\mathbf{Q}$ is a $2 N_{t} \times 2 N_{t}$ unitary matrix and $\mathbf{R}$ is a $2 N_{t} \times 2 N_{r}$ upper triangular matrix. Thus, multiplying $\mathbf{Q}^{H}$ by the receive signal $\mathbf{y}$ results in:

$$
\mathbf{x}=\mathbf{Q}^{H} \mathbf{y} \Longrightarrow \mathbf{x}=\mathbf{Q}^{H} \mathbf{H} \mathbf{s}+\mathbf{Q}^{H} \boldsymbol{\eta} \Longrightarrow \mathbf{x}=\mathbf{Q}^{H} \mathbf{Q R} \mathbf{s}+\mathbf{Q}^{H} \boldsymbol{\eta} \Longrightarrow \mathbf{x}=\mathbf{R s}+\tilde{\boldsymbol{\eta}}
$$

where $\tilde{\boldsymbol{\eta}}=\mathbf{Q}^{H} \boldsymbol{\eta}$ is a zero-mean complex Gaussian random vector and $\mathbf{Q}^{H} \mathbf{Q}=\mathbf{I}$.

The channel correlation matrix can be either square or tall. Initially, assuming a square $N_{a} \times N_{a}$ matrix $\mathbf{H}$, where $2 N_{t}=2 N_{r}=N_{a}$, we have:

$$
\begin{aligned}
& \mathbf{x}=\mathbf{R s}+\tilde{\boldsymbol{\eta}} \\
& {\left[\begin{array}{c}
x_{1} \\
x_{2} \\
\vdots \\
x_{N_{a}}
\end{array}\right]=\left[\begin{array}{cccc}
r_{1,1} & r_{1,2} & \cdots & r_{1, N_{a}} \\
0 & r_{2,2} & \cdots & r_{2, N_{a}} \\
\vdots & \vdots & \ddots & \vdots \\
0 & 0 & \cdots & r_{N_{a}, N_{a}}
\end{array}\right]\left[\begin{array}{c}
s_{1} \\
s_{2} \\
\vdots \\
s_{N_{a}}
\end{array}\right]+\left[\begin{array}{c}
\tilde{\eta}_{1} \\
\tilde{\eta}_{2} \\
\vdots \\
\tilde{\eta}_{N_{a}}
\end{array}\right]}
\end{aligned}
$$

Thus, we can write the recurrent equations in order to determine the soft decision $x_{N_{a}}$ to $x_{1}$ :

$$
\begin{aligned}
& x_{N_{a}}=r_{N_{a}, N_{a}} s_{N_{a}}+\tilde{\eta}_{N_{a}} \\
& x_{N_{a}-1}=r_{N_{a}-1, N_{a}} s_{N_{a}}+r_{N_{a}-1, N_{a}-1} s_{N_{a}-1}+\tilde{\eta}_{N_{a}-1}
\end{aligned}
$$

Now, assuming that a tall channel matrix $\mathbf{H}$, the same recurrent equations procedure of Eq. (2.23) can be followed, which a part of the received signals will be composed of non-useful information, i.e., that do not take into account the vector of estimated symbols. This part can be ignored, making the procedure similar to the case of square channel matrix.

Hence, $\tilde{s}_{N_{a}}$ can be detected from $x_{N_{a}}$ as follows:

$$
\text { Let } \quad \tilde{s}_{N_{a}}=\frac{x_{N_{a}}}{r_{N_{a}, N_{a}}}=s_{N_{a}}+\frac{\tilde{\eta}_{N_{a}}}{r_{N_{a}, N_{a}}}
$$

Then, the contribution of $\tilde{s}_{N_{a}}$ is to be canceled in detecting $\tilde{s}_{N_{a}-1}$ from $x_{N_{a}-1}$. This sequential detection procedure is terminated until all the data symbols of $\mathbf{s}$ are detected. The $n$th symbol of $\mathbf{s}$ can be detected after canceling $N_{a}-n$ data symbols as:

$$
\begin{aligned}
& u_{n}=x_{n}-\sum_{q=n+1}^{N_{a}} r_{n, q} \tilde{s}_{q}, \\
& \tilde{s}_{n}=\frac{u_{n}}{r_{n, n}}, \quad n \in\left\{1,2, \cdots, N_{a}-1\right\}
\end{aligned}
$$


Finally, the background noise can be taken into account in order to minimise the MSE, [61].

\subsubsection{MMSE-OSIC MIMO Detection}

Further performance improvement on the MMSE-SIC technique can be achieved through a properly ordering [62], [63], witch mitigate error propagation in the interference cancellation step. The ordering criterion is the minimization of the $\mathbf{Q}$ columns norm, which makes the detection be proceeded from the least noise corrupted symbol to the most. The form of the decomposition is simply:

$$
\mathbf{H P}=\mathbf{Q R}
$$

where matrix $\mathbf{P}$ is a permutation matrix, used to reorder the symbols after applying the SIC detection, Eq. (2.25), by multiplying it and the estimated symbol. Therefore the application of sorted QR Decomposition (SQRD) instead of the QR decomposition change the detector SIC into OSIC.

The SQRD procedure is summarized by the pseudocode in Algorithm 2.1.

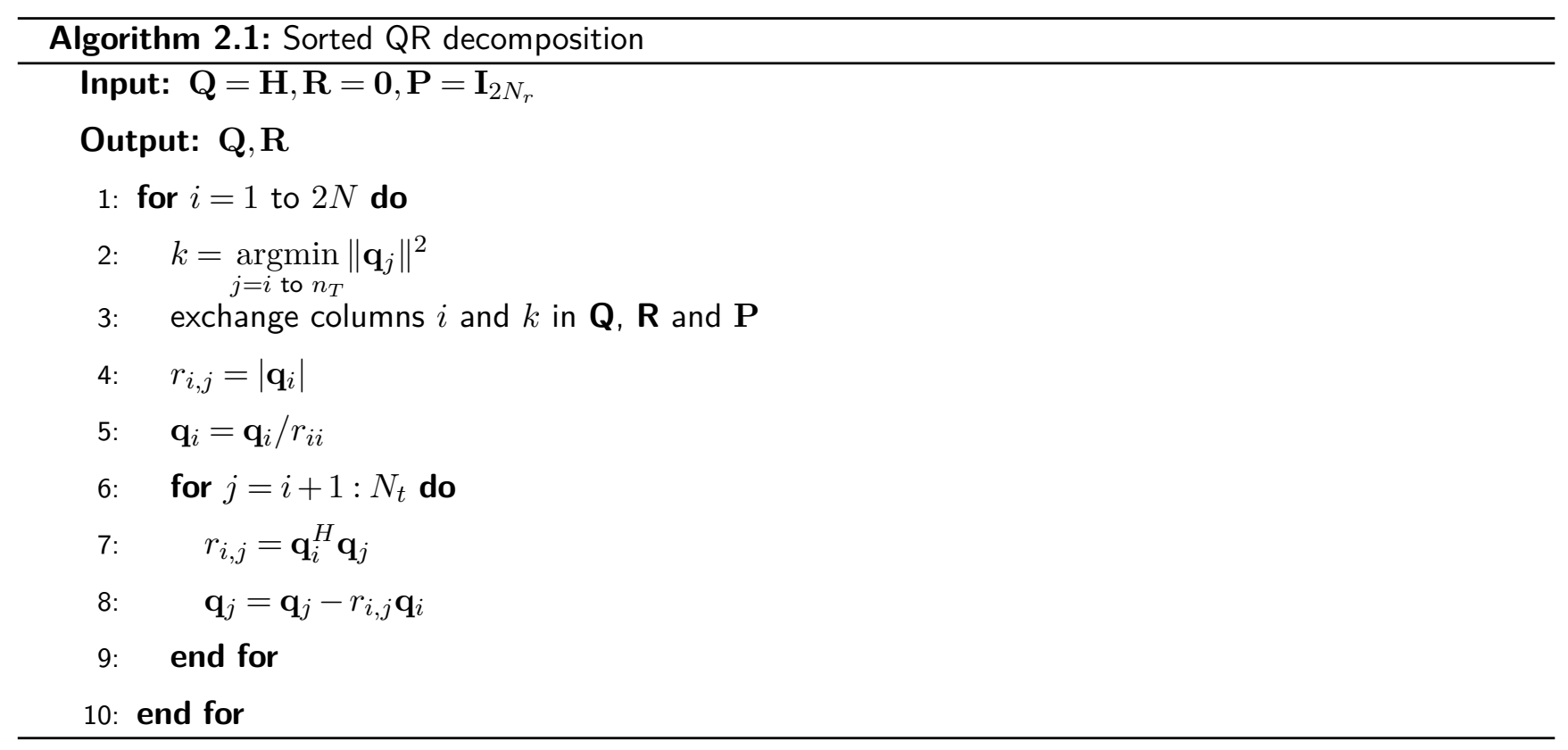

\subsubsection{Maximum Likelihood Detector}

The ML results in optimal performance and consists in finding the closest lattice point to the received signal $\mathbf{y}$ with the finite subset $\mathcal{A}$ as the search space:

$$
\mathbf{s}_{\mathrm{ML}}=\min _{\hat{\mathbf{s}} \in \mathcal{A}^{2 N_{t}}}\|\mathbf{y}-\mathbf{H} \hat{\mathbf{s}}\|^{2}
$$

However, the ML detector in Eq. (2.27) is exponentially complex in $N_{t}$, being prohibitive for large $N_{t} \cdot N_{r}$, which is the case of LS-MIMO systems. 


\section{Sphere Decoding}

The SD detector, proposed in [64] and based on the MIMO detection in [50,65], is based on a search on the points $\mathbf{s} \in \mathcal{A}^{2 N_{t}}$ of the mesh lying within a hypersphere of radius $r$, centered on the received vector $\mathbf{y}$. The delimitation, through the radius $r$, reduces the search space and, consequently, the computational complexity of the algorithm [51]. In this way, the strategy to choose the radius $r$ has a direct impact on the SD complexity, in this sense, the pruning method [66] stands out as one of the most used in the literature. The SD detector, with appropriate search radius, can achieve a performance similar to $M L$, and is often reported in the literature as the optimal threshold, in scenarios where obtaining ML becomes impractical. On the other hand, the major disadvantage of the SD technique consists of its computational complexity that is variable, stochastic and dependent on channel conditions and noise level, as well as the number of antennas and the modulation order [67]. Thus, according to the combination of these factors, the computational complexity of the SD can go from quadratic polynomial (best case) to exponential [68].

The basic operation of the SD algorithm can be described fundamentally through two steps: 1) preprocessing step; 2) tree search step. The preprocessing step consists of constructing the search tree structure. Initially, the channel matrix $\mathbf{H}$ is decomposed using the QR decomposition as in Eq. (2.20). Same of SIC procedure, the received signal vector, $\mathbf{y}$ of the Eq. (2.4), is left multiplied by $\mathbf{Q}^{H}$, which results in Eq. (2.21).

Based on Eq. (2.21), the ML detection criterion can be rewritten as:

$$
\begin{aligned}
\hat{\mathbf{s}}_{\mathrm{ML}} & =\underset{\mathbf{s} \in \mathcal{A}^{2 N_{t}}}{\arg \min }\|\mathbf{x}-\mathbf{R} \mathbf{s}\|^{2} \\
& =\underset{\mathbf{s} \in \mathcal{A}^{2 N_{t}}}{\arg \min } \sum_{i=1}^{N_{t}}\left|x_{i}-\sum_{j=1}^{N_{t}} r_{i, j} s_{j}\right|^{2}
\end{aligned}
$$

where $r_{i, j}$ is the element of the $i$ th row and $j$ th column of matrix $\mathbf{R}$.

Note from Eq. (2.28) that, due to the triangular condition of $\mathbf{R}$, the summation for calculating the $M L$ criterion can be given in a cumulative sequence, represented by a tree search of depth $2 N_{t}$. The search space is reduced by radius $r$, in order to reduce complexity. Therefore, the SD problem can be written as:

$$
\hat{\mathbf{s}}_{\mathrm{SD}}=\min _{\mathbf{s} \in \mathcal{A}^{2 N_{t}}}\left(\|\mathbf{x}-\mathbf{R s}\|^{2} \leq r^{2}\right)
$$

As mentioned before, the disadvantage of the SD algorithm lies in its computational complexity dependent on channel conditions, noise level, number of antennas and modulation order, which results in impracticable complexities under certain conditions of low SNR and/or high order modulation. Several SD-based strategies have been proposed, such as the fixed sphere decoder (FSD), [67], repeated tree search [69], single tree search $[70,71]$ and smart ordering and candidate adding (SOCA) [72].

\subsection{Lattice Reduction Based MIMO Detection}

The lattice (basis) reduction has been proposed in aiming to transform a regular basis to a nearly orthogonal one. In this sense, choosing the channel matrix $\mathbf{H}$ as a basis for a lattice, the MIMO problem can be treated 
as a lattice decoding problem.

A lattice can be generate by different basis (channel matrices), since the MIMO detection goal is reducing the noise and interference between multiple signals, it is convenient to find a matrix whose columns are nearly orthogonal to generate the same lattice. Hence, LR pre-processing technique can be applied to improve the MIMO detection performance; these methods are regarded as the LR-based detection for MIMO systems.

In order to deploy LR technique, the original constellation must be defined in terms of consecutive integers lattice. Consider two bases $\mathbf{H}$ and $\mathbf{G}$ that span the same lattice:

$$
\mathbf{H}=\mathbf{G U} \quad \text { or } \quad \mathbf{G}=\mathbf{H T},
$$

where $\mathbf{U}$ and $\mathbf{T}=\mathbf{U}^{-1}$ are a unimodular matrices. As a consequence the received signal in Eq. (2.4) can be rewritten as:

$$
\begin{gathered}
\mathbf{y}=\mathbf{H s}+\boldsymbol{\eta}=\mathbf{H T T}^{-1} \mathbf{s}+\boldsymbol{\eta}=\mathrm{Gz}+\boldsymbol{\eta}, \\
\text { where } \quad \mathrm{z}=\mathbf{T}^{-1} \mathbf{s}=\mathrm{Us} .
\end{gathered}
$$

Since the received signal can be treated as the lattice points spanned by the basis, lattice-based MIMO system detection can be developed aiming to reduce the complexity of conventional detectors. Hence, under lattice transform, $\mathbf{z}$ is initially detected using any MIMO detection technique, since the LR received signal of Eq. (2.31) can be treated equivalent to the MIMO received signal of Eq. (2.4); after that, the original symbols $\mathbf{s}$ can be obtained applying a combination of shifting and scaling operations [73].

\section{The Lenstra - Lenstra - Lovász Algorithm}

A well-known and efficient algorithm for LR is the Lenstra - Lenstra - Lovász (LLL) algorithm, proposed by Lenstra, Lenstra and Lovász in 1982 [74]. A basis $\tilde{\mathbf{A}}$ which can be decomposed by a QR decomposition as $\tilde{\mathbf{A}}=\tilde{\mathbf{Q}} \tilde{\mathbf{R}} \mathbf{T}^{-1}$ is named reduced LLL with parameter $\delta$ (which is in the range $\frac{1}{4}<\delta \leq 1$ ) if the following inequalities hold:

$$
\begin{aligned}
\left|\tilde{r}_{l, k}\right| & \leq \frac{1}{2}\left|\tilde{r}_{l, l}\right| \text { for } 1 \leq l<k \leq N_{t}, \\
\text { and } & \\
\delta \tilde{r}_{k-1, k-1}^{2} & \leq \tilde{r}_{k, k}^{2}+\tilde{r}_{k-1, k}^{2} \text { for } k=2, \ldots, N_{t}
\end{aligned}
$$

where $\mathbf{T}$ is a unimodular matrix, $\tilde{r}_{l, k}$ is the element of $l$-th row and $k$-th column of the upper triangular matrix $\tilde{\mathbf{R}}$.

If only the first inequality in (2.33) is fulfilled, the basis is called size reduced. Herein, the adopted LLL algorithm is based on [75] implementation and summarized by the pseudo-code in Algorithm 2.2. As shown in Algorithm 2.2, the parameter $\delta$ define the swap condition after the length reduction step and can be regarded as a strictness factor: the closer it is to 1 , the more orthogonal the reduced basis, in general, will be [76], on the other hand, the reduction tends to be slower. Thus, in summary, one can say the parameter $\delta$ influences the quality of the reduced basis. In this work, we have assumed $\delta=\frac{3}{4}$ as proposed in [74]. Notice that $\mathbf{Q}_{0}$, $\mathbf{R}_{0}$ and the permutation matrix $\mathbf{P}_{0}$, required by the algorithm, are obtained from Eq. (2.26). Besides, the 
Givens rotation matrix, $\Theta$, utilized in the Algorithm 2.2 (line 13) has a purpose of restore the upper triangular structure in $\tilde{\mathbf{R}}$.

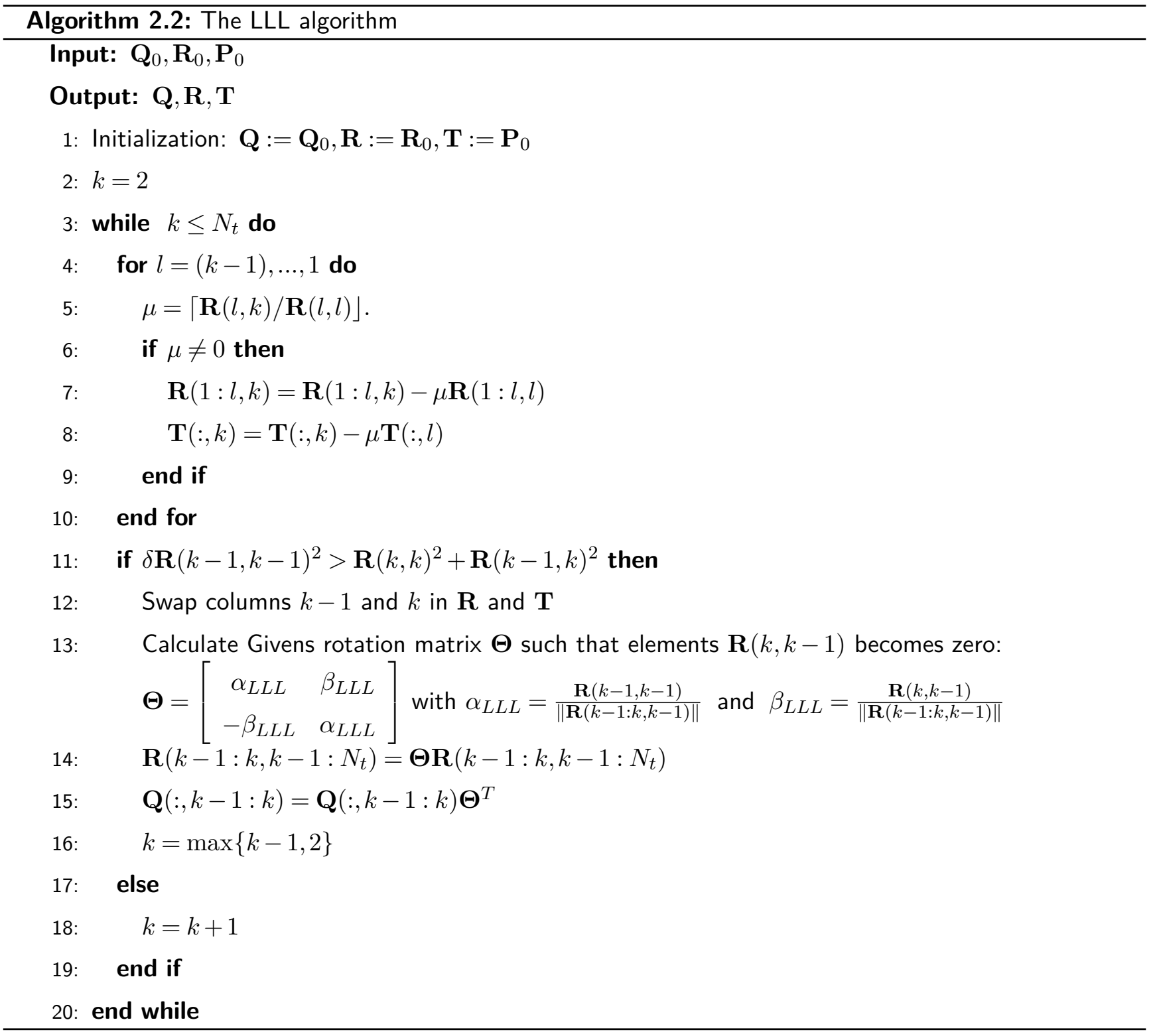

\subsection{Lattice Reduction aided MIMO Detectors in Correlated Channels with iCSI}

This section discusses the numerical results regarding the LR-assisted MIMO detectors in channels with antenna correlation and imperfect estimates of channel conditions. Moreover, in the case of LR-aided SD MIMO detector, it is proposed a simple procedure in order to reduce the computational complexity. In this first problem investigated, we considered conventional MIMO systems scenarios.

\subsection{LR-aided Sphere Decoder}

Considering the LR preprocessing, the received signal is rewritten as in Eq. (2.31) and $\mathbf{z}=\mathbf{T}^{-\mathbf{1}} \mathbf{s}=\mathbf{U} \mathbf{s}$ composes the transformed vector of transmitted symbols. Since $\mathbf{s} \in \mathcal{A} \in \mathcal{Z}^{2 N_{t}}$, then the range of $\mathbf{z}$ is denoted 
by $\mathbf{z} \in \tilde{\mathcal{A}}$, i.e. the image of $\mathcal{A}$ under $\mathbf{T}^{-1}$. However, the subset $\tilde{\mathcal{A}}$ is generally no longer of the simple hypercube structure as that of $\mathcal{A}$. Therefore, controlling the search range in $\tilde{\mathcal{A}}$ is a very complicated problem. To avoid this, we proposed a lookup table which contains the vectors-candidates already calculated at each layer. This procedure is intended to avoid the calculation of nodes already visited, which is the essence of the tree search. More details about the lookup table procedure is given in Subsection 2.6.1. On the other hand, the expensive computational cost of finding all possibilities for the transformed transmitted symbols vector, although not a specific calculation of the proposed procedure, can not be avoided and is of the order of $\mathcal{O}\left(M^{N_{t}}\right)$.

The primary search uses the initial radius based on the LR-aided Zero Forcing (LR-ZF) output, $\mathbf{z}_{\mathrm{LR}-\mathrm{LZF}}$; an analogous deterministic method is also deployed in [55], where the hypersphere radius is considered as the distance between the original received signal and the signal received using ZF detector. Thus, the primary search of the LR-SD for MIMO detections can be expressed by the following problem:

$$
\hat{\mathbf{z}}=\min _{\mathbf{c} \in \tilde{\mathcal{A}}}\left(\|\mathbf{y}-\mathbf{G} \mathbf{c}\|^{2} \leq r^{2}\right)
$$

where $\mathbf{c}$ represents the candidate vector on the LR reduced basis. The radius $r$ is initially expressed by:

$$
\begin{aligned}
\mathbf{z}_{\mathrm{LR}-\mathrm{LZF}} & =\mathbf{G}^{\dagger} \mathbf{y} \\
r^{2} & =\left\|\mathbf{y}-\mathbf{G} \mathbf{z}_{\mathrm{LR}-\mathrm{LZF}}\right\|^{2}
\end{aligned}
$$

Indeed, even with the computation of the LZF decoder, this primary search shows significant complexity reduction over the conventional SD of Eq. (2.29), due to the considerable decreasing in the sphere radius $r$. Nevertheless, the result $\hat{\mathbf{z}}$ not necessarily exists in the reduced basis domain $\tilde{\mathcal{A}}$, as stated in the Proposition 1.

Proposition 1. In lattice $L(\mathbf{G})=\mathbf{G} \mathbf{c} ; \mathbf{c} \in \mathcal{Z}^{2 N_{t}}$, suppose $\mathbf{G} \hat{\mathbf{z}}_{\mathrm{ML}}$ is the closest lattice point to a certain vector point $\mathbf{y}$ among the lattice points in subset $\left\{\mathbf{G} \mathbf{c}: \mathbf{c} \in \tilde{\mathcal{A}} \in \mathcal{Z}^{2 N_{t}}\right\}$. If there exists a lattice point $\mathbf{G} \hat{\mathbf{z}}$ which is closer to $\mathbf{y}$ than $\mathbf{G} \hat{\mathbf{z}}_{\mathrm{ML}}$, i.e., $\|\mathbf{y}-\mathbf{G} \hat{\mathbf{z}}\|^{2}<\left\|\mathbf{y}-\mathbf{G} \hat{\mathbf{z}}_{\mathrm{ML}}\right\|^{2}$, then $\hat{\mathbf{z}} \notin \tilde{\mathcal{A}}$ and, consequently, $\hat{\mathbf{s}}=\mathbf{T} \hat{\mathbf{z}} \notin \mathcal{A}$.

The primary search starts from the decision statistic $\mathbf{z}_{\mathrm{LR}-\mathrm{LZF}}$ as initial radius. The closest lattice point to $\mathbf{y}$ in the sphere, $\hat{\mathbf{z}} \in \tilde{\mathcal{A}}$, is the $\hat{\mathbf{z}}_{\mathrm{ML}}$. If none $\hat{\mathbf{z}} \in \tilde{\mathcal{A}}$ is found in the primary search, then a further search is required. In the next search, the squared radius need to be updated as:

$$
r^{2}=\left\|\mathbf{y}-\mathbf{G} \mathbf{z}_{\mathrm{LR}-\mathrm{LZF}}\right\|^{2}+\Delta
$$

where $\Delta$ is a constant increment. The selection of the squared radius increment $\Delta$ strongly affects the computational complexity, and its value has been obtained as a scaled variance of noise in such way that, a lattice point inside the hypersphere is found with a high probability. Let $\sigma^{2}$ be the variance of the entries $\eta \in \mathcal{Z}^{2 N_{r} \times 1}$, the squared radius increment is selected as $\Delta=\alpha N_{r} \sigma^{2}$, where $\alpha$ is calculated considering a high probability of a lattice point be found inside the hypersphere; determination of suitable values of $\alpha$ were proposed in this Doctoral Thesis [77] and can be found in Appendix A. With the updated radius, the search is proceed aiming to obtain the closest lattice point to $\mathbf{y}$ in the hypersphere. The closest point $\hat{\mathbf{z}} \in \tilde{\mathcal{A}}$ found must be the $M L$ detection result, $\hat{\mathbf{z}}_{\mathrm{ML}}$. If none lattice point is found, a further search is required again. Increase the sphere radius, $r^{2}$, by another $\Delta$ and perform a new SD search. This procedure is repeated until $\hat{\mathbf{z}}_{\mathrm{ML}}$ and, consequently, $\hat{\mathbf{s}}_{\mathrm{ML}}=\mathbf{T} \hat{\mathbf{z}}_{\mathrm{ML}} \in \mathcal{A}$ is obtained. The proposed LR-SD algorithm for MIMO detections 
is summarized by the pseudo-code in Algorithm 2.3. These further searches for $\hat{\mathbf{s}}_{\mathrm{ML}}$ will certainly lead to a complexity increase. In order to minimize this overhead two main procedures are proposed: a) optimization of $\Delta ; \mathrm{b})$ lookup table. The optimization of $\Delta$ is performed in order to avoid further searches, see Appendix A. The lookup table avoids the calculation of nodes already visited, so the complexity in subsequent searches is greatly reduced; this procedure is detailed in Subsection 2.6.1.

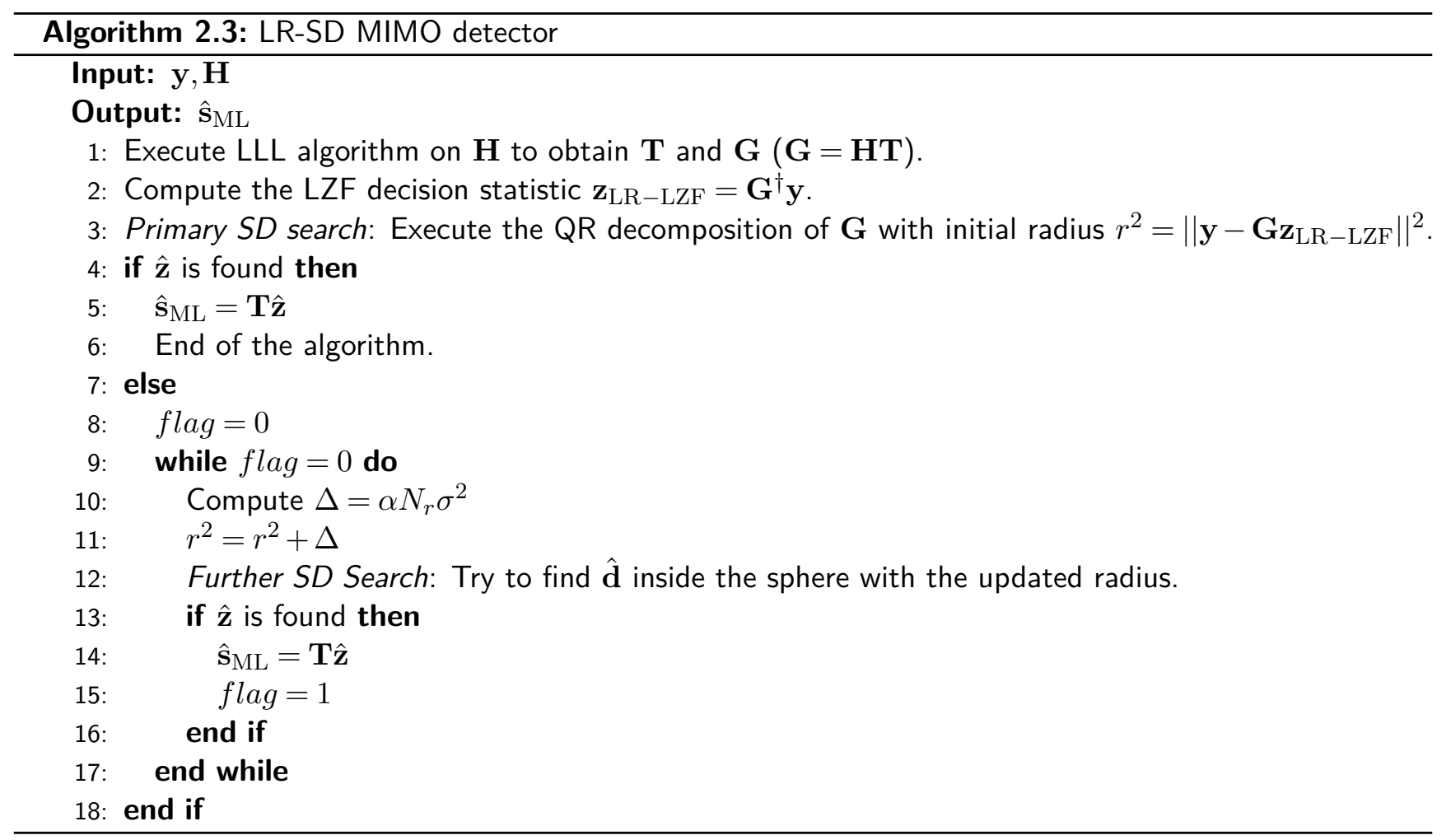

\subsubsection{Proposed Lookup Table Procedure}

One among main issues in the sphere decoding tree search is related to avoid partial Euclidean distance calculation of symbol vectors already calculated in past iterations. For example, considering two symbol vectors: $s_{1}=\left[\begin{array}{lllll}1 & 1 & -1 & 1\end{array}\right]^{\mathrm{T}}$ and $s_{2}=\left[\begin{array}{llll}-1 & -1 & -1 & 1\end{array}\right]^{\mathrm{T}}$. If the accumulated partial Euclidean distance (PED), starting from the last symbol (upper triangular matrix) on the last two bits of $s_{1}$, i.e. $\left[\begin{array}{ll}-1 & 1\end{array}\right]^{\mathrm{T}}$, exceeds the radius search, then considering the last two bits of $s_{2}$ are equal to $s_{1}$, will certainly exceed radius search, thus its calculation is not necessary.

In conventional SD, the tree search procedure is easily performed with simple loops due to the well defined boundary control related to the QAM constellation, as showed in Eq. (2.39). After the LR reduced basis transformation, the system model is considered as described in (2.31) and the boundary control of the transformed symbol vector $\mathbf{z}=\mathbf{T}^{-1} \mathbf{S}$ is not well defined as in conventional QAM constellation, as exemplified in Fig. 2.2, becoming a very complicated problem. 
As an example, consider a $2 \times 2$ MIMO with QSPK modulation. Let:

$$
\begin{gathered}
\mathbf{H}=\left[\begin{array}{cccc}
-1.2556 & -0.7481 & -0.1827 & 0.5916 \\
0.2331 & -0.4549 & -0.6305 & -0.3911 \\
0.1827 & -0.5916 & -1.2556 & -0.7481 \\
0.6305 & 0.3911 & 0.2331 & -0.4549
\end{array}\right] \\
\mathbf{T}=\left[\begin{array}{cccc}
1 & 0 & 0 & -1 \\
0 & 0 & 1 & 0 \\
0 & 1 & 1 & 0 \\
0 & 0 & 0 & 1
\end{array}\right]
\end{gathered}
$$

The matrix of possibilities of $\mathbf{s} \in\{-1,1\}^{2 K}$ is given by:

$$
\left[\begin{array}{cccccccccc}
-1 & 1 & -1 & 1 & -1 & 1 & -1 & 1 & \ldots & 1 \\
-1 & -1 & 1 & 1 & -1 & -1 & 1 & 1 & \ldots & 1 \\
-1 & -1 & -1 & -1 & 1 & 1 & 1 & 1 & \ldots & 1 \\
-1 & -1 & -1 & -1 & -1 & -1 & -1 & -1 & \ldots & 1
\end{array}\right]
$$

As showed in Eq. (2.39), the possibilities of $\mathbf{s}$ can be arranged in uniform tree search, as the conventional SD performs. When the constellation is transformed into the reduced basis, the LR-SD tree search becomes irregular between each layer, as showed in Fig. 2.2.

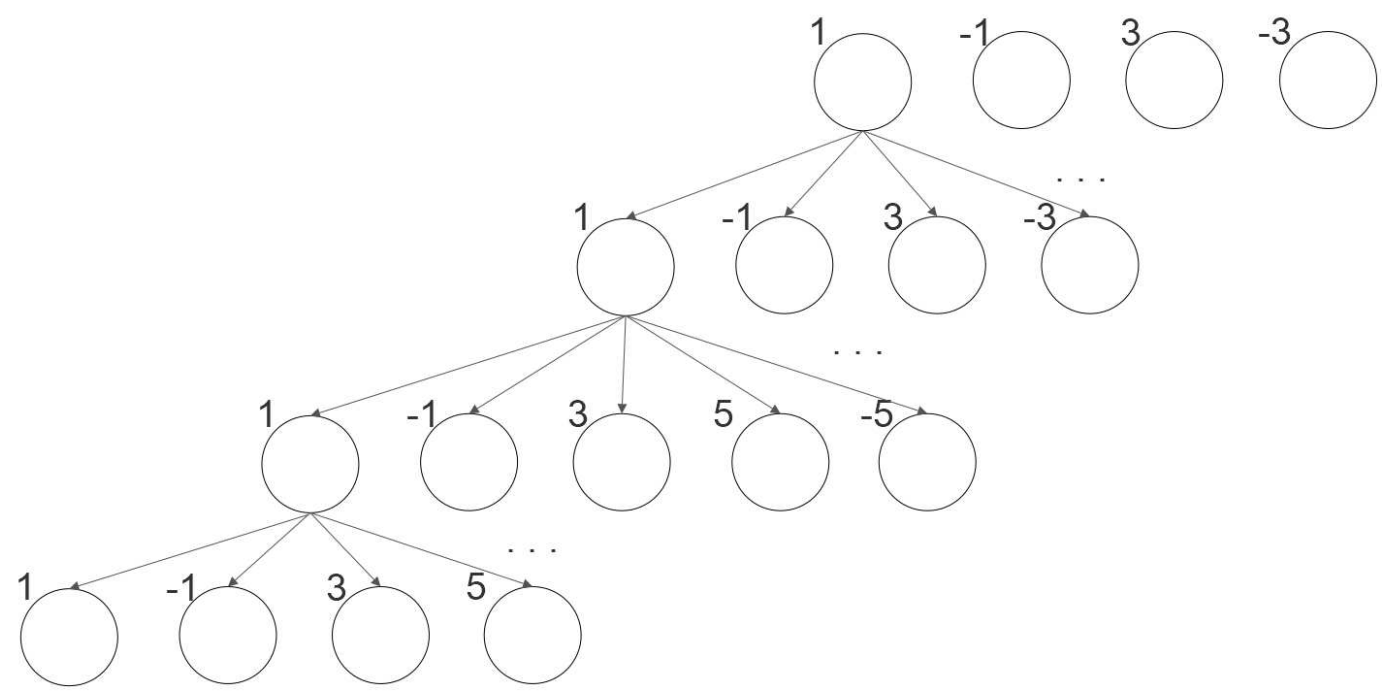

Figure 2.2: Example of the tree search expansion of the LR-SD.

Thus, the algorithm performs the tree search in all possible transformed symbol vectors, $\mathcal{S}_{\mathrm{LR}}=\left\{\mathbf{z}_{1}, \mathbf{z}_{2}, \ldots, \mathbf{z}_{n}\right\}$ (based on all transformed QAM symbol vectors), storing the accumulated PED in a lookup table, while using this table to avoid same calculations in others equal symbol vectors. The lookup table procedure is described by the pseudo-code shown in Algorithm 2.4. 


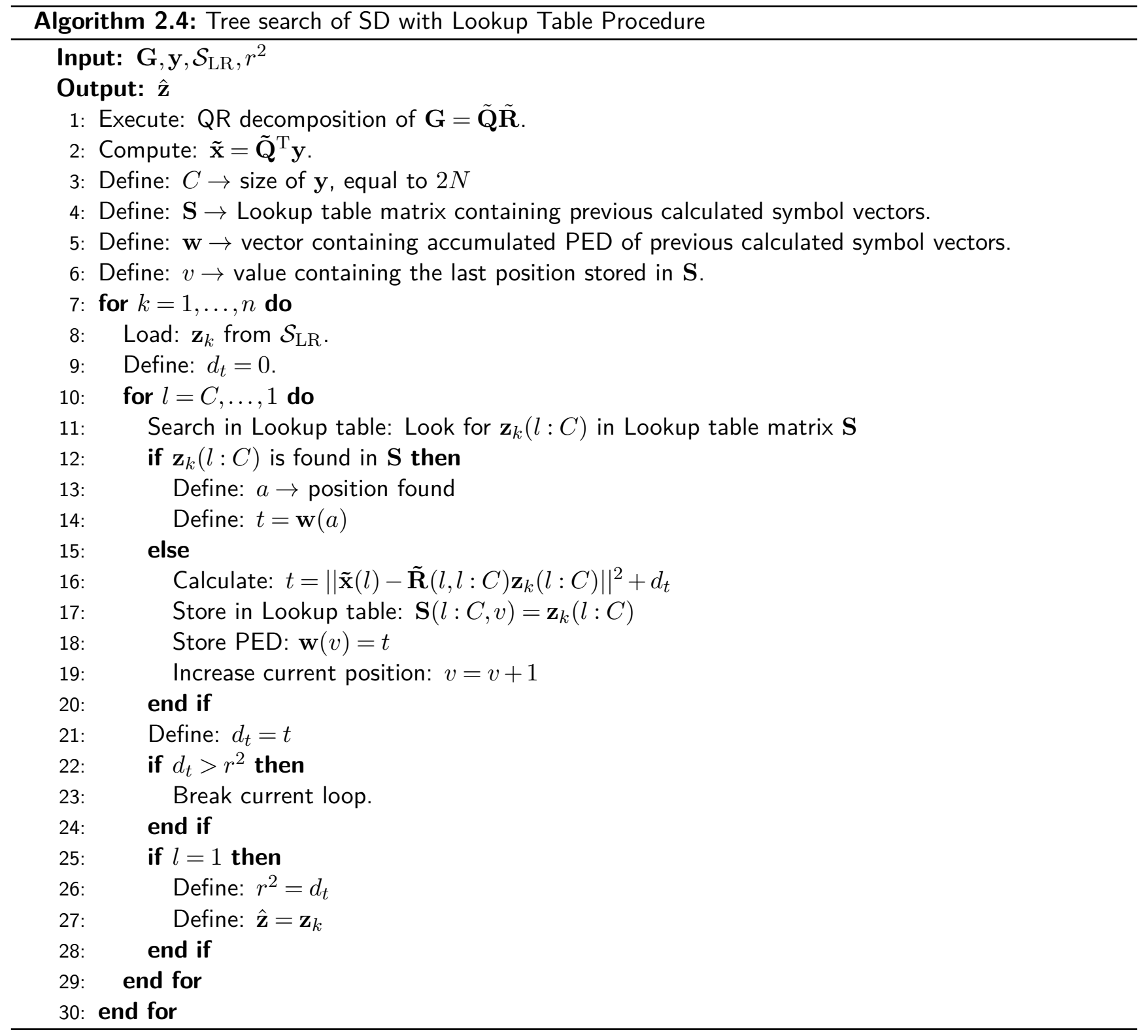




\subsection{Numerical Results}

In this section, performance in terms of BER versus SNR under perfect and channel error estimates is analyzed; further realistic MIMO detection performance analysis has been conducted considering channels correlated scenarios. Along this section, numerical Monte Carlo simulations (MCS) results are depicted and examined. Besides, 4-QAM modulation and Gray coding have been deployed across this section.

The MIMO detectors analyzed and their respective abbreviations in this section are presented in Table .

Table 2.1: Acronyms and descriptions of the investigated detectors

\begin{tabular}{rlc}
\hline Acronym & Description & Described in \\
\hline \hline ML & ML detector (optimal) & Subsection 2.3.4 \\
MMSE & Linear MMSE detector & Subsection 2.3.1 \\
MMSE-OSIC & MMSE with ordered SIC detector & Subsection 2.3.3 \\
LR-MMSE & MMSE detector with LR preprocessing & Subsection 2.3.1 and Section 2.4 \\
LR-MMSE-OSIC & MMSE with ordered SIC detector aided by LR & Subsection 2.3.3 and Section 2.4 \\
& SD detector aided by LR, using the proposed & Sections 2.4 and 2.6 \\
LR-SD & lookup table and $\alpha$ optimization procedures & \\
\hline \hline
\end{tabular}

The Fig. 2.3 compares the performance of the detectors under investigation in two scenarios: a) uncorrelated channels $(\rho=0.0)$ and b) highly correlated $(\rho=0.9)$. We consider $N_{t}=N_{r}=4$ antennas, 4-QAM modulation and pCSI. It may be noted in Fig. 2.3.(a) that even under perfect channel conditions (i.e., pCSI and uncorrelated antennas) the LR preprocessing technique applied to the MMSE and MMSE-OSIC detectors results in significant performance gain with the increase of the SNR: about $14 \mathrm{~dB}$ for the MMSE and $10 \mathrm{~dB}$ for the MMSE-OSIC, considering the BER range of $10^{-3}$. In the highly correlated scenario of Fig. 2.3.(b), one can conclude that the linear detector MMSE even with a joint OSIC strategy was not able to solve the detection problem. When these detectors receive LR assistance, there is a clear gain in performance and diversity, more precisely, the diversity order of these detectors equals the optimal ML. In addition, the OSIC aided by LR also results in a significant performance gap of about $7 \mathrm{~dB}$ against its pure LR-MMSE version. Regarding the LR-SD, as expected, its result was coincident with the optimal ML in both scenarios. Therefore, it is clear that the LR preprocessing technique is an interesting choice when aiming for a performance improvement in linear detectors, especially when there is a correlation between the antennas.

The condition of errors in the channel estimates is treated in Fig. 2.4. We consider a $4 \times 4 \mathrm{MIMO}$ system and uncorrelated channels. According to the results presented, it can be concluded that both the OSIC cancellation strategy and the LR technique have not been shown to be efficient when there is iCSI. In both the low, Fig. 2.4.(a), and high iCSI conditions, Fig. 2.4.(b), there was a similar performance degradation in all investigated detectors. 


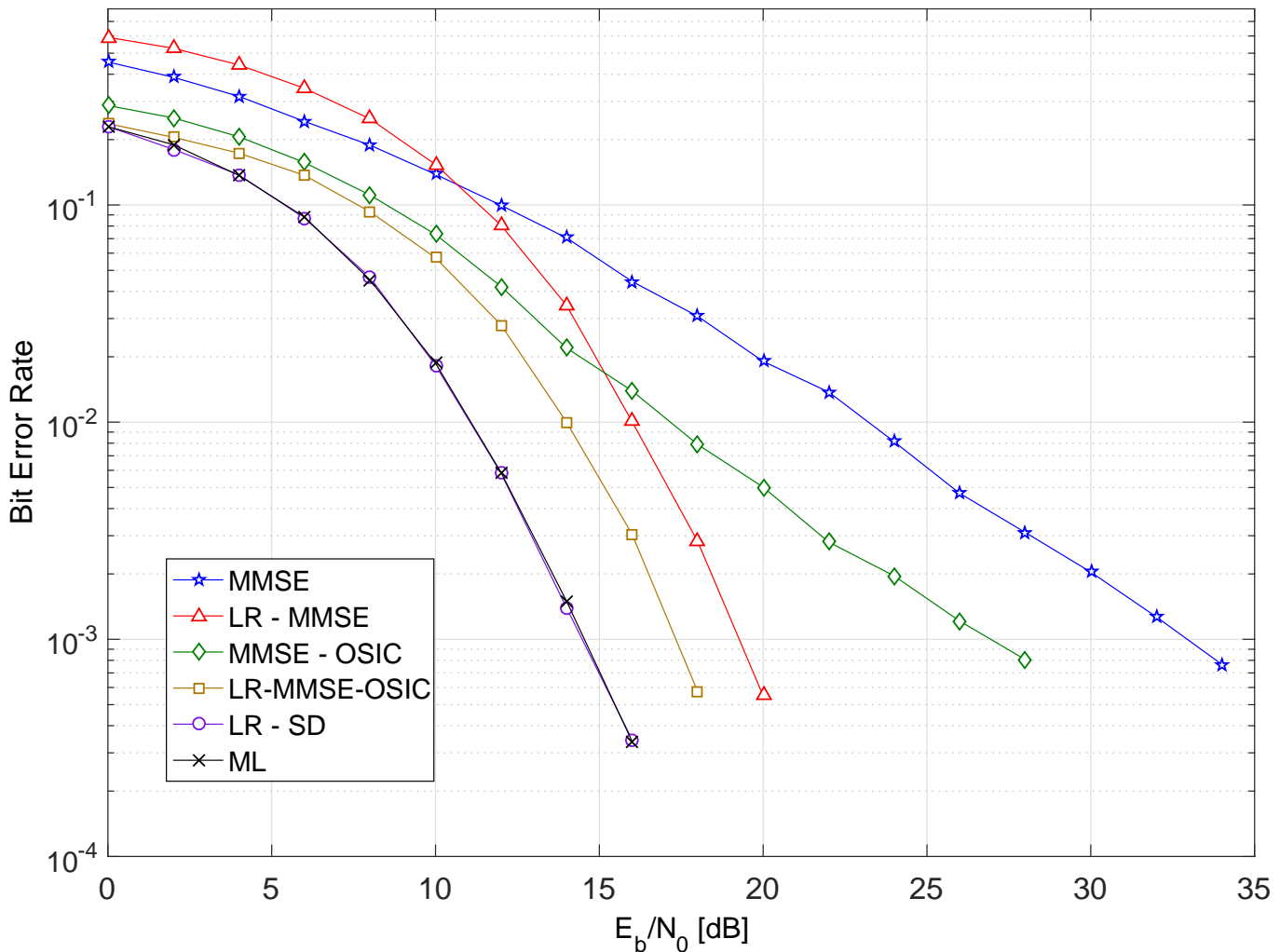

(a) Uncorrelated channel $(\rho=0.0)$

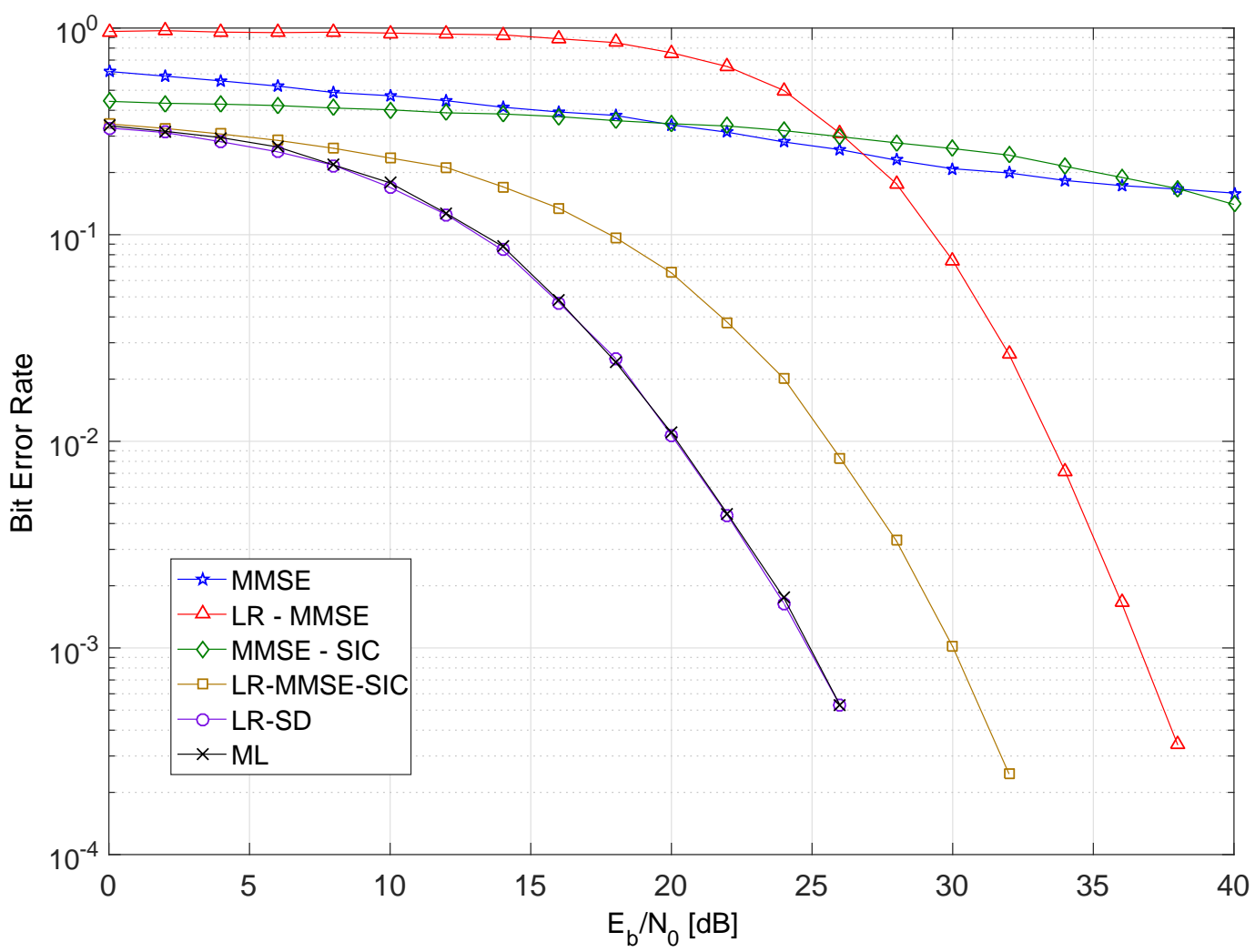

(b) Highly correlated channel $(\rho=0.9)$

Figure 2.3: BER performance for the $4 \times 4 \mathrm{MIMO}$ detectors under $4-\mathrm{QAM}, \mathrm{pCSI}$ and: a) uncorrelated channels, $\rho=0$; b) highly correlated channels, $\rho=0.9$. 


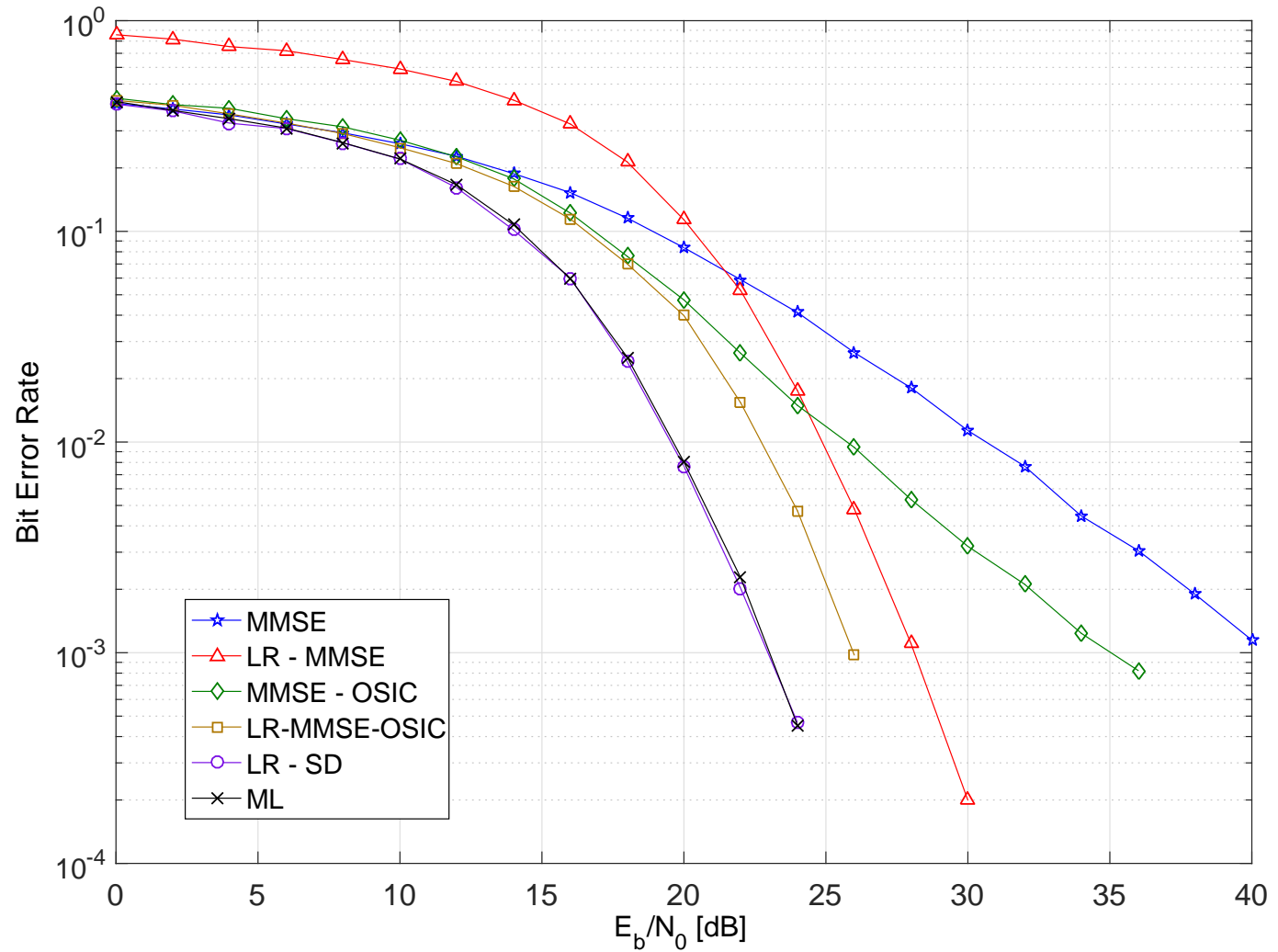

(a) low iCSI $\left(n_{\varepsilon}=2\right)$

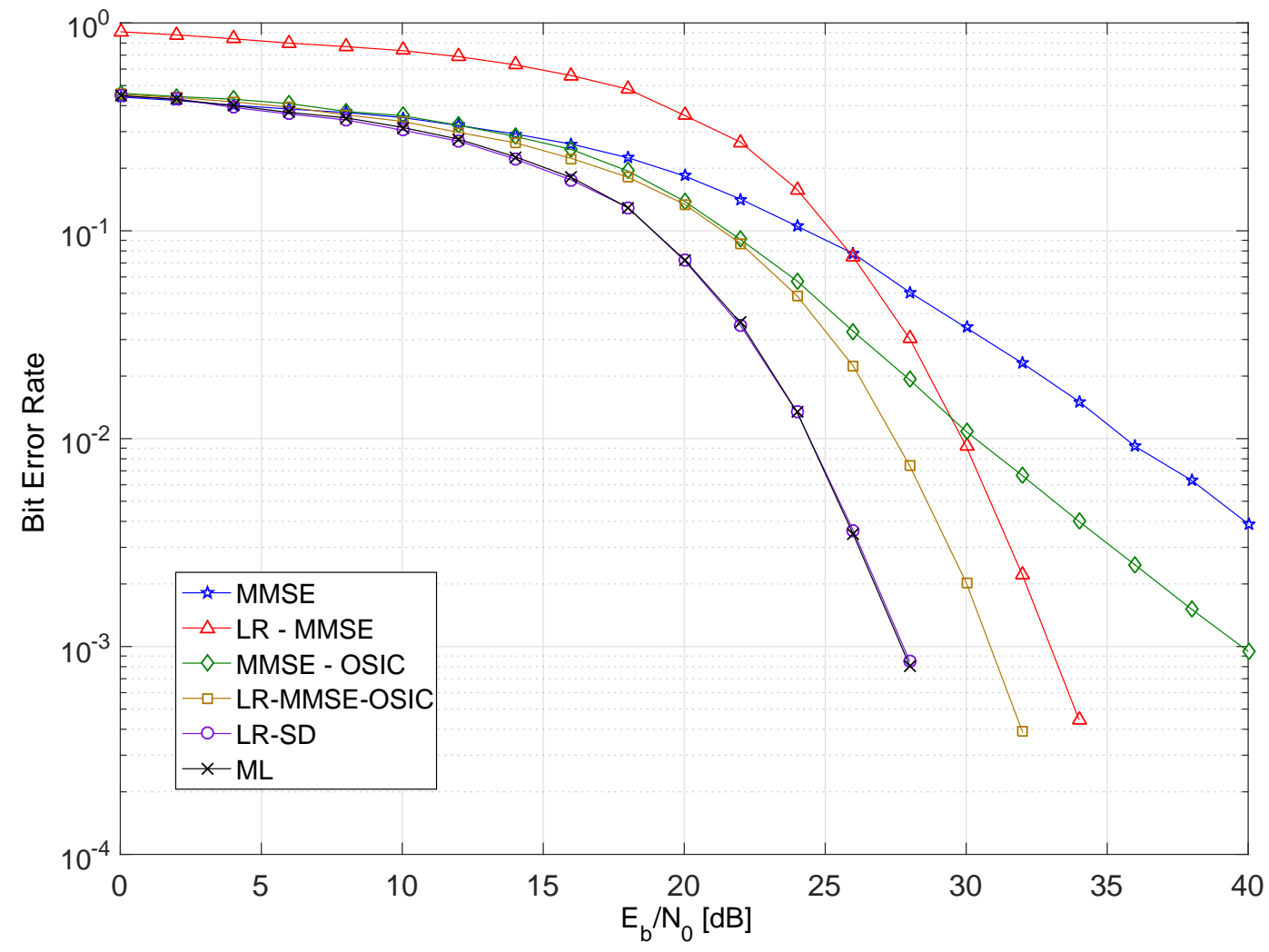

(b) high iCSI $\left(n_{\varepsilon}=10\right)$

Figure 2.4: Performance for the $4 \times 4 \mathrm{MIMO}$ detectors considering iCSI, uncorrelated channels $(\rho=0)$ and: a) low iCSI, $n_{\varepsilon}=3$; b) highly iCSI, $n_{\varepsilon}=10$. 


\subsection{Complexity Analysis}

In this section, MIMO detectors complexities are presented. Table I summarizes the overall complexity related to the analyzed MIMO detectors, considering $N_{t}=N_{r}=N_{g}$ and $M$ modulation order for the $M$-QAM. The complexity of the ML-MIMO detector is included as reference. The complexity is evaluated in terms of the total numbers of floating-point operations (flops), which one flop is defined as an addition, subtraction, multiplication or division between two floating points numbers, and matrix and vector operations flop count are based in [78]. Also, the complexity on the SQRD can be found in [63].

The complexity of LLL algorithm depends on the matrix size and also the antenna/channel correlation index $\rho$. In [79] the complexity of this algorithm was evaluated by numerical experiment, considering $\delta=\frac{3}{4}$ and equal number of $\mathrm{Tx}$ and $\mathrm{Rx}$ antennas. The function that describe the complexity in numbers of flops has been obtained by fitting, and given by:

$$
f_{L L L}\left(N_{g}, \rho\right)=\left(a e^{b \rho}+c\right) N_{g}^{3}
$$

where $a=5.018 \times 10^{-4}, b=13.48$ and $c=8.396$. It is worth noting that the computational complexity cost for the LLL algorithm increases substantially under large-array configurations and medium-high correlation index $(\rho \geq 0.5)$.

From Table 2.2, it can be observed that ML has a prohibitive exponential complexity in any practical MIMO system configuration with moderate or higher number of antennas for any channel correlated and estimation errors scenarios. On the other hand, MMSE detector offers a lower complexity, with polynomial order $\mathcal{O}\left(N_{g}^{3}\right)$. This complexity order is due to the matrix inversion operation. SIC-based MIMO detectors are capable to provide substantial performance improvement without increase the order of complexity regarding the MMSE MIMO detector; hence SIC-based MIMO topologies are able to offer a suitable BER performance-complexity tradeoff. Furthermore, despite the same polynomial complexity order $\mathcal{O}\left(N_{g}^{3}\right)$ achieved by LR-MMSE detectors, this MIMO detector topology presents a second complexity term that could become significant regard the first one, given by the LLL function, $f_{\mathrm{LLL}}$, which is dependent of the level of channel/antennas correlation and number of antennas, as predicted by (2.40). Under uncorrelated antennas scenarios, the LR-MMSE detectors complexity has dominated by the first term, and this technique can demonstrate an attractive performancecomplexity trade-off for these scenarios. Besides, under low to medium correlation indexes, LR-aided MIMO technique shows a affordable and manageable complexity, while preserving full diversity, which makes it a promising near-optimum MIMO transmitting scheme.

The computational complexity in terms of average number of FLOPS versus SNR for the MIMO detectors under uncorrelated and strongly correlated channels with $4 \times 4$ antennas are presented in Fig. 2.5. Indeed, Fig. 2.5.(a) depicts the complexity under uncorrelated channels, where one can notice that the proposed LR-SD strategy results in FLOPS complexity much lower than the ML, while the performance achieved is similarly optimal. Besides, the gap between sub-optimum detectors and their LR-aided versions are due to the $f_{\mathrm{LLL}}$ function computation. Regarding the MMSE-OSIC and LR-MMSE detectors, almost the same complexity in all SNR regions is found. On the other hand, as depicted in Fig. 2.3.(a) the performance of LR-MMSE is superior than MMSE-OSIC, specially in high SNR region, as the LR-MMSE can achieve the same diversity 
Table 2.2: Detectors Complexity

\begin{aligned} & \hline MIMO Detector Number of flops \\ & \hline \hline MMSE $\frac{26}{3} N_{g}^{3}+4 N_{g}^{2} \\ &$ MMSE-OSIC $\frac{40}{3} N_{g}^{3}+\frac{13}{3} N_{g}^{2}+\frac{25}{6} N_{g} \\ &$ LR-MMSE $\frac{26}{3} N_{g}^{3}+4 N_{g}^{2}+f_{\mathrm{LLL}}\left(N_{g}, \rho\right) \\ &$ LR-MMSE-OSIC $\frac{40}{3} N_{g}^{3}+\frac{13}{3} N_{g}^{2}+\frac{25}{6} N_{g}+f_{\mathrm{LLL}}\left(N_{g}, \rho\right) \\ &$ LR-SD $\frac{160}{3} N_{g}^{3}+56 N_{g}^{2}-4 N_{g}+f_{\mathrm{LLL}}\left(N_{g}, \rho\right) \\ &+f_{\text {avg }- \text { nodes }}\left(N_{g}, \rho, \gamma\right) \\ &$\hline $\mathrm{ML}\left(8 N_{g}^{2}+2 N_{g}\right) M^{N_{g}} \\ &$\hline\end{aligned}

order of the ML detector. Moreover, in Fig. 2.5.(b) the same scenario with strongly correlated channels are presented; it can be observed that the complexity of $f_{\text {LLL }}$ function becomes preponderant on the average number of FLOPS of these LR-aided suboptimal detectors. Also, due to the increased complexity imposed by the $f_{\text {LLL }}$ function, the OSIC algorithm complexity becomes negligible indicating LR-MMSE-OSIC a worth choice due to the better performance presented in Fig. 2.3.(b). It is also noticed that the proposed LR-SD also has a significant increase in its total complexity, again due to the expressive complexity increment from the LR technique; on the other hand, still results in complexity lower than ML.

\subsection{Illustrative results: conventional MIMO to LS-MIMO}

In this section, we intend to make a connection of MIMO scenarios, where it is considered a low amount of antennas, to LS-MIMO, in which number of antennas grows up to the order of hundreds. In addition, the transition between low and high system loadings and the resulted performances from the main linear detectors described in this chapter are discussed.

Fig. 2.6 illustrates the performance of the linear detectors in QPSK modulation, considering several scenarios of a) low system loading and b) high system loading. One can notice that the simple MF detection technique of treating interference from other transmitted signals as noise does not solve the detection problem either under low or high system loading conditions. In low system loading scenarios of Fig. 2.6.a, the MMSE and ZF detectors showed similar and good performances, moreover the resulted performance improves with increasing antennas, denoting that both strategies are able to deal with LS-MIMO detection problem when the system is under low loading. Increasing the system loading, i.e., $\beta \geq 0.75$ scenario of Fig. 2.6.b, one can notice that the ZF detector performance degrades in relation to the MMSE. Increasing the system loading, i.e., $\beta \geq 0.75$ scenario of Fig. 2.6.b, it may be noted that the ZF detector performance degrades in relation to the MMSE. This degradation can be gently observed when $\beta=0.75$, where it resulted in a performance gap between the detectors and more prominently in the full loading situation, where the ZF detector can not deal with the MIMO detection problem, resulting in similar performance to MF. In addition, one can notice that, in low and high system loading scenarios, the linear detection techniques ZF and MMSE present a performance gain with the increase of the number of antennas, demonstrating the potential for LS-MIMO schemes, however, when the loading is full, only the MMSE detector was able to demonstrate satisfactory performance. 


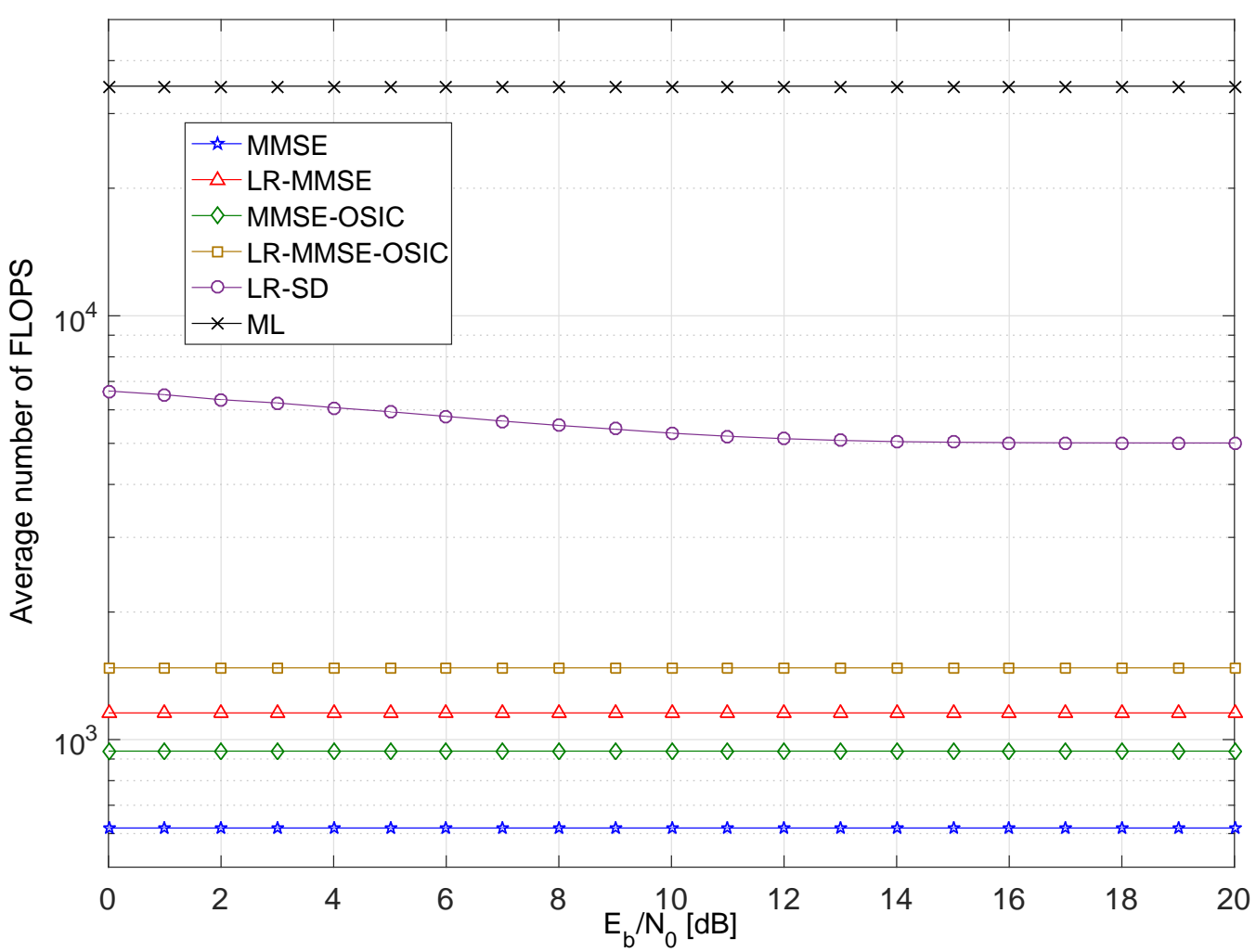

(a) Uncorrelated channel $(\rho=0.0)$

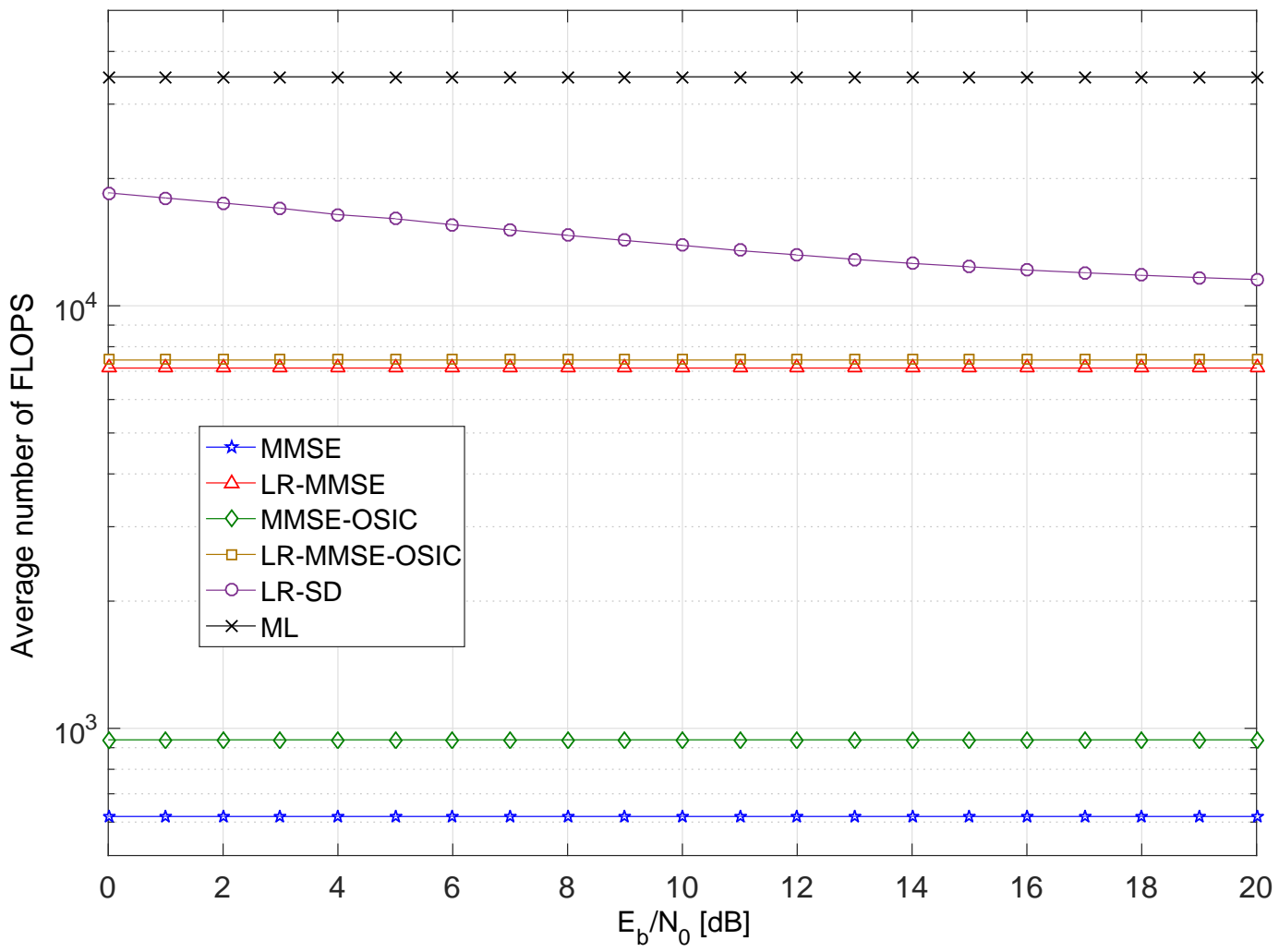

(b) Highly correlated channel $(\rho=0.9)$

Figure 2.5: Flop complexities for the analysed MIMO detectors with $4 \times 4$ antennas and pCSI in two scenarios: a) uncorrelated channels, $\rho=0$; b) highly correlated channels, $\rho=0.9$. 
In this sense, in the following chapters, where the LS-MIMO system is characterized and implemented through various detection techniques, the MMSE detector often appears as the initial solution, which can be iteratively improved by more advanced LS-MIMO detection strategies.

\subsection{Chapter conclusions}

In this chapter, the introductory MIMO system model as well as the conventional MIMO detection techniques were presented. In addition, the main results obtained with the application of the LR preprocessing technique were presented.

It is possible to conclude that the LR aid in linear detection in both correlated and uncorrelated channels results in performance gain by increasing the diversity order. When there is a high correlation between the antennas, the diversity order gain for the linear detectors is more conspicuous, leading the diversity order from a practically null condition to the optimal one. On the other hand, in these scenarios the LR complexity becomes preponderant in the total computation of these detectors FLOPS complexity.

Regarding the LR-SD with the proposed procedures of lookup table and optimization of the $\alpha$ parameter, as expected, an optimum performance was achieved, however with less complexity than the ML detector. On the other hand, in scenarios with strongly correlated antennas, the complexity gap between these detectors is reduced, due to the increased complexity of the LLL algorithm.

In the next chapter, the LS-MIMO system model is presented, which will serve as the basis for the main investigation developed in this Thesis. In addition, a comparative analysis of computational complexity among the most promising LS-MIMO detection techniques listed in the literature is presented. 


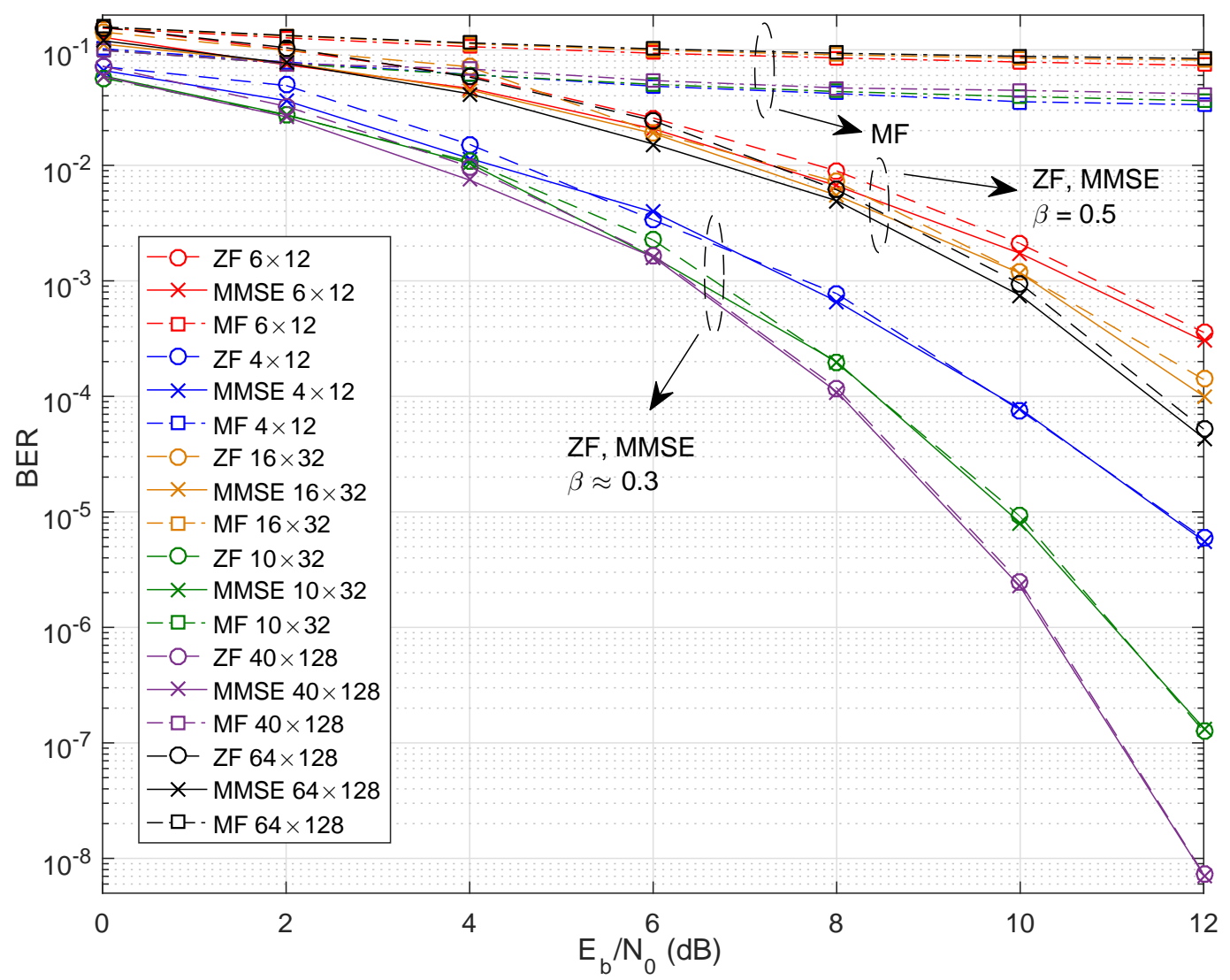

(a) low system loading

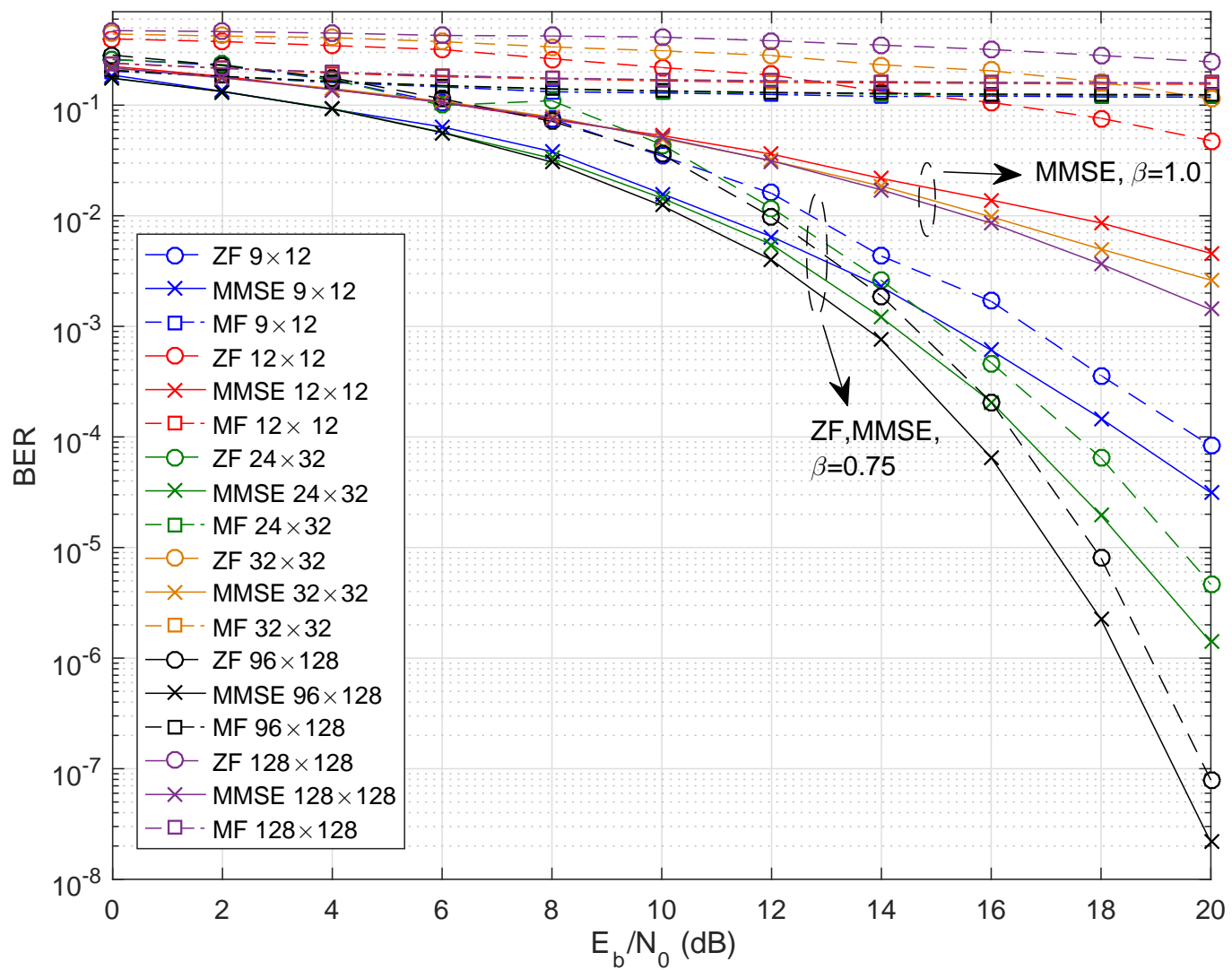

(b) high system loading

Figure 2.6: Performance of linear detectors MF, ZF and MMSE, in QPSK modulation, considering several antenna configurations: a) low system loading and b) high system loading scenarios. 


\section{System Model for LS-MIMO Systems}

This chapter describes the multi-user LS-MIMO system model, referring to the main scenario adopted in this work. The considered uplink channel (UL) system model is explained in addition to the computational complexities of the LS-MIMO detection techniques studied in this Thesis.

\subsection{System Model}

For simplicity, unicellular (single-cell) scenarios is considered, according to the model commonly adopted in the literature related to LS-MIMO detection [22, 80-84].

The considered UL single-cell MIMO communication system operating in multiplexing gain mode with $K$ active single-antenna users and $N$ receive antennas at the BS is disposed in Fig. 3.1.

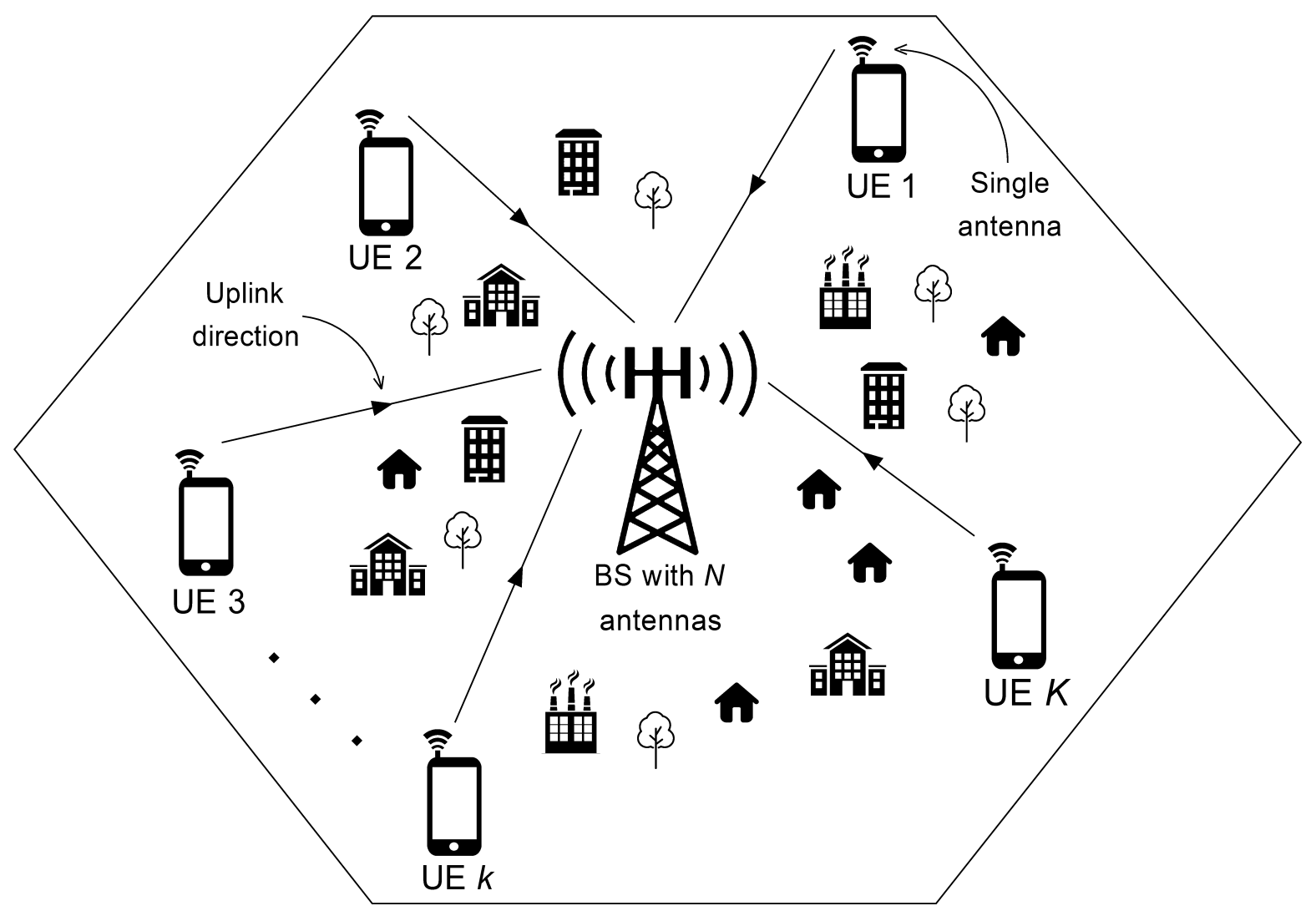

Figure 3.1: Single-cell uplink LS-MIMO communication system with $K$ single antenna UEs and a BS equipped with $N$ receive antennas.

The communication channel is similar to the conventional MIMO disposed in Section 2.2. It is assumed frequency-flat fading channel denoted by the complex matrix $\mathbf{H}_{c} \in \mathbb{C}^{N \times K}$. The elements of $\mathbf{H}_{c}$ are i.i.d. 
complex Gaussian random variables with zero mean and unit variance, i.e., $H_{c_{i, k}} \sim \mathcal{C N}[0 ; 1]$, where $H_{c_{i, k}}$ denotes the element in the $i$-th row and $k$-th column of the matrix $\mathbf{H}_{c}$. In other words, the fading coefficients matrix follow a Rayleigh distribution for amplitudes, representing NLOS communication, while the phase a uniform distribution in the interval $(0,2 \pi]$.

Let $\mathbf{s}_{c}$ be the $K \times 1$ complex vector corresponding to the $K$ symbols $M$-QAM transmitted over the singleantenna UEs, $\mathbf{s}_{c} \in \mathcal{A}_{c}^{K}$ where $\mathcal{A}_{c}$ denotes the QAM constellation adopted. The UL received signal, $y_{c_{i}}$, at the $i$-th $\mathrm{BS}$ antenna can be written as:

$$
\begin{aligned}
y_{c_{i}} & =\sum_{j=1}^{K} H_{c_{i, j}} s_{c_{j}}+\eta_{c_{i}}, \quad i=1, \ldots, N \\
& =\underbrace{H_{c_{i, k}} s_{c_{k}}}_{\text {desired signal }}+\underbrace{\sum_{j=1, j \neq k}^{K} H_{c_{i, j} s_{c_{j}}}}_{\text {intracellular interference }}+\underbrace{\eta_{c_{i}}}_{\text {AWGN }} .
\end{aligned}
$$

where $y_{c_{i}}$ denotes the $i$-th element of the complex received signal vector $\mathbf{y}_{c}, s_{c_{j}}$ is the $j$-th element of $\mathbf{s}_{c}$ and where $\eta_{c_{i}}$ denotes $i$-th element of the AWGN vector $\eta_{c}$. In matrix form, the received signal vector at the BS can be re-written as

$$
\mathbf{y}_{c}=\mathbf{H}_{c} \mathbf{s}_{c}+\boldsymbol{\eta}_{c}
$$

The system loading factor, $\beta$, is defined as:

$$
\beta=\frac{K}{N}
$$

The average received SNR at each receive antenna can be modeled as:

$$
\gamma=\frac{K P_{\mathrm{s}}}{\sigma^{2}}
$$

where $P_{\mathrm{S}}$ is the power of the received symbols. For simplicity, in Eq. (2.3) it is considered a perfect power control at the receiver, i.e., the large-scale fading effect is compensated in such a way that all $K$ users' signals are received with equal power at the $\mathrm{BS}$, and assumed equal to $K P_{\mathrm{s}}$, denoting the total sum power available at the transmitters [12]. The adopted unicellular multiuser LS-MIMO model can be seen as a point-to-point MIMO architecture, except that there is no possibility of correlation between the transmitting antennas, so that point-to-point MIMO detection algorithms can be applied.

An equivalent real-valued system model of the received signal can also be given, and is expressed as in Eq. (2.4).

For the QAM alphabet $\mathcal{A}_{c}$, the elements of $\mathrm{s}$ assume integer values from the underlying pulse-amplitude modulation (PAM) alphabet $\mathcal{A}$, i.e., $\mathrm{s} \in \mathcal{A}^{2 K}$.

\subsection{Duplexing protocols}

The system model presented in Section 3.1 considers the perfect knowledge of the channel parameters in the BS, however, it is known that, in practice, such parameters are not available and should be estimated, occupying the system's temporal resource for the estimation process. 
In the case of MU-LS-MIMO systems, the scheme for channel estimation is directly related to the adopted duplexing protocol, being the most used in such systems: time division duplexing (TDD) and frequency division duplexing (FDD). Both modes of transmission are considered full duplex.

The operation in each of these protocols is described below, and the advantages and disadvantages of each are also discussed.

\subsubsection{Time Division Duplexing}

In TDD mode, UL and DL (downlink) transmissions occupy distinct time bands, but both use the same frequency spectrum. Because they share the same band, the UL and DL channels have reciprocity (within the channel coherence time), i.e., estimating the CSI in one of the transmission channels, for example UL, there will be equivalence to the another, in this case DL.

The Figure 3.2 shows the time frame for channel estimation and data transmission DL and UL in the TDD mode, within a channel coherence time, $(\Delta t)_{\mathrm{C}}$ seconds, or $T=\frac{(\Delta t)_{\mathrm{C}}}{T_{s}}$ symbols; where $T_{s}$ is symbol period, in second. In the UL channel, since the BS requires the channel state information (CSI) to detect the signals, the process starts with the transmission of pilot sequences (PS) by each of the active $K$ users to BS, since the PSs should be orthogonal to each other, at least $K$ symbols are required. The BS performs the processing of these pilots, using a channel estimation technique, for CSI acquisition. The $K$ UEs then transmit data information to the BS. With the use of the estimated CSI, the BS performs the detection of the $K$ signals transmitted by the UEs, using one of the low complexity detection techniques, such as those discussed in the Chapters 4 and 5. In the DL channel, due to channel reciprocity, the estimated CSI in UL has equivalence in DL. Thus, the data information transmission by the BS can be precoded, however, for the detection of these signals by the UEs it is necessary to estimate the effective channel gain. For this, $K$ orthogonal pilot sequences are transmitted by BS, in which there is possibility of using beamforming since the CSI is already estimated. Again, at least $K$ symbols for training (sending the pilot sequences) DL are required. Alternatively, blind estimation can be used for effective channel gain, as recommended in [85], thus DL training is not required, which results in pilot overhead reduction. At the end, DL data information is transmitted to the $K$ UEs, where precoding techniques can be used. In total, at least $2 K$ symbols are required for UL and DL training and therefore $2 K<T$.

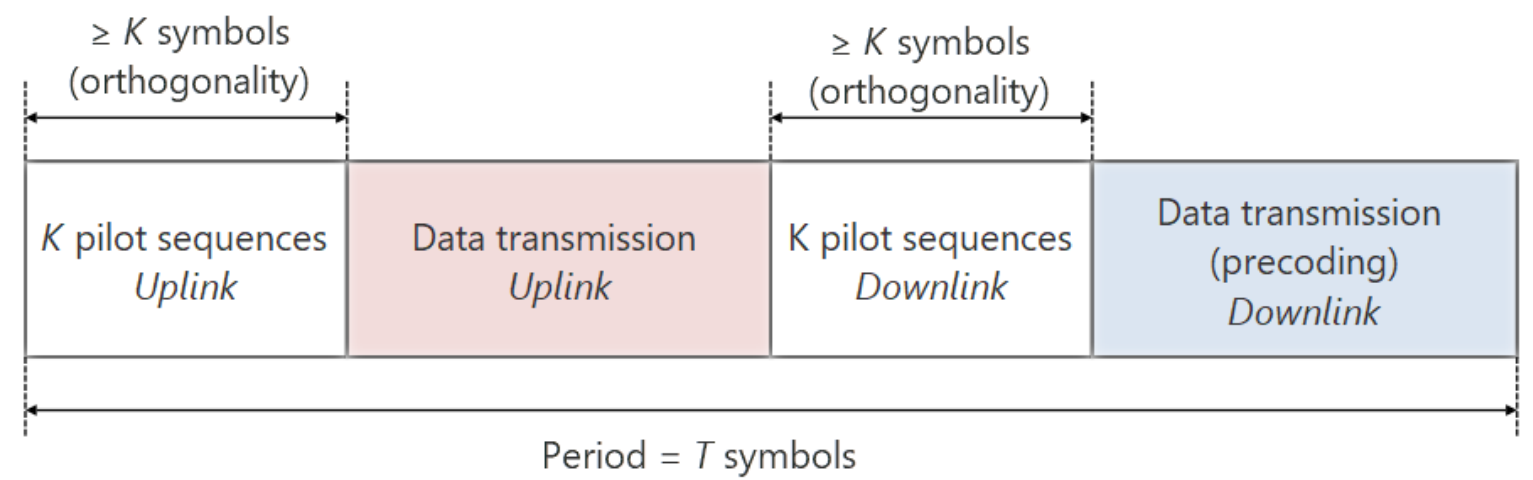

Figure 3.2: Temporal structure of transmission/estimation in a MU-LS-MIMO system in TDD mode. 
The main advantages and disadvantages of TDD mode in MU-LS-MIMO systems are listed below:

- TDD advantages

1. Reciprocity of uplink and downlink channels: significant advantage, especially in $\mathrm{DL}$, since there is no need for feedback, from UEs to BS, regarding CSI, necessary for precoding;

2. Ease of change in the uplink/downlink time relationship: depending on the system's requirement, one can easily change the time period intended for UL and DL transmission.

- TDD disadvantages

1. Pilot contamination: is the main drawback of TDD mode, representing a serious constraint on the overall system efficiency. It occurs due to the reuse of PSs in different users, in a multicell scenario. Currently, it is one of the most important topics within the MU-LS-MIMO subject and intense efforts are being made in the literature in order to combat the effect of the pilot contamination [86-90], being a promising field for studies;

2. Greater implementation difficulty (compared to FDD): due to the time division between the DL and UL channels, there is a need for greater accuracy in certain parameters, such as synchronization, guard intervals, among others.

\subsubsection{Frequency Division Duplexing}

In FDD mode, UL and DL transmissions occupy distinct frequency bands, so each channel is dedicated exclusively (full time) to the UL or DL transmission. In this way, there is simplification of implementation, since each channel is always "open". On the other hand, since they occupy different regions of the spectrum, there is no equivalence between the UL and DL channels, being necessary the estimation of the channel in both links.

Figure 3.3 shows the channel estimation scheme and data transmission for the UL and DL channels in FDD mode, considering that both channels have coherence times equal to $(\Delta t)_{\mathrm{C}}$ seconds, or $T=\frac{(\Delta t)_{\mathrm{C}}}{T_{s}}$ symbols; where $T_{s}$ is the symbol period, in seconds. In the UL channel, it begins with training with $K$ orthogonal PSs, requiring at least $K$ channel usage symbols. Then, UL data transmission from the $K$ UEs to the BS occurs. With the use of estimated CSIs in training, the BS detects the $K$ signals transmitted by the UEs. In addition, it is necessary that the UL channel perform the feedback of the $N K \mathrm{DL}$ channel estimates, which requires at least $N$ symbols. In the $\mathrm{DL}$ channel, there is no channel reciprocity and therefore the CSI should be estimated for use of precoding in the BS. Thus, $N$ orthogonal pilot sequences are transmitted by the $N$ BS antennas to the $K$ UEs. Each user will perform the estimation of $N$ channels based on received pilots. Next, there is a need for estimated CSI feedback through the UL, so BS can perform the precoding of the data information. With the CSI, the BS performs the precoding of the signals and the transmission of data symbols via DL. In total, at least $N+K$ symbols are required to use the UL channel and $N$ symbols for the downlink. In this way, it is required that $N+K<T$ for the $\mathrm{UL}$ and $N<T$ for the $\mathrm{DL}$, and thus the restriction in FDD mode is $N+K<T$. This is an immediate disadvantage in FDD, since pilot overhead depends on the number of $N$ antennas in BS, which is high for MU-LS-MIMO systems, causing high pilot overhead. 


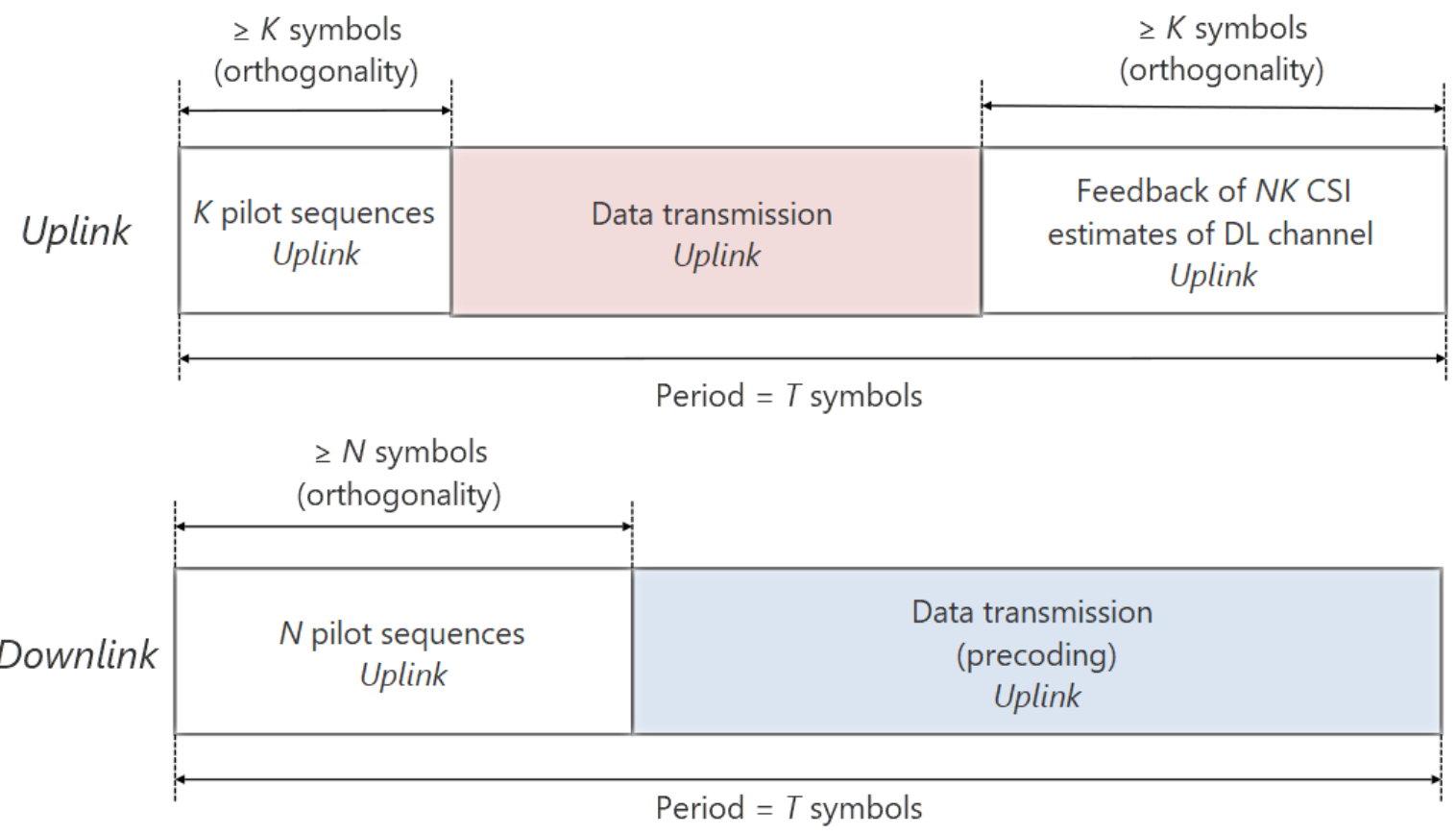

Figure 3.3: Temporal structure of transmission/estimation in a MU-LS-MIMO system in FDD mode.

Key advantages and disadvantages of FDD mode in MU-LS-MIMO systems include:

- FDD advantages

1. Pilot contamination effect can be bypassed: since the DL and UL channels are allocated in different frequency bands and there is no division of the channel coherence time between the transmissions, one has the possibility of using longer PSs, so that there is no repetition of PSs in adjacent cells. In addition, there is the possibility of using other strategies, such as allocation of different frequency bands between adjacent cells;

2. Simpler implementation in terms of synchronization and guarding periods (compared to TDD): since the allocation of each transmission path in different bands is sufficient, each channel remains constantly "open" to the UL or DL.

- FDD disadvantages

1. Estimation of both channels (DL and UL) and need for feedback: FDD mode disadvantage and the reason why most works in the literature assume the TDD mode in MU-LS-MIMO systems. Thus, for DL channel estimation, it is required that the BS send pilot signals and, after channel estimation by the UEs, the estimates must be returned to the BS for precoding in a time period substantially lower than the channel coherence time (to avoid pilot overhead). In the case of FDD MU-LS-MIMO, currently much of the efforts in the literature are about the DL channel estimation [91-94], being, also, a promising field for future studies;

2. Difficulty changing the uplink/downlink relationship: since the most common implementation would be dividing the band into two equal parts (for UL and $D L$ ), in case of higher demand for one of the transmission channels, the real-time change in this relationship is a complex process. 
The detection techniques investigated in this Thesis can be applied in both TDD and FDD modes since the PS training and channel estimation step are not considered. However, as will be shown in the Subsection 6.1 , there is a research direction in multicellular scenarios, in order to consider the effect of inter-cellular interference given by the pilot contamination. Therefore, the duplexing protocol adopted in this Doctoral research is the TDD.

\subsubsection{Computational Complexities of Detection Techniques}

Table 3.1 indicates the computational complexity of the detection techniques investigated during this research. The study and discussion of such techniques served as a basis for conjectures and contributions in the area of efficient detection in LS-MIMO systems, arranged in Chapters 4 and 5.

It can be seen from Table 3.1 that most of the detection techniques presented per-symbol symbol of the order $\mathcal{O}\left(K^{2}\right)$, which is considered an acceptable computational complexity for the LS-MIMO systems implementation. The complexity of the SD detector, according to $[67,68]$ is variable with the order of the constellation, denoted by $\mathcal{A}_{o}$, and by the parameter $\varsigma$ which represents the complexity with the channel conditions (or noise level) and therefore inversely proportional to the SNR, $\varsigma \propto \frac{1}{\gamma}$. As regards the per-symbol complexity of the FG-BP detector, it has been demonstrated in Chapter 4 (see also the published paper in Appendix $\mathrm{C}$ ) that it has a per-symbol complexity of $\mathcal{O}(N)$, which represents the lowest per-symbol complexity order detector among those investigated in this work. In addition, one can conclude that the heuristic detection techniques based on searches, i.e., LAS, RTS and LTS, present first per-symbol complexity order in their searches, being impacted by the quadratic complexity of the initial solution.

Table 3.1: Computational complexity of the detection techniques investigated in this work

\begin{tabular}{cc}
\hline Detectors & Per-symbol complexity \\
\hline \hline Linear ZF & $\mathcal{O}\left(K^{2}\right)$ \\
\hline Linear MMSE & $\mathcal{O}\left(K^{2}\right)$ \\
\hline MP based on MRF-BP & $\mathcal{O}\left(K^{2}\right)$ \\
\hline MP based on FG-BP & $\mathcal{O}(N)$ \\
\hline & Initial $(M M S E / Z F): \mathcal{O}\left(K^{2}\right)$ \\
LAS (heuristic) & LAS search: $\mathcal{O}(K)$ \\
& Total: $\mathcal{O}\left(K^{2}\right)$ \\
\hline & Initial $(M M S E / Z F): \mathcal{O}\left(K^{2}\right)$ \\
RTS (heuristic) & Total: $\mathcal{O}\left(K^{2}\right)$ \\
\hline & Initial $(M M S E / Z F): \mathcal{O}\left(K^{2}\right)$ \\
LTS (heuristic) & LAS search: $\mathcal{O}(K)$ \\
& Total: $\mathcal{O}\left(K^{2}\right)$ \\
\hline SD & $\mathcal{O}\left(\mathcal{A}_{o} \varsigma^{K} K^{2}\right)$ \\
\hline RID-MMSE & $\mathcal{O}(K)$ \\
\hline Conventional GS & $\mathcal{O}\left(K^{2}\right)$ \\
\hline MGS & $\mathcal{O}\left(K^{2}\right)$ \\
\hline \hline
\end{tabular}




\subsection{Chapter conclusions}

In this chapter the general system model was described, which will serve as a theoretical basis for the LS-MIMO detection techniques to be presented in the next chapters of this Thesis.

In the next chapter, the message passing detector is discussed both through different graphical models, focusing on the damping factor analysis, which represents one of the contributions of this Doctoral work. 


\section{Message Passing Detectors for Large Scale MIMO Systems: Damping Factor Analysis}

In this chapter, a MP detector based on BP algorithm for MRF and FG graphical models is analyzed under different LS-MIMO scenarios. The MD method is adapted to be applied in both MRF and FG approaches and an extensive analysis of such a method is performed in a variety of LS-MIMO scenarios, in order to compose a numerical approximation to propose an optimal DF value for MRF-BP.

Initially, a brief bibliographic review is addressed. Then, the MRF-BP and FG-BP detectors besides the respective MD strategy application are presented. Next, the main numerical results obtained are presented and the proposed optimal value determination for the DF is described. Finally, the remarks are made.

\subsection{Introduction}

BP based detectors have demonstrated a near optimal performance in LS-MIMO scenarios with low computational complexity [80, 95-97]. In [98], a Gaussian BP detector is proposed in a coded MIMO environment and its convergence is investigated under two different graph types, showing that the proposed Gaussian BP in fully connected pairwise graph has a faster convergence than ring type pairwise graph. In the work of [84], a Gaussian Message Passing Iterative Detection (GMPID) is considered in a massive multiuser MIMO system with LDPC decoding process; the convergence of GMPID detector is analyzed and demonstrated that it may not converge when system loading $\beta \geq(\sqrt{2}-1)^{2}$, in this sense, a new scale-and-add GMPID (SA-GMPID) is proposed which performs a faster and wide convergence range (for any $\beta \leq 1$ ).

Moreover, MP algorithms based on BP has been reported, in recent literature, as a promising detection procedure in single-carrier spatial modulation LS-MIMO systems [99]. Another efficient MP algorithms, based on BP technique, are such that LS-detectors inspired in graphical models, as factor graph or FG [20] and Markov random fields or MRF [100]. In some situations, BP algorithm may fail to converge, and if it does converge, the estimated marginal probabilities may be far from exact [101]. However, there are several methods in the literature to improve the convergence of BP algorithm, including message damping method $[102,103]$ and double loop methods $[104,105]$. The MD method applied to the MP algorithm based on MRF graphical model is presented in [20], where three types of damping are performed: message damping, belief damping and hybrid damping, showing that both message/belief damping significantly improves the performance without a relevant increase in computational complexity. In [80], MP detectors using MRF or FG approach are also presented and the MD method, applied exclusively in MRF-BP, demonstrated to achieve a relevant performance gain with a negligible complexity increment; besides, in the same work, a hybrid reactive 
tabu search BP is proposed in order to perform in high order QAM constellations. Although MD method has been proven to achieve a significant performance improvement, the DF values adopted in the previous works in the literature are valid only for specific system operation scenarios considered, while its extension for large MIMO schemes has not been investigated so far.

Table 4.1 below presents a summary of contributions, found in the literature, that make use of MP algorithms for signal detection in LS-MIMO systems.

Table 4.1: Synthesis of contributions in MP detectors for LS-MIMO.

\begin{tabular}{|c|c|c|}
\hline Year & Authors & Contributions \\
\hline$\overline{\overline{2011}}$ & $\begin{array}{c}\text { Goldberger et al. } \\
{[106]}\end{array}$ & $\begin{array}{l}\text { It is proposed a technique based on GTA and BP for MIMO detection in high order } \\
\text { constellations. The proposed technique demonstrates better performance results } \\
\text { when compared to MMSE and MMSE-SIC. }\end{array}$ \\
\hline 2011 & Som et al. [80] & $\begin{array}{l}\text { It is demonstrated that the use of MRF-BP and FG-BP detectors in LS-MIMO-ISI } \\
\text { (inter-symbol interference) channels results in almost optimal performance with low } \\
\text { complexity. In addition, it is shown that the performance tends to improve with } \\
\text { the increase of the number of antennas, which favors the application in LS-MIMO } \\
\text { scenarios. }\end{array}$ \\
\hline 2013 & Abiko et al. [107] & $\begin{array}{l}\text { It is considered the extrinsic information transfer (EXIT) analysis for validation and } \\
\text { convergence of iterative detection using BP in LS-MIMO scenarios. The results } \\
\text { indicate that there is convergence of performance of the BP detector when the } \\
\text { number of antennas is equal to } 100 \text {. }\end{array}$ \\
\hline 2014 & Wu et al. [108] & $\begin{array}{l}\text { It is proposed algorithms based on MP iterative approximation using Gaussian } \\
\text { approximation, expectation propagation, first order approximation, which demon- } \\
\text { strate acceptable performance versus computational complexity relation. }\end{array}$ \\
\hline 2015 & $\begin{array}{c}\text { Shengchu Wang et } \\
\text { al. [109] }\end{array}$ & $\begin{array}{l}\text { An MP detector based on the low-complexity GDL algorithm is developed. The } \\
\text { detector shows good performance in LS-MIMO scenarios based on the a priori } \\
\text { received signal distribution and hybrid measurements on the BS. }\end{array}$ \\
\hline 2016 & Liu et al. [84] & $\begin{array}{l}\text { A study on the convergence of of the GMPID for the MMSE detector in LS-MIMO } \\
\text { scenarios with low and medium loads is carried out. It is demonstrated that for } \\
\text { system loading greater than } \beta \geq(\sqrt{2}-1)^{2} \text {, the GMPID does not converge. It is } \\
\text { proposed an SA-GMPID detector, which has proven convergence for the MMSE to } \\
K / N<1 \text {, with no relevant complexity increase to the GMPID. }\end{array}$ \\
\hline
\end{tabular}

Due to the applicability and success of BP in LS-MIMO detection $[80,99,100,110]$, BP detectors based on a MRF and FG graphical models are considered in this Doctoral work. Furthermore, message damping method is applied and a BER performance $\times$ computational complexity analysis relating the DF value is performed. Our extensive analysis considers wide range of antennas configurations, matching large-scale MIMO, and SNR region scenarios, aiming to demonstrate the performance-complexity gain obtained with the MRF-BP detector deploying the proposed optimal damping factor.

\subsection{System Model and Basic Concepts}

The complex-valued system model adopted is described in Section 3.1. The transmitted symbols from the single-antenna UEs follow a BPSK modulation, i.e., $\mathbf{s}_{c} \in \mathcal{A}^{K}$, where $\mathcal{A}=\{-1,+1\}$. It is considered that the minimum spacing between the Rx antennas is obeyed, so that there is no correlation between antennas, $\rho_{\mathrm{Tx}}=0$; also, a pCSI is provided at the BS. 


\subsubsection{Graphical Models}

Graphical models (GMs) are graphs that indicate inter-dependencies between random variables [111]. Statistical distributions that exhibit some structure can generally be represented naturally and compactly using a $\mathrm{GM}$, that is the case of distributions of interest in MIMO systems (e.g., vector of received symbols). The GM structure often allows the statistical distribution of interest to be effectively used for inference, i.e., answering certain questions of interest using the distribution [12]. Three basic graphical models widely used to represent statistical distributions include Bayesian belief networks [112], Markov random fields [113] and factor graphs [114].

The MRF is an undirected graph whose vertices are random variables, which consists in a graph composed only of variable nodes. The variables are such that any variable is independent of all the other variables, given its neighbours. For instance, a MIMO system under multiplexing gain mode (Mux-MIMO) can be conveniently modeled through an MRF in which each transmitted symbol is represented by a variable node in the GM. Since each transmit antenna is used to transmit a separate symbol, there are $K$ nodes in such graph. Considering that every transmitted symbol interferes with every other transmitted symbol in a Mux-MIMO mode, the graph is fully connected.

Another type of GM that can be conveniently used in the representation of the LS-MIMO detection problem is the factor graph. FG are bipartite graphs, i.e., there are two types of nodes in a FG: variable nodes and function nodes (or observation nodes). Variable nodes are usually denoted as circles and function nodes are usually denoted as squares. A factor graph explicitly depicts the factorization of a function, typically a probability distribution, into several local functions; and each local function depends on a subset of the set of all variables. In the case of MIMO systems, the transmitted symbols can be denoted by the variable nodes and the received signal (at each receive antenna) by the observation nodes. At each type of node, a calculation is evaluated and transferred to the other type of node. This inter-transfer between variable and observation nodes is performed for a fixed number of iterations.

Therefore, the main difference between these models refers that MRF is a fully connected graphic with only variable nodes, whereas FG is a bipartite graph consisted by variable nodes and function (or observation) nodes. Also, the message update functions on each model are different.

\subsubsection{Belief Propagation}

Belief propagation is a technique that solves probabilistic inference problems usually implemented in graphical models. BP is a simple, yet highly effective, procedure that has been successfully employed in a variety of applications including computational biology, statistical signal/image processing, data mining, etc. The BP algorithm is widely recognized as an efficient tool that can be used to solve several problems, including wireless communications questions as well [111]. The goal is to detect a hidden input (e.g., transmitted symbol in MIMO systems) from its observed output (e.g., received signal in MIMO systems). The system can be represented as a GM and the system input detection is equivalent to carry out inference on the corresponding graph. More precisely, BP is a procedure used to compute the marginalization of functions by passing messages on a graphical model [12]. Due to its simplicity and efficiency, BP is the most common strategy adopted to 
implement message passing principle. Other message passing algorithms can be addressed as generalized distributive law [115] and sum-product algorithm [114].

\subsection{Message Passing via MRF for Large Scale MIMO Detection}

This section presents a BP based detector that employs message passing on a MRF [80]. Consider the system model in Eq. (3.1). The maximum a posteriori (MAP) detector takes the joint a posteriori distribution:

$$
p\left(\mathbf{s}_{c} \mid \mathbf{y}_{c}, \mathbf{H}_{c}\right) \propto p\left(\mathbf{y}_{c} \mid \mathbf{s}_{c}, \mathbf{H}_{c}\right) \cdot p(\mathbf{s})
$$

The MAP estimation of the bit $s_{i}, i=1, \ldots, K$ is given by

$$
\widehat{s}_{i}=\underset{a \in\{-1,+1\}}{\arg \max } p\left(s_{i}=a \mid \mathbf{y}_{c}, \mathbf{H}_{c}\right)
$$

whose complexity is exponential in $K$ [12].

Given $\mathbf{s}_{c}$ and $\mathbf{H}_{c}, \mathbf{y}_{c}$ is a complex Gaussian random vector with mean $\mathbf{H}_{c} \mathbf{s}_{c}$ and covariance $\sigma^{2} \mathbf{I}_{N}$. Thus,

$$
p\left(\mathbf{y}_{c} \mid \mathbf{s}_{c}, \mathbf{H}_{c}\right) \propto \exp \left(\frac{-\left\|\mathbf{y}_{c}-\mathbf{H}_{c} \mathbf{s}_{c}\right\|^{2}}{2 \sigma^{2}}\right)
$$

Assuming that the symbols in $\mathbf{s}_{c}$ are all independent, also a necessary condition for a MRF procedure [12]. Hence, a priori probability for the transmitted symbol vector is given by

$$
p\left(\mathbf{s}_{c}\right)=\prod_{i} p\left(s_{c_{i}}\right)
$$

From Eqs. (4.1), (4.3) and (4.4), the conditional probability function can be written as:

$$
\begin{aligned}
p\left(\mathbf{s}_{c} \mid \mathbf{y}_{c}, \mathbf{H}_{c}\right) & \propto \exp \left(\frac{-\left\|\mathbf{y}_{c}-\mathbf{H}_{c} \mathbf{s}_{c}\right\|^{2}}{2 \sigma^{2}}\right) \exp \left(\ln p\left(\mathbf{s}_{c}\right)\right)=\exp \left(\frac{-1}{2 \sigma^{2}}\left(\mathbf{y}_{c}-\mathbf{H}_{c} \mathbf{s}_{c}\right)^{\mathrm{H}}\left(\mathbf{y}_{c}-\mathbf{H}_{c} \mathbf{s}_{c}\right)\right) \prod_{i} \exp \left(\ln p\left(s_{c_{i}}\right)\right) \\
& \propto \exp \left(\frac{-1}{2 \sigma^{2}}\left(\mathbf{s}_{c}^{\mathrm{H}} \mathbf{H}_{c}^{\mathrm{H}} \mathbf{H}_{c} \mathbf{s}_{c}-2 \Re\left\{\mathbf{s}_{c}^{\mathrm{H}} \mathbf{H}_{c}^{\mathrm{H}} \mathbf{y}_{c}\right\}\right)\right) \prod_{i} \exp \left(\ln p\left(s_{c_{i}}\right)\right)
\end{aligned}
$$

Defining the channel correlation matrix $\mathbf{R}_{c}=\left(\frac{1}{\sigma^{2}}\right) \mathbf{H}_{c}^{\mathrm{H}} \mathbf{H}_{c}$ and $\mathbf{v}=\left(\frac{1}{\sigma^{2}}\right) \mathbf{H}_{c}^{\mathrm{H}} \mathbf{y}_{c}$, Eq. (4.5) can be rewritten as:

$$
\begin{aligned}
p\left(\mathbf{s}_{c} \mid \mathbf{y}_{c}, \mathbf{H}_{c}\right) & \propto \exp \left(-\sum_{i<j} \Re\left\{s_{c_{i}}^{*} r_{c_{i, j}} s_{c_{j}}\right\}\right) \exp \left(\sum_{i} \Re\left\{s_{c_{i}}^{*} v_{i}\right\}\right) \prod_{i} \exp \left(\ln p\left(s_{c_{i}}\right)\right) \\
& =\left(\prod_{i<j} \exp \left(-s_{c_{i}} \Re\left\{r_{c_{i, j}}\right\} s_{c_{j}}\right)\right)\left(\prod_{i} \exp \left(s_{c_{i}} \Re\left\{v_{i}\right\}+\ln p\left(s_{c_{i}}\right)\right)\right)
\end{aligned}
$$

where $v_{i}$ and $r_{c_{i, j}}$ are the elements of $\mathbf{v}$ and $\mathbf{R}_{c}$, respectively.

Analyzing Eq. (4.6), it is seen that the MRF of the MIMO system presents pair-wise interactions with 
the potentials ${ }^{1}$ defined by:

$$
\begin{gathered}
\psi_{i, j}\left(s_{c_{i}}, s_{c_{j}}\right)=\exp \left[-s_{c_{i}} \Re\left\{r_{c_{i, j}}\right\} s_{c_{j}}\right] \\
\phi_{i}\left(s_{c_{i}}\right)=\exp \left[s_{c_{i}} \Re\left\{v_{i}\right\}+\ln p\left(s_{c_{i}}\right)\right]
\end{gathered}
$$

The values of $\psi$ and $\phi$ define, respectively, the edge and self potentials of the MRF graphical model to which message passing algorithm is applied to compute the marginal probabilities of the variables. BP algorithm attempts to estimate the marginal probabilities of all the variables by way of passing messages between the local nodes.

A message from node $j$ to node $i$ is denoted by $m_{j, i}\left(s_{c_{i}}\right)$, and the belief at node $i$ is denoted $b_{i}\left(s_{c_{i}}\right)$, $s_{c_{i}} \in\{ \pm 1\}$. The belief $b_{i}\left(s_{c_{i}}\right)$ depends on how likely $s_{c_{i}}$ was transmitted. On the other hand, $m_{j, i}\left(s_{c_{i}}\right)$ depends on how likely that node $j$ evaluates $s_{c_{i}}$ was transmitted. The message from node $i$ to a neighboring node $j$ is then given by:

$$
\tilde{m}_{i, j}\left(s_{c_{j}}\right)=\sum_{s_{c_{i}}} \phi_{i}\left(s_{c_{i}}\right) \psi_{i, j}\left(s_{c_{i}}, s_{c_{j}}\right) \prod_{k \in \mathcal{B}(i) \backslash j} m_{k, i}\left(s_{c_{i}}\right)
$$

where $\mathcal{B}(i)$ denotes the set of all nodes neighboring the node $i$ and $\mathcal{B}(i) \backslash j$ denotes the same neighborhood, except the node $j$. Eq. (4.9) actually constitutes an iteration, as the message is defined in terms of the messages from other nodes. So, BP essentially involves computing the outgoing messages from a node to each of its neighbors using the local joint compatibility function and the incoming messages and transmitting them [12]. The algorithm terminates after a fixed number of iterations, $\mathcal{I}_{\mathrm{MRF}}$. In Fig. 4.1, a diagram describing in simplicity the operation of the MRF-BP described in this subsection is shown.

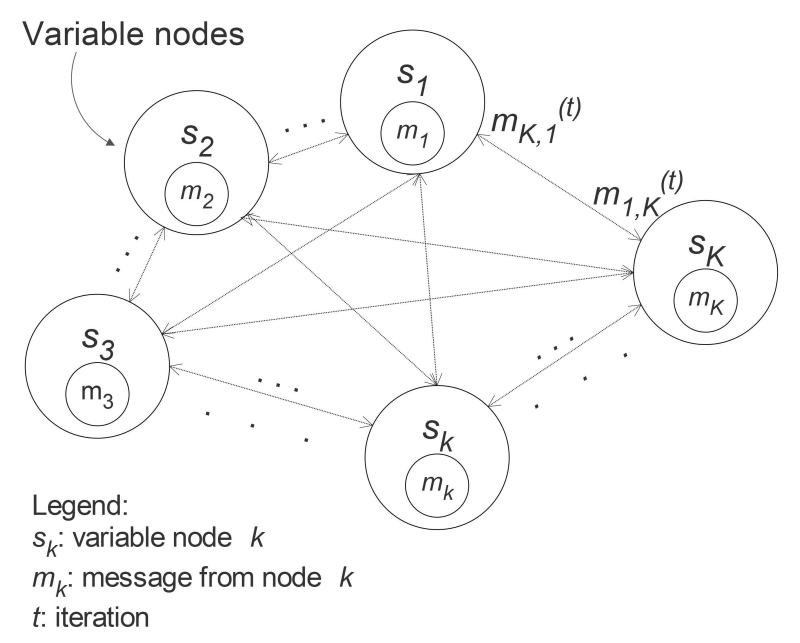

Figure 4.1: Diagram of MRF-BP, based on Markov random field graph model, where $m_{1, k}$ is the message from node 1 to node $k$ of Eq. (4.9).

\subsubsection{Message Damping on MRF-BP Detector}

The MD method can be used to improve the convergence of BP algorithm. The messages to be passed are computed as a weighted average of the message in the previous iteration and the message in the current iteration $[102,103]$. Thus, the damped message to be passed from node $i$ to node $j$ in iteration $t$, denoted

\footnotetext{
${ }^{1} \mathrm{~A}$ fully connected subgraph of an MRF is called a clique; the variables in an MRF, constrained by a compatibility function is known as a (clique) potential.
} 
by $m_{i, j}^{(t)}\left(s_{c_{j}}\right)$, is computed as a convex combination of the previous message and the current message as:

$$
m_{i, j}^{(t)}\left(s_{c_{j}}\right)=\alpha m_{i, j}^{(t-1)}\left(s_{c_{j}}\right)+(1-\alpha) \tilde{m}_{i, j}^{(t)}\left(s_{c_{j}}\right)
$$

where $\tilde{m}_{i, j}^{(t)}\left(x_{j}\right)$ and $m_{i, j}^{(t-1)}\left(x_{j}\right)$ denotes, respectively, the current message in iteration $t$ and the previous message in iteration $t-1$, and $\alpha \in(0,1]$ is referred as the damping factor. This simple damping of messages has been shown to be very effective in improving BP convergence and performance [102]. As shown in numerical results section, considering the LS-MIMO detection context, message damping method can improve performance significantly, without increasing the computational complexity. Furthermore, the analysis of DF in different LS-MIMO configurations is one of the contributions of this Doctoral work.

Damping of messages can be carried out in each iteration. The final belief about the variable $s_{c_{i}}$ is computed as:

$$
b_{i}\left(s_{c_{i}}\right) \propto \phi_{i}\left(s_{c_{i}}\right) \prod_{j \in \mathcal{B}(i)} m_{j, i}\left(s_{c_{j}}\right)
$$

In the case of a coded system, the soft output of the algorithm $b_{i}\left(s_{c_{i}}\right)$ can be directly fed to the decoder. A pseudocode for the MRF-BP described above is listed in Algorithm 4.1. 


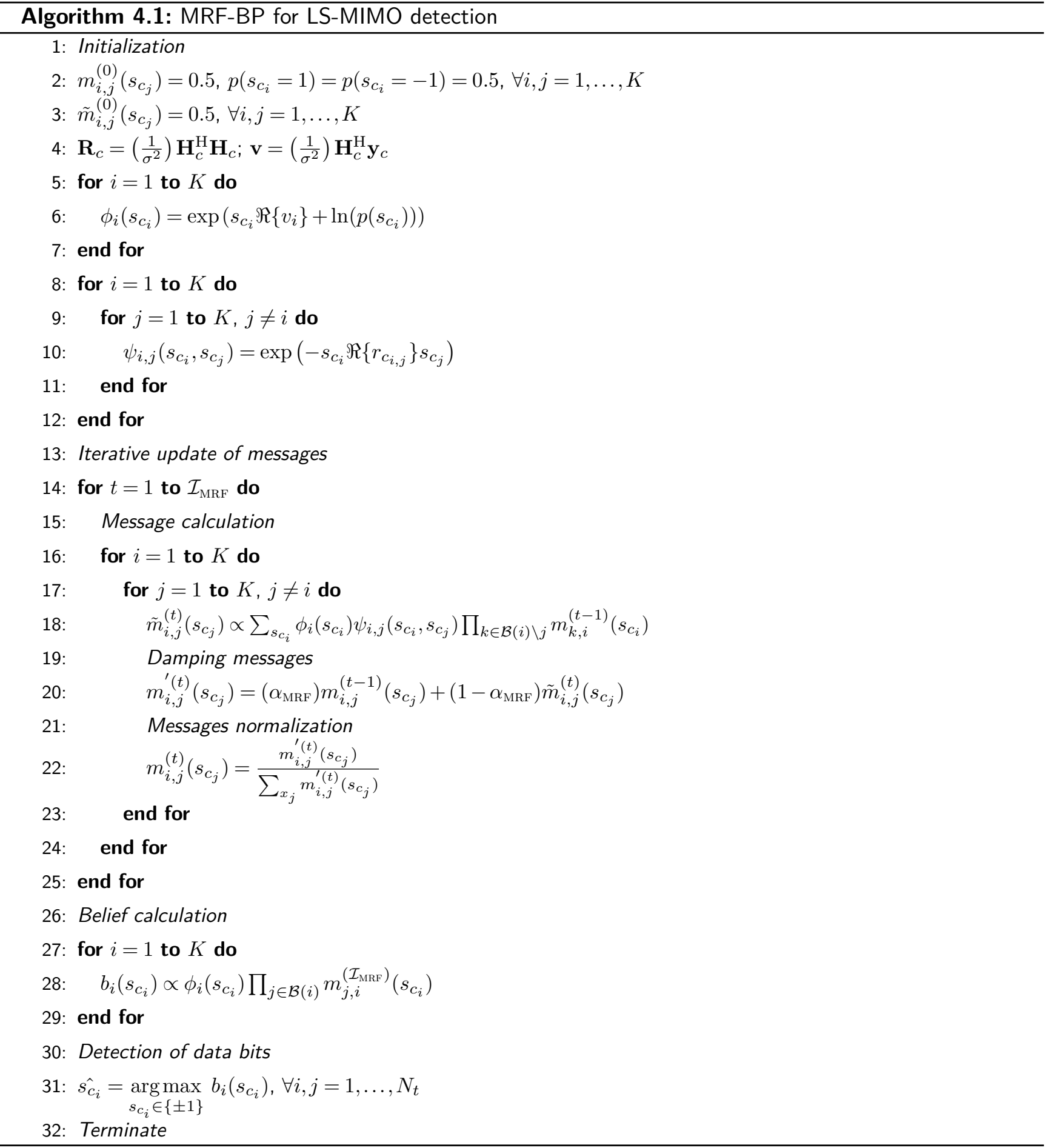

\subsection{Message Passing via FG for Large Scale MIMO Detection}

Considering each entry of the observation vector $\mathbf{y}_{c}$ as a function node (observation node) in a factor graph, and each transmitted symbol as a variable node. Considering the received signal from Eq. (3.1), the interferenceplus-noise term is given by:

$$
y_{c_{i}}=h_{c_{i, k}} s_{c_{k}}+\underbrace{\sum_{j=1, j \neq k}^{K} h_{c_{i, j}} s_{c_{j}}+\eta_{c_{i}}}_{\text {interference-plus-noise } \triangleq \lambda_{i, k}} \triangleq h_{c i, k} s_{c_{k}}+\lambda_{i, k}
$$


In order to reduce the computational complexity, the interference-plus-noise term can be approximated by a scalar Gaussian approximation (SGA) [80]. Thus, , $\lambda_{i, k}$, which refers to the message from the $i$ th observation node, $y_{c_{i}}$, to the $k$ th variable node, $s_{c_{k}}$, is modeled as $\mathbb{C B}\left(\mu_{\lambda_{i k}}, \sigma_{\lambda_{i k}}^{2}\right)$ with mean and variance given, respectively, by:

$$
\begin{array}{r}
\mu_{\lambda_{i k}}=\sum_{j=1, j \neq k}^{K} h_{c_{i, j}} \mathbb{E}\left\{s_{c_{j}}\right\} \\
\sigma_{\lambda_{i k}}^{2}=\sum_{j=1, j \neq k}^{K}\left|h_{c_{i, j}}\right| \operatorname{Var}\left\{s_{c_{j}}\right\}+\sigma^{2}
\end{array}
$$

where $\operatorname{Var}\{\cdot\}$ denotes the statistical variance operator.

Hence, the log-likelihood ratio (LLR) for the symbol $s_{c_{k}} \in\{+1,-1\}$ at the observation node $i$, denoted by $\Lambda_{i}^{k}$, can be expressed by

$$
\tilde{\Lambda}_{i}^{k}=\ln \frac{\mathrm{p}\left(y_{c_{i}} \mid \mathbf{H}_{c}, s_{c_{k}}=+1\right)}{\mathrm{p}\left(y_{c_{i}} \mid \mathbf{H}_{c}, s_{c_{k}}=-1\right)}=\frac{4}{\sigma_{\lambda_{i k}}^{2}} \Re\left\{h_{c_{i, k}}^{*}\left(y_{c_{i}}-\mu_{\lambda_{i k}}\right)\right\}
$$

From Eq. (4.15), the LLR values are computed at the observation nodes and passed (messaged) to the variable nodes. Using the LLR value, received through $i$ th observation node message, the $k$ th variable node computes the conditional probability of $s_{c_{k}}=+1$, given the received signal $\mathbf{y}_{c}$, as:

$$
\begin{aligned}
\tilde{\mathrm{p}}_{i}^{k+} & \triangleq \mathrm{p}_{i}\left(s_{c_{k}}=+1 \mid \mathbf{y}_{c}\right) \\
& =\frac{\exp \left(\sum_{l=1, l \neq i}^{N} \Lambda_{l}^{k}\right)}{1+\exp \left(\sum_{l=1, l \neq i}^{N} \Lambda_{l}^{k}\right)}
\end{aligned}
$$

Hence, the probabilities from Eq. (4.16) are passed back to the observation nodes. In Fig. 4.2, the graph model diagram describing in simplicity the operation of the FG-BP described in this subsection is shown.

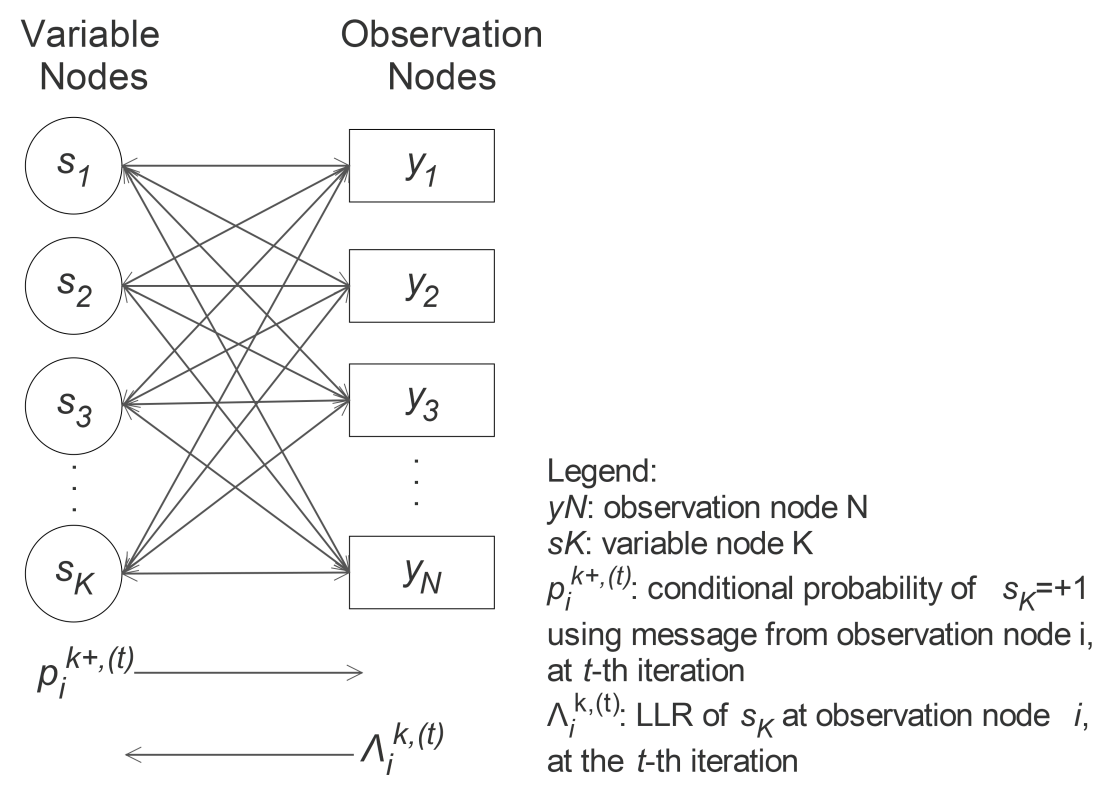

Figure 4.2: The graph model diagram of FG-BP, where $\mathrm{p}_{i}^{k+,(t)}$ is the conditional probability of Eq. (4.16) and $\Lambda_{i}^{k}$ is the LLR of Eq. (4.15). 
The message passing between observation nodes and variable nodes is carried out for a certain number of iterations, called $\mathcal{I}_{\mathrm{FG}}$. After that, the estimated symbol, $\hat{s}_{c_{k}}$, is detected as:

$$
\begin{aligned}
\tilde{s}_{c_{k}} & =\sum_{i=1}^{N} \Lambda_{i}^{k} \\
\hat{s}_{c_{k}} & =\operatorname{sgn}\left(\tilde{s}_{c_{k}}\right)
\end{aligned}
$$

Note that $\tilde{s}_{c_{k}}$ in Eq. (4.17) is a soft output from the LLR computation. In the case of a coded system, the soft output can be directly fed to the decoder. Moreover, from Eq. (4.18) the estimated symbol $\hat{s}_{c_{k}}$ is obtained as a hard-limiting of $\tilde{s}_{c_{k}}$, since a BPSK modulation is considered. Due to the factor graph model and the scalar Gaussian approximation of the interference, this algorithm is called FG-BP SGA; however, the last term is omitted in the remainder of this work for textual simplification.

\subsubsection{Message Damping on FG-BP Detector}

In this work, we proposed the application of the MD method in the FG-BP detector. Thus, the calculation of the LLR at the observation nodes and the probabilities at the variable nodes are given in its damped version by:

$$
\begin{aligned}
\Lambda_{i}^{k,(t)} & =\left(\alpha_{\mathrm{FG}}\right) \Lambda_{i}^{k,(t-1)}+\left(1-\alpha_{\mathrm{FG}}\right) \tilde{\Lambda}_{i}^{k,(t)} \\
p_{i}^{k+,(t)} & =\left(\beta_{\mathrm{FG}}\right) p_{i}^{k+,(t-1)}+\left(1-\beta_{\mathrm{FG}}\right) \tilde{p}_{i}^{k+,(t)}
\end{aligned}
$$

where $\alpha_{\mathrm{FG}}$ and $\beta_{\mathrm{FG}}$ are the DF for the FG-BP at the observation and variable nodes, respectively.

A pseudocode for the FG-BP algorithm described above is listed in Algorithm 4.2. 


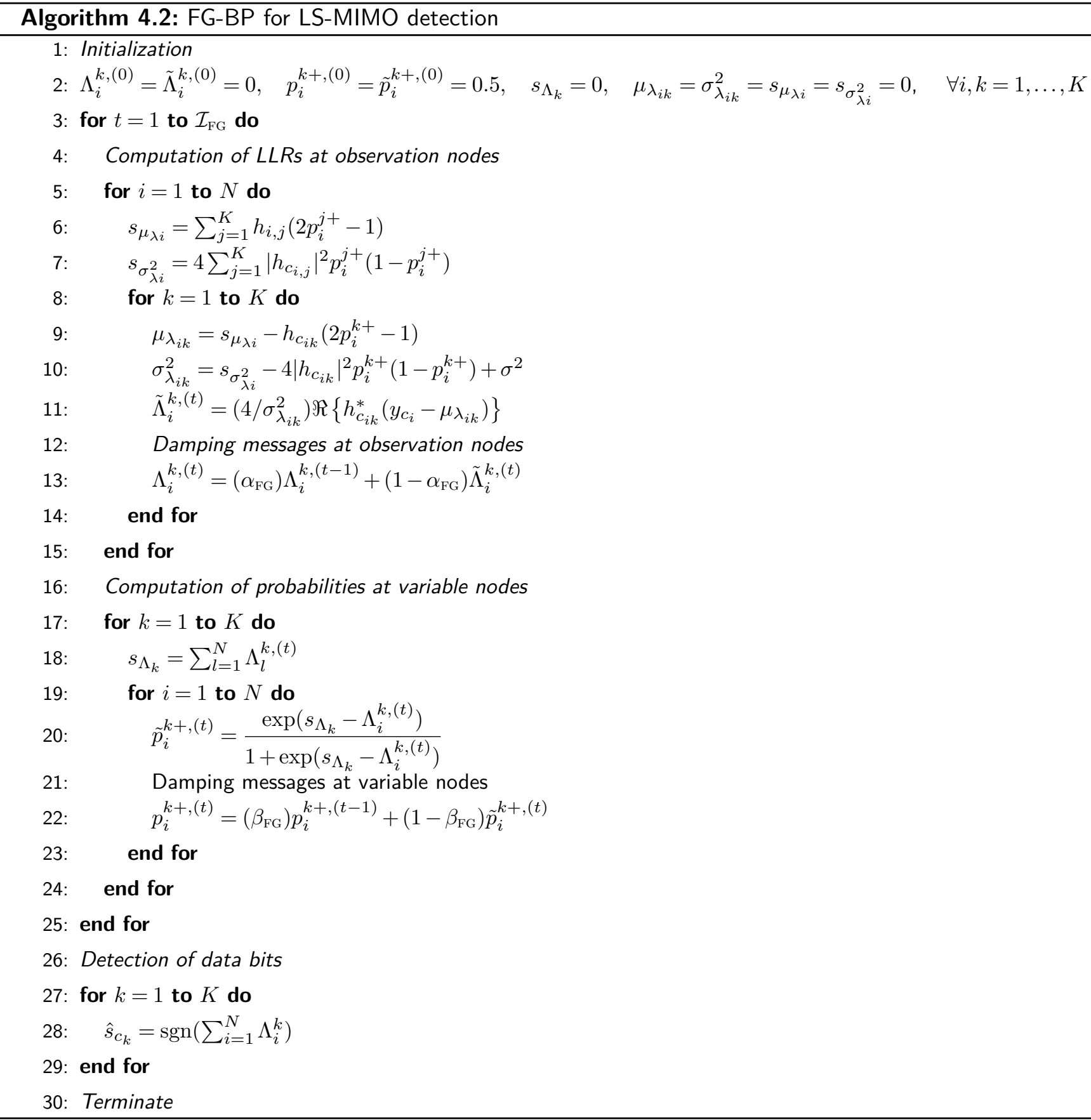

\subsection{Numerical Results}

In this section the uncoded BER performance related to the MRF-BP and FG-BP LS-MIMO detectors is evaluated through MCS. The simulations are performed assuming that $\mathrm{pCSI}$ is available at the receiver side and there is no antenna correlation. Besides, an extensive numerical analysis on the optimal value for the DF is carried out considering wide large-scale antennas scenarios and SNR regions. Finally, computational complexity of the MRF-BP and FG-BP algorithms are compared aiming to determine the best performancecomplexity tradeoff of both LS-MIMO detection approaches. For comparison purpose, the BER performance of a SISO transmission scheme operating under flat fading channel, as well as in purely AWGN channel were included in several graphs. Table 4.2 summarizes the main system and channel parameters values deployed in this section. 
Table 4.2: LS-MIMO system and channel parameters

\begin{tabular}{ll}
\hline Parameter & Value \\
\hline Link direction & Uplink $(\mathrm{UL})$ \\
\# Rx antennas & $N \in[20,500]$ \\
\# Mobile single-antenna users & $K \in[20,500]$ \\
System loading & $\beta=1.0$ \\
SNR ranges & $\gamma \in[0,10] \mathrm{dB}$ and $\gamma \in[6,14] \mathrm{dB}$ \\
\hline Channel type & Flat Rayleigh \\
Channel availability & Perfectly known at receiver \\
Modulation order & $\mathrm{BPSK}$ \\
\hline \# iterations MRF-BP & $\mathcal{I}_{\mathrm{MRF}} \in\{3 ; 4 ; 5\}$ \\
\# iterations FG-BP & $\mathcal{I}_{\mathrm{FG}} \in\{4 ; 5 ; 7\}$ \\
Damping Factor MRF-BP & $\alpha_{\mathrm{MRF}} \in[0,1]$ \\
Damping Factor FG-BP & $\alpha_{\mathrm{FG}} \in[0,1]$ and $\beta_{\mathrm{FG}} \in[0,1]$ \\
\hline
\end{tabular}

Fig. 4.3 depicts the impact of number of iterations $(\mathcal{I})$ over the performance of the both MRF-based and FG-based LS-MIMO detectors. Accordingly, Fig. 4.3.a.1) evidences the influence of the number of iterations $\left(\mathcal{I}_{\mathrm{MRF}}\right)$ of MRF-BP algorithm on the BER performance at $\gamma=10 \mathrm{~dB}$ without damping messages $\left(\alpha_{\mathrm{MRF}}=0\right)$. One can notice the occurrence of a significant performance gain in the first four iterations of Algorithm 4.1. Besides, with the increasing number of antennas, the performance of MRF-BP detector improves accordingly. For all antennas configuration depicted in this graph, the performance gain occurs, mainly, in the first four iterations, after that, there is no relevant performance gain, i.e., no relevant information is carried out in neighborhood messages. On the other hand, Fig. 4.3.b.1) presents the influence of the number of iterations, $\mathcal{I}_{\mathrm{FG}}$, on FG-BP BER performance at $\gamma_{=} 10 \mathrm{~dB}$ without MD method, i.e. $\alpha_{\mathrm{FG}}=\beta_{\mathrm{FG}}=0$. For low number of antennas, $K=N=20$, it can be noted that only the seven first iterations represents a BER performance gain; more precisely, in the first five iterations occurs the main performance gain. As the number of antennas increases, it is noticeable that the fifth iteration becomes less relevant in BER performance; indeed, the majority performance gain is obtained into the first four iterations, whereas when $K=N=200$ the convergence is fully achieved in the fourth iteration. Furthermore, one can notice that when the MD technique is not applied, the error rates reached by the FG-BP detector are significantly lower compared to the MRF-BP for the same amount of antennas.

Also, an analysis of MD impact on the algorithm iterations can be performed for the MRF-BP and FG-BP, as disposed, respectively, in Figs. 4.3.a.2) and 4.3.b.2) operating at $\gamma_{\mathrm{dB}}=10 \mathrm{~dB}$. The DF values adopted in these results are described in Subsection 4.5.1 for MRF-BP; while for FG-BP, they represent the best results found during the simulations performed, for details see the full version [116] in the Appendix C. Comparing the MRF-BP to the case without MD, Fig. 4.3.a.1), it is remarkable that the performance level reached after the convergence is almost two decades superior adopting optimal MD values. Besides, one can notice that the convergence of algorithm is improved, in the sense that the performance shows a stable asymptotic condition. Moreover, the detector operating under $200 \times 200$ antennas configuration reached its convergence with less iterations, more precisely, in the third iteration the convergence is almost totally achieved against four iterations required when $\alpha_{\mathrm{MRF}}=0$ case. Regarding the FG-BP detector, the resulting performance by applying the MD to the variable or observation nodes was analogous [116]. In this sense, the MD is applied 
only at variable nodes, i.e., $\alpha_{\mathrm{FG}}=0$ and $\beta_{\mathrm{FG}} \neq 0$. Thus, it can be observed from Fig. 4.3.b.2) that a relevant performance gain in damped FG-BP detector occurs only in low number of antennas scenario $(K=N=20)$. In medium to high antennas schemes, the performance gain, provided by the MD in the FG-BP along the $\mathcal{I}_{\mathrm{FG}}$ iterations, is almost null. Moreover, in $20 \times 20$ case, the convergence is reached only in the seventh iteration, which is similar to the undamped case; in the schemes with higher number of antennas there was also no change in the convergence of the algorithm. Comparing MRF-BP to the FG-BP algorithm updating process, the MRF-BP with $\alpha_{\mathrm{MRF}}^{\text {best }}$ is able to achieve convergence with less iterations in all scenarios, as disposed in Table 4.3. This reduction in the number of iterations of the MRF-BP detector shows an initial advantage of this detector, however, a detailed analysis of the performance-complexity relation is carried out in the Subsection 4.5.2.

Table 4.3: Convergence comparison between MRF-BP and FG-BP, according to Figure 4.3

\begin{tabular}{c|cc|cc}
\hline \# Antennas & MRP-BP description & Convergence & FG-BP description & Convergence \\
\hline$K=N=20$ & Damped MRF-BP & $\mathcal{I}_{\mathrm{MRF}}=4$ & Damped FG-BP & $\mathcal{I}_{\mathrm{FG}}=7$ \\
$K=N=50$ & Damped MRF-BP & $\mathcal{I}_{\mathrm{MRF}}=4$ & Undamped FG-BP & $\mathcal{I}_{\mathrm{FG}}=5$ \\
$K=N=100$ & Damped MRF-BP & $\mathcal{I}_{\mathrm{MRF}}=4$ & Undamped FG-BP & $\mathcal{I}_{\mathrm{FG}}=5$ \\
$K=N=200$ & Damped MRF-BP & $\mathcal{I}_{\mathrm{MRF}}=3$ & Undamped FG-BP & $\mathcal{I}_{\mathrm{FG}}=4$ \\
\hline
\end{tabular}

\subsubsection{Optimal Damping Factor Determination $\left(\alpha^{\text {best }}\right)$}

As the gain of the MD method was negligible in FG-BP detector (see Appendix C for in-depth analysis), this section focuses on getting an optimal DF value to the MRF-BP.

Considering the MD impact, Figs. 4.4 depicts the resulted BER performance due to the DF variation under medium SNR regions. A MRF-BP detector with $0 \leq \alpha_{\mathrm{MRF}} \leq 1$ is considered for 20 and 100 antennas. For the two antennas configurations, one can notice that, the performance gain with message damping is remarkable, as can be seen by comparing the undamped $\left(\alpha_{\mathrm{MRF}}=0\right)$ with the damped condition $\left(\alpha_{\mathrm{MRF}}>0\right)$, and increases in higher SNR regions, while an optimal $\alpha_{\mathrm{MRF}}^{\text {best }}$ (best BER performance) can be defined for each SNR value. Indeed, for $\gamma_{\mathrm{dB}}=6 \mathrm{~dB}$ the performance gain with damping $\left(\alpha_{\mathrm{MRF}} \neq 0\right)$ is less than one decade; on the other hand, in the scenario with $\gamma_{\mathrm{dB}}=12 \mathrm{~dB}$, the performance gain with a suitable choice of the DF value is $\approx 2$ for $K=N=20$ antennas. Moreover, specific conclusions regarding the scenario a) $20 \times 20$ antennas: in higher SNR region, the best DF, i.e., associated with the lowest BER, has a lower value and tends be more responsive to $\alpha_{\mathrm{MRF}}$ variations. Looking at the implicit curve of $\gamma_{\mathrm{dB}}=6 \mathrm{~dB}$, the performance gain variation from $0.25 \leq \alpha_{\mathrm{MRF}} \leq 0.45$ is negligible; while in the case of $14 \mathrm{~dB}$, the BER performance degradation from $\alpha_{\mathrm{MRF}}=0.05$ to 0.20 is more than one decade. Furthermore, the performance becomes worse regarding no damping messages case $\left(\alpha_{\mathrm{MRF}}=0\right)$ from $\alpha_{\mathrm{MRF}} \geq 0.35$ in $\gamma_{\mathrm{dB}}=14 \mathrm{~dB}$ the same situation occurs from $\alpha_{\mathrm{MRF}} \geq 0.80$ when $\gamma_{\mathrm{dB}}=6 \mathrm{~dB}$. Therefore, the DF value needs to be accurately chosen, specially when the system operates in medium/high SNR regions.

From Fig. 4.4.b), it is distinguishable that, when $N_{t}$ of LS-MIMO system increases, e.g., from $20 \times 20$ to $100 \times 100$ antennas, the BER performance behavior with damping factor demonstrates constant (flat condition) at greater intervals of $\alpha_{\mathrm{MRF}}$. One can conclude that, the difficult task to accurately choose the DF value in 

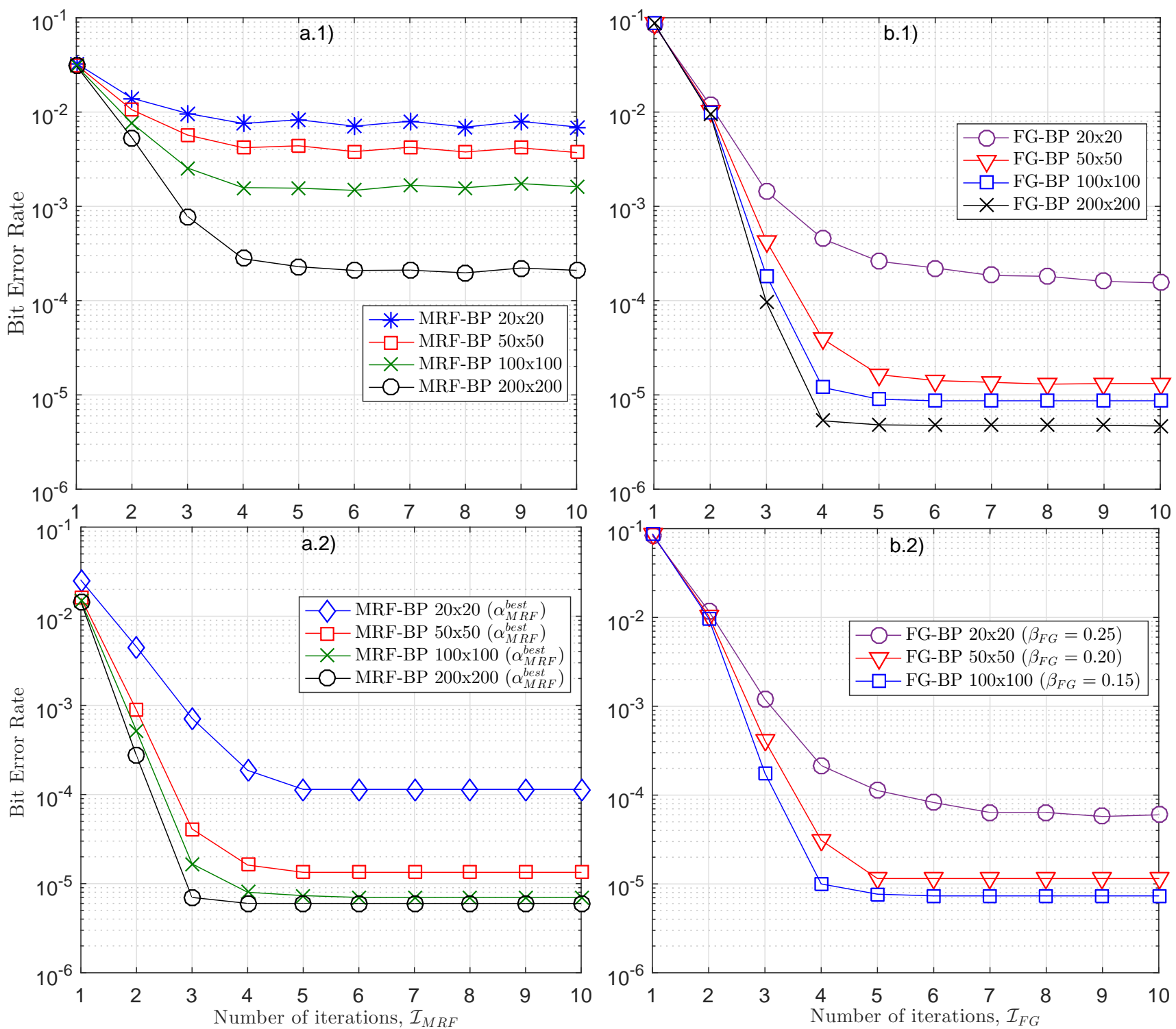

a) MRF-BP detector (Algorithm 4.1)

b) FG-BP detector (Algorithm 4.2)

Figure 4.3: Influence of the number of iterations $\mathcal{I}$ on the BER performance; both detectors operate under $\gamma=10 \mathrm{~dB}$ and the scenarios are split in: a.1) MRF-BP without MD $\left(\alpha_{\mathrm{MRF}}=0\right)$; b.1) FG-BP without MD $\left(\alpha_{\mathrm{FG}}=\beta_{\mathrm{FG}}=0\right)$; a.2) MRF-BP with optimal MD, from Eq. (4.21); b.2) FG-BP with best results found of MD. 


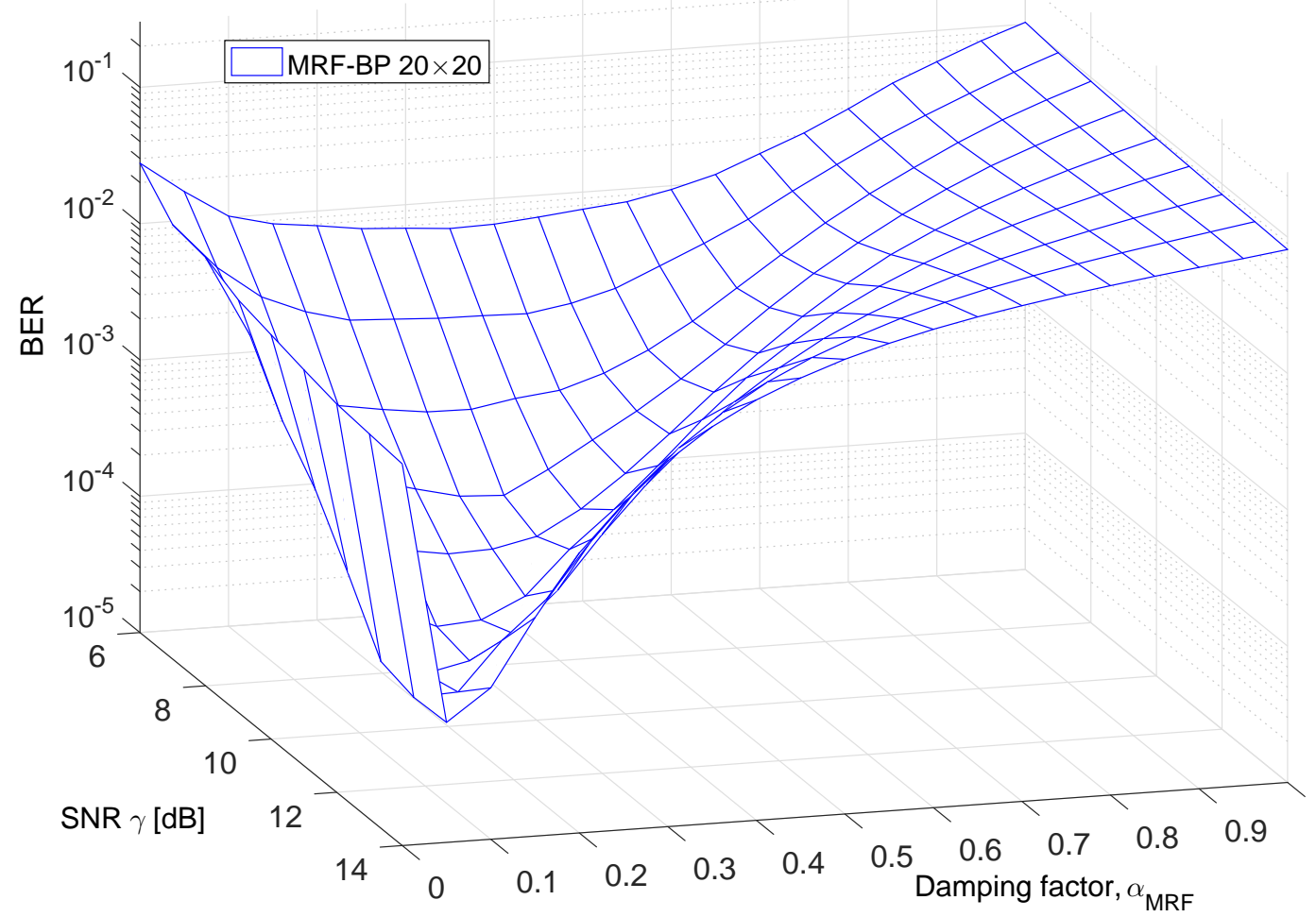

(a)

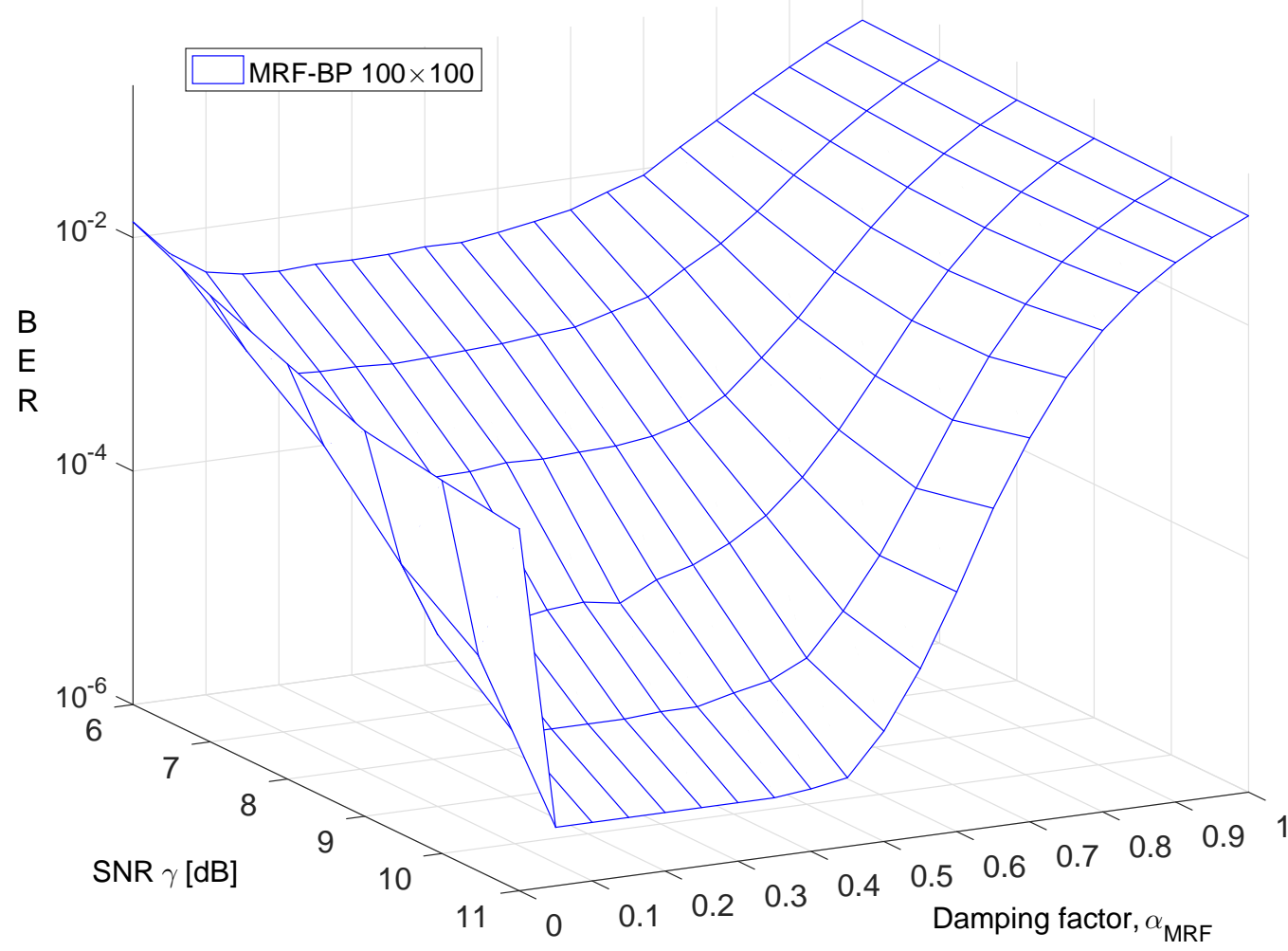

(b)

Figure 4.4: BER performance of a MRF-BP LS-MIMO system with $\mathcal{I}_{\mathrm{MRF}}=5$ as a function of different SNR scenarios and MRF-BP damping factors, $\alpha_{\mathrm{MRF}}$ : a) $20 \times 20$; b) $100 \times 100$ antennas 
small number of LS-MIMO antennas scenario, i.e., $20 \times 20$, is relaxed with increasing number of antennas. Thus, the application of message damping, especially in high SNR region, is more suitable in LS-MIMO systems due to the flat BER performance response to DF variation.

In Fig. 4.5 the best DF values $\alpha_{\mathrm{MRF}}^{\text {best }}$ are analyzed from the perspective of number of antennas $K=N$ and SNR $\gamma$ values. The necessary number of iterations for convergence differs from each antennas configuration scenario, according the analysis performed in Fig. 4.3.a.2), i.e., $\mathcal{I}_{\mathrm{MRF}}=5$ iterations for $N=20, \mathcal{I}_{\mathrm{MRF}}=4$ for $N \in\{50,100\}$, and $\mathcal{I}_{\mathrm{MRF}}=3$ for $N=200$. One can notice that, the best DF value tends to decrease in higher SNR regions and/or with increasing number of antennas. Despite the decrease in DF value, it is important to emphasize, regarding to Fig. 4.4 , that a minor increase in $\alpha_{\mathrm{MRF}}$, i.e., from 0 to 0.1 , resulted in great performance gain, especially in high SNR regions.

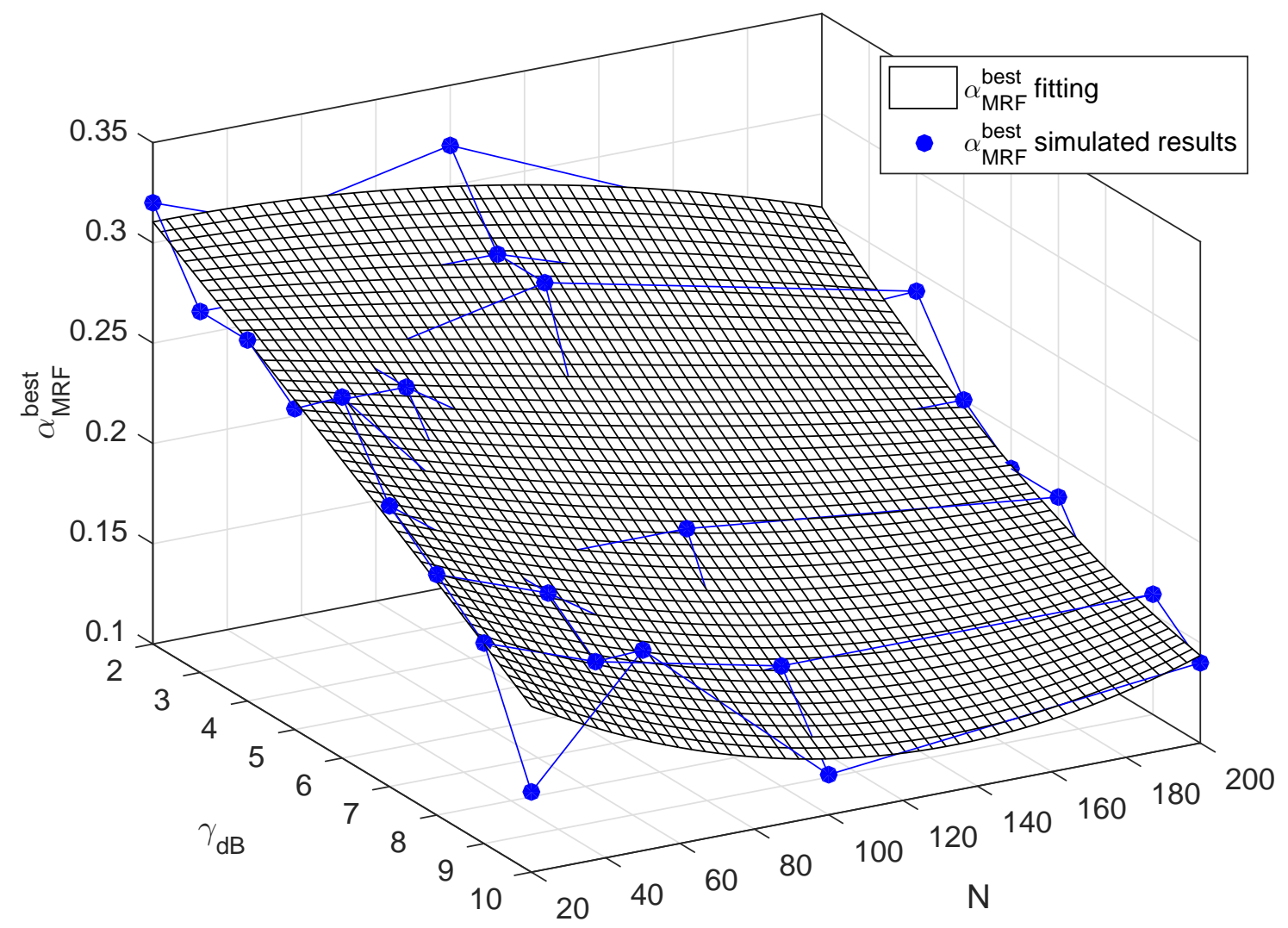

Figure 4.5: 3th order polynomial data fitting based on simulated results.

In order to provide a wide $\alpha_{\mathrm{MRF}}^{\text {best }}$ determination, a data fitting is carried out on Fig. 4.5. We have obtained a polynomial data fitting for $\alpha_{\mathrm{MRF}}^{\text {best }}$ as a polynomial surface of 3th order in $\gamma_{\mathrm{dB}}$ and 2th order in $N$ antennas, resulting in a root mean squared error (RMSE) of RMSE $=0.02$, and given by

$$
\alpha_{\mathrm{MRF}}^{\text {best }}=p_{00}+p_{10} \gamma_{\mathrm{dB}}+p_{01} N+p_{11} \gamma_{\mathrm{dB}} N+p_{20} \gamma_{\mathrm{dB}}^{2}+p_{02} N^{2}+p_{12} \gamma_{\mathrm{dB}} N^{2}+p_{21} \gamma_{\mathrm{dB}}^{2} N+p_{30} \gamma_{\mathrm{dB}}^{3}
$$

where the coefficients and quality of the resulting fitting are presented in Table 4.4. 
Table 4.4: Parameters of data fitting function: $\alpha_{\mathrm{MRF}}^{\text {best }}(\gamma, N)$, eq. (4.21)

\begin{tabular}{cl}
\hline Coefficient & Value \\
\hline$p_{00}$ & +0.33100 \\
$p_{10}$ & -0.01036 \\
$p_{01}$ & $+5.41100 \cdot 10^{-4}$ \\
$p_{11}$ & $-2.43800 \cdot 10^{-4}$ \\
$p_{20}$ & $-1.75800 \cdot 10^{-4}$ \\
$p_{02}$ & $-0.03419 \cdot 10^{-4}$ \\
$p_{12}$ & $+0.80930 \cdot 10^{-6}$ \\
$p_{21}$ & $+0.65220 \cdot 10^{-6}$ \\
$p_{30}$ & $-0.42090 \cdot 10^{-6}$ \\
\hline RMSE & 0.0207 \\
Coef. Determ. $\left(r^{2}\right)$ & 0.8913 \\
\hline
\end{tabular}

\section{Message Damping method with $\alpha_{\mathrm{mrf}}^{\text {best }}$ applied to MRF-BF Detector}

Considering that MD procedure results in a relevant BER performance gain only in MRF-BP detector and the analysis performed around the $\alpha_{\mathrm{MRF}}^{\text {best }}$, a BER comparison between FG-BP (without MD in medium to

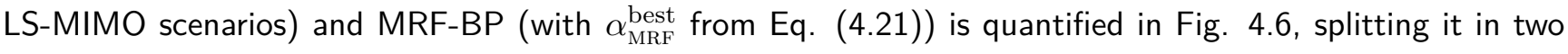
scenarios: a) low to medium number of antennas, $b$ ) high number of antennas. One can conclude that the MRF-BP with optimal DF value performs a marginally equal performance to the FG-BP detector. Compared to the result in [20], the MRF-BP with proposed $\alpha_{\mathrm{MRF}}^{\text {best }}$ reached marginally same performance with less number of antennas, besides that, the MRF-BP with optimal DF outperforms the FG-BP when a $50 \times 50$ antennas scenario is deployed. With large number of antennas, Fig. 4.6.b), the FG approach shows a slightly better BER performance compared to MRF-BP. On the other hand, the iterations required for convergence is lower in MRF approach in all scenarios. Indeed, in LS-MIMO scenarios, the MRF with optimal MD converges with one less iteration compared to the FG scheme. More evidently, regarding Fig. 4.3, in $200 \times 200$ case, MRF with optimal DF converges with 3 iterations, against 4 iterations of FG-BP, which can lead to a more interesting performance versus complexity tradeoff for MRF approach. An in-depth discussion of computational complexity is developed in Subsection 4.5.2.

In the sequel the computational complexity of both detectors are evaluated, demonstrating that, under specific problem sizes, the MRF-BP algorithm can outperform the FG-BP in terms of both computational complexity (lower) and BER performance (lower). On the other hand, with increasing number of antennas, FG-BP tends to present a lower computational complexity than MRF-BP massive MIMO detector.

\subsubsection{Computational Complexity}

The computational complexity is described in terms of floating-point operations (flops), in which one flop denotes the computational complexity of the complex mathematical operations: addition, subtraction, multiplication or division. In the case of exponential function, an approximation through Taylor Series with 18 terms has been considered. Table 4.5 describes the per-symbol computational complexity involved in each step of MRF-BP and FG-BP algorithms. The asymptotic per-symbol complexity of the Algorithm 4.1 is of the order 


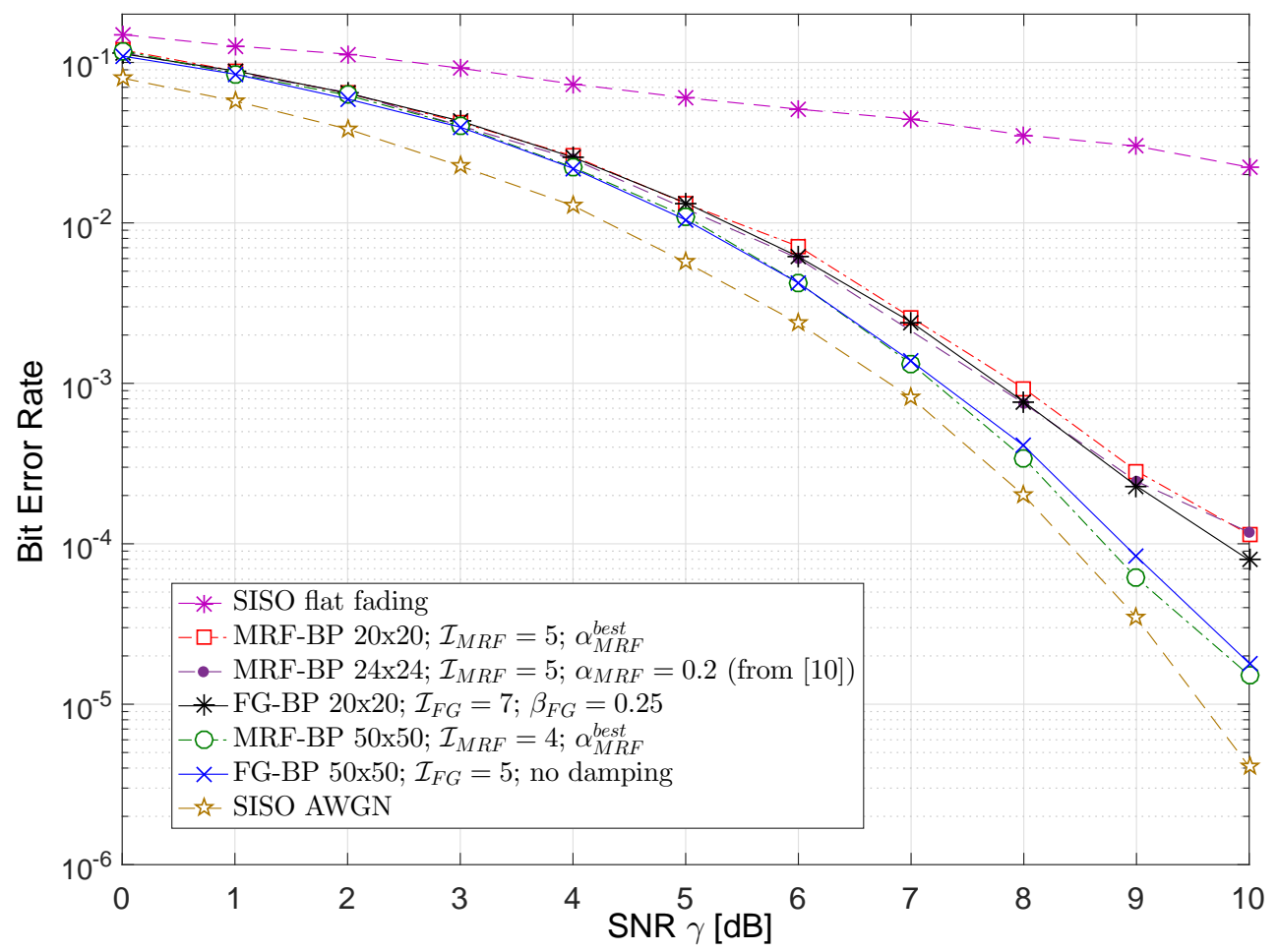

(a) Low-medium number of antennas

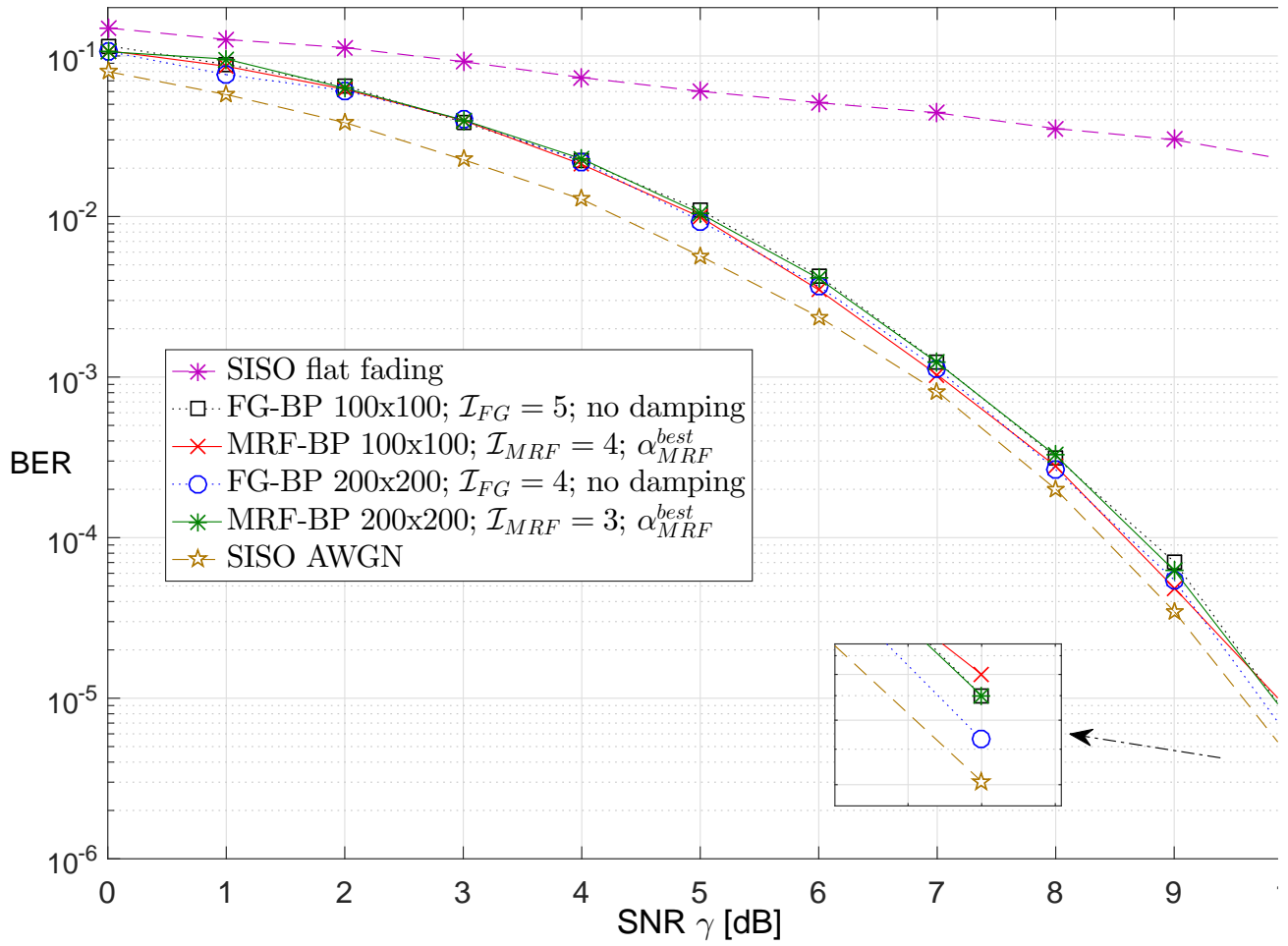

(b) High number of antennas

Figure 4.6: BER performance of the implemented MRF-BP detector with the best DF value and FG-BP detector without MD, split in two scenarios: a) low to medium number of antennas, b) high number of antennas. 
of $\mathcal{O}\left(K^{2}\right)$, while the Algorithm 4.2 complexity is about $\mathcal{O}(N)$. It is important to note that message damping does not increase substantially the computational complexity of the analyzed algorithms, as discussed in the sequel. In addition, since the LS-MIMO scenarios are part of the scope of this work, and the FG-BP detector benefited from the MD technique only with a low number of antennas, this section will consider the undamped version of the FG-BP detector, i.e., $\alpha_{\mathrm{FG}}=\beta_{\mathrm{FG}}=0$, during the computational complexity simulations.

Table 4.5: Per-symbol Computational Complexity of MRF-BP and FG-BP Algorithms.

\begin{tabular}{ccc}
\hline \multicolumn{4}{c}{ Procedure } & Step & Complexity \\
\hline \hline & Algorithm & $4.1-$ MRF-BP \\
\hline $\mathbf{R}_{c}$ & line 4 & $N-1$ \\
$\mathbf{v}$ & line 4 & $N-1$ \\
$\phi, \quad$ Eq. (4.7) & line 6 & 718 \\
$\psi, \quad$ Eq. (4.8) & line 10 & $360(K-1)$ \\
Messages update & lines 15 to 25 & $\left(4 K^{2}+4 K-8\right) \cdot \mathcal{I}_{\mathrm{MRF}}$ \\
Message damping & line 20 & $4(K-1) \cdot \mathcal{I}_{\mathrm{MRF}}$ \\
Belief calculation & line 28 & $N$ \\
\hline Total Complexity MRF-BP & $360 K+3 N+356+\mathcal{I}_{\mathrm{MRF}}\left(4 K^{2}+8 K-12\right)$ \\
\hline \hline
\end{tabular}

Algorithm 4.2 - FG-BP

\begin{tabular}{|c|c|c|}
\hline \multicolumn{3}{|c|}{ Computation of LLRs at observation nodes } \\
\hline$s_{\mu_{\lambda_{i}}}$ & line 6 & $\left(4 N-\frac{N}{K}\right) \cdot \mathcal{I}_{\mathrm{FG}}$ \\
\hline$s_{\sigma_{\lambda_{i}}^{2}}^{i}$ & line 7 & $5 N \cdot \mathcal{I}_{\mathrm{FG}}$ \\
\hline Eq. (4.13) & line 9 & $3 N \cdot \mathcal{I}_{\mathrm{FG}}$ \\
\hline$\sigma_{i k}^{2}, \quad$ Eq. (4.14) & line 10 & $7 N \cdot \mathcal{I}_{\mathrm{FG}}$ \\
\hline LLR calculation, $\tilde{\Lambda}_{i}^{k,(t)}$ & line 11 & $4 N \cdot \mathcal{I}_{\mathrm{FG}}$ \\
\hline Message damping, $\alpha_{\mathrm{FG}}$ & line 13 & $4 N \cdot \mathcal{I}_{\mathrm{FG}}$ \\
\hline \multicolumn{3}{|c|}{ Computation of probabilities at variable nodes } \\
\hline$s_{\Lambda_{k}}$ & line 18 & $(N-1) \cdot \mathcal{I}_{\mathrm{FG}}$ \\
\hline Probabilities, $\tilde{p}_{i}^{k+,(t)}$ & line 20 & $720 N \cdot \mathcal{I}_{\mathrm{FG}}$ \\
\hline Message damping, $\beta_{\mathrm{FG}}$ & line 22 & $4 N \cdot \mathcal{I}_{\mathrm{FG}}$ \\
\hline Detection of data bits & line 28 & $(N-1)$ \\
\hline Total Complexity - FG-BP & $N-$ & $\left.52 N-\frac{N}{K}-1\right)$ \\
\hline
\end{tabular}

In Fig. 4.7.(a), the computational complexity increment of MD method in MRF-BP detector is evaluated. One can notice that, the complexity increment of MD is very low compared to the overall MRF-BP detector complexity, whereas in the worst case points that the percent complexity increment holds below of $2.5 \%$ (when $K=N=20$ antennas and $\mathcal{I}_{\mathrm{MRF}}=5$ iterations). Furthermore, one can conclude that with increase number of antennas, the percent increment of flops tends to a low constant floor $(\approx 0.2 \%)$, which can be considered negligible and denotes an interesting application of MD in MRF-BP under LS-MIMO scenarios.

In Fig. 4.7.(b) a numerical computational complexity comparison between the two MP-based LS-MIMO detectors are performed relating the number of antennas and iterations on BP algorithm. It is noticeable that for $N \leq 160$ antennas (in both $\mathcal{I}_{\mathrm{MRF}}=\mathcal{I}_{\mathrm{FG}}=4$ and $\mathcal{I}_{\mathrm{MRF}}=\mathcal{I}_{\mathrm{FG}}=5$ ) the MRF-BP algorithm results in less computational complexity, although the difference becomes progressively smaller with the increase of 
antennas, due to the quadratic complexity order of $\mathcal{O}\left(K^{2}\right)$ of MRF-BP against $\mathcal{O}(N)$ from FG-BP. In terms of performance, under antenna configuration of $20 \times 20$ or $50 \times 50$, the MRF-BP approach demonstrated better BER performance than FG. Under higher number of antennas $-N=100$ and 200 schemes - damped MRF-BP and undamped FG-BP detectors have resulted in a marginally different performance, with a slight advantage for the FG-BP approach. Equaling the number of iterations, in $200 \times 200$ case, FG-BP performs with less complexity than MRF-BP; on the other hand, since optimal DF in MRF leads to an iteration reduction, MRF$\mathrm{BP}$ after convergence $\left(\mathcal{I}_{\mathrm{MRF}}=3\right)$ shows a marginally lower complexity than $\mathrm{FG}-\mathrm{BP}$ with $\mathcal{I}_{\mathrm{FG}}=4$ iterations. Finally, with the increase in the number of antennas, i.e., $N_{t}=N_{r}>200$, FG scheme performs with a less computational complexity than MRF due to its 1 th order complexity in $N$.

\subsection{Chapter conclusions}

In this chapter, a detector for large scale MIMO systems based on message passing MRF graphical model has been extensively analyzed. More specifically, the impact of the message damping method on the BER performance was evaluated and it has demonstrated a promising performance versus complexity tradeoff gain for MRF-BP detector, especially in LS-MIMO scenarios.

Furthermore, an optimal damping factor for MRF-BP LS-MIMO detector is proposed and the numerical results for this damped detector have demonstrated promising performance-complexity tradeoff, since damping messages procedure just negligibly increases the overall computational complexity while providing a significant performance gain in the MRF-BP detection. Considering the algorithm convergence, the message damping method resulted in faster MRF-BP convergence, with one less iterations (total of $\mathcal{I}_{\mathrm{MRF}}=3$ against $\mathcal{I}_{\mathrm{MRF}}=$ 4 without damping case) in large-scale MIMO configuration, i.e., $200 \times 200$. Such results demonstrate a consistent gain, not only in performance, but also in the computational complexity, thanks to the application of the MD procedure in the MRF-BP algorithm.

Besides, under large scale antenna scenarios, damping factor value choose is facilitated due to the flat BER performance response to a relative wide $\alpha_{\mathrm{MRF}}$ values interval. The FG-BP approach with a massive number of antennas has demonstrated a performance close to the optimal SISO AWGN, even without message damping. Also, the same detector presented a BER performance gain with increasing number of antennas, demonstrating also be an attractive LS-MIMO detector. On the other hand, the MD method applied on FG-BP algorithm did not result in relevant performance gain in medium to high number of antennas scenarios; whereas a reasonable performance gain was reached in low antennas scheme. Comparing both massive MIMO detectors operating under their best performance condition, MRF-BP with optimal DF value demonstrated a marginally equal performance than FG approach.

The computational complexity of MRF-BP algorithm results in a quadratic order with the number of transmit antennas, while FG-BP algorithm presents a first order complexity regarding the Rx antennas. Nevertheless, under small to medium/high number of antennas scenarios ( $K=N \leq 160)$, MRF approach attains a lower complexity than FG one.

Specifically, the main contributions refer to: 


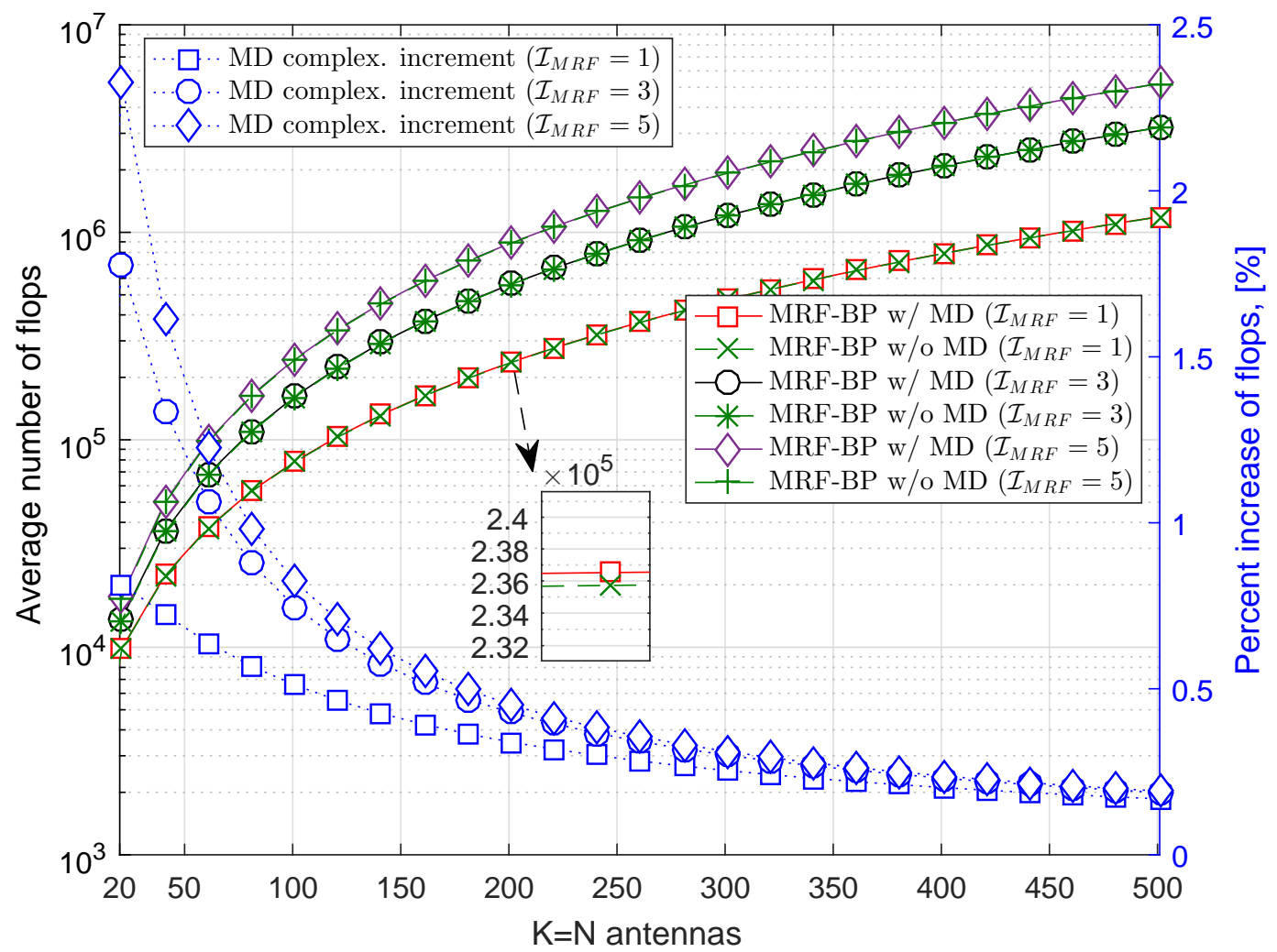

(a) Percent increase of complexity due to the MD method

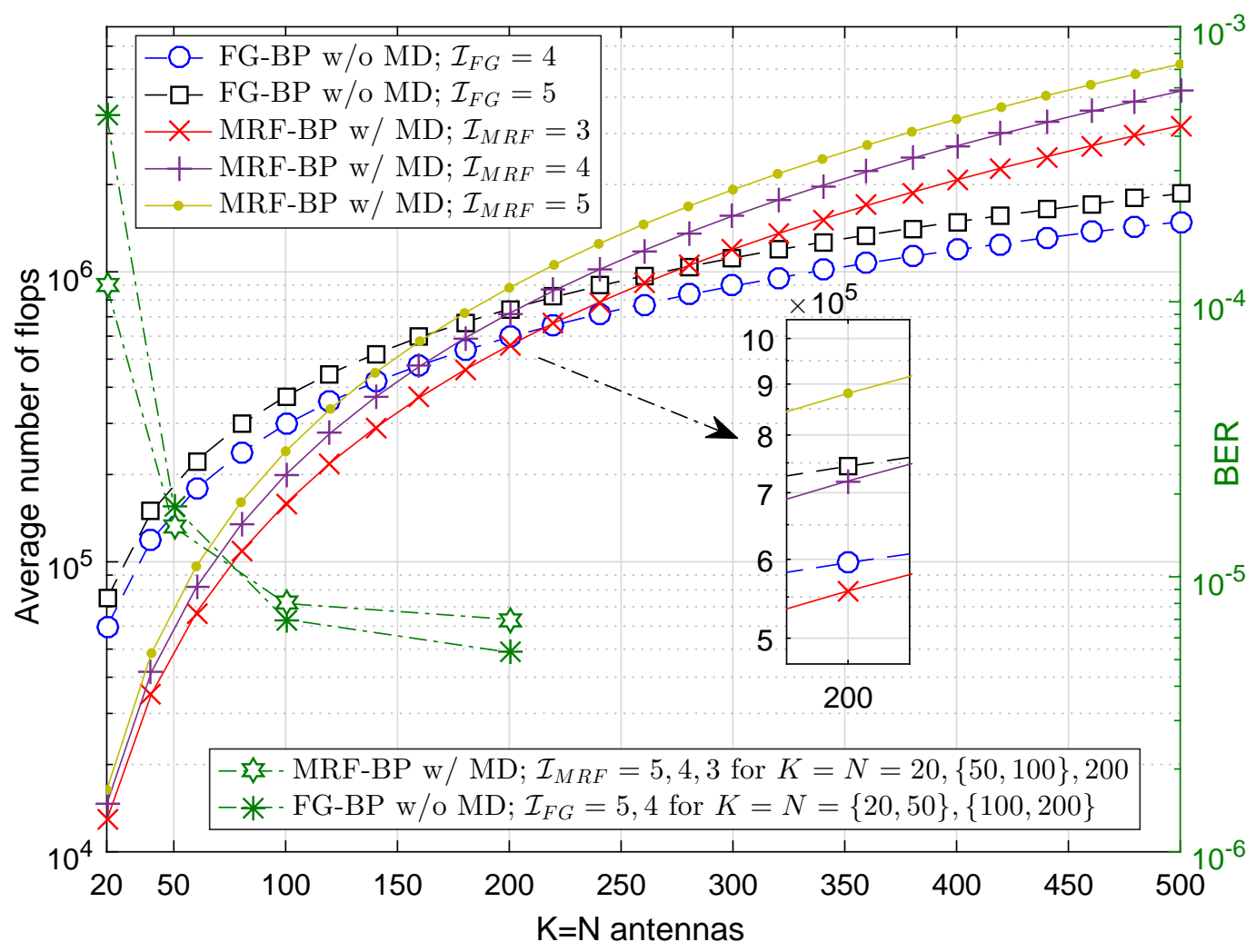

(b) Performance versus complexity comparison

Figure 4.7: Analysis of computational complexity, in terms of average number of flops, operating under large-scale MIMO scenarios, $K=N$ antennas and different number of algorithm iterations, $\mathcal{I} \in\{3,4,5\}$, carried out in two aspects: a) percent increment of complexity of MD method applied in MRF-BP detector;

b) comparison of complexity and respective BER among MRF-BP and FG-BP detectors, $\gamma_{\mathrm{dB}}=10 \mathrm{~dB}$. 
- Application of MD method in FG-BP LS-MIMO detector in two variations: MD at variable nodes and MD at function nodes;

- Extensive performance and complexity analyses of MRF-BP and FG-BP detectors considering a DF range variation under a different number of antennas configurations, especially including LS-MIMO scenarios;

- Numerical analysis of the impact of MD method on MRF-BP convergence, in order to determine a complexity reduction of the algorithm;

- Proposition of an optimal DF function for MRF-BP, namely $\alpha_{\mathrm{MRF}}^{\text {best }}$, considering a given $\gamma$ and number of antennas, based on a polynomial data fitting.

In the next chapter, two approaches for reducing the impact of the noisy solution from Mixed Gibbs Sampling detector are proposed and analyzed, which represents one of the main contributions of this Doctoral work. 


\section{Reducing the Noisy Solution Impact of Mixed Gibbs Sampling Detector in High-Order Modulation Large-Scale MIMO Systems}

In the previous chapter is considered a low order modulation, i.e., BPSK, LS-MIMO system. In this chapter, high order modulation is adopted where two approaches, named aMGS and $d$-sMGS, are proposed to mitigate the impact of noisy solution on MGS detector. These strategies aim to improve the performance and convergence of the algorithm in LS-MIMO systems operating in high modulation order.

Initially, a brief bibliographic review is presented. Then a review on the MGS detector, in addition to the description of the two approaches proposed during this Doctoral Thesis are addressed. At the end, the main numerical results obtained are discussed and the final considerations are made.

\subsection{Introduction}

The Markov Chain Monte Carlo (MCMC) was introduced in MIMO and digital communications in [117, 118]. The application of Gibbs Sampling-based (GS-based) MCMC on detection problem in MIMO systems was first studied in [118], while in [2] a strategy of mixing between the conventional GS solution and a random or noisy solution was proposed, which is controlled by a mixing ratio parameter and is called Mixed GS or MGS. The MGS has been shown to solve the stalling problem of the GS detector in low order of modulation, i.e., 4-QAM. With the modulation order increase, the multiple restarts (MR) technique is proposed, which restarts the algorithm with a new initial solution, taking advantage of the random evolution of the algorithm and can result in a better cost solution. The MGS-MR detector showed near-optimal performance in 16QAM modulation, however, in high modulation order the noisy solution interferes with the convergence of the algorithm, requiring an extra strategy to avoid the impact of this solution. In order to minimize this impact, we proposed in this Doctoral work two approaches that resulted in convergence/performance gain, especially in scenarios with high-order modulation and high system loading, with no extra computational complexity.

Also in [2], the choice of mixing ratio parameter is determined by the inverse of the number of real dimensions of the system, i.e., for a system with complex modulation alphabet, $q=1 / 2 K$. The optimal choice in [2] was based on Markov chains theory considering low dimension systems. However, in our contribution we demonstrate by numerical simulation that the choice of mixing ratio parameter is also dependent on the system loading and the number of samples deployed in coordinate updating process; thus, it is not always coincident with the inverse of the number of real dimensions. 
In [119] an optimization on mixing time was introduced to accelerate the finding of the optimal solution. Numerical simulation results demonstrated that the choice of mixing time based on SNR can improve convergence, although the stalling problem persisted when a fixed mixing time is adopted. Also, the results do not considered the performance behaviour in high-order modulation schemes. More recently, the MCMC detector with $\mathrm{QR}$ decomposition was addressed in [37, 120], which can reduce the number of operations due to the lower triangular matrix feature. Besides, based on the multiple random parallel Markov chains, the work in [121] proposes a MR strategy through parallel chains; such strategy reduced the algorithm's running time compared to MGS-MR, despite the increasing of the number of real operations per symbol. Moreover, in [122] a MCMC detector using successive over-relaxation is proposed in order to improve the convergence rate, although $M$-QAM modulation orders higher than $M=16$ were not investigated.

\subsection{Review of Mixed Gibbs Sampling detection}

The LS-MIMO detector MGS proposed in [2] is revisited in this subsection, which is based on the motivation to solve the stalling problem presented in the conventional GS detector.

In this chapter, the real-valued system model adopted is described in Subsection 3.1. The transmitted symbols from the single-antenna UEs follow a QAM modulation, thus the elements of real-valued transmitted symbols vector $\mathbf{s}$ assume integer values from the underlying PAM alphabet $\mathcal{A}$, i.e., $\mathbf{s} \in \mathcal{A}^{2 K}$. It is considered that the minimum spacing between the Rx antennas is obeyed, so that there is no correlation between antennas, i.e. $\rho_{\mathrm{Tx}}=0$; also, a $\mathrm{pCSI}$ is provided at the BS.

To sample the estimated symbol at each position, a target distribution [123] is evaluated, which is given by:

$$
p\left(\hat{s}_{1}, \hat{s}_{2}, \ldots, \hat{s}_{2 K} \mid \mathbf{y}, \mathbf{H}\right) \propto \exp \left(-\frac{\|\mathbf{y}-\mathbf{H s}\|^{2}}{\alpha^{2} \sigma^{2}}\right)
$$

where $\hat{s}_{i}$ denotes the $i$-th position of the estimated symbols vector $\hat{\mathbf{s}}, \alpha$ denotes a positive parameter, which tunes the mixing time of the Markov chain [123] and is also called as temperature. The conventional Gibbs sampling detector does not include the $\alpha$ parameter in its sample process, and thus can be viewed as a special case when $\alpha=1$. A larger temperature speeds up the mixing and aims to reduce the higher moments of the number of iterations when finding the correct solution. However, as stated in [2], the stalling problem persists even with large $\alpha$.

The MGS detector utilizes a mixing of: a) Conventional Gibbs sampling (i.e., $\alpha=1$ ); and $\mathbf{b}$ ) the infinite temperature version of (5.1) (i.e., $\alpha \rightarrow \infty$ ), resulting in a random and uniform sample from all the possibilities, called a noisy or random solution in this paper. In this way, the MGS follows a sampling distribution given by:

$$
p\left(\hat{s}_{1}, \ldots, \hat{s}_{2 K} \mid \mathbf{y}, \mathbf{H}\right) \sim(1-q) \psi\left(\alpha_{1}\right)+q \psi\left(\alpha_{2}\right)
$$

and

$$
\psi(\alpha)=\exp \left(-\frac{\|\mathbf{y}-\mathbf{H} \hat{\mathbf{s}}\|^{2}}{\alpha^{2} \sigma^{2}}\right),
$$

where $q$ denotes the mixing ratio. The MGS detector of [2] considers the $\alpha_{1}=1, \alpha_{2} \rightarrow \infty$ combination, which results in a near-ML performance, overcoming the stalling problem of the GS, being also a simple 
implementation choice. On the other hand, in high-order modulation, such as 64-QAM and 256-QAM, the noisy solution interferes in the algorithm's convergence, since there are a large number of symbols in the constellation and a simple random solution in this signal space has a high possibility of being far from the real solution, which causes the algorithm to require more iterations for convergence. In this sense, the proposed $d$-sMGS detector acts to mitigate this harmful effect.

Regarding the mixing ratio parameter $q$, in [2] an analysis in low order QAM constellations is carried out and its suitable value choice is presented as the inverse of the number of dimensions in the system, i.e., $q=\frac{1}{2 K}$.

In the MGS algorithm, an initial solution $\hat{\mathbf{s}}^{(t=0)}$ is considered for the estimated symbols vector, where $t$ represents the current iteration. Indeed, the initial solution may be chosen either by a random symbols vector or as the output of a linear low-complexity detector, such as ZF or MMSE. The index $i$, in addition to the position of the vector $\hat{\mathbf{s}}$, also denotes the coordinate referring to the MGS algorithm, where $i=1,2, \ldots, 2 \mathrm{~K}$. Therefore, each iteration requires $2 K$ coordinate updating. At each iteration, updating the $2 K$ coordinates is performed by sampling the distributions given by:

$$
\hat{s}_{i}^{(t)} \sim p\left(\hat{s}_{i} \mid \hat{s}_{1}^{(t)}, \ldots, \hat{s}_{i-1}^{(t)}, \hat{s}_{i+1}^{(t-1)}, \ldots, \hat{s}_{2 K}^{(t-1)}, \mathbf{y}, \mathbf{H}\right)
$$

One can notice that by (5.4) each updated coordinate is fed, in the same iteration, to the next coordinate.

The probability of the $i$-th symbol assuming the value $a_{j} \in \mathcal{A}, \forall j=1, \ldots,|\mathcal{A}|$ can be written as:

$$
p\left(\hat{s}_{i}=a_{j} \mid \hat{\mathbf{s}}_{i-1}, \mathbf{y}, \mathbf{H}\right)=\frac{\exp \left(-\frac{\left\|\mathbf{y}-\mathbf{H} \hat{\mathbf{s}}_{i, j}\right\|^{2}}{\alpha^{2} \sigma^{2}}\right)}{\sum_{l=1}^{|\mathcal{A}|} \exp \left(-\frac{\left\|\mathbf{y}-\mathbf{H} \hat{\mathbf{s}}_{i, l}\right\|^{2}}{\alpha^{2} \sigma^{2}}\right)},
$$

where the cardinality of set $\mathcal{A}$ is expressed as $|\mathcal{A}|$, while $\hat{\mathbf{s}}_{i, j}$ denotes the vector $\hat{\mathbf{s}}^{(t)}$ with its $i$-th position changed to the symbol $a_{j}$.

The sampling process based on (5.5) can lead to a numerical limitation due to the exponential function. In this sense, such implementation was carried out through a logarithmic intermediate step, as:

$$
\begin{aligned}
& \log \left(p\left(\hat{s}_{i}=a_{j} \mid \hat{\mathbf{s}}_{i-1}, \mathbf{y}, \mathbf{H}\right)\right)= \\
& =f(i, j)-\left[f_{0}^{\text {ord }}+\log \left(1+\sum_{m=1}^{|\mathcal{A}|-1} \exp \left(f_{m}^{\text {ord }}-f_{0}^{\text {ord }}\right)\right)\right] \\
& =g(i, j)
\end{aligned}
$$

where $f(i, j)=-\frac{\left\|\mathbf{y}-\mathbf{H} \hat{\mathbf{s}}_{i, j}\right\|^{2}}{\alpha^{2} \sigma^{2}}$ and $f_{i}^{\text {ord }}$ is $i$-th position of $\mathbf{f}$ in descending order, for $i=1, \ldots,|\mathcal{A}|$. A practical and computationally efficient evaluation of MGS target Function is summarized in the Algorithm 5.1.

The MGS algorithm ends after a certain amount of iterations, and the vector of estimated symbols is chosen as the vector that presented the lowest ML cost, considering all iterations. In the next subsections, the additional strategy of MR [2] and the stopping criteria for the iterations and the restarts are addressed. 


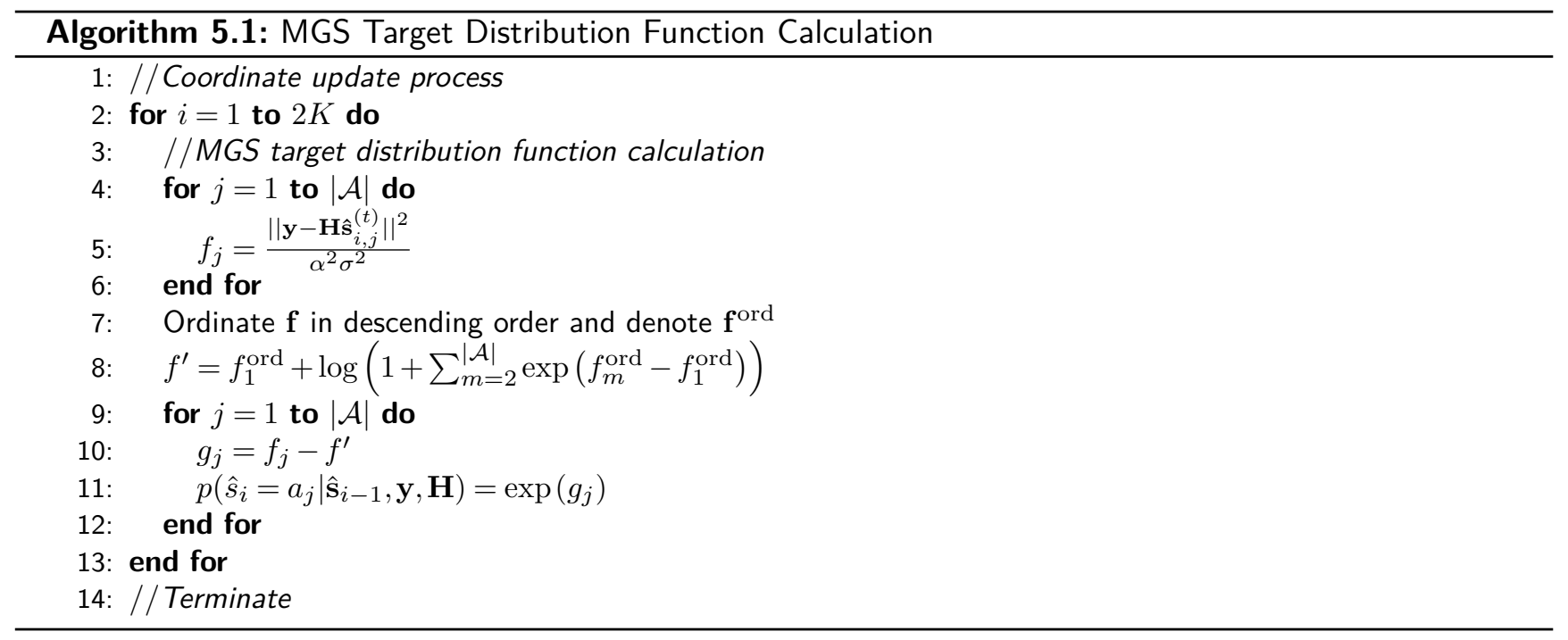

\subsubsection{Multiple restarts}

In medium QAM order modulations, such as 16-QAM, the mixing strategy of MGS is unable to achieve nearoptimal performance [52] in a reasonable number of iterations, while MR procedure, as proposed in [2] has demonstrated promising results, leading the MGS-MR under 16-QAM to near-optimal performance.

In the proposed aMGS and $d$-sMGS detectors the MR strategy is also incorporated, namely aMGS-MR and $d$-sMGS-MR detectors. Thus, the Algorithms 5.2 and 5.3 run either a maximal number of restarts $R_{\max }$ times or it is limited by a stopping criterion and the lowest cost found considering all restarts is the final solution. As discussed in Section 4.5, the MR strategy can improve the convergence of the algorithm compared to the same number of iterations in a single execution, resulting in a better performance-complexity tradeoff.

\subsubsection{Stopping criterion}

Given that the mixing strategy provides the local minimum escaping feature, the evolution of the cost function values across iterations becomes unpredictable and the optimal solution can be found before the maximum number of iterations $\mathcal{I}$ has been reached, as illustrated in Fig. 5.1. It is noticeable that the aMGS algorithm found the optimal solution, approximately, in the fifth iteration. After that, iterations become a waste of computational resources. In this sense, an efficient stopping criterion is paramount in reducing the complexity of the MGS-based detectors.

Similarly, the decision to set a restart in the algorithm requires a criterion definition, since the optimal solution may already have been found, not requiring an extra execution of the algorithm. Hence, MR strategy must be balanced aiming to achieve a better performance-complexity tradeoff.

Stopping criteria have been proposed in the literature. For instance, in [2], the stopping criterion is based on the difference between the best ML cost found so far and the noise variance. Moreover, the QAM constellation size could be taken into account. The main idea in [2] is to stop the detection iterations if a maximum number of iterations $\mathcal{I}$ is attained or if the iteration in stalling mode is larger than a maximum of $\Theta_{s}$ iterations. 


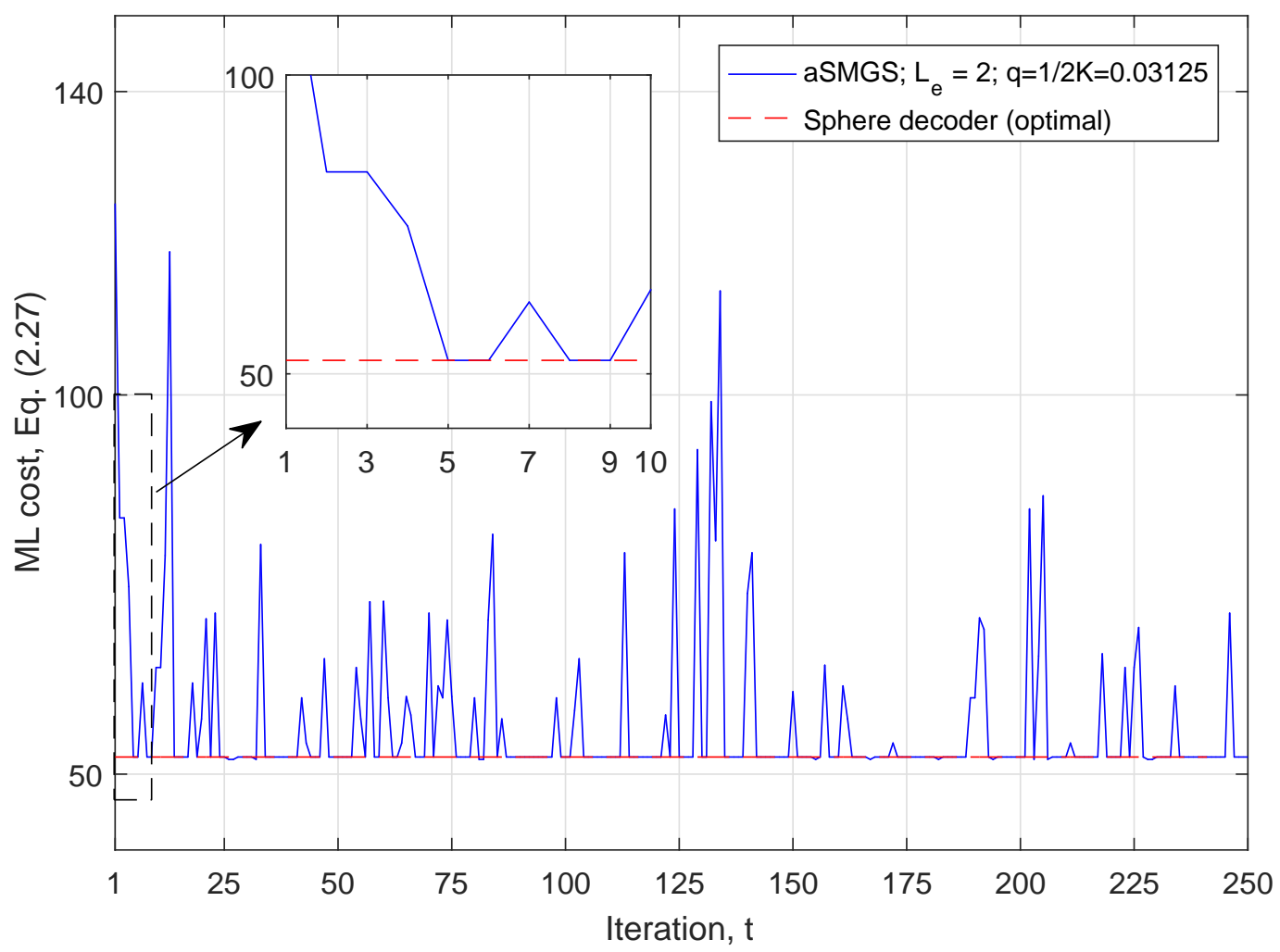

Figure 5.1: $M L$ cost function evolution during the aMGS algorithm's iterations and the optimal result (sphere decoder); $K=M=16, \gamma=10 \mathrm{~dB}, \mathcal{I}=250$ iterations, and 4-QAM modulation.

Assume the estimated symbol vector, in the $t$-th iteration, is $\hat{\mathbf{s}}^{(t)}$. The quality metric of $\hat{\mathbf{s}}^{(t)}$ is defined as

$$
\phi\left(\hat{\mathbf{s}}^{(t)}\right)=\frac{\left\|\mathbf{y}-\mathbf{H} \hat{\mathbf{s}}^{(t)}\right\|^{2}-N \sigma^{2}}{\sqrt{N} \sigma^{2}}
$$

Hence, the stalling limit for iterations, $\Theta_{s}$, is given by

$$
\Theta_{s}\left(\phi\left(\hat{\mathbf{s}}^{(t)}\right)\right)=c_{s} \cdot e^{\phi\left(\hat{\mathbf{s}}^{(t)}\right)}
$$

where $c_{s}$ is a constant depending upon the $M$-QAM constellation size, which increases with $M$. Although (5.8) is suitable as a stopping criterion, a minimum number of iterations $c_{\min }$ must be defined to ensure the quality of symbol detection. Therefore, $\Theta_{s}$ can be rewritten as

$$
\begin{aligned}
\Theta_{s}\left(\phi\left(\hat{\mathbf{s}}^{(t)}\right)\right) & =\left\lceil\max \left(c_{\min }, c_{s} \cdot e^{\phi\left(\hat{\mathbf{s}}^{(t)}\right)}\right)\right\rceil \\
\text { with } \quad c_{s} & =c_{1} \log _{2}(M)
\end{aligned}
$$

where $c_{1}$ is a tunning constant which defines the allowed number of iterations in stalling mode.

For the MR strategy, the criterion set the allowable number of restarts $\Theta_{r}$, which also is based on quality metric $\phi\left(\hat{\mathbf{s}}^{(t)}\right)$ :

$$
\begin{aligned}
\Theta_{r}\left(\phi\left(\hat{\mathbf{s}}^{(t)}\right)\right) & =\left\lceil\max \left(0, c_{r} \cdot \phi\left(\hat{\mathbf{s}}^{(t)}\right)\right)\right\rceil+1 \\
\text { with } \quad c_{r} & =c_{2} \log _{2}(M)
\end{aligned}
$$

and $c_{2}$ is the tuning constant adjusting the maximum number of restarts. 
At the end of each restart, $\Theta_{r}$ is computed and checked if the actual number of repetitions is less than $\Theta_{r}$. If yes, go to another run of the algorithm; else, output the solution vector with the minimum cost so far as the final solution.

For the aMGS and $d$-sMGS detectors presented below, aMGS and $d$-sMGS, we also assume the stop criteria described in this subsection.

\subsection{Reducing the Impact of Noisy Solution}

Originally, the mixture between the target distribution function solution and the random solution, proposed by MGS detector of [2], attempted to escape local minima that degrade system performance. In fact, this procedure showed to significantly improves the performance, specially in low-order modulation scenarios, as 4 or 16-QAM. On the other hand, in high-order modulation systems, the large number of symbols causes the random solution to degrade the convergence of the algorithm since it is based on a coordinate update process which requires the global solution, thus one or more positions that consider a random solution (probably erroneous and far from the real solution) interfere in the convergence in the other positions and, consequently, in the global one. This condition is aggravated in high-dimension problems, i.e., combining high-order modulations and number of antennas, which is the case of interest in this work.

In this sense, two approaches that tries to alleviate the harmful impact of the noisy solution are described below. Fig. 5.2 summarizes the coordinate update process on the aMGS and $d$-sMGS detectors. Each detection scheme uses its proper mixing and target function. For the aMGS, the mixing function at $i$-th coordinate and $m$-th sample is given by $\rho_{m, i}(\cdot)$, described in Eq. (5.13), while its target and random functions are given, respectively, by $\xi(\cdot)$ and $\psi(\cdot)$, which are defined in Eqs. (5.11) and (5.3). For the $d$-sMGS approach, the mixing function at $i$-th coordinate is given by $\zeta_{i}(\cdot)$, Eq. (5.16), and its target, $\xi(\cdot)$, and random, $v(\cdot)$, functions are defined in Eqs. (5.11) and (5.17), respectively.

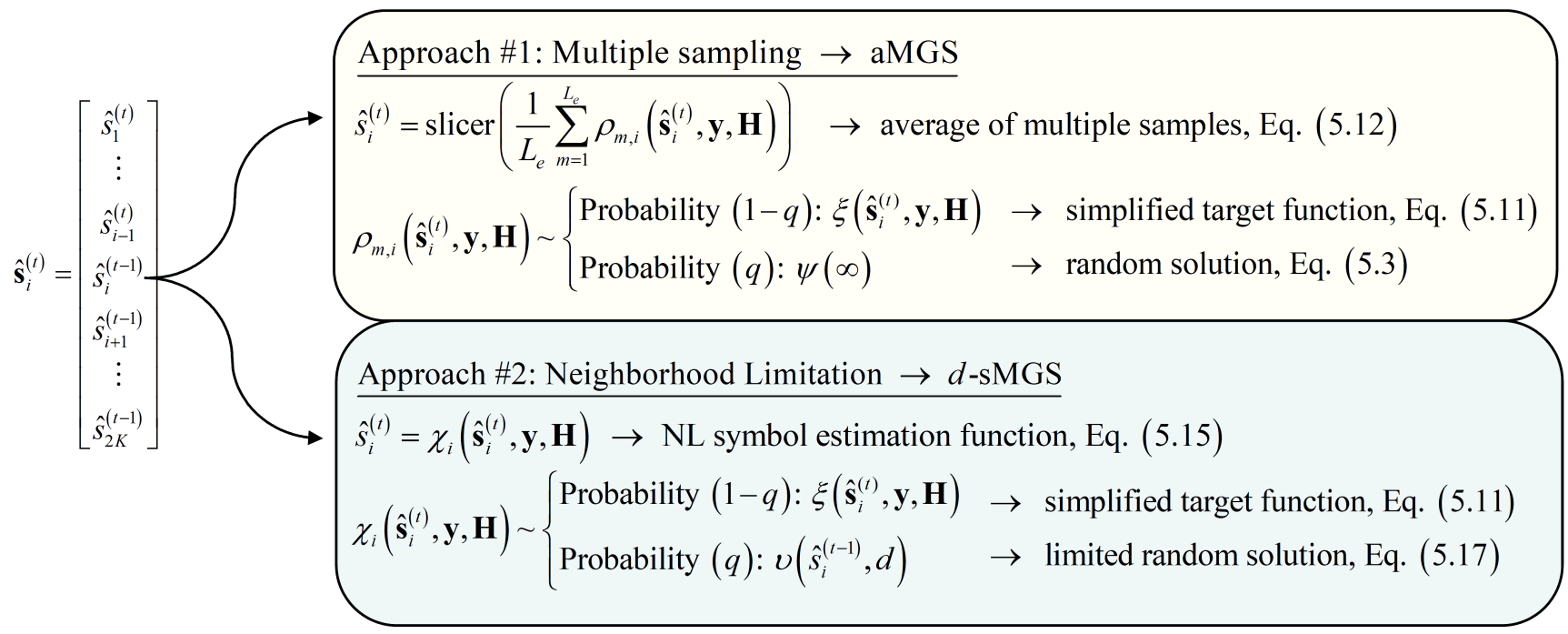

Figure 5.2: A brief description of the coordinate update process on the aMGS and the proposed $d$-sMGS detectors. 


\subsubsection{Approach \#1: Averaged MGS LS-MIMO detector}

A novel approach for the MGS, namely aMGS was proposed during this Doctoral Thesis, which is based on the following improvements:

1. Averaged Multiple Sampling on Each Coordinate: differently from the single sampling strategy [2], the proposed aMGS employs an average between $L_{e}$ number of samples (realizations) in each coordinate during the update process. The proposed strategy tries to alleviate the problem which arise when the noisiest solution of $\alpha_{2} \rightarrow \infty$ is randomly chosen in a given coordinate during the MGS mixing step. By employing the MS calculation, an intermediate (averaged) point between the target function symbol and the random symbol is more likely to be chosen, instead of a pure random symbol. As a result, the benefit of local minima escape is maintained, while the negative impact on the algorithm's convergence is smoothed.

2. Target Function Simplification: to reduce the computational complexity related to the calculation of the target function in (5.5), we adopted a minimum ML cost approach. This simplification performs fewer mathematical operations, since the $\|\mathbf{y}-\mathbf{H} \hat{\mathbf{s}}\|$ computation is already performed in (5.5). The aMGS target function calculates $\xi\left(\hat{\mathbf{s}}_{i}^{(t)}, \mathbf{y}, \mathbf{H}\right) \in \mathcal{A}$ which chooses, in the $i$-th position, the $j$-th symbol that minimizes the Euclidian distance. Thus, it is evaluated in the $t$-th iteration as:

$$
\xi\left(\hat{\mathbf{s}}_{i}^{(t)}, \mathbf{y}, \mathbf{H}\right)=\arg \min _{a_{j} \in \mathcal{A}}\left\|\mathbf{y}-\mathbf{H} \hat{\mathbf{s}}_{i, j}\right\|
$$

where $\hat{\mathbf{s}}_{i}^{(t)}$ denotes the updated estimated symbol vector until the $(i-1)$ position at the $t$-th iteration, whereas the other remaining $i,(i+1), \ldots, 2 K$ positions assume the values from the previous iteration, i.e.,

$$
\hat{\mathbf{s}}_{i}^{(t)}=\left[\hat{s}_{1}^{(t)}, \ldots, \hat{s}_{i-1}^{(t)}, \hat{s}_{i}^{(t-1)}, \ldots, \hat{s}_{2 K}^{(t-1)}\right]^{T}
$$

Remark 5.3.1 (aMGS performance-complexity). Compared to (5.5), the calculation of (5.11) performs fewer operations while achieving the same bit error rate (BER) performance, as depicted in Fig. 5.3. For illustration purpose, a 4-QAM and 16-QAM, $K=16$ and $N=16$ system is deployed, with $\mathcal{I}=8 K \sqrt{M}, q=1 / 2 K$ and $R_{\max }=50$ [2].

\section{MS in coordinate update process}

The coordinate update process of aMGS is defined by:

$$
\hat{s}_{i}^{(t)}=\frac{1}{L_{\mathrm{e}}} \sum_{m=1}^{L_{\mathrm{e}}} \rho_{m, i}\left(\hat{\mathbf{s}}_{i}^{(t)}, \mathbf{y}, \mathbf{H}\right),
$$

where $L_{e}$ is the number of samples (realizations), and the random variable (r.v.) $\rho_{m, i}$ is a mixture of two r.v. with weight given by the mixing ratio $q$, defined by:

$$
\rho_{m, i}\left(\hat{\mathbf{s}}_{i}^{(t)}, \mathbf{y}, \mathbf{H}\right) \sim(1-q) \cdot \xi\left(\hat{\mathbf{s}}_{i}^{(t)}, \mathbf{y}, \mathbf{H}\right)+q \cdot \psi(\infty)
$$




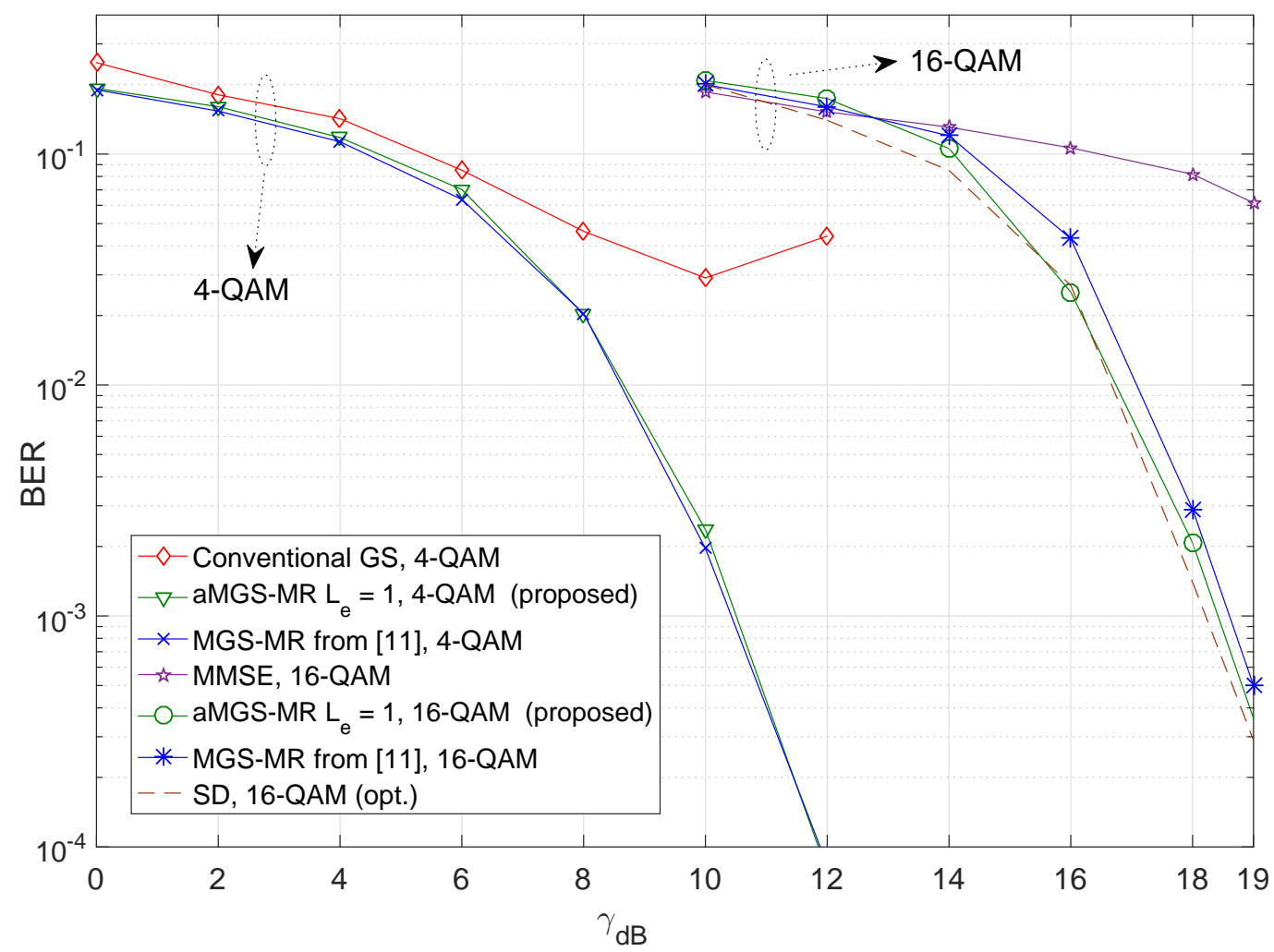

Figure 5.3: An illustrative example showing the stalling effect of conventional GS and denoting that the proposed simplification of the target function of aMGS does not degrade the LS-MIMO performance:

$K=N=16$ antennas, $L_{e}=1$ sample, $\mathcal{I}=512$ iterations, $R_{\max }=50$, 4-QAM and 16-QAM.

It is important to note that, being (5.11) a deterministic function, during the $L_{e}$ realizations on each coordinate, (5.11) is calculated only once, when $m=1$. After that, each $m$ realization has the computational cost of generating a random number (relative to the mixing ratio).

At the end of algorithm iterations, the vector with the lowest cost is assumed the best global solution. Due to the mean operation, a slicer for $M$-QAM constellation is needed at the end of the detection procedure. Thus,

$$
\hat{\mathbf{s}}_{\text {best }}=\operatorname{slicer}\left(\hat{\mathbf{s}}_{\mathrm{f}-\text { best }}\right),
$$

where $\hat{\mathbf{s}}_{\mathrm{f} \text {-best }}$ is the "floating-best" solution which represents the estimated vector related to the best global cost attained after $\mathcal{I}$ iterations, and $\hat{\mathbf{s}}_{\text {best }}$ is the final estimated symbol vector. A pseudocode for the aMGS is described in Algorithm 5.2.

\subsubsection{Approach \#2: Simplified MGS with Neighborhood Limitation LS-MIMO detector}

As extension of the first proposed aMGS solution, we also proposed an different approach which is based on a simply neighborhood limitation of distance $d$ in the random solution and is named $d$-sMGS LS-MIMO detector. The term simplified refers to the simplified target function of Eq. 5.11, which is also employed in this scheme.

The proposed $d$-sMGS detector acts in the symbol constellation performing a neighborhood limitation $(\mathrm{NL})$, with distance $d$ in relation to the symbol estimated in the previous iteration, when sorting the random symbol. This procedure showed to significantly improves the convergence when a modulation of high-order 


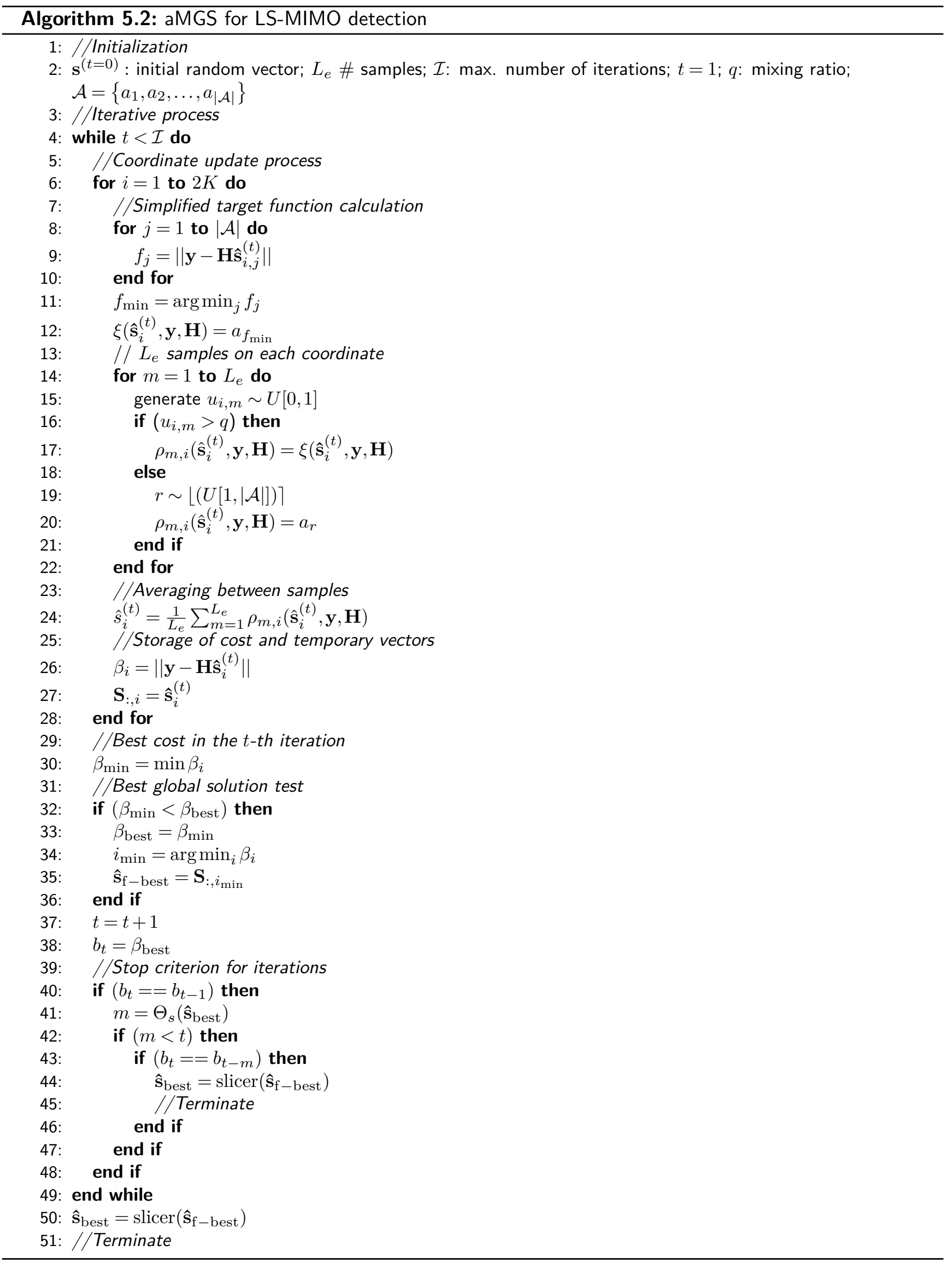


is considered, as disposed in section 4.5, and presents the lowest per-symbol complexity among MGS and aMGS, since it considers the simplified target function (overcoming the MGS in mathematical operations) and performs a single sample (overcoming the multiple sampling aMGS), as showed in Section 2.8.

\section{NL in coordinate update process}

The $d$-sMGS coordinate update process is based on a mixture between the simplified target function, Eq. 5.11, and a limited random solution. Thus, the estimated symbol in the $t$-iteration at the $i$-th coordinate is given by:

$$
\hat{s}_{i}^{(t)}=\zeta_{i}\left(\hat{\mathbf{s}}_{i}^{(t)}, \mathbf{y}, \mathbf{H}\right)
$$

where $\zeta_{i}(\cdot)$ is the mixed r.v. with weight $q$, defined by:

$$
\zeta_{i}\left(\hat{\mathbf{s}}_{i}^{(t)}, \mathbf{y}, \mathbf{H}\right) \sim(1-q) \cdot \xi\left(\hat{\mathbf{s}}_{i}^{(t)}, \mathbf{y}, \mathbf{H}\right)+q \cdot v\left(\hat{s}_{i}^{(t-1)}, d\right)
$$

the r.v. $v\left(\hat{s}_{i}^{(t-1)}, d\right)$ denotes an uniform sorted symbol in the constellation neighborhood of $\hat{s}_{i}^{(t-1)}$, with distance $d$.

In this algorithm, the neighborhood of the current solution $\hat{s}_{i}^{(t-1)}$ is defined as

$$
\mathcal{D}\left(\hat{s}_{i}^{(t-1)}, d\right)=\left\{s^{\prime} \in \mathcal{A} \mid \kappa_{d}\left(\hat{s}_{i}^{(t-1)}, s^{\prime}\right) \leq d\right\}
$$

where $\kappa_{d}$ is the symbol distance function in the real-valued constellation considered, for example, let $\mathcal{A}=$ $\{-7,-5,-3,-1,+1,+3,+5,+7\}, \hat{s}_{i}^{(t-1)}=-3$ and $s^{\prime}=+1$, then the symbol distance function results in $\kappa_{d}\left(\hat{s}_{i}^{(t-1)}, s^{\prime}\right)=2$.

Thus, the r.v. $v\left(\hat{s}_{i}^{(t-1)}, d\right)$ samples from a discrete uniform distribution on the set $\mathcal{D}\left(\hat{s}_{i}^{(t-1)}, d\right)=$ $\left\{n_{1}, \ldots, n_{|\mathcal{D}|}\right\}$.

A pseudocode for the proposed $d$-sMGS is described in Algorithm 5.3. The multiple restarts additional strategy is omitted, since it simply restarts the algorithm with another initial solution.

\subsection{Computational Complexity}

The computational complexity is described in terms of real number operations (rops), in which one rop denotes the computational complexity of the real mathematical operations: addition, subtraction, multiplication or division. For the exponential and logarithmic functions, an approximation through Taylor Series with 18 terms has been considered to calculate the computational complexity. For the generation of random numbers, the linear congruent method is considered, in which to generate a random number three rops are required: addition, multiplication and division (mod operation) [124]. Table 5.1 describes the per-symbol computational complexity, $\mathcal{C}_{T}$, involved in each step of aMGS algorithm. Additionally, the total complexity per-symbol of the MGS has been evaluated. The $\mathcal{C}_{I}$ denotes the per-symbol complexity of the initial solution, which is adopted as the linear MMSE detector for MGS, aMGS and $d$-sMGS detection schemes. The per-symbol complexity of the MMSE is also showed in Table 5.1 [125]. 


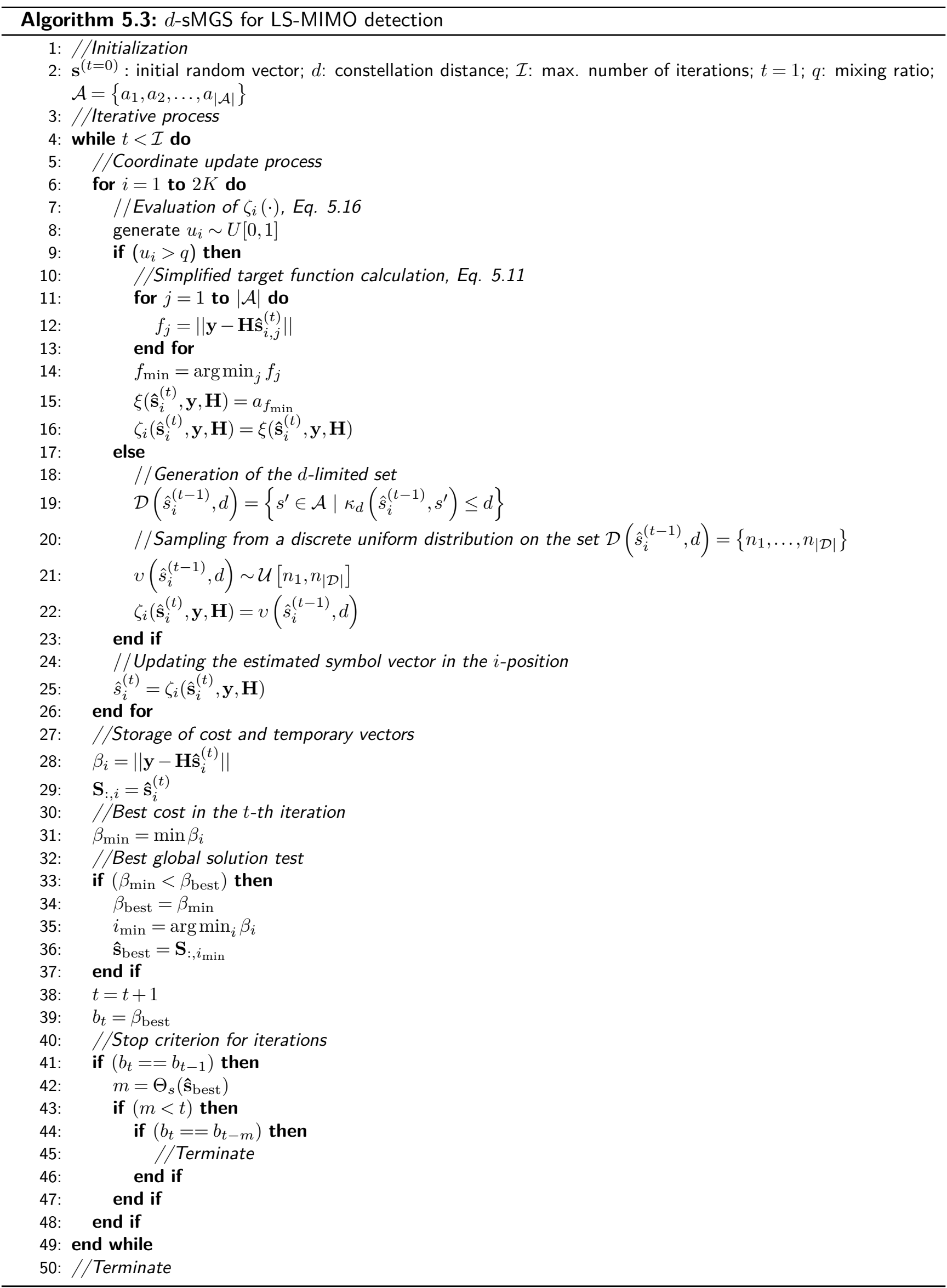


From Table 5.1, one can notice that the $d$-sMGS algorithm, aMGS and MGS algorithms have the same asymptotic per-symbol complexity order of $\mathcal{O}\left(K^{2}\right)$, although the conventional MGS algorithm may require an additional complexity dependent on constellation size due to the exponential function, which is represented by the cardinality $|\mathcal{A}|$. On the other hand, the additional complexity due to the averaged strategy of the aMGS represents a negligible impact, since it requires only a generation of a random number for each $L_{e}$ sample and a division for the mean operation, which is equal to $\left(3 L_{e}+1\right)$ rops per iteration, while such additional complexity is not dependent on the problem size. Regarding the $d$-sMGS algorithm, it combines advantages of both by using a single sample such as the MGS and the simplified aMGS target function. The complexity increment given by the neighborhood constraint is considered negligible, since the symbol is already previously estimated and such procedure represents only a random sampling in a restricted vector.

It is important to emphasize that the complexity of the $d$-sMGS, aMGS and MGS algorithms is defined by the number of iterations, which is controlled by the stopping criterion $\Theta_{s}$, with the upper limit $\mathcal{I}$. Similarly, the amount of restarts is controlled by $\Theta_{r}$, with an upper limit $R_{\max }$. In terms of complexity, the MR procedure can be interpreted as an extra amount of iterations necessary for each new restart. In this sense, an $\mathcal{I}_{\text {eff }}$ is considered in Table 5.1, which denotes the total amount of iterations (including all restarts) performed at each symbol period. Since MCS method is employed in simulations, in Section 4.5 a mean value of $\mathcal{I}_{\text {eff }}$ considering all realizations is evaluated and is called effective number of iterations (ENI):

$$
\mathrm{ENI}=\frac{1}{T} \sum_{i=1}^{T} \mathcal{I}_{\mathrm{eff}, i}
$$

where $T$ denotes the total number of realizations (symbol periods) during the simulation and $\mathcal{I}_{\text {eff }, i}$ denotes the $\mathcal{I}_{\text {eff }}$ in the $i$ th realization.

\subsubsection{Quality Metric}

Due to the large number of parameters involved in the MGS-based LS-MIMO detectors, a simple performancecomplexity tradeoff metric is defined herein aiming to establish a fair comparison analysis among different MIMO detection strategies:

$$
\chi\left(\mathrm{BER}, \mathcal{C}_{T}\right)=-\frac{10 \log _{10}(\mathrm{BER})}{10^{-8} \cdot \mathcal{C}_{T}}=-\frac{\mathrm{BER}_{\mathrm{dB}}}{10^{-8} \cdot \mathcal{C}_{T}}
$$

where $\mathrm{BER}_{\mathrm{dB}}$ denotes the bit error rate in $\mathrm{dB}$. Hence, the quality detection metric $\chi\left(\mathrm{BER}, \mathcal{C}_{T}\right)$ tries to capture the detector efficiency in providing performance improvement against complexity increasing. Higher values of $\chi(\cdot)$ imply more efficient and effective LS-MIMO detector.

\subsection{Numerical results and discussion}

In this section the uncoded BER performance related to the $d$-sMGS algorithm for LS-MIMO detection is evaluated through MCS. The simulations are performed for a large-scale MIMO operating in multiplexing mode, assuming uncorrelated antennas and that a pCSI is available at the receiver side. Table 5.2 summarizes the main system and channel parameter values deployed in this section. 
Table 5.1: Per-symbol Computational Complexity of aMGS, conventional MGS and MMSE Algorithms.

\begin{tabular}{|c|c|c|}
\hline Procedure & Step & Complexity \\
\hline \multicolumn{3}{|c|}{$d$-MGS - Algorithm 5.3} \\
\hline Target function calculation & lines $11-16$ & $16 K N-4 N+|\mathcal{A}|(16 N+2)$ \\
\hline Generation of the $d$-limited set & line 19 & negligible \\
\hline Cost computation at each coordinate & line 28 & $20 N$ \\
\hline$\Theta_{s}, \quad$ Eq. $(5.9)$ & line 41 & $\frac{24}{K}$ \\
\hline \multicolumn{3}{|c|}{$\mathcal{C}_{T}=\mathcal{C}_{I}+\mathcal{I}_{\text {eff }}\left[16 K N+16 N+|\mathcal{A}|(16 N+2)+\frac{24}{K}\right]$} \\
\hline \multicolumn{3}{|c|}{ aMGS - Algorithm 5.2} \\
\hline Target function calculation & lines $8-12$ & $16 K N-4 N+|\mathcal{A}|(16 N+2)$ \\
\hline Averaging between samples & line 24 & $3 L_{e}+1$ \\
\hline Cost computation at each coordinate & line 26 & $20 N$ \\
\hline$\Theta_{s}, \quad$ Eq. (5.9) & line 41 & $\frac{24}{K}$ \\
\hline Total per-symbol complexity: & $\mathcal{C}_{T}=\mathcal{C}_{I}+\mathcal{I}_{\text {eff }}$ & {$\left[16 K N+16 N+|\mathcal{A}|(16 N+2)+\left(3 L_{e}+1\right)+\frac{24}{K}\right.$} \\
\hline
\end{tabular}

\section{MGS - Target distribution function calculation on Algorithm 5.1}

\begin{tabular}{rcc} 
Target distribution function calculation & lines 4-6 & $16 K N-4 N+|\mathcal{A}|(16 N+12)$ \\
Evaluation of each symbol probability & lines 8-12 & $1238|\mathcal{A}|$ \\
Cost computation of estimated vector & $\frac{10 N}{K}$ \\
$\Theta_{s}, \quad$ Eq. (5.9) & $\frac{24}{K}$ \\
\hline
\end{tabular}

Total per-symbol complexity: $\quad \mathcal{C}_{T}=\mathcal{C}_{I}+\mathcal{I}_{\text {eff }}\left[16 K N-4 N+|\mathcal{A}|(16 N+1450)+\frac{10 N+24}{K}\right]$

\section{MMSE Algorithm}

Total per-symbol complexity: $\quad \mathcal{C}_{T}=\frac{1}{6} K^{2}+\frac{3}{2} N K+\frac{3}{2} N+\frac{5}{6}$ 
For the stopping criterion parameters, we have adopted $c_{1}=10, c_{2}=1.0$, and $c_{\min }=10$ [3]. A detailed discussion of these values can be found in the Appendix D.

This numerical simulation section has been divided into two main parts: in Subsection 5.5.1, a discussion about the main parameters of the MGS-based detectors is performed, namely: mixing ratio $q$ and number of samples $L_{e}$ of the aMGS, and maximum number of iterations $\mathcal{I}$ for the $d$-sMGS; in subsection 5.5.2, we present numerical results of performance and computational complexity of the proposed detectors against the conventional MGS technique.

Table 5.2: LS-MIMO system and channel parameters.

\begin{tabular}{|c|c|}
\hline Parameter & Value \\
\hline \multicolumn{2}{|c|}{ LS-MIMO system } \\
\hline Link direction & UL \\
\hline \# Rx antennas (BS) & $N \in\{64,128\}$ \\
\hline $\begin{array}{l}\text { \# Tx antennas (UEs) } \\
\text { (single user-antenna) }\end{array}$ & $K \in\{48,96\}$ \\
\hline System loading & $\beta=\frac{K}{N} \in[0.3125,0.90625]$ \\
\hline Modulation order & 64-QAM \\
\hline SNR ranges & $\gamma \in[0,25] \mathrm{dB}$ \\
\hline \multicolumn{2}{|c|}{ Channel } \\
\hline Channel type & Flat Rayleigh \\
\hline Channel availability & Perfectly known at receiver \\
\hline \multicolumn{2}{|c|}{ aMGS-MR detector } \\
\hline Max. number of iterations & $\mathcal{I} \in\{3000,6000,9000\}$ \\
\hline Max. number of restarts & $R_{\max } \in\{5,10\}$ \\
\hline Number of aMGS samples & $L_{e} \in\{1,2,4,8\}$ \\
\hline Mixing ratio & $q \in\left\{\frac{1}{2 K}, \frac{1}{3 K}, \frac{1}{4 K}\right\}$ \\
\hline Stop criterion parameters & $c_{1}=10 ; c_{2}=1 ; c_{\min }=10$ \\
\hline \multicolumn{2}{|c|}{$d$-sMGS-MR detector } \\
\hline Max. number of iterations & $\mathcal{I}=8 K \sqrt{M}$ \\
\hline Max. number of restarts & $R_{\max }=20$ \\
\hline NL distance & $d \in\{1,2,3\}$ \\
\hline Mixing ratio & $q=\frac{1}{2 K}$ \\
\hline Stop criterion parameters & $c_{1}=10 ; c_{2}=1 ; c_{\min }=10$ \\
\hline
\end{tabular}

\subsubsection{Parameters discussion}

The aMGS-MR BER performance for different mixing ratios $q=\{1 / 2 K, 1 / 3 K, 1 / 4 K\}$, considering $R_{\max }=$ $\{1,5,10\}$, is presented in Figs. 5.4 and 5.5 for each fixed $L_{e} \in\{1,2,4,8\}$ samples scenario. The number of 
users is equal to $K=96$ while $N=128 \mathrm{BS}$ antennas $(\beta=0.75)$. The system is operating under medium-high $\mathrm{SNR}, \gamma_{\mathrm{dB}}=25 \mathrm{~dB}$. First, it is evident that the choice of different mixing ratio values impact both performance and complexity (represented by the ENI quantity at convergence). In addition, one can notice that the large amount of $L_{e}=8$ samples becomes harmful to the algorithm, once convergence is achieved with larger ENI. Among the other results, the best performance-complexity tradeoff is presented with $L_{e}=2$ samples and $q=1 / 4 K$, which results in: $\left.\chi\right|_{L_{e}=2}=44.89$; against $\left.\chi\right|_{L_{e}=4}=37.85$ with 4 samples and $q=1 / 2 K$; and $\left.\chi\right|_{L_{e}=1}=39.66$ with 1 sample and $q=1 / 4 K$. A detailed analysis of the aMGS performance/complexity gain in relation to the mixing ratio and the number of samples can be found in Subsection 5.1.1 of Appendix D.

It can also be concluded that with increasing number of samples $L_{e}$, the curve represented by $q=1 / 2 \mathrm{~K}$ has its convergence improved, resulting in less complexity. That is, when the impact of the noisy solution is reduced, the choice of $q=1 / 2 \mathrm{~K}$ is presented as the best performance-complexity tradeoff. In this sense, the value $q=1 / 2 K$ is adopted for the detector $d$-sMGS.

Through the complete analysis carried out in Appendix D, the parameter values summarized in Table 5.3 have been adopted for the aMGS in the reminder of this work. For the MGS-MR, the following parameters have been adopted: $q=1 / 2 K, \mathcal{I}=8 K \sqrt{M}, R_{\max }=50, c_{1}=10$ and $c_{2}=0.5$ [2].

Table 5.3: Best parameters for aMGS-MR detector, presented in [3]

\begin{tabular}{r|r|cccc}
\hline & & \multicolumn{4}{|c}{ \# aMGS samples, $\boldsymbol{L}_{\boldsymbol{e}}$} \\
Parameter & BS antennas & $\mathbf{1}$ & $\mathbf{2}$ & $\mathbf{4}$ & $\mathbf{8}$ \\
\hline \hline Mixing & $N<=64:$ & $\frac{1}{4 K}$ & $\frac{1}{4 K}$ & $\frac{1}{3 K}$ & $\frac{1}{2 K}$ \\
ratio, $\boldsymbol{q}$ & $N>64:$ & $\frac{1}{4 K}$ & $\frac{1}{4 K}$ & $\frac{1}{2 K}$ & $\frac{1}{2 K}$ \\
\hline Max. \# Iterations, $\boldsymbol{I}$ & \multicolumn{4}{|c}{3000} \\
\hline \multicolumn{2}{r|}{ Max. Restarts, $R_{\max }$} & \multicolumn{4}{|c}{10} \\
\hline Iterations stop criterion, $c_{1}$ & \multicolumn{4}{|c}{1} \\
Restarts stop criterion, $c_{2}$ & \multicolumn{4}{|c}{} \\
\hline
\end{tabular}

In Fig. 5.6 the convergence of the aMGS algorithm adopting best $q$ values, from Table 5.3, is analyzed against the average rops complexity, with $96 \times 128$ antennas and 64 -QAM. For comparison purpose, a single sampling result using the optimal mixing ratio value as proposed in [2], i.e., $q=1 / 2 K$ (curve [E]) is also included. One can notice that a less number of samples has shown to be beneficial in this LS-MIMO scenario, since the single sample case presented the best performance combined to the lowest asymptotic complexity, followed by the two $\left(L_{e}=2\right)$ and four-fold $\left(L_{e}=4\right)$ sampling case. Nevertheless, due to a slightly convergence gain observed with $L_{e}=2$ samples, the tradeoff metric for $L_{e}=1$ is found to be $\left.\chi\right|_{L_{e}=1}=39.83$ against $\left.\chi\right|_{L_{e}=2}=44.22$ with $L_{e}=2$ samples.

Regarding the $d$-sMGS, we focus on finding the maximum number of iterations $\mathcal{I}$ aiming at maximizing tradeoff performance $\times$ complexity. In the literature, the quantity $\mathcal{I}=8 K \sqrt{M}$ adopted in [2] is quite reasonable since it takes into account the number of active users and the modulation order. In this sense, Figure 5.7 shows the performance convergence of the proposed algorithm with the increase of the maximum number of iterations. We considered $K=N=16$ antennas in 64-QAM with NL distance $d=\{1,2,3\}$ and used the 


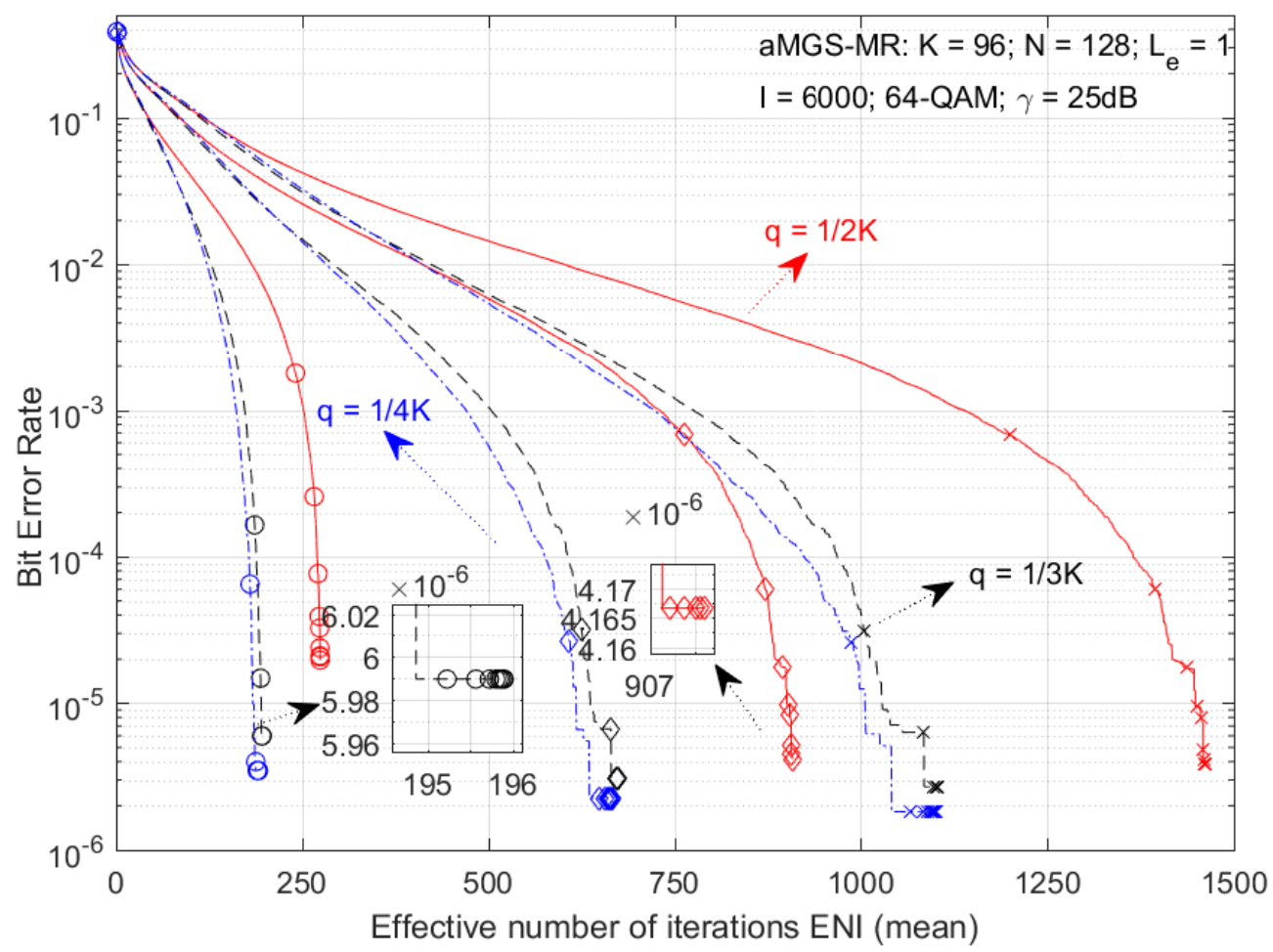

(a) $96 \times 128, L_{e}=1$

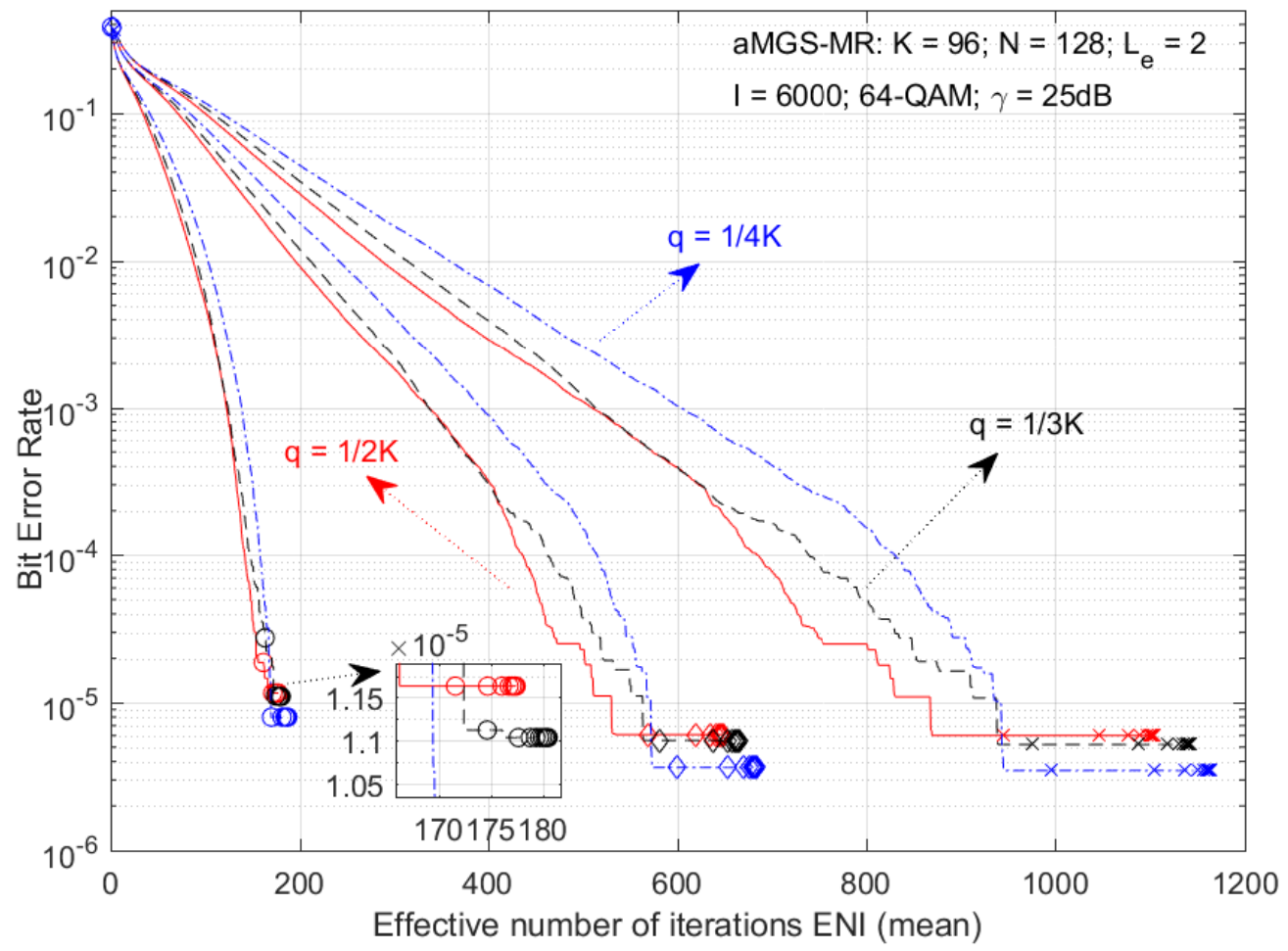

(b) $96 \times 128, L_{e}=2$

\begin{tabular}{|c|c|c|}
\hline $\begin{array}{l}-R_{\text {max }}=1 ; q=1 / 2 \mathrm{~K} \\
\diamond-R_{\text {max }}=5 ; q=1 / 2 \mathrm{~K} \\
\times-R_{\text {max }}=10 ; q=1 / 2 \mathrm{~K}\end{array}$ & $\begin{array}{l}-R_{\text {max }}=1 ; q=1 / 3 \mathrm{~K} \\
\checkmark-R_{\text {max }}=5 ; q=1 / 3 \mathrm{~K} \\
\times-R_{\text {max }}=10 ; q=1 / 3 \mathrm{~K}\end{array}$ & $\begin{array}{l}-\mathrm{R}_{\text {max }}=1 ; \mathrm{q}=1 / 4 \mathrm{~K} \\
-\mathrm{R}_{\text {max }}=5 ; \mathrm{q}=1 / 4 \mathrm{~K} \\
\times \leftarrow \mathrm{R}_{\text {max }}=10 ; \mathrm{q}=1 / 4 \mathrm{~K}\end{array}$ \\
\hline
\end{tabular}

Figure 5.4: BER performance convergence of different mixing ratios, $q$, of aMGS in medium number of antennas scenario $(K=96, N=128)$ at $\gamma_{\mathrm{dB}}=25 \mathrm{~dB}, 64$-QAM, $R_{\max }=\{1,5,10\}, \mathcal{I}=6000$ and low number of $L_{e}$ samples: a) $L_{e}=1$, b) $L_{e}=2$. 


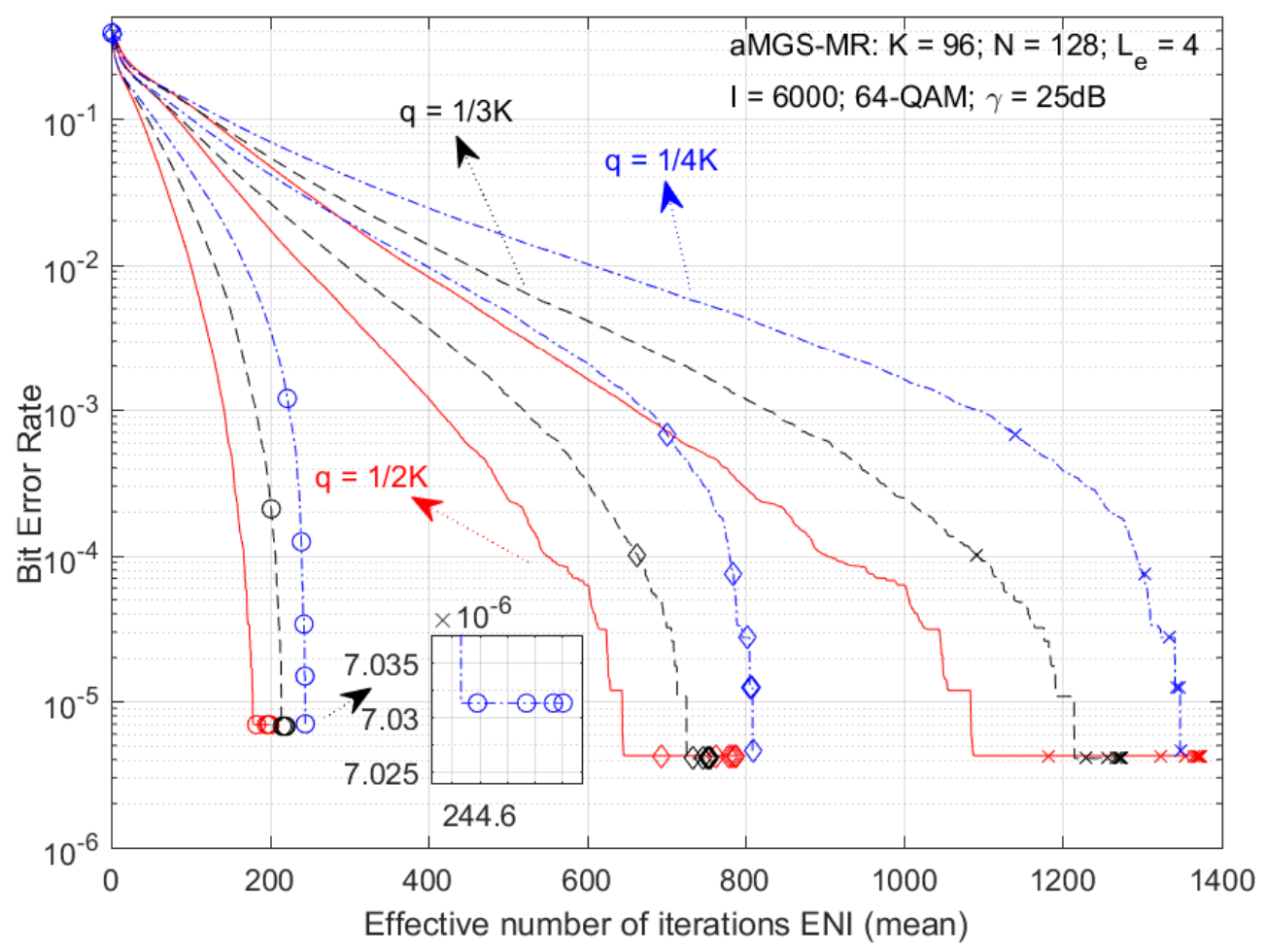

(a) $96 \times 128, L_{e}=4$

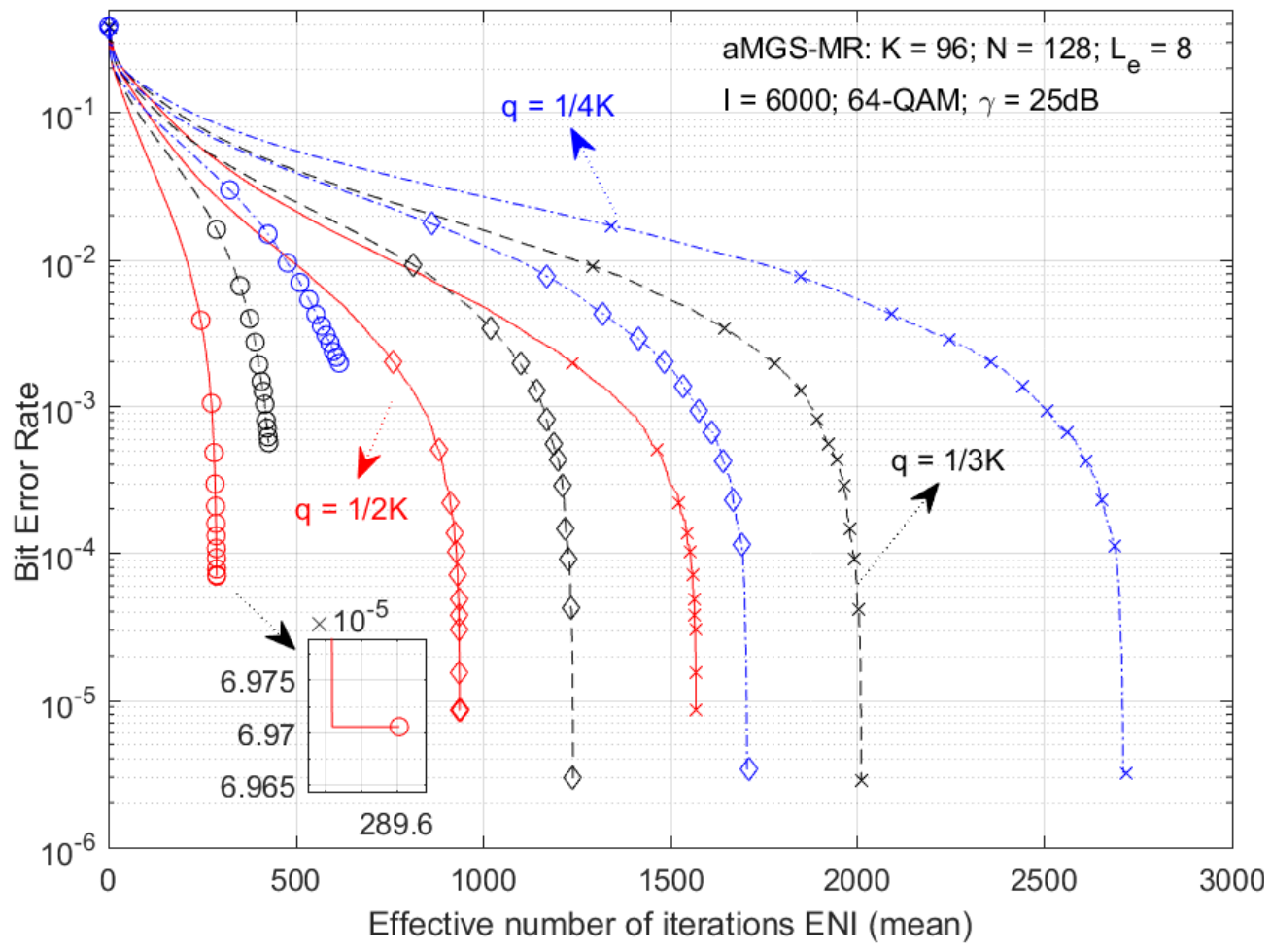

(b) $96 \times 128, L_{e}=8$

\begin{tabular}{|c|c|c|}
\hline $\begin{array}{l}-R_{\text {max }}=1 ; q=1 / 2 \mathrm{~K} \\
\checkmark-R_{\text {max }}=5 ; q=1 / 2 \mathrm{~K} \\
\times-R_{\text {max }}=10 ; q=1 / 2 \mathrm{~K}\end{array}$ & $\begin{array}{l}-\mathrm{R}_{\text {max }}=1 ; \mathrm{q}=1 / 3 \mathrm{~K} \\
\diamond-\mathrm{R}_{\text {max }}=5 ; \mathrm{q}=1 / 3 \mathrm{~K} \\
\times-\mathrm{R}_{\text {max }}=10 ; \mathrm{q}=1 / 3 \mathrm{~K}\end{array}$ & 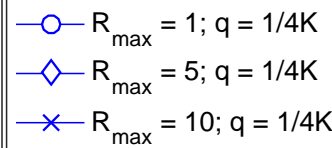 \\
\hline
\end{tabular}

Figure 5.5: BER performance convergence of different mixing ratios, $q$, of aMGS in medium number of antennas scenario $(K=96, N=128)$ at $\gamma_{\mathrm{dB}}=25 \mathrm{~dB}, 64$-QAM, $R_{\max }=\{1,5,10\}, \mathcal{I}=6000$ and high number of $L_{e}$ samples: a) $L_{e}=4$, b) $L_{e}=8$. 


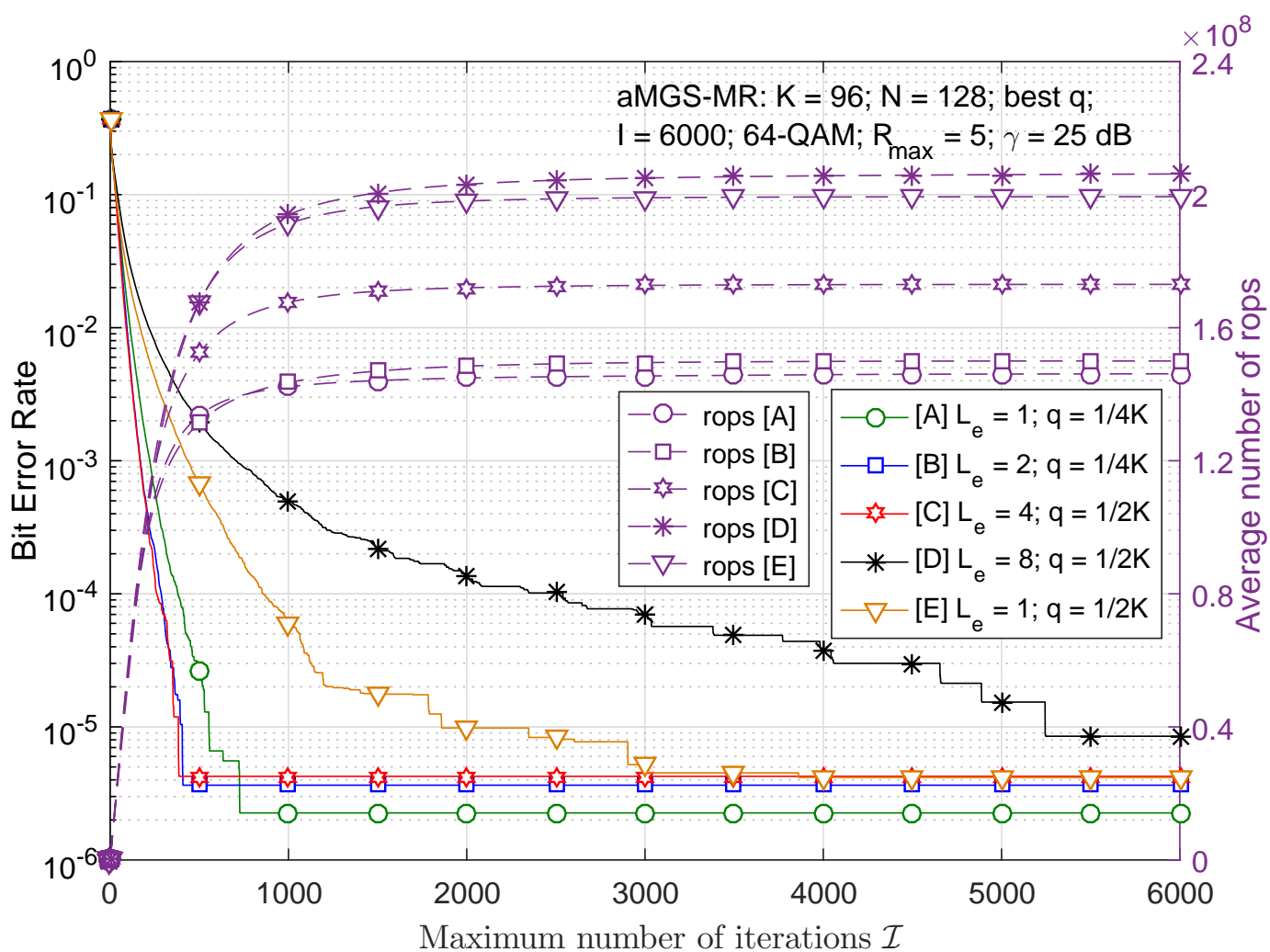

Figure 5.6: BER performance and complexity vs. convergence for the aMGS algorithm considering 64-QAM modulation, best mixing ratio $q$ (curves [A] to [D]) from Table 5.3 and optimal value as proposed in [2], i.e., $q=1 / 2 K$ and $L_{e}=1$ (curve [E]).

parameter $a$ to denote the maximum number of iterations, so that $\mathcal{I}=a K \sqrt{M}=128 a$. It can be clearly seen that the increase in the NL distance is not beneficial to the algorithm's performance, which is easily explained by the fact that, with increasing $d$, the neighborhood of the random solution increases, approaching the condition of unrestricted solution in the constellation, retaking its negative impact on the algorithm's convergence. Thus, observing the 1-sMGS curve, it can be seen that its convergence is reached with $a$ equal to 8 , which coincides with the result adopted in [2]. Therefore, this value $\mathcal{I}=8 K \sqrt{M}$ will be adopted for the proposed $d$-sMGS detector in the reminder of this work.

\subsubsection{Analysis on the proposed approaches}

The Fig. 5.8 shows the SNR vs. performance/computational complexity of the addressed detectors. A high system loading, i.e., $\beta \approx 0.9$, in 64-QAM modulation is adopted with: a) $K=58, N=64$ and b) $K=87, N=96$ antennas. One can notice in Fig. 5.8.(a) that both proposed detectors presented significant performance gain in the region of high SNR in relation to the other detectors, equivalent to approximately one decade against the second best performance detector aMGS-MR with $L_{e}=8$ samples. Differently from that observed previously, the increase in the NL distance did not cause a loss of performance, since the 2-sMGS detector resulted in a marginally similar performance to the 1-sMGS. Thus, it denotes a tendency that the increase of the NL distance can be beneficial in scenarios with greater number of antennas, such as LS-MIMO. Related to the computational complexity, it can be observed that the complexity of the 1-sMGS, 2-sMGS and aMGS detectors with $L_{e}=2,4$ and 8 samples are marginally equivalent, although the aMGS with 8 samples presented the least number of rops (excluding the linear MMSE detector). Considering that both $d$-sMGS and 


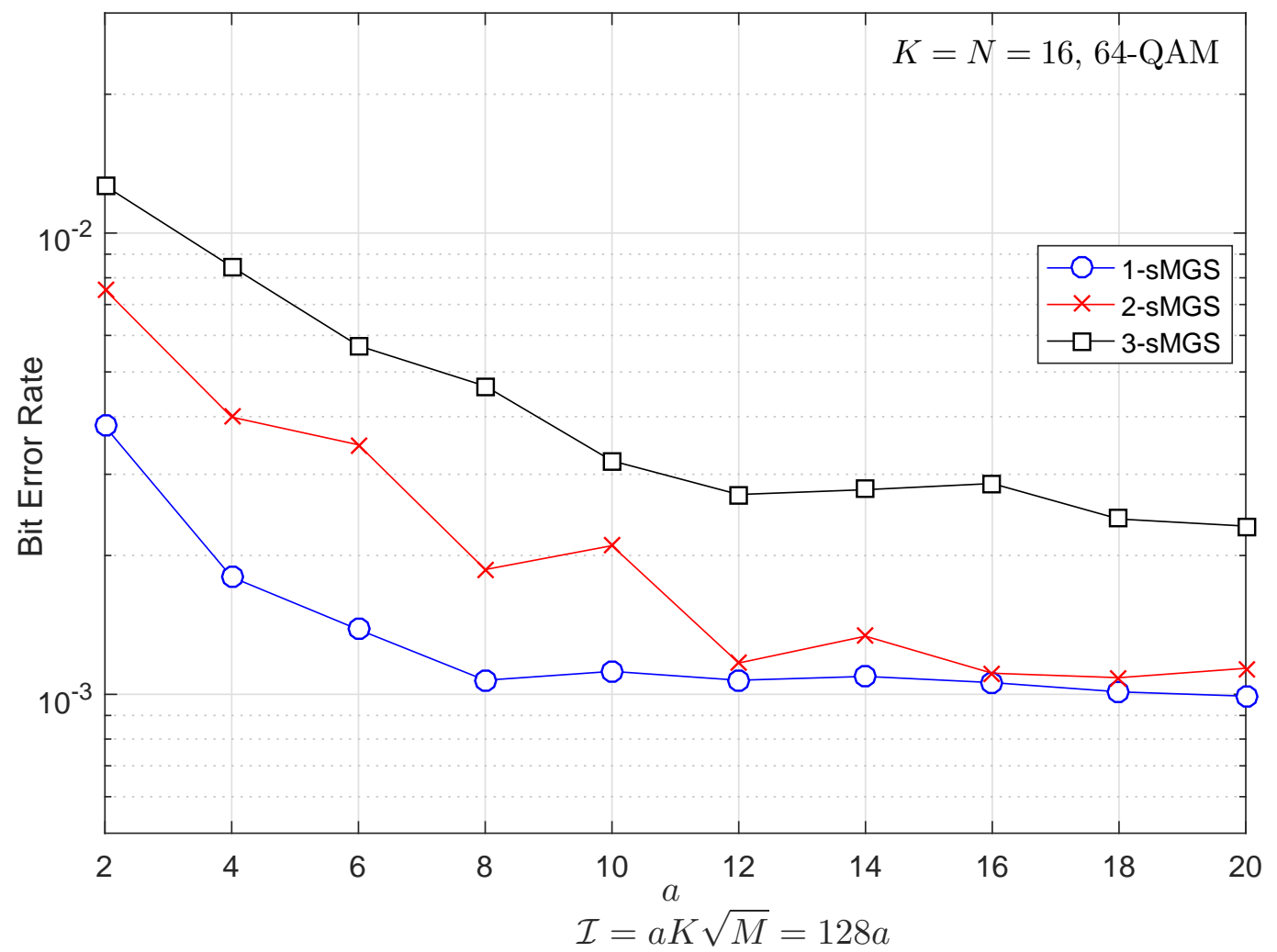

Figure 5.7: Performance convergence against the maximum number of iterations $\mathcal{I}$ of the proposed $d$-sMGS detector, with $16 \times 16$ antennas in 64-QAM modulation among different NL distance values. The number of iterations is related to the parameter $a$, were $\mathcal{I}=a K \sqrt{M}$.

aMGS have marginally the same complexity per iteration, it can be concluded that the MS strategy converged with fewer iterations, on the other hand, with inferior performance to that reached by $d$-sMGS.

With increasing antenna numbers, Fig. 5.8.(b), it is reiterated the hypothesis that the increase of the NL distance results in a performance gain. Also, it is noticeable a significant performance gain in the 4-sample aMGS detector, surpassing the result with $L_{e}=8$, which corroborates the same hypothesis, i.e., a smaller restriction in the noisy solution becomes beneficial with the increase in the number of antennas. In fact, in the region of high SNR, $\gamma_{\mathrm{dB}}=25 \mathrm{~dB}$, it can be seen that the 2-sMGS and aMGS with $L_{e}=4$ achieve similar performance, although in the medium SNR region $\left(\gamma_{\mathrm{dB}}=23 \mathrm{~dB}\right)$, the proposed $d$-sMGS still appears superior. With respect to the complexity in terms of rops, it is noticed that the 2-sMGS-MR and aMGS-MR detectors with $L_{e}=4$ and 8 samples presented a marginally equal complexity in $\gamma_{\mathrm{dB}}=25 \mathrm{~dB}$; however, the least complexity is reached again by the aMGS, specially in medium SNR region $\left(\gamma_{\mathrm{dB}}=[21,23] \mathrm{dB}\right)$. Therefore, it can be concluded that the proposed $d$-sMGS detection technique presented the best performance in both scenarios, and the smaller restriction of neighborhood with $d=2$ was a more interesting choice with increasing number of antennas. In addition, there was no significant increase of complexity compared to the MS detector aMGS. In other words, the complexity of the 2-sMGS detector was marginally equal to the lowest complexity techniques: aMGS with $L_{e}=4$ and 8 samples.

A system loading analysis against BER and rops complexity is depicted in Fig. 5.9 under $\gamma_{\mathrm{dB}}=25 \mathrm{~dB}$. It may be first noted that at high loading, i.e., $\beta \approx 0.9$, the NL-based detection scheme showed a significant gain in performance over the aMGS. In the other regions, there is no clearly outstanding technique, however, 


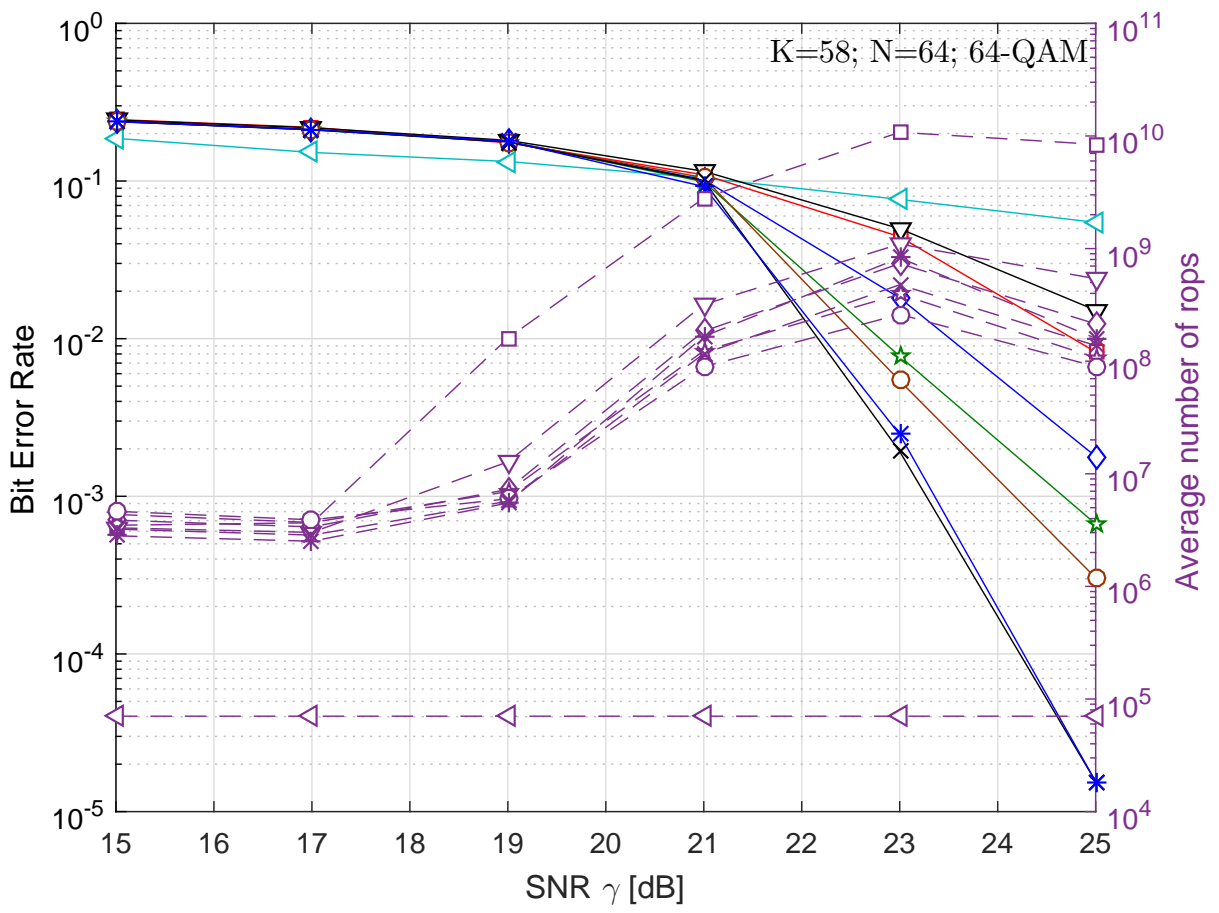

(a) $58 \times 64$

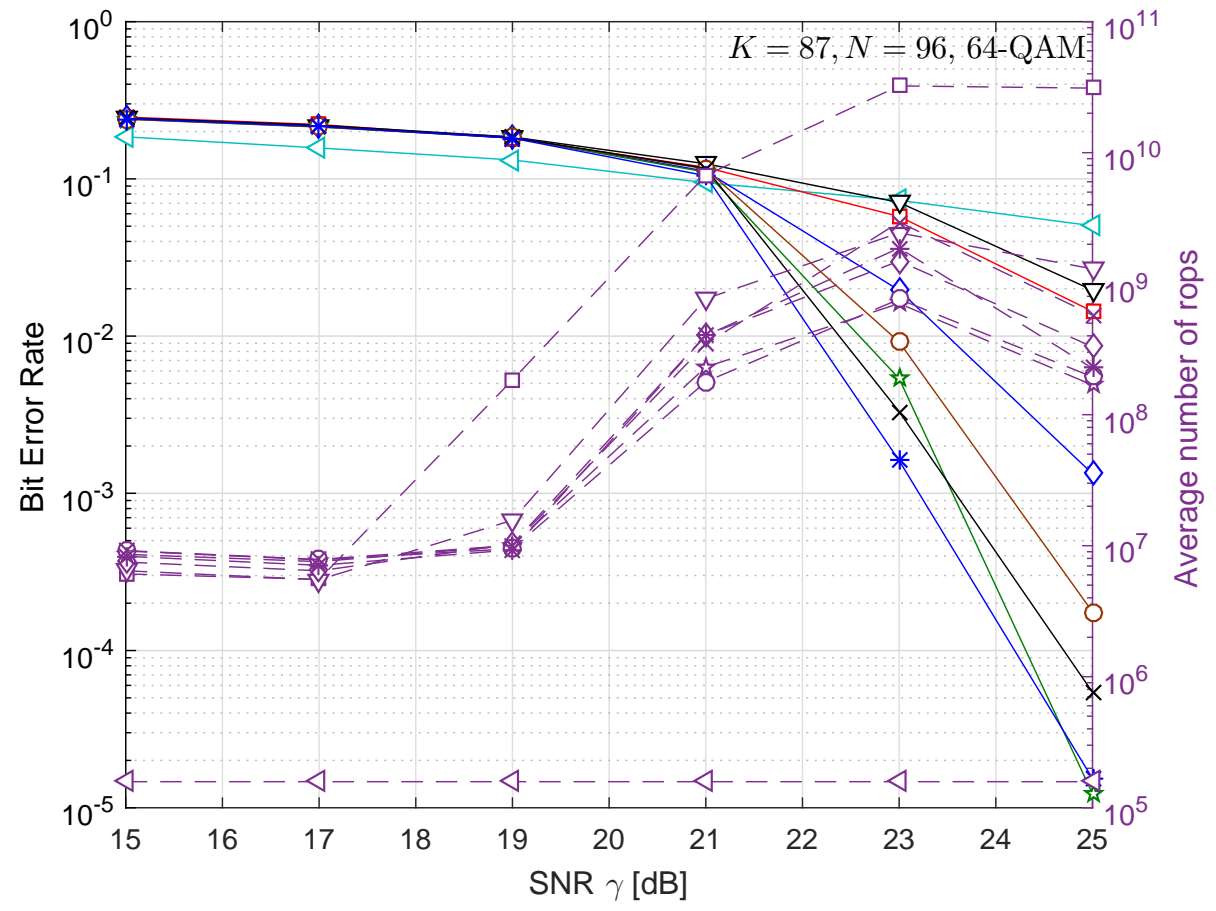

(b) $87 \times 96$

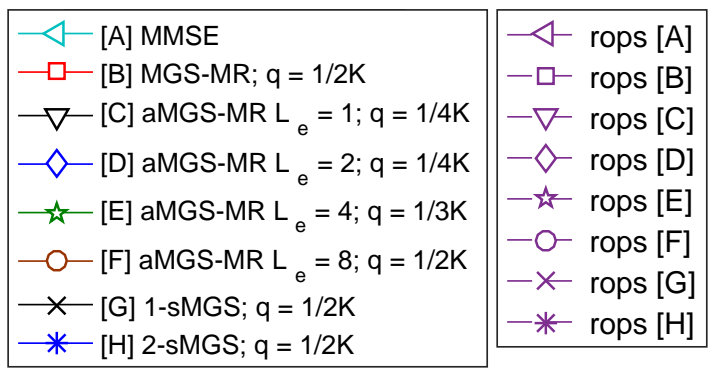

Figure 5.8: SNR vs. BER performance/Average number of rops in $d$-sMGS-MR detector against aMGS-MR approach and MGS-MR. Parameters: $K=58, N=64,64-\mathrm{QAM}, \beta \approx 0.9$. 
a lower restriction in the noisy solution demonstrated better results, which are represented by the 2-sMGS overpassing the 1-sMGS and aMGS with $L_{e}=1$ or 2 in front of the $L_{e}=4$ and 8 samples. In relation to the computational complexity with $N=64$ antennas (Fig. 5.9.(b), one can notice that in the mediumhigh loading region $(\beta \geq 0.75)$, the proposed $d$-sMGS strategy presented less complexity both with respect to multiple sampling aMGS and conventional MGS. In the medium-low system loading results $(\beta \leq 0.5)$, MS schemes presented lower computational complexity. Therefore, one can highlight the superiority of the NL strategy in both performance and complexity in medium-high loading configurations, demonstrating the potential of this strategy when the LS-MIMO system operates under high loading crowded scenarios. This can be explained as the number of mobile users increases, approaching the full-loading system condition $\beta \rightarrow 1$, the set of possible symbol combinations becomes larger, such that the noisy solution from the mixture has its negative effect aggravated, affecting the algorithm's convergence; whereas the NL strategy is able to mitigate this effect, having a beneficial effect on the convergence which results in improvement in performance and computational complexity. In a medium-low loading configurations, the aMGS with small number of samples appears as a more interesting choice, resulting in the lowest complexities among MGS-based detectors and superior performance.

With the increasing number of antennas at $N=128$, Fig. 5.10, the system loading analysis reflects a clear superiority of the 2-sMGS detector in high loading configurations, both in performance and in complexity. This performance behavior corroborates the hypotheses raised in Fig. 5.8 regarding performance improvement with increasing NL distance. On the other hand, in medium-low loading, the complexity of $d$-sMGS was shown to be greater than all aMGS configurations, equating only to the conventional MGS-MR.

\subsection{Chapter conclusions}

Two MGS-based approaches for high-order QAM modulation in large-scale MIMO systems have been proposed. Both approaches have demonstrated capability to mitigate the impact caused by the random symbol from the mixture, which is aggravated either in near full-loading system condition or when a medium to high-order modulation is deployed. Specifically, the main contributions refer to:

- we propose an averaged multiple sampling (MS), namely aMGS, strategy in order to improve the MGS convergence rate operating under higher-order modulation and large scale MIMO regime. The proposed strategy deploys multiple samples per coordinate and performs a simple mean to estimate each coordinate symbol to alleviate the impact caused by the random solution, i.e. infinite mixing time, employed in the mixture used by the MGS detector;

- a NL strategy is proposed aiming at improving the MGS convergence rate operating under higher-order modulation and large scale MIMO regime. The proposed strategy, called $d$-sMGS ( $d$-simplified MGS), performs a NL in the random solution coming from the mixture used by the MGS detector. As a result, the impact caused by this noisy solution is mitigated and the convergence is increased;

- lastly, we propose a simplification on the target function aiming at reducing its computational complexity, while maintaining the performance. Since the target function is computational burden, requir- 


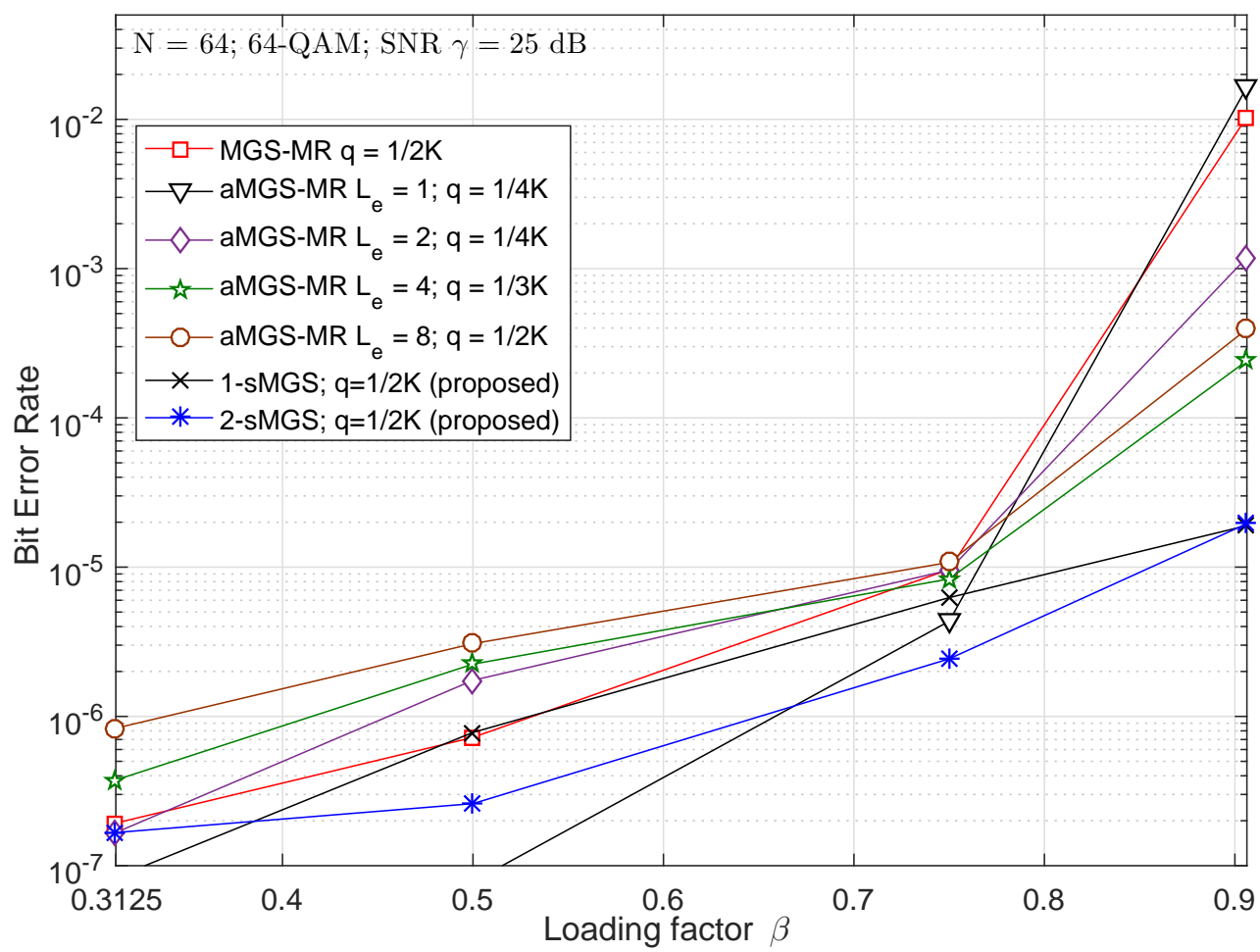

(a) Performance, $N=64$

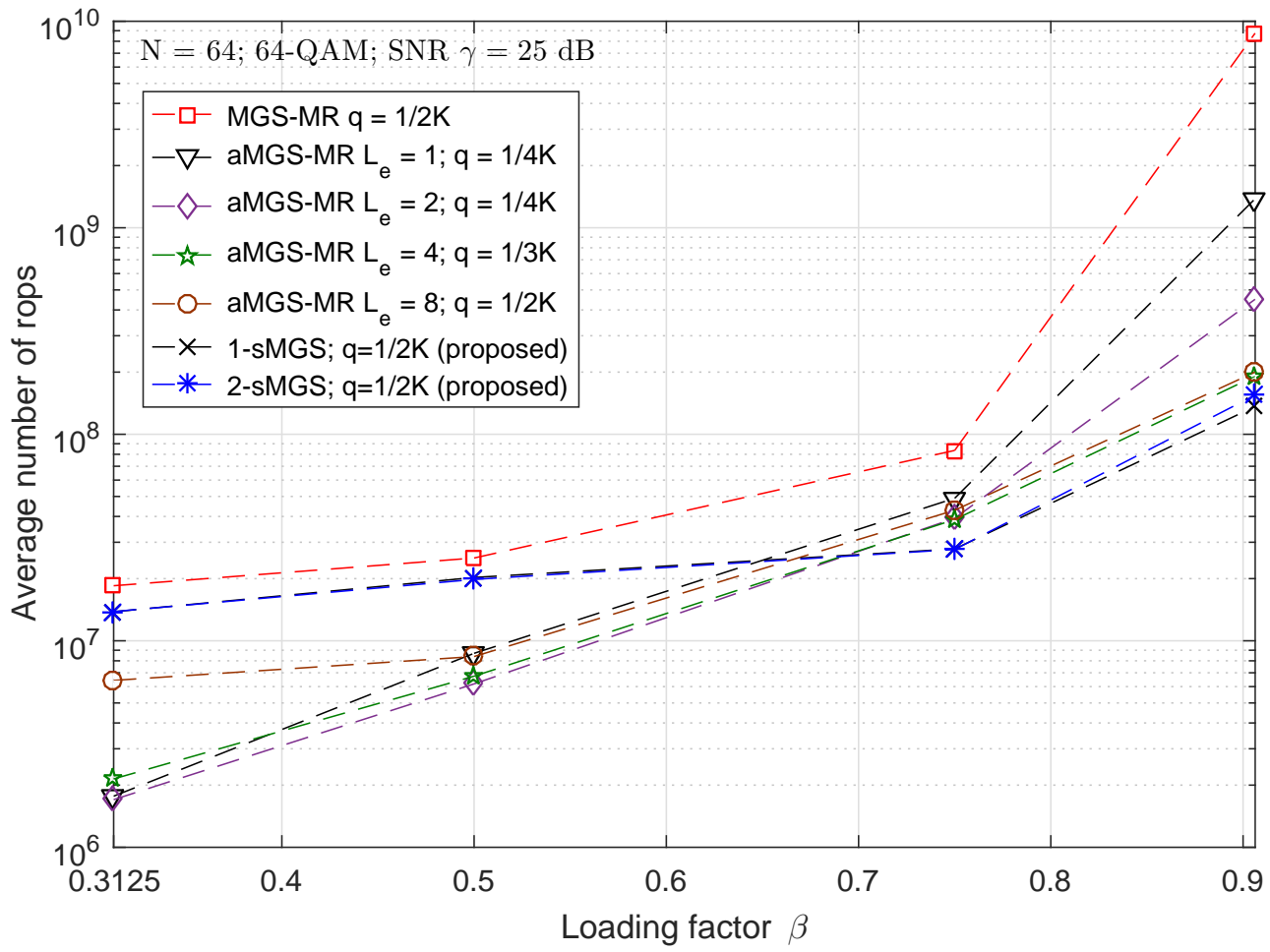

(b) Complexity, $N=64$

Figure 5.9: Performance and complexity versus system loading, considering $\gamma_{\mathrm{dB}}=25 \mathrm{~dB}, 64-\mathrm{QAM}$ : a) Performance for $N=64$; b) Average rop complexity for $N=64$. 


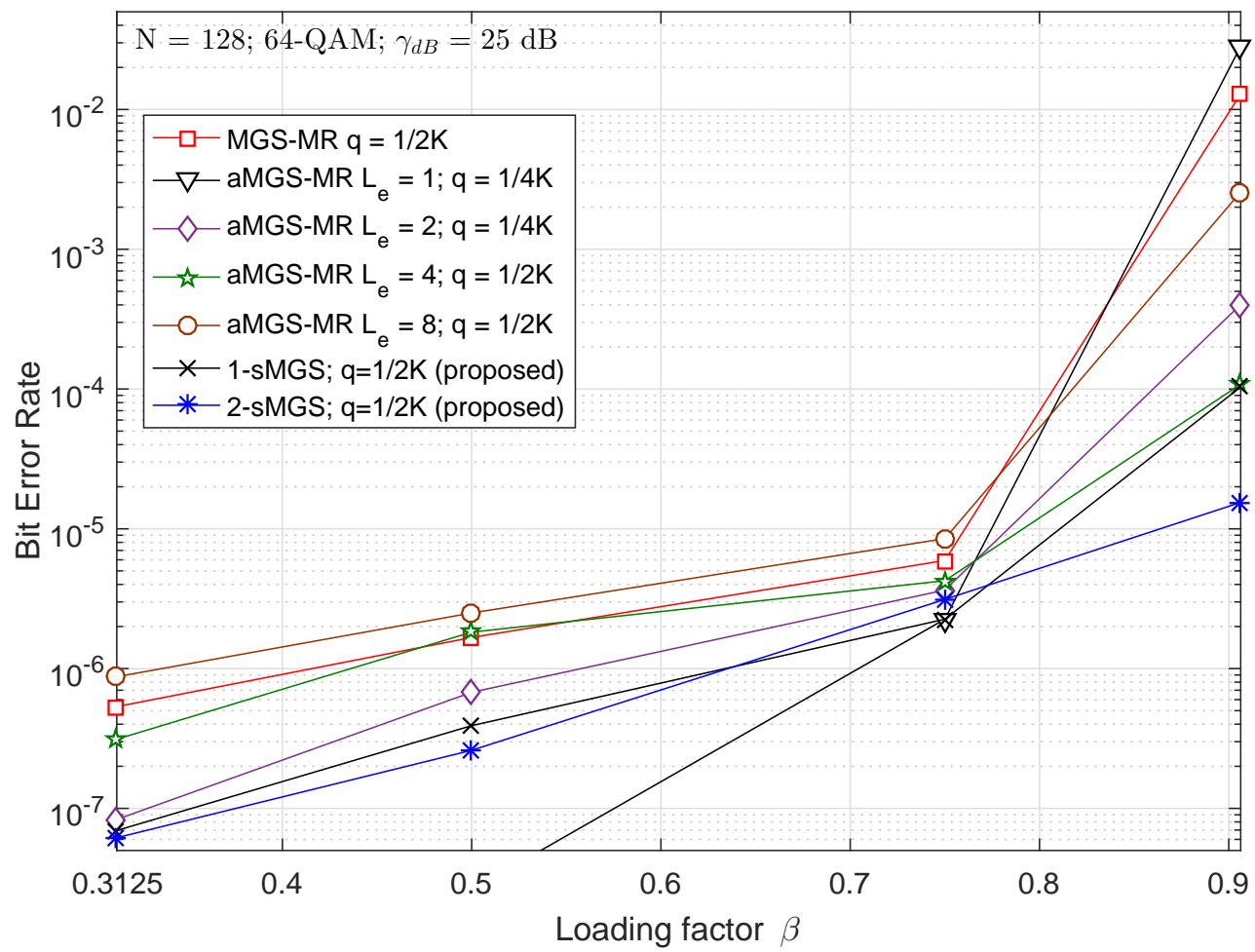

(a) Performance, $N=128$

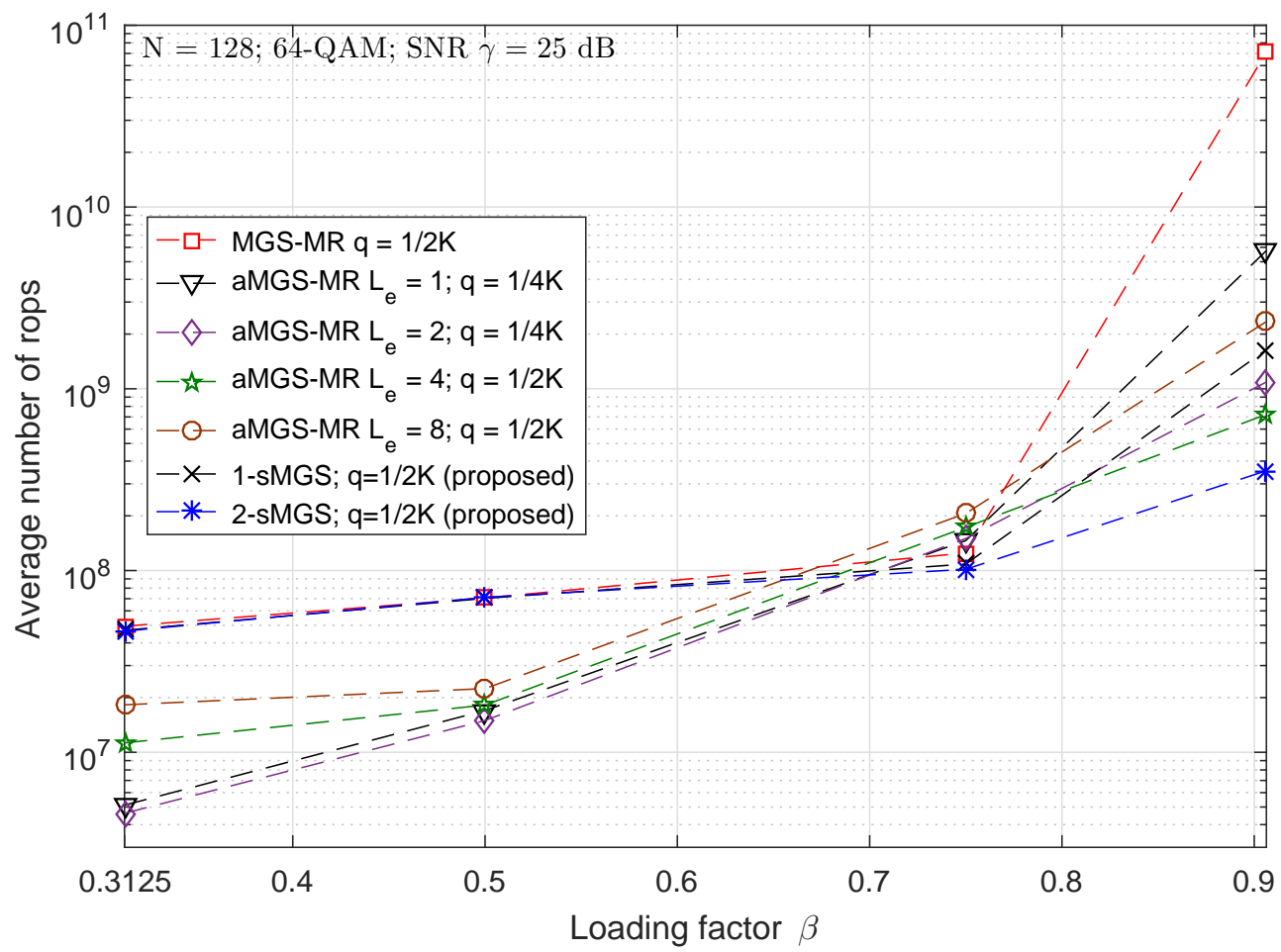

(b) Complexity, $N=128$

Figure 5.10: Performance and complexity versus system loading, considering $\gamma_{\mathrm{dB}}=25 \mathrm{~dB}, 64-\mathrm{QAM}$ : a) Performance for $N=128$; b) Average rop complexity for $N=128$. 
ing $2 K$ times per iteration, our proposition is that the target function can be directly based on the maximum-likelihood cost minimization, which avoids the exponential function computation, resulting in a complexity decreasing.

\subsubsection{Approach \#1: aMGS}

The first approach, namely aMGS, is based on multiple-samples on coordinate updating process, whereas suitable tradeoff metric values have been found for a wide LS-MIMO system configuration scenarios when the number of samples is set in-between $L_{e}=1$ and $L_{e}=8$. Furthermore, a simplification in the target function is hold, aiming at reducing complexity by avoiding the exponential calculation. The proposed target function is based on ML cost minimization and requires less mathematical operations than the original MGS, while no degradation in performance is observed.

Also, our numerical analysis for the mixing ratio parameter optimization considering a wide range of system scenarios has demonstrated that the best mixing ratio value is determined by the system loading and by the quantity of samples of the aMGS as well. As a result, we have found different optimal values than $q=1 / 2 \mathrm{~K}$ reported previously by [2]. Moreover, when the LS-MIMO systems runs in low to medium system loading (i.e., $\beta \leq 0.5)$, the first approach aMGS is shown to be a more interesting option, resulting in significantly reduced complexity compared to $d$-sMGS or MGS.

\subsubsection{Approach \#2: $d$-sMGS}

The second approach refers to a neighborhood limited $d$-sMGS detector for large-scale MIMO systems, which based on the neighborhood constraint of the noisy solution at a distance of $d$. The proposed $d$-sMGS detection scheme demonstrated better performance and lowest computational complexity against aMGS and conventional MGS detectors when a high system loading condition (i.e., $\beta \geq 0.75$ ) is deployed and medium-high SNR is present. These advantages are especially obtained when a large number of antennas is deployed, which meets the requirements of LS-MIMO systems. Furthermore, with increasing the number of dimensions, i.e., increasing number of antennas and/or modulation order, a smaller restriction of 2-sMGS was shown to be a more interesting choice than 1-sMGS.

In the next chapter, the main conclusions, from the contributions to the weaknesses found, regarding the 4 works developed in this Doctoral Thesis are discussed. 


\section{Conclusions}

This thesis investigated the main signal detection techniques in LS-MIMO systems, regarding the criteria of computational complexity and performance. Furthermore, new strategies were proposed in the operation of some detection techniques that fundamentally resulted in a better performance $\times$ complexity tradeoff, being these the main contributions of this thesis. The main results achieved are summarized below.

In the first problem investigated, a performance-complexity analysis was performed regarding the LR preprocessing technique considering channels with imperfect estimates of the channel conditions and under a correlation effect between the Tx and Rx antennas. Linear detectors were also aided by OSIC technique, in addition to the LR-SD detector, which a lookup table procedure was proposed aiming complexity reduction. It has been shown by numerical results that the LR technique results in performance improvement by increasing the diversity gain in linear detectors, in both uncorrelated and highly correlated scenarios. In the cases of strongly correlated channels, the performance gain was remarkable, on the other hand, there was also a considerable increase in the total complexity of the algorithms, caused by the LLL algorithm calculation. As for the LR-SD detector, this resulted in optimal performance in all scenarios investigated and with computational complexity lower than ML. In channels with iCSI, both the LR-assisted linear detectors and the LR-SD suffered significant performance degradations, demonstrating little robustness in these scenarios.

In the second work resulting from this Doctoral Thesis, LS-MIMO detectors by message passing through graphical models were investigated. An extensive analysis was performed on the DF parameter, and an optimum calculation of its value was proposed, based on numerical results adjusted by curve fitting. It has been demonstrated that the DF has a great impact not only on the detector's performance, but also on its complexity, through an improvement in the algorithm convergence. The MRF-BP detector using the proposed $\alpha_{\mathrm{MRF}}$ resulted in less complexity than the FG-BP disposed in the literature, in scenarios with $K=N<160$ antennas. With less than 75 antennas, MRF-BP with $\alpha_{\mathrm{MRF}}$ resulted in both better performance and lower complexity compared to FG-BP.

The third detection technique investigated was MGS. It was noticed that, in high modulation orders, the MGS algorithm disposed in the literature suffers negative impact of the noisy solution coming from the mixing strategy. In this sense, two approaches were proposed in order to mitigate this effect: by multiple samples and by the neighborhood limitation of the random solution. In addition, a simplification in the calculation of the objective function was proposed, aiming at a reduction in the number of operations. It has been shown that both techniques proposed are able to mitigate the effect of the random solution, however, with different results. The first aMGS approach resulted in superiority in both performance and complexity over MGS in highly loaded scenarios; and in less complexity, with similar performance, in systems with low- 
medium loads. Thus, it has been shown that the use of multiple samples results in improved convergence of the algorithm by counteracting the negative effect of the noisy solution. On the other hand, the aMGS detector is significantly surpassed by the d-sMGS, proposed in a work extension, when high loading LS-MIMO $(\beta \geq 0.75)$ scenarios are considered. In these scenarios, the performance gain of the d-sMGS was remarkable with even lower computational complexity. However, with system loading less than 0.65 , aMGS resulted in a significant reduction of complexity and better performance than d-sMGS. Thus, it is concluded that the $d$ sMGS neighborhood constraint has been shown to be a more effective technique in denser and larger scenarios because it is a more abrupt way of limiting the reach of the random solution. The technique of multiple aMGS samples, because it is a more variable way (considering the average of a quantity of random samples) of limitation of the random solution, showed superiority in less dense scenarios, in which the negative effect of the noise solution is more subtle.

\subsection{Future Research Directions}

With the development of this Doctoral Thesis, some points were identified as possible improvements in the formulation of the central signal detection problem, as well as other promising techniques in terms of performance-complexity for LS-MIMO systems, which are listed below:

\section{- More realistic system model}

- Imperfect channel estimation: it is known that channel estimation in practical systems is performed through estimation algorithms, and perfect knowledge of channel conditions is impractical. In this sense, the consideration of a channel estimator portrays an improvement, in the sense of making the channel more realistic, in relation to the scenario adopted in this Thesis.

- Imperfect power control: another problem identified in the adopted LS-MIMO system model, which is also commonly found in the literature [22, 80-84], refers to the consideration of the same power received in BS by all active users. This is clearly a non-realistic situation and adoption of a model in which to consider an imperfect power control represents an improvement in the system model to be considered.

- Multi-cellular environment: as mentioned, pilot contamination refers to one of the main problems faced in large-scale MIMO systems and is represented by inter-cellular interference. Therefore, this effect is only evaluated when considering multi-cellular scenarios. The adoption of a multi-cellular scenario is an important requirement aiming the direction of this research.

\section{- Promising LS-MIMO detection techniques}

- MMSE approximation: MMSE iterative approximation techniques, such as the RID mentioned in this Thesis, appear as strategies of very low complexity and with near-optimal performance in low system loading scenarios, which is a condition applicable in massive MIMO systems. In this sense, the investigation of such techniques, still possibly helping more elaborate strategies, such as heuristics, results in another possible direction of this research. 
- Error localization: error localization techniques, such as the generalized orthogonal matching pursuit (GOMP) [126], can exploit the sparse signal processing structure via compressive sensing (CS), which results in performance gain. These techniques also require very low complexity and are promising in the actual LS-MIMO signal detection scenario. 


\section{Bibliography}

[1] CISCO. Cisco Visual Networking Index: Forecast and Trends, 2017-2022. [S.I.], 2018.

[2] DATTA, T.; KUMAR, N. A.; CHOCKALINGAM, A.; RAJAN, B. S. A novel monte-carlo-sampling-based receiver for large-scale uplink multiuser mimo systems. IEEE Transactions on Vehicular Technology, v. 62, n. 7, p. 3019-3038, Sept. 2013. ISSN 0018-9545.

[3] MUSSI, A. M.; ABRãO, T. Multiple restarts mixed gibbs sampling detector for large-scale antenna systems. IET Signal Processing, Institution of Engineering and Technology, November 2018. ISSN 1751-9675. Disponível em: <https://digital-library.theiet.org/content/journals/10.1049/iet-spr.2018.5206>.

[4] ERICSSON. Ericsson Mobility Report. [S.I.], 2018.

[5] ALAMOUTI, S. A simple transmit diversity technique for wireless communications. Selected Areas in Communications, IEEE Journal on, v. 16, n. 8, p. 1451 -1458, out. 1998.

[6] FOSCHINI, G. J.; GANS, M. J. On limits of wireless communications in a fading environment when using multiple antennas. Wireless Personal Communications, v. 6, p. 311-335, 1998.

[7] TELATAR, E. Capacity of multi-antenna gaussian channels. Eur. Trans. Telecomm., Wiley Subscription Services, Inc., A Wiley Company, v. 10, n. 6, p. 585-595, 1999. Disponível em: <http://dx.doi.org/10.1002/ett.4460100604>.

[8] MARZETTA, T. Noncooperative cellular wireless with unlimited numbers of base station antennas. Wireless Communications, IEEE Transactions on, v. 9, n. 11, p. 3590-3600, 2010. ISSN 1536-1276.

[9] TELATAR, E. Capacity of multi-antenna gaussian channels. European Transactions on Telecommunications, Wiley Subscription Services, Inc., A Wiley Company, v. 10, n. 6, p. 585-595, 1999. ISSN 1541-8251.

[10] HOYDIS, J.; BRINK, S. ten; DEBBAH, M. Massive mimo in the ul/dl of cellular networks: How many antennas do we need? Selected Areas in Communications, IEEE Journal on, v. 31, n. 2, p. 160-171, 2013. ISSN 0733-8716.

[11] RUSEK, F.; PERSSON, D.; LAU, B. K.; LARSSON, E.; MARZETTA, T.; EDFORS, O.; TUFVESSON, F. Scaling up mimo: Opportunities and challenges with very large arrays. Signal Processing Magazine, IEEE, v. 30 , n. 1 , p. $40-60,2013$. ISSN 1053-5888.

[12] CHOCKALINGAM, A.; RAJAN, B. Large MIMO Systems. United Kingdom: Cambridge University Press, 2014.

[13] LU, L.; LI, G. Y.; SWINDLEHURST, A. L.; ASHIKHMIN, A.; ZHANG, R. An overview of massive mimo: Benefits and challenges. IEEE Journal of Selected Topics in Signal Processing, v. 8, n. 5, p. 742-758, Oct. 2014. ISSN 1932-4553.

[14] TULINO, A. M.; VERDú, S. Random matrix theory and wireless communications. Foundations and Trends in Communications and Information Theory, v. 1, n. 1, p. 1-182, 2004. ISSN 1567-2190. Disponível em: <http://dx.doi.org/10.1561/0100000001>.

[15] NAM, Y.-H.; NG, B. L.; SAYANA, K.; LI, Y.; ZHANG, J.; KIM, Y.; LEE, J. Full-dimension mimo (fd-mimo) for next generation cellular technology. Communications Magazine, IEEE, v. 51, n. 6, p. 172-179, 2013. ISSN 0163-6804. 
[16] ZHANG, J.; WEN, C.-K.; JIN, S.; GAO, X.; WONG, K.-K. On capacity of large-scale mimo multiple access channels with distributed sets of correlated antennas. Selected Areas in Communications, IEEE Journal on, v. 31, n. 2, p. 133-148, 2013. ISSN 0733-8716.

[17] GESBERT, D.; KOUNTOURIS, M.; HEATH, R.; CHAE, C.-B.; SALZER, T. Shifting the mimo paradigm. Signal Processing Magazine, IEEE, v. 24, n. 5, p. 36-46, 2007. ISSN 1053-5888.

[18] VARDHAN, K.; MOHAMMED, S.; CHOCKALINGAM, A.; RAJAN, B. A low-complexity detector for large mimo systems and multicarrier cdma systems. Selected Areas in Communications, IEEE Journal on, v. 26, n. 3 , p. $473-485,2008$. ISSN 0733-8716.

[19] RAJAN, B.; MOHAMMED, S.; CHOCKALINGAM, A.; SRINIDHI, N. Low-complexity near-ml decoding of large non-orthogonal stbcs using reactive tabu search. In: Information Theory, 2009. ISIT 2009. IEEE International Symposium on. [S.I.: s.n.], 2009. p. 1993-1997.

[20] SOM, P.; DATTA, T.; CHOCKALINGAM, A.; RAJAN, B. S. Improved large-mimo detection based on damped belief propagation. In: Information Theory (ITW 2010, Cairo), 2010 IEEE Information Theory Workshop on. [S.I.: s.n.], 6-8 Jan. 2010. p. 1-5.

[21] SRINIDHI, N.; DATTA, T.; CHOCKALINGAM, A.; RAJAN, B. Layered tabu search algorithm for large-mimo detection and a lower bound on $\mathrm{ml}$ performance. Communications, IEEE Transactions on, v. 59, n. 11 , p. 2955-2963, 2011. ISSN 0090-6778.

[22] GUO, R.; LI, X.; FU, W.; HEI, Y. Low-complexity signal detection based on relaxation iteration method in massive mimo systems. China Communications, v. 12, n. Supplement, p. 1-8, December 2015. ISSN 1673-5447.

[23] HEMESI, H.; ABDIPOUR, A.; MOHAMMADI, A. Analytical modeling of mimo-ofdm system in the presence of nonlinear power amplifier with memory. IEEE Transactions on Communications, v. 61, n. 1, p. 155-163, 2013. ISSN 0090-6778.

[24] HE, L.; WANG, J.; SONG, J. On generalized spatial modulation aided millimeter wave mimo: Spectral efficiency analysis and hybrid precoder design. IEEE Transactions on Wireless Communications, v. 16, n. 11, p. 7658-7671, 2017. ISSN 1536-1276.

[25] RAJASHEKAR, R.; HANZO, L. Iterative matrix decomposition aided block diagonalization for mm-wave multiuser mimo systems. IEEE Transactions on Wireless Communications, v. 16, n. 3, p. 1372-1384, 20117. ISSN 1536-1276.

[26] LI, Z.; HAN, S.; MOLISCH, A. F. User-centric virtual sectorization for millimeter-wave massive mimo downlink. IEEE Transactions on Wireless Communications, v. 17, n. 1, p. 445-460, 2018. ISSN 1536-1276.

[27] XUE, Q.; FANG, X.; XIAO, M.; MUMTAZ, S.; RODRIGUEZ, J. Beam management for millimeter-wave beamspace mu-mimo systems. IEEE Transactions on Communications, v. 67, n. 1, p. 205-217, 2019. ISSN 0090-6778.

[28] NADEEM, Q.; KAMMOUN, A.; DEBBAH, M.; ALOUINI, M. Design of $5 \mathrm{~g}$ full dimension massive mimo systems. IEEE Transactions on Communications, v. 66, n. 2, p. 726-740, 2018. ISSN 0090-6778.

[29] LUO, S.; LI, Y. A dual-band antenna array with mutual coupling reduction using 3d metamaterial structures. In: 2018 International Symposium on Antennas and Propagation (ISAP). [S.I.: s.n.], 2018. p. 1-2.

[30] GAO, F.; SU, W.; SHAN, R.; ZHU, W.; HE, K.; WANG, L. 3d coverage optimization research on 5g massive mimo antenna array. In: 2018 IEEE International Symposium on Electromagnetic Compatibility and 2018 IEEE Asia-Pacific Symposium on Electromagnetic Compatibility (EMC/APEMC). [S.I.: s.n.], 2018. p. 94-97.

[31] ROSHNA, T. K.; DEEPAK, U.; SAJITHA, V. R.; VASUDEVAN, K.; MOHANAN, P. A compact uwb mimo antenna with reflector to enhance isolation. IEEE Transactions on Antennas and Propagation, v. 63, n. 4, p. 1873-1877, 2015. ISSN 0018-926X. 
[32] LI, Z.; DU, Z.; GONG, K. Compact reconfigurable antenna array for adaptive mimo systems. IEEE Antennas and Wireless Propagation Letters, v. 8, p. 1317-1320, 2009. ISSN 1536-1225.

[33] HUI, H. T. Influence of antenna characteristics on mimo systems with compact monopole arrays. IEEE Antennas and Wireless Propagation Letters, v. 8, p. 133-136, 2009. ISSN 1536-1225.

[34] DING, K.; GAO, C.; QU, D.; YIN, Q. Compact broadband mimo antenna with parasitic strip. IEEE Antennas and Wireless Propagation Letters, v. 16, p. 2349-2353, 2017. ISSN 1536-1225.

[35] PATChARAMANEEPAKORN, P.; WU, S.; WANG, C. X.; AGGOUNE, H.; ALWAKEEL, M.; GE, X.; RENZO, M. D. Spectral, Energy and Economic Efficiency of $5 G$ Multi-cell Massive MIMO Systems with Generalized Spatial Modulation. 2016. 1-1 p.

[36] WANG, S.; LI, Y.; WANG, J. Multiuser detection in massive spatial modulation mimo with low-resolution adcs. IEEE Transactions on Wireless Communications, v. 14, n. 4, p. 2156-2168, April 2015. ISSN 1536-1276.

[37] YANG, P.; XIAO, Y.; GUAN, Y. L.; HARI, K. V. S.; CHOCKALINGAM, A.; SUGIURA, S.; HAAS, H.; RENZO, M. D.; MASOUROS, C.; LIU, Z.; XIAO, L.; LI, S.; HANZO, L. Single-Carrier Spatial Modulation: A Promising Design for Large-Scale Broadband Antenna Systems. 2016. 1-1 p.

[38] YANG, F.; CAI, P.; QIAN, H.; LUO, X. Pilot contamination in massive mimo induced by timing and frequency errors. IEEE Transactions on Wireless Communications, v. 17, n. 7, p. 4477-4492, 2018. ISSN $1536-1276$.

[39] VERENZUELA, D.; BJöRNSON, E.; SANGUINETTI, L. Spectral and energy efficiency of superimposed pilots in uplink massive mimo. IEEE Transactions on Wireless Communications, v. 17, n. 11, p. 7099-7115, 2018. ISSN 1536-1276.

[40] SOHN, J.; YOON, S. W.; MOON, J. On reusing pilots among interfering cells in massive mimo. IEEE Transactions on Wireless Communications, v. 16, n. 12, p. 8092-8104, 2017. ISSN 1536-1276.

[41] MUPPIRISETTY, L. S.; CHARALAMBOUS, T.; KAROUT, J.; FODOR, G.; WYMEERSCH, H. Location-aided pilot contamination avoidance for massive mimo systems. IEEE Transactions on Wireless Communications, v. 17, n. 4, p. 2662-2674, 2018. ISSN 1536-1276.

[42] ZENG, J.; LIN, J.; WANG, Z. Low complexity message passing detection algorithm for large-scale mimo systems. IEEE Wireless Communications Letters, v. 7, n. 5, p. 708-711, 2018. ISSN 2162-2337.

[43] MANDLOI, M.; BHATIA, V. Low-complexity near-optimal iterative sequential detection for uplink massive mimo systems. IEEE Communications Letters, v. 21, n. 3, p. 568-571, 2017. ISSN 1089-7798.

[44] MANDLOI, M.; BHATIA, V. Error recovery based low-complexity detection for uplink massive mimo systems. IEEE Wireless Communications Letters, v. 6, n. 3, p. 302-305, 2017. ISSN 2162-2337.

[45] KATO, K.; FUKAWA, K.; YAMADA, R.; SUZUKI, H.; SUYAMA, S. Low-complexity mimo signal detection employing multistream constrained search. IEEE Transactions on Vehicular Technology, v. 67, n. 2, p. 1217-1230, 2018. ISSN 0018-9545.

[46] JIN, F.; LIU, Q.; LIU, H.; WU, P. A low complexity signal detection scheme based on improved newton iteration for massive mimo systems. IEEE Communications Letters, v. 23, n. 4, p. 748-751, 2019. ISSN 1089-7798.

[47] WÜBBEN, D.; SEETHALER, D.; JALDÉN, J.; MATZ, G. Lattice reduction-a survey with applications in wireless communications. IEEE Signal Processing Magazine, v. 28, n. 3, p. 70-91, 2011.

[48] MOSTAGI, Y. M.; ABRãO., T. Lattice-reduction-aided over guided search mimo detectors. International Journal of Satellite Communications Policy and Management, p. 142-154, 2012.

[49] KOBAYASHI, R. T.; CIRIACO, F.; aO, T. A. Efficient near-optimum detectors for large mimo systems under correlated channels. Wirel. Pers. Commun., Kluwer Academic Publishers, Hingham, MA, USA, v. 83, n. 2, p. 1287-1311, jul. 2015. ISSN 0929-6212. Disponível em: <http://dx.doi.org/10.1007/s11277-015$2450-y>$. 
[50] HASSIBI, B.; VIKALO, H. On the expected complexity of sphere decoding. In: Signals, Systems and Computers, 2001. Conference Record of the Thirty-Fifth Asilomar Conference on. [S.I.: s.n.], 2001. v. 2, p. 1051-1055 vol.2. ISSN 1058-6393.

[51] HASSIBI, B.; VIKALO, H. On the sphere-decoding algorithm i. expected complexity. Signal Processing, IEEE Transactions on, v. 53, n. 8, p. 2806-2818, 2005. ISSN 1053-587X.

[52] DAMEN, M.; GAMAL, H. E.; CAIRE, G. On maximum-likelihood detection and the search for the closest lattice point. Information Theory, IEEE Transactions on, v. 49, n. 10, p. 2389-2402, 2003. ISSN 0018-9448.

[53] SINGH, A.; ELIA, P.; JALDEN, J. Achieving a vanishing snr gap to exact lattice decoding at a subexponential complexity. Information Theory, IEEE Transactions on, v. 58, n. 6, p. 3692-3707, 2012. ISSN 0018-9448.

[54] KIM, H.; PARK, J.; LEE, H.; KIM, J. Near-ml mimo detection algorithm with Ir-aided fixed-complexity tree searching. Communications Letters, IEEE, v. 18, n. 12, p. 2221-2224, 2014. ISSN 1089-7798.

[55] QIAO, S. Integer least squares: sphere decoding and the III algorithm. In: Proceedings of the 2008 $C<\sup >3</$ sup $>S<$ sup $>2</$ sup $>E$ conference. Montreal, Quebec, Canada: ACM, 2008. p. 23-28.

[56] BUDIHAL, S.; BANAKAR, R. Complexity analysis of lattice reduction aided increasing radius algorithm in mimo systems. In: Computational Intelligence and Information Technology, 2013. CIIT 2013. Third International Conference on. [S.I.: s.n.], 2013. p. 360-366.

[57] MONTEIRO, F. Lattices in MIMO spatial multiplexing: detection and geometry. Tese (Doutorado), 2012.

[58] WANG, J.; LI, M.; ZHANG, Y.; ZHOU, Q. Effect of channel estimation error on the mutual information of mimo fading channels. In: Wireless Communications, Networking and Mobile Computing, 2008. WiCOM '08. 4th International Conference on. [S.I.: s.n.], 2008. p. 1-4.

[59] ZELST, A. V.; HAMMERSCHMIDT, J. S. A single coefficient spatial correlation model for multiple-input multiple-output (mimo) radio channels. In: in Proc. URSI XXVIIth General Assembly. [S.I.: s.n.], 2002. p. 1-4.

[60] KAY, S. M. Fundamentals of Statistical Signal Processing: Estimation Theory. Upper Saddle River, NJ, USA: Prentice-Hall, Inc., 1993. ISBN 0-13-345711-7.

[61] BAI, L.; CHOI, J. Low complexity MIMO detection. [S.I.]: Springer Verlag, 2012.

[62] WüBBEN, D.; BöHNKE, R.; RINAS, J.; KAMMEYER, K.-D.; KüHN, V. Efficient algorithm for decoding layered space-time codes. IEE Electronic Letters, v. 37, n. 22, p. 1348-1350, Nov 2001.

[63] WÜBBEN, D.; BÖHNKE, R.; KÜHN, V.; KAMMEYER, K.-D. MMSE extension of V-BLAST based on sorted QR decomposition. In: IEEE Semiannual Vehicular Technology Conference (VTC2003-Fall). Orlando, Florida, USA: [s.n.], 2003. p. 1-5.

[64] FINCKE, U.; POHST, M. Improved methods for calculating vectors of short length in a lattice, including a complexity analysis. Mathematics of Computation, American Mathematical Society, v. 44, n. 170, p. 463-471, 1985. ISSN 00255718, 10886842. Disponível em: <http://www.jstor.org/stable/2007966>.

[65] VITERBO, E.; BOUTROS, J. A universal lattice code decoder for fading channels. IEEE Transactions on Information Theory, v. 45, n. 5, p. 1639-1642, Jul 1999. ISSN 0018-9448.

[66] GOWAIKAR, R.; HASSIBI, B. Statistical pruning for near-maximum likelihood decoding. IEEE Transactions on Signal Processing, v. 55, n. 6, p. 2661-2675, June 2007. ISSN 1053-587X.

[67] BARBERO, L.; THOMPSON, J. Fixing the complexity of the sphere decoder for mimo detection. Wireless Communications, IEEE Transactions on, v. 7, n. 6, p. 2131-2142, 2008. ISSN 1536-1276. 
[68] JALDEN, J.; OTTERSTEN, B. On the complexity of sphere decoding in digital communications. Signal Processing, IEEE Transactions on, v. 53, n. 4, p. 1474-1484, April 2005. ISSN 1053-587X.

[69] WANG, R.; GIANNAKIS, G. B. Approaching mimo channel capacity with reduced-complexity soft sphere decoding. In: Wireless Communications and Networking Conference, 2004. WCNC. 2004 IEEE. [S.I.: s.n.], 21-25 March 2004. v. 3, p. 1620-1625 Vol.3. ISSN 1525-3511.

[70] STUDER, C.; BURG, A.; BOLCSKEI, H. Soft-output sphere decoding: algorithms and vlsi implementation. IEEE Journal on Selected Areas in Communications, v. 26, n. 2, p. 290-300, February 2008. ISSN 0733-8716.

[71] STUDER, C.; BOLCSKEI, H. Soft-input soft-output single tree-search sphere decoding. IEEE Transactions on Information Theory, v. 56, n. 10, p. 4827-4842, Oct. 2010. ISSN 0018-9448.

[72] MILLINER, D. L.; ZIMMERMANN, E.; BARRY, J. R.; FETTWEIS, G. A fixed-complexity smart candidate adding algorithm for soft-output mimo detection. IEEE Journal of Selected Topics in Signal Processing, v. 3, n. 6, p. 1016-1025, Dec. 2009. ISSN 1932-4553.

[73] MILFORD, D.; SANDELL, M. Simplified quantisation in a reduced-lattice mimo decoder. Communications Letters, IEEE, v. 15, n. 7, p. 725-727, July 2011. ISSN 1089-7798.

[74] LENSTRA, A. K.; LENSTRA, H. W. Factoring polynomials with rational coefficients. Muth. Ann, p. 515-534, 1982.

[75] WüBBEN, D.; BöHNKE, R.; KüHN, V.; KAMMEYER, K. dirk. Mmse-based lattice-reduction for near-ml detection of mimo systems. In: in ITG Workshop on Smart Antennas. [S.I.: s.n.], 2004. p. 18-19.

[76] GAMA, N.; NGUYEN, P. Q. Predicting lattice reduction. In: Proceedings of the Theory and Applications of Cryptographic Techniques 27th Annual International Conference on Advances in Cryptology. Berlin, Heidelberg: Springer-Verlag, 2008. (EUROCRYPT'08), p. 31-51. ISBN 3-540-78966-9, 978-3-540-78966-6. Disponível em: <http://dl.acm.org/citation.cfm?id=1788414.1788417>.

[77] MUSSI, A. M.; COSTA, B. F.; ABRãO, T. Efficient lattice reduction aided detectors under realistic mimo channels. Wireless Personal Communications, 2017.

[78] GOLUB, G. H.; LOAN, C. F. V. Matrix Computations. Maryland, USA: Johns Hopkins University Press, 1996.

[79] KOBAYASHI, R. T.; aO, T. A. Ordered mmse-sic via sorted qr decomposition in ill conditioned large-scale mimo channels. Telecommun. Syst., Kluwer Academic Publishers, Norwell, MA, USA, v. 63, n. 2, p. 335-346, out. 2016. ISSN 1018-4864. Disponível em: <http://dx.doi.org/10.1007/s11235-015-0123-5>.

[80] SOM, P.; DATTA, T.; SRINIDHI, N.; CHOCKALINGAM, A.; RAJAN, B. S. Low-complexity detection in large-dimension mimo-isi channels using graphical models. IEEE Journal of Selected Topics in Signal Processing, v. 5, n. 8, p. 1497-1511, Dec. 2011. ISSN 1932-4553.

[81] NARASIMHAN, T. L.; RAVITEJA, P.; CHOCKALINGAM, A. Large-scale multiuser sm-mimo versus massive mimo. In: Information Theory and Applications Workshop (ITA), 2014. [S.I.: s.n.], 9-14 Feb. 2014. p. 1-9.

[82] GAO, X.; DAI, L.; YUEN, C.; ZHANG, Y. Low-complexity mmse signal detection based on richardson method for large-scale mimo systems. In: 2014 IEEE 80th Vehicular Technology Conference (VTC2014-Fall). [S.I.: s.n.], 14-17 Sept. 2014. p. 1-5. ISSN 1090-3038.

[83] JEON, C.; MALEKI, A.; STUDER, C. On the performance of mismatched data detection in large mimo systems. In: 2016 IEEE International Symposium on Information Theory (ISIT). [S.I.: s.n.], 10-15 July 2016. p. $180-184$.

[84] LIU, L.; YUEN, C.; GUAN, Y. L.; LI, Y.; SU, Y. Convergence analysis and assurance for gaussian message passing iterative detector in massive mu-mimo systems. IEEE Transactions on Wireless Communications, v. 15 , n. 9, p. 6487-6501, Sept. 2016. ISSN 1536-1276. 
[85] NGO, H. Q.; LARSSON, E. G. Blind estimation of effective downlink channel gains in massive mimo. In: 2015 IEEE International Conference on Acoustics, Speech and Signal Processing (ICASSP). [S.I.: s.n.], 19-24 April 2015. p. 2919-2923. ISSN 1520-6149.

[86] ROGALiN, R.; BURSALIOGLU, O. Y.; PAPAdOPOULOS, H.; CAIRE, G.; MOLISCH, A. F.; MICHALOLIAKOS, A.; BALAN, V.; PSOUNIS, K. Scalable synchronization and reciprocity calibration for distributed multiuser mimo. IEEE Transactions on Wireless Communications, v. 13, n. 4, p. 1815-1831, April 2014. ISSN 1536-1276.

[87] XIANG, Z.; TAO, M.; WANG, X. Massive mimo multicasting in noncooperative cellular networks. IEEE Journal on Selected Areas in Communications, v. 32, n. 6, p. 1180-1193, June 2014. ISSN 0733-8716.

[88] ZAREI, S.; GERSTACKER, W.; SCHOBER, R. I/q imbalance aware widely-linear precoding for downlink massive mimo systems. In: 2014 IEEE Globecom Workshops (GC Wkshps). [S.I.: s.n.], 8-12 Dec. 2014. p. 301-307. ISSN 2166-0077.

[89] JIN, S.; LI, M.; HUANG, Y.; DU, Y.; GAO, X. Pilot scheduling schemes for multi-cell massive multiple-input multiple-output transmission. IET Communications, v. 9, n. 5, p. 689-700, 326 2015. ISSN 1751-8628.

[90] AHMADI, H.; FARHANG, A.; MARCHETTI, N.; MACKENZIE, A. A game theoretic approach for pilot contamination avoidance in massive mimo. IEEE Wireless Communications Letters, v. 5, n. 1, p. 12-15, Feb. 2016. ISSN 2162-2337.

[91] YU, Y.; GU, D. Enhanced mu-mimo downlink transmission in the fdd-based distributed antennas system. IEEE Communications Letters, v. 16, n. 1, p. 37-39, January 2012. ISSN 1089-7798.

[92] RAO, X.; LAU, V. K. N. Distributed compressive csit estimation and feedback for fdd multi-user massive mimo systems. IEEE Transactions on Signal Processing, v. 62, n. 12, p. 3261-3271, June15, 2014. ISSN 1053-587X.

[93] CHOI, J.; LOVE, D. J.; BIDIGARE, P. Downlink training techniques for fdd massive mimo systems: Open-loop and closed-loop training with memory. IEEE Journal of Selected Topics in Signal Processing, v. 8, n. 5 , p. 802-814, Oct. 2014. ISSN 1932-4553.

[94] SHEN, W.; DAI, L.; SHIM, B.; MUMTAZ, S.; WANG, Z. Joint csit acquisition based on low-rank matrix completion for fdd massive mimo systems. IEEE Communications Letters, v. 19, n. 12, p. 2178-2181, Dec. 2015. ISSN 1089-7798.

[95] YUE, Z.; GUO, Q.; XIANG, W. Complex gaussian belief propagation algorithms for distributed multicell multiuser mimo detection with imperfect channel state information. In: 2014 IEEE 25th Annual International Symposium on Personal, Indoor, and Mobile Radio Communication (PIMRC). [S.I.: s.n.], 2-5 Sept. 2014. p. 1902-1907. ISSN 2166-9570.

[96] SU, Q.; WU, Y. C. Convergence analysis of the variance in gaussian belief propagation. IEEE Transactions on Signal Processing, v. 62, n. 19, p. 5119-5131, Oct.1, 2014. ISSN 1053-587X.

[97] TAKAHASHI, T.; IBI, S.; OHGANE, T.; SAMPEI, S. On normalized belief of gaussian bp in correlated large mimo channels. In: 2016 International Symposium on Information Theory and Its Applications (ISITA). [S.I.: s.n.], Oct. 30 2016-Nov. 2 2016. p. 458-462.

[98] YOON, S.; CHAE, C. B. Low-complexity mimo detection based on belief propagation over pairwise graphs. IEEE Transactions on Vehicular Technology, v. 63, n. 5, p. 2363-2377, Jun 2014. ISSN 0018-9545.

[99] YANG, P.; XIAO, Y.; GUAN, Y. L.; HARI, K. V. S.; CHOCKALINGAM, A.; SUGIURA, S.; HAAS, H.; RENZO, M. D.; MASOUROS, C.; LIU, Z.; XIAO, L.; LI, S.; HANZO, L. Single-Carrier Spatial Modulation: A Promising Design for Large-Scale Broadband Antenna Systems. 2016. 1-1 p.

[100]SUNEEL, M.; SOM, P.; CHOCKALINGAM, A.; RAJAN, B. S. Belief propagation based decoding of large non-orthogonal stbcs. In: Information Theory, 2009. ISIT 2009. IEEE International Symposium on. [S.I.: s.n.], June 28 2009-July 3 2009. p. 2003-2007. 
[101]MOOIJ, J. M.; KAPPEN, H. J. Sufficient conditions for convergence of the sum-product algorithm. IEEE Transactions on Information Theory, v. 53, n. 12, p. 4422-4437, Dec. 2007. ISSN 0018-9448.

[102]MURPHY, K. P.; WEISS, Y.; JORDAN, M. I. Loopy belief propagation for approximate inference: An empirical study. In: Proc. Fifteenth Conference on Uncertainty in Artificial Intelligence. San Francisco: [s.n.], 1999. p. 467-475. ISBN 1-55860-614-9.

[103]PRETTI, M. A message-passing algorithm with damping. Journal of Statistical Mechanics: Theory and Experiment, IOP Publishing, v. 2005, n. 11, p. P11008, 2005.

[104]YUILLE, A. L. A double-loop algorithm to minimize the bethe and kikuchi free energies. NEURAL COMPUTATION, v. 14, p. 2002, 2001.

[105]HESKES, T.; ALBERS, K.; KAPPEN, B. Approximate inference and constrained optimization. In: Proceedings of the Nineteenth Conference on Uncertainty in Artificial Intelligence. San Francisco, CA, USA: Morgan Kaufmann Publishers Inc., 2003. (UAI'03), p. 313-320. ISBN 0-127-05664-5. Disponível em: $<$ http://dl.acm.org/citation.cfm?id=2100584.2100622>.

[106]GOLDBERGER, J.; LESHEM, A. Mimo detection for high-order qam based on a gaussian tree approximation. IEEE Transactions on Information Theory, v. 57, n. 8, p. 4973-4982, Aug. 2011. ISSN 0018-9448.

[107]ABIKO, T.; FUKUDA, W.; NISHIMURA, T.; OHGANE, T.; OGAWA, Y.; OHWATARI, Y.; KISHIYAMA, $\mathrm{Y}$. An exit chart analysis for belief-propagation based detection in a large-scale mimo system. In: Vehicular Technology Conference (VTC Spring), 2013 IEEE 77th. [S.I.: s.n.], 2-5 June 2013. p. 1-5. ISSN 1550-2252.

[108]WU, S.; KUANG, L.; NI, Z.; LU, J.; HUANG, D.; GUO, Q. Low-complexity iterative detection for large-scale multiuser mimo-ofdm systems using approximate message passing. IEEE Journal of Selected Topics in Signal Processing, v. 8, n. 5, p. 902-915, Oct. 2014. ISSN 1932-4553.

[109]SHENGCHU, W.; LI, Y.; WANG, J. Large-scale antenna system with massive one-bit integrated energy and information receivers. In: 2015 IEEE International Conference on Communications (ICC). [S.I.: s.n.], 8-12 June 2015. p. 2024-2029. ISSN 1550-3607.

[110]NARASIMHAN, T. L.; CHOCKALINGAM, A.; RAJAN, B. S. Factor graph based joint detection/decoding for Idpc coded large-mimo systems. In: Vehicular Technology Conference (VTC Spring), 2012 IEEE 75th. [S.I.: s.n.], 6-9 May 2012. p. 1-5. ISSN 1550-2252.

[111]FREY, B. J. Graphical Models for Machine Learning and Digital Communication. Cambridge, MA, USA: MIT Press, 1998. ISBN 0-262-06202-X.

[112]HECKERMAN, D.; WELlMAN, M. P. Bayesian networks. Commun. ACM, ACM, New York, NY, USA, v. 38, n. 3, p. 27-30, mar. 1995. ISSN 0001-0782. Disponível em: $<$ http://doi.acm.org/10.1145/203330.203336>.

[113]GRIFFEATH, D. Introduction to Markov Random Fields, in Denumerable Markov Chains, J. G. Kerney, J. L. Snell and A.W. Knupp. Second. New York: Springer-Verlag, 1976.

[114]KSCHISCHANG, F. R.; FREY, B. J.; LOELIGER, H. A. Factor graphs and the sum-product algorithm. IEEE Transactions on Information Theory, v. 47, n. 2, p. 498-519, Feb 2001. ISSN 0018-9448.

[115]AJI, S. M.; MCELIECE, R. J. The generalized distributive law. IEEE Transactions on Information Theory, v. 46, n. 2, p. 325-343, Mar 2000. ISSN 0018-9448.

[116]MUSSI, A. M.; ABRãO, T. Message passing detection for large-scale mimo systems: damping factor analysis. IET Signal Processing, Institution of Engineering and Technology, v. 11, p. 923-935(12), October 2017. ISSN 1751-9675. Disponível em: <https://digital-library.theiet.org/content/journals/10.1049/ietspr.2017.0148>. 
[117]CHEN, R.; LIU, J. S.; WANG, X. Convergence analyses and comparisons of Markov chain Monte Carlo algorithms in digital communications. IEEE Transactions on Signal Processing, v. 50, n. 2, p. 255-270, Feb 2002. ISSN 1053-587X.

[118]FARHANG-BOROUJENY, B.; ZHU, H.; SHI, Z. Markov chain Monte Carlo algorithms for CDMA and MIMO communication systems. IEEE Transactions on Signal Processing, v. 54, n. 5, p. 1896-1909, May 2006. ISSN 1053-587X.

[119]HASSIBI, B.; HANSEN, M.; DIMAKIS, A. G.; ALSHAMARY, H. A. J.; XU, W. Optimized Markov chain Monte Carlo for signal detection in MIMO systems: An analysis of the stationary distribution and mixing time. IEEE Transactions on Signal Processing, v. 62, n. 17, p. 4436-4450, Sept 2014. ISSN 1053-587X.

[120]MANDLOI, M.; BHATIA, V. Layered Gibbs sampling algorithm for near-optimal detection in large-MIMO systems. In: 2017 IEEE Wireless Communications and Networking Conference (WCNC). [S.I.: s.n.], 2017. p. 1-6.

[121]GAO, C.; XU, J.; TAO, X.; QIN, Z. An improved mixed Gibbs sampling algorithm based on multiple random parallel Markov chains for massive MIMO systems. In: 2016 IEEE 27th Annual International Symposium on Personal, Indoor, and Mobile Radio Communications (PIMRC). [S.I.: s.n.], 2016. p. 1-5.

[122] CHOI, J. An MCMC-MIMO detector as a stochastic linear system solver using successive overrelexation. IEEE Transactions on Wireless Communications, v. 15, n. 2, p. 1445-1455, 2016. ISSN 1536-1276.

[123]HANSEN, M.; HASSIBI, B.; DIMAKIS, A. G.; XU, W. Near-optimal detection in MIMO systems using Gibbs sampling. In: GLOBECOM 2009 - 2009 IEEE Global Telecommunications Conference. [S.I.: s.n.], Nov. p. 1-6. ISSN 1930-529X.

[124]KNUTH, D. E. The Art of Computer Programming, Volume 1 (3rd Ed.): Fundamental Algorithms. Redwood City, CA, USA: Addison Wesley Longman Publishing Co., Inc., 1997. ISBN 0-201-89683-4.

[125]LIU, T. Some results for the fast mmse-sic detection in spatially multiplexed mimo systems. IEEE Transactions on Wireless Communications, v. 8, n. 11, p. 5443-5448, November 2009. ISSN 1536-1276.

[126]WANG, J.; KWON, S.; SHIM, B. Generalized orthogonal matching pursuit. IEEE Transactions on Signal Processing, v. 60, n. 12, p. 6202-6216, 2012. ISSN 1053-587X. 


\section{APPENDIX A - Full paper published in the journal "Wireless Personal Communications (WIRE)", October 2016}

Title: Efficient lattice reduction aided detectors under realistic MIMO channels.

Authors: Alex Miyamoto Mussi, Bruno Felipe Costa and Taufik Abrão.

Journal: Wireless Personal Communications (WIRE) (ISSN 0929-6212).

Classification (Qualis-CAPES 2016): B1 (Engenharias IV).

Impact Factor (2017): 1.200.

Publication Date: October 2016.

DOI: $10.1007 /$ s11277-016-3807-6 


\title{
Efficient Lattice Reduction Aided Detectors Under Realistic MIMO Channels
}

\author{
Alex Miyamoto Mussi ${ }^{1} \cdot$ Bruno Felipe $\operatorname{Costa}^{1} \cdot$ \\ Taufik Abrão 1
}

Published online: 13 October 2016

(C) Springer Science+Business Media New York 2016

\begin{abstract}
This contribution analyses the performance of efficient multiple-input-multipleoutput (MIMO) detectors under correlated channels and imperfect coefficients channel estimation. A number of signal detection principles and techniques, including the minimum mean squared error detector with and without ordered successive interference cancellation; the sphere decoding MIMO detection, as well as promising near-orthogonal transformation techniques combined with these detectors, namely the lattice reduction and the $\mathrm{QR}$ decomposition are analysed under the perspective of complexity-performance tradeoff. While in most of available works perfect channel state information and uncorrelated channels have been considered, herein the complexity-performance tradeoff has been analysed and compared with the maximum likelihood (ML) limit under specific but practical scenarios of interest, namely: high spectral efficiency scenario; channel error estimates; channel/antenna correlation; combined channel errors and correlated channels. Under performance-complexity perspective, the optimum MLMIMO detector is deployed as reference aiming to evaluate the efficiency and performance degradation of those sub-optimal MIMO detectors operating under hostile channel conditions.
\end{abstract}

Keywords Multiple-input-multiple-output (MIMO) - Complexity · Lattice reduction $(\mathrm{LR}) \cdot$ Minimum mean squared error (MMSE) - Ordered successive interference cancellation (OSIC) · Maximum likelihood (ML) · Sphere decoder (SD)

\section{Introduction}

Systems with multiple transmitting antennas and multiple receiving antennas (MIMO) present a remarkable spectral efficiency and/or are able to improve the performance and reliability of wireless communication by deploying multiples antennas at both transmitter

Taufik Abrão

taufik@uel.br

Alex Miyamoto Mussi

alexmmussi@gmail.com

1 Department of Electrical Engineering, State University of Londrina, Londrina, Brazil 
and receiver side [1]. In a spatial multiplexing gain configuration, parallel data streams are transmitted using multiple antennas in order to increase the spectral efficiency at the cost of increasing complexity for data detection at the receiver [2]. The MIMO system suffers influences from many effects that can degrade the performance, and consequently reduce its capacity. The knowledge of the channel state information (CSI) is of fundamental importance for acceptable system operation, and it can be used at the receiver, transmitter, or both sides, depending on the MIMO architecture chosen. Under realistic scenarios, the CSI cannot be perfectly estimated, and therefore the information available contains errors. The impact of the imperfect CSI estimation over the MIMO precoder design and respective MIMO performance is investigated in [3, 4].

Another important effect to be considered in realistic MIMO scenarios is the channel correlation between antennas. The correlation between antennas has a great impact on the bit error rate (BER) performance, specially when linear structures of detection are deployed [5]. Some important works have analysed the capacity gain of MIMO systems assuming independent fading channel, which are in practice difficult to obtain due to physical constraints (spacing between antennas), or specially in scenarios with a massive numbers of antennas, where it is physically difficult to design antenna array with enough spacing between antenna elements in order to guarantee no correlation. For instance, recently, the effect of antenna correlation on the performance of massive MIMO systems has been investigated in [6].

Among well-established MIMO detectors, the linear zero forcing (LZF) is known by completely cancel the interference between antennas [7], at the expense of increasing significantly the background noise for badly-conditioned channel matrix. At this point, the minimum mean squared error (MMSE) detector can be seen as a better alternative, since it takes into account the noise power during the symbol detection process. Besides, the successive interference cancellation (SIC) detector performs the detection layer-by-layer, using either a LZF or MMSE strategy, and canceling the interference from the previously detected symbols [8]. Since errors at the detection of the first layers can be propagated along the algorithm, a remarkable improvement on performance can be achieved detecting the most reliable antennas first, which features the ordered SIC (OSIC) MIMO detectors [9]. As these linear sub-optimum detection techniques present a performance clearly inferior to the maximum likelihood (ML) detector.

The improvement in MIMO performance-complexity trade-off obtained by the lattice reduction (LR) pre-processing technique is due to the quasi-orthogonalisation of the MIMO channel achieved with the LR technique, reducing considerably the detector processing task. The LR is a mathematical concept deployed to solve many problems involving lattice points. For instance, in the MIMO signal detection problem, the LR can be used to improve the channel matrix conditioning, thus allowing the use of simpler detector structures [2]; in other words, reduction in computational complexity can be achieved with the LR technique aggregation while maintain overall system performance acceptable [10, 11].

The sphere decoding (SD) can achieve the exact ML detection performance, but requires considerable amount of computations compared with various suboptimal detections. Furthermore, in scenarios with large number of transmit and receive antennas with high order modulation (e.g., 64-, 128-QAM, and so on) the SD becomes impracticable. To reduce the overall SD complexity, a lattice-reduction-aided sphere decoding (LR-SD) is proposed in this contribution. Since the SD achieves optimal performance, the LR-SD would not result in performance improvement; nevertheless the LR pre-processing technique would aim in the SD complexity reduction, specially under large dimensions of 
detection, e.g., considering MIMO channels with high order modulations and number of $\mathrm{Tx}-\mathrm{Rx}$ antennas.

The concept of LR-SD for MIMO detections has been mentioned in recent literature, particularly in [12-16]. In [12, 13], lattice reduction is considered not being useful for the sphere decoding for MIMO detections, which is a closest lattice vector problem (CVP) over a (finite) subset of the infinite lattice. The reason given is that the lattice transforming matrix often destroys the properties of the subset, and the result of LR-SD, which is the closest lattice point to the received signal point, is not necessarily the ML detection result, which is the closest lattice point to the received signal point with the finite subset as the search space. The authors of [14] believe that although the LR-aided SD for MIMO detections can achieve the ML detection BER performance, it can not offer the complexity reduction because the search may be repeated many times to exclude the undesired lattice points. However, recent theoretical analysis in [15] confirmed that the LR-SD for MIMO detections is able to reduce the computational complexity significantly, but the authors admit it assumes a performance gap to the ML detection because sometimes its search result is not equal to the ML detection result. The computational complexity reduction achieved by the LR-SD detector compared to the conventional SD is also pointed out in $[17,18]$; the reason is that the preprocessing technique is able to reduce the total number of search paths. Also, the LR-SD is used in [16], but the exact ML performance can not be achieved essentially owing to the same reason as presented in [15]. Notice that in most of the studies involving LR-SD MIMO detector perfect channel condition scenarios (perfect CSI and uncorrelated channels) have been considered. Specifically in this work the LR-SD MIMO detector is analysed under hostile channel conditions.

The contribution of this work consists in quantify the impact on the MIMO system performance when lattice reduction technique is deployed to mitigate the effects of channel correlation and error estimates. More precisely, LR technique is applied to improve the MIMO detector performance under correlated and imperfect channel estimates, generic $M$ QAM modulation order and number of transmit and receive antennas. Moreover, in the case of LR-aided sphere decoding MIMO detector, namely LR-SD-MIMO detector, we have proposed a simple procedure in order to reduce the computational complexity, which in certain operation conditions implies in a full tree search (specially under low SNR scenarios); hence, the application of LR preprocessing technique is able to reduce substantially such three search overhead. Both LR-aided MMSE, i.e., with and without ordered SIC, namely LR-MMSE and LR-MMSE-OSIC, respectively, as well as the LRSD-MIMO detectors are analysed and compared in terms of performance-complexity taking into consideration the following aspects:

(a) Different numbers of transmit and receive antennas, $N_{t}$ and $N_{r}$, respectively;

(b) Different levels of antenna correlation $\rho$;

(c) Different levels of channel coefficient error estimate, $n_{\varepsilon}$;

(d) Imperfect CSI knowledge under correlated channels.

This paper is organised as follows: in Sect. 2, we describe the MIMO system model with focus on channel error estimation and antenna correlation. In Sect. 3, we revisit classical sub-optimal MIMO detectors, while Sect. 4 presents the LR-aided MIMO detectors and the proposed LR-SD-MIMO detector. Numerical results obtained via Monte-Carlo simulation for several system and channel configuration scenarios are discussed in Sect. 5. The complexity analysis comparing those MIMO detectors is developed in Sect. 6. Conclusions remarks are summarised in Sect. 7. 


\section{MIMO System Model}

In this contribution we consider a complex baseband linear transmission system with nonline-of-sight (NLOS), $N_{t}$ inputs and $N_{r}$ outputs corrupted by additive white Gaussian noise (AWGN). The matrix model for the system under investigation is

$$
\mathbf{y}=\mathbf{H s}+\boldsymbol{\eta}
$$

where $\mathbf{H}$ represents an $N_{r} \times N_{t}$ fading coefficients matrix following a Rayleigh distribution (for amplitudes) representing NLOS point-to-point communication, $\mathbf{s}$ is vector of data symbols and $\boldsymbol{\eta}$ is the independent white noise vector samples with Gaussian distribution with:

$$
\mathbb{E}\left[\eta^{2}\right]=N_{0}=\sigma_{\eta}^{2}=\frac{N_{t} E_{s}}{\gamma}
$$

where $E_{s}$ denotes the average energy of the transmitted symbols, $N_{0}$ is the noise power spectral density which is equal to the variance of entries $\boldsymbol{\eta}$; and $\gamma$ is the average SNR per transmit antenna.

\subsection{Correlated MIMO Channels}

One important class of MIMO channel model assumes that the correlation between the transmit antennas (Tx) is independent of the correlation among receive antennas $(\mathrm{Rx})$; hence, admitting a MIMO channel Rayleigh flat-fading, [19], one can express the fading coefficients matrix:

$$
\mathbf{H}=\sqrt{\mathbf{R}_{\mathrm{H}, \mathrm{Rx}}} \mathbf{G} \sqrt{\mathbf{R}_{\mathrm{H}, \mathrm{Tx}}}
$$

where $\mathbf{G} \in \mathbb{C}^{\mathrm{NrxNt}}$ is an independent identically distributed (i.i.d.) complex Gaussian zeromean unit variance elements. The correlation matrices $\mathbf{R}_{\mathrm{H}, \mathrm{Tx}} \in \mathbb{R}_{\mathrm{Nt} \times \mathrm{Nt}}$ and $\mathbf{R}_{\mathrm{H}, \mathrm{Rx}} \in \mathbb{R}_{\mathrm{Nr} \text { Nr }}$ denote correlation observed among the transmitter antennas and receiver antennas, respectively. Assuming in this work that the Tx and Rx antennas are equally separated, equal numbers of antennas and equal correlation matrix $\mathbf{R}_{\mathrm{H}, \mathrm{Rx}}=\mathbf{R}_{\mathrm{H}, \mathrm{Tx}}=\mathbf{R}_{\mathrm{H}}$. Hence, the matrix $\mathbf{R}_{\mathrm{H}}$, can be written as:

$$
\mathbf{R}_{\mathrm{H}}=\left[\begin{array}{ccccc}
1 & \rho & \rho^{4} & \cdots & \rho^{\left(n_{T}-1\right)^{2}} \\
\rho & 1 & \rho & \cdots & \vdots \\
\rho^{4} & \rho & 1 & \cdots & \rho^{4} \\
\vdots & \vdots & \vdots & \ddots & \rho \\
\rho^{\left(n_{T}-1\right)^{2}} & \cdots & \rho^{4} & \rho & 1
\end{array}\right]
$$

where $\rho$ is the normalised correlation index. Note that a totally uncorrelated scenario means $\rho=0$, while a fully correlated scenario implies $\rho=1$.

\subsection{MIMO Channel Error Estimation}

The estimation of the Gaussian channel matrix in the receiver is not perfect, therefore it is necessary consider this effect on the overall system performance. The estimated channel matrix $\widehat{\mathbf{H}}$ can be simply modelled as: 


$$
\widehat{\mathbf{H}}^{\prime}=\mathbf{H}+\boldsymbol{\varepsilon}
$$

where $\boldsymbol{\varepsilon}=\boldsymbol{\varepsilon}_{i}+j \boldsymbol{\varepsilon}_{q}$ is a stochastic complex error, with in-phase and quadrature components assumed i.i.d. Gaussian variables, with $\boldsymbol{\varepsilon}_{i}$ and $\boldsymbol{\varepsilon}_{q} \sim \mathcal{N}\left\{0, \sigma_{\epsilon}^{2} / 2\right\}$. It is important to emphasize that this channel error estimation model comprises both module and phase, being the last more harmful for the detection process. Also, this estimation must be normalised by $\left(1+\sigma_{\epsilon}^{2}\right)^{-1}$, as suggested by [20]; therefore the estimated MIMO channel matrix becomes:

$$
\widehat{\mathbf{H}}=\frac{\mathbf{H}+\boldsymbol{\varepsilon}}{1+\sigma_{\varepsilon}^{2}}
$$

This normalisation is necessary aiming to not change the original average power channel characteristics. Furthermore, in this modelling, the variance $\sigma_{\varepsilon}^{2}$ is assumed inversely proportional to the SNR:

$$
\sigma_{\varepsilon}^{2}=\frac{n_{\varepsilon}}{\gamma}
$$

where $n_{\varepsilon}$ represents the channel error factor.

\section{Conventional MIMO Detectors}

In the sequel, classical MIMO detectors found in the literature are revisited, including the minimum mean squared error (MMSE) criterion, successive interference cancellation method, and QR decomposition-based MIMO detectors.

\subsection{MMSE MIMO Detection}

In order to reduce the impact of fading and background noise, the MMSE detector employ a linear filter that can take into account the channel matrix and the background noise effect as well. The MMSE filter can be found by minimizing the mean-square error (MSE) as [21]:

$$
\begin{aligned}
\mathbf{W}_{\mathrm{mmse}} & =\underset{\mathbf{W}}{\operatorname{argmin}} \mathbb{E}\left[\left\|\mathbf{s}-\mathbf{W}^{\mathbf{H}} \mathbf{y}\right\|^{2}\right] \\
& =\left(\mathbb{E}\left[\mathbf{y} \mathbf{y}^{H}\right]\right)^{-1} \mathbb{E}\left[\mathbf{y} \mathbf{s}^{H}\right] \\
& =\mathbf{H}\left(\mathbf{H}^{H} \mathbf{H}+\frac{N_{0}}{E_{s}} \mathbf{I}\right)^{-1}
\end{aligned}
$$

where $\mathbf{I}$ is a $N_{t} \times N_{t}$ identity matrix and $\mathbb{E}[\cdot]$ is the statistical expectation operator. The resulting estimated symbol vector can be written as:

$$
\mathbf{s}_{\mathrm{mmse}}=\mathbf{W}_{\mathrm{mmse}}^{H} \mathbf{y}
$$

\subsection{MMSE-SIC MIMO Detection}

The MMSE-SIC MIMO detector is performed from the decomposition of the channel matrix $\mathbf{H}$ and assuming that $\mathbf{H}$ is square or tall, where $N_{t} \leq N_{r}$. Hence, applying for instance the QR factorisation on the channel matrix $\mathbf{H}$ : 


$$
\mathbf{H}=\mathbf{Q R}
$$

where $\mathbf{Q}$ is a $N_{t} \times N_{t}$ unitary matrix and $\mathbf{R}$ is a $N_{t} \times N_{r}$ upper triangular matrix. Hence, multiplying $\mathbf{Q}^{H}$ by the receive signal $\mathbf{y}$ we can written:

$$
\mathbf{x}=\mathbf{Q}^{H} \mathbf{y} \mathbf{x}=\mathbf{R s}+\mathbf{Q}^{H} \boldsymbol{\eta}
$$

where $\tilde{\boldsymbol{\eta}}=\mathbf{Q}^{H} \boldsymbol{\eta}$ is a zero-mean complex Gaussian random vector. Notice that $\mathbf{Q}^{H} \boldsymbol{\eta}$ and $\tilde{\boldsymbol{\eta}}$ share the same statistical properties.

The channel correlation matrix can be either square or tall. Initially, assuming a square $M \times M$ matrix $\mathbf{H}$, we have:

$$
\begin{gathered}
\mathbf{x}=\mathbf{R s}+\tilde{\boldsymbol{\eta}} \\
{\left[\begin{array}{c}
x_{1} \\
x_{2} \\
\vdots \\
x_{M}
\end{array}\right]=\left[\begin{array}{cccc}
r_{1,1} & r_{1,2} & \cdots & r_{1, M} \\
0 & r_{2,2} & \cdots & r_{2, M} \\
\vdots & \vdots & \ddots & \vdots \\
0 & 0 & \cdots & r_{M, M}
\end{array}\right]\left[\begin{array}{c}
s_{1} \\
s_{2} \\
\vdots \\
s_{M}
\end{array}\right]+\left[\begin{array}{c}
\tilde{\eta}_{1} \\
\tilde{\eta}_{2} \\
\vdots \\
\tilde{\eta}_{M}
\end{array}\right]}
\end{gathered}
$$

Thus, we can write the recurrent equations in order to determine the soft decision $x_{M}$ to $x_{1}$ :

$$
\begin{gathered}
x_{M}=r_{M, M} s_{M}+\tilde{\eta}_{M} \\
x_{M-1}=r_{M-1, M} s_{M}+r_{M-1, M-1} s_{M-1}+\tilde{\eta}_{M-1}
\end{gathered}
$$

Now assuming that the matrix $\mathbf{H}$ is tall with $N \times M$ dimension and $N \geq M$, we have

$$
\left[\begin{array}{c}
x_{1} \\
x_{2} \\
\vdots \\
x_{M} \\
x_{M+1} \\
\vdots \\
x_{N}
\end{array}\right]=\left[\begin{array}{cccc}
r_{1,1} & r_{1,2} & \cdots & r_{1, M} \\
0 & r_{2,2} & \cdots & r_{2, M} \\
\vdots & \vdots & \ddots & \vdots \\
0 & 0 & \cdots & r_{M, M} \\
0 & 0 & \cdots & 0 \\
\vdots & \vdots & \vdots & \vdots \\
0 & 0 & \cdots & 0
\end{array}\right]\left[\begin{array}{c}
s_{1} \\
s_{2} \\
\vdots \\
s_{M}
\end{array}\right]+\left[\begin{array}{c}
\tilde{\eta}_{1} \\
\tilde{\eta}_{2} \\
\vdots \\
\tilde{\eta}_{M} \\
\tilde{\eta}_{M+1} \\
\vdots \\
\tilde{\eta}_{N}
\end{array}\right]
$$

Therefore, the soft decision $x_{N}$ to $x_{1}$ can be recursively determined as:

$$
\begin{gathered}
x_{N}=\tilde{\eta}_{N} \\
\vdots \\
x_{M+1}=\tilde{\eta}_{M+1} \\
x_{M}=r_{M, M} s_{M}+\tilde{\eta}_{M} \\
x_{M-1}=r_{M-1, M} s_{M}+r_{M-1, M-1} s_{M-1}+\tilde{\eta}_{M-1}
\end{gathered}
$$

Since the received signals $x_{M+1}, x_{M+2}, \ldots, x_{N}$ do not have any useful information, we can simply ignore them. Hence, Eqs. (13) and (15) are equivalents. 


\subsubsection{Successive Interference Cancelation (SIC) Step}

Firstly, $s_{M}$ can be detected from $x_{M}$ as follows:

$$
\text { Let } \quad \tilde{s}_{M}=\frac{x_{M}}{r_{M, M}}=s_{M}+\frac{\tilde{\eta}_{M}}{r_{M, M}}
$$

Then, the contribution of $\tilde{s}_{M}$ is to be canceled in detecting $s_{M-1}$ from $x_{M-1}$. This sequential detection procedure is terminated until all the data symbols of $\mathbf{s}$ are detected. The $m$ th symbol of $\mathbf{s}$ can be detected after cancelling $M-m$ data symbols as:

$$
\begin{aligned}
& u_{m}=x_{m}-\sum_{q=m+1}^{M} r_{m, q} \tilde{s}_{q} \\
& s_{m}=\frac{u_{m}}{r_{m, m}}, \quad m \in\{1,2, \ldots, M-1\}
\end{aligned}
$$

Finally, the background noise can be taken into account in order to minimise the mean square error, [21].

- Extended channel matrix: $\mathbf{H}_{\mathrm{ex}}=\left[\begin{array}{ll}\mathbf{H}^{T} & \sqrt{\frac{N_{0}}{E_{s}}} \mathbf{I}\end{array}\right]^{T} ;$

- Extended receive signal: $\mathbf{y}_{\mathrm{ex}}=\left[\begin{array}{ll}\mathbf{y}^{T} & \boldsymbol{0}^{T}\end{array}\right]^{T}$;

- Extended noise AWGN: $\mathbf{n}_{\mathrm{ex}}=\left[\begin{array}{ll}\mathbf{n}^{T} & -\sqrt{\frac{N_{0}}{E_{s}}} \mathbf{s}^{T}\end{array}\right]^{T}$.

\subsection{MMSE-OSIC MIMO Detection}

Further performance improvement on the MIMO MMSE-SIC technique can be achieved through a properly ordering $[9,22]$, witch avoid error propagation in the interference cancellation step. The ordering criterion is the minimization of the $\mathbf{Q}$ columns norm, which makes the detection be proceeded from the least noise corrupted symbol to the most. The form of the decomposition is simply:

$$
\mathbf{H P}=\mathbf{Q R}
$$

where matrix $\mathbf{P}$ is a permutation matrix, used to reorder the symbols after applying the SIC detection, Eq. (17), by multiplying it and the estimated symbol. Therefore the application of sorted QR Decomposition (SQRD) instead of the QR decomposition change the detector SIC into OSIC.

\subsubsection{Sorted QR Decomposition (SQRD)}

The sorted QR decomposition (SQRD) is summarised by the pseudo-code in Algorithm 1. 


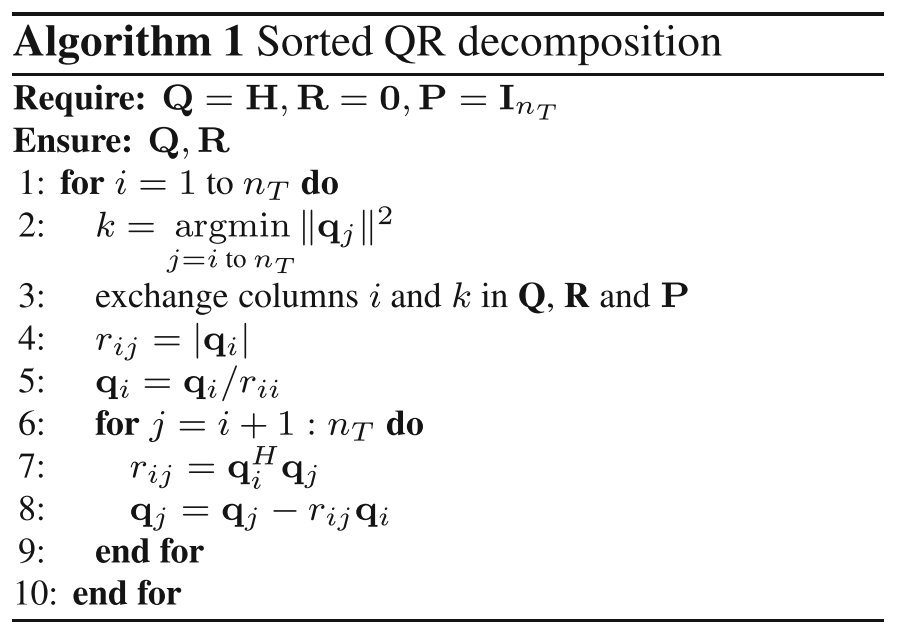

\subsection{Sphere Decoder (SD) Principle}

To simplify the analysis, let us reformulate the complex number signal model of Eq. (1) into a real number signal model:

$$
\begin{aligned}
\mathbf{y}_{r} & =\mathbf{A} \mathbf{s}_{r}+\eta_{r} \\
{\left[\begin{array}{c}
\mathfrak{R}\{\mathbf{y}\} \\
\mathfrak{J}\{\mathbf{y}\}
\end{array}\right] } & =\left[\begin{array}{cc}
\mathfrak{R}\{\mathbf{H}\} & -\mathfrak{J}\{\mathbf{H}\} \\
\mathfrak{I}\{\mathbf{H}\} & \mathfrak{R}\{\mathbf{H}\}
\end{array}\right]\left[\begin{array}{c}
\mathfrak{R}\{\mathbf{s}\} \\
\mathfrak{I}\{\mathbf{s}\}
\end{array}\right]+\left[\begin{array}{c}
\mathfrak{R}\{\eta\} \\
\mathfrak{I}\{\eta\}
\end{array}\right]
\end{aligned}
$$

where $\mathbf{y}_{r} \in \mathcal{R}^{M}, \mathbf{s}_{r} \in \mathcal{Z}^{N}, \eta_{r} \in \mathcal{R}^{M}$, and $\mathbf{A} \in \mathcal{R}^{M \times N}$ with $N=2 N_{t}$ and $M=2 N_{r}$. The $\mathbf{s}_{r}$ belongs to a finite subset of the infinite integer vector space $\mathcal{Z}^{N}$, denoted by $\mathbf{s} \in \Lambda \in \mathcal{Z}^{N}$.

Let $L(\mathbf{A})=\mathbf{A z} ; \mathbf{z} \in \mathcal{Z}^{N}$ denote the lattice with $\mathbf{A}$ as its basis. The ML MIMO detection problem consists in finding the closest lattice point to the received signal $\mathbf{y}_{r}$ with the finite subset $\Lambda$ as the search space:

$$
\hat{\mathbf{s}}_{\mathrm{ML}}=\min _{\mathbf{z} \in \Lambda \in \mathcal{Z}^{N}}\left\|\mathbf{y}_{r}-\mathbf{A z}\right\|^{2}
$$

In order to reduce the computational complexity of the ML detection, the search in Eq. (20) can be limited within a hypersphere of a certain radius $\xi$, which is referred to sphere decoding, and expressed as:

$$
\hat{\mathbf{S}}_{\mathrm{ML}}=\min _{\mathbf{z} \in \Lambda \in \mathcal{Z}^{N}}\left(\left\|\mathbf{y}_{r}-\mathbf{A z}\right\|^{2} \leq \xi^{2}\right)
$$

\section{Lattice Reduction Based MIMO Detection}

The lattice (basis) reduction or LR has been proposed in aiming to transform a regular basis to a nearly orthogonal one. Choosing the channel matrix $\mathbf{H}$ as a basis for a lattice, the MIMO problem can be treated as a lattice decoding problem. The lattice concept is explored in the sequel; after that, the MIMO LR-aided detector is discussed in details.

\subsection{Lattices}

Let $\mathbf{L}$ be a $2 \times 2$ matrix and $\mathbf{u}=\left[u_{1} u_{2}\right]^{T}$ a $2 \times 1$ vector. A lattice $\Lambda_{L}$ is the set of points: 


$$
\boldsymbol{\Lambda}_{L}=\left\{\mathbf{L u} \mid u_{1}, u_{2} \in \mathbb{Z}[i]\right\}
$$

where $\mathbb{Z}[i]$ is the set of Gaussian integers. The set of Gaussian integers is the complex numbers $\alpha=a+b i$ whose components $a$ and $b$ are both integers [23]. $\mathbf{L}$ is called a generator matrix for the lattice $\boldsymbol{\Lambda}_{L}$. The minimum distance of $\boldsymbol{\Lambda}_{L}$ is defined as:

$$
d_{\min }^{2}\left(\boldsymbol{\Lambda}_{L}\right)=\min _{\mathbf{u} \neq \mathbf{v}}\|\mathbf{L}(\mathbf{u}-\mathbf{v})\|^{2}
$$

where $\mathbf{u}$ and $\mathbf{v}$ are Gaussian integer vectors. From the definition of $\boldsymbol{\Lambda}_{L}$ are infinitely different bases in a lattice and they all span the same lattice $\boldsymbol{\Lambda}_{L}$. Assume that $\boldsymbol{L}^{\prime}$ is another basis for $\boldsymbol{\Lambda}_{L}$. Then follows that $\boldsymbol{L}^{\prime}=\mathbf{L Z}$, where $\mathbf{Z}$ is a unimodular matrix; therefore, $\mathbf{Z}$ has Gaussian integers entries and $\operatorname{det}(\mathbf{Z}) \in\{ \pm 1, \pm i\}$. From the definition of $d_{\text {min }}^{2}\left(\boldsymbol{\Lambda}_{L}\right)$ follows that:

$$
d_{\min }^{2}\left(\boldsymbol{\Lambda}_{\mathrm{QLZ}}\right)=d_{\min }^{2}\left(\boldsymbol{\Lambda}_{L}\right)
$$

where $\mathbf{Q}$ is a unitary matrix. A matrix $\mathbf{U} \in M_{n}$ is said to be unitary if $\mathbf{U}^{H} \mathbf{U}=\mathbf{I}$, where $M_{n}$ is the set of matrix $n \times n$ [24].

\subsection{MIMO System with Lattice}

Let the basis $\mathbf{B}$ consisting of $M$ real-valued linearly independent basis vectors given by

$$
\mathbf{B}=\left\{\mathbf{b}_{1}, \mathbf{b}_{2}, \ldots, \mathbf{b}_{M}\right\} .
$$

Since a lattice can be generated from an integer linear combination of a basis, with $\mathbf{B}$, we can have a lattice defined by

$$
\Lambda=\left\{\mathbf{u} \mid \mathbf{u}=\sum_{m=1}^{M} \mathbf{b}_{m} z_{m}, \quad z_{m} \in \mathbb{Z}[i]\right\} .
$$

Hence, adopting $\mathbf{H}$ as a basis and $\mathbf{s}$ to produce an integer linear combination of the basis, the $\mathbf{y}$ becomes a vector in the lattice generated by the basis $\mathbf{H}$.

\subsection{The Lenstra-Lenstra-Lovász (LLL) Algorithm}

A well-known and powerful algorithm for lattice reduction is the LLL algorithm, proposed by Lenstra, Lenstra and Lovász in 1982 [25]. A basis $\tilde{\mathbf{A}}$ which can be decomposed by a QR decomposition as $\tilde{\mathbf{A}}=\tilde{\mathbf{Q}} \tilde{R} \mathbf{T}^{-1}$ is named reduced LLL with parameter $\delta$ in the range $\frac{1}{4}<\delta \leq 1$ if the following inequalities hold:

$$
\begin{aligned}
& \left|\tilde{r}_{l, k}\right| \leq \frac{1}{2}\left|\tilde{r}_{l, l}\right| \text { for } 1 \leq l<k \leq m, \\
& \text { and } \\
& \delta \tilde{r}_{k-1, k-1}^{2} \leq \tilde{r}_{k, k}^{2}+\tilde{r}_{k-1, k}^{2} \text { for } k=2, \ldots, m
\end{aligned}
$$

where $\mathbf{T}$ is a unimodular matrix, $\tilde{r}_{l, k}$ is the element of $l$-th row and $k$-th column of the upper triangular matrix $\tilde{\mathbf{R}}$.

If only the first inequality in (27) is fulfilled, the basis is called size reduced. Herein, the adopted LLL algorithm is based on [26] implementation and summarised by the pseudo- 
code in Algorithm 2. As shown in Algorithm 2, the parameter $\delta$ define the swap condition after the length reduction step and can be regarded as a strictness factor: the closer it is to 1 , the more orthogonal the reduced basis will be, in general [27], on the other hand, the reduction tends to be slower. Thus, in summary, one can say the parameter $\delta$ influences the quality of the reduced basis. In this paper, we have assumed $\delta=\frac{3}{4}$ as proposed in [25]. Notice that $\mathbf{Q}, \mathbf{R}$ and the permutation matrix $\mathbf{P}$, required by the algorithm, are obtained from (18). Besides, the Givens rotation matrix, $\Theta$, utilised in the Algorithm 2 (line 13) has a purpose of restore the upper triangular structure in $\tilde{\mathbf{R}}$.

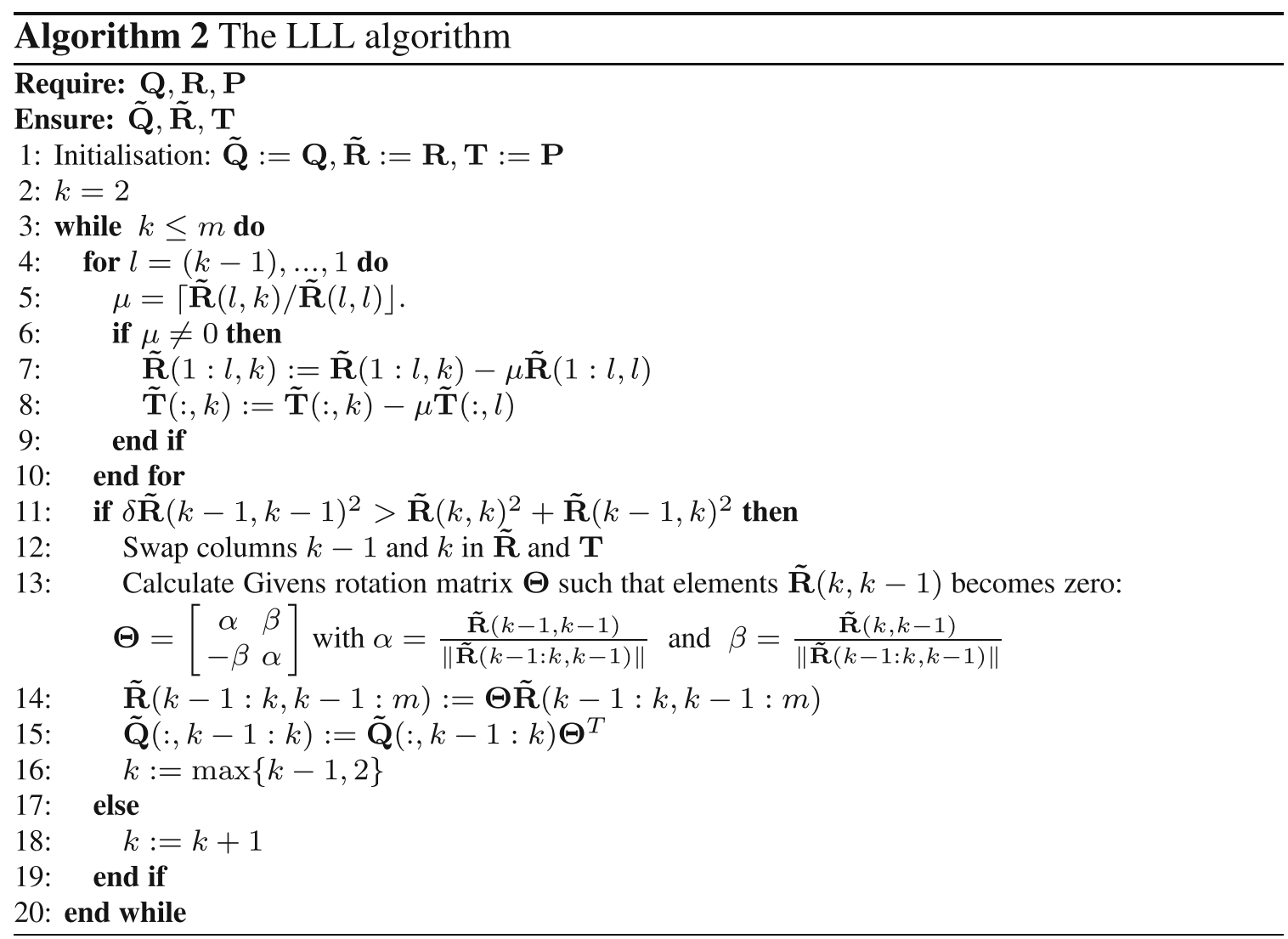

\subsubsection{Computational Complexity of LLL Algorithm}

The complexity of LLL algorithm depends on the matrix size and also the antenna/channel correlation index $\rho$. In [28] the complexity of this algorithm was evaluated by numerical experiment, considering $N_{t}=N_{r}=N$ and $\delta=\frac{3}{4}$. The function that describe the complexity in numbers of flops has been obtained by fitting, and given by:

$$
f_{L L L}(N, \rho)=\left(a e^{b \rho}+c\right) N^{3}
$$

where $a=5.018 \times 10^{-4}, b=13.48$ and $c=8.396$. It is worth noting that the computational complexity cost for the LLL algorithm increases substantially under large-array configurations and medium-high correlation index $(\rho \geq 0.5)$. Further analysis on the MIMO detectors complexity is provided in Sect. 6. 


\subsection{LR-Aided MIMO Detectors}

Since a lattice can be generate by different bases or channel matrices, with the goal of reducing the noise and interference between multiple signals, it is convenient to find a matrix whose columns are nearly orthogonal to generate the same lattice. Hence, LR technique can be applied to improve the MIMO detection performance; these methods are regarded as the LR-based detection for MIMO systems.

In order to deploy LR technique, the original constellation must be defined in terms of consecutive integers lattice. Consider two bases $\mathbf{H}$ and $\mathbf{G}$ that span the same lattice; it also shown that

$$
\mathbf{H}=\mathbf{G U}
$$

where $\mathbf{U}$ and $\mathbf{T}=\mathbf{U}^{-1}$ are a unimodular matrices. As a consequence the received signal in (1) can be rewritten as:

$$
\begin{gathered}
\mathbf{y}=\mathbf{H s}+\boldsymbol{\eta}=\mathbf{H T T}^{-1} \mathbf{s}+\boldsymbol{\eta}=\mathbf{G z}+\boldsymbol{\eta}, \\
\text { where } \quad \mathbf{z}=\mathbf{T}^{-1} \mathbf{s}=\mathbf{U s} .
\end{gathered}
$$

Since the received signal can be treated as the lattice points spanned by the basis, lattice-based MIMO system detection can be developed aiming to reduce the complexity of conventional detectors. Hence, under lattice transform, $\mathbf{z}$ is initially detected; after that, the original symbols s can be obtained applying a combination of shifting and scaling operations, Eq. (34), as described in the sequel, in order to correctly estimate $\mathbf{z}$ and then de-mapping deploying (31).

\subsubsection{LR-Aided Linear Detectors}

The LR-based MIMO linear detection can be carried out initially detecting $\mathbf{z}$ by applying linear filtering in the received signal vector; disregarding the background noise we have:

$$
\mathbf{z}=\mathbf{W}^{H} \mathbf{y},
$$

where for the LR-MMSE detector the linear filter is describe by

$$
\mathbf{W}^{H}=\left(\mathbf{G}^{H} \mathbf{G}+\frac{N_{0}}{E_{s}} \mathbf{U}^{-H} \mathbf{U}^{-1}\right)^{-1} \mathbf{G}^{H}
$$

\subsubsection{Shift and Scale Method}

After computing soft decisions on $\mathbf{z}$ it is necessary to estimate the correct symbols. The solution to this quantisation problem is to use a combination of shifting and scaling operations to ensure that both the original $\mathbf{s}$ and the reduced constellation $\mathbf{z}$ are defined in terms of consecutive integer lattices [29]. The shifting and scaling operations is given by:

$$
\widehat{\mathbf{z}}=2\left\lceil\frac{1}{2}\left(\mathbf{z}-\beta^{\prime} \boldsymbol{\imath}\right)\right\rfloor+\beta^{\prime} \boldsymbol{\imath}
$$

where $\lceil\cdot\rfloor$ represent rounding to the nearest integer. Hence, the original symbols $\mathbf{s}$ can be obtained applying (34) and then de-mapping in (31). The steps to achieve shifting and scaling equation (34) is explained in the following. 
In the LR-aided scheme the constellation symbols are related to a consecutive integer lattice $\mathbf{x}$ by:

$$
s_{i}=\alpha x_{i}+\beta
$$

where $s_{i}$ is a complex integer, $\alpha$ is a scalar and $\beta$ a complex offset. The symbol vector can be represent as

$$
\mathbf{s}=\alpha \mathbf{x}+\beta \mathbf{1}
$$

where $\mathbf{s}$ is a vector of symbols in the complex integer lattice and $\mathbf{1}$ is a vector of ones. Another equivalent representation for $\mathbf{s}$ is normalising by $\frac{2}{\alpha}$ :

$$
\mathbf{s}=2 \mathbf{x}+\beta^{\prime} \mathbf{1}
$$

where $\beta^{\prime}=\left(\frac{2 \beta}{\alpha}\right)$, assuming the value $1+j$ for all M-QAM constellations. From (31) we can write

$$
\begin{gathered}
\mathbf{T}^{-1} \mathbf{S}=\mathbf{T}^{-1}\left(2 \mathbf{x}+\beta^{\prime} \mathbf{1}\right) \\
\mathbf{z}=2 \breve{\mathbf{x}}+\beta^{\prime} \boldsymbol{\imath}
\end{gathered}
$$

where $\breve{\mathbf{x}}=\mathbf{T}^{-1} \mathbf{x}$ and the row-sum vector $\boldsymbol{\imath}=\mathbf{T}^{-1} \boldsymbol{l}$. Isolating the $\breve{\mathbf{x}}$ we have

$$
\breve{\mathbf{x}}=\frac{1}{2}\left(\mathbf{z}-\beta^{\prime} \boldsymbol{\imath}\right)
$$

and symbol estimate in the reduced lattice should be based on quantisation in the consecutive integer lattice basis

$$
\widehat{\overrightarrow{\mathbf{x}}}=\left\lceil\frac{1}{2}\left(\mathbf{z}-\beta^{\prime} \mathbf{\imath}\right)\right\rfloor
$$

Finally, from (38) the estimate in the reduced lattice follows:

$$
\widehat{\mathbf{z}}=2\left\lceil\frac{1}{2}\left(\mathbf{z}-\beta^{\prime} \boldsymbol{\imath}\right)\right\rfloor+\beta^{\prime} \boldsymbol{\imath}
$$

The core of LR-MMSE MIMO detection process is described by the pseudo-code shown in Algorithm 3.

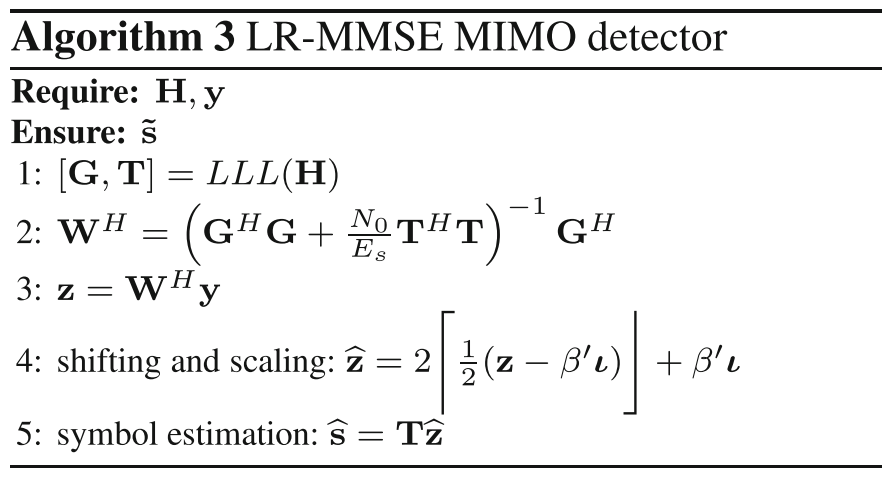




\subsubsection{LR-Aided Sphere Decoding}

By using the lattice reduction algorithm such as the LLL algorithm on the basis $\mathbf{A}$, a reduced basis $\tilde{\mathbf{A}}$ and a unimodular matrix $\mathbf{U}$ can be found such that:

$$
\tilde{\mathbf{A}}=\mathbf{A} \mathbf{T}
$$

Notice that the unimodular matrix $\mathbf{T}$ and its inverse $\mathbf{U}=\mathbf{T}^{-1}$ are composed only by integer values and $\operatorname{det}(\mathbf{T})$ is equal to 1 or -1 . Using the unimodular matrix $\mathbf{T}$, a transformed symbol vector $\mathbf{d}=\mathbf{T}^{-1} \mathbf{s}_{r}=\mathbf{U} \mathbf{s}_{r}$ is obtained in order to perform the transformation in a reduced basis, expressed as

$$
\begin{aligned}
\mathbf{y}_{r} & =\mathbf{A} \mathbf{s}_{r}+\eta_{r}=\mathbf{A} \mathbf{I} \mathbf{s}_{r}+\eta_{r}=\mathbf{A} \mathbf{T} \mathbf{T}^{-1} \mathbf{s}_{r}+\eta_{r} \\
& =\tilde{\mathbf{A}} d+\eta_{r}
\end{aligned}
$$

where I denotes the identity matrix.

Since $\mathbf{s}_{r} \in \Lambda \in \mathcal{Z}^{N}$, then the range of $\mathbf{d}$ is denoted by $\mathbf{d} \in \tilde{\Lambda}$, the image of $\Lambda$ under $\mathbf{T}^{-1}$. However, the subset $\tilde{\Lambda}$ is generally no longer of the simple hypercube structure as that of $\Lambda$. Therefore, controlling the search range in $\tilde{\Lambda}$ is a very complicated problem. To avoid this, a lookup table is built containing the vectors-candidates already calculated at each layer. This procedure is intended to avoid the calculation of nodes already visited, which is the essence of the tree search. More details about the lookup table procedure is given in 1 .

The primary search uses the initial radius based on the LR-aided Linear Zero Forcing (LR-LZF) decision; this deterministic method is also deployed in [17], where the hypersphere radius is considered as the distance between the original received signal and the signal received using LZF detector. Thus, the primary search of the LR-SD for MIMO detections can be expressed by the following problem:

$$
\hat{\mathbf{d}}=\min _{\mathbf{z} \in \tilde{\Lambda}}\left(\left\|\mathbf{y}_{r}-\tilde{\mathbf{A}} z\right\|^{2} \leq \xi^{2}\right)
$$

where $\xi^{2}$ is initially expressed by:

$$
\begin{aligned}
& \mathbf{d}_{\mathrm{LR}-\mathrm{LZF}}=\tilde{\mathbf{A}}^{\dagger} \mathbf{y}_{r} \\
& \xi^{2}=\left\|\mathbf{y}_{r}-\tilde{\mathbf{A}} \mathbf{d}_{\mathrm{LR}-\mathrm{LZF}}\right\|^{2}
\end{aligned}
$$

Indeed, this primary search shows significant complexity reduction over the conventional SD of Eq. (21), due to the considerable decreasing in the sphere radius $\xi$. Nevertheless, the result $\hat{\mathbf{d}}$ not necessarily exists in the reduced basis domain $\tilde{\Lambda}$, as stated in the Proposition 1 .

Proposition 1 In lattice $L(\mathbf{A})=\mathbf{A z} ; \mathbf{z} \in \mathcal{Z}^{N}$, suppose $\mathbf{A} \hat{\mathbf{d}}_{\mathrm{ML}}$ is the closest lattice point to a certain vector point $\mathbf{y}_{r}$ among the lattice points in subset $\left\{\mathbf{A z}: \mathbf{z} \in \Lambda \in \mathcal{Z}^{N}\right\}$. If there exists a lattice point Âd which is closer to $\mathbf{y}_{r}$ than A $\hat{\mathbf{d}}_{\mathrm{ML}}$, i.e., $\left\|\mathbf{y}_{r}-\mathbf{A} \hat{\mathbf{d}}\right\|^{2}<\left\|\mathbf{y}_{r}-\mathbf{A} \hat{\mathbf{d}}_{\mathrm{ML}}\right\|^{2}$, then $\hat{\mathbf{d}} \notin \tilde{\Lambda}$ and, consequently, $\hat{\mathbf{s}}=\mathbf{T} \hat{\mathbf{d}} \notin \Lambda$.

The primary search starts from the decision statistic $\mathbf{d}_{\mathrm{LR}-\mathrm{LZF}}$ as initial radius. The closest lattice point to $\mathbf{y}_{r}$ in the sphere, $\hat{\mathbf{d}} \in \tilde{\Lambda}$, is the $\hat{\mathbf{d}}_{\mathrm{ML}}$. If none $\hat{\mathbf{d}} \in \tilde{\mathbf{A}}$ is found in the primary search, then a further search is required. In the next search, the squared radius need to be updated as: 


$$
\xi^{2}=\left\|\mathbf{y}_{r}-\tilde{\mathbf{A}} \mathbf{d}_{\mathrm{LR}-\mathrm{LZF}}\right\|^{2}+\Delta
$$

where $\Delta$ is a constant increment. The selection of the squared radius increment $\Delta$ strongly affects the computational complexity, and its value has been obtained as a scaled variance of noise in such way that, a lattice point inside the hypersphere is found with a high probability. Let $\sigma_{\eta}^{2}$ be the variance of the entries $\eta_{r} \in \mathcal{R}^{N \times 1}$, the squared radius increment is selected as $\Delta=\alpha N \sigma_{\eta}^{2}$, where $\alpha$ is calculated considering a high probability of a lattice point be found inside the hypersphere; determination of suitable values of $\alpha$ is provided in 1 .

With the updated radius, the search is proceed aiming to obtain the closest lattice point to $\mathbf{y}_{r}$ in the hypersphere. The closest point $\hat{\mathbf{d}} \in \tilde{\Lambda}$ found must be the ML detection result, $\hat{\mathbf{d}}_{\mathrm{ML}}$. If none lattice point is found, a further search is required again. Increase the sphere radius, $\xi^{2}$, by another $\Delta$ and perform a new sphere search. This procedure is repeated until $\hat{\mathbf{d}}_{\mathrm{ML}}$ and, consequently, $\hat{\mathbf{s}}_{\mathrm{ML}}=\mathbf{T} \hat{\mathbf{d}}_{\mathrm{ML}} \in \Lambda$ is obtained. The proposed LR-SD algorithm for MIMO detections is summarised by the pseudo-code in Algorithm 4.

These further searches for $\hat{\mathbf{s}}_{\mathrm{ML}}$ will certainly lead to a complexity increase. In order to minimise this overhead two main procedures are performed herein: (a) optimisation of $\Delta$; (b) lookup table. The optimisation of $\Delta$ was performed in order to avoid further searches, see 1 . The lookup table avoid the calculation of nodes already visited, thus, the complexity in the further searches is greatly decreased.

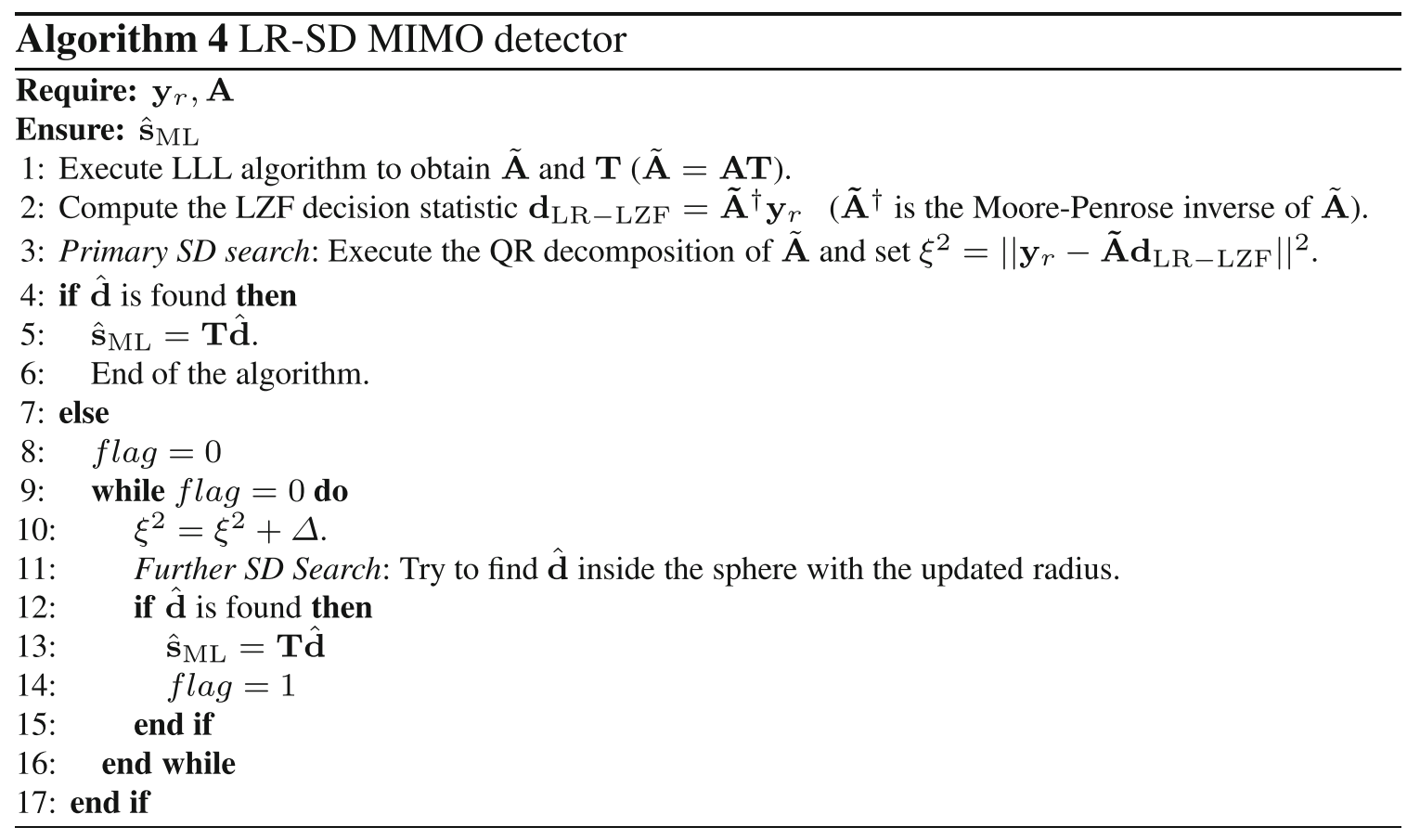

\section{Numerical Results}

In this section, performance in terms of BER versus SNR under perfect and channel error estimates is analysed; further realistic MIMO detection performance analysis has been conducted considering channels correlated scenarios. Along this section, numerical MonteCarlo simulations (MCS) results are depicted and examined. Different system and channel configuration scenarios have been considered: 
(a) Perfect CSI knowledge available at receiver side $\left(n_{\varepsilon}=0\right)$, but not at the transmitter side, and uncorrelated antennas;

(b) Perfect CSI and different levels of antenna correlation $(\rho>0)$;

(c) Imperfect CSI knowledge $\left(n_{\varepsilon}>0\right)$ under uncorrelated channels $(\rho=0)$;

(d) Imperfect CSI knowledge under correlated channels.

Besides, 4-QAM modulation and Gray coding have been deployed across this section; hence, the analysed BER and SER in this section are equivalent.

Figure 1 depicts the BER performance of different detectors for a 4-QAM MIMO system with $N_{t}=N_{r}=2$ and $N_{t}=N_{r}=4$ antennas with perfect channel estimation, i.e., the channel error factor $n_{\varepsilon}=0$, under uncorrelated channels $(\rho=0)$. In Fig. 1a, the performance of MMSE-OSIC detector for a MIMO $2 \times 2$ shows a slightly improvement compared with the MMSE detector, although this detector also presents slightly higher complexity, as shown in Sect. 6; however, the performance of theses detectors, due to the noise enhancement, is poor in comparison to ML. In contrast, the LR-aided MIMO detectors clearly outperform the others MIMO detectors, remarkably in the high SNR region, indicating that the LR technique is robust against the channel fading effect, specially in high SNR region, although its performance in low SNR region has proved worse, specially with the increase of the numbers of antennas, as shown in Fig. 1 b $4 \times 4$. The LRMMSE-OSIC detector achieves near-ML performance specially in the high SNR regime, where the additive noise is negligible. The LR-SD achieves the ML performance in both cases (Fig. 1a, b); this result is expected since the LR aided SD search performed in this work obtain the lowest euclidean distance vector inside the reduced basis domain. On the other hand, with $2 \times 2$ antennas, the LR-SD detector experiment an excessive

(a) MIMO $2 \times 2$

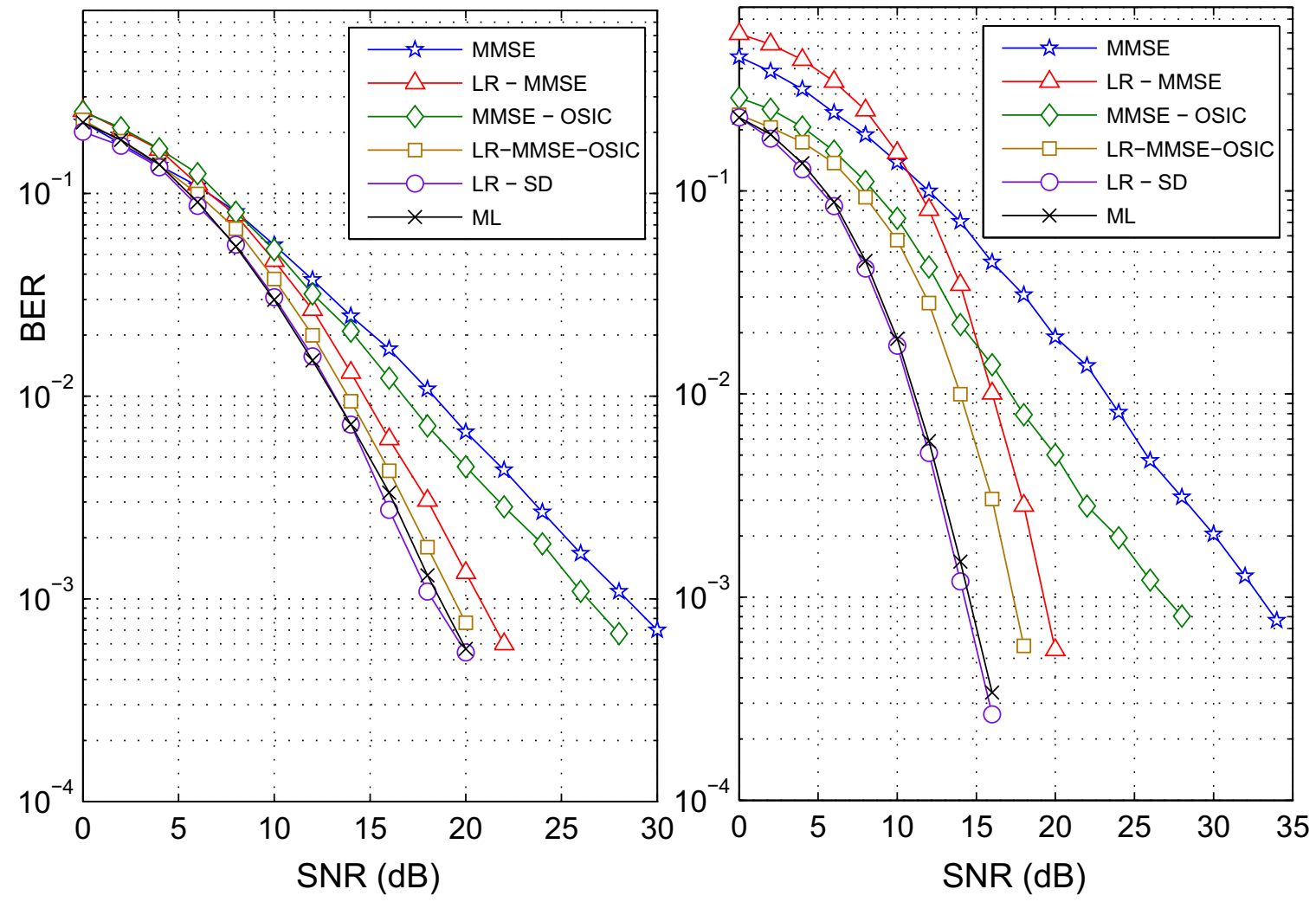

Fig. 1 BER performance for the MIMO detectors under 4-QAM, perfect channel estimation and uncorrelated channels for a $2 \times 2$ antennas; b $4 \times 4$ antennas 

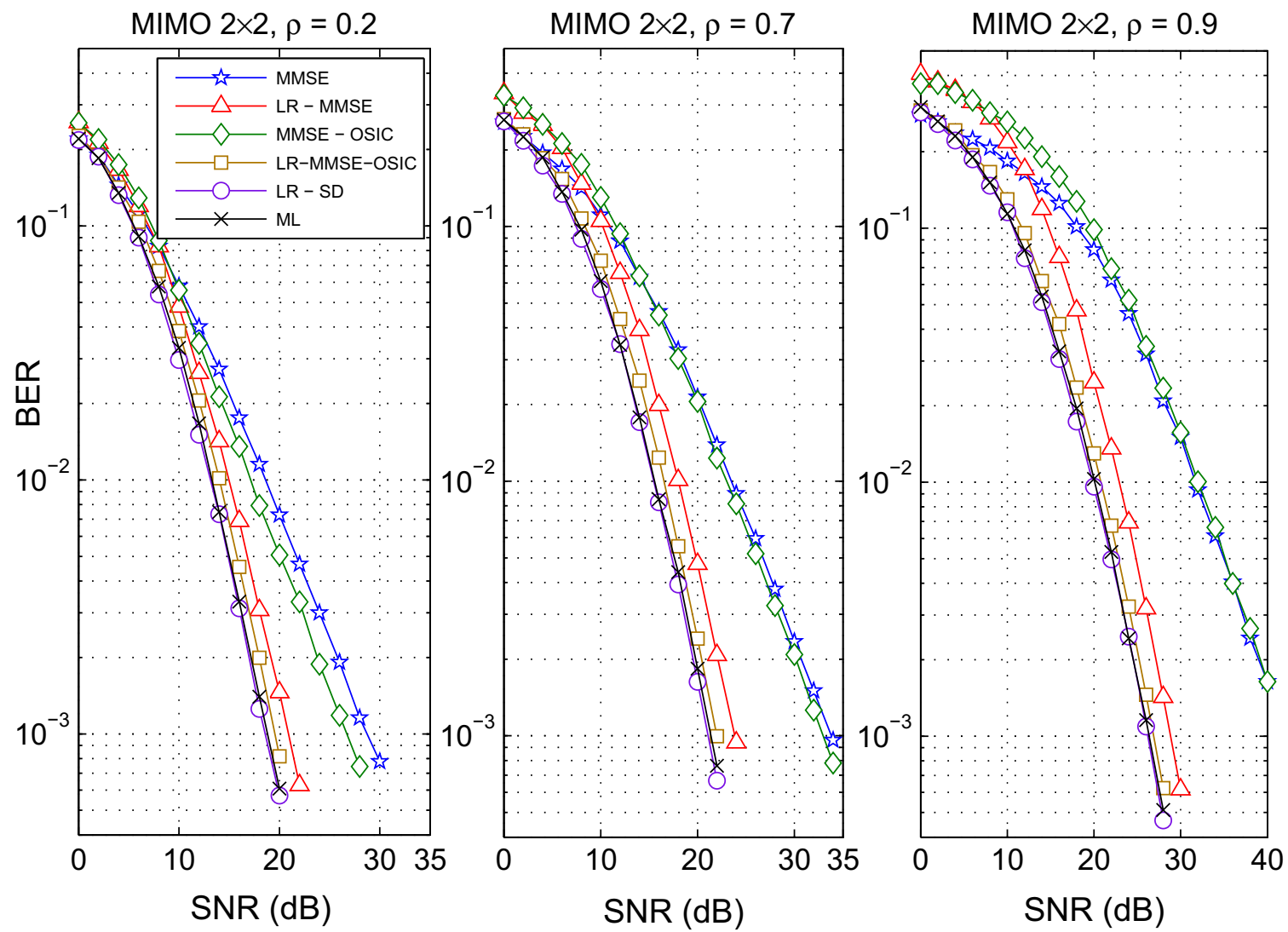

Fig. 2 BER performance for the MIMO detectors under correlated channels with $2 \times 2$ antennas

computational complexity, of same order of ML, in both uncorrelated and correlated channels; denoting this detector is not applicable in very low number of antennas scenarios; indeed, complexity analysis is discussed in Sect. 6. Moreover, under $2 \times 2$ configuration the performance gap between LR-SD and LR-MMSE-OSIC detectors is negligible, demonstrating that the LR-MMSE-OSIC detector should represent a better choice under very lower number of antennas scenarios.

Figures 2 and 3 depict the BER performance of the detectors for a 4-QAM MIMO system with $N_{t}=N_{r}=2$ and $N_{t}=N_{r}=4$ antennas, respectively, and perfect channel estimation $\left(n_{\varepsilon}=0\right)$, but considering three different levels of channel correlation, low, high and very high, $\rho=[0.2 ; 0.7 ; 0.9]$. The performance of the MIMO detectors under channels weakly correlated $(\rho=0.2)$ results very close to the uncorrelated channel condition, although with the increase of the levels of correlation implies in an increasing degradation in terms of BER performance. The MIMO detection with the aid of lattice-reduced technique has demonstrated robustness against inter-antenna interference. Elaborating further, in strongly correlated channels and reduced number of antennas, the detectors ML and LR-aided MMSE-OSIC in high SNR region present very similar performance; the same behaviour happens with MMSE and MMSE-OSIC detectors. Although with low number of antennas and very high channel correlation, the computational complexity for LR-aided MMSE detectors results in the same order of ML due to the exhaustive calculation of $f_{\mathrm{LLL}}\left(N_{t}, \rho\right)$, as discussed in Sect. 6. Under the same channel and system scenario but with $4 \times 4$ antennas, as depicted in Fig. 3, one can observe that the jointly effects, i.e. the increasing level of correlation and inter-antenna interference, provoke a remarkable degradation in the BER performance, specially for the MMSE and MMSE-OSIC detectors, which under strongly correlated channels results in a really poor performance. 

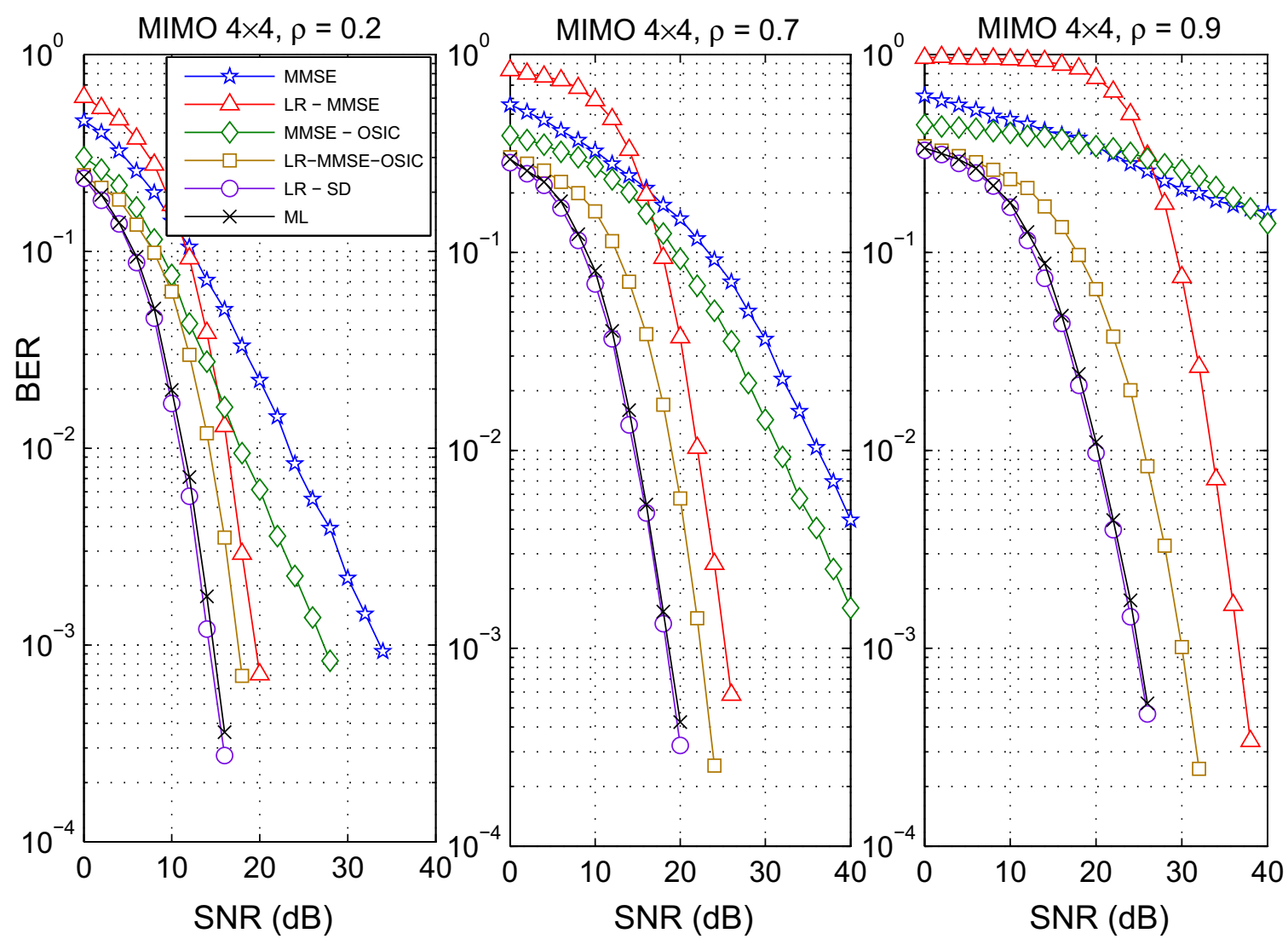

Fig. 3 BER performance for the MIMO detectors under correlated channels with $4 \times 4$ antennas

In the context of Fig. 3 yet, combining strongly correlated channels with a higher number of antennas it is noticeable that under low-medium SNR regions the LR-MMSEbased MIMO detector shows a higher performance degradation regarding the LR-MMSEOSIC. The reason is that the LR technique applied to MIMO systems, specially under large number of antennas condition, makes them more sensitive to the noise and inter-antenna interference, as discussed in [5]. However, this performance degradation effect does not occurs with LR-MMSE-OSIC because the OSIC substantially mitigates the interference effect via ordered successive cancellation.

Moreover, from Fig. 3, one can conclude that the LR-MMSE-OSIC for increasing number of antennas presents a slight performance degradation regarding the ML-MIMO (3-5 dB for increasing $\rho$ ) but with much less complexity, while LR-MMSE-OSIC detector is able to offer the same diversity order, as indicated by the slope of BER performance curve in high SNR region. Furthermore, notice that the LR-SD detector achieved in all correlation and $4 \times 4$ number of antennas scenarios the exact ML performance but with a lower complexity compared to the ML exhaustive search, as discussed in Sect. 6. Moreover the performance results for $2 \times 2$ and $4 \times 4$ MIMO schemes indicate a clear trend in terms of gap degradation between these detectors, inducing the conclusion that in large MIMO schemes (tens to hundreds antennas) the performance of the LRMMSE-OSIC should be increasingly worse; therefore, the LR-SD detector evince to be a worth option for large MIMO systems.

Figures 4 and 5 show the BER performance of the detectors for a 4-QAM MIMO system with $N_{t}=N_{r}=2$ and $N_{t}=N_{r}=4$ antennas, respectively, now considering uncorrelated channels $(\rho=0)$ but under different channel error factors, $n_{\varepsilon}=[1.0 ; 3.0 ; 7.0 ; 10.0]$. One can notice that with increasing CSI error levels the 
A. M. Mussi et al.
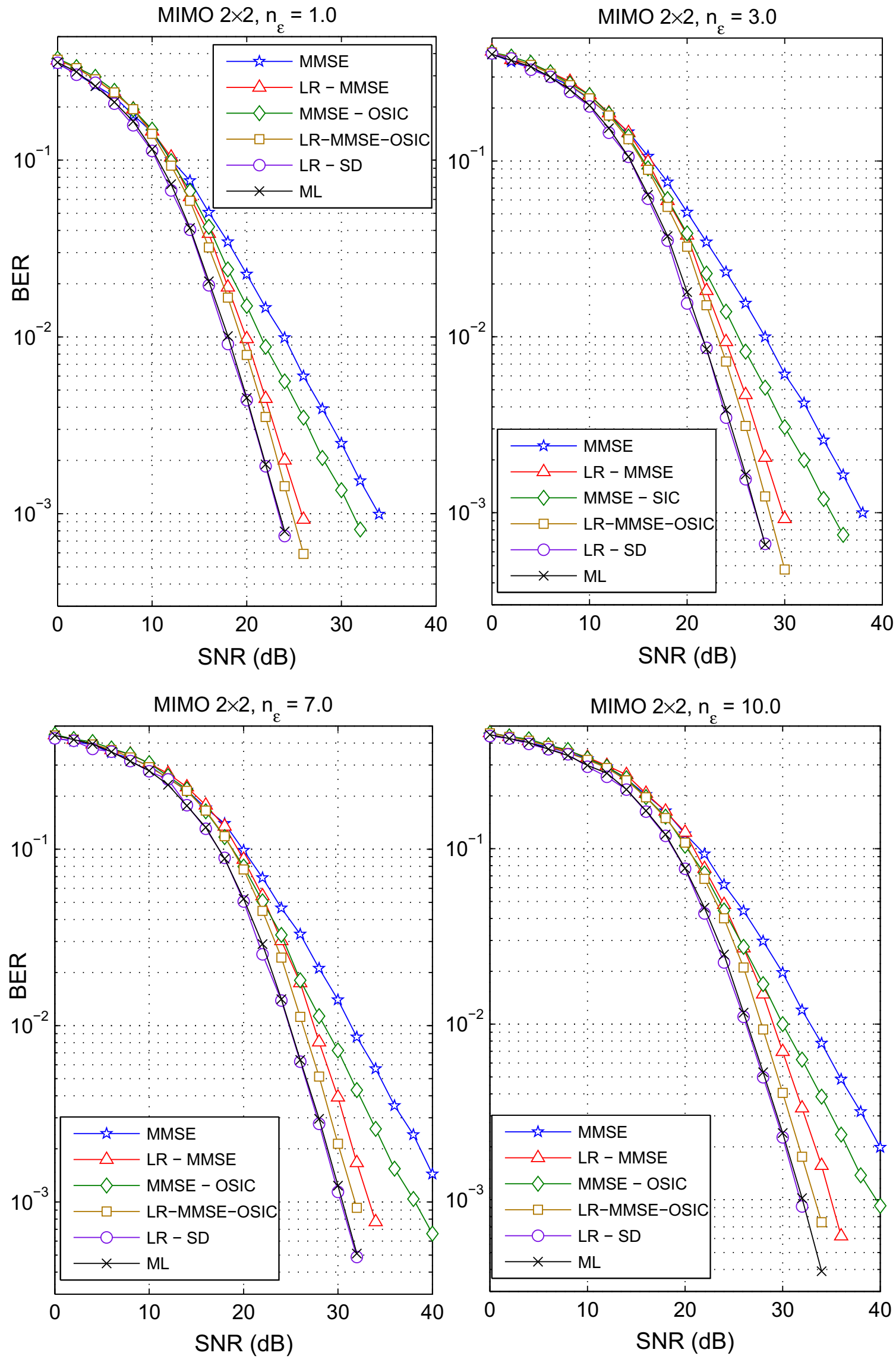

Fig. 4 BER performance for the $2 \times 2$ MIMO detectors with channel errors estimation $\left(n_{\varepsilon}>0\right)$ and uncorrelated antennas $\rho=0$ 
degradation of BER performance is remarkable for all analyzed MIMO detectors. The same degradation observed in the LR-based MIMO detectors and the linear detectors indicates that the LR pre-processing technique does not mitigate completely the CSI estimation errors. Moreover, in Fig. 4 one can observe from the low $\left(n_{\varepsilon}=1.0\right)$ to the very high $\left(n_{\varepsilon}=10.0\right)$ channel error factor scenarios an increasingly performance gap and diversity lost between LR-MMSE and LR-MMSE-OSIC, denoting that the OSIC technique is able to slightly mitigate the performance degradation in very high channel error estimation scenarios. In Fig. 5, the same BER degradation in all implemented MIMO detectors can be observed, representing that the LR can not mitigate the CSI estimation errors in this scenario with a higher number of antennas. Additionaly, with increasing CSI estimation errors, the SNR region when LR-MMSE shows better performance than MMSE becomes higher. In other words, the diversity gain, proportionate by the LR preprocessing technique, is expressed in higher SNR regions with increasing CSI estimation errors, denoting the devasting effects of these estimation errors. As expected, even with strong CSI estimation errors, in both cases (Figs. 4, 5) the LR-SD achieved the ML performance.

For comparison purpose, Fig. 6 put in perspective the impact of errors in CSI considering just the LR-MMSE-OSIC MIMO detector, once its achieved the best performance among the analysed linear detectors. Also, in this scenario is considered uncorrelated channels in order to analyse the isolated effect of CSI estimation errors. It is noticeable that errors in channel estimates are devastating in terms of BER performance degradation, independent on the number of antennas. On the other hand, it is worth to note that the diversity gain remains the same from low to very high channel errors estimation for both $2 \times 2$ and $4 \times 4$ antennas, demonstrating the robustness of the LR-MMSE-OSIC MIMO detection technique. Besides, the BER degradation demonstrates to be more responsive in lower CSI estimation errors, in order that $n_{\varepsilon}$ from 0.5 to 1.0 degraded $3 \mathrm{~dB}$ in BER performance, the same degradation is observed with a longer gap in higher values of CSI estimation error (i.e., $n_{\varepsilon}=7.0$ to $n_{\varepsilon}=10.0$ ).

Figure 7 put in perspective the joint effect of antenna correlation and CSI error estimation on the BER performance degradation, considering 4-QAM MIMO system with $N_{t}=N_{r}=2$. The linear and LR-aided MIMO BER degradations have been calculated for $\rho=[0 ; 0.2 ; 0.5 ; 0.7 ; 0.9]$ combined to $n_{\varepsilon}=[0 ; 1.0 ; 3.0 ; 5.0 ; 7.0]$. In Fig. 7a the LRMMSE-OSIC MIMO detector performance is analysed for different SNR levels and antena correlation, while CSI errors are parameterised via $n_{\varepsilon}$. As expected, depending on the level of channel correlation and CSI error the impact on the BER degradation would be remarkable. Complementary, in Fig. $7 \mathrm{~b}$ the joint effects of channel correlation and CSI error estimates on the BER degradation of various MIMO detectors are analysed considering a fixed $\mathrm{SNR}=20 \mathrm{~dB}$. Notice that for all analysed MIMO detectors the performance degradation is more affected by the error estimation (when $n_{\varepsilon}$ is high), than antenna correlation (high $\rho$ ), which indicate that the effect of channel error estimation on the BER is more devastating than the channel correlation.

\section{Complexity Analysis}

In this section, MIMO detector complexities are compared in terms of floating-point operations per second (flops). Table 1 summarises the overall complexity related to the analysed MIMO detectors, considering $N_{t}=N_{r}=N_{a}$ and $M$ modulation order for the M-QAM. The complexity of the ML-MIMO detector is included as reference. The 
A. M. Mussi et al.
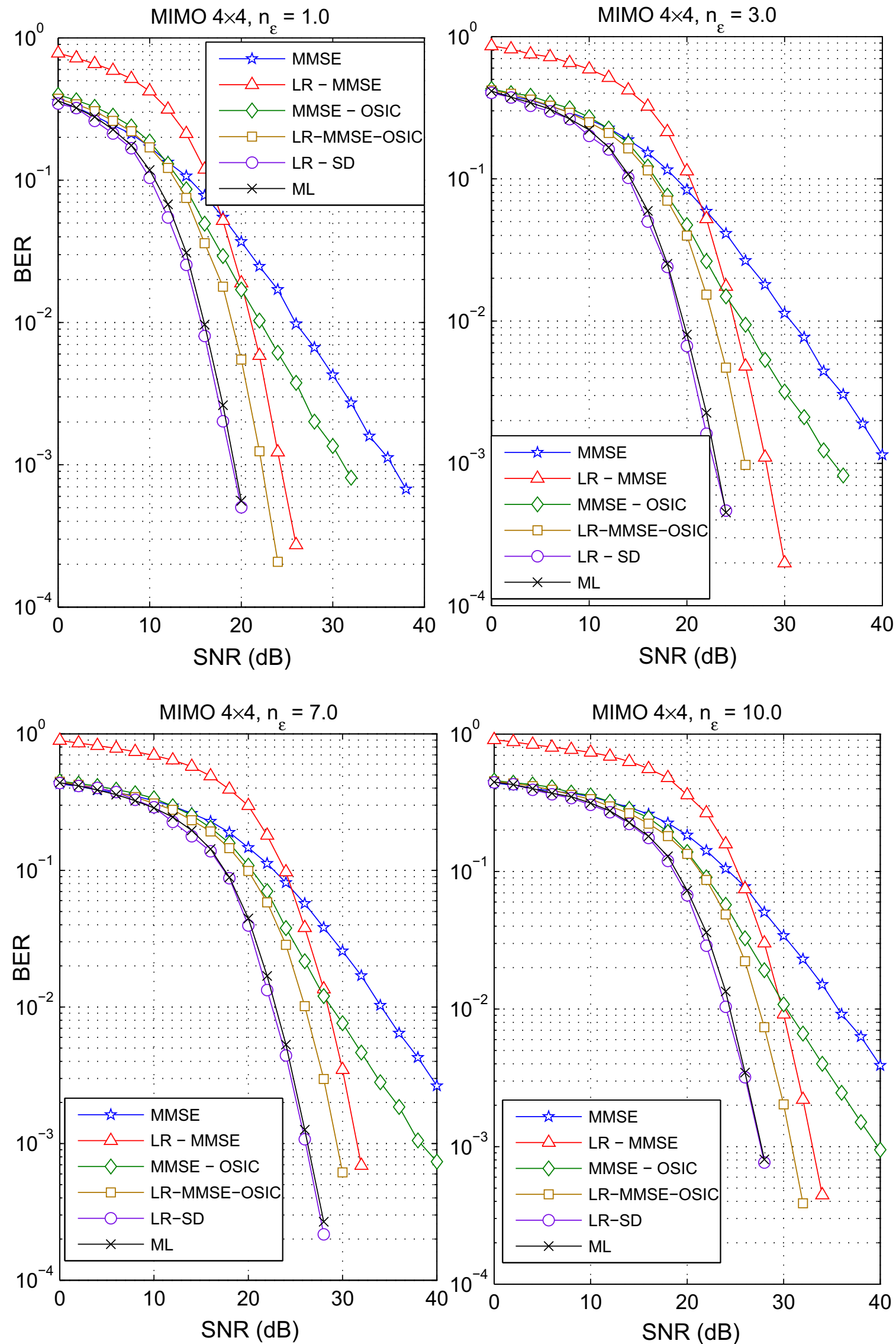

Fig. 5 BER performance for the $4 \times 4$ MIMO detectors with channel errors estimation $\left(n_{\varepsilon}>0\right)$ and uncorrelated antennas, $\rho=0$ 
(a) LR-MMSE-OSIC MIMO $2 \times 2$

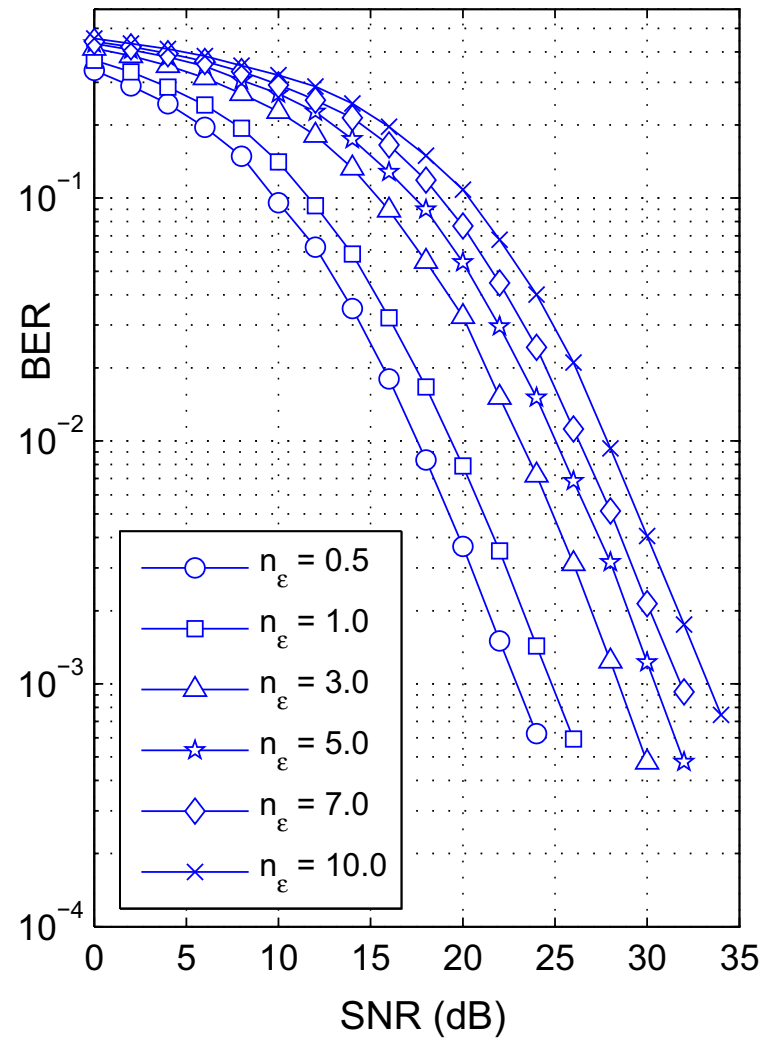

(b) LR-MMSE-OSIC MIMO $4 \times 4$

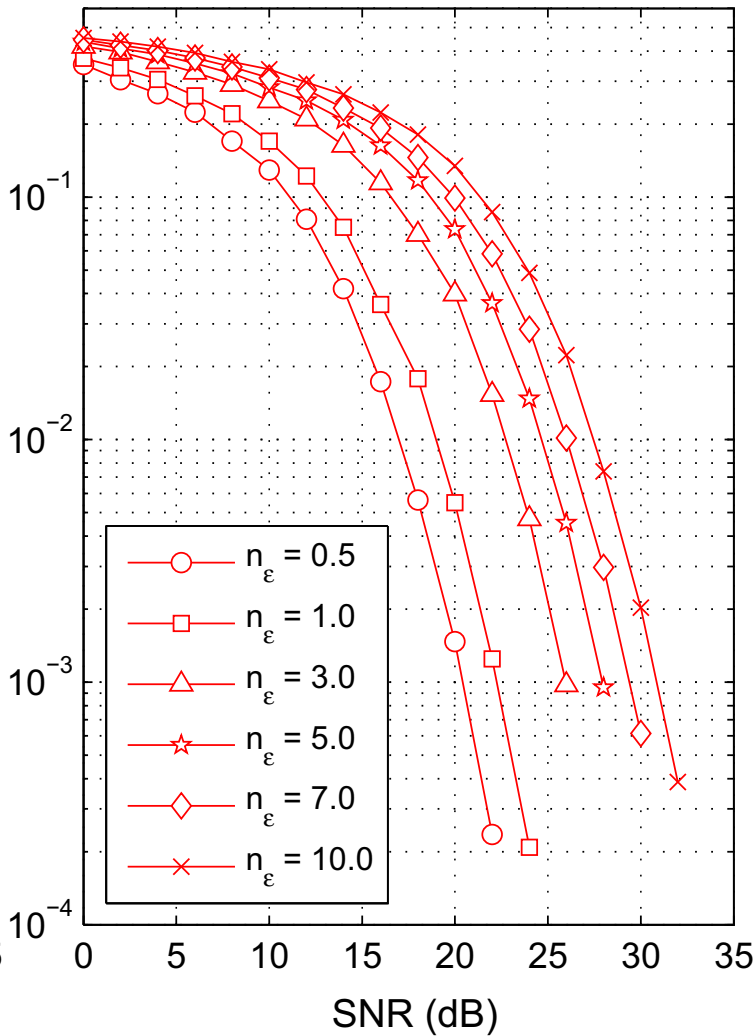

Fig. 6 BER performance degradation due to channel error estimations for the LR-MMSE-OSIC MIMO detector under uncorrelated channels: a $2 \times 2$ antennas; b $4 \times 4$ antennas

complexity is evaluated in terms of the total numbers of floating-point operations (flops), which one flop is defined as an addition, subtraction, multiplication or division between two floating points numbers, and matrix and vector operations flop count are based in [30]. Also, the complexity on the SQRD can be found in [9].

From Table 1, it can be observed that ML has a prohibitive exponential complexity in any practical MIMO system configuration with moderate or higher number of antennas for any channel correlated and estimation errors scenarios. On the other hand, MMSE detector offers a lower complexity, with polynomial order $\mathcal{O}\left(N^{3}\right)$. This complexity order is due to the matrix inversion operation. SIC-based MIMO detectors are capable to provide substantial performance improvement without increase the order of complexity regarding the MMSE MIMO detector; hence SIC-based MIMO topologies are able to offer a suitable BER performance-complexity tradeoff. Furthermore, despite the same polynomial complexity order $\mathcal{O}\left(N^{3}\right)$ achieved by LR-MMSE detectors, this MIMO detector topology presents a second complexity term that could become significant regard the first one, given by the LLL function, $f_{\mathrm{LLL}}$, which is dependent of the level of channel/antennas correlation and number of antennas, as predicted by (28). Under uncorrelated channel/antennas scenarios, the LR-MMSE detectors complexity has dominated by the first term, and this technique can demonstrate an attractive performance-complexity trade-off for these scenarios. Besides, under low to medium correlation indexes, LR-aided MIMO technique shows a affordable and manageable complexity, while preserving full diversity, which makes it a promising near-optimum MIMO transmitting scheme. 
A. M. Mussi et al.
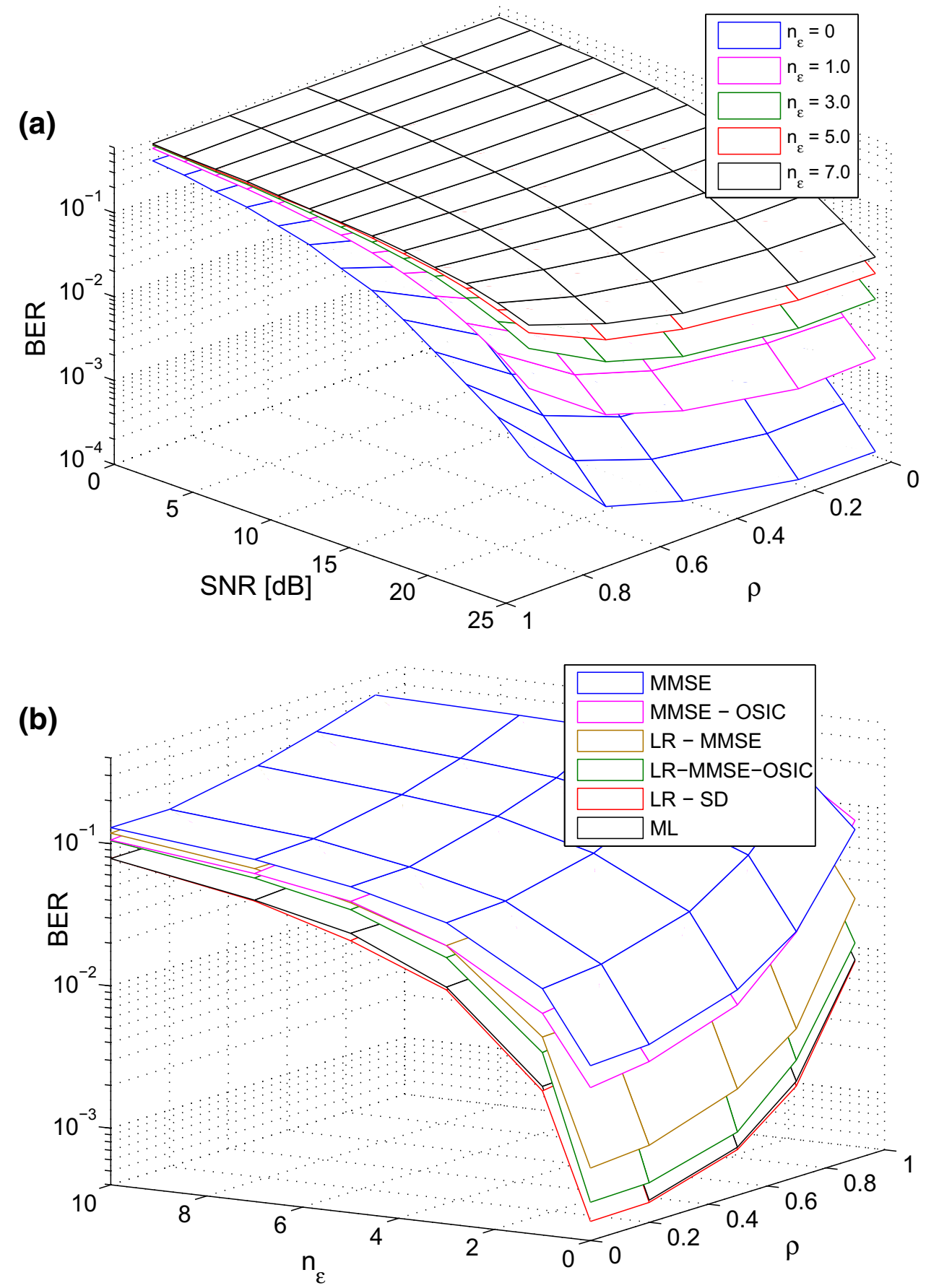

Fig. 7 BER performance degradation under different levels of CSI estimation errors and correlation for $2 \times 2$ antennas. a LR-MMSE-OSIC MIMO detector for different SNRs and correlation; b All considered MIMO detectors with SNR $=20 \mathrm{~dB}$

\subsection{LR-SD Complexity}

The complexity of the LR-SD detector, particularly for 4-QAM, presents the same polynomial complexity order $\mathcal{O}\left(N^{3}\right)$ added to two other functions, $f_{\text {LLL }}$ from the lattice reduction algorithm and $f_{\text {avg-nodes }}$ which is a numerical evaluated complexity relating the number of transmit antennas, the channel correlation and the average SNR per transmit antenna. These three variables affects the complexity of the SD search in such way that the number of visited nodes varies. Moreover, with the knowledge of these variables the 
Table 1 Detectors complexity

\begin{tabular}{ll}
\hline MIMO detector & Number of flops \\
\hline MMSE & $\frac{26}{3} N_{a}^{3}+4 N_{a}^{2}$ \\
MMSE-OSIC & $\frac{40}{3} N_{a}^{3}+\frac{13}{3} N_{a}^{2}+\frac{25}{6} N_{a}$ \\
LR-MMSE & $\frac{26}{3} N_{a}^{3}+4 N_{a}^{2}+f_{\mathrm{LLL}}\left(N_{a}, \rho\right)$ \\
LR-MMSE-OSIC & $\frac{40}{3} N_{a}^{3}+\frac{13}{3} N_{a}^{2}+\frac{25}{6} N_{a}+f_{\mathrm{LLL}}\left(N_{a}, \rho\right)$ \\
LR-SD & $\frac{160}{3} N_{a}^{3}+56 N_{a}^{2}-4 N_{a}+f_{\mathrm{LLL}}\left(N_{a}, \rho\right)+f_{\text {avg-nodes }}\left(N_{a}, \rho, \gamma\right)$ \\
ML & $\left(8 N_{a}^{2}+2 N_{a}\right) M^{N_{a}}$ \\
\hline
\end{tabular}

computational complexity of the sphere decoding search is still stochastic and dependent on the channel conditions and noise level, [31]. Thus, we have numerically evaluated the average number of visited nodes in each layer of the tree, based on these three variables, using Monte-Carlo simulation. The assortment for each layer of the tree is related to the SD search property (triangular upper matrix, R), where each layer results in different size problems, consequently, different number of operations. The results have been obtained using 10,000 channel realisations with $N_{t}$ uncoded complex symbols transmitted in every channel realisation.

\subsubsection{Average Number of Visited Nodes, $\mathcal{N}_{v}$}

Figures 8 and 9 depict the average number of visited nodes $\mathcal{N}_{v}$ in the SNR range from 0 to $20 \mathrm{~dB}$ with $2 \times 2$ and $4 \times 4$ antennas, respectively, obtained by simulation. Figure $8 \mathrm{a}$ describes the $\mathcal{N}_{v}$ for an uncorrelated channel $(\rho=0)$; one can notice that in low SNR region the average nodes visited is higher while $\mathcal{N}_{v}$ decreases, assuming an asymptotic behaviour when SNR increases (high SNR regime). It is also observed that the $\mathcal{N}_{v}$ in the 4th layer presents a stable course among the variation of the SNR due to the organisation of the symbols in the tree search, meaning that the 4th layer is the first layer visited (due to the characteristic of the upper triangular $\mathbf{R}$ matrix). The same behaviour for the number of visited nodes can be observed in Fig. 8b where a strongly correlated channel $(\rho=0.9)$ is considered. Also, it is noted that the under high correlated channels, the asymptotic $\mathcal{N}_{v}$ region tend to occur in higher SNR region, i.e., beyond $20 \mathrm{~dB}$.

The same analysis is depicted in Fig. 9 for $4 \times 4$ antennas. It can be observed the same behaviour for the average number of visited nodes found in Fig. 8 when the SNR increases, but with a higher SNR values. Hence, one can conclude that the number of transmit antennas affect the average number of visited nodes, obviously $\mathcal{N}_{v}$ increases with number of antennas. Besides, in Fig. 9b the asymptotic behaviour of the average number of nodes visited tend to befall in a high SNR region, nevertheless in a much higher SNR region than exposed in Fig. 8b, which indicates that the channel correlation has an impact as great as the number of antennas in the average number of visited nodes. 
A. M. Mussi et al.

(a) LR-aided SD $2 \times 2, \rho=0$

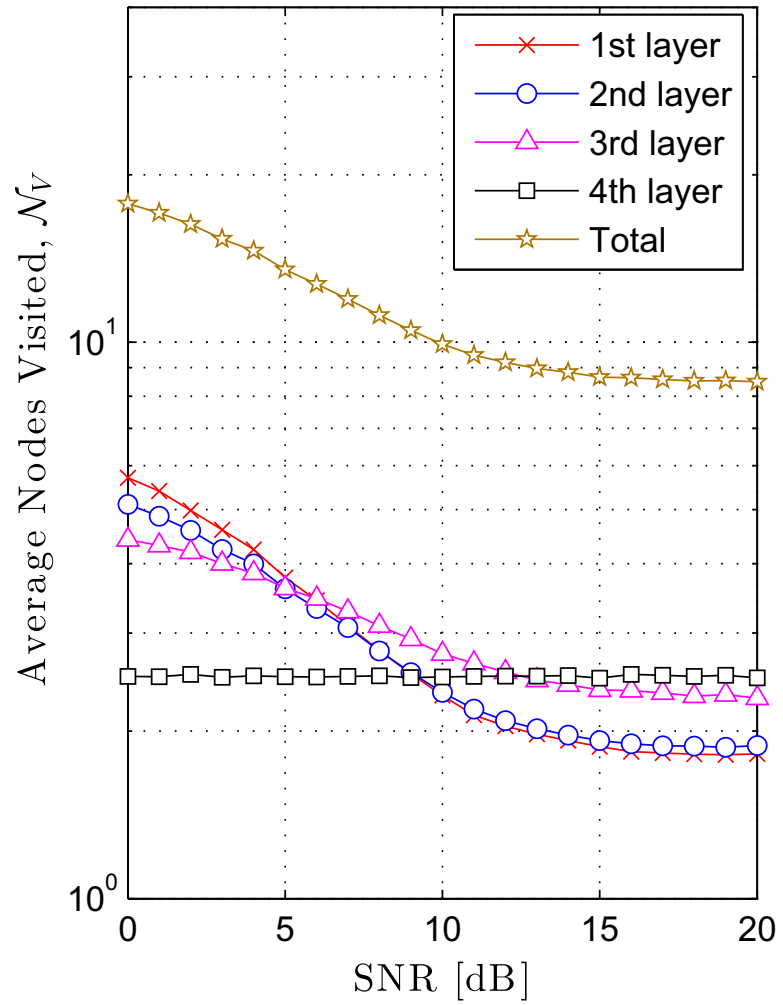

(b) LR-aided SD $2 \times 2, \rho=0.9$

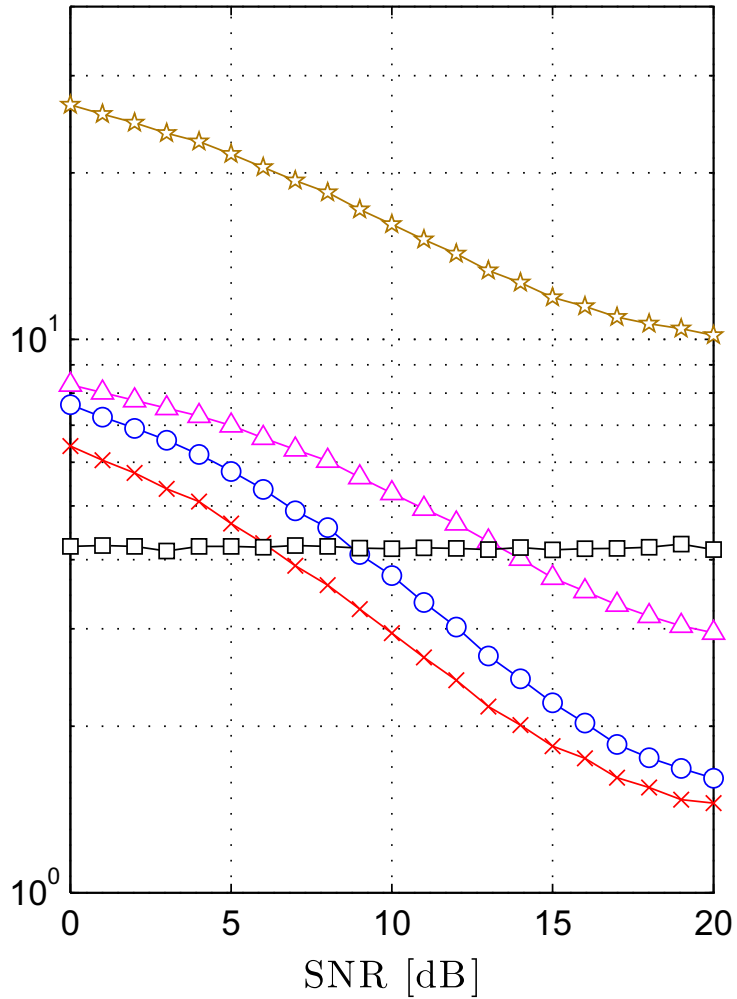

Fig. 8 Average number of visited nodes for the LR-SD MIMO detector with $2 \times 2$ antennas

(a)

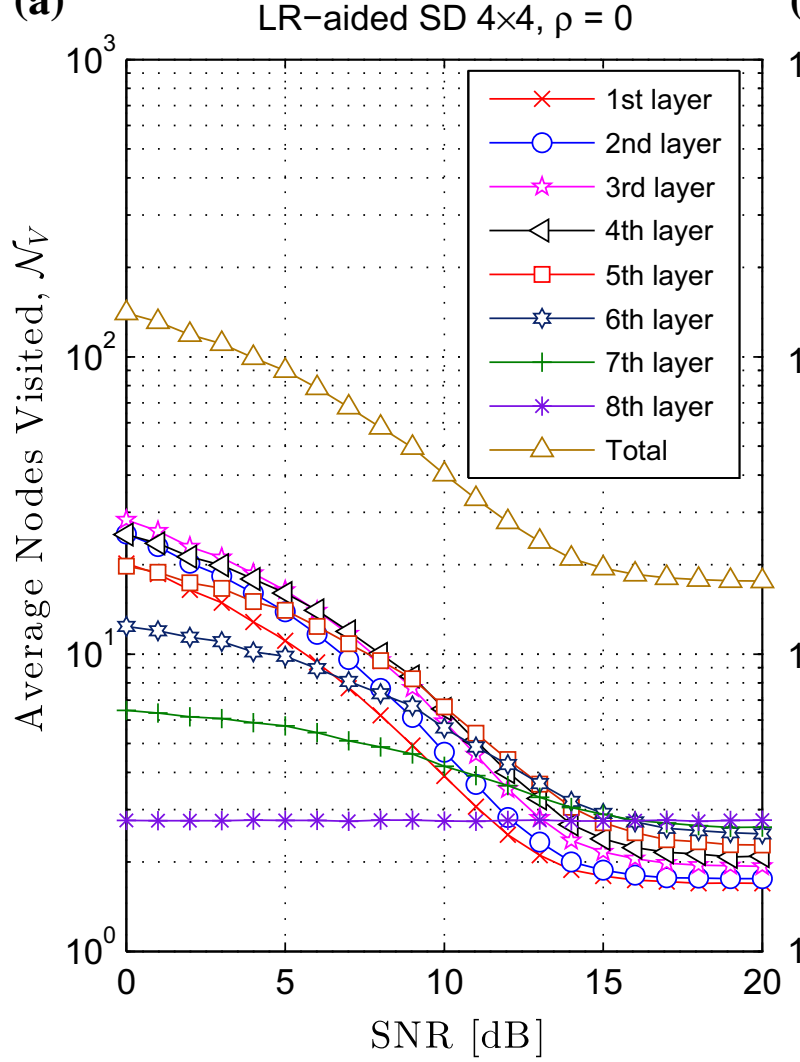

(b)

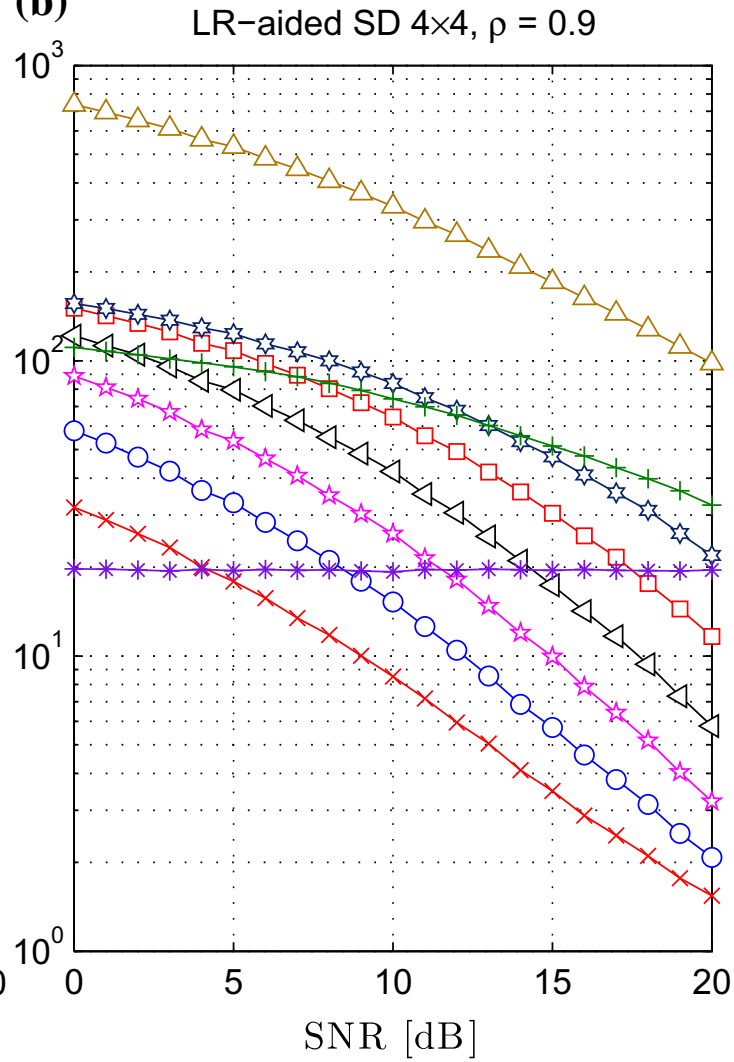

Fig. 9 Average number of visited nodes for the LR-SD MIMO detector with $4 \times 4$ antennas 
(a) $\mathrm{MIMO} 2 \times 2, \rho=0$

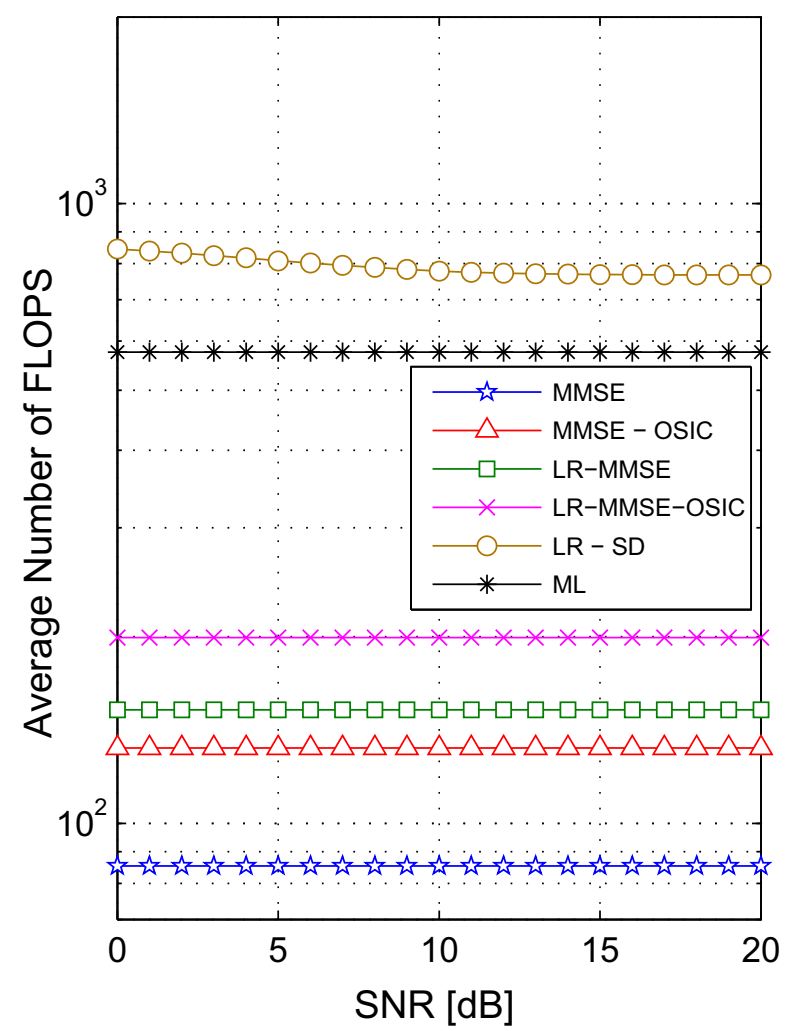

(b) $\mathrm{MIMO} 2 \times 2, \rho=0.9$

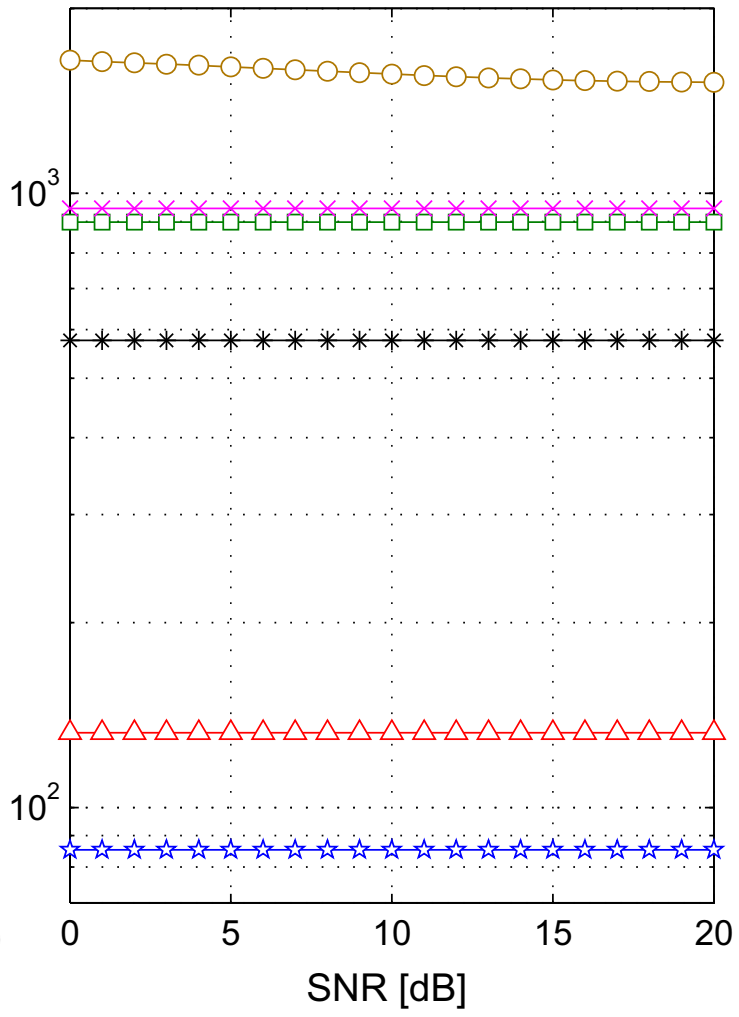

Fig. 10 Flop complexities for the analysed MIMO detectors: $2 \times 2$ antennas

\subsubsection{Average Number of FLOPS}

The computational complexity in terms of average number of FLOPS versus SNR for the MIMO detectors under uncorrelated and highly correlated channels with $2 \times 2$ and $4 \times 4$ antennas are presented in Figs. 10 and 11, respectively. Indeed, Fig. 10a depicts the complexity under uncorrelated channels, where one can notice that for a small problem dimension the average complexity of the LR-SD MIMO detector results greater than ML due to the reduced number of antennas $(2 \times 2)$ and modulation order $(4-\mathrm{QAM})$, indicating that it is not worth the application of this detector under low dimension scenarios. The gap between sub-optimum detectors and their LR-aided versions are due to the $f_{\text {LLL }}$ function computation while it does not represent a significant impact on the overall complexity. In fact, the MMSE-OSIC and LR-MMSE has almost the same complexity in all SNR regions. On the other hand, as depicted in Fig. 1a the performance of LR-MMSE is superior than MMSE-OSIC, specially in high SNR region, as the LR-MMSE can achieve the same diversity order of the ML detector. Moreover, in Fig. 10b the same scenario with strongly correlated channels are presented; it can be observed that the complexity of $f_{\text {LLL }}$ function becomes preponderant on the average number of FLOPS of these LR-aided suboptimal detectors; besides, those LR-aided MIMO detectors results in a higher complexity than ML detector. One can conclude that the application of LR preprocessing in these suboptimal detectors with low $2 \times 2$ number of antennas, low modulation order combined to a strongly correlated channels did not shown effectiveness, due to the high complexity required by the LLL algorithm under these conditions.

However, just increase the number of antennas to $4 \times 4$ is enough to become more attractive and effective the sub-optimum LR-aided MIMO detectors. Figure 11a, b depict 
(a) MIMO $4 \times 4, \rho=0$

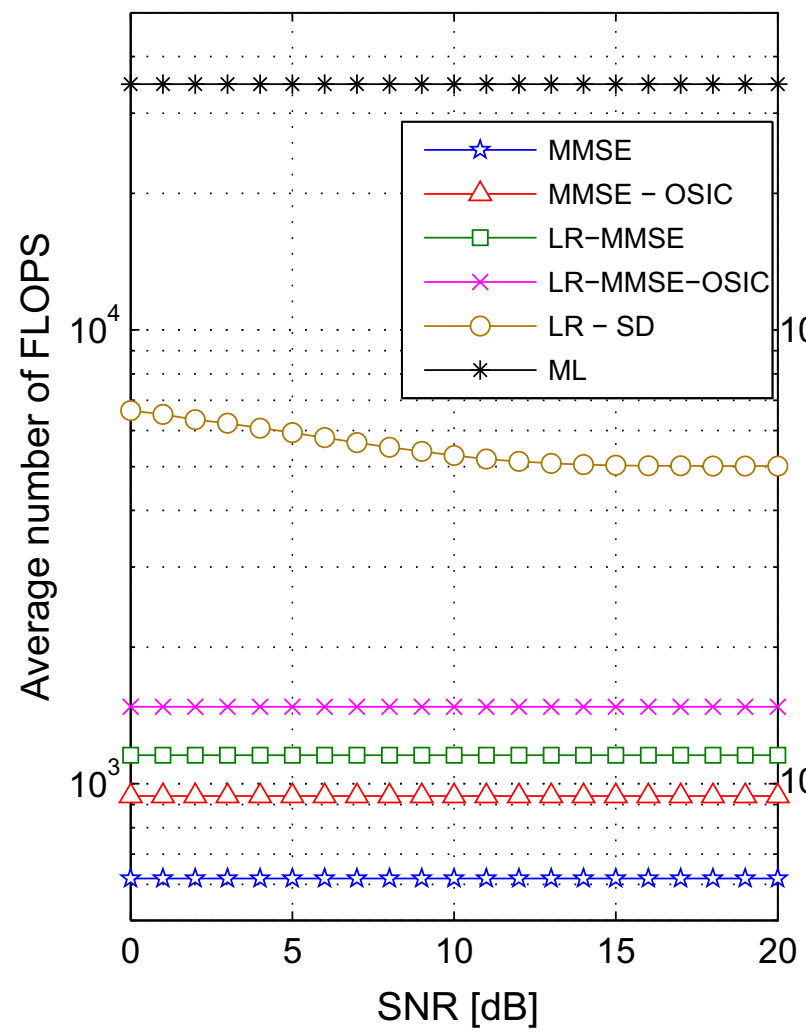

(b) $\mathrm{MIMO} 4 \times 4, \rho=0.9$

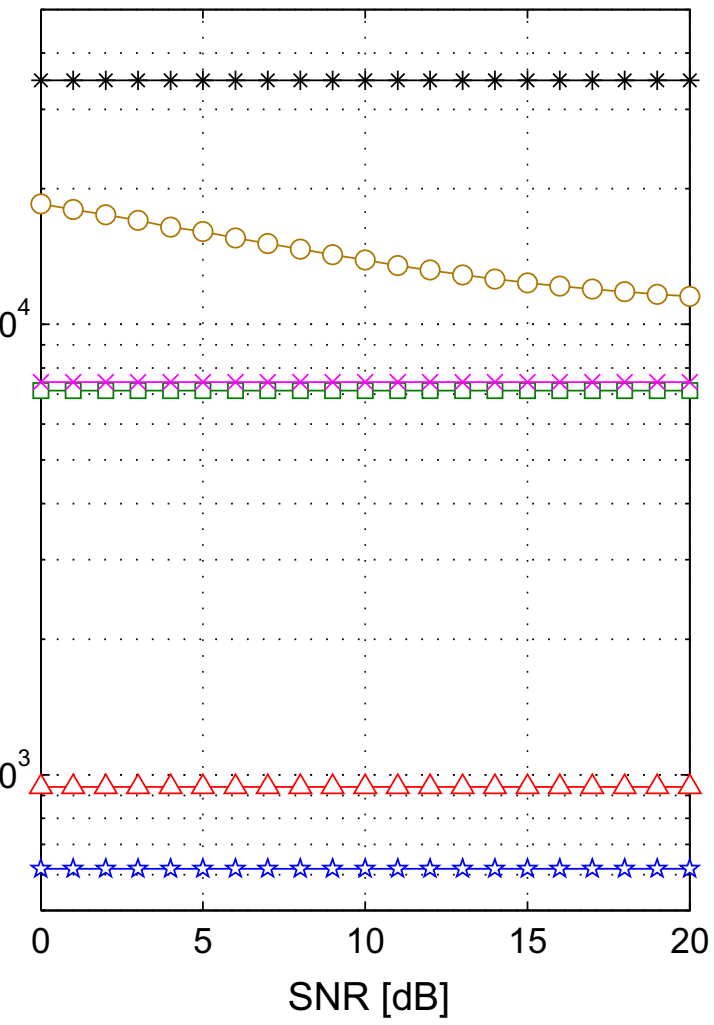

Fig. 11 Flop complexities for the analysed MIMO detectors: $4 \times 4$ antennas

the $4 \times 4$ MIMO detectors FLOPS complexity under uncorrelated $(\rho=0)$ and strongly correlated channels $(\rho=0.9)$, respectively. It is straightforward notice that the ML complexity becomes preponderant among the detectors investigated while LR-SD detector presents a manageable and competitive computational complexity due to the increased number of antennas. Once again, the complexity required by the LLL algorithm does not offer a great impact on the overall complexity of the LR-MMSE detectors. On the other hand, in Fig. $11 \mathrm{~b}$ the additional complexity of $f_{\text {LLL }}$ function is significant, nevertheless it is still lower than ML exhaustive search. Also, due to the increased complexity imposed by the $f_{\mathrm{LLL}}$ function, the OSIC algorithm complexity becomes negligible indicating LRMMSE-OSIC a worth choice due to the better performance presented in Fig. 3. Going further, regarding the to LR-MMSE-OSIC, the LR-SD detector denotes a marginal gap in terms of FLOPS complexity, specially in high SNR region, but achieving optimal performance. Therefore the LR-SD presents a viable MIMO detection option specially under strongly correlated channels due to the attainable ML performance with a computational complexity comparable to the LR-MMSE detectors.

\section{Conclusions}

The correlated channels effects have been demonstrated very significant on the MIMO system performance equipped with polynomial-complexity sub-optimal detectors. Among the analysed MIMO detectors, the LR-aided technique has been demonstrated very useful aiming to improve the performance of those sub-optimal MIMO detectors under antenna correlation. Indeed, numerical results and analyses for channel correlation effect on the 
BER performance of LR-aided MIMO system equipped with different detectors have indicated notable gains in terms of performance and robustness when compared with the respective version without LR-aided detection. However, the MMSE and MMSE-OSIC MIMO detectors have shown a remarkable performance degradation in strongly correlated channels, indicating, in addition, that detectors aided by lattice reduction technique can effectively be an alternative to this degraded scenario, although the complexity introduced by the LLL algorithm has a significant impact on the overall computational complexity under high correlation scenarios, specially in very low number of antennas scenarios where LR-aided MIMO detectors have resulted substantial complexity of the same order of ML exhaustive search. Moreover, among the linear detectors, the LR-MMSE MIMO OSIC detector has achieved the smaller degradation due to the devastating effect of the combined effects of channel correlation and channel error estimation. Indeed, the LR-SD MIMO detector operating under hostile channel conditions achieved optimal performance in all scenarios at a cost of increasing computational complexity with the increment of channel correlation.

Our finding indicates that the LR-SD MIMO detector operating under channel correlation scenarios with medium-large number of $\mathrm{Tx}-\mathrm{Rx}$ antennas and medium-high SNR region is able to achieve the best performance-complexity tradeoff among those analysed MIMO detectors.

Acknowledgments This work was supported in part by the National Council for Scientific and Technological Development (CNPq) of Brazil under Grants 202340/2011-2, and in part by Londrina State University-Paraná State Government (UEL).

\section{Appendix 1: Lookup Table Procedure}

One among main issues in the sphere decoding tree search is related to avoid partial Euclidian distance calculation of symbol vectors already calculated in past iterations. For example, considering two symbol vectors: $s_{1}=\left[\begin{array}{lllll}1 & 1 & -1 & 1\end{array}\right]^{\mathrm{T}} \quad$ and $s_{2}=\left[\begin{array}{llll}-1 & -1 & -1 & 1\end{array}\right]^{\mathrm{T}}$. If the accumulated partial Euclidian distance (PED), starting from the last symbol (upper triangular matrix) on the last two bits of $s_{1}$, i.e. $\left[\begin{array}{ll}-1 & 1\end{array}\right]^{\mathrm{T}}$, exceeds the radius search, then considering the last two bits of $s_{2}$, equal to $s_{1}$, will also exceed radius search, then its calculation is not necessary.

In conventional SD, the tree search procedure is easily performed with simple loops due to the well defined boundary control related to the QAM constellation. Hence, after the LR reduced basis transformation, the system model is considered as described in (43). The boundary control of the transformed symbol vector $\mathbf{d}=\mathbf{T}^{-1} \mathbf{s}_{r}$ is not well defined as in conventional QAM constellation, and becomes a very complicated problem. Thus, the algorithm performs the tree search in all possible transformed symbol vectors, $\mathcal{S}_{\mathrm{LR}}=$ $\left\{\mathbf{d}_{1}, \mathbf{d}_{2}, \ldots, \mathbf{d}_{n}\right\}$ (based on all transformed QAM symbol vectors), storing the accumulated PED in a lookup table, while using this table to avoid same calculations in others equal symbol vectors. The lookup table procedure is described by the pseudo-code shown in Algorithm 5. 


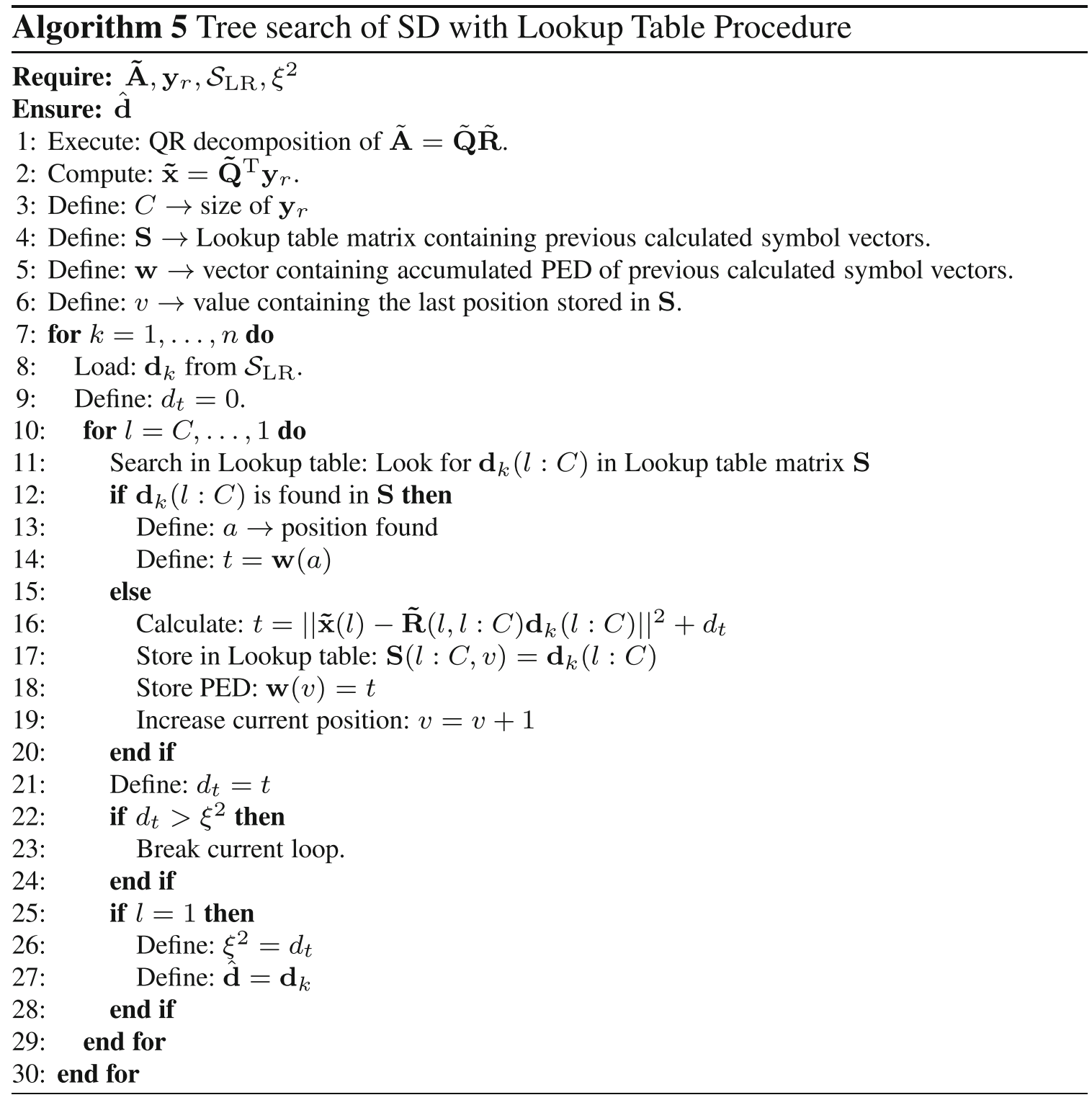

\section{Appendix 2: Optimisation of Squared Radius Increment $\Delta$ in LR-SD Detector}

Given the MIMO system model expressed in (1), with the assumption of NLOS channel, and channels amplitude with Rayleigh distribution and AWGN, the expression $\frac{\|\mathbf{y}-\mathbf{H s}\|^{2}}{\sigma_{\eta}^{2}}$ can be modelled as a Chi-square random variable with $N_{t}$ degrees of freedom, as discussed in [13]. Using this definition and considering the LR-SD system model presented in Sect. 4.4.3, the probability of a lattice point be found inside a hypersphere of squared radius, $\xi_{0}^{2}=\left\|\mathbf{y}_{r}-\tilde{\mathbf{A}} \hat{\mathbf{d}}\right\|$, is denoted by: 


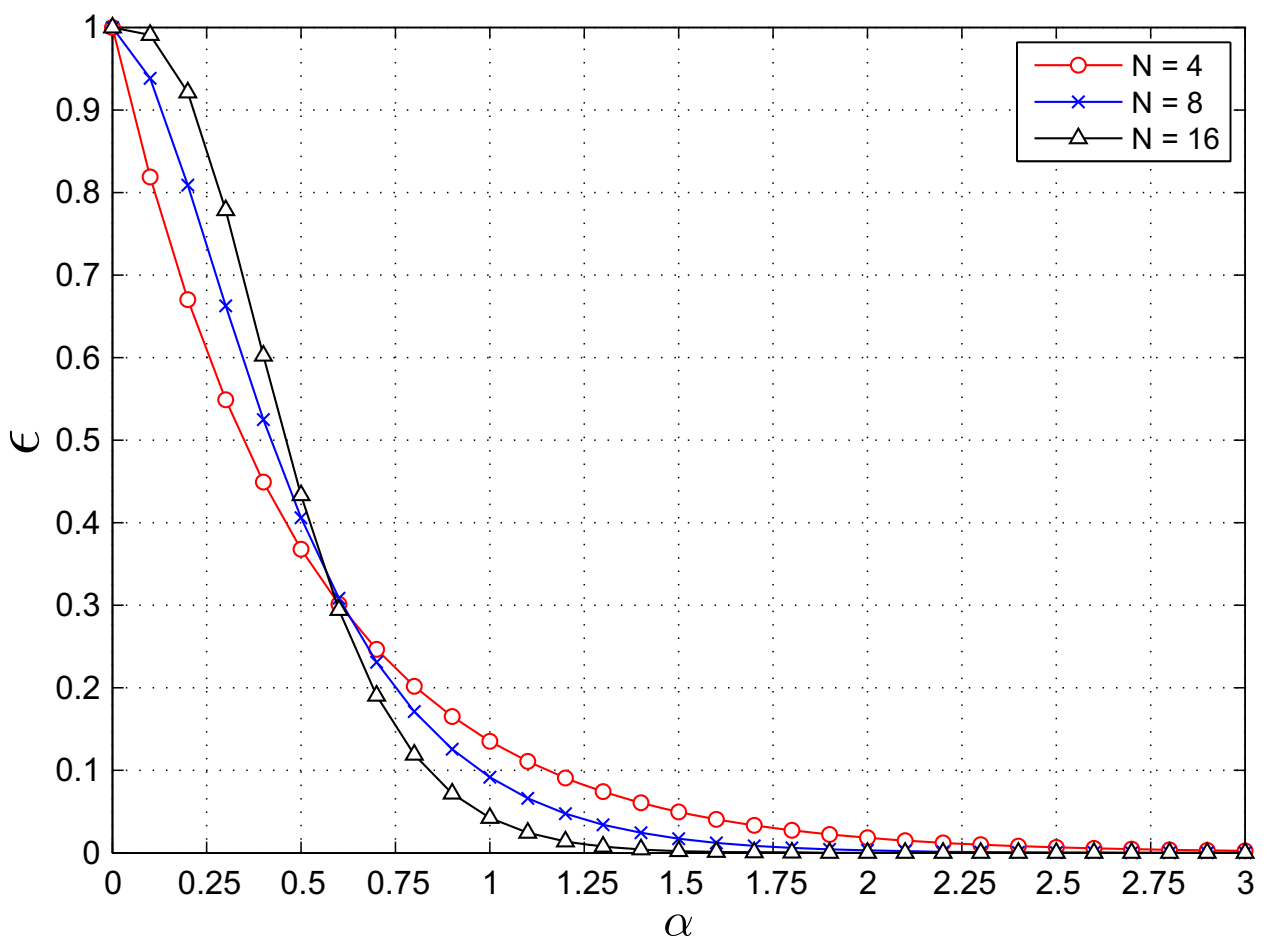

Fig. 12 Relation between $\alpha$ and the null probabilty, $\epsilon$, with different numbers of receive antennas $N=2 N_{t}=4,8$ or 16

$$
\int_{0}^{\frac{\xi_{0}^{2}}{\sigma_{\eta}^{2}}} \frac{\lambda^{\frac{N}{2}-1}}{2^{\frac{N}{2}} \Gamma\left(\frac{N}{2}\right)} e^{-\frac{\lambda}{2}} \mathrm{~d} \lambda=1-\epsilon
$$

where the integrant in (47) is the probability density function (PDF) of a Chi-square random variable with $N$ degrees of freedom and $\Gamma(\cdot)$ is the Gamma function, [32].

Assuming the squared radius increment as a scaled variance noise $\Delta=\alpha N \sigma_{\eta}^{2}$ and replacing the squared radius, $\xi_{0}^{2}$, by $\Delta$ in (47):

$$
\int_{0}^{\alpha N} \frac{\lambda^{\frac{N}{2}-1}}{2^{\frac{N}{2}} \Gamma\left(\frac{N}{2}\right)} e^{-\frac{\lambda}{2}} \mathrm{~d} \lambda=1-\epsilon
$$

In (48) the value of $\alpha$ can be obtained conditioning a high probability of a lattice point is found inside the hypersphere, i.e., with a very small value of the null probability, $\epsilon$. In Fig. 12, it is depicted the relation $\alpha$ versus $\epsilon$ by applying (48) for $N=2 N_{t}=4,8$ or 16 antennas. Hence, one can choice $\alpha>1.25(N=16)$, or $\alpha>1.60(N=8)$ or $\alpha>2.25$ $(N=4)$ to guarantee a high probability of lattice point be found inside the hypersphere.

\section{References}

1. Foschini, G. J., \& Gans, M. J. (1998). On limits of wireless communications in a fading environment when using multiple antennas. Wireless Personal Communications, 6, 311-335.

2. Wübben, D., Seethaler, D., Jaldén, J., \& Matz, G. (2011). Lattice reduction-a survey with applications in wireless communications. IEEE Signal Processing Magazine, 28(3), 70-91.

3. Andalibi, Z., Nguyen, H. H., \& Salt, J. E. (2013). Precoder design for BICM-MIMO systems under channel estimation error. Wireless Personal Communications, 72(4), 2823-2835. 
4. Yeh, C.-J., Wang, L.-C., \& Wu, J.-Y. (2011). On the performance of receive ZF MIMO broadcast systems with channel estimation errors. In 2011 IEEE international conference on communications (ICC), June 2011, pp. 1-5.

5. Valente, R. A., Filho, J. C. M., \& Abrão, T. (2014). LR-aided MIMO detectors under correlated and imperfectly estimated channels. Wireless Personal Communications, 77(1), 173-196.

6. Fang, X., Fang, S., Ying, N., Cao, H., \& Liu, C. (2013). The performance of massive MIMO systems under correlated channel. In 2013 19th IEEE international conference on networks (ICON), Dec 2013, pp. $1-4$.

7. Wübben, D., Böhnke, R., Kühn, V., \& Kammeyer, K.-D. (2004). Near-maximum-likelihood detection of MIMO systems using MMSE-based lattice reduction. In IEEE international conference on communications, Vol. 2, June 2004, pp. 798-802.

8. Böhnke, R., Wübben, D., Kühn, V., Kammeyer, K.-D. (2003). Reduced complexity MMSE detection for BLASTarchitectures. In GLOBECOM'03. IEEE, 2003, pp. 2258-2262.

9. Wübben, D., Böhnke, R., Kühn, V., \& Kammeyer, K.-D. (2003). MMSE extension of V-BLAST based on sorted QRdecomposition. In IEEE semiannual vehicular technology conference (VTC2003-fall), Orlando, Florida, USA, Oct. 2003, pp. 1-5.

10. Mostagi, Y. M., \& Abrão, T. (2012). Lattice-reduction-aided over guided search mimo detectors. International Journal of Satellite Communications Policy and Management, 2, 142-154.

11. Kobayashi, R. T., \& Abrão, T. (2015). Ordered MMSE-SIC via sorted QR decomposition in ill conditioned large-scale MIMO channels. Telecommunication systems, pp. 1-12.

12. Hassibi, B., \& Vikalo, H. (2001). On the expected complexity of spheredecoding. In Conference record of the 35th asilomar conference on signals, systems and computers, 2001, Vol. 2, pp. 1051-1055.

13. Hassibi, B., \& Vikalo, H. (2005). On the sphere-decoding algorithm I. Expected complexity. IEEE Transactions on Signal Processing, 53(8), 2806-2818.

14. Damen, M., El Gamal, H., \& Caire, G. (2003). On maximum-likelihood detection and the search for the closest lattice point. IEEE Transactions on Information Theory, 49(10), 2389-2402.

15. Singh, A., Elia, P., \& Jalden, J. (2012). Achieving a vanishing snr gap to exact lattice decoding at a subexponential complexity. IEEE Transactions on Information Theory, 58(6), 3692-3707.

16. Kim, H., Park, J., Lee, H., \& Kim, J. (2014). Near-ML MIMO detection algorithm with LR-aided fixedcomplexity tree searching. IEEE Communications Letters, 18(12), 2221-2224.

17. Qiao, S. (2008). Integer least squares: Sphere decoding and the LLL algorithm. In Proceedings of the 2008 C3S2E conference, ser. C3S2E '08 (pp. 23-28). New York: ACM.

18. Budihal, S., \& Banakar, R. (2013). Complexity analysis of latticereduction aided increasing radius algorithm in MIMO systems. In3rd International conference on computational intelligence and information technology, 2013. CIIT 2013, pp. 360-366.

19. Zelst, A. V., \& Hammerschmidt, J. S. (2002). A single coefficient spatial correlation model for multipleinput multiple-output (MIMO) radio channels. In Proceedings of URSI XXVIIth general assembly, pp. $1-4$.

20. Wang, J., Li, M., Zhang, Y., \& Zhou, Q. (2008). Effect of channel estimation error on the mutual information of mimo fading channels. In 4th International conference on wireless communications, networking and mobile computing, 2008. WiCOM '08, Oct 2008, pp. 1-4.

21. Bai, L., \& Choi, J. (2012). Low complexity MIMO detection. New York: Springer.

22. Wübben, D., Böhnke, R., Rinas, J., Kammeyer, K.-D., \& Kühn, V. (2001). Efficient algorithm for decoding layered space-time codes. IEE Electronic Letters, 37(22), 1348-1350.

23. James, G. (2010). Modern engineering mathematics. New York: Pearson Education.

24. Horn, R. A., \& Johnson, C. R. (1985). Matrix analysis. Cambridge: Cambridge University Press.

25. Lenstra, A. K., \& Lenstra, H. W. (1982). Factoring polynomials with rational coefficients, Mathematische Annalen, 261(4), 515-534.

26. Wübben, D., Böhnke, R., Kühn, V., \& dirk Kammeyer, K. (2004). Mmse-based lattice-reduction for near-ml detection of mimo systems. In ITG workshop on smart antennas, pp. 18-19.

27. Gama, N., \& Nguyen, P. Q. (2008). Predicting lattice reduction (pp. 31-51). Berlin: Springer. [Online] doi:10.1007/978-3-540-78967-3_3

28. Kobayashi, R. T., Ciriaco, F., \& Abrão, T. (2015). Efficient near-optimum detectors for large MIMO systems under correlated channels. Wireless Personal Communications, 83(2), 1287-1311.

29. Milford, D., \& Sandell, M. (2011). Simplified quantisation in a reduced-lattice mimo decoder. IEEE Communications Letters, 15(7), 725-727.

30. Golub, G. H., \& Loan, C. F. V. (1996). Matrix computations. Baltimore: Johns Hopkins University Press.

31. Barbero, L., \& Thompson, J. (2008). Fixing the complexity of the sphere decoder for mimo detection. IEEE Transactions on Wireless Communications, 7(6), 2131-2142. 
32. Papoulis, A., \& Pillai, S. U. (2002). Probability, random variables and stochastic processes. Europe: McGraw-Hill.

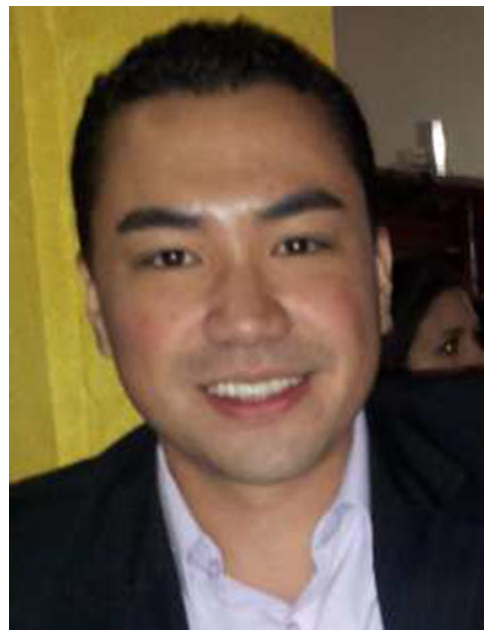

Alex Miyamoto Mussi received his B.S., M. Sc. degress, all in Electrical Engineering, Londrina State University (UEL), PR, Brazil, in 2010 and 2012, respectively. Currently, he is a Ph.D. student at Polytechnic School of the University of São Paulo, São Paulo, Brazil, where he is developing a doctoral thesis in efficient detection techniques in Large MIMO systems. Since November 2011, he works as a Professor of the Control and Industrial Processes Department of Instituto Federal do Paraná, Assis Chateaubriand, PR, Brazil. Since 2011, he is affiliated to the Brazilian Society of Telecommunications (SBrT). His area of expertise involves wireless communications and signal processing, more specially, multi-user detection and estimation on Large MIMO systems.

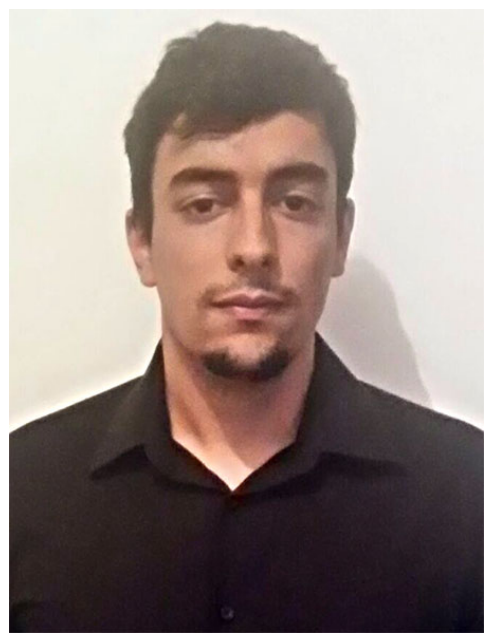

Bruno Felipe Costa received his B. S. degree in Electrical Engineering, Londrina State University (UEL), PR, Brazil in 2016, he worked in detection techniques in MIMO systems during his graduation. His area of expertise involves wireless communication and signal processing, more specially in MIMO systems.

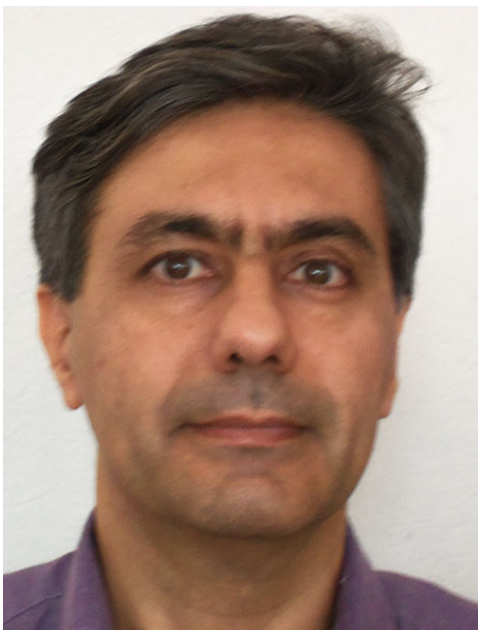

Taufik Abrão received the B.S., M.Sc., and Ph.D. degrees in electrical engineering from the Polytechnic School of the University of São Paulo, São Paulo, Brazil, in 1992, 1996, and 2001, respectively. Since March 1997, he has been with the Communications Group, Department of Electrical Engineering, Londrina State University, Paraná, Brazil, where he is currently an Associate Professor in Telecommunications and head of Telecomm \& Signal Processing Lab. In 2012, he was an Academic Visitor with the Communications, Signal Processing and Control Research Group, University of Southampton, Southampton, U.K. From 2007 to 2008, he was a Post-doctoral Researcher with the Department of Signal Theory and Communications, Polytechnic University of Catalonia (TSC/UPC), Barcelona, Spain. He has participated in several projects funded by government agencies and industrial companies. He is involved in editorial board activities of six journals in the communication area and he has served as TCP member in several symposium and conferences. He has been served as an Editor for the IEEE Communications Surveys \& Tutorials since 2013 the IET Journal of Engineering since 2014 and IEEE Access since 2016. He is a member of SBrT and a senior member of IEEE. His current research interests include communications and signal processing, specially the multi-user detection and estimation, MC-CDMA and MIMO systems, cooperative communication and relaying, resource allocation, 
as well as heuristic and convex optimization aspects of $3 \mathrm{G}$ and $4 \mathrm{G}$ wireless systems. He has supervised 20 MSc and $02 \mathrm{PhD}$ students, co-authored nine book chapters on mobile radio communications and more than two hundred research papers published in specialized/international journals and conferences. (http://www. uel.br/pessoal/taufik). 


\section{APPENDIX B - Full paper published in conference " $X X X I V$} Simpósio Brasileiro de Telecomunicações" (SBrT 2016), August 2016

Title: Damping Factor Analysis on Large Scale MIMO Detector Based on Message Passing. Authors: Alex Miyamoto Mussi and Taufik Abrão.

Conference: XXXIV Simpósio Brasileiro de Telecomunicações.

Publication Date: August 2016. 


\title{
Damping Factor Analysis on Large Scale MIMO Detector Based on Message Passing
}

\author{
Alex Miyamoto Mussi and Taufik Abrão
}

\begin{abstract}
A message passing detector based on belief propagation (BP) algorithm for Markov random field (MRF) graphical model, named MRF-BP, is analysed under large scale MIMO scenarios. The contribution of this work consists of the analysis of message damping method, applied to such MRF-BP detector. A damping factor variation under different number of antennas configuration and SNR regions is considered; BER performance and computational complexity are evaluated over different scenarios. Numerical Results lead to a great performance gain with message damping with no extra computational complexity, although with low number of antennas the damping factor value needs carefully be chosen. Besides, based on the proposed analysis an optimal value for the damping factor is evaluated for different number of antennas scenarios.
\end{abstract}

Keywords - Low complexity MIMO detector; message passing; damping messages; Markov random fields; graphical models.

\section{INTRODUCTION}

Recently, it has been proposed and analyzed communication structures that use tens to hundreds of antennas in transmission and reception of signals, termed large-scale MIMO (LSMIMO) structures, and also called massive MIMO or full dimension MIMO. Such structures hold the same benefits as conventional MIMO, however, in large-scale. More properly LS-MIMO is defined as a transmission/reception design using typically several tens or even hundreds of antennas in at least one of the communication terminals, usually in the BS [1], [2]. The reduced dimensions of user equipments (UEs) suggests a single antenna arrangement in each UE; on the other hand, a huge amount of the antennas is installed in each BS. In an asymptotic model, an infinite number of antennas at the BS is assumed in a LS-MIMO scheme, resulting in paramount advantages: a) effects of noise background and fast fading channel disappear; b) the transmission rate and the number of UEs become independent of cell size; c) spectral efficiency becomes independent of the system bandwidth; and d) power required for transmission of bit tends to zero [3].

However, these advantages are fully achieved since this unlimited number of antennas at the BS meets a fixed number of UEs. These results become very interesting in scenarios with very erroneous channel estimates due to the high noise power (very low SNR): a sufficient increase in the number of BS antennas in a LS-MIMO system is capable of mitigating harmful effect of error in the channel estimation. On the other hand, in multicellular LS-MIMO systems the use of training pilot sequences for channel estimation purpose imposes a intercellular interference in different cells. The problem is

Alex Miyamoto Mussi, PhD student at EPUSP - University of São Paulo and Professor at Federal Institute of Paraná, Assis Chateaubriand, Brazil, e-mail: alex.mussi@ifpr.edu.br; Taufik Abrão, Department of Electrical Engineering, State University of Londrina, Brazil, e-mail: taufik@uel.br called pilot contamination, persisting even in asymptotic BS antennas scenarios [3].

The main advantage achieved with the LS-MIMO scheme refers to high capacity/spectral efficiency [3], [4]; however, with a high number of antennas at BS, the computational complexity of data detection tend to grow proportionally. In this sense, a low computational complexity detector emerges as an essential requirement in LS-MIMO systems, still developing key role to reap the benefits of their high spectral efficiencies. Many low complexity detection procedures for LS-MIMO has been proposed in recent literature, including LS-MIMO detectors based on a) local neighborhood search, such as likelihood ascent search (LAS) algorithm [5], and reactive tabu search (RTS) algorithm [6]; b) promising belief propagation (BP) based algorithms, such that LS-detectors inspired in graphical models, as factor graph (FG) [7] and Markov random field (MRF) [8].

BP based detectors have demonstrated a near optimal performance in LS-MIMO scenarios with low computational complexity [9]. Moreover message passing (MP) algorithms based on BP has been reported, in recent literature, as a promising detection procedure in single-carrier spatial modulation LS-MIMO systems [10]. In some situations, BP algorithm may fail to converge, and if it does converge, the estimated marginals may be far from exact [11]. However, there are several methods in the literature to improve the convergence of BP algorithm, including message damping method [12], [13] and double loop methods [14], [15]. In this work, graphical models and BP procedures are deployed to aid the efficient detection in LS-MIMO systems.

\section{A. Graphical Models}

GMs are graphs that indicate inter-dependencies between random variables [16]. Distributions that exhibit some structure can generally be represented naturally and compactly using a graphical model, that is the case of distributions of interest in MIMO systems (e.g., vector of received symbols). The graphical model structure often allows the statistical distribution of interest to be used effectively for inference, i.e., answering certain questions of interest using the distribution [17]. Three basic graphical models widely used to represent statistical distributions include Bayesian belief networks [18], Markov random fields [19], and factor graphs [20].

A MRF is an undirected graph whose vertices are random variables. The variables are such that any variable is independent of all the other variables, given its neighbors. For instance, a V-BLAST MIMO system can be conveniently represented as a MRF with a node for every symbol. Since every transmit antenna is used to transmit a separate symbol, there are $N_{t}$ nodes in such a graph. Since every transmitted 
XXXIV SIMPÓSIO BRASILEIRO DE TELECOMUNICAÇÕES - SBrT2016, AUGUST 30 TO SEPTEMBER 02, SANTARÉM, PA

symbol interferes with every other transmitted symbol in VBLAST, the graph is fully connected.

\section{B. Belief Propagation}

Belief propagation (BP) is a technique that solves probabilistic inference problems usually implemented in graphical models. BP is a simple, yet highly effective, procedure that has been successfully employed in a variety of applications including computational biology, statistical signal/image processing, data mining, etc. The BP algorithm is now widely recognized an efficient tool that can be used to solve several problems, including communications problems as well [16]. The goal is to detect a hidden input (e.g., transmitted symbol in MIMO systems) from its observed output (e.g., received signal in MIMO systems). The system can be represented as a graphical model and the detection of the system input is equivalent to carry out inference on the corresponding graph. More precisely, BP is a procedure used to compute the marginalization of functions by passing messages on a graphical model [17]. Due to its simplicity and efficiency, BP is the most common strategy adopted to implement message passing principle. Other message passing algorithms can be adressed as generalized distributive law (GDL) [21] and sumproduct algorithm [20].

Due to the applicability and success of BP in LS-MIMO detection [8], [9], [22], [10], a BP detector based on a MRF graphical model is considered in this work. Furthermore, message damping (MD) method is applied and a bit error rate (BER) performance $\times$ damping factor analysis with different antennas configurations in various signal-to-noise ratio (SNR) scenarios is developed in order to evaluate an optimal damping factor value, pointed as the main contribution of this work.

This paper is organized as follow. Section I presents the adopted model for MIMO system, while MRF-based message passing for LS-MIMO detection is discussed in section III. Numerical results are analysed in section IV. Conclusion remarks are provided in section $\mathrm{V}$.

\section{SYSTEM MODEL}

Consider a V-BLAST MIMO communication system with $N_{t}$ transmit antennas and $N_{r}$ receive antennas, for simplicity, the channel is assumed to be a frequency-flat fading channel, characterized by the channel matrix $\mathbf{H}$. The elements of $\mathbf{H}$ are all independent complex Gaussian random variables with zero mean and unit variance. Let $\mathbf{x}$ be the $N_{t} \times 1$ vector corresponding to the BPSK symbols transmitted over the $N_{t}$ transmit antennas, $x \in\{-1,+1\}^{N_{t}}$. The additive white Gaussian noise (AWGN) at any receive antenna is assumed to be a complex Gaussian random variable with zero mean and variance $\sigma^{2}$. The matrix model for the system under investigation is

$$
\mathbf{y}=\mathbf{H x}+\boldsymbol{\eta}
$$

where $\mathbf{H}$ represents an $N_{r} \times N_{t}$ fading coefficients matrix following a Rayleigh distribution (for amplitudes) representing NLOS (non-line-of-sight) communication and $\boldsymbol{\eta}$ is the noise vector samples with:

$$
\mathbb{E}\left[\eta^{2}\right]=N_{0}=\sigma^{2}=\frac{N_{t} \varepsilon_{\mathrm{x}}}{\gamma}
$$

where $\varepsilon_{\mathrm{x}}$ denotes the average energy of the transmitted symbols, $N_{0}$ is the noise power spectral density which is equal to the variance of $\boldsymbol{\eta}$ entries; and $\gamma$ is the average SNR per transmit antenna.

\section{Message PASSING VIA MRF FOR LS-MIMO}

This section presents a BP based detector that employs message passing on an MRF [9]. Consider the system model in Eq. (1). The maximum a posteriori (MAP) detector takes the joint a posterior distribution:

$$
p(\mathbf{x} \mid \mathbf{y}, \mathbf{H}) \propto p(\mathbf{y} \mid \mathbf{x}, \mathbf{H}) p(\mathbf{x})
$$

The MAP estimate of the bit $x_{i}, i=1, \ldots, N_{t}$ is given by

$$
\hat{x}_{i}=\underset{a \in\{-1,+1\}}{\arg \max } p\left(x_{i}=a \mid \mathbf{y}, \mathbf{H}\right)
$$

whose complexity is exponential in $N_{t}$ [17].

Given $\mathbf{x}$ and $\mathbf{H}, \mathbf{y}$ is a complex Gaussian random vector with mean $\mathbf{H x}$ and covariance $\sigma^{2} \mathbf{I}_{N_{r}}$. Thus,

$$
p(\mathbf{y} \mid \mathbf{x}, \mathbf{H}) \propto \exp \left(\frac{-\|\mathbf{y}-\mathbf{H} \mathbf{x}\|^{2}}{2 \sigma^{2}}\right)
$$

Assuming that the symbols in $\mathbf{x}$ are all independent, also necessary for a Markov random fields procedure [17], hence, a priori probability for the transmitted symbol is given by

$$
p(\mathbf{x})=\prod_{i} p\left(x_{i}\right)
$$

From Eqs. (3), (5) and (6), the conditional probability function can be written as:

$$
\begin{aligned}
p(\mathbf{x} \mid \mathbf{y}, \mathbf{H}) & \propto e^{-\frac{1}{2 \sigma^{2}}(\mathbf{y}-\mathbf{H} \mathbf{x})^{\mathrm{H}}(\mathbf{y}-\mathbf{H} \mathbf{x})} \prod_{i} e^{\ln p\left(x_{i}\right)} \\
& \propto e^{\frac{-1}{2 \sigma^{2}}\left(\mathbf{x}^{\mathrm{H}} \mathbf{H}^{\mathrm{H}} \mathbf{H} \mathbf{x}-2 \mathcal{R}\left\{\mathbf{x}^{\mathrm{H}} \mathbf{H}^{\mathrm{H}} \mathbf{y}\right\}\right)} \prod_{i} e^{\ln p\left(x_{i}\right)}
\end{aligned}
$$

Defining $\mathbf{R}=\left(\frac{1}{\sigma^{2}}\right) \mathbf{H}^{\mathrm{H}} \mathbf{H}$ and $\mathbf{z}=\left(\frac{1}{\sigma^{2}}\right) \mathbf{H}^{\mathrm{H}} \mathbf{y}$, Eq. (7) can be rewritten as

$$
\begin{aligned}
p(\mathbf{x} \mid \mathbf{y}, \mathbf{H}) & \propto e^{-\sum_{i<j} \mathcal{R}\left\{x_{i}^{*} R_{i j} x_{j}\right\}} e^{\sum_{i} \mathcal{R}\left\{x_{i}^{*} z_{i}\right\}} \prod_{i} \exp \left(\ln p\left(x_{i}\right)\right) \\
& =\prod_{i<j} e^{-x_{i} \mathcal{R}\left\{R_{i j}\right\} x_{j}} \prod_{i} e^{x_{i} \mathcal{R}\left\{z_{i}\right\}+\ln p\left(x_{i}\right)}
\end{aligned}
$$

where $z_{i}$ and $R_{i j}$ are the elements of $\mathbf{z}$ and $\mathbf{R}$, respectively, and $\mathcal{R}(\cdot)$ denotes the real part of a complex number. Analyzing Eq. (8), it is seen that the MRF of the MIMO system presents pair-wise interactions with the potentials ${ }^{1}$ defined by:

$$
\begin{aligned}
& \psi_{i, j}\left(x_{i}, x_{j}\right)=\exp \left[-x_{i} \mathcal{R}\left\{R_{i j}\right\} x_{j}\right] \\
& \phi_{i}\left(x_{i}\right)=\exp \left[x_{i} \mathcal{R}\left\{z_{i}\right\}+\ln p\left(x_{i}\right)\right]
\end{aligned}
$$

The values of $\psi$ and $\phi$ define, respectively, the edge and self potentials of the MRF graphical model to which message passing algorithm is applied to compute the marginal probabilities of the variables. BP algorithm attempts to estimate the marginal probabilities of all the variables by way of passing messages between the local nodes.

\footnotetext{
${ }^{1}$ A fully connected subgraph of an MRF is called a clique; the variables in an MRF constrained by a compatibility function is known as a (clique) potential.
} 
XXXIV SIMPÓSIO BRASILEIRO DE TELECOMUNICAÇÕES - SBrT2016, AUGUST 30 TO SEPTEMBER 02, SANTARÉM, PA

A message from node $j$ to node $i$ is denoted by $m_{j, i}\left(x_{i}\right)$, and belief at node $i$ is denoted $b_{i}\left(x_{i}\right), x_{i} \in\{ \pm 1\}$. The belief $b_{i}\left(x_{i}\right)$ depends on how likely $x_{i}$ was transmitted. On the other hand, $m_{j, i}\left(x_{i}\right)$ depends on how likely that node $j$ evaluates $x_{i}$ was transmitted. The message from node $i$ to a neighboring node $j$ is then given by:

$$
m_{i, j}\left(x_{j}\right)=\sum_{x_{i}} \phi_{i}\left(x_{i}\right) \psi_{i, j}\left(x_{i}, x_{j}\right) \prod_{k \in \mathcal{N}(i) \backslash j} m_{k, i}\left(x_{i}\right)
$$

where $\mathcal{N}(i)$ denotes the set of all nodes neighboring the node $i$ and $\mathcal{N}(i) \backslash j$ denotes the same neighborhood, except the node $j$. Eq. (11) actually constitutes an iteration, as the message is defined in terms of the messages from other nodes. So, BP essentially involves computing the outgoing messages from a node to each of its neighbors using the local joint compatibility function and the incoming messages and transmitting them [17]. The algorithm terminates after a fixed number of iterations.

\section{A. Message Damping}

The MD method can be used to improve the convergence of BP algorithm. The messages to be passed are computed as a weighted average of the message in the previous iteration and the message in the current iteration [12], [13]. Thus, the damped message to be passed from node $i$ to node $j$ in iteration $t$, denoted by $m_{i, j}^{(t)}\left(x_{j}\right)$, is computed as a convex combination of the previous message and the current message as:

$$
m_{i, j}^{(t)}\left(x_{j}\right)=\alpha m_{i, j}^{(t-1)}\left(x_{j}\right)+(1-\alpha) \tilde{m}_{i, j}^{(t)}\left(x_{i}\right)
$$

where $\tilde{m}_{i, j}^{(t)}\left(x_{j}\right)$ and $m_{i, j}^{(t-1)}\left(x_{j}\right)$ denotes, respectively, the current message in iteration $t$ and the previous message in iteration $t-1$, and $\alpha \in(0,1]$ is referred as the damping factor (DF). This simple damping of messages has been shown to be very effective in improving $\mathrm{BP}$ convergence and performance [12]. As shown in numerical results section, considering the LS-MIMO detection context, message damping method can improve performance significantly, without increasing the computational complexity. Furthermore, the analysis of damping factor in different LS-MIMO configurations is the main contribution of this work.

Damping of messages can be carried out in each iteration. The final belief about the variable $x_{i}$ is computed as:

$$
b_{i}\left(x_{i}\right) \propto \phi_{i}\left(x_{i}\right) \prod_{j \in \mathcal{N}(i)} m_{j, i}\left(x_{j}\right)
$$

In the case of a coded system, the soft output of the algorithm $b_{i}\left(x_{i}\right)$ can be directly fed to the decoder. A pseudocode for the MRF BP described above is listed in Algorithm 1.

\section{Simulation Results}

In this section the uncoded bit error rate (BER) performance related to the MRF-BP algorithm for LS-MIMO detection is evaluated through Monte Carlo simulations. The simulations are performed for a V-BLAST MIMO configuration and assuming that a perfect channel state information (CSI) is available at the receiver side. For comparison purpose, the BER performance of a single-input single-output (SISO) transmission scheme operating in flat fading, as well as in purely AWGN channels were included in several graphs. Table I summarizes the main system and channel parameter values deployed in this section. Also, the number of transmit antennas per mobile user in all scenarios is fixed in $N_{t u}=1$ antenna

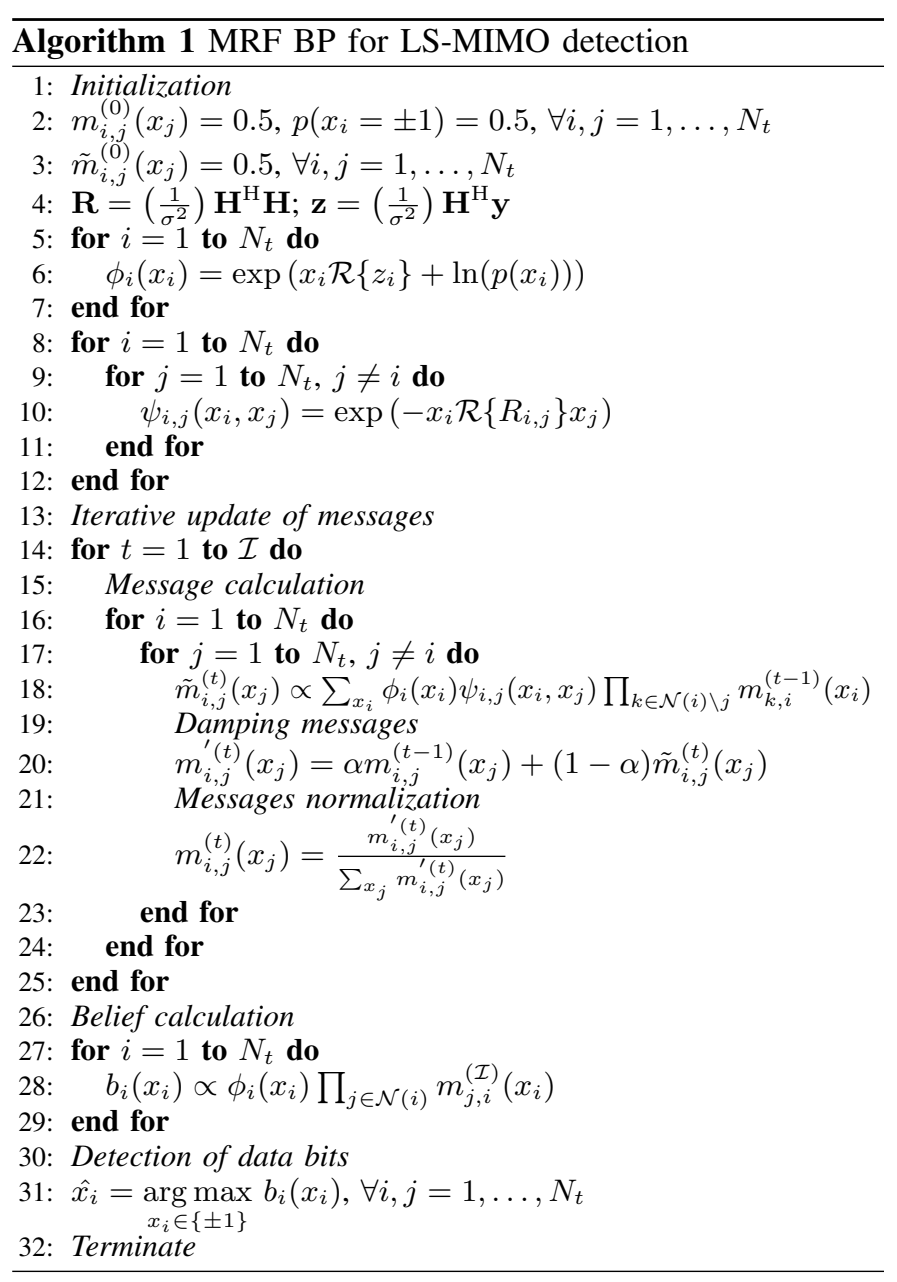

In Fig. 1 the BER performance of the implemented MRFBP algorithm is showed for various $N_{t}=N_{r}=U$ antennas configurations. Moreover, a number of iterations $\mathcal{I}=4$ in BP algorithm were adopted. From Fig. 1.a), one can conclude that under few BP iterations, the LS-MIMO MRF-BP performance tends to the SISO AWGN system performance when the number of antennas increasing, achieving such performance in medium SNR when $N_{t}=500$ antennas. Indeed, with $500 \times 500$ antennas configuration, the BER performance and diversity gain achieved by MRF-BP detector is very close to that of SISO AWGN bound leading to a conclusion that, in such large antennas configuration, the performance of MRFBP detector reaches the asymptotic LS condition and an increasing in $N_{t}=N_{r}$ would not result in a relevant performance gain. From these results, it can be concluded that the MRF-BP detector demonstrates promising BER performance in large-scale MIMO systems. Fig. 1.b) shows the MRF-BP detector with optimal DF value, evaluated from numerical results presented in Figs. 3 and 4. The performance and diversity gain with damping messages is noticeable in all antenna configuration. 
XXXIV SIMPÓSIO BRASILEIRO DE TELECOMUNICAÇÕES - SBrT2016, AUGUST 30 TO SEPTEMBER 02, SANTARÉM, PA

Fig. 2 evidences the influence of the number of iterations $(\mathcal{I})$ of BP algorithm on the BER performance at $\mathrm{SNR}=10 \mathrm{~dB}$ without damping messages, i.e., adopting $\alpha=0$. One can notice the occurrence of a significant performance gain in the first four iterations of Algorithm 1. Besides, with the increasing number of antennas, the performance of MRF-BP detector improves accordingly. For all antennas configuration depicted in this graph, the performance gain occurs, mainly, in the first four iterations, after that, there is no relevant performance gain, i.e., no relevant information is carried out in neighborhood messages.

TABLE I

LS-MIMO SYSTEM AND CHANNEL PARAMETERS

\begin{tabular}{ll}
\hline Parameter & Value \\
\hline Link direction & Uplink (UL) \\
\# Rx antennas & $N_{r} \in[20 ; 50 ; 100 ; 500]$ \\
\# Mobile users & $U=N_{r}$ \\
\# Tx antennas (per user) & $N_{t u}=1$ \\
\# Tx antennas (total) & $N_{t}=U . N_{t u}$ \\
\hline Channel type & flat Rayleigh \\
Channel availability & Perfectly known at receiver \\
Modulation order & BPSK \\
\hline \# iterations MRF-BP & $\mathcal{I} \in\{4,5\}$ \\
\hline
\end{tabular}

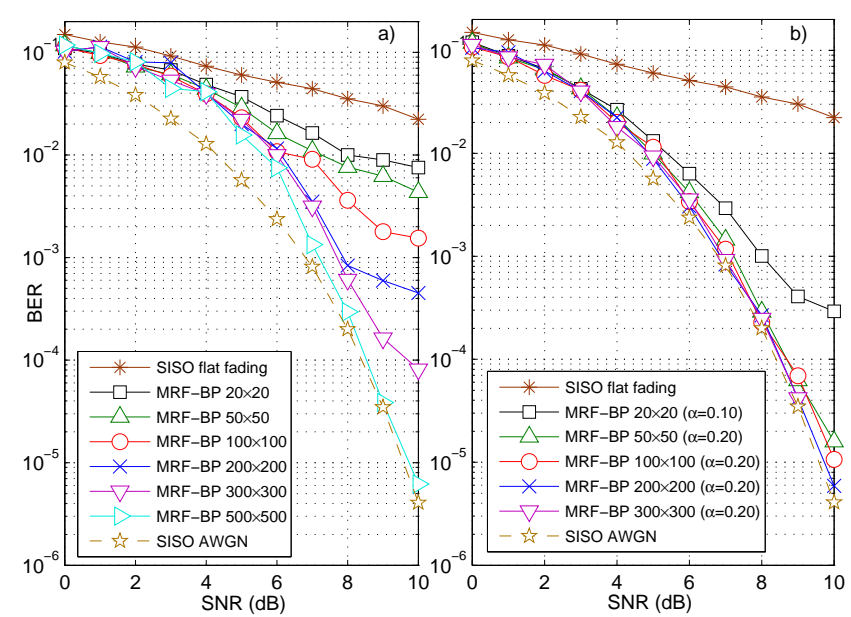

Fig. 1. BER performance of the implemented MRF-BP detector with various $N_{t}=N_{r}$ antennas and $4 \mathrm{BP}$ iterations; a) no dampling messages and b) with optimal DF value.

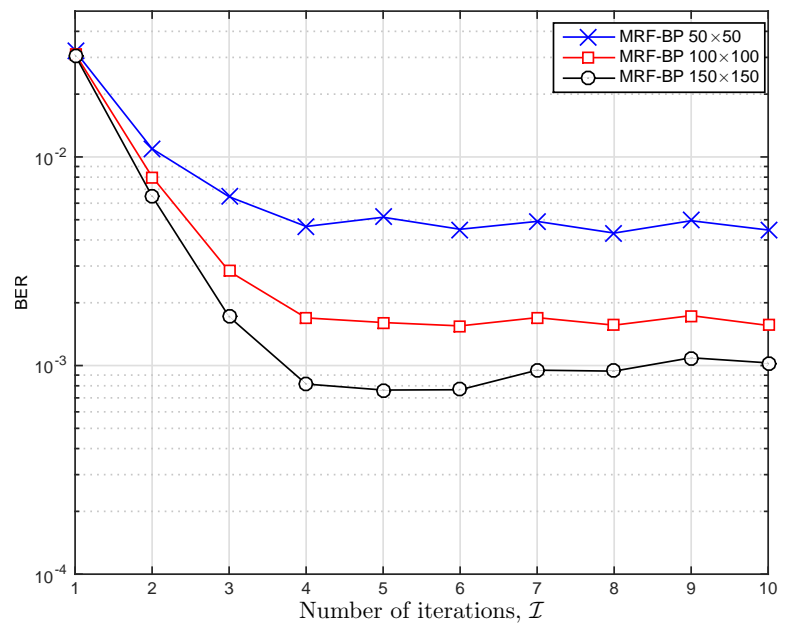

Fig. 2. BP iterations effect on the BER performance; $\mathrm{SNR}=10 \mathrm{~dB}$.

Figs. 3 shows the message damping impact on BER per- formance considering medium-high SNR regions. A MRF-BP detector with message damping variation in the range $0 \leq \alpha \leq$ 1 is considered for $a$ ) $20 \times 20$ and $b$ ) $50 \times 50$ antennas. For both antennas configuration, one can notice that, the performance gain with message damping increases in higher SNR regions; in SNR equal to $6 \mathrm{~dB}$ the performance gain with damping ( $\alpha \neq 0$ ) is less than one decade; on the other hand, in the scenario with $\mathrm{SNR}=12 \mathrm{~dB}$ the performance gain with message damping is approximately 2 and 3 decades for $N_{t}=20$ and 50 antennas, respectively. Specific conclusions regarding the scenario $a$ ) $N_{t}=20$ antennas is that in higher SNR region, the best damping factor, i.e., associated with the lowest BER, has a lower value and tends be more responsive to $\alpha$ variations. Looking at the implicit curve of $\mathrm{SNR}=6 \mathrm{~dB}$, the performance variation from $0.25 \leq \alpha \leq 0.45$ is negligible; in the case of $14 \mathrm{~dB}$, the BER performance degradation from $\alpha=0.05$ to 0.20 is more than one decade. Furthermore, the performance becomes worse regarding no damping messages case $(\alpha=0)$ from $\alpha \geq 0.35$ in $\mathrm{SNR}=14 \mathrm{~dB}$; the same situation occurs from $\alpha \geq 0.80$ when $\mathrm{SNR}=6 \mathrm{~dB}$. Therefore, the $\mathrm{DF}$ value needs to be accurately chosen, specially in medium/high SNR regions.

a)
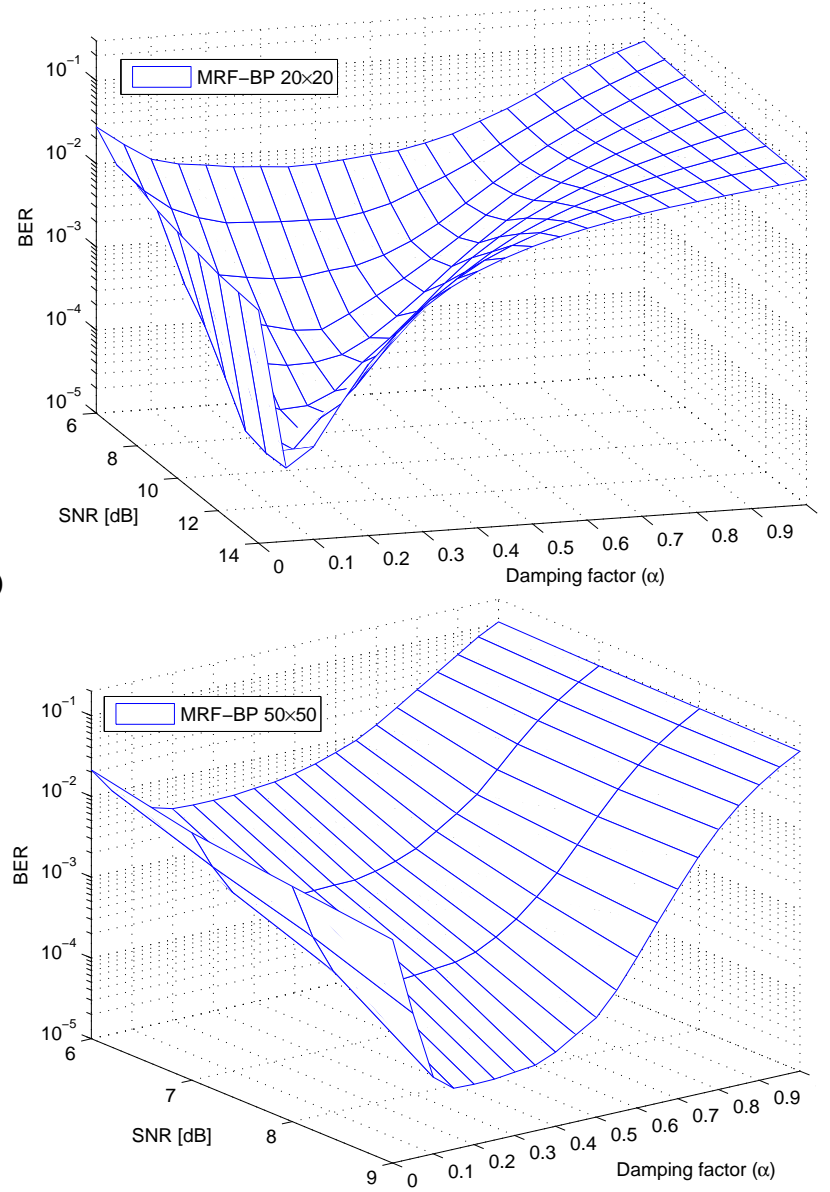

b)

Fig. 3. MRF-BP LS-MIMO performance, $\mathcal{I}=5$, as a function of different SNR scenarios and damping factor $\alpha$ : a) $20 \times 20$; b) $50 \times 50$ antennas

From Fig. 4, it is distinguishable that, when $N_{t}$ of LSMIMO system increases, e.g., from $20 \times 20$ to $100 \times 100$ antennas, the BER performance behaviour with damping factor demonstrates constant (flat condition) at greater intervals of $\alpha$. 
The same way that, with $50 \times 50$ antennas the performance is flatter than $20 \times 20$ case (Fig. 3.a and 3.b). One can conclude that, the difficult task to accurately choose the DF value in small number of LS-MIMO antennas scenario, i.e., $20 \times 20$, is relaxed with increasing number of antennas. Thus, the application of message damping, specially in high SNR region, is more suitable in LS-MIMO systems due to the flat BER performance response to $\mathrm{DF}$ variation.

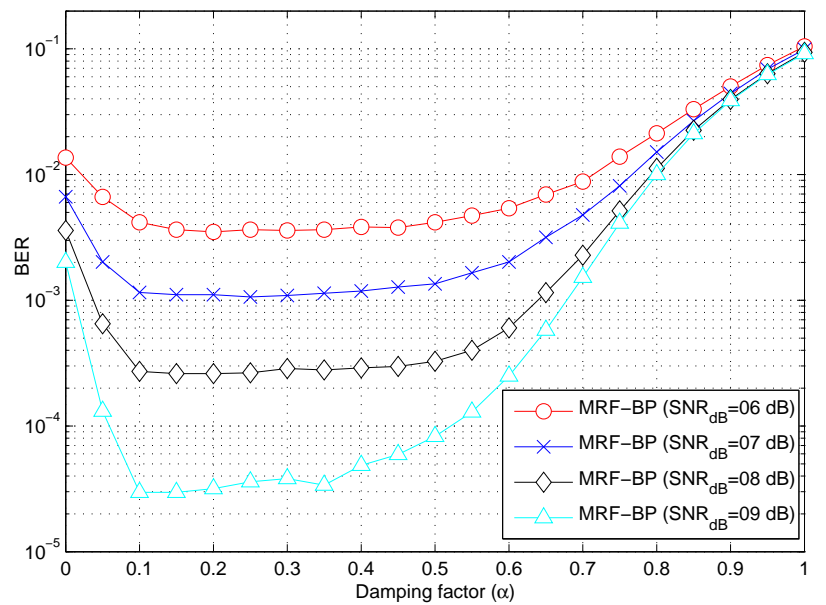

Fig. 4. Variation of damping factor on the BER performance of a MRF-BP $100 \times 100$ considering different SNR scenarios; $\mathcal{I}=5$.

\section{A. Computational Complexity of MRF-BP LS-MIMO detector}

The computational complexity is described in terms of floating-point operations, which one floating-point operation denotes the computational complexity of the real mathematical operations: addition, subtraction, multiplication or division. Table II describes the per-symbol computational complexity involved in each step of MRF-BP algorithm. The overall persymbol complexity of the Algorithm 1 is about $\mathcal{O}\left(N_{t}^{2}\right)$. It is important to note that message damping does not increase the computational complexity of the MRF-BP algorithm.

TABLE II

COMPUTATIONAL COMPLEXITY OF MRF-BP ALGORITHM.

\begin{tabular}{ccc}
\hline Procedure & Algoritm 1 & Per-symbol Complexity \\
\hline $\mathbf{R}$ & line 4 & $N_{r}-1$ \\
$\mathbf{z}$ & line 4 & $N_{r}-1$ \\
$\phi, \quad$ eq. $(9)$ & line 6 & 4 \\
$\psi, \quad$ eq. $(10)$ & line 10 & $12 N_{t}$ \\
Messages update & lines 15 to 25 & $\left(4 N_{t}^{2}+4 N_{t}-8\right) \cdot \mathcal{I}$ \\
Message damping & line 20 & $\frac{4}{N_{t}}($ negligible $)$ \\
\hline
\end{tabular}

\section{CONCLUSIONS}

A detector for LS-MIMO systems based on message passing MRF graphical model and BP algorithm was analyzed. More specifically, message damping method impact on the BER performance was evaluated and has demonstrated a promising method for message passing detectors, specially in LS-MIMO scenarios. Numerical results for the MRF-BP LS-MIMO detector has demonstrated promising performance $\times$ complexity tradeoff, since damping messages procedure just increases marginally the overall computational complexity while providing a significant performance gain. Besides, under large scale antenna scenarios, the DF value choose is facilitated due to the flat BER performance response to $\alpha$ variation.

\section{REFERENCES}

[1] J. Hoydis, S. ten Brink, and M. Debbah, "Massive mimo in the ul/dl of cellular networks: How many antennas do we need?" Selected Areas in Communications, IEEE Journal on, vol. 31, no. 2, pp. 160-171, 2013.

[2] F. Rusek, D. Persson, B. K. Lau, E. Larsson, T. Marzetta, O. Edfors, and F. Tufvesson, "Scaling up mimo: Opportunities and challenges with very large arrays," Signal Processing Magazine, IEEE, vol. 30, no. 1, pp. 40-60, 2013.

[3] T. Marzetta, "Noncooperative cellular wireless with unlimited numbers of base station antennas," Wireless Communications, IEEE Transactions on, vol. 9, no. 11, pp. 3590-3600, 2010.

[4] K. Zheng, L. Zhao, J. Mei, B. Shao, W. Xiang, and L. Hanzo, "Survey of large-scale mimo systems," IEEE Communications Surveys \& Tutorials, vol. 17, no. 3, pp. 1738-1760, thirdquarter 2015.

[5] K. Vardhan, S. Mohammed, A. Chockalingam, and B. Rajan, "A lowcomplexity detector for large mimo systems and multicarrier cdma systems," Selected Areas in Communications, IEEE Journal on, vol. 26, no. 3, pp. 473-485, 2008.

[6] B. Rajan, S. Mohammed, A. Chockalingam, and N. Srinidhi, "Lowcomplexity near-ml decoding of large non-orthogonal stbcs using reactive tabu search," in Information Theory, 2009. ISIT 2009. IEEE International Symposium on, 2009, pp. 1993-1997.

[7] P. Som, T. Datta, A. Chockalingam, and B. S. Rajan, "Improved largemimo detection based on damped belief propagation," in Information Theory (ITW 2010, Cairo), 2010 IEEE Information Theory Workshop on, 6-8 Jan. 2010, pp. 1-5.

[8] M. Suneel, P. Som, A. Chockalingam, and B. S. Rajan, "Belief propagation based decoding of large non-orthogonal stbcs," in Information Theory, 2009. ISIT 2009. IEEE International Symposium on, June 28 2009-July 3 2009, pp. 2003-2007.

[9] P. Som, T. Datta, N. Srinidhi, A. Chockalingam, and B. S. Rajan, "Lowcomplexity detection in large-dimension mimo-isi channels using graphical models," IEEE Journal of Selected Topics in Signal Processing, vol. 5, no. 8, pp. 1497-1511, Dec. 2011.

[10] P. Yang, Y. Xiao, Y. L. Guan, K. V. S. Hari, A. Chockalingam, S. Sugiura, H. Haas, M. D. Renzo, C. Masouros, Z. Liu, L. Xiao, S. Li, and L. Hanzo, "Single-carrier spatial modulation: A promising design for large-scale broadband antenna systems," pp. 1-1, 2016.

[11] J. M. Mooij and H. J. Kappen, "Sufficient conditions for convergence of the sum-product algorithm," IEEE Transactions on Information Theory, vol. 53, no. 12, pp. 4422-4437, Dec. 2007.

[12] K. P. Murphy, Y. Weiss, and M. I. Jordan, "Loopy belief propagation for approximate inference: An empirical study," in Proc. Fifteenth Conference on Uncertainty in Artificial Intelligence, San Francisco, 1999, pp. 467-475.

[13] M. Pretti, "A message-passing algorithm with damping," Journal of Statistical Mechanics: Theory and Experiment, vol. 2005, no. 11, p. P11008, 2005.

[14] A. L. Yuille, "A double-loop algorithm to minimize the bethe and kikuchi free energies," NEURAL COMPUTATION, vol. 14, p. 2002, 2001.

[15] T. Heskes, K. Albers, and B. Kappen, "Approximate inference and constrained optimization," in Proceedings of the Nineteenth Conference on Uncertainty in Artificial Intelligence, ser. UAI'03. San Francisco, CA, USA: Morgan Kaufmann Publishers Inc., 2003, pp. 313-320.

[16] B. J. Frey, Graphical Models for Machine Learning and Digital Communication. Cambridge, MA, USA: MIT Press, 1998.

[17] A. Chockalingam and B. Rajan, Large MIMO Systems. Cambridge University Press, 2014.

[18] D. Heckerman and M. P. Wellman, "Bayesian networks," Commun. $A C M$, vol. 38, no. 3, pp. 27-30, Mar. 1995.

[19] D. Griffeath, Introduction to Markov Random Fields, in Denumerable Markov Chains, J. G. Kerney, J. L. Snell and A.W. Knupp, 2nd ed. New York: Springer-Verlag, 1976.

[20] F. R. Kschischang, B. J. Frey, and H. A. Loeliger, "Factor graphs and the sum-product algorithm," IEEE Transactions on Information Theory, vol. 47, no. 2, pp. 498-519, Feb 2001.

[21] S. M. Aji and R. J. McEliece, "The generalized distributive law," IEEE Transactions on Information Theory, vol. 46, no. 2, pp. 325-343, Mar 2000.

[22] T. L. Narasimhan, A. Chockalingam, and B. S. Rajan, "Factor graph based joint detection/decoding for ldpc coded large-mimo systems," in Vehicular Technology Conference (VTC Spring), 2012 IEEE 75th, 6-9 May 2012, pp. 1-5. 


\section{APPENDIX C - Full paper published in the journal "IET} Signal Processing", July 2017

Title: Message passing detection for large-scale MIMO systems: damping factor analysis.

Authors: Alex Miyamoto Mussi and Taufik Abrão.

Journal: IET Signal Processing (ISSN 1751-9675).

Classification (Qualis-CAPES 2016): A2 (Engenharias IV).

Impact Factor (2017): 1.250.

Publication Date: July 2017.

DOI: $10.1049 /$ iet-spr.2017.0148 


\section{Message passing detection for large-scale MIMO systems: damping factor analysis} systems: damping factor analysis
ISSN 1751-9675

Received on 10th April 2017 Revised 25th May 2017 Accepted on 2nd June 2017 E-First on 18th July 2017 doi: 10.1049/iet-spr.2017.0148 www.ietdl.org

\author{
Alex Miyamoto Mussi ${ }^{1,2}$, Taufik Abrão ${ }^{3} 凶$ \\ ${ }^{1}$ Federal Institute of Paraná, Assis Chateaubriand, Paraná, Brazil \\ ${ }^{2}$ (PhD student), EPUSP - University of São Paulo, São Paulo, Brazil \\ ${ }^{3}$ Electrical Engineering Department, Londrina State University, Londrina, Paraná, Brazil \\ 凶-mail: taufik@uel.br
}

\begin{abstract}
A message passing detector based on belief propagation (BP) algorithm for Markov random fields (MRF-BP) and factor graph (FG-BP) graphical models is analysed under different large-scale (LS) multiple-input multiple-output (MIMO) scenarios, including system parameters, such as damping factor (DF), number of users and number of antennas, from $N=20$ to 200 antennas. Specifically, the DF variation under different number of antennas configuration and signal-to-noise ratio (SNR) regions is extensively evaluated; bit error rate (BER) performance and computational complexity are assessed over different scenarios. Numerical results lead to a great performance gain with damped MRF-BP approach, overcoming FG-BP scheme in specific scenarios, with no extra computational complexity. Also, message damping (MD) method resulted in faster convergence of MRF-BP algorithm in LS scenarios, evidencing that, besides the performance gain, MD technique can lead to a computational complexity reduction. Specifically under low number of transmit antennas scenarios, the DF value needs to be carefully chosen. Furthermore, based on the proposed analysis, optimal value for the DF is determined considering wide LS antennas scenarios and SNR regions.
\end{abstract}

\section{Introduction}

In the last decade, technological advances in wireless and cellular communication have enabled progressive reductions in size and power consumption of mobile terminals. At the same time, the availability of multimedia services is supplying an increasing demand from users and coverage areas and it is predicted that this trend should continue in the coming years [1]. However, physical resources (e.g. spectrum, time and users spatial distribution) necessary for wireless data transmission remain the same. Therefore, the wireless communication systems need to be innovative in the sense as to coordinate coverage area, being more efficient in bandwidth, power and able to operate in different scenarios: urban, suburban and rural, interior and exterior, with maintenance of quality of service.

In a point-to-point multiple antennas (MIMO) communication, the channel capacity increases linearly with the minimum number of transmitting and receiving antennas [2, 3]. Recently, it has been proposed and analysed communication structures that use tens to hundreds of antennas in transmission and reception of signals, termed large-scale MIMO (LS-MIMO) structures, and also called massive MIMO or full-dimension MIMO. Such structures hold the same benefits as conventional MIMO, however, in large scale. More properly, LS-MIMO is defined as a transmission/reception design using typically several tens or even hundreds of antennas in at least one of the communication terminals, usually in the base station (BS) $[4,5]$. The reduced dimensions of user equipments (UEs) suggest a single antenna arrangement in each UE; on the other hand, a huge amount of antennas is installed in each BS. In an asymptotic model, an infinite number of antennas at the BS is assumed in an LS-MIMO scheme, resulting in paramount advantages: (i) effects of noise background and fast fading channel disappear, (ii) the transmission rate and the number of UEs become independent of cell size, (iii) spectral efficiency becomes independent of the system bandwidth and (iv) power required for transmission of bit tends to zero [6].

However, these advantages are fully achieved as long as this unlimited number of antennas at the BS meets a fixed number of UEs. These results become very interesting in scenarios with poor channel estimates due to the high noise power, meaning very low signal-to-noise ratio (SNR): a sufficient increase in the number of BS antennas in an LS-MIMO system is capable of mitigating harmful effects of error in the channel estimation. On the other hand, in multicellular LS-MIMO systems the use of training pilot sequences for channel estimation purpose imposes an intercellular interference in different cells. The problem is called pilot contamination, persisting even in asymptotic BS antennas scenarios [6].

The main advantage achieved with the LS-MIMO scheme refers to high capacity/spectral efficiency $[6,7]$; however, with a high number of antennas at BS, the computational complexity of data detection tends to grow proportionally. In this sense, a low computational complexity detector emerges as an essential requirement in LS-MIMO systems, still developing key role to reap the benefits of their high spectral efficiencies. Many low complexity detection procedures for LS-MIMO has been proposed in recent literature, including LS-MIMO detectors based on (i) local neighbourhood search, such as likelihood ascent search algorithm [8], and reactive tabu search (RTS) algorithm [9]; (ii) promising message passing (MP) algorithms, based on belief propagation (BP) technique, such that LS-detectors inspired in graphical models (GMs), as factor graph (FG) [10] and Markov random fields (MRF) [11]. The main difference between these models refers that MRF is a fully connected graphic with only variable nodes, whereas FG is a bipartite graph consisted by variable nodes and function (or observation) nodes. Also, the message update functions on each model are different.

BP-based detectors have demonstrated a near optimal performance in LS-MIMO scenarios with low computational complexity [12-15]. In [16], a Gaussian BP detector is proposed in a coded MIMO environment and its convergence is investigated under two different graph types, showing that the proposed Gaussian BP in fully connected pairwise graph has a faster convergence than ring-type pairwise graph. In the work of [17], a Gaussian message passing iterative detection (GMPID) is considered in a massive multiuser MIMO system with LDPC decoding process; the convergence of GMPID detector is analysed and demonstrated that it may not converge when system loading 
$\beta \geq(\sqrt{2}-1)^{2}$, in this sense, a new scale-and-add GMPID (SAGMPID) is proposed which performs a faster and wide convergence range (for any $\beta \leq 1$ ).

Moreover, MP algorithms based on BP has been reported, in recent literature, as a promising detection procedure in singlecarrier spatial modulation LS-MIMO systems [18]. In some situations, BP algorithm may fail to converge, and if it does converge, the estimated marginal probabilities may be far from exact [19]. However, there are several methods in the literature to improve the convergence of BP algorithm, including message damping (MD) method [20, 21] and double loop methods [22, 23]. The MD method applied to the MP algorithm based on MRF GM (MRF-BP) is presented in [10], where three types of damping are performed: MD, belief damping and hybrid damping, showing that both message/belief damping significantly improves the performance without a relevant increase in computational complexity. In [12], MP detectors using MRF or FG approach are also presented and the MD method demonstrated to achieve a relevant performance gain with a negligible complexity increment; besides, in the same work, a hybrid reactive tabu search BP (RTS$\mathrm{BP}$ ) is proposed in order to perform in high-order QAM constellations. Although MD method has been proved to achieve a significant performance improvement, the damping factor (DF) values adopted in the previous works in the literature are valid only for specific system operation scenarios considered, while its extension for large MIMO schemes has not been investigated so far.

Due to the applicability and success of BP in LS-MIMO detection [11, 12, 18, 24], BP detectors based on an MRF and FG GMs are considered in this work. Furthermore, $M D$ method is applied and a bit error rate (BER) performance $\times$ computational complexity analysis relating the DF value is performed. Our extensive analysis considers wide range of antennas configurations, matching LS MIMO, and SNR region scenarios, aiming to demonstrate the performance-complexity gain obtained with the MRF-BP detector deploying the optimal DF. Also, a damped FG$\mathrm{BP}$ detector is proposed with two different damping types: $\mathrm{MD}$ at variable nodes and MD at observation nodes. The main contributions of this work are fourfold:

- Application of MD method in FG-BP LS-MIMO detector in two variations: $\mathrm{MD}$ at variable nodes and $\mathrm{MD}$ at function nodes;

- Extensive performance and complexity analyses of MRF-BP and FG-BP detectors considering a DF range variation under a different number of antennas configurations, especially including LS-MIMO scenarios;

- Numerical analysis of the impact of MD method on MRF-BP convergence, in order to determine a complexity reduction of the algorithm;

- Proposition of an optimal DF function for MRF-BP, considering a given $\gamma_{\mathrm{dB}}$ and number of $N=N_{\mathrm{t}}=N_{\mathrm{r}}$ antennas, based on a polynomial data fitting.

This paper is organised as follows: Section 2 presents some basic concepts on GMs and BP technique, as well as the adopted LS MIMO system model. The MRF- and FG-based MP for LSMIMO detection are discussed in Sections 3 and 4, respectively. Numerical results are analysed in Section 5. Finally, conclusion remarks are provided in Section 6.

\section{System model and basic concepts}

\subsection{Graphical models}

GMs are graphs that indicate inter-dependencies between random variables [25]. Distributions that exhibit some structure can generally be represented naturally and compactly using a GM, that is the case of distributions of interest in MIMO systems (e.g. vector of received symbols). The GM structure often allows the statistical distribution of interest to be effectively used for inference, i.e. answering certain questions of interest using the distribution [26]. Three basic GMs widely used to represent statistical distributions include Bayesian belief networks [27], MRF [28] and FGs [29].
The MRF is an undirected graph whose vertices are random variables, which consists in a graph composed only of variable nodes. The variables are such that any variable is independent of all the other variables, given its neighbours. For instance, an MIMO system under multiplexing gain mode (Mux-MIMO) can be conveniently modelled through an MRF in which each transmitted symbol is represented by a variable node in the GM. Since each transmit antenna is used to transmit a separate symbol, there are $N_{\mathrm{t}}$ nodes in such graph. Considering that every transmitted symbol interferes with every other transmitted symbol in a Mux-MIMO mode, the graph is fully connected, as illustrated in the top part of Fig. 1. In [12], an MRF-BP detector for LS antennas aiming to deal with inter-symbol interference (ISI) MIMO channels is proposed as an attractive choice for LS-MIMO schemes since the performance achieved is increasingly closer to the optimum detection for increasing number of antennas.

On the other hand, the MD method aiding the MRF-BP detector was only analysed from the perspective of the fixed number of antennas in a specific SNR point scenario. In the present work, an in-depth analysis is developed on MD method for MRF-BP detector, considering scenarios that assume variations in the number of antennas (typical to LS MIMO) and SNR regions, in order to determine the optimal DF value, namely $\alpha_{\mathrm{MRF}}^{\text {best }}$, for each peculiar LS configuration scenario.

Factor graphs are GMs as bipartite graphs, i.e. there are two kinds of nodes in a FG: variable nodes and function nodes (or observation nodes). Variable nodes are usually denoted as circles and function nodes are usually denoted as squares. An FG explicitly depicts the factorisation of a function, typically a probability distribution, into several local functions; and each local function depends on a subset of the set of all variables.

In the case of MIMO systems, the transmitted symbols can be denoted by the variable nodes and the received signal (at each receive antenna) by the observation nodes. At each type of node, a calculation is evaluated and transferred to the other type of node. This inter-transfer between variable and observation nodes is performed for a fixed number of iterations. A diagram for this FG principle applied to an MIMO system, with four transmit and four receive antennas, is depicted in Fig. 2. Also in [12], an FG-BP detector for LS-MIMO-ISI channels is proposed and the results showed to be an interesting option for LS-MIMO schemes, since the performance achieved is increasingly closer to optimum detection for increasing number of antennas, same as MRF-BP detector. However, authors of [12] do not consider performing the MD method in FG-BP detection. Hence, our work proposes a dual use for MD method in FG-BP algorithm and an analysis is performed on the MD method for FG-BP detector considering different number of massive antennas and SNR regions.

\subsection{Belief propagation}

$\mathrm{BP}$ is a technique that solves probabilistic inference problems usually implemented in GMs. BP is a simple, yet highly effective, procedure that has been successfully employed in a variety of applications including computational biology, statistical signal/ image processing, data mining, etc. The BP algorithm is now widely recognised as an efficient tool that can be used to solve several problems, including communications issues as well [25]. The goal is to detect a hidden input (e.g. transmitted symbol in MIMO systems) from its observed output (e.g. received signal in MIMO systems). The system can be represented as a GM and the detection of the system input is equivalent to carry out inference on the corresponding graph. More precisely, BP is a procedure used to compute the marginalisation of functions by passing messages on a GM [26]. Due to its simplicity and efficiency, BP is the most common strategy adopted to implement MP principle. Other MP algorithms can be addressed as generalised distributive law [30] and sum-product algorithm [29].

\subsection{System model}

Consider an MIMO communication system operating in MuxMIMO mode with $N_{\mathrm{t}}$ transmit antennas and $N_{\mathrm{r}}$ receive antennas, 


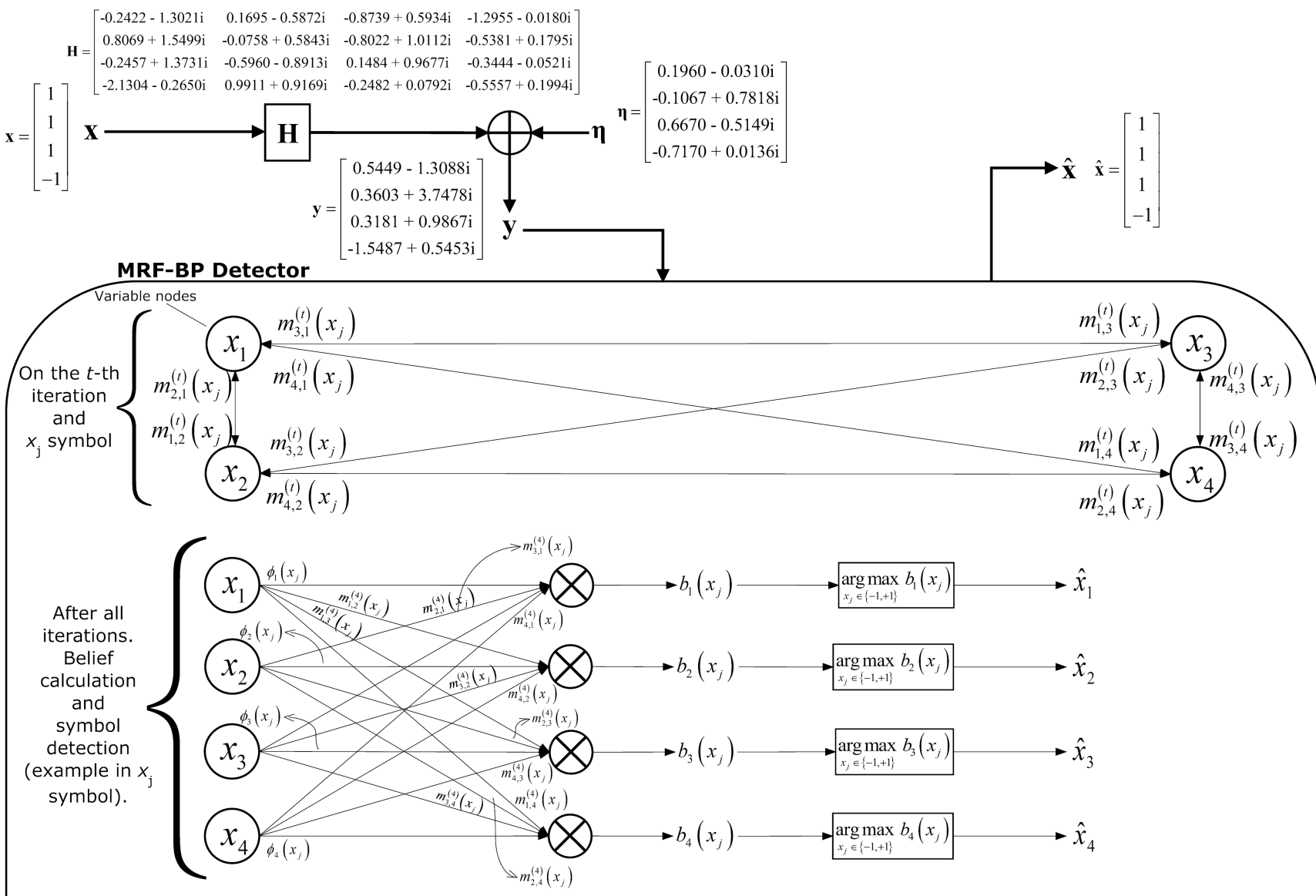

\begin{tabular}{|c|c|c|c|c|c|c|c|c|c|c|c|c|c|c|c|c|c|c|c|}
\hline Variables & \multicolumn{3}{|c|}{$\begin{array}{l}\text { Initialization } \\
\qquad(t=0)\end{array}$} & \multicolumn{4}{|c|}{$\begin{array}{l}\text { 1st iteration } \\
\quad(t=1)\end{array}$} & \multicolumn{4}{|c|}{$\begin{array}{l}\text { 2nd iteration } \\
\qquad(t=2)\end{array}$} & \multicolumn{4}{|c|}{$\begin{array}{l}\text { 3rd iteration } \\
\qquad(t=3)\end{array}$} & \multicolumn{4}{|c|}{$\begin{array}{l}\text { 4th iteration } \\
\qquad(t=4)\end{array}$} \\
\hline $\begin{array}{c}\phi\left(x_{i}=-1\right) \\
\quad(\text { line 6) }\end{array}$ & \multicolumn{3}{|c|}{$\left[\begin{array}{llll}0 & 0 & 0 & 0\end{array}\right]^{\mathrm{T}}$} & & & & & \multicolumn{4}{|c|}{ - } & \multicolumn{4}{|c|}{ - } & \multicolumn{4}{|c|}{ - } \\
\hline $\begin{array}{c}\phi\left(x_{i}=1\right) \\
\text { (line 6) }\end{array}$ & \multicolumn{3}{|c|}{$\left[\begin{array}{llll}9.78 .10^{7} & 0 & 0 & 0\end{array}\right]^{\mathrm{T}}$} & \multicolumn{4}{|c|}{-} & \multicolumn{4}{|c|}{-} & \multicolumn{4}{|c|}{-} & \multicolumn{4}{|c|}{-} \\
\hline $\begin{array}{c}\psi\left(x_{i}=-1, x_{j}=-1\right) \\
\psi\left(x_{i}=1, x_{j}=1\right) \\
\text { (line 10) }\end{array}$ & {$\left[\begin{array}{cc}0 & 18.91 \\
18.91 & 0 \\
0.03 & 4.61 \\
0.12 & 1.32\end{array}\right.$} & $\begin{array}{c}0.03 \\
4.61 \\
0 \\
0.05\end{array}$ & $\left.\begin{array}{c}0.12 \\
1.32 \\
0.05 \\
0\end{array}\right]$ & \multicolumn{4}{|c|}{ - } & \multicolumn{4}{|c|}{-} & \multicolumn{4}{|c|}{ - } & \multicolumn{4}{|c|}{ - } \\
\hline $\begin{array}{c}\psi\left(x_{i}=-1, x_{j}=1\right) \\
\psi\left(x_{i}=1, x_{j}=-1\right) \\
\quad \text { (line 10) }\end{array}$ & $\begin{array}{cc}0 & 0.05 \\
0.05 & 0 \\
30.13 & 0.21 \\
8.09 & 0.75\end{array}$ & $\begin{array}{c}30.13 \\
0.21 \\
0 \\
16.75\end{array}$ & $\left.\begin{array}{c}8.09 \\
0.75 \\
16.75 \\
0\end{array}\right]$ & \multicolumn{4}{|c|}{ - } & \multicolumn{4}{|c|}{ - } & \multicolumn{4}{|c|}{ - } & \multicolumn{4}{|c|}{ - } \\
\hline $\begin{array}{c}m^{(t)}\left(x_{j}=-1\right) \\
\text { (line 22) }\end{array}$ & {$\left[\begin{array}{ll}0.5 & 0.5 \\
0.5 & 0.5 \\
0.5 & 0.5 \\
0.5 & 0.5\end{array}\right.$} & $\begin{array}{l}0.5 \\
0.5 \\
0.5 \\
0.5\end{array}$ & $\left.\begin{array}{l}0.5 \\
0.5 \\
0.5 \\
0.5\end{array}\right]$ & {$\left[\begin{array}{l}0.10 \\
0.14 \\
0.90 \\
0.79\end{array}\right.$} & $\begin{array}{l}0.10 \\
0.10 \\
0.14 \\
0.42\end{array}$ & $\begin{array}{l}0.90 \\
0.17 \\
0.10 \\
0.79\end{array}$ & $\left.\begin{array}{l}0.89 \\
0.40 \\
0.90 \\
0.10\end{array}\right]$ & {$\left[\begin{array}{l}0.02 \\
0.04 \\
0.98 \\
0.59\end{array}\right.$} & $\begin{array}{l}0.02 \\
0.02 \\
0.06 \\
0.57\end{array}$ & $\begin{array}{l}0.98 \\
0.07 \\
0.02 \\
0.61\end{array}$ & $\left.\begin{array}{l}0.97 \\
0.37 \\
0.98 \\
0.02\end{array}\right]$ & {$\left[\begin{array}{l}0.00 \\
0.01 \\
0.99 \\
0.30\end{array}\right.$} & $\begin{array}{l}0.01 \\
0.00 \\
0.05 \\
0.62\end{array}$ & $\begin{array}{l}0.99 \\
0.05 \\
0.00 \\
0.35\end{array}$ & $\left.\begin{array}{l}0.98 \\
0.36 \\
0.99 \\
0.00\end{array}\right]$ & {$\left[\begin{array}{l}0.00 \\
0.01 \\
1.00 \\
0.14\end{array}\right.$} & $\begin{array}{l}0.00 \\
0.00 \\
0.05 \\
0.64\end{array}$ & $\begin{array}{l}1.00 \\
0.05 \\
0.00 \\
0.22\end{array}$ & $\left.\begin{array}{l}0.98 \\
0.36 \\
1.00 \\
0.00\end{array}\right]$ \\
\hline $\begin{array}{c}m^{(t)}\left(x_{j}=1\right) \\
\text { (line 22) }\end{array}$ & {$\left[\begin{array}{ll}0.5 & 0.5 \\
0.5 & 0.5 \\
0.5 & 0.5 \\
0.5 & 0.5\end{array}\right.$} & $\begin{array}{l}0.5 \\
0.5 \\
0.5 \\
0.5\end{array}$ & $\left.\begin{array}{l}0.5 \\
0.5 \\
0.5 \\
0.5\end{array}\right]$ & {$\left[\begin{array}{l}0.10 \\
0.86 \\
0.10 \\
0.21\end{array}\right.$} & $\begin{array}{l}0.90 \\
0.10 \\
0.86 \\
0.58\end{array}$ & $\begin{array}{l}0.10 \\
0.83 \\
0.10 \\
0.21\end{array}$ & $\left.\begin{array}{l}0.11 \\
0.60 \\
0.10 \\
0.10\end{array}\right]$ & {$\left[\begin{array}{l}0.02 \\
0.03 \\
0.02 \\
0.41\end{array}\right.$} & $\begin{array}{l}0.98 \\
0.02 \\
0.94 \\
0.43\end{array}$ & $\begin{array}{l}0.02 \\
0.93 \\
0.02 \\
0.39\end{array}$ & $\left.\begin{array}{l}0.03 \\
0.63 \\
0.02 \\
0.02\end{array}\right]$ & {$\left[\begin{array}{l}0.00 \\
0.99 \\
0.01 \\
0.70\end{array}\right.$} & $\begin{array}{l}0.99 \\
0.00 \\
0.95 \\
0.38\end{array}$ & $\begin{array}{l}0.01 \\
0.95 \\
0.00 \\
0.65\end{array}$ & $\left.\begin{array}{l}0.02 \\
0.64 \\
0.01 \\
0.00\end{array}\right]$ & $\begin{array}{l}0.00 \\
0.99 \\
0.00 \\
0.86\end{array}$ & $\begin{array}{l}1.00 \\
0.00 \\
0.95 \\
0.36\end{array}$ & $\begin{array}{l}0.00 \\
0.95 \\
0.00 \\
0.78\end{array}$ & $\left.\begin{array}{l}0.02 \\
0.64 \\
0.00 \\
0.00\end{array}\right]$ \\
\hline $\begin{array}{c}\hat{\mathbf{x}} \\
\text { (line 31) }\end{array}$ & & \multicolumn{4}{|c|}{$\left[\begin{array}{c}1 \\
1 \\
1 \\
-1\end{array}\right]$} & \multicolumn{4}{|c|}{$\left[\begin{array}{c}1 \\
1 \\
1 \\
-1\end{array}\right]$} & \multicolumn{4}{|c|}{$\left[\begin{array}{c}1 \\
1 \\
1 \\
-1\end{array}\right]$} & \multicolumn{4}{|c|}{$\left[\begin{array}{c}1 \\
1 \\
1 \\
-1\end{array}\right]$} \\
\hline
\end{tabular}

Fig. 1 MRF GM exemplifying an MIMO system operating under multiplexing mode with $N_{t}=4$ transmit antennas, $N_{r}=4$ receive antennas and $\mathscr{I}_{M R F}=4$ iterations. This numerical example of MRF-BP detector (Algorithm 1 (Fig. 3), Section 3.1) details the values in message update process. The interconnections between antennas represent inter-antenna interference. A fully connected subgraph of an MRF is called a clique; variables constrained by a compatibility function is named potential 


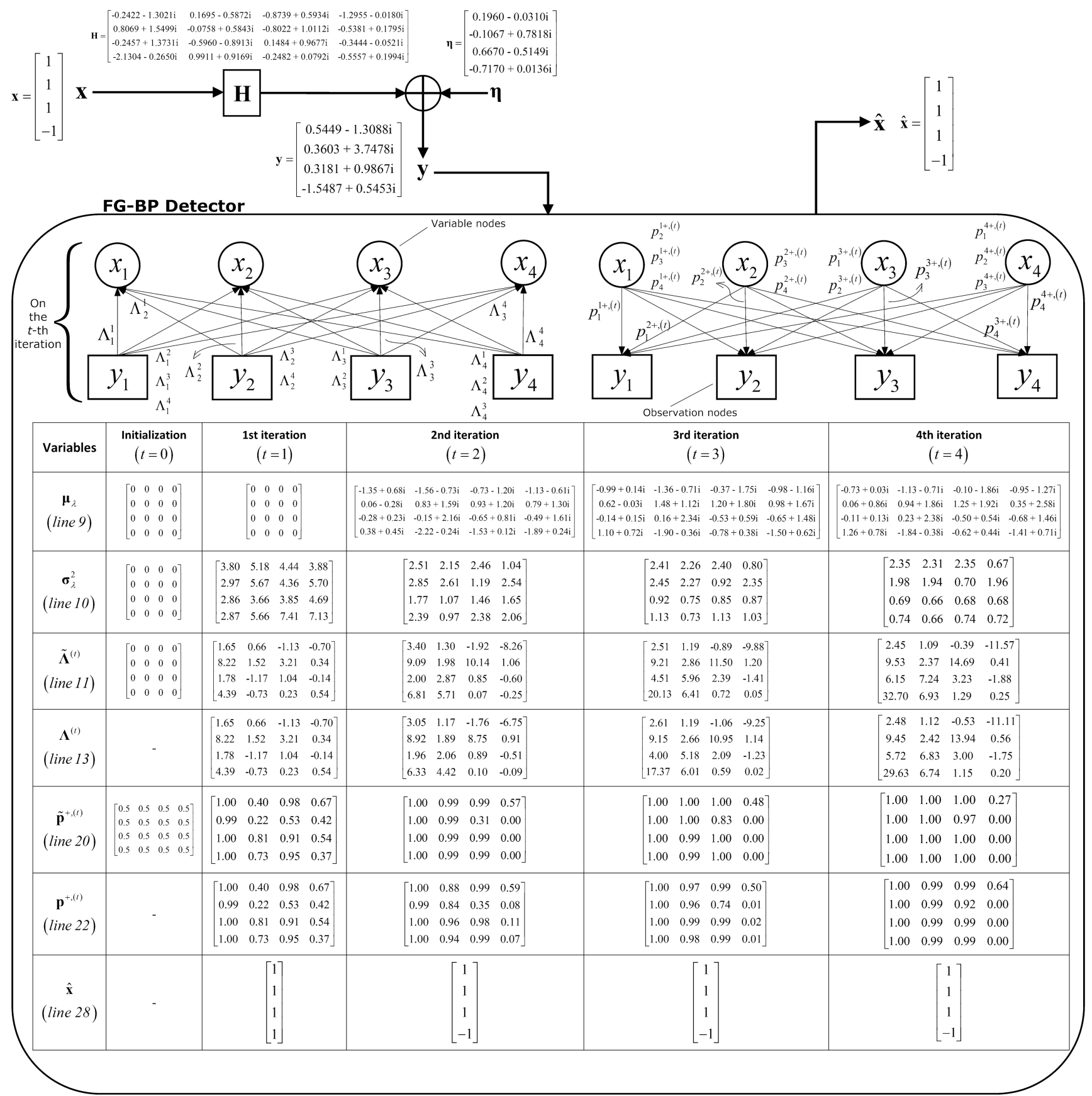

Fig. 2 FG-BP GM exemplifying an MIMO system with $N_{t}=N_{r}=4$ operating under multiplexing mode. Numerical example for $\mathscr{I}_{F G}=4$ iterations of FG-BP detector via Algorithm 2 (Fig. 4) (details in Section 4). Observation nodes evaluate calculation of interest and transfer messages to variable nodes and viceversa

for simplicity, the channel is assumed to be a frequency-flat fading channel, characterised by the channel matrix $\boldsymbol{H}$. The elements of $\boldsymbol{H}$ are all independent complex Gaussian random variables with zero mean and unit variance. Let $\boldsymbol{x}$ be the $N_{\mathrm{t}} \times 1$ vector corresponding to the binary phase-shift keying (BPSK) symbols transmitted over the $N_{\mathrm{t}}$ transmit antennas, $\boldsymbol{x} \in\{-1,+1\}^{N_{\mathrm{t}}}$. The additive white Gaussian noise (AWGN) at any receive antenna is assumed to be a complex Gaussian random variable with zero mean and variance $\sigma^{2}$. The matrix model for the system under investigation is

$$
y=H x+\eta
$$

where $\boldsymbol{H}$ represents an $N_{\mathrm{r}} \times N_{\mathrm{t}}$ fading coefficients matrix following a Rayleigh distribution (for amplitudes), representing a non-lineof-sight communication, and $\boldsymbol{\eta}$ is the noise vector samples with variance:

$$
\mathbb{E}\left[\eta^{2}\right]=N_{0}=\sigma^{2}=N_{\mathrm{t}} \frac{\varepsilon_{\mathrm{x}}}{\gamma}
$$

where $\varepsilon_{\mathrm{X}}$ denotes the average energy of the transmitted symbols, $N_{0}$ is the noise power spectral density and $\gamma$ is the average SNR per transmit antenna.

\section{MP via MRF for LS MIMO detection}

This section presents a BP-based detector that employs MP on an MRF [12]. Consider the system model in (1). The maximum $a$ posteriori (MAP) detector takes the joint a posteriori distribution:

$$
p(\boldsymbol{x} \mid \boldsymbol{y}, \boldsymbol{H}) \propto p(\boldsymbol{y} \mid \boldsymbol{x}, \boldsymbol{H}) p(\boldsymbol{x})
$$

The MAP estimation of the bit $x_{i}, i=1, \ldots, N_{\mathrm{t}}$ is given by

$$
\hat{x}_{i}=\underset{a \in\{-1,+1\}}{\arg \max } p\left(x_{i}=a \mid \boldsymbol{y}, \boldsymbol{H}\right)
$$


whose complexity is exponential in $N_{\mathrm{t}}$ [26].

Given $\boldsymbol{x}$ and $\boldsymbol{H}, \boldsymbol{y}$ is a complex Gaussian random vector with mean $\boldsymbol{H} \boldsymbol{x}$ and covariance $\sigma^{2} \boldsymbol{I}_{N_{\mathrm{r}}}$. Thus,

$$
p(\boldsymbol{y} \mid \boldsymbol{x}, \boldsymbol{H}) \propto \exp \left(\frac{-\|\boldsymbol{y}-\boldsymbol{H} \boldsymbol{x}\|^{2}}{2 \sigma^{2}}\right)
$$

Assuming that the symbols in $\boldsymbol{x}$ are all independent, also necessary for an MRF procedure [26]. Hence, a priori probability for the transmitted symbol vector is given by

$$
p(\boldsymbol{x})=\prod_{i} p\left(x_{i}\right)
$$

From (3), (5) and (6), the conditional probability function can be written as (7). Defining the channel correlation matrix $\boldsymbol{R}=\left(1 / \sigma^{2}\right) \boldsymbol{H}^{\mathrm{H}} \boldsymbol{H}$ and $z=\left(1 / \sigma^{2}\right) \boldsymbol{H}^{\mathrm{H}} \boldsymbol{y},(7)$ can be rewritten as (8).

$$
\begin{aligned}
p(\boldsymbol{x} \mid \boldsymbol{y}, \boldsymbol{H}) & \propto \exp \left(\frac{-\|\boldsymbol{y}-\boldsymbol{H} \boldsymbol{x}\|^{2}}{2 \sigma^{2}}\right) \exp (\ln p(\boldsymbol{x})) \\
& =\exp \left(\frac{-1}{2 \sigma^{2}}(\boldsymbol{y}-\boldsymbol{H} \boldsymbol{x})^{\mathrm{H}}(\boldsymbol{y}-\boldsymbol{H} \boldsymbol{x})\right) \prod_{i} \exp \left(\ln p\left(x_{i}\right)\right) \\
& \propto \exp \left(\frac{-1}{2 \sigma^{2}}\left(\boldsymbol{x}^{\mathrm{H}} \boldsymbol{H}^{\mathrm{H}} \boldsymbol{H} \boldsymbol{x}-2 \mathscr{R}\left\{\boldsymbol{x}^{\mathrm{H}} \boldsymbol{H}^{\mathrm{H}} \boldsymbol{y}\right\}\right)\right) \prod_{i} \exp \left(\ln p\left(x_{i}\right)\right)
\end{aligned}
$$

(see (8)) where $z_{i}$ and $R_{i j}$ are the elements of $\boldsymbol{z}$ and $\boldsymbol{R}$, respectively, and $\mathscr{R}(\cdot)$ denotes the real part of a complex number.

A fully connected subgraph of an MRF is called a clique; the variables in an MRF constrained by a compatibility function is known as a (clique) potential. Analysing (8), it is seen that the MRF of the MIMO system presents pairwise interactions with the potentials defined by

$$
\begin{aligned}
& \psi_{i, j}\left(x_{i}, x_{j}\right)=\exp \left[-x_{i} \mathscr{R}\left\{R_{i j}\right\} x_{j}\right] \\
& \phi_{i}\left(x_{i}\right)=\exp \left[x_{i} \mathscr{R}\left\{z_{i}\right\}+\ln p\left(x_{i}\right)\right]
\end{aligned}
$$

The values of $\psi$ and $\phi$ define, respectively, the edge and selfpotentials of the MRF GM to which MP algorithm is applied to compute the marginal probabilities of the variables. BP algorithm attempts to estimate the marginal probabilities of all the variables by way of passing messages between the local nodes.

A message from node $j$ to node $i$ is denoted by $m_{j, i}\left(x_{i}\right)$, and belief at node $i$ is denoted $b_{i}\left(x_{i}\right), x_{i} \in\{ \pm 1\}$. The belief $b_{i}\left(x_{i}\right)$ depends on how likely $x_{i}$ was transmitted. On the other hand, $m_{j, i}\left(x_{i}\right)$ depends on how likely that node $j$ evaluates $x_{i}$ was transmitted. The message from node $i$ to a neighbouring node $j$ is then given by

$$
m_{i, j}\left(x_{j}\right)=\sum_{x_{i}} \phi_{i}\left(x_{i}\right) \psi_{i, j}\left(x_{i}, x_{j}\right) \prod_{k \in \mathcal{N}(i) \backslash j} m_{k, i}\left(x_{i}\right)
$$

where $\mathcal{N}(i)$ denotes the set of all nodes neighbouring the node $i$ and $\mathcal{N}(i) \backslash j$ denotes the same neighbourhood, except the node $j$. Equation (11) actually constitutes an iteration, as the message is defined in terms of the messages from other nodes. So, BP essentially involves computing the outgoing messages from a node to each of its neighbours using the local joint compatibility function and the incoming messages and transmitting them [26]. The algorithm terminates after a fixed number of iterations, $\mathscr{I}_{\text {MRF }}$.

\subsection{Message damping on MRF-BP detector}

The MD method can be used to improve the convergence of BP algorithm. The messages to be passed are computed as a weighted average of the message in the previous iteration and the message in the current iteration $[20,21]$. Thus, the damped message to be passed from node $i$ to node $j$ in iteration $t$, denoted by $m_{i, j}^{(t)}\left(x_{j}\right)$, is computed as a convex combination of the previous message and the current message as

$$
m_{i, j}^{(t)}\left(x_{j}\right)=\alpha m_{i, j}^{(t-1)}\left(x_{j}\right)+(1-\alpha) \tilde{m}_{i, j}^{(t)}\left(x_{j}\right)
$$

where $\tilde{m}_{i, j}^{(t)}\left(x_{j}\right)$ and $m_{i, j}^{(t-1)}\left(x_{j}\right)$ denote, respectively, the current message in iteration $t$ and the previous message in iteration $t-1$, and $\alpha \in(0,1]$ is referred as the DF. This simple damping of messages has been shown to be very effective in improving BP convergence and performance [20]. As shown in numerical results section, considering the LS-MIMO detection context, MD method can improve performance significantly, without increasing the computational complexity. Furthermore, the analysis of DF in different LS-MIMO configurations is the main contribution of this work.

Damping of messages can be carried out in each iteration. The final belief about the variable $x_{i}$ is computed as

$$
b_{i}\left(x_{i}\right) \propto \phi_{i}\left(x_{i}\right) \prod_{j \in \mathscr{N}(i)} m_{j, i}\left(x_{j}\right)
$$

In the case of a coded system, the soft output of the algorithm $b_{i}\left(x_{i}\right)$ can be directly fed to the decoder. A pseudocode for the MRF-BP described above is listed in Algorithm 1 (see Fig. 3).

\subsection{Numerical example - Algorithm 1 (Fig. 3) for LS-MIMO}

A simple but helpful numerical example of MRF-BP algorithm is sketched in Fig. 1. Let's consider an MIMO system with $N_{\mathrm{t}}=N_{\mathrm{r}}=4$ during a given symbol period. The algorithm runs with $\mathscr{I}_{\mathrm{MRF}}=4$ and MD is equal to $\alpha_{\mathrm{MRF}}=0.2$. With the knowledge of $\boldsymbol{H}$ and $\boldsymbol{y}$, and assuming equal symbol a priori probability, $\phi_{i}\left(x_{i}\right)$ and $\psi_{i, j}\left(x_{i}, x_{j}\right) \quad \forall i, j=1, \ldots, N_{\mathrm{t}}$ are pre-calculated (before algorithm iterations) for each $\left(x_{i}, x_{j}\right)$ possible symbol in the BPSK constellation considered. Thereafter, the iterative message update process begins. Each variable node performs and transfers its $m_{i, j}^{(t)}\left(x_{j}\right)$ message considering all other variable node's message. The message passed to the other nodes are also damped and normalised. After $\mathscr{I}_{\mathrm{MRF}}=4$ iterations, the algorithm performs the belief calculation and the hard decision of data bits. In this example, if the MRF-BP algorithm stopped even in the first iteration, none of four transmitted symbols would be resulted in error (high SNR region).

\section{MP via FG for LS MIMO detection}

Considering the same model expressed in (1) and BPSK modulation. Treat each entry of the observation vector $\boldsymbol{y}$ as a function node (observation node) in a FG, and each transmitted symbol as a variable node. The received signal $y_{i}$ can be written as

$$
y_{i}=\sum_{j=1}^{N_{\mathrm{t}}} H_{i, j} x_{j}+\eta_{i}=H_{i, k} x_{k}+\underbrace{\sum_{j=1, j \neq k}^{N_{\mathrm{t}}} H_{i, j} x_{j}}_{\text {interference }}+\eta_{i}
$$

$$
\begin{aligned}
p(\boldsymbol{x} \mid \boldsymbol{y}, \boldsymbol{H}) & \propto \exp \left(-\sum_{i<j} \mathscr{R}\left\{x_{i}^{*} R_{i j} x_{j}\right\}\right) \exp \left(\sum_{i} \mathscr{R}\left\{x_{i}^{*} z_{i}\right\}\right) \prod_{i} \exp \left(\ln p\left(x_{i}\right)\right) \\
& =\left(\prod_{i<j} \exp \left(-x_{i} \mathscr{R}\left\{R_{i j}\right\} x_{j}\right)\right)\left(\prod_{i} \exp \left(x_{i} \mathscr{R}\left\{z_{i}\right\}+\ln p\left(x_{i}\right)\right)\right)
\end{aligned}
$$


1: Initialization

2: $m_{i, j}^{(0)}\left(x_{j}\right)=0.5, \quad p\left(x_{i}=1\right)=p\left(x_{i}=-1\right)=0.5$,

$\forall i, j=1, \ldots, N_{t}$

3: $\tilde{m}_{i, j}^{(0)}\left(x_{j}\right)=0.5, \forall i, j=1, \ldots, N_{t}$

4: $\mathbf{R}=\left(\frac{1}{\sigma^{2}}\right) \mathbf{H}^{\mathrm{H}} \mathbf{H} ; \mathbf{z}=\left(\frac{1}{\sigma^{2}}\right) \mathbf{H}^{\mathrm{H}} \mathbf{y}$

5: for $i=1$ to $N_{t}$ do

$\phi_{i}\left(x_{i}\right)=\exp \left(x_{i} \mathcal{R}\left\{z_{i}\right\}+\ln \left(p\left(x_{i}\right)\right)\right)$

end for

for $i=1$ to $N_{t}$ do

for $j=1$ to $N_{t}, j \neq i$ do

$\psi_{i, j}\left(x_{i}, x_{j}\right)=\exp \left(-x_{i} \mathcal{R}\left\{R_{i, j}\right\} x_{j}\right)$

$$
\text { end for }
$$

end for

13: Iterative update of messages

4: for $t=1$ to $\mathcal{I}_{\mathrm{MRF}}$ do

Message calculation

for $i=1$ to $N_{t}$ do

for $j=1$ to $N_{t}, j \neq i$ do

$\tilde{m}_{i, j}^{(t)}\left(x_{j}\right) \propto \sum_{x_{i}} \phi_{i}\left(x_{i}\right) \psi_{i, j}\left(x_{i}, x_{j}\right) \prod_{k \in \mathcal{N}(i) \backslash j} m_{k, i}^{(t-1)}\left(x_{i}\right)$

Damping messages

$m_{i, j}^{\prime(t)}\left(x_{j}\right)=\left(\alpha_{\mathrm{MRF}}\right) m_{i, j}^{(t-1)}\left(x_{j}\right)+(1-$

$\left.\alpha_{\mathrm{MRF}}\right) \tilde{m}_{i, j}^{(t)}\left(x_{j}\right)$

21: $\quad$ Messages normalization

22: $\quad m_{i, j}^{(t)}\left(x_{j}\right)=\frac{m_{i, j}^{\prime(t)}\left(x_{j}\right)}{\sum_{x_{j}} m_{i, j}^{\prime(t)}\left(x_{j}\right)}$

23: $\quad$ end for

24: end for

25: end for

26: Belief calculation

27: for $i=1$ to $N_{t}$ do

28: $\quad b_{i}\left(x_{i}\right) \propto \phi_{i}\left(x_{i}\right) \prod_{j \in \mathcal{N}(i)} m_{j, i}^{\left(\mathcal{I}_{\text {MRF }}\right)}\left(x_{i}\right)$

29: end for

30: Detection of data bits

31: $\hat{x_{i}}=\underset{\arg \max }{\operatorname{ar}} b_{i}\left(x_{i}\right), \forall i, j=1, \ldots, N_{t}$

32: Terminate

$$
\begin{aligned}
& x_{i} \in\{ \pm 1\} \\
& \text { inate }
\end{aligned}
$$

Fig. 3 Algorithm 1: MRF-BP for LS-MIMO detection

To reduce the computational complexity, the calculation of the message from the $i$ th observation node, $y_{i}$, to the $k$ th variable node, $x_{k}$, can be approximated by the following scalar Gaussian approximation (SGA) for the interference-plus-noise [12]:

$$
y_{i}=H_{i, k} x_{k}+\underbrace{\sum_{j=1, j \neq k}^{N_{\mathrm{t}}} H_{i, j} x_{j}+\eta_{i}}_{\triangleq \lambda_{i, k}} \triangleq H_{i, k} x_{k}+\lambda_{i, k}
$$

where the interference-plus-noise term, $\lambda_{i, k}$, is modelled as $\mathbb{C} \mathcal{N}\left(\mu_{\lambda_{i k}}, \sigma_{\lambda_{i k}}^{2}\right)$ with mean and variance given, respectively, by

$$
\begin{gathered}
\mu_{i k}=\sum_{j=1, j \neq k}^{N_{\mathrm{t}}} H_{i, j} \mathbb{E}\left(x_{j}\right) \\
\sigma_{\lambda_{i k}}^{2}=\sum_{j=1, j \neq k}^{N_{\mathrm{t}}}\left|H_{i, j}\right| \operatorname{Var}\left(x_{j}\right)+\sigma^{2}
\end{gathered}
$$

Hence, the log-likelihood ratio (LLR) for the symbol $x_{k} \in\{+1,-1\}$ at the observation node $i$, denoted by $\Lambda_{i}^{k}$, can be expressed by

$$
\begin{aligned}
\Lambda_{i}^{k} & =\log \frac{p\left(y_{i} \mid \boldsymbol{H}, x_{k}=+1\right)}{p\left(y_{i} \mid \boldsymbol{H}, x_{k}=-1\right)} \\
& =\frac{4}{\sigma_{\lambda_{i k}}^{2}} \mathscr{R}\left(H_{i, k}^{*}\left(y_{i}-\mu_{\lambda_{i k}}\right)\right)
\end{aligned}
$$

From (18), the LLR values are computed at the observation nodes and passed (messaged) to the variable nodes. Using the LLR value, received through $i$ th observation node message, the $k$ th variable node compute the conditional probability of $x_{k}=+1$, given the received signal $\boldsymbol{y}$, as

$$
\begin{aligned}
p_{i}^{k+} & \triangleq p_{i}\left(x_{k}=+1 \mid \boldsymbol{y}\right) \\
& =\frac{\exp \left(\sum_{l=1, l \neq i}^{N_{r}} \Lambda_{l}^{k}\right)}{1+\exp \left(\sum_{l=1, l \neq i}^{N_{r}} \Lambda_{l}^{k}\right)}
\end{aligned}
$$

Hence, the probabilities from (20) are passed back to the observation nodes. Fig. 2 illustrates the MP in FG discussed in this subsection. The MP between observation nodes and variable nodes is carried out for a certain number of iterations, called $\mathscr{I}_{\mathrm{FG}}$. After that, the estimated symbol, $\hat{x}_{k}$, is detected as

$$
\begin{aligned}
& \tilde{x}_{k}=\sum_{i=1}^{N_{\mathrm{r}}} \Lambda_{i}^{k} \\
& \hat{x}_{k}=\operatorname{sgn}\left(\tilde{x}_{k}\right)
\end{aligned}
$$

Note that $\tilde{x}_{k}$ in (21) is a soft output from the LLR computation. In the case of a coded system, the soft output can be directly fed to the decoder. Moreover, from (22), the estimated symbol $\hat{x}_{k}$ is obtained as a hard limiting of $\tilde{x}_{k}$. Due to the FG model and the SGA of the interference, this algorithm is called FG-BP SGA; however, the last term is omitted in the remainder of this paper for textual simplification. A pseudocode for the FG-BP algorithm described above is listed in Algorithm 2 (see Fig. 4).

\subsection{Numerical example - Algorithm 2 (Fig. 4) for LS-MIMO}

To gain insight into the FG-BP message updating process, a simple numerical example is offered through Fig. 2. Again, consider an MIMO system with $N_{\mathrm{t}}=N_{\mathrm{r}}=4$ during a given symbol period. The algorithm runs with $\mathscr{I}_{\mathrm{FG}}=4$ and $\mathrm{MD}$ is equal to $\alpha_{\mathrm{FG}}=\beta_{\mathrm{FG}}=0.2$. With the knowledge of $\boldsymbol{H}$ and $\boldsymbol{y}$, and assuming equal symbol a priori probability, $\mu_{\lambda_{i k}}$ and $\sigma_{\lambda_{i k}}^{2} \forall i=1, \ldots, N_{\mathrm{r}} ; k=1, \ldots, N_{\mathrm{t}}$ are calculated at the observation nodes. After, the LLR value, $\tilde{\Lambda}_{i}^{k,(t)}$, can be performed, damped $\left(\Lambda_{i}^{k,(t)}\right)$ and finally transferred to the variable nodes. Then, the probability of transmitted symbols, $p_{i}^{k+,(t)}$, is calculated in variable nodes using LLR values and messaged back to the observation nodes. This loop is performed for $\mathscr{I}_{\mathrm{FG}}=4$ iterations. One can notice that, in this example, if the FG-BP algorithm stopped in the first iteration, one of four transmitted symbols would be resulted in error.

\section{Numerical simulation results}

In this section, the uncoded BER performance related to the MRF$\mathrm{BP}$ and FG-BP algorithms for LS-MIMO detection is evaluated through Monte-Carlo simulations. The simulations are performed for a Mux-MIMO mode and assuming that a perfect channel state information is available at the receiver side. Besides, an extensive numerical analysis on the optimal value for the DF is carried out considering wide LS antennas scenarios and SNR regions. Finally, computational complexity of the MRF-BP and FG-BP algorithms is compared aiming with determine the best performancecomplexity tradeoff of both LS-MIMO detection approaches. Table 1 summarises the main system and channel parameters values deployed in this section.

\subsection{BER performance analysis for the MRF-BP $\times F G-B P$ detectors in LS-MIMO scenarios}

For comparison purpose, the BER performance of a single-input single-output (SISO) transmission scheme operating under flat 


\section{1: Initialization}

2: $\Lambda_{i}^{k,(0)}=\tilde{\Lambda}_{i}^{k,(0)}=0, \quad p_{i}^{k+,(0)}=\tilde{p}_{i}^{k+,(0)}=0.5, \quad s_{\Lambda_{k}}=$

$0, \quad \mu_{\lambda_{i k}}=\sigma_{\lambda_{i k}}^{2}=s_{\mu_{\lambda i}}=s_{\sigma_{\lambda i}^{2}}=0, \quad \forall i, k=1, \ldots, N_{t}$

3: for $t=1$ to $\mathcal{I}_{\mathrm{FG}}$ do

4: $\quad$ Computation of LLRs at observation nodes

5: $\quad$ for $i=1$ to $N_{r}$ do

6: $\quad s_{\mu_{\lambda i}}=\sum_{j=1}^{N_{t}} h_{i j}\left(2 p_{i}^{j+}-1\right)$

7: $\quad s_{\sigma_{\lambda i}^{2}}=4 \sum_{j=1}^{N_{t}}\left|h_{i j}\right|^{2} p_{i}^{j+}\left(1-p_{i}^{j+}\right)$

8: $\quad$ for $k=1$ to $N_{t}$ do

9: $\quad \mu_{\lambda_{i k}}=s_{\mu_{\lambda i}}-h_{i k}\left(2 p_{i}^{k+}-1\right)$

10: $\quad \sigma_{\lambda_{i k}}^{2}=s_{\sigma_{\lambda i}^{2}}-4\left|h_{i k}\right|^{2} p_{i}^{k+}\left(1-p_{i}^{k+}\right)+\sigma^{2}$

11: $\quad \tilde{\Lambda}_{i}^{k,(t)}=\left(4 / \sigma_{\lambda_{i k}}^{2}\right) \mathcal{R}\left(h_{i k}^{*}\left(y_{i}-\mu_{\lambda_{i k}}\right)\right)$

12: $\quad$ Damping messages at observation nodes

13: $\quad \Lambda_{i}^{k,(t)}=\left(\alpha_{\mathrm{FG}}\right) \Lambda_{i}^{k,(t-1)}+\left(1-\alpha_{\mathrm{FG}}\right) \tilde{\Lambda}_{i}^{k,(t)}$

14: $\quad$ end for

15: end for

16: Computation of probabilities at variable nodes

17: $\quad$ for $k=1$ to $N_{t}$ do

18: $\quad s_{\Lambda_{k}}=\sum_{l=1}^{N_{r}} \Lambda_{l}^{k,(t)}$

19: $\quad$ for $i=1$ to $N_{r}$ do

20: $\quad \tilde{p}_{i}^{k+,(t)}=\frac{\exp \left(s_{\Lambda_{k}}-\Lambda_{i}^{k,(t)}\right)}{1+\exp \left(s_{\Lambda_{k}}-\Lambda_{i}^{k,(t)}\right)}$

21: $\quad$ Damping messages at variable nodes

22: $\quad p_{i}^{k+,(t)}=\left(\beta_{\mathrm{FG}}\right) p_{i}^{k+,(t-1)}+\left(1-\beta_{\mathrm{FG}}\right) \tilde{p}_{i}^{k+,(t)}$

23: end for

24: end for

25: end for

26: Detection of data bits

27: for $k=1$ to $N_{t}$ do

28: $\quad \hat{x}_{k}=\operatorname{sgn}\left(\sum_{i=1}^{N_{r}} \Lambda_{i}^{k}\right)$

29: end for

30: Terminate

Fig. 4 Algorithm 2: FG-BP for massive MIMO detection

fading channel, as well as in purely AWGN channel, was included in several graphs.

In Fig. 5, the BER performance of the implemented MP detectors is depicted for various $N_{\mathrm{t}}=N_{\mathrm{r}}=U$ antennas configurations, where the number of transmit antennas per mobile user in all scenarios is fixed in $N_{\text {tu }}=1$ antenna. Moreover, the number of iterations, $\mathscr{I}_{\mathrm{MRF}}$ and $\mathscr{I}_{\mathrm{FG}}$, adopted in BP algorithm accords with the convergence analysis performed in Fig. 6. From Fig. 5, one can conclude that even under a few BP iterations, both MRF-BP and FG-BP LS-MIMO detectors performance improves substantially when the number of antennas increases; besides, with $N_{\mathrm{t}}=N_{\mathrm{r}}=100$ the FG-BP detector achieved BER performance close to SISO AWGN system performance. Indeed, in all cases, the MP detector based on FG produces better BER performance than the MRF-based one; moreover, the performance gap between these detectors increases with the SNR. In other words, the FG-BP detector has resulted in a higher diversity gain than the MRF-BP detector with low-to-medium number of antennas, i.e. from tens to 100 antennas. With high number of antennas, FG-BP achieved better performance than MRF-BP in $200 \times 200$ and $300 \times 300$ scenarios; moreover, with $500 \times 500$ antennas both LS-MIMO detectors achieved SISO AWGN bound while demonstrated the same performance in medium-to-high SNR region (i.e. from 7 to $>10 \mathrm{~dB}$ ), although in low-to-medium SNR region the FG-based detector has indicated better performance than MRF-based detector. From these results, it is noticeable that the FG-BP detector demonstrates better BER performance than MRF-BP detector in almost all scenarios, although both detectors have a promising performance in LS MIMO systems, i.e. both detectors are able to achieve SISO AWGN bound performance when $N_{\mathrm{t}}=N_{\mathrm{r}} \geq 500$ antennas.

Fig. 6 depicts the impact of number of iterations $(\mathscr{I})$ over the performance of the both MRF- and FG-based LS-MIMO detectors. Accordingly, Fig. $6 a .1$ evidences the influence of the number of
Table 1 LS-MIMO system and channel parameters

\begin{tabular}{lc}
\hline Parameter & Value \\
\hline link direction & uplink (UL) \\
no. of Rx antennas & $N_{\mathrm{r}} \in\{20 ; 50 ; 100 ; 200 ; 300 ; 500\}$ \\
no. of mobile users & $U \in\{20 ; 50 ; 60 ; 100 ; 140 ; 200 ; 300 ; 500\}$ \\
no. of Tx antennas (per user) & $N_{\mathrm{tu}}=1$ \\
no. of Tx antennas (total) & $N_{\mathrm{t}}=U \cdot N_{\mathrm{tu}}$ \\
system loading & $\beta \in\{0.3 ; 0.5 ; 0.7 ; 1.0\}$ \\
SNR ranges & $\gamma_{\mathrm{dB}} \in[0,10] \mathrm{dB}$ and $\gamma_{\mathrm{dB}} \in[6,14] \mathrm{dB}$ \\
channel type & flat Rayleigh \\
channel availability & perfectly known at receiver \\
modulation order & $\mathrm{BPSK}$ \\
no. of iterations MRF-BP & $\mathscr{J}_{\mathrm{MRF}} \in\{3 ; 4 ; 5\}$ \\
no. of iterations FG-BP & $\mathscr{I}_{\mathrm{FG}} \in\{4 ; 5 ; 7\}$ \\
damping factor MRF-BP & $\alpha_{\mathrm{MRF}} \in[0,1]$ \\
damping factor FG-BP & $\alpha_{\mathrm{FG}} \in[0,1]$ and $\beta_{\mathrm{FG}} \in[0,1]$ \\
\hline
\end{tabular}

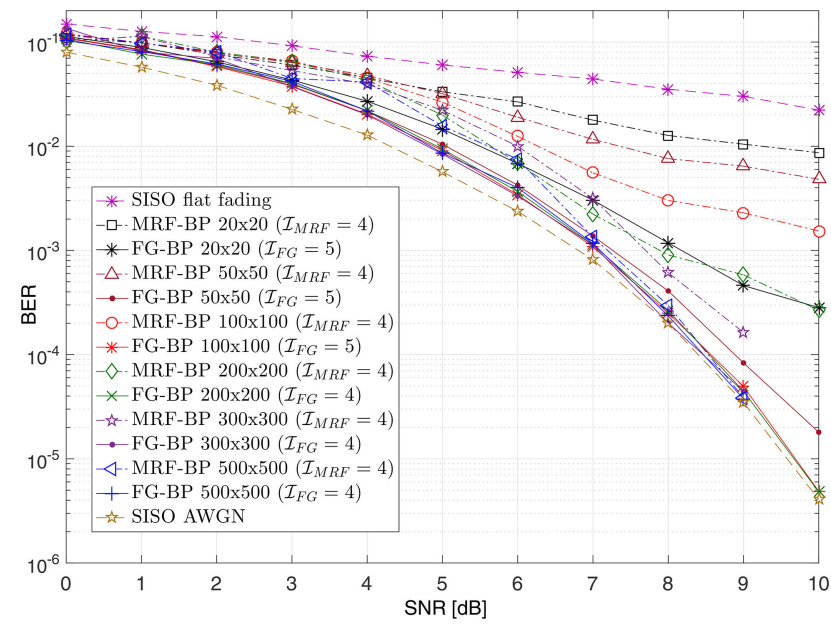

Fig. 5 BER performance of the implemented MRF-BP detector under flat Rayleigh channels and various $N_{t}=N_{r}$ antennas; without $M D$

iterations $\left(\mathscr{I}_{\mathrm{MRF}}\right)$ of MRF-BP algorithm on the BER performance at $\gamma_{\mathrm{dB}}=10 \mathrm{~dB}$ without damping messages $\left(\alpha_{\mathrm{MRF}}=0\right)$. One can notice the occurrence of a significant performance gain in the first four iterations of Algorithm 1 (Fig. 3). Besides, with the increasing number of antennas, the performance of MRF-BP detector improves accordingly. For all antennas configuration depicted in this graph, the performance gain occurs, mainly, in the first four iterations, after that, there is no relevant performance gain, i.e. no relevant information is carried out in neighbourhood messages. On the other hand, Fig. $6 b .1$ presents the influence of the number of iterations, $\mathscr{I}_{\mathrm{FG}}$, on BER performance at $\gamma_{\mathrm{dB}}=10 \mathrm{~dB}$ with $\alpha_{\mathrm{FG}}=\beta_{\mathrm{FG}}=0$. For low number of antennas, $N_{\mathrm{t}}=N_{\mathrm{r}}=20$, it can be noted that only the seven first iterations represents a BER performance gain; more precisely, in the first five iterations occurs the main performance gain. As the number of antennas increases, it is noticeable that the fifth iteration becomes less relevant in BER performance; indeed, the majority of the performance gain is obtained into the first four iterations, whereas when $N_{\mathrm{t}}=N_{\mathrm{r}}=200$ the convergence is fully achieved in the fourth iteration.

Also, an analysis of MD impact on the algorithm iterations can be performed, as disposed in Figs. $6 a .2$ and $b .2$ operating at $\gamma_{\mathrm{dB}}=10 \mathrm{~dB}$. The DF values adopted in these results are described in Section 5.2. Comparing the MRF-BP to the case without MD, Fig. $6 a .1$, one can notice that the convergence of algorithm is improved, in the sense that the performance shows a stable asymptotic condition. Moreover, the detector operating under $200 \times 200$ antennas configuration reached its convergence with less iterations, more precisely, in the third iteration the convergence is almost totally achieved against four iterations required in $\alpha_{\mathrm{MRF}}=0$ 

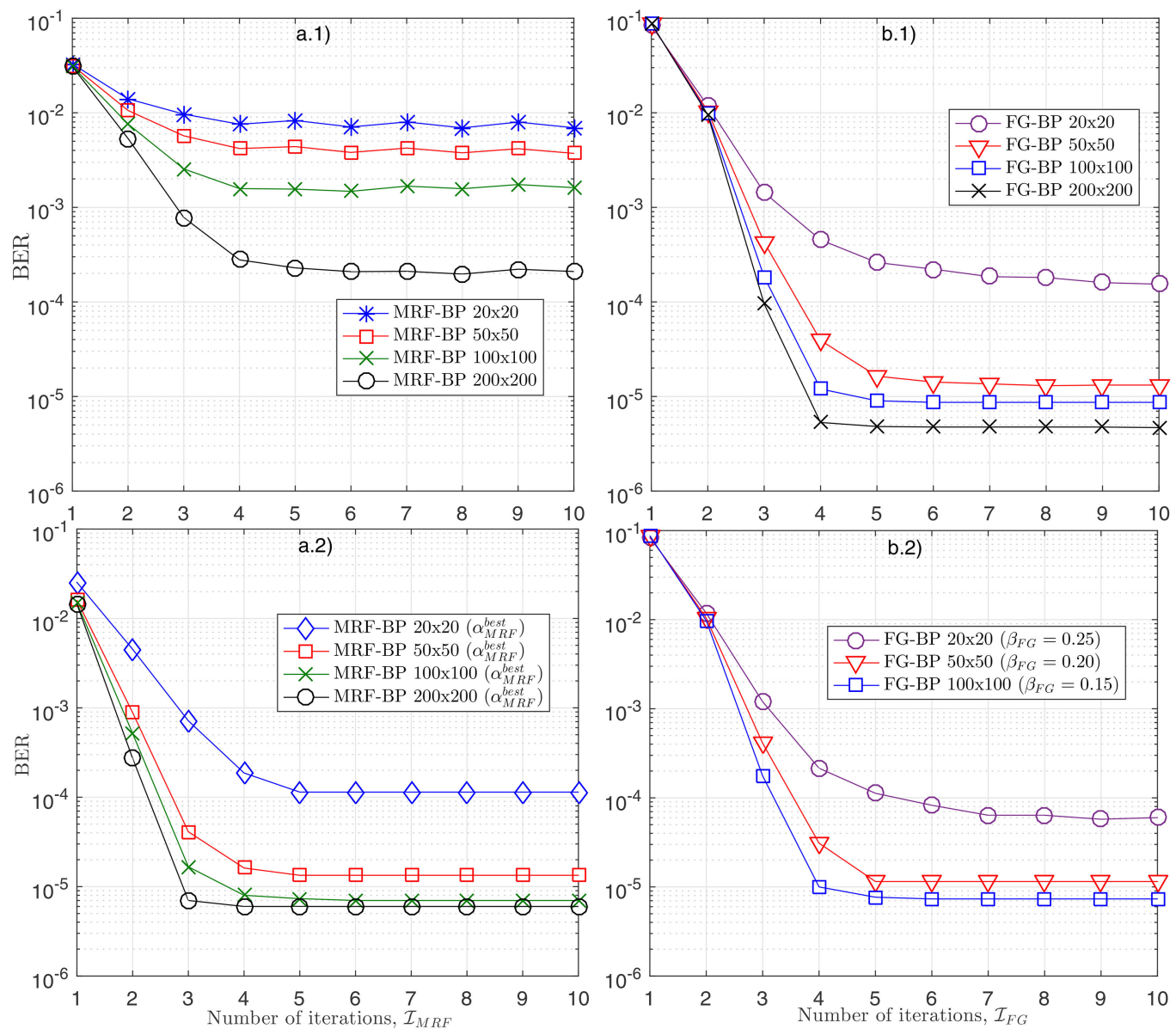

Fig. 6 Influence of the number of iterations $\mathscr{I}$ on the BER performance; both detectors operate under $\gamma_{d B}=10 \mathrm{~dB}$ and the scenarios are split in: (a.1) MRF$B P$ without $M D\left(\alpha_{M R F}=0\right)$, (b.1) FG-BP without $M D\left(\alpha_{F G}=\beta_{F G}=0\right)$, (a.2) MRF-BP with optimal MD, from (22), (b.2) FG-BP with best results of $M D$, from Fig. 8

(a) MRF-BP detector (Algorithm 1 (Fig. 3)), (b) FG-BP detector (Algorithm 2 (Fig. 4))

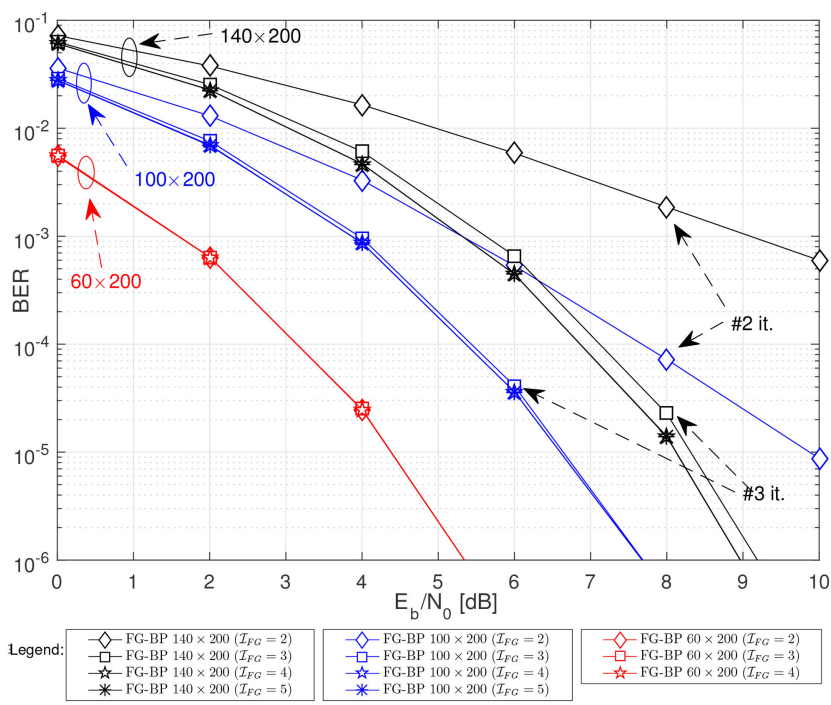

Fig. 7 BER of the FG-BP detector under different under-loading system $\left(N_{t}<N_{r}\right)$ over the iterations of the algorithm; $\alpha_{F G}=\beta_{F G}=0$

case. Besides, as mentioned previously, the performance level reached after the convergence is almost two decades superior adopting optimal MD values. Regarding the FG-BP detector, the resulting performance by applying the MD to the variable or observation nodes was analogous, as provided in Fig. 10. In this sense, the MD is applied only at variable nodes, i.e. $\alpha_{\mathrm{FG}}=0$ and $\beta_{\mathrm{FG}} \neq 0$. Thus, it can be observed from Fig. $6 b .2$ that a relevant performance gain in damped FG-BP detector occurs only in low number of antennas scenario $\left(N_{\mathrm{t}}=N_{\mathrm{r}}=20\right)$. In medium-to-high antennas schemes, the performance gain, provided by the MD at the observation nodes, along the $\mathscr{I}_{\mathrm{FG}}$ iterations is almost null. Moreover, in $20 \times 20$ case, the convergence is reached only in the seventh iteration; in the schemes with higher number of antennas there was no change in the convergence of the algorithm. Comparing MRF-BP to the FG-BP algorithm updating process, the MRF-BP with $\alpha_{\mathrm{MRF}}^{\text {best }}$ is able to achieve convergence with less iterations in all scenarios:

- $N_{\mathrm{t}}=N_{\mathrm{r}}=20:$ Damped MRF-BP with $\mathscr{I}_{\mathrm{MRF}}=4$ against damped FG-BP with $\mathscr{I}_{\mathrm{FG}}=7$.

- $N_{\mathrm{t}}=N_{\mathrm{r}}=50:$ Damped MRF-BP with $\mathscr{I}_{\mathrm{MRF}}=4$ against undamped FG-BP with $\mathscr{I}_{\mathrm{FG}}=5$.

- $N_{\mathrm{t}}=N_{\mathrm{r}}=100:$ Damped MRF-BP with $\mathscr{I}_{\mathrm{MRF}}=4$ against undamped FG-BP with $\mathscr{I}_{\mathrm{FG}}=5$.

- $N_{\mathrm{t}}=N_{\mathrm{r}}=200$ : Damped MRF-BP with $\mathscr{I}_{\mathrm{MRF}}=3$ against undamped FG-BP with $\mathscr{I}_{\mathrm{FG}}=4$.

This reduction in the number of iterations of the MRF-BP detector becomes specially interesting, since both detectors presented almost equal performance in LS antenna scenarios, Fig. 11.

In practical LS MIMO scenarios, the system loading usually works very below than $100 \%$ and the performance of FG-BP detector in these cases are showed in Fig. 7. One can notice that, for the low loading case, i.e., $\beta=N_{\mathrm{t}} / N_{\mathrm{r}}=0.3$, the FG-BP algorithm converges faster, compared to full loading scenario, and needed only two iterations to reach its best performance. As long as $\beta$ increases, more iterations are needed for the convergence, seeing that when $\beta=0.5$ the convergence is reached with $\mathscr{I}_{\mathrm{FG}}=3$ and for $\beta=0.7$ is totally achieved with $\mathscr{I}_{\mathrm{FG}}=4$ iterations. 

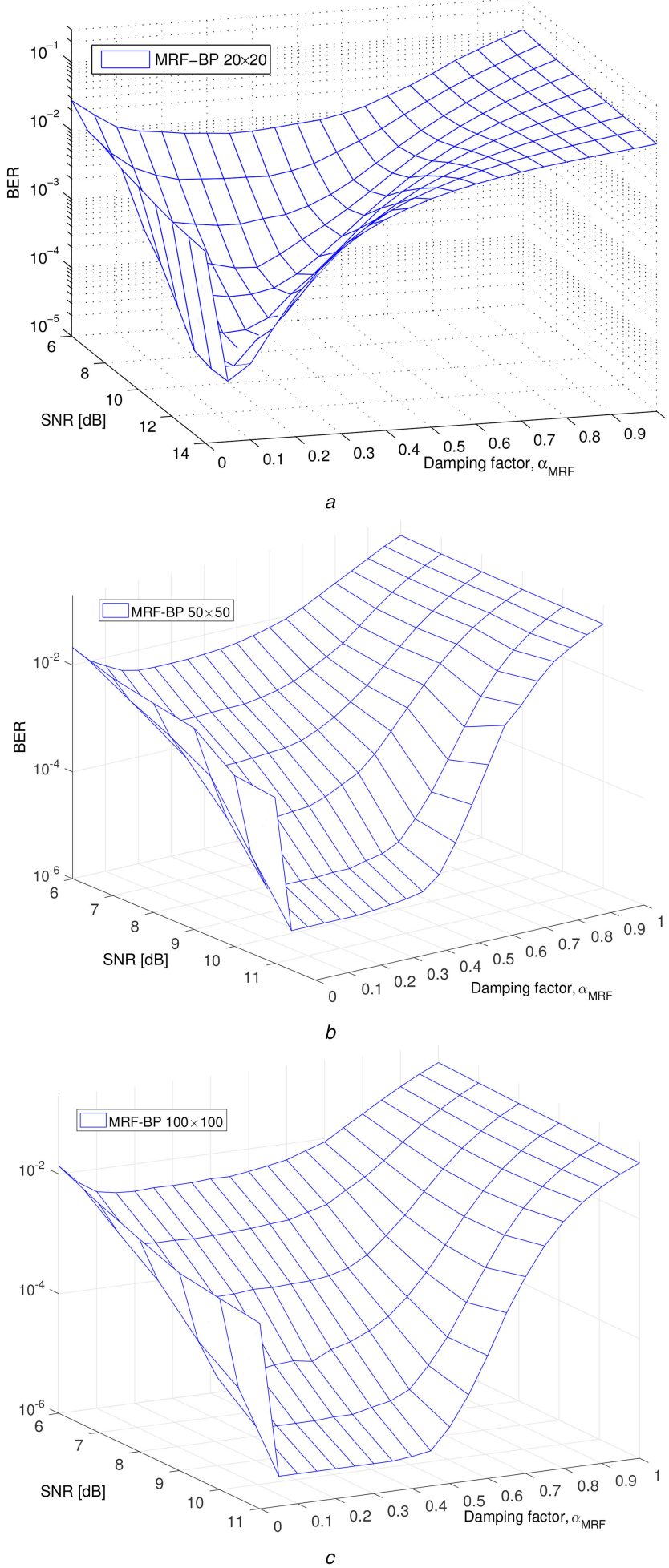

Fig. 8 BER performance of an MRF-BP LS-MIMO system with $\mathscr{I}_{M R F}=5$ as a function of different $S N R$ scenarios and MRF-BP DFs, $\alpha_{M R F}$ (a) $20 \times 20$, (b) $50 \times 50$, (c) $100 \times 100$ antennas

\subsection{Optimal value determination for the damping factor $\left(\alpha^{\text {best }}\right)$}

Now, considering the MD impact, Fig. 8 depicts the resulted BER performance due to the DF variation under medium SNR regions. An MRF-BP detector with $0 \leq \alpha_{\mathrm{MRF}} \leq 1$ is considered for 20,50 and 100 antennas. For the three antennas configurations, one can notice that, the performance gain with MD increases in higher SNR regions, while an optimal $\alpha_{\mathrm{MRF}}^{\text {best }}$ (best BER performance) can be defined for each SNR value. Indeed, for $\gamma_{\mathrm{dB}}=6 \mathrm{~dB}$ the performance gain with damping $\left(\alpha_{\mathrm{MRF}} \neq 0\right)$ is less than one decade; on the other hand, in the scenario with $\gamma_{\mathrm{dB}}=12 \mathrm{~dB}$, the

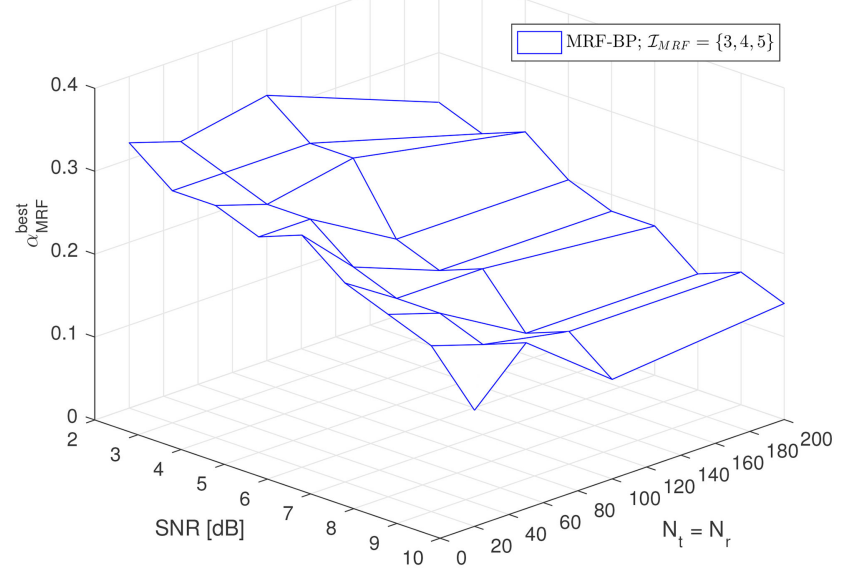

a

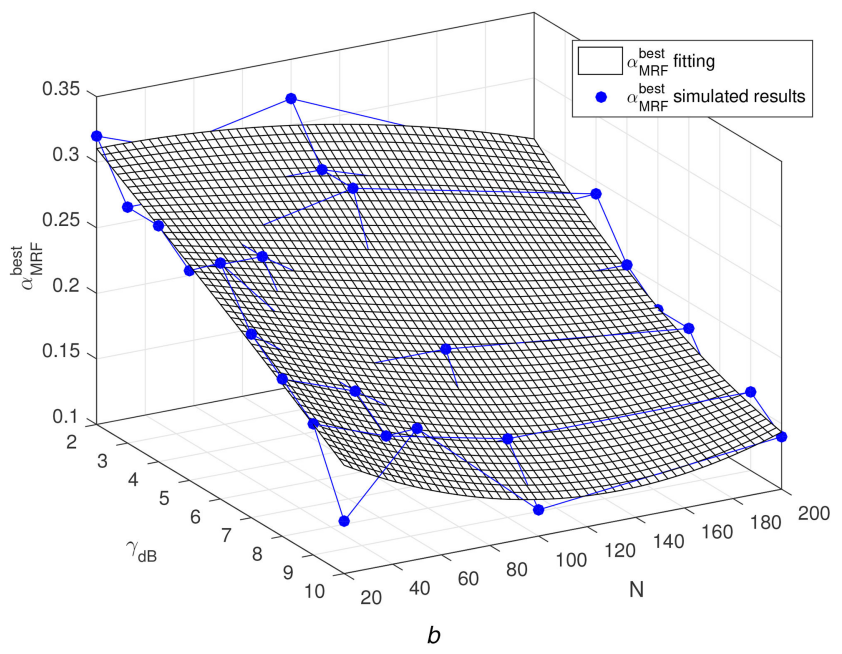

Fig. 9 Best DF values per SNR point and number of antennas $N$ for the $M R F-B P$ detector

(a) Simulated results, $\mathscr{I}_{\mathrm{MRF}} \in\{5,4,3\}$ for $N \in\{20 ; 50 ; 100 ; 200\}$, (b) Third-order polynomial data fitting

performance gain with a suitable choice of the DF value is $\simeq 2$ and 3 decades for $N_{\mathrm{t}}=20$ and 50 antennas, respectively. Moreover, specific conclusions regarding the scenario a) $N_{\mathrm{t}}=20$ antennas is that in higher SNR region, the best DF, i.e. associated with the lowest BER, has a lower value and tends to be more responsive to $\alpha_{\mathrm{MRF}}$ variations. Looking at the implicit curve of $\gamma_{\mathrm{dB}}=6 \mathrm{~dB}$, the performance gain variation from $0.25 \leq \alpha_{\mathrm{MRF}} \leq 0.45$ is negligible; while in the case of $14 \mathrm{~dB}$, the BER performance degradation from $\alpha_{\mathrm{MRF}}=0.05-0.20$ is more than one decade. Furthermore, the performance becomes worse regarding no damping messages case $\left(\alpha_{\mathrm{MRF}}=0\right)$ from $\alpha_{\mathrm{MRF}} \geq 0.35$ in $\gamma_{\mathrm{dB}}=14 \mathrm{~dB}$ the same situation occurs from $\alpha_{\mathrm{MRF}} \geq 0.80$ when $\gamma_{\mathrm{dB}}=6 \mathrm{~dB}$. Therefore, the DF value needs to be accurately chosen, specially when the system operates in medium/high SNR regions.

From Fig. $8 c$ ), it is distinguishable that, when $N_{\mathrm{t}}$ of LS-MIMO system increases, e.g. from $20 \times 20$ to $100 \times 100$ antennas, the BER performance behaviour with DF demonstrates constant (flat condition) at greater intervals of $\alpha_{\mathrm{MRF}}$. The same way that with $50 \times 50$ antennas the performance is flatter than $20 \times 20$ case (Figs. $8 a$ and $b$, respectively). One can conclude that the difficult task to accurately choose the DF value in small number of LSMIMO antennas scenario, i.e. $20 \times 20$, is relaxed with increasing number of antennas. Thus, the application of MD, especially in high SNR region, is more suitable in LS-MIMO systems due to the flat BER performance response to DF variation.

In Fig. 9, the best DF values $\alpha_{\mathrm{MRF}}^{\text {best }}$ are analysed from the perspective of number of antennas $N=N_{\mathrm{t}}=N_{\mathrm{r}}$ and SNR $\gamma_{\mathrm{dB}}$ values. The necessary number of iterations for convergence differs 
Table 2 Parameters of data fitting function: $\alpha_{\mathrm{MRF}}^{\text {best }}\left(\gamma_{\mathrm{dB}}, N\right),(22)$

\begin{tabular}{ll}
\hline Coefficient & Value \\
\hline$p_{00}$ & +0.33100 \\
$p_{10}$ & -0.01036 \\
$p_{01}$ & $+5.41100 \times 10^{-4}$ \\
$p_{11}$ & $-2.43800 \times 10^{-4}$ \\
$p_{20}$ & $-1.75800 \times 10^{-4}$ \\
$p_{02}$ & $-0.03419 \times 10^{-4}$ \\
$p_{12}$ & $+0.80930 \times 10^{-6}$ \\
$p_{21}$ & $+0.65220 \times 10^{-6}$ \\
$p_{30}$ & $-0.42090 \times 10^{-6}$ \\
rmse & 0.0207 \\
Coef. determ. $\left(r^{2}\right)$ & 0.8913 \\
\hline
\end{tabular}

from each antennas configuration scenario, according the analysis performed in Fig. $6 a .2$, i.e. $\mathscr{I}_{\mathrm{MRF}}=5$ iterations for $N=20$, $\mathscr{I}_{\mathrm{MRF}}=4$ for $N \in\{50,100\}$, and $\mathscr{J}_{\mathrm{MRF}}=3$ for $N=200$. One can notice that the best DF value tends to decrease in higher SNR regions and/or with increasing number of antennas. Despite the decrease in DF value, it is important to emphasise, regarding to Fig. 8, that a minor increase in $\alpha_{\mathrm{MRF}}$, i.e. from 0 to 0.1 , resulted in great performance gain, especially in high SNR regions.

To provide a wide $\alpha_{\mathrm{MRF}}^{\text {best }}$ determination, a data fitting is carried out on Fig. 9. We have obtained a polynomial data fitting for $\alpha_{\mathrm{MRF}}^{\text {best }}$ as a polynomial surface of the third order in $\gamma_{\mathrm{dB}}$ and second order in $N$ antennas, resulting in a root mean-squared error of RMSE $=0.02$, and given by

$$
\begin{aligned}
\alpha_{\mathrm{MRF}}^{\text {best }}= & p_{00}+p_{10} \gamma_{\mathrm{dB}}+p_{01} N+p_{11} \gamma_{\mathrm{dB}} N+p_{20} \gamma_{\mathrm{dB}}^{2} \\
& +p_{02} N^{2}+p_{12} \gamma_{\mathrm{dB}} N^{2}+p_{21} \gamma_{\mathrm{dB}}^{2} N+p_{30} \gamma_{\mathrm{dB}}^{3}
\end{aligned}
$$

where the coefficients and quality of the resulting fitting are presented in Table 2.

Fig. 10 demonstrates the impact on BER performance of MD method applied to the observation and variable nodes $\left(\alpha_{\mathrm{FG}}\right.$ and $\beta_{\mathrm{FG}}$, respectively) of FG-BP algorithm, considering different number of antennas and SNR values. From such BER 3D-graphics, one can notice that the MD applied to the FG-BP algorithm demonstrates equivalent performance behaviour when applied at observation or variable nodes and provides a reasonable BER performance gain only in the low antennas case, Figs. $10 a$ and $b$. In medium and high number of antennas scenarios, Figs. $10 c$ and $d$, the MD method does not imply performance gain, since the BER performance from $\beta_{\mathrm{FG}}=0$ does not improve with increasing $\beta_{\mathrm{FG}}$; the same behaviour was observed with $\alpha_{\mathrm{FG}}$ and for this reason its result was omitted. Considering that this work is focused on massive MIMO scenarios, where the FG-BP detector does not benefit from the MD technique, the undamped version of the FG-BP detector in medium and high antennas scenarios will be considered in the reminder of this paper, whereas in low number of antennas scenario, the $\beta_{\mathrm{FG}}=0.25$ value, obtained by simulation as the one with the highest performance gain, will be considered.

5.2.1 MD method with optimal damping factor applied to MRF-BF detector: Considering that MD procedure results in a relevant BER performance gain only in MRF-BP detector and the analysis performed around $\alpha_{\mathrm{MRF}}^{\text {best }}$, a BER comparison between FGBP (without MD in medium to LS-MIMO scenarios) and MRF-BP (with $\alpha_{\mathrm{MRF}}^{\text {best }}$ ) is quantified in Fig. 11, splitting it into two scenarios: (a) low-to-medium number of antennas, (b) high number of antennas. One can conclude that the MRF-BP with optimal DF value performs a marginally equal performance to the FG-BP detector. Compared to the result in [10], the MRF-BP with proposed $\alpha_{\mathrm{MRF}}^{\text {best }}$ reached marginally same performance with less number of antennas, besides that, the FG-BP with MD at variable nodes, proposed in this work, outperforms both MRF-BP damped detectors in this low antennas scenario. With large number of antennas, Fig. $11 b$, the FG approach shows a slightly better BER performance compared with MRF-BP. On the other hand, the iterations required for convergence is lower in MRF approach in all scenarios. In LS-MIMO scenarios, the MRF with optimal MD converges with one less iteration compared with the FG scheme. More evidently, regarding Fig. 6, in $200 \times 200$ case, MRF with optimal MD converges with three iterations, against four iterations of FG-BP, which can lead to a more interesting performance versus complexity tradeoff for MRF approach. An in-depth discussion of computational complexity is developed in Section 5.3.

In the sequel, the computational complexity of both detectors is evaluated, demonstrating that, under specific problem sizes, the MRF-BP algorithm can outperform the FG-BP in terms of both computational complexity (lower) and BER performance (lower). On the other hand, with increasing number of antennas, FG-BP tends to present a lower computational complexity than MRF-BP massive MIMO detector.

\subsection{Computational complexity}

The computational complexity is described in terms of floatingpoint operations (flops), in which one flop denotes the computational complexity of the complex mathematical operations: addition, subtraction, multiplication or division. In the case of exponential function, an approximation through Taylor Series with 18 terms has been considered. Table 3 describes the per-symbol computational complexity involved in each step of MRF-BP and FG-BP algorithms. The asymptotic per-symbol complexity of the Algorithm 1 (Fig. 3) is of the order of $\mathcal{O}\left(N_{\mathrm{t}}^{2}\right)$, while the Algorithm 2 (Fig. 4) complexity is about $\mathcal{O}\left(N_{\mathrm{r}}\right)$. It is important to note that $M D$ does not increase substantially the computational complexity of the analysed algorithm, as discussed in the sequel. In addition, since the LS-MIMO scenarios are part of the scope of this work, and the FG-BP detector benefited from the MD technique only with a low number of antennas, this section will consider the undamped version of the FG-BP detector, i.e. $\alpha_{\mathrm{FG}}=\beta_{\mathrm{FG}}=0$.

In Fig. 12a, the computational complexity increment of MD method in MRF-BP detector is evaluated. One can notice that the complexity increment of MD is very low compared with the overall MRF-BP detector complexity, whereas in the worst case points a percentual complexity increment holds below of $2.5 \%$ (when $N_{\mathrm{t}}=N_{\mathrm{r}}=20$ antennas and $\mathscr{I}_{\mathrm{MRF}}=5$ iterations). Furthermore, one can conclude that with increase number of antennas, the percentual complexity increment tends to a low constant floor $(\simeq 0.2 \%)$, which can be considered negligible and denotes an interesting application of MD in MRF-BP under LSMIMO scenarios.

In Fig. 12b, a numerical computational complexity comparisons between the two MP-based LS-MIMO detectors are performed relating the number of antennas and iterations on BP algorithm. It is noticeable that for $N \leq 160$ antennas (in both $\mathscr{I}_{\mathrm{MRF}}=\mathscr{I}_{\mathrm{FG}}=4$ and $\mathscr{I}_{\mathrm{MRF}}=\mathscr{I}_{\mathrm{FG}}=5$ ) the MRF-BP algorithm results in less 


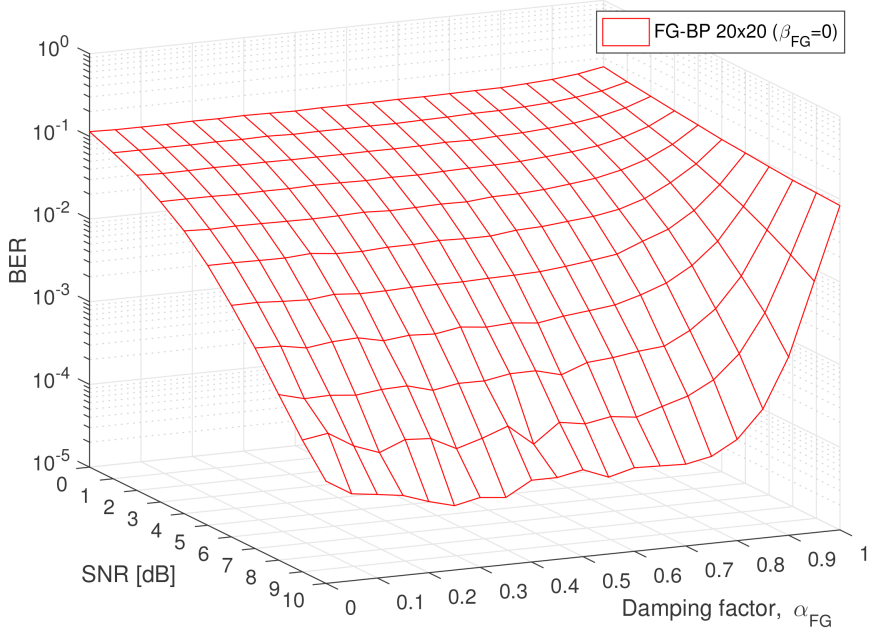

a

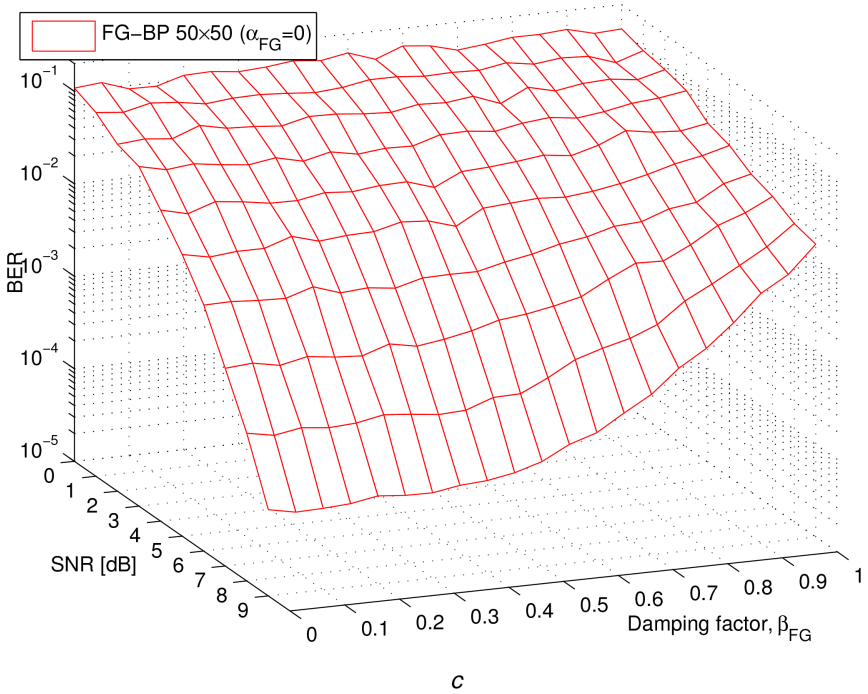

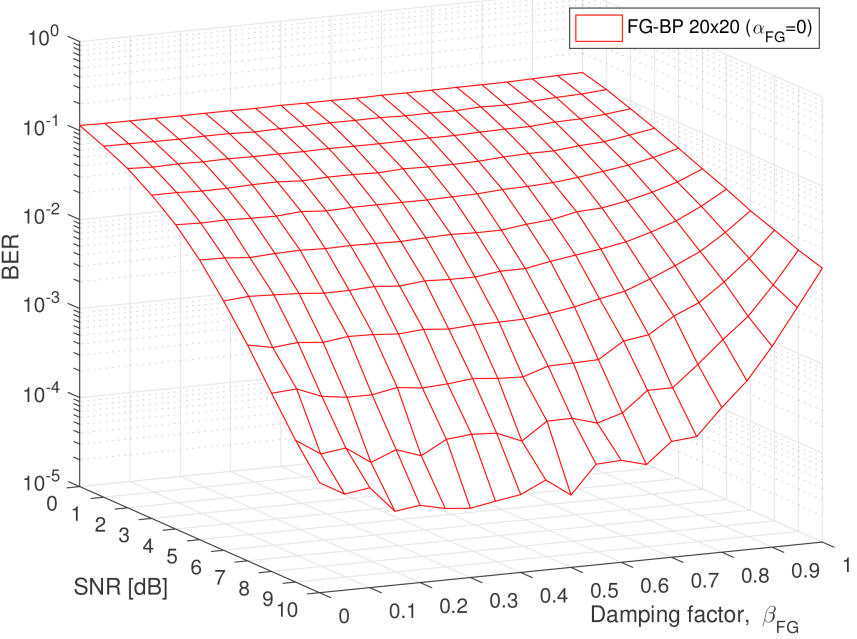

$b$

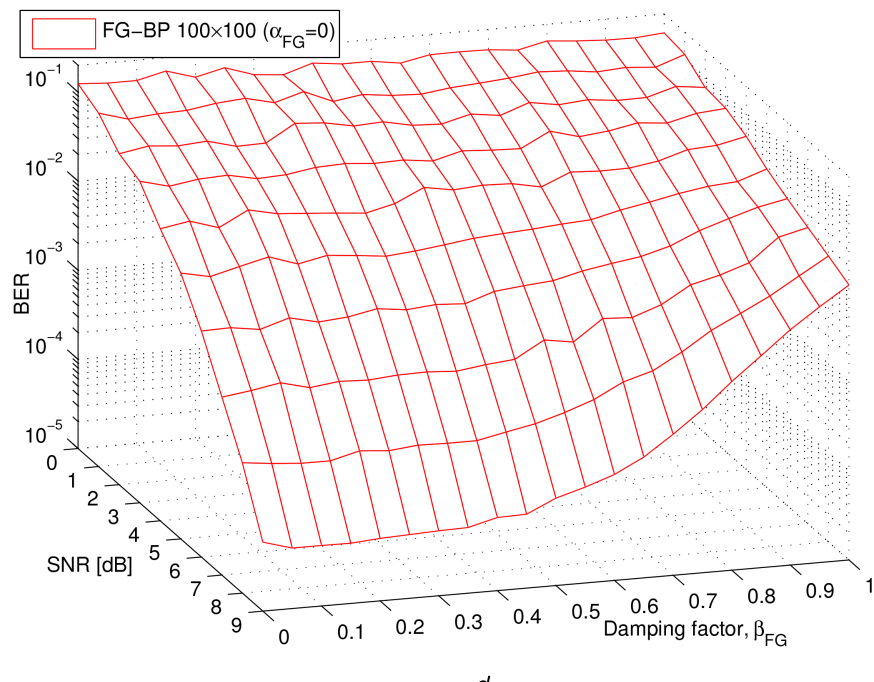

d

Fig. 10 BER performance of an FG-BP LS-MIMO system with $\mathscr{I}_{F G}=5$ as a function of different SNR scenarios

( $\boldsymbol{a}$ and $\boldsymbol{b}) N_{\mathrm{t}}=N_{\mathrm{r}}=20$ with DF at observation and variable nodes, respectively; (c and d) DF only at variable nodes $\left(\beta_{\mathrm{FG}}\right)$ with $N_{\mathrm{t}}=N_{\mathrm{r}}=50$ and $N_{\mathrm{t}}=N_{\mathrm{r}}=100$, respectively
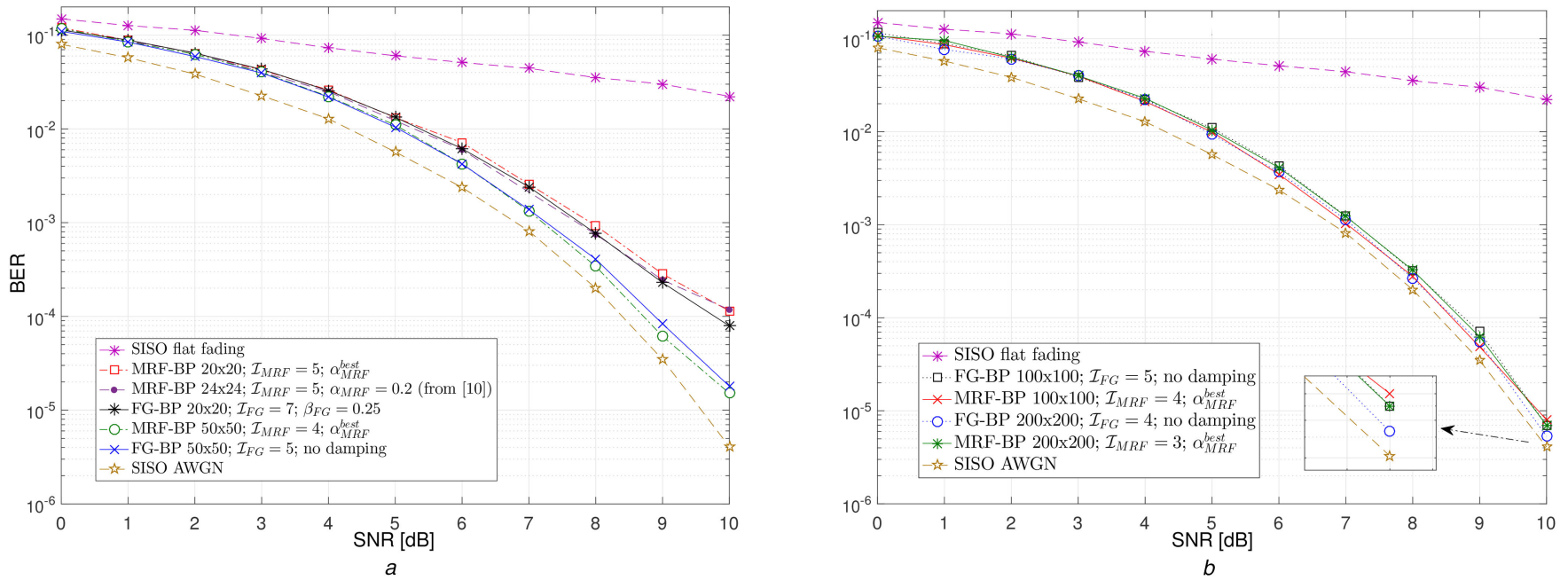

Fig. 11 BER performance of the implemented MRF-BP detector with the best DF value and FG-BP detector without MD, split into two scenarios (a) Low-to-medium number of antennas, (b) High number of antennas

computational complexity, although the difference becomes progressively smaller with the increase of antennas, due to the quadratic complexity order of $\mathcal{O}\left(N_{\mathrm{t}}^{2}\right)$ of MRF-BP against $\mathcal{O}\left(N_{\mathrm{r}}\right)$ from FG-BP. In terms of performance, under antenna configuration of $20 \times 20$ or $50 \times 50$, the MRF-BP approach demonstrated better BER performance than FG. Under higher number of antennas -
$N=100$ and 200 schemes - damped MRF-BP and undamped FG$\mathrm{BP}$ detectors have resulted in a marginally different performance, with a slight advantage for the FG-BP approach. Equalling the number of iterations, in $200 \times 200$ case, FG-BP performs with less complexity than MRF-BP; on the other hand, since optimal DF in MRF leads to an iteration reduction, MRF-BP after convergence 
Table 3 Per-symbol computational complexity of MRF-BP and FG-BP algorithms

\begin{tabular}{|c|c|c|}
\hline Procedure & Step & Complexity \\
\hline \multicolumn{3}{|c|}{ Algorithm 1 (Fig. 3) - MRF-BP } \\
\hline$R$ & line 4 & $N_{\mathrm{r}}-1$ \\
\hline$z$ & line 4 & $N_{\mathrm{r}}-1$ \\
\hline$\phi,(9)$ & line 6 & 718 \\
\hline$\psi,(10)$ & line 10 & $360\left(N_{\mathrm{t}}-1\right)$ \\
\hline messages update & I.15-25 & $\left(4 N_{\mathrm{t}}^{2}+4 N_{\mathrm{t}}-8\right) \cdot \mathscr{I}_{\mathrm{MRF}}$ \\
\hline MD & line 20 & $4\left(N_{\mathrm{t}}-1\right) \cdot \mathscr{I}_{\mathrm{MRF}}$ \\
\hline belief calculation & line 28 & $N_{\mathrm{r}}$ \\
\hline \multicolumn{3}{|c|}{ total complexity MRF-BP: $360 N_{\mathrm{t}}+3 N_{\mathrm{r}}+356+\cdots+\mathscr{J}_{\mathrm{MRF}}\left(4 N_{\mathrm{t}}^{2}+8 N_{\mathrm{t}}-12\right)$} \\
\hline \multicolumn{3}{|c|}{ Algorithm 2 (Fig. 4) - FG-BP } \\
\hline \multicolumn{3}{|c|}{ Computation of LLRs at observation nodes } \\
\hline$s_{\mu_{z i}}$ & line 6 & $\left(4 N_{\mathrm{r}}-\frac{N_{\mathrm{r}}}{N_{\mathrm{t}}}\right) \cdot \mathscr{J}_{\mathrm{FG}}$ \\
\hline$s_{\sigma_{z i}^{2}}^{2}$ & line 7 & $5 N_{\mathrm{r}} \cdot \mathscr{I}_{\mathrm{FG}}$ \\
\hline$\mu_{i k},(16)$ & line 9 & $3 N_{\mathrm{r}} \cdot \mathscr{I}_{\mathrm{FG}}$ \\
\hline$\sigma_{i k}^{2},(17)$ & line 10 & $7 N_{\mathrm{r}} \cdot \mathscr{I}_{\mathrm{FG}}$ \\
\hline LLR calculation, $\tilde{\Lambda}_{i}^{k,(t)}$ & line 11 & $4 N_{\mathrm{r}} \cdot \mathscr{I}_{\mathrm{FG}}$ \\
\hline $\mathrm{MD}, \alpha_{\mathrm{FG}}$ & line 13 & $4 N_{\mathrm{r}} \cdot \mathscr{I}_{\mathrm{FG}}$ \\
\hline \multicolumn{3}{|c|}{ Computation of probabilities at variable nodes } \\
\hline$s_{\Lambda_{k}}$ & line 18 & $\left(N_{\mathrm{r}}-1\right) \cdot \mathscr{I}_{\mathrm{FG}}$ \\
\hline probabilities, $\tilde{p}_{i}^{k+,(t)}$ & line 20 & $720 N_{\mathrm{r}} \cdot \mathscr{I}_{\mathrm{FG}}$ \\
\hline $\mathrm{MD}, \beta f g$ & line 22 & $4 N_{\mathrm{r}} \cdot \mathscr{I}_{\mathrm{FG}}$ \\
\hline detection of data bits & line 28 & $\left(N_{\mathrm{r}}-1\right)$ \\
\hline
\end{tabular}

$\left(\mathscr{J}_{\mathrm{MRF}}=3\right)$ shows a marginally lower complexity than FG-BP with $\mathscr{I}_{\mathrm{FG}}=4$ iterations. Finally, with the increase in the number of antennas, i.e. $N_{\mathrm{t}}=N_{\mathrm{r}}>200$, FG scheme performs with a less computational complexity than MRF due to its first-order complexity in $N_{\mathrm{r}}$.

\section{Conclusions}

A detector for LS MIMO systems based on MP MRF GM and BP algorithm has been extensively analysed. More specifically, the impact of the MD method on the BER performance was evaluated and it has demonstrated a promising performance versus complexity tradeoff gain for MP detectors based on MRF GM, especially in LS-MIMO scenarios.

Numerical results for the damped MRF-BP LS-MIMO detector have demonstrated promising performance-complexity tradeoff, since damping messages procedure just negligibly increases the overall computational complexity while providing a significant performance gain in the MRF-BP detection. Considering the algorithm convergence, the MD method resulted in faster MRF-BP convergence, with one less iterations (total of $\mathscr{I}_{\mathrm{MRF}}=3$ against $\mathscr{I}_{\mathrm{MRF}}=4$ without damping case) in LS MIMO configuration, i.e. $200 \times 200$. Such results demonstrate a consistent gain, not only in performance but also in the computational complexity, thanks to the application of the MD procedure in the MRF-BP algorithm.

Besides, under LS antenna scenarios, DF value choose is facilitated due to the flat BER performance response to a relative wide $\alpha_{\mathrm{MRF}}$ values interval. The FG-BP approach with a massive number of antennas has demonstrated a performance close to the optimal SISO AWGN, even without MD. Also, the same detector presented a BER performance gain with increasing number of antennas, demonstrating also be an attractive LS-MIMO detector. On the other hand, the MD method applied on FG-BP algorithm did not result in relevant performance gain in medium-to-high number of antennas scenarios, whereas a reasonable performance gain was reached in low antennas scheme. Comparing both massive MIMO detectors operating under their best performance condition, MRF-BP with optimal DF value demonstrated a marginally equal performance than $\mathrm{FG}$ approach.

The computational complexity of MRF-BP algorithm results in a quadratic order with the number of transmit antennas, while FGBP algorithm presents a first-order complexity regarding the analogue variable at the receiver side. Nevertheless, under smallto-medium/high number of antennas scenarios, MRF approach attains a lower complexity than FG one.

\section{Acknowledgments}

This work has been partially supported by the National Council for Scientific and Technological Development $(\mathrm{CNPq})$ of Brazil under grant 304066/2015-0, by the Londrina State University (UEL), by the Federal Institute of Paraná (IFPR) and the Paraná State Government. All the agencies are gratefully acknowledged. 

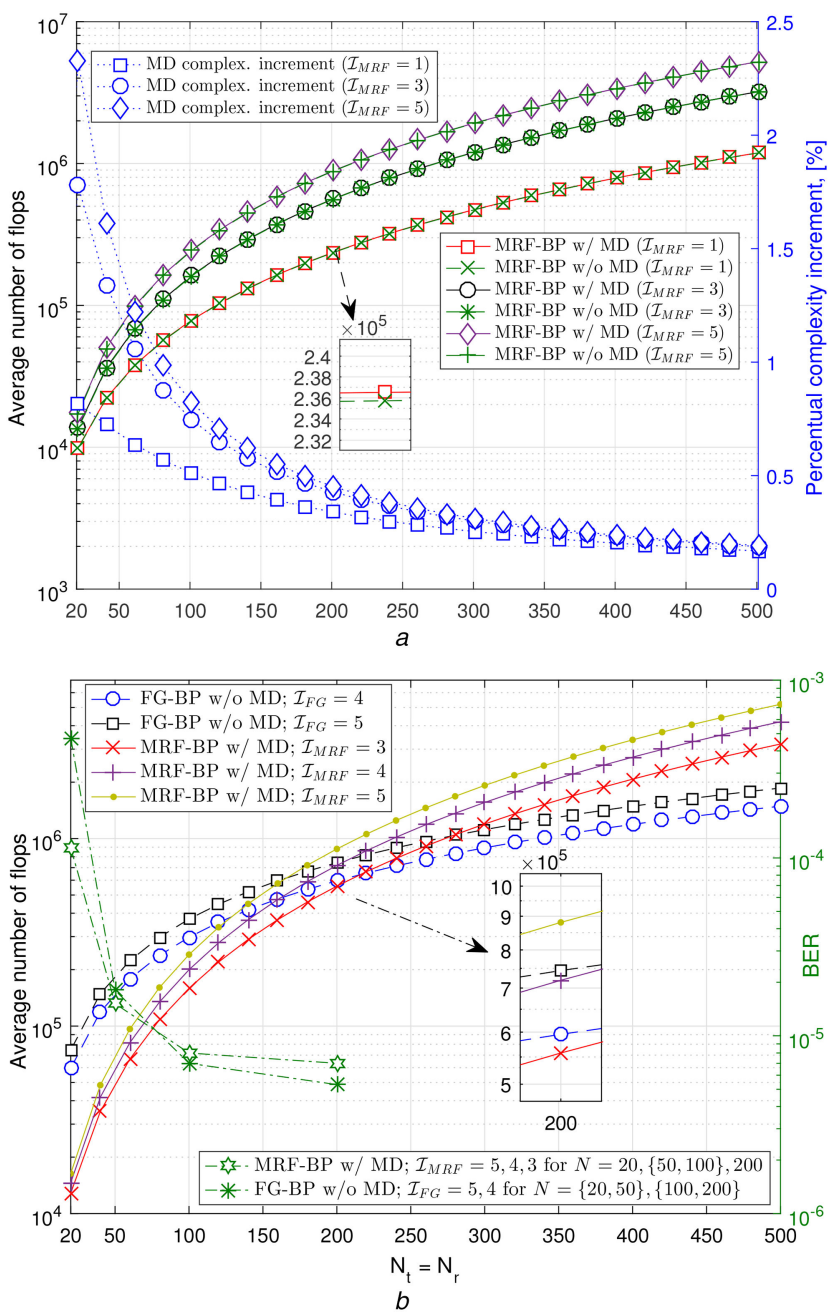

Fig. 12 Analysis of computational complexity, in terms of average number of flops, operating under LS MIMO scenarios, $N_{\mathrm{t}}=N_{\mathrm{r}}$ antennas and different number of algorithm iterations, $\mathscr{I} \in\{3,4,5\}$, carried out in two strands

(a) Percentual complexity increment of MD method applied in MRF-BP detector (b) Comparison of complexity and respective BER among MRF-BP and FG-BP detectors, $\gamma_{\mathrm{dB}}=10 \mathrm{~dB}$

\section{References}

[1] Ericsson, A.: 'Traffic and market report: on the pulse of the networked society'. Technical Report 198/287 01-FGB 101 220, Ericsson, Stockholm, Sweden, 2012

[2] Telatar, E.: 'Capacity of multi-antenna Gaussian channels', Eur. Trans. Telecommun., 1999, 10, (6), pp. 585-595. Available at http://dx.doi.org/ $10.1002 /$ ett.4460100604

[3] Foschini, G.J., Gans, M.J.: 'On limits of wireless communications in a fading environment when using multiple antennas', Wirel. Pers. Commun., 1998, 6, pp. 311-335

[4] Hoydis, J., ten Brink, S., Debbah, M.: 'Massive MIMO in the UL/DL of cellular networks: How many antennas do we need?', IEEE J. Sel. Areas Commun., 2013, 31, (2), pp. 160-171

[5] Rusek, F., Persson, D., Lau, B.K., et al.: 'Scaling up MIMO: opportunities and challenges with very large arrays', IEEE Signal Process. Mag., 2013, 30, (1), pp. $40-60$
[6] Marzetta, T.L.: 'Noncooperative cellular wireless with unlimited numbers of base station antennas', IEEE Trans. Wirel. Commun., 2010, 9, (11), pp. 35903600

[7] Zheng, K., Zhao, L., Mei, J., et al.: 'Survey of large-scale MIMO systems', IEEE Commun. Surv. Tutor., 2015, 17, (3), pp. 1738-1760

[8] Vardhan, K., Mohammed, S.K., Chockalingam, A., et al.: 'A low-complexity detector for large MIMO systems and multicarrier CDMA systems', IEEE $J$ Sel. Areas Commun., 2008, 26, (3), pp. 473-485

[9] Rajan, B.S., Mohammed, S.K., Chockalingam, A., et al.: 'Low-complexity near-ml decoding of large non-orthogonal STBCS using reactive TABU search'. IEEE Int. Symp. Information Theory, 2009. ISIT 2009., 2009, pp. 1993-1997

[10] Som, P., Datta, T., Chockalingam, A., et al.: 'Improved large-MIMO detection based on damped belief propagation'. 2010 IEEE Information Theory Workshop on Information Theory (ITW 2010, Cairo), 6-8 January 2010, pp. $1-5$

[11] Suneel, M., Som, P., Chockalingam, A., et al: 'Belief propagation based decoding of large non-orthogonal STBCS'. IEEE Int Symp. Information Theory, 2009. ISIT 2009, June 28-July 32009 , pp. 2003-2007

[12] Som, P., Datta, T., Srinidhi, N., et al.: 'Low-complexity detection in largedimension MIMO-ISI channels using graphical models', IEEE J. Sel. Topics Signal Process., 2011, 5, (8), pp. 1497-1511

[13] Yue, Z., Guo, Q., Xiang, W.: 'Complex Gaussian belief propagation algorithms for distributed multicell multiuser MIMO detection with imperfect channel state information'. 2014 IEEE 25th Annual Int. Symp. on Personal, Indoor, and Mobile Radio Communication (PIMRC), 2-5 September 2014, pp. 1902-1907

[14] Su, Q., Wu, Y.C.: 'Convergence analysis of the variance in Gaussian belief propagation', IEEE Trans. Signal Process., 2014, 62, (19), pp. 5119-5131

[15] Takahashi, T., Ibi, S., Ohgane, T., et al.: 'On normalized belief of Gaussian BP in correlated large MIMO channels'. 2016 Int. Symp. Information Theory and Its Applications (ISITA), October 30-November 2 2016, pp. 458-462

[16] Yoon, S., Chae, C.B.: 'Low-complexity MIMO detection based on belief propagation over pairwise graphs', IEEE Trans. Veh. Technol., 2014, 63, (5) pp. 2363-2377

[17] Liu, L., Yuen, C., Guan, Y.L et al. 'Convergence analysis and assurance for Gaussian message passing iterative detector in massive MU-MIMO systems', IEEE Trans. Wirel. Commun., 2016, 15, (9), pp. 6487-6501

[18] Yang, P., Xiao, Y., Guan, Y.L., et al.: 'Single-carrier SM-MIMO: a promising design for broadband large-scale antenna systems', IEEE Commun. Surv. Tutor., 2016, 18, (3), pp. 1687-1716

[19] Mooij, J.M., Kappen, H.J.: 'Sufficient conditions for convergence of the sumproduct algorithm', IEEE Trans. Inf. Theory, 2007, 53, (12), pp. 4422-4437

[20] Murphy, K.P., Weiss, Y., Jordan, M.I.: 'Loopy belief propagation for approximate inference: An empirical study'. Proc. Fifteenth Conf. on Uncertainty in Artificial Intelligence, San Francisco, 1999, pp. 467-475

[21] Pretti, M.: 'A message-passing algorithm with damping', J. Stat. Mech. Theory Exp., 2005, 2005, (11), p. P11008

[22] Yuille, A.L.: 'A double-loop algorithm to minimize the Bethe and Kikuchi free energies', Neural Comput., 2001, 14, p. 2002

[23] Heskes, T., Albers, K., Kappen, B.: 'Approximate inference and constrained optimization'. Proc. of the Nineteenth Conf. on Uncertainty in Artificial Intelligence. UAI'03, San Francisco, CA, USA, 2003, pp. 313-320. Available from: http://dl.acm.org/citation.cfm?id=2100584.2100622

[24] Narasimhan, T.L., Chockalingam, A., Rajan, B.S.: 'Factor graph based joint detection/decoding for LDPC coded large-MIMO systems'. 2012 IEEE 75th Vehicular Technology Conf. (VTC Spring), 6-9 May 2012, pp. 1-5

[25] Frey, B.J.: 'Graphical models for machine learning and digital communication' (MIT Press, Cambridge, MA, USA, 1998)

[26] Chockalingam, A., Rajan, B.S.: 'Large MIMO systems' (Cambridge University Press, 2014)

[27] Heckerman, D., Wellman, M.P.: 'Bayesian networks', Commun. ACM, 1995 38, (3), pp. 27-30. Available at http://doi.acm.org/10.1145/203330.203336

[28] Griffeath, D.: 'Introduction to Markov random fields', in Kerney, J.G., Snell, J.L., Knupp, A.W. (Eds.): 'Denumerable Markov chains' (Springer-Verlag, New York, 1976, 2nd edn.)

[29] Kschischang, F.R., Frey, B.J., Loeliger, H.A.: 'Factor graphs and the sumproduct algorithm', IEEE Trans. Inf. Theory, 2001, 47, (2), pp. 498-519

[30] Aji, S.M., McEliece, R.J.: 'The generalized distributive law', IEEE Trans. Inf. Theory, 2000, 46, (2), pp. 325-343 


\section{APPENDIX D - Full paper published in the journal "IET Signal Processing", November 2018}

Title: Multiple restarts mixed Gibbs sampling detector for large-scale antenna systems.

Authors: Alex Miyamoto Mussi and Taufik Abrão.

Journal: IET Signal Processing (ISSN 1751-9675).

Classification (Qualis-CAPES 2016): A2 (Engenharias IV).

Impact Factor (2017): 1.25.

Publication Date: November 2018.

DOI: $10.1049 /$ iet-spr.2018.5206 


\title{
Multiple restarts mixed Gibbs sampling detector for large-scale antenna systems
}

ISSN 1751-9675

Received on 4th June 2018 Revised 22nd September 2018 Accepted on 1st November 2018 doi: 10.1049/iet-spr.2018.5206 www.ietdl.org

\author{
Alex Miyamoto Mussi', Taufik Abrao $2,3 \otimes$ \\ ${ }^{1}$ Electrical Engineering Department, State University of Londrina, Rod. Celso Garcia Cid - PR445, Po.Box 10.011. CEP:86057-970, Londrina, \\ $P R$, Brazil \\ Q1 ${ }^{2}$ Federal Institute of Paraná Assis Chateaubriand, Brazil \\ ${ }^{3}$ University of São Paulo, Brazil \\ 凶E-mail: taufik@uel.br
}

\begin{abstract}
This work proposes a low-complexity detector for medium- and high-order modulation large-scale multiple-input multiple-output (LS-MIMO) systems based on the set of Markov chain Monte-Carlo techniques. Such efficient signal detection algorithm is based on the mixed Gibbs sampling with multiple restarts (MGS-MR) strategy with sample-averaged approach during the coordinate updating process, named averaged MGS (aMGS). The proposed strategy applies multiple samples average procedure to restrict the range of the random solution, which comes from the mixture proposed by the original MGS. Numerical simulation results considering higher-order $M$-QAM demonstrated that the proposed detection method can substantially improve the convergence of the MGS-MR algorithm, while no extra computational complexity is required. The proposed aMGS-based detector suitable for medium- and high-order modulation LS-MIMO further exhibits improved performance when the system loading is high, i.e. when $(K / N) \geq 0.75$. In addition, the proposed numerical simulation analyses have shown that the optimal value of the mixing ratio parameter can vary regarding system and channel configuration scenarios, resulting somewhat different from the $1 / 2 \mathrm{~K}$ value disseminated in the literature.
\end{abstract} Q2

\section{Introduction}

Recently, communication schemes that use tens to hundreds of antennas in transmission and reception of signals, termed largescale multiple-input multiple-output (LS-MIMO), has been of interest in the telecommunications research area. Such structures hold the same benefits as conventional MIMO, however on a larger scale. More properly, LS-MIMO is defined as a transmission/ reception design using typically several tens or even hundreds of antennas in at least one of the communication terminals, usually in the base station (BS) [1, 2]. The reduced dimensions of user equipments (UEs) suggest a single antenna arrangement in each $\mathrm{UE}$; on the other hand, a very large number of antennas are installed in each BS. Asymptotically, i.e. when the number of antennas $N$ at the BS increases to infinite while the ratio $K / N$ holds constant, where $K$ is the number of single-antenna UEs, the LSMIMO system results in paramount advantages: (i) effects of noise background and fast fading channel disappear; (ii) the transmission rate and the number of UEs become independent of cell size; (iii) spectral efficiency becomes independent of the system bandwidth; and (iv) power required for transmission of bit tends to zero [3].

These results become very interesting in scenarios with poor channel estimates due to the high noise power, meaning very low signal-to-noise ratio (SNR): a sufficient increase in the number of BS antennas in a LS-MIMO system is capable of mitigating harmful effects of error in the channel estimation. On the other hand, in multicellular LS-MIMO systems the use of training pilot sequences for channel estimation purpose imposes an intercellular interference. The problem is called pilot contamination, persisting even in asymptotic BS antennas scenarios [3].

The LS-MIMO high capacity/spectral efficiency comes with a price; as the number of antennas at BS increases, the computational complexity of data detection tends to grow proportionally. In this sense, a low computational complexity detector emerges as an essential requirement in LS-MIMO systems. Many low-complexity LS-MIMO detectors have been proposed in recent literature, including detectors based on (i) local neighbourhood search, such as likelihood ascent search algorithm [4], and reactive tabu search algorithm [5]; (ii) message passing algorithms, based on belief propagation technique, such that LS-detectors inspired in graphical models, as factor graph [6] and Markov random fields [7]; (iii) minimum mean square error (MMSE) approximation techniques $[8,9]$, which result in low complexity at the price of achieving good performance only at low system loading factor; (iv) Markov chain Monte Carlo (MCMC) techniques, which are based on Gibbs sampling (GS) [10], emerging as a promising approach to deal with LS-MIMO structures [11-15], since such techniques demonstrate a near-optimum performance while require a moderate complexity (quadratic order), also presenting a simple and effective way to solve the large-scale detection problem.

The MCMC was introduced in MIMO and digital communications in $[10,16]$. The application of GS-based MCMC on detection problem in MIMO systems was first studied in [10], while in [11] a mixed GS (MGS) was proposed to solve the stalling problem attached to the conventional GS detector, additionally a multiple restarts (MR) strategy was proposed in order to deal with a convergence problem for modulation orders $M \geq 16$, called MGS-MR. Also in [11], the choice of mixing ratio parameter is determined by the inverse of the number of real dimensions of the system, i.e. for a system with complex modulation alphabet, $q=1 / 2 K$. The optimal choice in [11] was based on Markov chains theory considering low dimension systems. However, in our current contribution we demonstrate by numerical simulation that the choice of mixing ratio parameter is also dependent on the system loading and the number of samples deployed in coordinate updating process; thus, it is not always coincident with the inverse of the number of real dimensions.

In [17], an optimisation on mixing time was introduced to accelerate the finding of the optimal solution. Numerical simulation results demonstrated that the choice of mixing time based on SNR can improve convergence, although the stalling problem persisted when a fixed mixing time is adopted. Also, the results do not consider the performance behaviour in high-order modulation schemes. More recently, the MCMC detector with QR decomposition was addressed in [14, 18], which can reduce the number of operations due to the lower triangular matrix feature. Besides, based on the multiple random parallel Markov chains, the work in [13] proposes a MR strategy through parallel chains; such 


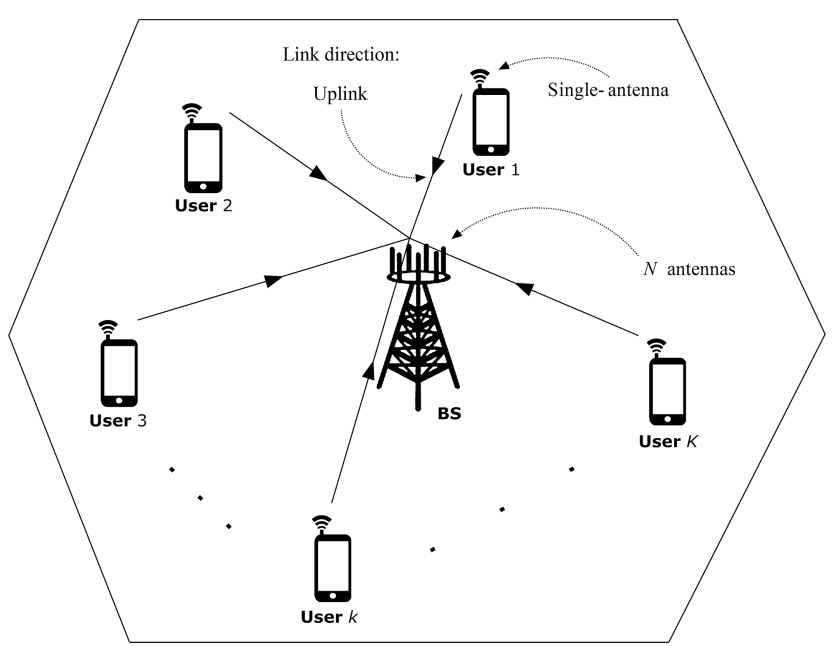

Fig. 1 Single-cell uplink LS-MIMO communication system with K singleantenna UEs and a $B S$ with $N$ receive antennas

strategy reduced the algorithm's running time compared to MGS$\mathrm{MR}$, despite the increasing of the number of real operations per symbol. Moreover, in [12] a MCMC detector using successive over-relaxation is proposed in order to improve the convergence rate, although $M$-QAM modulation orders higher than $M=16$ were not investigated.

The contribution of this work is threefold: (i) we propose an averaged multiple sampling (MS) strategy in order to improve the MGS convergence rate operating under higher-order modulation and large-scale MIMO regime. The proposed strategy deploys multiple samples per coordinate and performs a simple mean to estimate each coordinate symbol to alleviate the impact caused by the random solution, i.e. infinite mixing time, employed in the mixture used by the MGS detector. (ii) extensive numerical analysis regarding the optimal mixing ratio value, evidencing its dependence w.r.t. the number of antennas and the quantity of averaged samples; thus, we show that the optimal mixing ratio value is not equal to the inverse of the number of real dimensions, as stated in [11]. (iii) lastly, we propose a simplification on the target function aiming at reducing its computational complexity, while maintaining the performance. Since the target function is computational burden, requiring $2 K$ times per iteration, our proposition is that the target function can be directly based on the maximum-likelihood (ML) cost minimisation, which avoids the exponential function computation, resulting in a complexity decreasing.

The remainder of this paper is organised as follows. Section 2 presents the adopted large-scale MIMO system model. A review on the MGS technique is presented in Section 3 and the proposed averaged MGS (aMGS) detection scheme suitable for LS-MIMO is developed in Section 4. Extensive numerical simulation results are analysed in Section 5. Conclusion remarks are provided in Section 6.

\section{System model}

For simplicity of analysis, let us consider an uplink (UL) singlecell MIMO communication system operating in multiplexing gain mode with $K$ active single-antenna users and $N$ receive antennas at the BS (Fig. 1). Since the purpose of this work is to investigate the performance $\times$ complexity tradeoff of suitable LS-MIMO detection schemes, we assume the availability of the channel state information at the BS, aiming to consider the pure efficiency of each detection technique; therefore, the pilot training stage and the respective pilot contamination effect have not taken into account in such context.

Furthermore, for simplicity, the radio channel is assumed to be frequency-flat fading, characterised by the complex channel matrix $\boldsymbol{H}_{c} \in \mathbb{C}^{N \times K}$. The elements of $\boldsymbol{H}_{c}$ are all independent complex Gaussian random variables with zero mean and unit variance, $H_{c_{i, k}} \sim \mathscr{C} \mathcal{N}[0 ; 1]$. Let $\boldsymbol{s}_{c}$ be the $K \times 1$ complex vector corresponding to the $K$ symbols $M$-QAM transmitted over the transmit antennas, $\boldsymbol{s}_{c} \in \mathbb{A}_{c}^{K}$ where $\mathbb{A}_{c}$ denotes the QAM constellation. The received signal $y_{c_{i}}$ at the $i$ th $\mathrm{BS}$ antenna, considering the UL direction and that the BS is interested on the signal from user $k$, can be written as

$$
\begin{aligned}
y_{c_{i}} & =\sum_{j=1}^{K} H_{c_{i, j}} s_{c_{j}}+\eta_{c_{i}}, \quad i=1, \ldots, N \\
& =\underbrace{H_{c_{i, k} s_{c_{k}}}}_{\text {desired signal }}+\underbrace{\sum_{j=1, j \neq k}^{K} H_{c_{i, j} s_{c_{j}}}}_{\text {intracellular interference }}+\underbrace{\eta_{c_{i}}}_{\mathrm{AWGN}} .
\end{aligned}
$$

Using matrix notation, the received signal vector at the BS equipped with $N$ antennas is re-written as

$$
\boldsymbol{y}_{c}=\boldsymbol{H}_{c} \boldsymbol{s}_{c}+\boldsymbol{\eta}_{c}
$$

where $\boldsymbol{\eta}_{c}$ is the additive white Gaussian noise, vector, assumed to be a complex Gaussian random variable with zero mean and variance given by

$$
\mathbb{E}\left[\boldsymbol{\eta}_{c} \boldsymbol{\eta}_{c}^{\mathrm{H}}\right]=\sigma^{2} \boldsymbol{I}_{N}
$$

where $\sigma^{2}$ is the noise variance at each receive antenna.

The system loading factor, $\beta$, is defined as

$$
\beta=\frac{K}{N}
$$

The average received SNR at each receive antenna can be modelled as

$$
\gamma=\frac{K P_{\mathrm{s}}}{\sigma^{2}}
$$

where $P_{\mathrm{s}}$ is the power of the received symbols. For simplicity, it is assumed that the symbols of all users have their transmit power properly adjusted so that all the symbols reach the BS with the same power. Indeed, in (5) it was considered that the large-scale fading effect has been compensated in such a way that all $K$ users' signals are received with equal power at the BS, and assumed equal to $K P_{\mathrm{s}}$, denoting the total sum power available at the transmitters [19].

In this work, a real-valued system model [20] corresponding to (2) is adopted, which is given by

$$
\boldsymbol{y}=\boldsymbol{H} \boldsymbol{s}+\boldsymbol{\eta}
$$

where $\boldsymbol{y} \in \mathbb{R}^{2 N \times 1}, \boldsymbol{H} \in \mathbb{R}^{2 N \times 2 K}, \boldsymbol{s} \in \mathbb{R}^{2 K \times 1}, \boldsymbol{\eta} \in \mathbb{R}^{2 N \times 1}$, and defined as

$$
\begin{aligned}
\boldsymbol{H} & =\left[\begin{array}{cc}
\mathscr{R}\left(\boldsymbol{H}_{c}\right) & -\mathscr{I}\left(\boldsymbol{H}_{c}\right) \\
\mathscr{I}\left(\boldsymbol{H}_{c}\right) & \mathscr{R}\left(\boldsymbol{H}_{c}\right)
\end{array}\right] \\
\boldsymbol{s} & =\left[\begin{array}{c}
\mathscr{R}\left(s_{c}\right) \\
\mathscr{I}\left(s_{c}\right)
\end{array}\right], \quad \boldsymbol{\eta}=\left[\begin{array}{l}
\mathscr{R}\left(\boldsymbol{\eta}_{c}\right) \\
\mathscr{I}\left(\boldsymbol{\eta}_{c}\right)
\end{array}\right], \quad \boldsymbol{y}=\left[\begin{array}{c}
\mathscr{R}\left(\boldsymbol{y}_{c}\right) \\
\mathscr{I}\left(\boldsymbol{y}_{c}\right)
\end{array}\right]
\end{aligned}
$$

For the QAM alphabet $\mathbb{A}_{c}$, the elements of $s$ assume integer values from the underlying pulse-amplitude modulation alphabet $\mathbb{A}$, i.e. $s \in \mathbb{A}^{2 K}$.

The ML decision rule is given by

$$
\boldsymbol{s}_{\mathrm{ML}}=\underset{\hat{\boldsymbol{s}} \in \mathbb{A}^{2 K}}{\arg \min }\|\boldsymbol{y}-\boldsymbol{H} \hat{\boldsymbol{s}}\|^{2}
$$

However, the ML detector in (8) is exponentially complex in $K$, being prohibitive for large $K \cdot N$, which is the case of LS-MIMO systems. 


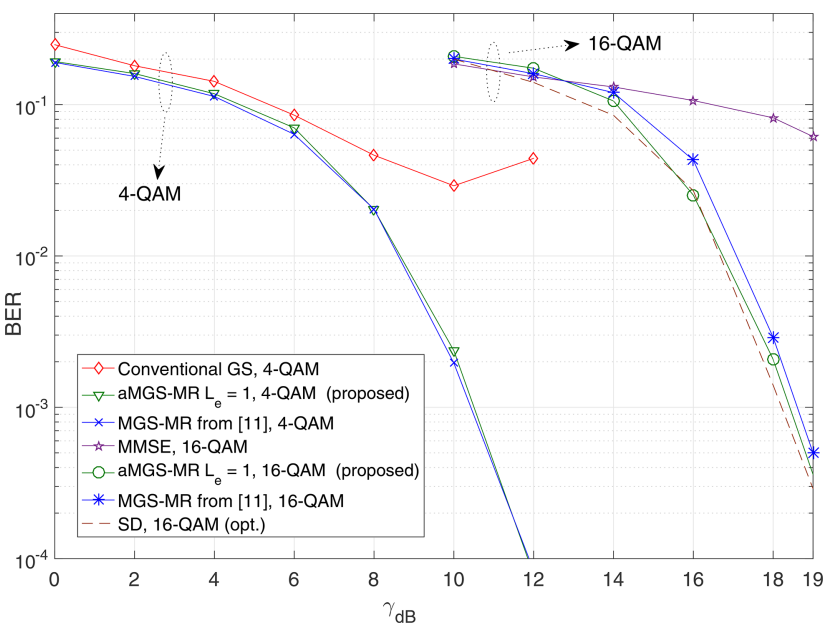

Fig. 2 Illustrative example showing the stalling effect of conventional GS and denoting that the proposed simplification of the target function of aMGS does not degrade the LS-MIMO performance: $K=N=16$ antennas, $L_{e}=1$ sample, $\mathscr{I}=512$ iterations, $R_{\max }=50,4-Q A M$ and 16 $Q A M$

\section{Review of mixed Gibbs sampling detection}

A brief review on the conventional GS [10] and on the MGS [11] is performed in this subsection.

In conventional GS detection, an initial solution $\hat{\boldsymbol{s}}^{(t=0)}$ is set for the estimated symbols vector, where $t$ represents the current iteration. The initial solution can be either a random vector or an output from linear low complexity detectors, such as matched filter, zero-forcing or MMSE [20]. The index $i$, in addition to the position of the vector $\hat{s}$, also denotes the coordinate referring to the MGS algorithm, where $i=1,2, \ldots, 2 K$. Therefore, each iteration requires $2 K$ coordinate updating. At each iteration, updating the $2 K$ coordinates is done by sampling the following distributions:

$$
\hat{s}_{i}^{(t)} \sim p\left(\hat{s}_{i} \mid \hat{s}_{1}^{(t)}, \ldots, \hat{s}_{i-1}^{(t)}, \hat{s}_{i+1}^{(t-1)}, \ldots, \hat{s}_{2 K}^{(t-1)}, \boldsymbol{y}, \boldsymbol{H}\right),
$$

and the target distribution for Gibbs-sampling-based detection is given by

$$
p\left(\hat{s}_{1}, \hat{s}_{2}, \ldots, \hat{s}_{2 K} \mid \boldsymbol{y}, \boldsymbol{H}\right) \propto \exp \left(-\frac{\|\boldsymbol{y}-\boldsymbol{H} \boldsymbol{s}\|^{2}}{\sigma^{2}}\right),
$$

One can notice that by (9) each updated coordinate is fed, in the same iteration, to the next coordinate. The algorithm ends after a certain amount of iterations, and the vector of estimated symbols is chosen as the vector that presented the lowest ML cost, considering all iterations.

A known problem with the given conventional GS detector is the stalling problem, which results in BER floor at high SNR regions [10]. This is shown in Fig. 2 where a 4-QAM, $K=16$ users and $N=16 \mathrm{BS}$ antennas $(16 \times 16)$ configuration is deployed, with 512 iterations and random initial solution: the performance of the conventional GS becomes degraded in the SNR region after 6 $\mathrm{dB}$ and more predominantly after $10 \mathrm{~dB}$, when a BER reversion occurs. The stalling problem appears because the algorithm becomes trapped in local low-performance solutions.

Based on the motivation to solve the stalling problem presented in the conventional GS, the LS-MIMO detector MGS was proposed in [11].

First, the MGS target distribution uses a positive parameter $\alpha$, which tunes the mixing time of the Markov chain [21]. The parameter $\alpha$ multiplies with the variance of the Gaussian-like distribution, thus defining its effective variance; the parameter is also called as temperature. Therefore, the target distribution of interest is given by

$$
p\left(\hat{s}_{1}, \hat{s}_{2}, \ldots, \hat{s}_{2 K} \mid \boldsymbol{y}, \boldsymbol{H}\right) \propto \exp \left(-\frac{\|\boldsymbol{y}-\boldsymbol{H} \boldsymbol{s}\|^{2}}{\alpha^{2} \sigma^{2}}\right),
$$

One can notice by (11) that the conventional GS can be viewed as a special case when $\alpha=1$. A larger temperature speeds up the mixing and aims to reduce the higher moments of the number of iterations when finding the correct solution. However, as stated in [11], the stalling problem persists even with large $\alpha$.

In order to overcome the stalling problem, the MGS detection strategy utilises a mixing of: (i) conventional GS (i.e. $\alpha=1$ ); and (ii) the noisiest version of (11), which employs infinite effective variance by taking $\alpha=\infty$, resulting in a random and uniform sample from all the possibilities. In this way, the MGS distribution for sampling purpose is given by

$$
p\left(\hat{s}_{1}, \ldots, \hat{s}_{2 K} \mid \boldsymbol{y}, \boldsymbol{H}\right) \sim(1-q) \psi\left(\alpha_{1}\right)+q \psi\left(\alpha_{2}\right)
$$

and

$$
\psi(\alpha)=\exp \left(-\frac{\|\boldsymbol{y}-\boldsymbol{H} \hat{\boldsymbol{s}}\|^{2}}{\alpha^{2} \sigma^{2}}\right)
$$

where $q 1 p t \in[0,1 p t 1 p t 1]$ is the mixing ratio, denoting the probability of considering the random uniform sampling solution, $\psi\left(\alpha_{2}=\infty\right)$.

The MGS detector considers the $\alpha_{1}=1, \alpha_{2}=\infty$ combination, which results in a near-ML performance, overcoming the stalling problem of the GS, being also a simple implementation choice. Additionally, in [11] a theoretical analysis of the mixing ratio $q$ effect operating under a small number of antennas and low-order QAM constellations was performed; the suitable value of $q$ was found as the inverse of the number of dimensions of the system, i.e. $q=(1 / 2 K)$. However, our numerical analysis carried out in Section 5 demonstrated that the optimal mixing ratio value under higherorder QAM modulation and large-scale regime can differ significantly from that value depending on the system configuration and channel scenarios.

The probability of the $i$ th symbol taking the value $a_{j}$ can be written as

$$
\begin{aligned}
p\left(\hat{s}_{i}\right. & \left.=a_{j} \mid \hat{\boldsymbol{s}}_{i-1}, \boldsymbol{y}, \boldsymbol{H}\right) \\
& =\frac{\exp \left(-\left(\left\|\boldsymbol{y}-\boldsymbol{H} \hat{\boldsymbol{s}}_{i, j}\right\|^{2} / \alpha^{2} \sigma^{2}\right)\right)}{\sum_{l=1}^{|\mathbb{A}|} \exp \left(-\left(\left\|\boldsymbol{y}-\boldsymbol{H} \hat{\boldsymbol{s}}_{i, l}\right\|^{2} / \alpha^{2} \sigma^{2}\right)\right)},
\end{aligned}
$$

where $a_{j} \in \mathbb{A}, \forall j=1, \ldots,|\mathbb{A}|$. The cardinality of set $\mathbb{A}$ is expressed as $|\mathbb{A}|$, while $\hat{\boldsymbol{s}}_{i, j}$ denotes the vector $\hat{\boldsymbol{s}}^{(t)}$ with its $i$ th position changed to the symbol $a_{j}$.

The sampling process based on (14) can lead to a numerical limitation due to the exponential function. In this sense, such implementation was carried out through a logarithmic intermediate step, as

$$
\begin{aligned}
& \log \left(p\left(\hat{s}_{i}=a_{j} \mid \hat{\boldsymbol{s}}_{i-1}, \boldsymbol{y}, \boldsymbol{H}\right)\right) \\
& \quad=f(i, j)-\left[f_{0}^{\text {ord }}+\log \left(1+\sum_{m=1}^{|\mathbb{A}|-1} \exp \left(f_{m}^{\text {ord }}-f_{0}^{\text {ord }}\right)\right)\right] \\
& \quad=g(i, j)
\end{aligned}
$$

where $f(i, j)=-\left(\left\|\boldsymbol{y}-\boldsymbol{H} \hat{\boldsymbol{s}}_{i, j}\right\|^{2} / \alpha^{2} \sigma^{2}\right)$ and $f_{i}^{\text {ord }}$ is $i$ th position of $\boldsymbol{f}$ in descending order, for $i=1, \ldots,|\mathbb{A}|$. A practical and computationally efficient evaluation of MGS target function is summarised in Algorithm 1 (see Fig. 3). Q3

\section{Proposed aMGS LS-MIMO detector}

A novel approach for the MGS, namely aMGS is proposed herein, which is based on the following improvements: 
i. Averaged MS on each coordinate: Differently from the single sampling strategy [11], the proposed aMGS employs an average between $L_{e}$ number of samples (realisations) in each coordinate during the update process. The proposed strategy tries to alleviate the problem which arises when the noisiest solution of $\alpha_{2}=\infty$ is randomly chosen in a given coordinate during the MGS mixing step. The mixing is a kind of local minima escaping strategy; on the other hand, it can lead solutions to regions 'far' from optimum, affecting the algorithm's convergence, especially under high-dimension problems, i.e. combining high number of antennas and medium-/high-order modulations. By employing the MS calculation, an intermediate (averaged) point between the target function symbol and the random symbol is more likely to be chosen, instead of a pure random symbol. As a result, the benefit of local minima escape is maintained, while the negative impact on the algorithm's convergence is smoothed.

ii. Target function simplification: To reduce the computational complexity related to the calculation of the target function in (14), we adopted a minimum ML cost approach. This simplification performs fewer mathematical operations, since the $\|\boldsymbol{y}-\boldsymbol{H} \hat{\boldsymbol{s}}\|$ computation is already performed in (14). The aMGS target function calculates $\xi\left(\hat{\boldsymbol{s}}_{i}^{(t)}, \boldsymbol{y}, \boldsymbol{H}\right) \in \mathbb{A}$ which chooses, in the $i$ th position, the $j$ th symbol that minimises the Euclidian distance. Thus, it is evaluated in the $t$ th iteration as

$$
\xi\left(\hat{\boldsymbol{s}}_{i}^{(t)}, \boldsymbol{y}, \boldsymbol{H}\right)=\underset{a_{j} \in \mathbb{A}}{\arg \min }\left\|\boldsymbol{y}-\boldsymbol{H} \hat{\boldsymbol{s}}_{i, j}\right\|,
$$

where $\hat{\boldsymbol{s}}_{i}^{(t)}$ denotes the updated estimated symbol vector until the $(i-1)$ position at the $t$ th iteration, whereas the other remaining $i,(i+1), \ldots, 2 K$ positions assume the values from the previous iteration, i.e.

$$
\hat{\boldsymbol{s}}_{i}^{(t)}=\left[\hat{s}_{1}^{(t)}, \ldots, \hat{s}_{i-1}^{(t)}, \hat{s}_{i}^{(t-1)}, \ldots, \hat{s}_{2 K}^{(t-1)}\right]^{\mathrm{T}}
$$

Remark 1: (aMGS performance complexity): Compared to (14), the calculation of (16) performs fewer operations while achieving the same bit error rate (BER) performance, as depicted in Fig. 2. For illustration purpose, a 4-QAM and 16-QAM, $K=16$ and $N=16$ system is deployed, with $\mathscr{I}=8 K \sqrt{M}, q=1 / 2 K$ and $R_{\max }=50[11]$.

\subsection{Coordinate updating process}

The coordinate update process of aMGS is defined by

$$
\hat{s}_{i}^{(t)}=\frac{1}{L_{\mathrm{e}}} \sum_{m=1}^{L_{\mathrm{e}}} \rho_{m, i}\left(\hat{\boldsymbol{s}}_{i}^{(t)}, \boldsymbol{y}, \boldsymbol{H}\right),
$$

where $L_{e}$ is the number of samples (realisations), and the random variable (r.v.) $\rho_{m, i}$ is a mixture of two r.v. with weight given by the mixing ratio $q$, defined by

$$
\rho_{m, i}\left(\hat{\boldsymbol{s}}_{i}^{(t)}, \boldsymbol{y}, \boldsymbol{H}\right) \sim(1-q) \cdot \xi\left(\hat{\boldsymbol{s}}_{i}^{(t)}, \boldsymbol{y}, \boldsymbol{H}\right)+q \cdot \psi(\infty)
$$

It is important to note that, being (16) a deterministic function, during the $L_{e}$ realisations on each coordinate, (16) is calculated only once, when $m=1$. After that, each $m$ realisation has the computational cost of generating a random number (relative to the mixing ratio).

At the end of algorithm iterations, the vector with the lowest cost is assumed the best global solution. Due to the mean operation, a slicer for $M$-QAM constellation is needed at the end of the detection procedure. Thus

$$
\hat{\boldsymbol{s}}_{\text {best }}=\operatorname{slicer}\left(\hat{\boldsymbol{s}}_{\mathrm{f}-\text { best }}\right) \text {, }
$$

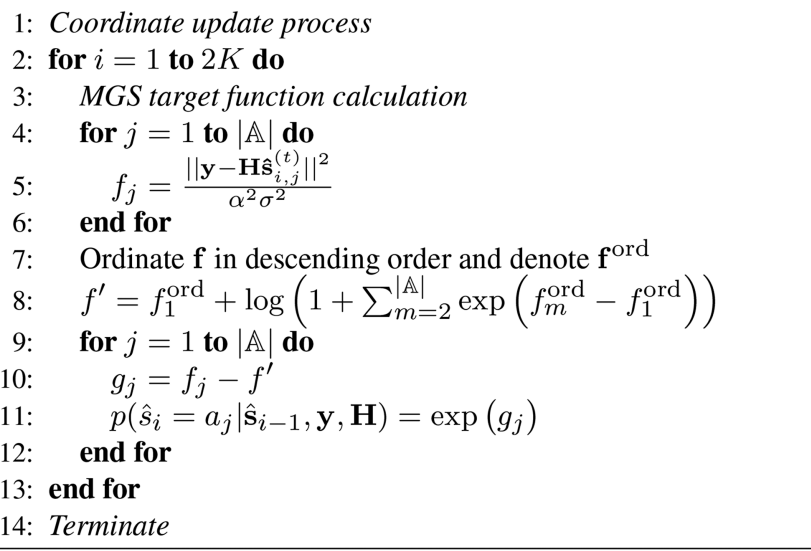

Fig. 3 Algorithm 1: MGS target function calculation

where $\hat{\boldsymbol{s}}_{\mathrm{f} \text {-best }}$ is the 'floating-best' solution which represents the estimated vector related to the best global cost attained after $\mathscr{I}$ iterations, and $\hat{\boldsymbol{s}}_{\text {best }}$ is the final estimated symbol vector. A pseudocode for the proposed aMGS is described in Algorithm 2 (see Fig. 4). Specific features of the proposed algorithm, such as $\mathrm{MR}$, adopted stopping criterion and resulted complexity are exploited in the next subsections.

\subsection{Multiple restarts}

In medium QAM-order modulations, such as 16-QAM, 64-QAM, the mixing strategy of MGS is unable to achieve near-optimal performance [22] in a reasonable number of iterations, while MR procedure, as proposed in [11] has demonstrated promising results, leading the MGS-MR under 16-QAM or 64-QAM to near-optimal performance.

In our proposed aMGS, the MR strategy is also incorporated to the aMGS, namely aMGS-MR detector. Thus, Algorithm 2 (Fig. 4) runs either a maximal number of restarts $R_{\max }$ times or it is limited by a stopping criterion, while the lowest cost found considering all restarts is the final solution. As discussed in Section 5, the MR strategy can improve the convergence of the aMGS algorithm compared to the same number of iterations in a single execution, resulting in a better performance-complexity tradeoff.

\subsection{Stopping criterion}

Given that the mixing strategy provides the local minimum escaping feature, the evolution of the cost function values across iterations becomes unpredictable and the optimal solution can be found before the maximum number of iterations $\mathscr{I}$ has been reached, as illustrated in Fig. 5. It is noticeable that the aMGS algorithm found the optimal solution, approximately, in the fifth iteration. After that, iterations become a waste of computational resources. In this sense, an efficient stopping criterion is paramount in reducing the complexity of the aMGS detector.

Similarly, the decision to set a restart in the algorithm requires a criterion definition, since the optimal solution could be found in the first execution of the algorithm, not requiring an MR procedure. Hence, MR strategy must be balanced aiming to achieve a better performance-complexity tradeoff.

Stopping criteria have been proposed in the literature. For instance, in [11], the stopping criterion is based on the difference between the best ML cost found so far and the noise variance. Additionally, the QAM constellation size could be taken into account. The main idea in [11] is to stop the detection iterations if a maximum number of iterations is attained or if the iteration in stalling mode is larger than a maximum of $\Theta_{s}$ iterations. We assume the stop criterion similar to that adopted in [11], either to control the number of iterations or the restarts, as described below.

Assume the estimated symbol vector, in the $t$ th iteration, is $\hat{\boldsymbol{s}}^{(t)}$. The quality metric of $\hat{\boldsymbol{s}}^{(t)}$ is defined as 


$$
\phi\left(\hat{\boldsymbol{s}}^{(t)}\right)=\frac{\left\|\boldsymbol{y}-\boldsymbol{H} \hat{\boldsymbol{s}}^{(t)}\right\|^{2}-N \sigma^{2}}{\sqrt{N} \sigma^{2}}
$$

Hence, the stalling limit, $\Theta_{s}$, is given by

$$
\Theta_{s}\left(\phi\left(\hat{\boldsymbol{s}}^{(t)}\right)\right)=c_{s} \cdot e^{\phi\left(\hat{\boldsymbol{s}}^{(t)}\right)}
$$

where $c_{s}$ is a constant depending on the $M$-QAM constellation size, which increases with $M$. Although (21) is suitable as a stopping criterion, a minimum number of iterations $c_{\min }$ must be defined to ensure the quality of symbol detection. Therefore, $\Theta_{s}$ can be rewritten as

$$
\begin{aligned}
\Theta_{s}\left(\phi\left(\hat{\boldsymbol{s}}^{(t)}\right)\right) & =\left\lceil\max \left(c_{\min }, c_{s} \cdot e^{\phi\left(\hat{\boldsymbol{s}}^{(t)}\right)}\right)\right\rceil \\
\text { with } \quad c_{s} & =c_{1} \log _{2}(M)
\end{aligned}
$$

where $c_{1}$ is a tuning constant which defines the allowed number of iterations in stalling mode and $\lceil x\rceil$ is the minimum integer that is larger than $x$.

For the MR strategy, the criterion sets the allowable number of restarts $\Theta_{r}$, which also is based on $\phi\left(\hat{\boldsymbol{s}}^{(t)}\right)$

$$
\begin{aligned}
\Theta_{r}\left(\phi\left(\hat{\boldsymbol{s}}^{(t)}\right)\right)=\left\lceil\max \left(0, c_{r} \cdot \phi\left(\hat{\boldsymbol{s}}^{(t)}\right)\right)\right\rceil+1 \\
\text { with } \quad c_{r}=c_{2} \log _{2}(M),
\end{aligned}
$$

and $c_{2}$ is the tuning constant adjusting the maximum number of restarts.

At the end of each restart, $\Theta_{r}$ is computed and checked if the actual number of repetitions is less than $\Theta_{r}$. If yes, go to another run of the aMGS-MR algorithm; else, output the solution vector with the minimum cost so far as the final solution.

\subsection{Complexity}

The computational complexity is described in terms of real number of operations (rops), in which one rop denotes the computational complexity of the real mathematical operations: addition, subtraction, multiplication or division. For the exponential and logarithmic functions, an approximation through Taylor Series with 18 terms has been considered to calculate the computational complexity. Table 1 describes the per-symbol computational complexity, $\mathscr{C}_{T}$, involved in each step of aMGS algorithm. Additionally, the total complexity per-symbol of the MGS has been evaluated. The $\mathscr{C}_{I}$ denotes the per-symbol complexity of the initial solution, which is adopted as the linear MMSE detector for both aMGS and MGS detection schemes. The per-symbol complexity of the MMSE is also shown in Table 1 [23].

From Table 1, one can notice that the aMGS and MGS algorithms have the same asymptotic per-symbol complexity order of $\mathcal{O}\left(K^{2}\right)$, although the conventional MGS algorithm may require an additional complexity dependent on constellation size due to the exponential function, which is represented by the cardinality $|\mathbb{A}|$. On the other hand, the additional complexity due to the averaged strategy of the aMGS represents a negligible impact, since it requires only $\left(2 L_{e}+2\right)$ rops per iteration, while such additional complexity is not dependent on the problem size.

It is important to emphasise that the complexity of the aMGS and MGS algorithms is defined by the number of iterations, which is controlled by the stopping criterion $\Theta_{s}$, with the upper limit $\mathscr{I}$. Similarly, the amount of restarts is controlled by $\Theta_{r}$, with an upper limit $R_{\max }$. In terms of complexity, the MR procedure can be interpreted as an extra amount of iterations necessary for each new restart. In this sense, the $\mathscr{I}_{\text {eff }}$ is considered in Table 1, denoting the total amount of iterations (including all restarts) performed at each symbol period detection. Since Monte-Carlo method is deployed in the simulations, in Section 5 a mean value of $\mathscr{J}_{\text {eff }}$ considering all realisations is evaluated and is called effective number of iterations (ENI)
1: Initialization

2: $\hat{\mathbf{s}}^{(t=0)}$ : initial random vector; $L_{e} \#$ samples; $\mathcal{I}$ : max. number of iterations; $t=1 ; q$ mixing ratio; $\mathbb{A}=\left\{a_{1}, a_{2}, \ldots, a_{|\mathbb{A}|}\right\}$

3: Iterative process

4: while $t<\mathcal{I}$ do

Coordinate update process

for $i=1$ to $2 K$ do

Simplified target function calculation

for $j=1$ to $|\mathbb{A}|$ do

$f_{j}=\left\|\mathbf{y}-\mathbf{H} \hat{\mathbf{s}}_{i, j}^{(t)}\right\|$

end for

$f_{\min }=\arg \min _{j} f_{j}$

$\xi_{\min }=a_{j_{\min }}$

$L_{e}$ samples on each coordinate

for $l=1$ to $L_{e}$ do

generate $u_{i, l} \sim U[0,1]$

if $\left(u_{i, l}>q\right)$ then

$W_{l}=x i_{\min }$

else

$r \sim \operatorname{round}(U[1,|\mathbb{A}|])$

$W_{l}=a_{r}$

end if

end for

Averaging between samples

$\hat{s}_{i}^{(t)}=\frac{1}{L_{e}} \sum_{l=1}^{L_{e}} W_{l}$

Storage of cost and temporary vectors

$\epsilon_{i}=\left\|\mathbf{y}-\mathbf{H} \hat{\mathbf{s}}_{i}^{(t)}\right\|$

$\mathbf{S}_{:, i}=\hat{\mathbf{s}}_{i}^{(t)}$

end for

Best cost in the $t$-th iteration

$\epsilon_{\min }=\min \epsilon_{i}$

Best global solution test

if $\left(\epsilon_{\min }<\epsilon_{\text {best }}\right)$ then

$\epsilon_{\text {best }}=\epsilon_{\min }$

$i_{\min }=\arg \min _{i} \epsilon_{i}$

$\hat{\mathbf{s}}_{\mathrm{f}-\text { best }}=\mathbf{S}_{:, i_{\text {min }}}$

end if

$t=t+1$

$b_{t}=\epsilon_{\text {best }}$

Stop criterion for iterations

if $\left(b_{t}==b_{t-1}\right)$ then

$m=\Theta_{s}\left(\hat{\mathbf{s}}_{\text {best }}\right)$

if $(m<t)$ then

if $\left(b_{t}==b_{t-m}\right)$ then

$\hat{\mathbf{s}}_{\text {best }}=\operatorname{slicer}\left(\hat{\mathbf{s}}_{\mathrm{f}-\text { best }}\right)$

Terminate

end if

end if

end if

end while

0: $\hat{\mathbf{s}}_{\text {best }}=\operatorname{slicer}\left(\hat{\mathbf{s}}_{\mathrm{f}}\right.$-best $)$

51: Terminate

Fig. 4 Algorithm 2: aMGS for LS-MIMO detection

$$
\mathrm{ENI}=\sum_{i=1}^{T} \mathscr{I}_{\mathrm{eff}, i}
$$

where $T$ denotes the total number of realisations (symbol periods) during the simulation, and $\mathscr{I}_{\text {eff, } i}$ denotes the effective number of iterations deployed in the $i$ th realisation.

\subsection{Quality metric}

Due to the large number of parameters involved in the aMGS-MR LS-MIMO detector under medium-high $M$-QAM order modulation, a simple performance-complexity tradeoff metric is defined herein aiming to establish a fair comparison analysis among different MIMO detection strategies 


$$
\chi\left(\mathrm{BER}, \mathscr{C}_{T}\right)=-\frac{10 \log _{10}(\mathrm{BER})}{10^{-8} \cdot \mathscr{C}_{T}}=-\frac{\mathrm{BER}_{\mathrm{dB}}}{10^{-8} \cdot \mathscr{C}_{T}}
$$

where $\mathrm{BER}_{\mathrm{dB}}$ denotes the bit error rate in $\mathrm{dB}$. Hence, the quality detection metric $\chi\left(\mathrm{BER}, \mathscr{C}_{T}\right)$ captures the detector efficiency in quantifying performance improvement against complexity increasing. Higher values of $\chi(\cdot)$ imply more efficient and effective LS-MIMO detector.

\section{Simulation results}

In this section, the uncoded BER performance related to the aMGS algorithm for LS-MIMO detection is evaluated through Monte Carlo simulations. The simulations are performed for a large-scale MIMO operating in multiplexing mode and assuming that a perfect channel state information is available at the receiver side. Table 2 summarises the main system and channel parameter values deployed in this section.

This numerical simulation section has been divided into three main parts: in Section 5.1 the best input parameters for the proposed detection algorithm have been determined numerically; in Section 5.2, the main performance indexes and corresponding computational complexities have been compared by deploying the input parameters found in Section 5.1; and, finally, in Section 5.3 an asymptotic analysis regarding the number of antennas is performed to evaluate the aMGS-MR performance in other LSMIMO scenarios.

\subsection{Best input aMGS parameters}

In this subsection, the input parameters of the aMGS-MR detector are examined aiming at numerically finding its optimum values. For each specific scenario, the parameters were inspected in order to determine its optimal value, generalising as many scenarios as possible. The analysed input aMGS parameters include:

- Stopping criterion parameters: $c_{1}, c_{2}$;

- Mixing ratio, $q$;
- Averaged sampling, $L_{e}$;

- Maximum number of iterations, $\mathscr{I}$;

- Maximum number of restarts, $R_{\max }$;

For the stopping criterion parameters, considering a single and MS strategy, a brief analysis has been carried out in the Appendix, aiming to establish the best tradeoff values for $c_{1}$ and $c_{2}$. As a result, in the remainder of this work we have adopted $c_{1}=10$, $c_{2}=1.0$ and $c_{\min }=10$.

5.1.1 Mixing ratio and averaged samples: The BER performance for different mixing ratios $q=\{1 / 2 K, 1 / 3 K, 1 / 4 K\}$, considering $R_{\max }=\{1,5,10\}$, is performed in Fig. 6 for each fixed $L_{e} \in\{1,2,4,8\}$ samples scenario. The number of users is set to $K=48$ while $N=64$ antennas $(\beta=0.75)$. The system is operating

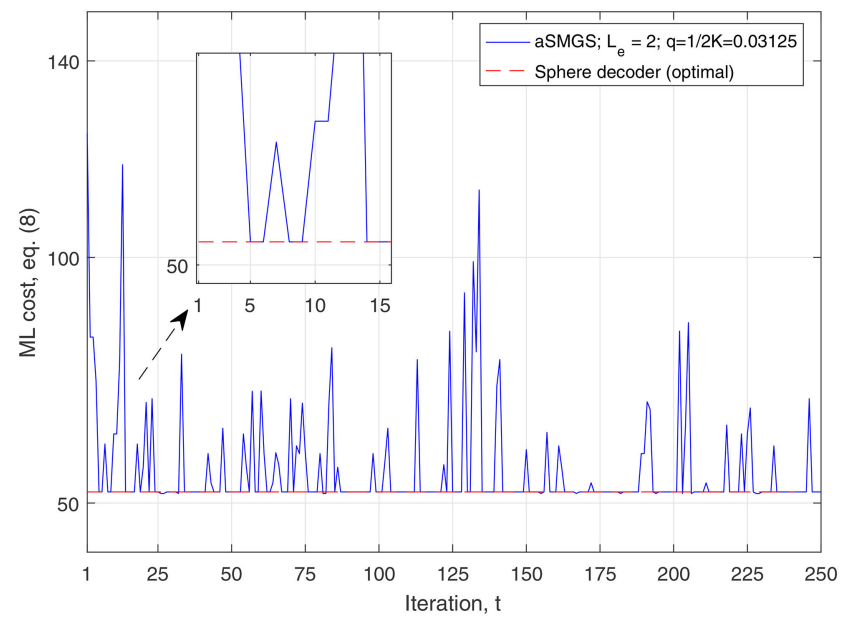

Fig. $5 M L$ cost function evolution during the aMGS algorithm's iterations and the optimal result (sphere decoder); $K=M=16, E_{b} / N_{0}=10 \mathrm{~dB}$, $\mathscr{I}=250$ iterations, and 4-QAM modulation

Table 1 Per-symbol computational complexity of aMGS, conventional MGS and MMSE algorithms

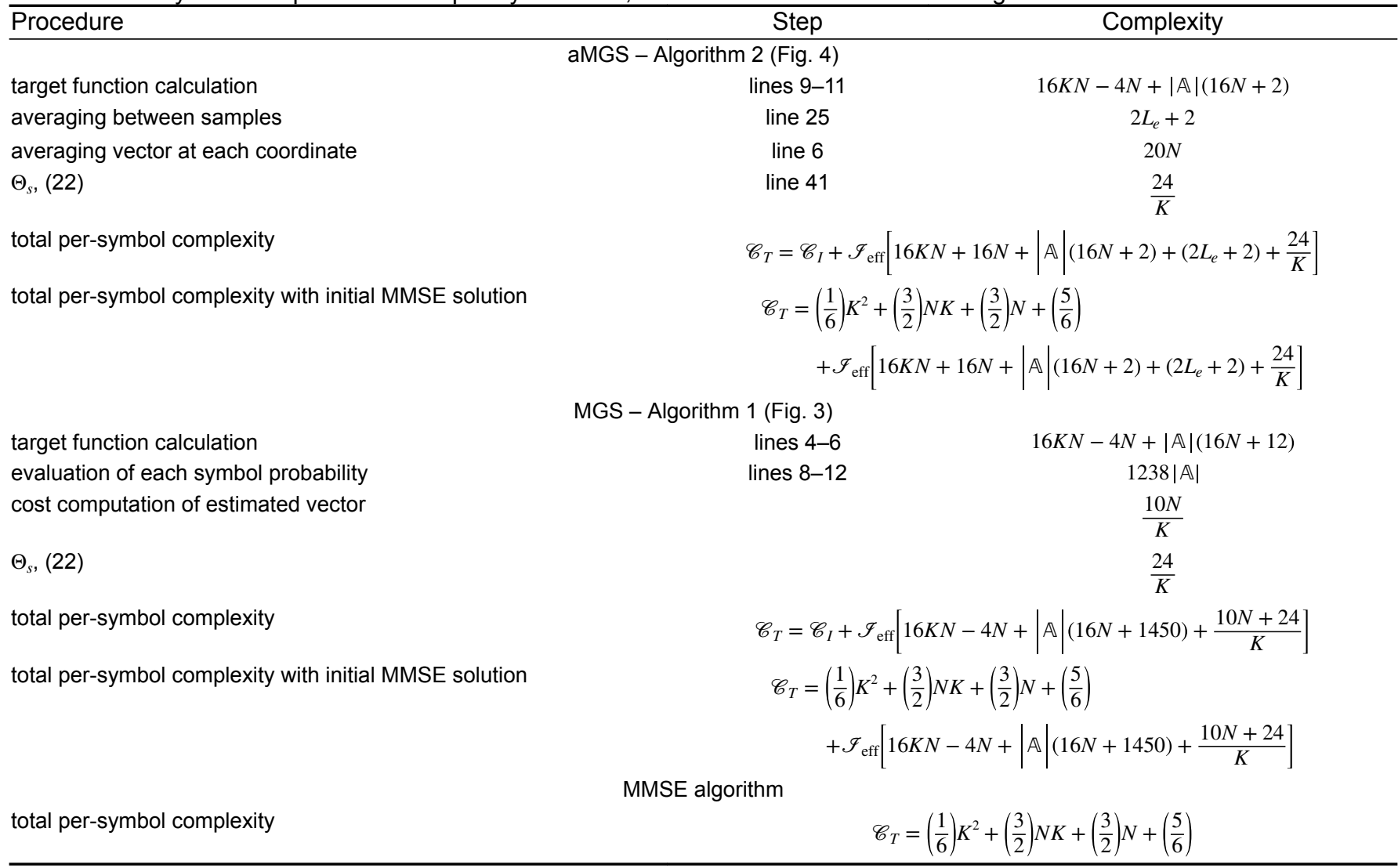


under medium SNR, $\gamma_{\mathrm{dB}}=25 \mathrm{~dB}$. Also, assuming the complexity is linear with the number of iterations (see Table 1), a similarly complexity analysis can be held with respect to the ENI.

From all sampling strategies presented in Fig. 6, it is also noticeable that $q=1 / 2 \mathrm{~K}$ presents the largest ENI complexity, and the worst performance (for the same $R_{\max }$ ), except when $L_{e}=8$ samples. Such bunch of numerical results demonstrates that $q=1 / 2 K$ is not the optimal mixing ratio value, even in a single sampling scenario, as stated in [11]. One can also conclude that the use of MS process provides a significant complexity reduction, indicated by the decreasing ENI at convergence from single (Fig. $6 a$ ) to MS scenarios (Figs. 6b-d), with a marginally performance loss.

Among all system scenarios subsumed under Fig. 6, two main analysis relating to the proposed MS technique can be highlighted in the following remarks.

Remark 2: (MR provides a limited BER-ENI tradeoff gain): Regardless of mixing ratio and quantity of samples, the BER performance from the $R_{\max }=5$ to 10 is marginally the same, while the gap in terms of ENI is significant (around 60\% more). It shows that, in these configurations, specially under $\beta<1$ system loading condition, the algorithm convergence is improved and the performance gain obtained by the MR technique is reduced; hence, convergence is reached with few restarts $(\simeq 5)$, leading to irrelevant performance improvement after that. Moreover, comparing with no restarts configuration, i.e. $R_{\max }=1$, one can conclude that a high number of samples (Fig. $6 d$ ) results in a better performance $\times$ complexity tradeoff than single sample case (Fig. $6 a$ ) - since a lower BER is achieved at practically the same ENI - evidencing the direct performance improvement of the proposed MS strategy.

Remark 3: (MS strategy $L_{e} \propto q$ improves BER-ENI): The performance $\times$ complexity tradeoff for the mixing ratio $q$ demonstrated to be directly proportional to the number of $L_{e}$ samples: a greater mixing ratio is beneficial for a large number of samples, and vice-versa. From $L_{e}=1$ to $L_{e}=8$, it can be noticed a progressive BER-ENI tradeoff gain in $q=1 / 2 K$ curves, contrariwise, $q=1 / 4 K$ curves show a gradual tradeoff loss with the increasing number of samples. It evidences that a greater number of samples mitigates the impact of the random symbol, $\psi(\infty)$ : a higher $q$ implies that the random symbol will more probably occur and increasing number of samples smoothes this effect, at the same time, a low $q$ combined to a high $L_{e}$ tends to null the mixing strategy, degrading the performance. It can be

Table 2 LS-MIMO system and channel parameters

\begin{tabular}{|c|c|}
\hline Parameter & Value \\
\hline \multicolumn{2}{|c|}{ LS-MIMO system } \\
\hline link direction & uplink (UL) \\
\hline number of Rx antennas (BS) & $N \in\{64,128\}$ \\
\hline $\begin{array}{l}\text { number of Tx antennas (MTs) (single } \\
\text { user-antenna) }\end{array}$ & $K \in\{48,96\}$ \\
\hline system loading & $\beta \in[0.3125,0.90625]$ \\
\hline modulation order & 64-QAM \\
\hline SNR ranges & $\gamma_{\mathrm{dB}} \in[0,25] \mathrm{dB}$ \\
\hline number of realisations & $T \in[5000,15000]$ \\
\hline \multicolumn{2}{|c|}{ Channel } \\
\hline channel type & flat Rayleigh \\
\hline channel availability & perfectly known at receiver \\
\hline \multicolumn{2}{|c|}{ aMGS-MR detector } \\
\hline max. number of iterations & $\mathscr{I} \in\{3000,6000,9000\}$ \\
\hline max. number of restarts & $R_{\max } \in\{5,10\}$ \\
\hline number of aMGS samples & $L_{e} \in\{1,2,4,8\}$ \\
\hline mixing ratio & $q \in\left\{\frac{1}{2 K}, \frac{1}{3 K}, \frac{1}{4 K}\right\}$ \\
\hline stop criterion parameters & $c_{1}=10 ; c_{2}=1 ; c_{\min }=10$ \\
\hline
\end{tabular}

explained: the summation terms of (17) can either assume values from the target function, $\boldsymbol{\xi}\left(\hat{\boldsymbol{s}}_{i}^{(t)}, \boldsymbol{y}, \boldsymbol{H}\right)$, or the random solution, depending on $q$; in a $L_{e}=1$ single sampling scheme, the random solution can be fully adopted (since there will be no other terms in the summation), whereas, in a MS strategy, it is unlikely that all terms come from the random solution [Since $(1-q) \gg q$ in (18).] Q4and the result tends towards target function with increasing $L_{e}$.

5.1.2 Tuning mixing ratio and number of sampling: Table 3 summarises the configuration which resulted in best BER-ENI tradeoff considering each $L_{e}$ samples scenario analysed in Fig. 6. Among all scenarios, the best BER-ENI tradeoff can be found with $L_{e}=4$ samples combined to $q=1 / 3 K$ and $R_{\max }=5$, in which a suitable BER performance is achieved under ENI $<600$.

The performance with increasing number of antennas to $K=96$ and $N=128(\beta=0.75)$ is depicted in Fig. 7. Compared to the lower number of antennas scenario of Fig. 6 , similar analysis and conclusions can be held, except that the use of a higher number of samples $\left(L_{e}=8\right)$ demonstrated a harmful effect w.r.t. the aMGS convergence, denoted by the ENI increasing, whereas the BER performance is only marginally improved. It evidences that, in large antenna scenarios, the impact of random symbols from the mixture does not strongly affect the algorithm's convergence as seen with less antennas context, i.e. $K=48$ and $N<64$. Thus, the averaged MS strategy has its benefit reduced when $K, N \rightarrow \infty$. Even so, considering the adopted quality metric, the best tradeoff in this large antenna scenario was reached by using $L_{e}=2$ samples and mixing ratio $q=1 / 4 K$. The most promising tradeoff for each configuration of Fig. 7 are listed in Table 4.

Through the analysis performed in this subsection, the parameter values summarised in Table 5 have been adopted in the sequel.

Remark 4: (Tuning parameters): The input parameters for the aMGS-MR detector are selected based on the performance $x$ complexity tradeoff criterium. Tables 4 and 5 reveal the best tradeoff in the large antenna scenarios, i.e. $\beta \geq 0.75$ and $N \geq 64$, is reached by using number of sampling $L_{e}=2$ and mixing ratio factor $q=1 / 4 K$. As a result, different optimal values than $q=1 / 2 K$ reported in [11] have been found herein.

\subsection{Performance-complexity tradeoff}

In Fig. 8, the convergence of the aMGS algorithm adopting best $q$ values is analysed against the average rops complexity, with $48 \times 64$ and $96 \times 128$ antennas, respectively, and 64-QAM. For comparison purpose, it is also included a single sampling result using the optimal mixing ratio value as proposed in [11], i.e. $q=1 / 2 K$ (curve [E]). One can notice that the use of mixing ratio $q=1 / 4 K$ has resulted in better performance-complexity tradeoff when compared to the MGS with $q=1 / 2 K$ of [11] in both scenarios. It is also noticed that for the most promising performance results, the maximum number of iterations $\mathscr{I}=3000$ showed to comply with the aMGS-MR performance convergence. In medium-large antennas scenario of Fig. $8 a$, the single sample case exhibits the best end performance, slightly overpassing the MS strategy. On the other hand, as expected, the convergence with multiple samples is improved (as observed in curves [B] and [C]) compared to the single sample case, which resulted in complexity reduction. In summary, comparing the performance-complexity tradeoff between the best results in multiple $\left(L_{e}=4\right.$; curve $\left.[\mathrm{C}]\right)$ and single $\left(L_{e}=1, q=1 / 4 K\right.$; curve [A]) cases, the MS strategy is found to achieve a higher tradeoff metric

$$
\left.\chi\right|_{L_{e}=4}=-\frac{10 \log _{10}\left(8.30 .10^{-6}\right)}{3.607 .10^{7} / 10^{8}}=140.86
$$

against the single-sample strategy 

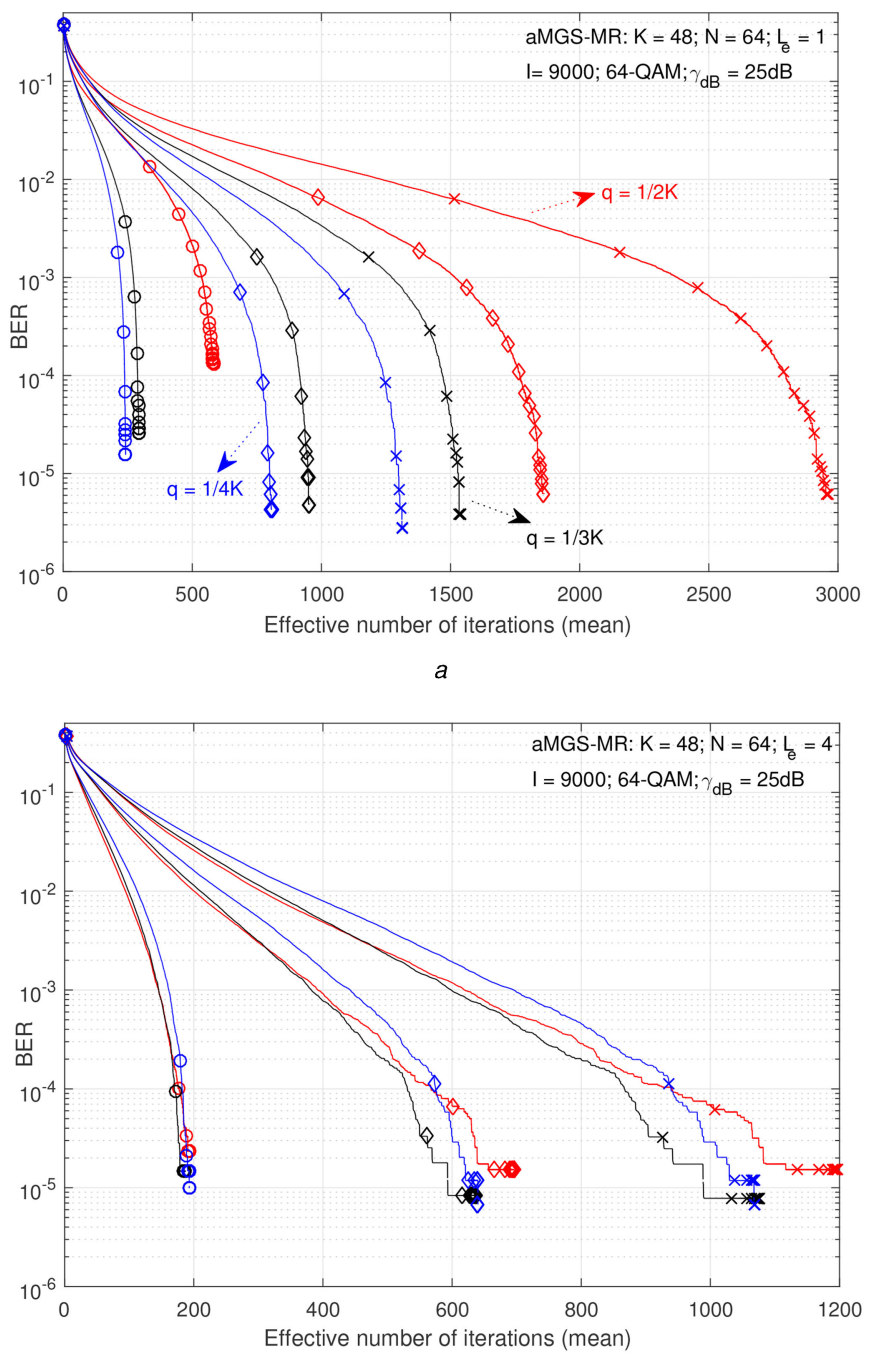
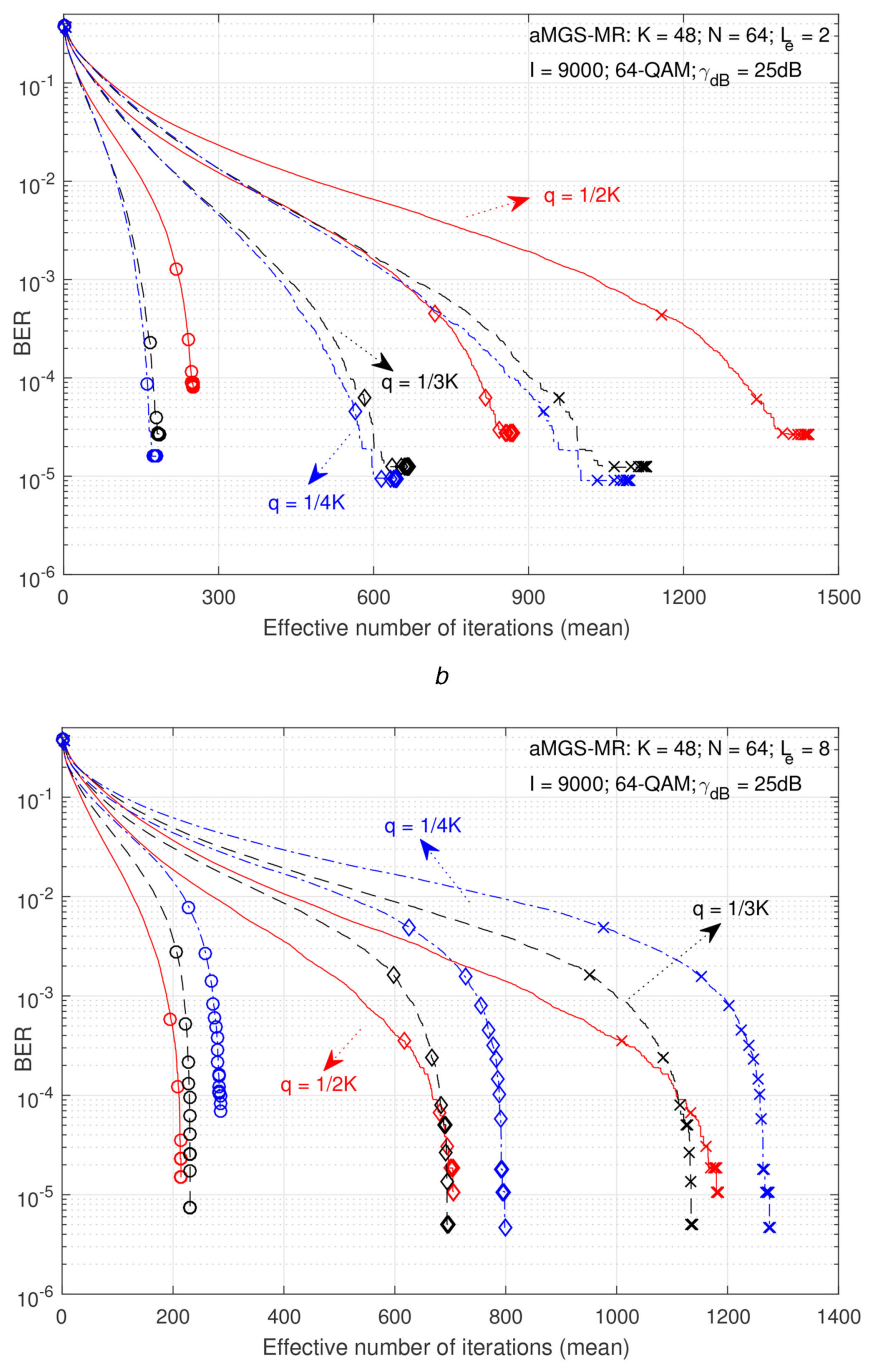

d

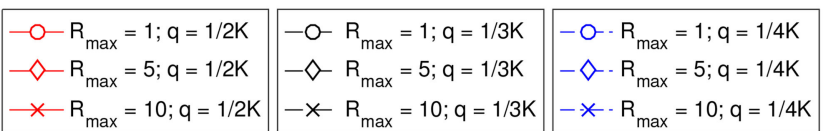

Fig. 6 BER performance convergence of different mixing ratios, $q$, of aMGS in medium number of antennas scenario $(K=48$, $N=64)$ at $\gamma_{d B}=25 d B$, $R_{\max }=\{1,5,10\}, \mathscr{I}=9000$ and different $L_{e}$ samples

(a) $L_{e}=1$, (b) $L_{e}=2$, (c) $L_{e}=4$, (d) $L_{e}=8$

Table 3 Best aMGS parameter values for $K=48$ and $N=64$

\begin{tabular}{|c|c|c|c|c|c|c|}
\hline Fig. & $L_{e}$ & $R_{\max }$ & $q$ & $\operatorname{BER}\left(10^{-6}\right)$ & ENI & $\chi$ \\
\hline Fig. $6 a$ & 1 & 5 & $\frac{1}{4 K}$ & 4.35 & 807 & 109.42 \\
\hline Fig. $6 b$ & 2 & 5 & $\frac{1}{4 K}$ & 9.5 & 600 & 137.85 \\
\hline Fig. $6 c$ & 4 & 5 & $\frac{1}{3 K}$ & 8.3 & 594 & 140.86 \\
\hline Fig. $6 c$ & 4 & 5 & $\frac{1}{4 K}$ & 6.8 & 640 & 132.97 \\
\hline Fig. $6 d$ & 8 & 5 & $\frac{1}{3 K}$ & 5.0 & 695 & 125.61 \\
\hline
\end{tabular}

$$
\left.\chi\right|_{L_{e}=1}=-\frac{10 \log _{10}\left(4.35 \cdot 10^{-6}\right)}{4.90 .10^{7} / 10^{8}}=108.42
$$

With increasing number of antennas, $K=96$ and $N=128$ (Fig. $8 b$ ), a lower number of samples has shown to be beneficial, since the single sample case presented the best performance combined to the lowest asymptotic complexity, followed by the twice $\left(L_{e}=2\right)$ sampling case. Nevertheless, due to a slightly convergence gain observed when using $L_{e}=2$ samples, the tradeoff metric for $L_{e}=1$ is found to be $\left.\chi\right|_{L_{e}=1}=39.83$ against $\left.\chi\right|_{L_{e}=2}=44.22$ with $L_{e}=2$ samples.

Fig. 9 depicts the BER performance across SNR regions; also, the averaged complexity, in terms of number of real operations (rops), is included. The system operates with parameters from Table 5 in 64-QAM with a higher system loading condition of $\beta \simeq 0.9$ : (i) $K=58, N=64$ and (ii) $K=87, N=96$. One can 

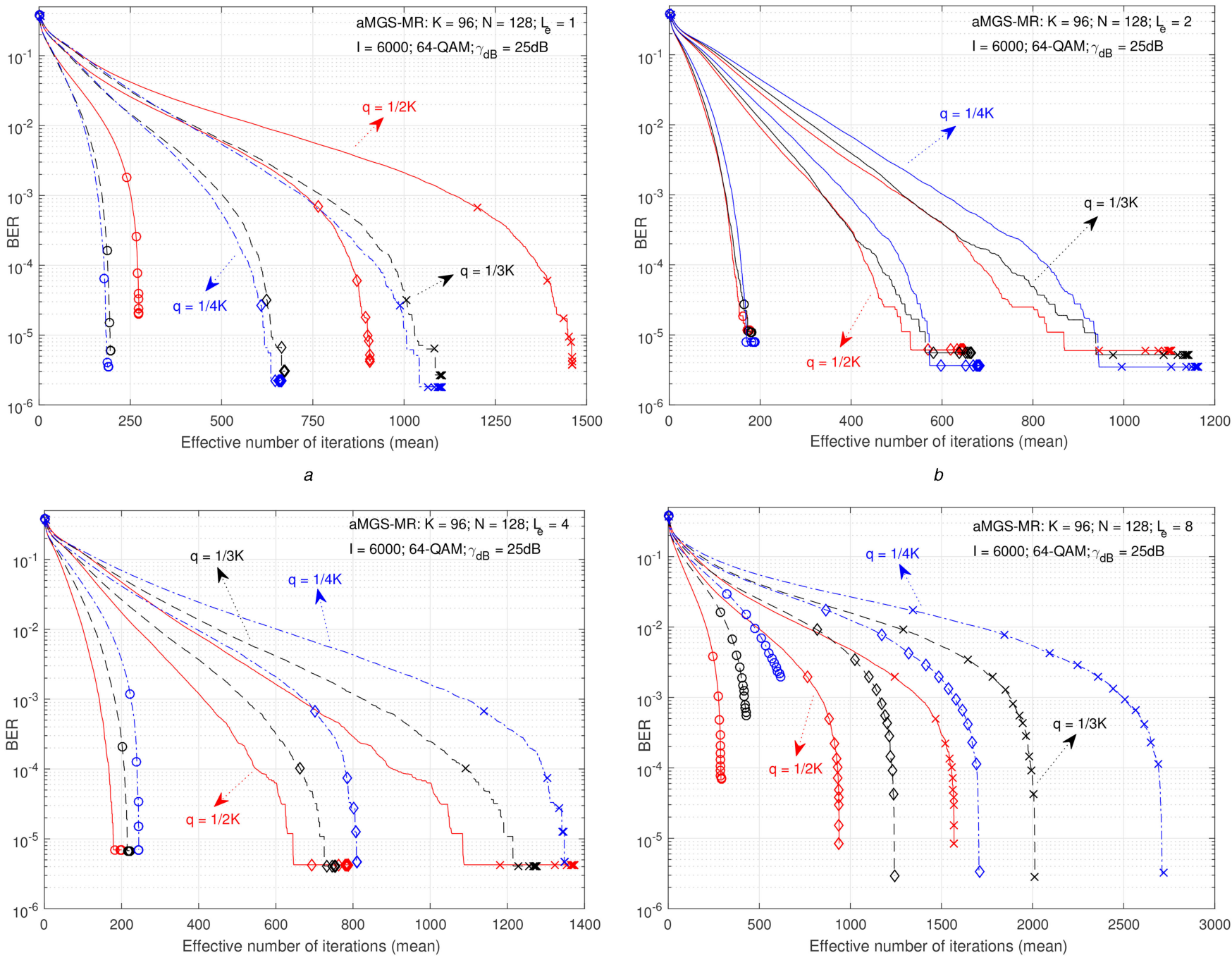

$c$

d

\begin{tabular}{|c|c|c|}
\hline $\begin{array}{l}-O-R_{\text {max }}=1 ; q=1 / 2 K \\
\checkmark-R_{\text {max }}=5 ; q=1 / 2 K \\
\times-R_{\text {max }}=10 ; q=1 / 2 K\end{array}$ & $\begin{array}{l}-O-R_{\max }=1 ; q=1 / 3 \mathrm{~K} \\
-\checkmark-R_{\text {max }}=5 ; q=1 / 3 \mathrm{~K} \\
-\times R_{\max }=10 ; q=1 / 3 \mathrm{~K}\end{array}$ & $\begin{array}{l}-O \cdot R_{\max }=1 ; q=1 / 4 K \\
-\diamond \cdot R_{\text {max }}=5 ; q=1 / 4 K \\
-* \cdot R_{\max }=10 ; q=1 / 4 K\end{array}$ \\
\hline
\end{tabular}

Fig. 7 BER performance convergence of different mixing ratios, $q$, of aMGS in medium number of antennas scenario $(K=96$, $N=128)$ at $\gamma_{d B}=25 d B$, $R_{\max }=\{1,5,10\}, \mathscr{I}=6000$ and different $L_{e}$ samples

(a) $L_{e}=1$, (b) $L_{e}=2$, (c) $L_{e}=4$, (d) $L_{e}=8$

Table 4 Best aMGS parameter values for $K=96$ and $N=128$

\begin{tabular}{|c|c|c|c|c|c|c|}
\hline Fig. & $L_{e}$ & $R_{\max }$ & $q$ & $\operatorname{BER}\left(10^{-6}\right)$ & ENI & $\chi$ \\
\hline Fig. $7 a$ & 1 & 5 & $\frac{1}{4 K}$ & 2.26 & 648 & 39.66 \\
\hline Fig. $7 b$ & 2 & 5 & $\frac{1}{2 K}$ & 6.16 & 531 & 44.66 \\
\hline Fig. $7 b$ & 2 & 5 & $\frac{1}{4 K}$ & 3.60 & 552 & 44.89 \\
\hline Fig. $7 c$ & 4 & 5 & $\frac{1}{2 K}$ & 4.25 & 646 & 37.85 \\
\hline Fig. $7 d$ & 8 & 5 & $\frac{1}{2 K}$ & 8.51 & 938 & 24.60 \\
\hline Fig. $7 d$ & 8 & 5 & $\frac{1}{3 K}$ & 2.95 & 1241 & 20.28 \\
\hline
\end{tabular}

notice that, in this system loading condition, the MS strategy results in BER performance gain and complexity reduction, specially with increasing SNR. Besides that, in Fig. 9a), the highest number of samples result, i.e. $L_{e}=8$, showed the best performance and lowest complexity among all aMGS-MR and MGS-MR presented. Another important conclusion with increasing the number of antennas (Fig. $9 b$ ) is that the $L_{e}=4$ samples performance resulted in the best performance, overcoming the $L_{e}=8$, which denotes its potential for LS-MIMO scenarios with more than a hundred antennas.

A system loading analysis against BER and rops complexity is depicted in Fig. 10 under $\gamma_{\mathrm{dB}}=25 \mathrm{~dB}$. Differently of the aMGS- 
Table 5 Best performance versus complexity tradeoff parameters for aMGS-MR detector

\begin{tabular}{|c|c|c|c|c|c|}
\hline \multirow[t]{2}{*}{ Parameter } & \multirow[t]{2}{*}{$K \times N$} & \multicolumn{4}{|c|}{ No. of aMGS samples, $L_{e}$} \\
\hline & & 1 & 2 & 4 & 8 \\
\hline mixing & $48 \times 64:$ & $\frac{1}{4 K}$ & $\frac{1}{4 K}$ & $\frac{1}{3 K}$ & $\frac{1}{2 K}$ \\
\hline ratio, $q$ & $96 \times 128:$ & $\frac{1}{4 K}$ & $\frac{1}{4 K}$ & $\frac{1}{2 K}$ & $\frac{1}{2 K}$ \\
\hline Max. no. of iterations, I & & \multicolumn{4}{|c|}{3000} \\
\hline Max. restarts, $R_{\max }$ & & \multicolumn{4}{|c|}{5} \\
\hline iterations stop criterion, $c_{1}$ & & \multicolumn{4}{|c|}{10} \\
\hline restarts stop criterion, $c_{2}$ & & \multicolumn{4}{|c|}{1} \\
\hline
\end{tabular}
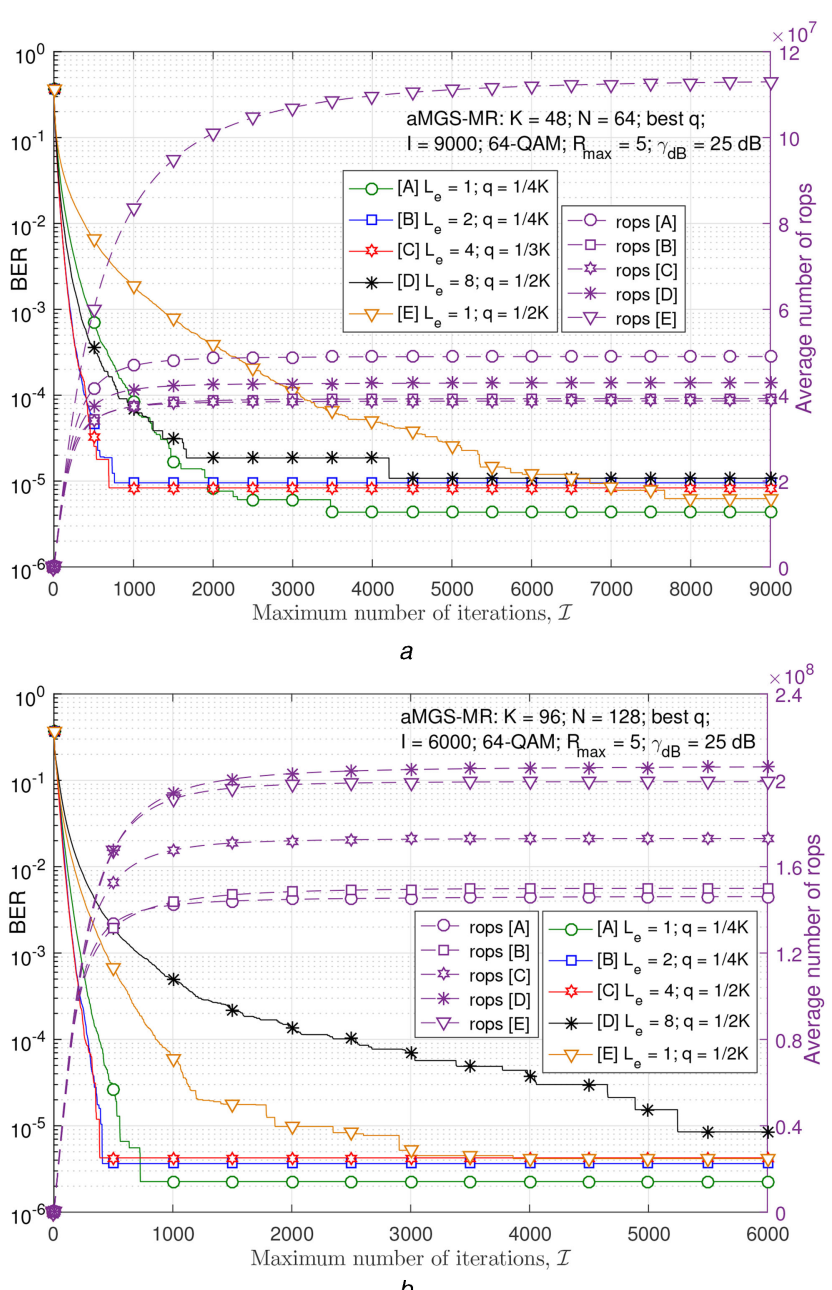

Fig. 8 BER performance and complexity versus convergence for the aMGS algorithm considering 64-QAM modulation, $\beta=0.75$, best mixing ratio $q$ (curves [A] to [D]) and optimal value as proposed in [11], i.e. $q=1 / 2 K$ with $L_{e}=1$ (curve [E])

(a) $48 \times 64$, (b) $96 \times 128$

MR, the MGS-MR detector is set to $\mathscr{I}=8 K \sqrt{M}$ and $R_{\max }=50$ [11]. One can conclude that, under high system loading, i.e. $\beta \geq 0.8$, the MS strategy is benefited more significantly, both in terms of performance and computational complexity, demonstrating the potential of this strategy when the LS-MIMO system operates under high loading crowded scenarios. This can be explained as the number of mobile users increases, approaching the full-loading system condition $\beta \rightarrow 1$, the set of possible symbol combinations becomes larger, such that the noisy solution from the mixture has its negative effect aggravated, affecting the algorithm's convergence; whereas the MS strategy is able to mitigate this effect, having a beneficial effect on the convergence which results in improvement in performance and complexity reduction. In both scenarios of $N=64$ and $N=128$ BS antennas, the best tradeoff
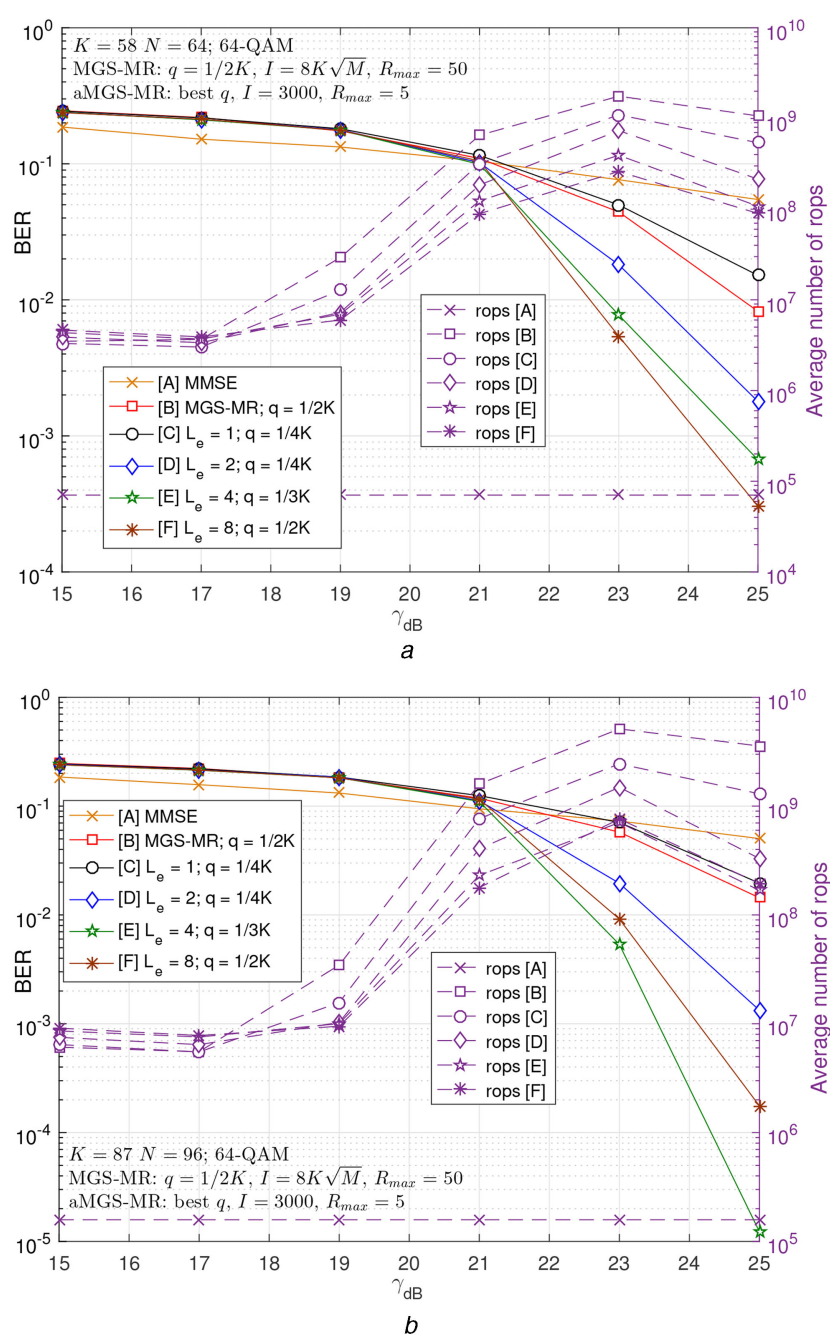

Fig. 9 SNR versus BER performance/average number of rops in aMGS$M R$ detector using best $q$ option on each $L_{e}$ case, 64-QAM, $\beta \simeq 0.9$, $R_{\max }=5$ restarts and for the MGS-MR: $q=1 / 2 K, R_{\max }=50[11]$

(a) $58 \times 64$, (b) $87 \times 96$

scheme under high system loading condition is found to be with $L_{e}=4$ samples, which resulted in the lowest BER performance and computational complexity.

It is also noticeable in Fig. 10 that the performance gap between the MGS-MR, with a single sample, and the aMGS-MR with $L_{e}=1$ is due to the mixing ratio feature. As demonstrated in Section 5.1.1, the optimal $q=1 / 4 K$ value results in better performance when a single sample is considered.

\subsection{Asymptotic analysis with the number of antennas}

The BER performance of the aMGS detector in different LSMIMO scenarios is analysed under increasing number of antennas and samples. Results presented in Fig. 11 were simulated considering a wide range of number of antennas, 


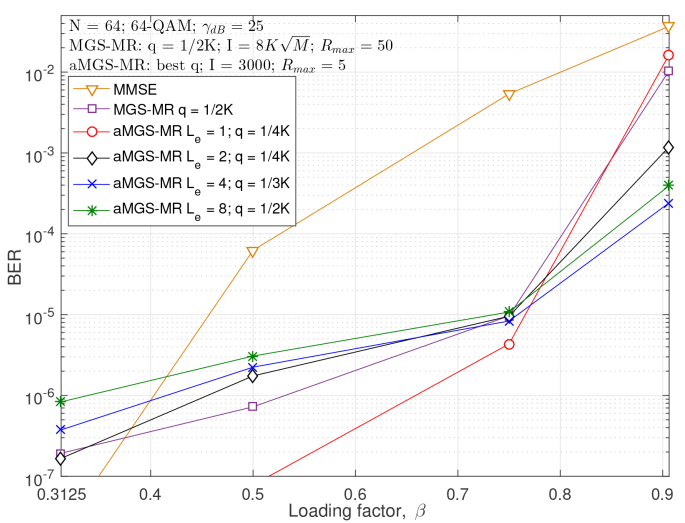

a

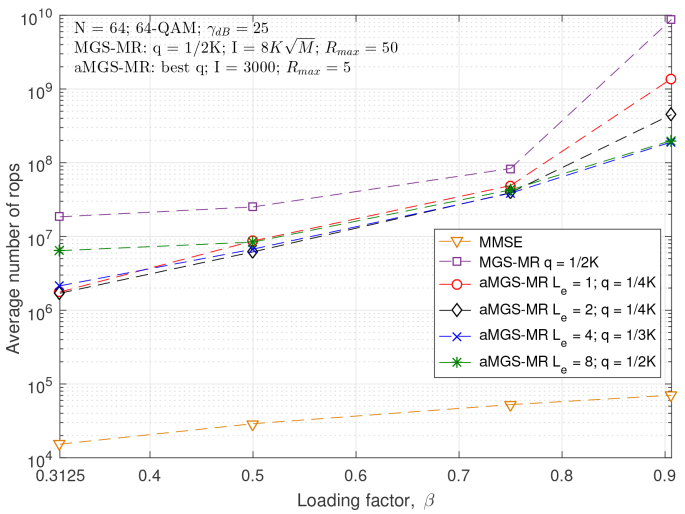

$c$

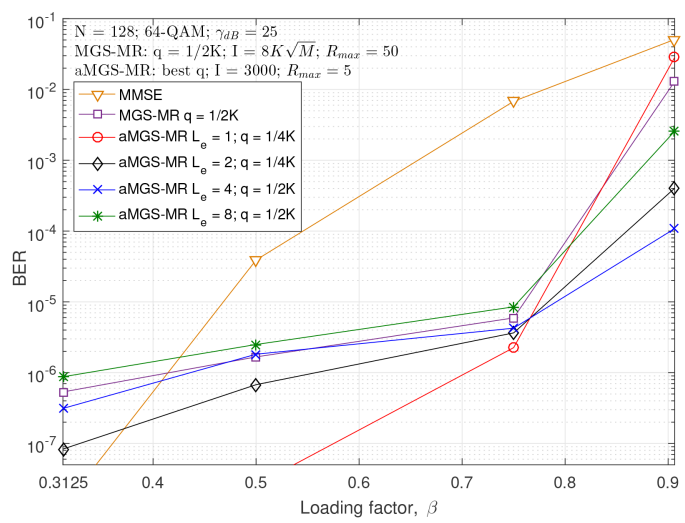

$b$

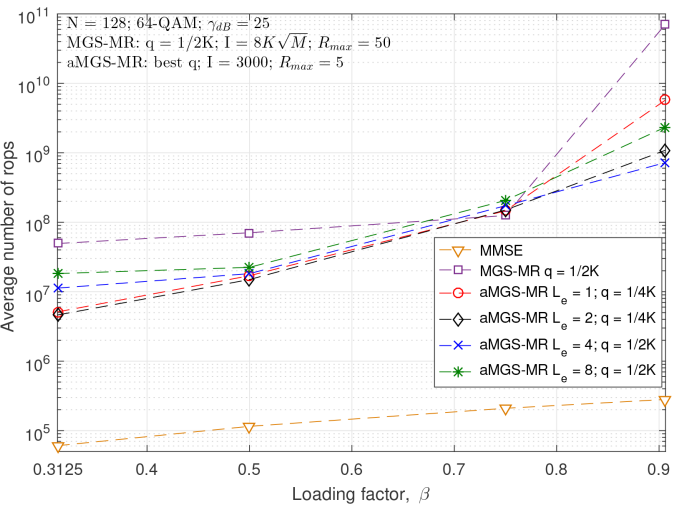

$d$

Fig. 10 Performance and complexity versus system loading, considering $\gamma_{d B}=25 \mathrm{~dB}, 64-Q A M, R_{\max }=5, \mathscr{I}=3000$

(a) Performance for $N=64$, (b) Performance for $N=128$ antennas, (c) Average rop complexity for $N=64$, (d) Average rop complexity for $N=128$

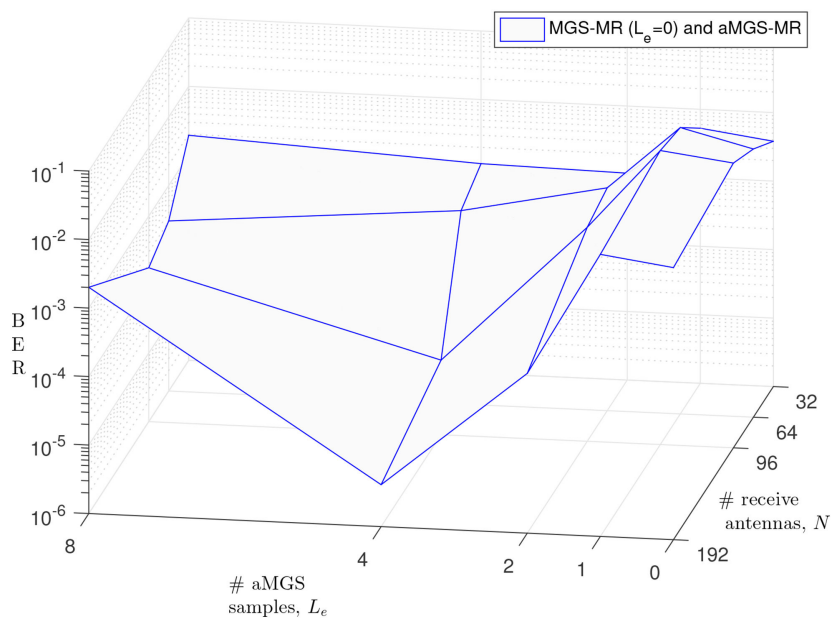

Fig. 11 Three-dimensional figure comparing BER performance $\times$ number of receive antennas $\times L_{e}$ samples of the aMGS-MR schemes and MGS-MR (displayed in $L_{e}=0$ ), 64-QAM, $\beta \simeq 0.9, \gamma_{d B}=25 d B$

$N=\{32,64,96,192\}$, high system loading $\beta \simeq 0.9$, medium $\gamma_{\mathrm{dB}}=25 \mathrm{~dB}$ and 64-QAM. It is instructive to confirm that the performance remarkably varies with the number of samples in MS strategy, $L_{e}=\{1,2,4,8\}$, while the MGS-MR detector is described by the $L_{e}=0$ line. The number of restarts and mixing ratio values for the aMGS-MR follow Table 5, while for the MGS-MR was considered $R_{\max }=5$ and $q=1 / 2 K$ [11]. One can notice that the MGS-MR and aMGS-MR with $L_{e}=1$ does not provide a good BER performance compared to the aMGS-MR with MS cases, and its degradation in performance becomes more prevalent under a large number of antennas. Indeed, with an increasing number of antennas, one can conclude that the aMGS-MR with $L_{e}=4$ samples becomes the most interesting strategy, denoting a performance gain of more than a decade in relation to the others.
As a result, the MS strategy with $L_{e}=4$ samples appears to be the most suitable choice in other LS-MIMO scenarios with hundreds of antennas, high system loading and medium/high modulation orders.

\section{Conclusions}

aMGS detector for large-scale MIMO systems has been proposed based on multiple-samples on coordinate updating process. The proposed aMGS LS-MIMO detection scheme has demonstrated capability to mitigate the impact caused by the random symbol from the mixture, which is aggravated either in near full-loading system condition or when a medium or high-order modulation is deployed. Moreover, suitable tradeoff metric values have been found for a wide LS-MIMO system configuration scenarios when the number of samples is set in-between $L_{e}=1$ and $L_{e}=4$. Furthermore, a simplification in the target function is hold, aiming at reducing complexity by avoiding the exponential calculation. The proposed target function is based on ML cost minimisation and requires less mathematical operations than the original MGS, while no degradation in performance is observed.

Our numerical analysis for the mixing ratio parameter optimisation considering a wide range of system scenarios has demonstrated that the best mixing ratio value is determined by the system loading and by the quantity of samples of the aMGS as well. As a result, we have found different optimal values than $q=1 / 2 K$ reported previously by Datta et al. [11].

The modifications in MGS technique proposed herein have demonstrated effectiveness in achieving improvements in the detection algorithm's convergence, which results in significant gains in both performance and complexity aspects, specially when the system loading is high $(\beta \geq 0.75)$, compared to the original MGS technique. These advantages can be achieved without an increase in complexity, since the aMGS strategy is based on simple average operations and simplification procedure. On the other hand, when a low system loading is considered, the MS strategy resulted in a performance degradation. Finally, in LS-MIMO 
scenarios with more than hundreds of antennas and high system loading, a choice of $L_{e}=4$ samples with $q=1 / 2 K$ showed to be the most interesting.

\section{Acknowledgments}

This work has been partially supported by the National Council for Scientific and Technological Development (CNPq) of Brazil under grants 304066/2015-0; by the Londrina State University (UEL), by the Federal Institute of Parana (IFPR), by the University of Sao Paulo (USP) and the Parana State Government. All the agencies are gratefully acknowledged.

\section{References}

[1] Hoydis, J., ten Brink, S., Debbah, M. 'Massive MIMO in the UL/DL of cellular networks: how many antennas do we need?', IEEE J. Sel. Areas Commun., 2013, 31, (2), pp. 160-171

[2] Rusek, F., Persson, D., Lau, B.K., et al.: 'Scaling up MIMO: opportunities and challenges with very large arrays', IEEE Signal Process. Mag., 2013, 30, (1), pp. 40-60

[3] Marzetta, T.: 'Noncooperative cellular wireless with unlimited numbers of base station antennas', IEEE Trans. Wirel. Commun., 2010, 9, (11), pp. 35903600

[4] Vardhan, K., Mohammed, S., Chockalingam, A., et al: 'A low-complexity detector for large MIMO systems and multicarrier CDMA systems', IEEE $J$. Sel. Areas Commun., 2008, 26, (3), pp. 473-485

[5] Rajan, B., Mohammed, S., Chockalingam, A., et al.: 'Low-complexity nearML decoding of large non-orthogonal STBCs using reactive tabu search' IEEE Int. Symp. on Information Theory, 2009. ISIT 2009, 2009, pp. 19931997

Q5 [6] Som, P., Datta, T., Chockalingam, A., et al.: 'Improved large-MIMO detection based on damped belief propagation'. 2010 IEEE Information Theory Workshop on Information Theory (ITW 2010, Cairo), 6-8 January 2010, pp. $1-5$

[7] Suneel, M., Som, P., Chockalingam, A., et al:: 'Belief propagation based decoding of large non-orthogonal STBCs'. IEEE Int. Symp. on Information Theory, 2009. ISIT 2009, 28 June 2009-3 July 2009, pp. 2003-2007

[8] Tang, C., Liu, C., Yuan, L., et al.: 'High precision low complexity matrix inversion based on Newton iteration for data detection in the massive MIMO', IEEE Commun. Lett., 2016, 20, (3), pp. 490-493

[9] Thanos, A., Paliouras, V.: 'Hardware trade-offs for massive MIMO uplink detection based on Newton iteration method'. 2017 6th Int. Conf. on Modern Circuits and Systems Technologies (MOCAST), 2017, pp. 1-4

[10] Farhang-Boroujeny, B., Zhu, H., Shi, Z.: 'Markov chain Monte Carlo algorithms for CDMA and MIMO communication systems', IEEE Trans. Signal Process., 2006, 54, (5), pp. 1896-1909

[11] Datta, T., Kumar, N.A., Chockalingam, A., et al.: 'A novel Monte-Carlosampling-based receiver for large-scale uplink multiuser MIMO systems', IEEE Trans. Veh. Technol., 2013, 62, (7), pp. 3019-3038

[12] Choi, J.: 'An MCMC-MIMO detector as a stochastic linear system solver using successive overrelexation', IEEE Trans. Wirel. Commun., 2016, 15, (2), pp. $1445-1455$

[13] Gao, C., Xu, J., Tao, X., et al.: 'An improved mixed Gibbs sampling algorithm based on multiple random parallel Markov chains for massive MIMO systems'. 2016 IEEE 27th Annual Int. Symp. on Personal, Indoor, and Mobile Radio Communications (PIMRC), 2016, pp. 1-5

[14] Mandloi, M., Bhatia, V.: 'Layered Gibbs sampling algorithm for near-optimal detection in large-MIMO systems'. 2017 IEEE Wireless Communications and Networking Conf. (WCNC), 2017, pp. 1-6

[15] Martino, L., Elvira, V., Camps-Valls, G.: 'The recycling Gibbs sampler for efficient learning', Digit. Signal Process., 2018, 74, pp. 1-13

[16] Chen, R., Liu, J.S., Wang, X.: 'Convergence analyses and comparisons of Markov chain Monte Carlo algorithms in digital communications', IEEE Trans. Signal Process., 2002, 50, (2), pp. 255-270

[17] Hassibi, B., Hansen, M., Dimakis, A.G., et al.: 'Optimized Markov chain Monte Carlo for signal detection in MIMO systems: an analysis of the stationary distribution and mixing time', IEEE Trans. Signal Process., 2014, 62, (17), pp. 4436-4450

[18] Yang, Y., Peng, H., Zhang, D., et al.: 'Markov chain Monte Carlo-based separation of paired carrier multiple access signals', IEEE Commun. Lett., 2016, 20, (11), pp. 2209-2212

[19] Chockalingam, A., Rajan, B.: 'Large MIMO systems' (Cambridge University Press, 2014)

Q6 [20] Monteiro, F.: 'Lattices in MIMO spatial multiplexing: detection and geometry'. PhD dissertation, University of Cambridge, UK, 2012

[21] Hansen, M., Hassibi, B., Dimakis, A.G., et al.: 'Near-optimal detection in MIMO systems using Gibbs sampling', GLOBECOM 2009 - 2009 IEEE Global Telecommunications Conf., November 2009, pp. 1-6

[22] Damen, M., El Gamal, H., Caire, G.: 'On maximum-likelihood detection and the search for the closest lattice point', IEEE Trans. Inf. Theory, 2003, 49, (10), pp. 2389-2402

[23] Liu, T.: 'Some results for the fast MMSE-SIC detection in spatially multiplexed MIMO systems', IEEE Trans. Wirel. Commun., 2009, 8, (11), pp. $5443-5448$

\section{Appendix}

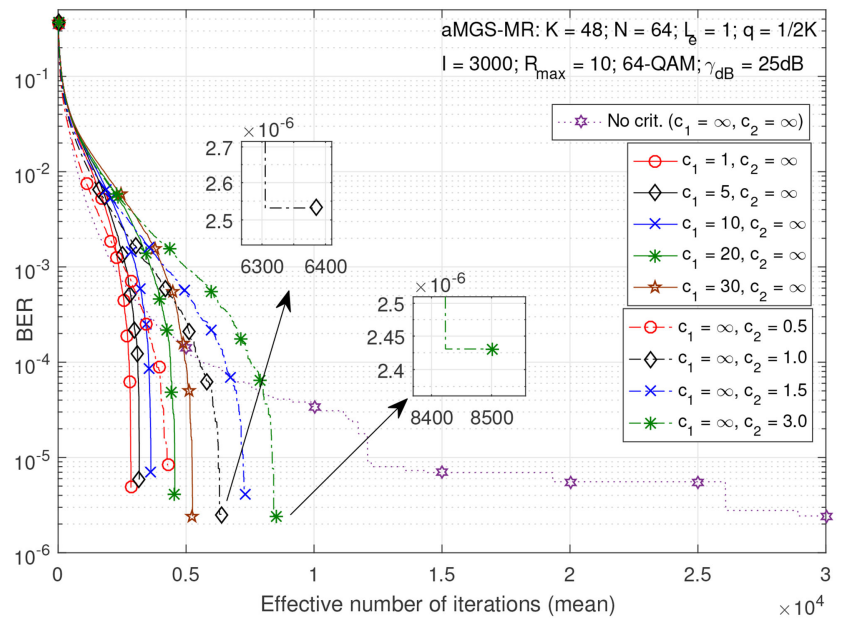

a

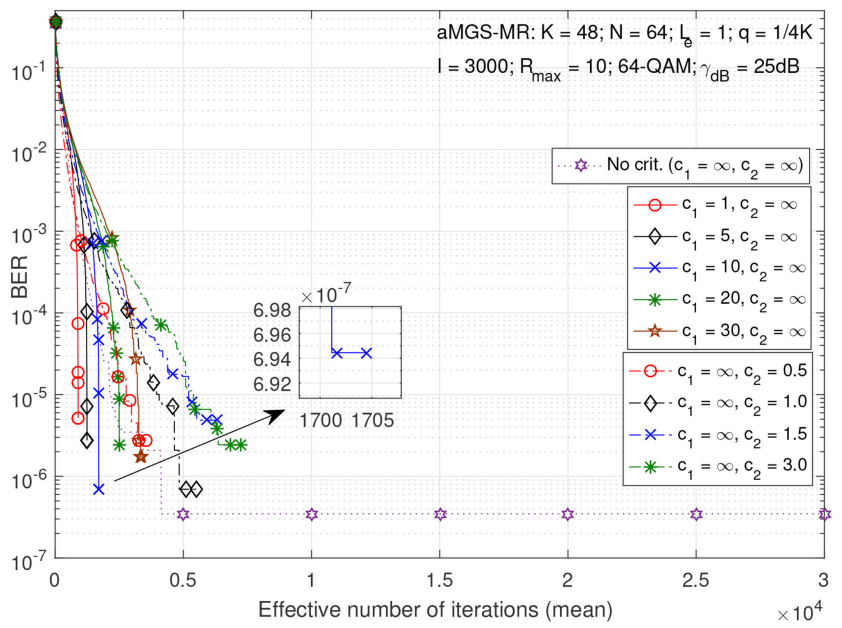

$b$

Fig. 12 BER performance versus ENI convergence for several stop criterion parameter combinations. $K=48, N=64 ; \gamma_{d B}=25 \mathrm{~dB} ; 64-Q A M$; $R_{\max }=10, L_{e}=1$, sample and two mixing ratios

(a) $48 \times 64, q=1 / 2 K, \gamma_{\mathrm{dB}}=25 \mathrm{~dB}, 64-\mathrm{QAM}$, (b) $48 \times 64, q=1 / 4 K, \gamma_{\mathrm{dB}}=25 \mathrm{~dB}$, 64-QAM

\subsection{Stopping criterion parameters}

In Fig. 12, the stop criterion is applied under different values of $c_{1}$, which tunes the maximum number of iterations in stalling mode, and $c_{2}$, which rules the maximum amount of restarts. For the $c_{\min }$ parameter, the same value of [11] was adopted, i.e. $c_{\min }=10$. The maximum number of iterations is set $\mathscr{I}=3000$ and $R_{\max }=10$ restarts. The system is operating under 64-QAM modulation and $\gamma_{\mathrm{dB}}=25 \mathrm{~dB}$, with $K=48, N=64$ and $L_{e}=1$ samples. It is worth noticing that all curves have reached their convergence, so that even with an increasing in the number of iterations $\mathscr{F}$, the performance would not be significantly changed.

For the stopping criterion analysis, the simulations are performed aiming to analyse each parameter individually. Thus, for the parameter that is not under analysis a large (infinite) value is assumed. The mixing ratio in Fig. $12 a$ is set to $q=1 / 2 \mathrm{~K}$. As expected, in the absence of stopping criterion the algorithm's convergence is remarkably retarded, while a marginally improved performance when compared to the results with stopping criterion is attained. Moreover, considering stopping criterion, the best performance-complexity tradeoff is obtained with $c_{1}=1$. Although a better performance is perceived when $c_{1}=30$, the ENI almost doubled, denoting a worse performance versus complexity tradeoff. Indeed, increasing $c_{1}$, a slightly better performance is reached, since more iterations in stalling mode is allowed; on the other hand, the averaged number of iterations is almost doubled. 
Regarding the restarting stopping criterion, the choice of $c_{2}=1.0$ is clearly the best tradeoff among those compared, since a significant performance gain is observed from $c_{2}=0.5$ and less ENI is required to achieve the same convergence if compared to $c_{2}=1.5$ or 3.0 .

With mixing ratio $q=1 / 4 K$ in Fig. $12 b$, it is noteworthy that the combination of $c_{1}=10$ and $c_{2}=1.0$ denotes the best tradeoff in terms of iterations and restarts, respectively; indeed, the first, with an expressive performance gain regarding $c_{1}=1$ or 5 , with a marginal ENI increment; the second, analogously with respect to $c_{2}=0.5$.

Finally, when $c_{1}=1.0$ results in best tradeoff in Fig. $12 a$, while the choice of $c_{1}=10$ achieved marginally same performance and ENI. On the other hand, in Fig. $12 b$ scenario, $c_{1}=1.0$ showed a significant performance loss compared to the case of $c_{1}=10$. From this analysis, we adopted in the remainder of this work $c_{1}=10$ and $c_{2}=1.0$ as stop criterion for iterations and restarts, respectively. 


\section{APPENDIX E - Full paper under R1 review in the journal "EURASIP Journal on Advances in Signal Processing", January 2019}

Title: Mitigating the noisy solution impact of mixed Gibbs sampling detector in high-order modulation largescale MIMO systems.

Authors: Alex Miyamoto Mussi and Taufik Abrão.

Journal: EURASIP Journal on Advances in Signal Processing (ISSN 1687-6180).

Classification (Qualis-CAPES 2016): A2 (Engenharias IV).

Impact Factor (2017): 1.639

Submission Date (R1 version): January 2019. 


\section{EURASIP Journal on Advances in Signal Processing Mitigating the Noisy Solution Impact of Mixed Gibbs Sampling Detector in High-Order Modulation Large-Scale MIMO Systems --Manuscript Draft--}

\section{Manuscript Number:}

Full Title:

Article Type:

Funding Information:

\begin{tabular}{l}
\hline Abstract: \\
\hline Corresponding Author: \\
\hline Corresponding Author Secondary \\
Information: \\
\hline Corresponding Author's Institution: \\
\hline Corresponding Author's Secondary \\
Institution: \\
\hline
\end{tabular}

First Author:

First Author Secondary Information:

Order of Authors:

Order of Authors Secondary Information:

Response to Reviewers:
JSIG-D-18-00184R1

Mitigating the Noisy Solution Impact of Mixed Gibbs Sampling Detector in High-Order Modulation Large-Scale MIMO Systems

Research

A neighborhood restricted Mixed Gibbs Sampling (MGS) based approach is proposed for low-complexity high-order modulation large-scale Multiple-Input Multiple-Output (LS-MIMO) detection. The proposed LS-MIMO detector applies a neighborhood limitation (NL) on the noisy solution from the MGS at a distance $d$ - thus, named dsimplified MGS (d-sMGS) - in order to mitigate its impact, which can be harmful when a high order modulation is considered. Numerical simulation results considering 64-QAM demonstrated that the proposed detection method can substantially improve the MGS algorithm convergence, whereas no extra computational complexity per iteration is required. The proposed d-sMGS-based detector suitable for high-order modulation LSMIMO further exhibits improved performance $\times$ complexity tradeoff when the system loading is high, i.e., when $\mathrm{K} / \mathrm{N} \geq 0.75$. Also, with increasing the number of dimensions, i.e., increasing number of antennas and/or modulation order, a smaller restriction of 2sMGS was shown to be a more interesting choice than 1-sMGS.

Alex Miyamoto Mussi, M.Sc.

Instituto Federal de Educacao Ciencia e Tecnologia do Parana

Assis Chateaubriand, Paraná BRAZIL

Instituto Federal de Educacao Ciencia e Tecnologia do Parana

Alex Miyamoto Mussi, M.Sc.

Alex Miyamoto Mussi, M.Sc.

Taufik Abrão, Ph.D.

\#Response to editor:

We appreciate the editorial assistance to our work. We would like to thank the Associate Editor and Reviewers for the valuable time and comments provided to this article. We emphasize that we are totally committed to all the observations made in this review stage and, once again, we appreciate the comments that have further enriched the work. In addition, we would like to clarify that during the review period, the work was extended and enriched seeking novelties and more efficient techniques to solve the problem addressed. As a result, this revised version was supplemented by a novel detection technique based on neighborhood limitation, called \$d\$-sMGS. Such technique demonstrated a performance gain over the multiple sampling strategy, aMGS, without any increase in computational complexity. In addition, the multiple sampling aMGS technique was published in November 2018 and is cited in the revised manuscript. We hope the changes we've made are sufficient for a positive final response from your highly appreciated review panel.

We have also added a supplementary document file for a detailed response letter to the reviewers. This document is intended to facilitate the review process and clarify the response to the reviewers. 
\#Reviewer 1:

The authors thank for the compliments to the work and for the time spent reviewing the article. In addition, we underline the importance of the valuable comments provided by the reviewer. The changes in the text regarding the questions are in the green color. We appreciate the comentary provided. In fact, the gain provided by the use of multiple samples is, in certain situations, marginal. However, under favorable conditions of high loading and high modulation order, it becomes significant over the conventional MGS (see Figures 5 and 6 of the revised version).

The reason why the number of iterations increases subtancially when the number of samples grows is given by the fact that, when taking the average of more samples (taking into account that $\$(1-q)>>q$ ), the result becomes close to the symbol coming from the target function, which almost nullify the contribution of the random symbol (remember that the random symbol was proposed in the MGS as local minimum escape). Therefore, a large number of samples (tens to hundreds) would make the aMGS approximately in a conventional GS (nullifying the MGS mixture), which would result in algorithm convergence/performance losses. Therefore, it is noticed that the use of multiple samples becomes tricky, since many samples may be detrimental to the algorithm and few samples may not result in an expressive performance gain. In this sense, a new detection procedure, based on the neighborhood limitation of the random solution, is proposed in this work. This proposal proved to be more efficient than the aMGS, resulting in better performance and similar complexity.

The selection of a random solution becomes detrimental in systems with high order of modulation, since there are a large number of symbols in the constellation and the choice by a random symbol makes it probably far from the real solution. This hinders the convergence of the algorithm since it is based on coordinate updates and each of these coordinates is updated based on the global solution, and some of these positions with an (very far) erroneous solution, the overall convergence of the algorithm ends up being affected. We added an introductory text to Section 4, which portrays this important explanation alerted by the reviewer.

We thanks the important note given. In fact, the reviewer is correct, we realized that the choice of mixing ratio has little impact on performance. Since this is intimately linked to the probability of choice of the noisy solution, a technique of minimizing the impact of this noisy solution (which is the focus of this work) is sufficient to minimize the effect of the mixing ratio. Thus, the inverse of the number of real dimensions becomes acceptable. This modification has been included for the novel proposed detector $\$ \mathrm{~d} \$-$ sMGS. The adopted value for the $\$ \mathrm{~d} \$$-sMGS mixing ratio is given in 2th paragraph of Subsection 6.1.\}

Icolg\{Regarding the practical determination of the mixing ratio value, an asymptotic numerical analysis is developed in [14] resulting in the mixing ratio $\$ q=1 / 2 \mathrm{~K} \$$ and $\$ L_{-} e=4 \$$ samples as the most suitable choice in LS-MIMO scenarios with hundreds of antennas, high system loading and medium/high modulation order.

We added in the second paragraph of Section 2 the missing definitions.

There was a typo and we apologize. In fact, it is wrong. The intention was a \$|xi_\{lrm $\min \} \$$. Besides that, we clarify that the pseudocode had some of its variables changed in order to make it more intuitive with the equation presented in the work.

\#Reviewer 2:

The authors are grateful for the time spent in reading and reviewing the article, as well as the important observations made to improve the work. The changes in the text regarding your questions are in the orange color.

In fact, the work does not originate from a theoretical source but rather from an operational aspect of the MGS algorithm. On the other hand, a solution is proposed and demonstrates significant results under high order modulation conditions (Fig. 5 of the revised version) and high loading (Fig. 6).

With respect to algorithm 2, we clarify that the pseudocode had some of its variables changed in order to make it more intuitive with the equation presented in the work. Besides that, more comments were added to the pseudocode.

We appreciate the reviewer observation. In fact, the simplification in the objective function is a minimization of Euclidean distance, which is also widely used in the literature, but because it is an inherent concept in the area, it was not possible to find an adequate reference.

Yes, the "target function" means the "target distribution function" in [10]. The term 
"distribution". The term "distribution" was omitted, since the simplified target function is deterministic and no longer a distribution as the target distribution function of [10]. We thanks for your comentary. Indeed, the multiple sampling strategy when mitigating the noisy solution also runs the risk of nullifying this solution if many samples are employed. When a mean among many terms from a r.v. with probabilities $\$ q \$$ and $\$(1-$ q) $\$$-- with $\$ q<<(1-q) \$--$ are considered, there is tendency to an average value in which the term with probability $\$ q \$$ is nullified. In this sense, the noisy solution would be ineffective and the condition of stalling problem could happen, since the mixing of the MGS is a strategy to specifically combat it. We have added a sentence with this explanation in the paragraph above of subsection 4.1 .

In Algorithm 2, the simplified target function in calculated in lines 8--12 and is used in line 17 , where it is set in the $\$ m \$$-th sample according to the sorted $\$ \backslash m u \_\{i, m\} \$$ and mixing ratio $\$ q \$$. The simplified target function acts no more as a distribution, but as minimization of the Euclidean distance in the \$i\$-th coordinate (position). In this way, the symbol resulting from the simplified target function will be what minimizes the Euclidean distance within the considered constellation.

In fact, the mixing ratio information in this figure was omitted and was equal to $\$ q=1$ / $2 \mathrm{~K} \$$. However, the related Figure 2, denoting the performance validation obtained with the simplified objective function, was removed since its validation can be found in [14]. The reviewer is correct, the related performance gain is not reasonable. A possible explanation is given by the fact that the algorithm works with a random mixing, which makes its evolution/convergence variable, also adding the stop criteria effect. In any case, this "unexplained gain" was not related as a gain/benefit of the simplified target function.

There was a typo and we apologize. In fact, it is wrong. The intention was a \$\xi_\{lrm $\min \}$. In previous version, \$W_I\$ represented the \$m\$-th sample \$ $\left\{\mid r h o \_\{m, i\}\right.$ $\left(\operatorname{Ihat}\{\operatorname{lmathbf}\{\mathrm{s}\}\}^{\wedge}\{(\mathrm{t})\}_{\_}\{\mathrm{i}\}, \operatorname{Imathbf}\{\mathrm{y}\}\right.$, Imathbf $\left.\left.\{\mathrm{H}\}\right)\right\} \$$. For a better understanding, we changed in the pseudocode the variable $\$ W \_$I according to the equation given in the body of the text (Eq. 20). The complexities of single and multiple (averaged) sampling are not the same, although its difference is marginal: refers to the mathematical operations of generating random values in equal quantity of samples, besides addition and division operations, representing the arithmetic mean.

We thanks for the careful observation. We have changed in Table 1 the term "Algorithm 1" to "Target distribution function calculation on Algorithm 1". We have added two figures of BER performance vs. SNR: Fig. 5.a and 5.b. 


\title{
Mitigating the Noisy Solution Impact of Mixed Gibbs Sampling Detector in High-Order Modulation Large-Scale MIMO Systems
}

\author{
Alex M Mussi ${ }^{1,2 *}$ and Taufik Abrão ${ }^{3}$
}

\begin{abstract}
A neighborhood restricted Mixed Gibbs Sampling (MGS) based approach is proposed for low-complexity high-order modulation large-scale Multiple-Input Multiple-Output (LS-MIMO) detection. The proposed LS-MIMO detector applies a neighborhood limitation (NL) on the noisy solution from the MGS at a distance $d$ - thus, named $d$-simplified MGS ( $d$-sMGS) - in order to mitigate its impact, which can be harmful when a high order modulation is considered. Numerical simulation results considering 64-QAM demonstrated that the proposed detection method can substantially improve the MGS algorithm convergence, whereas no extra computational complexity per iteration is required. The proposed $d$-sMGS-based detector suitable for high-order modulation LS-MIMO further exhibits improved performance $\times$ complexity tradeoff when the system loading is high, i.e., when $\frac{K}{N} \geq 0.75$. Also, with increasing the number of dimensions, i.e., increasing number of antennas and/or modulation order, a smaller restriction of 2-sMGS was shown to be a more interesting choice than 1-sMGS.
\end{abstract}

Keywords: Massive MIMO; low complexity detector; Markov chain Monte Carlo; Gibbs sampling

Reviewer 1: green

Reviewer 2: orange

Other modifications: blue

\section{Introduction}

In order to meet the demands of high transmission capacity, high reliability and spectral and energy efficiency requirements of modern wireless communication systems, the multiple input and output (MIMO) technique has been proposed and considered an appropriate solution due to to their ability to provide multiplexing and diversity gains without the need for additional spectral features. These advantages are further enhanced by large-scale use, called Large-Scale MIMO (LS-MIMO), which has important application in fifth generation $(5 \mathrm{G})$ wireless communications. Such structures hold the same benefits as conventional MIMO, however on a larger scale. More properly, LS-MIMO is defined as a transmission/reception design using typically several tens or even hundreds of antennas in at

${ }^{*}$ Correspondence: alex.mussi@ifpr.edu.br

${ }^{1}$ Federal Institute of Paraná, Cívica Avenue 475, 85935-000 Assis Chateaubriand, Brazil

Full list of author information is available at the end of the article least one of the communication terminals, usually in the base station (BS) $[1,2]$. This turns out to be convenient for the systems in question, since the reduced dimensions of user equipments (UEs) suggest a single antenna arrangement in each UE; on the other hand, a huge amount of antennas need to be is installed in each BS.

However, the LS-MIMO high capacity/spectral efficiency comes with a price: as the number of antennas at BS increases, the computational complexity of data detection tends to grow proportionally. Hence, efficient and low-complexity symbol detection techniques becomes critical as the processing of large numbers of signals can become a system bottleneck. It is well known that maximum likelihood (ML) detection could provide optimum symbol detection, but its high complexity forbids it from a practical implementation for MIMO systems. Therefore, sub-optimal linear and non-linear detectors with low complexity are often employed. Many low-complexity LS-MIMO detectors have been proposed in recent literature, including detectors based on a) local neighborhood search, such as likelihood ascent search (LAS) algorithm [3], and reactive tabu search (RTS) algorithm [4]; b) message pass- 
ing (MP) algorithms, based on belief propagation (BP) technique, such that LS-detectors inspired in graphical models, as factor graph (FG) [5] and Markov random fields (MRF) [6]; c) minimum mean square error (MMSE) approximation techniques [7, 8], which result in low-complexity at the price of achieving good performance only at low system loading factor; d) Markov Chain Monte Carlo (MCMC) techniques, which are based on Gibbs Sampling (GS) [9] and its variations $[10,11,12,13]$, emerging as a promising approach to deal with LS-MIMO structures, since such techniques demonstrate a near-optimum performance while require a low-moderate complexity (quadratic order) and also presenting a simple and effective way to solve the large-scale detection problem.

From the GS based techniques, in [10] a strategy of mixing between the conventional GS solution and a random or noisy solution was proposed, which is controlled by a mixing ratio parameter and is called Mixed GS (MGS). The MGS has been shown to solve the stalling problem of the GS detector in low order of modulation, i.e., 4-QAM. With the modulation order increasement, the multiple restarts (MR) technique is proposed, which restarts the algorithm with a new initial solution, taking advantage of the random evolution of the algorithm and can result in a better cost solution. The MGS-MR detector showed near-optimal performance in 16-QAM modulation, however, in high modulation order the noisy solution interferes with the convergence of the algorithm, requiring an extra strategy to avoid the impact of this solution. In [14] is proposed the use of multiple samples, called averaged MGS (aMSG), in order to minimize this impact, besides a simplification in the target distribution function. Numerical results demonstrate a convergence improvement in high order modulation and high system loading, on the other hand, the choice of sample amount and mixing ratio tends to be difficult. In the present work, a strategy for reducing the solution is also addressed, through a limitation in the neighborhood of the random solution, which presented superior performance to the aMGS, with marginally similar computational complexity.

Also related to the MGS detector, in [15] an optimization on mixing time was introduced to accelerate the finding of the optimal solution. Numerical results demonstrated that a mixing time dynamic choice based on SNR can improve convergence, although the stalling problem persisted when a fixed mixing time is adopted. Besides that, these results did not considered the performance behavior in high-order modulation systems. A QR decomposition approach within the MCMC detector was addressed in [16, 17], which demonstrated to reduce the number of operations due to the lower triangular matrix feature. Furthermore, based on the concept of multiple random parallel Markov chains, work in [18] proposes a MR strategy through parallel chains; such strategy reduced the algorithm's running time compared to MGS-MR, despite the increasing of the number of real operations per symbol.

The contribution of this work follows: i) a neighborhood limitation (NL) strategy is proposed aiming at improving the MGS convergence rate operating under higher-order modulation and large scale MIMO regime. The proposed strategy, called $d$-sMGS ( $d$-simplified MGS), performs a NL in the random solution coming from the mixture used by the MGS detector. As a result, the impact caused by this noisy solution is mitigated and the convergence is increased. ii) an analysis of the performance $\times$ complexity tradeoff is carried out among the proposed $d$-sMGS, the conventional MGS [10] and the aMGS (averaged MGS) [14], which the latter is an approach that also aims to alleviate the impact caused by the random solution, although the procedure is based on multiple sampling (MS) strategy, which samples the estimated symbol multiple times and performs a mean operation to obtain the result.

The remainder of this paper is organized as follows. Section 2 presents the adopted large-scale MIMO system model. A review on the MGS technique is presented in section 3 and the MGS based approaches with noisy solution reduced impact are discussed in section 4, while the aMGS approach is described in subsection 4.1 and the proposed simplified MGS with NL detector for LS-MIMO is developed in subsection 4.1. Computational complexity are presented in section 5 and extensive numerical simulation results are analyzed in section 6 . Conclusion remarks are provided in section 7 .

\section{System model and problem formulation}

We consider an uplink (UL) single-cell MIMO communication system operating in multiplexing gain mode with $K$ active single-antenna users and $N$ receive antennas at the base station (BS), as disposed Fig. 1. We mainly investigate the performance $\times$ complexity tradeoff of suitable LS-MIMO detection schemes and, for simplicity, the availability of the channel state information at the BS is considered, which also aims to reach the pure efficiency of each detection technique. Thus, the pilot training stage and the respective pilot contamination effect have not taken into account in such context.

Moreover, for simplicity, the communication channel is assumed to be frequency-flat fading, compound 
by the complex channel matrix $\mathbf{H}_{c} \in \mathbb{C}^{N \times K}$. The elements of $\mathbf{H}_{c}$ are all independent complex Gaussian random variables with zero mean and unit variance, i.e., $H_{c_{i, k}} \sim \mathcal{C N}[0 ; 1]$, where $H_{c_{i, k}}$ denotes the element in the $i$-th row and $k$-th column of the matrix $\mathbf{H}_{c}$. Let $\mathbf{s}_{c}$ be the $K \times 1$ complex vector corresponding to the $K$ symbols $M$-QAM transmitted over the single-antenna users, $\mathbf{s}_{c} \in \mathbb{A}_{c}^{K}$ where $\mathbb{A}_{c}$ denotes the QAM constellation adopted. The UL received signal, $y_{c_{i}}$, at the $i$-th BS antenna can be written as:

$$
\begin{aligned}
y_{c_{i}} & =\sum_{j=1}^{K} H_{c_{i, j}} s_{c_{j}}+\eta_{c_{i}}, \quad i=1, \ldots, N \\
& =\underbrace{H_{c_{i, k} s_{c_{k}}}}_{\text {desired signal }}+\underbrace{\sum_{j=1, j \neq k}^{K} H_{c_{i, j} s_{c_{j}}}}_{\text {intracellular interference }}+\underbrace{\eta_{c_{i}}}_{\text {AWGN }} .
\end{aligned}
$$

where $y_{c_{i}}$ denotes the $i$-th element of the complex received signal vector $\mathbf{y}_{c}$ and $s_{c_{j}}$ is the $j$-th element of $\mathbf{s}_{c}$. In matrix form, the received signal vector at the $\mathrm{BS}$ is re-written as

$$
\mathbf{y}_{c}=\mathbf{H}_{c} \mathbf{s}_{c}+\boldsymbol{\eta}_{c}
$$

where $\boldsymbol{\eta}_{c}$ denotes the additive white Gaussian noise (AWGN) vector, assumed to be a complex Gaussian random variable with zero mean and variance given by:

$$
\mathbb{E}\left[\boldsymbol{\eta}_{c} \boldsymbol{\eta}_{c}^{H}\right]=\sigma^{2} \mathbf{I}_{N}
$$

where $\sigma^{2}$ is the noise variance at each receive antenna.

The average received SNR at each receive antenna can be modelled as:

$$
\gamma=\frac{K P_{\mathrm{s}}}{\sigma^{2}}
$$

where $P_{\mathrm{s}}$ is the power of the received symbols. For simplicity, in Eq. (4) it is considered that the largescale fading effect has been compensated in such a way that all $K$ users' signals are received with equal power at the BS, and assumed equal to $K P_{\mathrm{s}}$, denoting the total sum power available at the transmitters [19].

In this work, a real-valued system model corresponding to (2) is adopted, which is given by:

$$
\mathbf{y}=\mathbf{H s}+\boldsymbol{\eta}
$$

where $\mathbf{y} \in \mathbb{R}^{2 N \times 1}, \mathbf{H} \in \mathbb{R}^{2 N \times 2 K}, \mathbf{s} \in \mathbb{R}^{2 K \times 1}, \boldsymbol{\eta} \in$ $\mathbb{R}^{2 N \times 1}$, and defined as:

$$
\begin{aligned}
\mathbf{H} & =\left[\begin{array}{ll}
\mathcal{R}\left(\mathbf{H}_{c}\right) & -\mathcal{I}\left(\mathbf{H}_{c}\right) \\
\mathcal{I}\left(\mathbf{H}_{c}\right) & \mathcal{R}\left(\mathbf{H}_{c}\right)
\end{array}\right] \\
\mathbf{s} & =\left[\begin{array}{l}
\mathcal{R}\left(\mathbf{s}_{c}\right) \\
\mathcal{I}\left(\mathbf{s}_{c}\right)
\end{array}\right], \quad \boldsymbol{\eta}=\left[\begin{array}{l}
\mathcal{R}\left(\boldsymbol{\eta}_{c}\right) \\
\mathcal{I}\left(\boldsymbol{\eta}_{c}\right)
\end{array}\right], \quad \mathbf{y}=\left[\begin{array}{c}
\mathcal{R}\left(\mathbf{y}_{c}\right) \\
\mathcal{I}\left(\mathbf{y}_{c}\right)
\end{array}\right]
\end{aligned}
$$

For the QAM complex alphabet $\mathbb{A}_{c}$, the elements of $\mathbf{s}$ assume integer values from the underlying pulseamplitude modulation (PAM) alphabet $\mathbb{A}$, i.e., $\mathbf{s} \in$ $\mathbb{A}^{2 K}$.

The maximum-likelihood (ML) decision rule is given by

$$
\mathbf{s}_{\mathrm{ML}}=\underset{\hat{\mathbf{s}} \in \mathbb{A}^{2 K}}{\arg \min }\|\mathbf{y}-\mathbf{H} \hat{\mathbf{s}}\|^{2}
$$

However, the ML detector in (7) is exponentially complex in $K$, being prohibitive for large $K \cdot N$, which is the case of LS-MIMO systems [19].

\section{Conventional method: review of Mixed Gibbs Sampling detection}

The LS-MIMO detector Mixed Gibbs Sampling (MGS) proposed in [10] is revisited in this subsection, which is based on the motivation to solve the stalling problem presented in the conventional GS detector.

To sample the estimated symbol at each position, a target distribution [20] is evaluated, which is given by:

$$
p\left(\hat{s}_{1}, \hat{s}_{2}, \ldots, \hat{s}_{2 K} \mid \mathbf{y}, \mathbf{H}\right) \propto \exp \left(-\frac{\|\mathbf{y}-\mathbf{H} \mathbf{s}\|^{2}}{\alpha^{2} \sigma^{2}}\right)
$$

where $\hat{s}_{i}$ denotes the $i$-th position of the estimated symbols vector $\hat{\mathbf{s}}, \alpha$ denotes a positive parameter, which tunes the mixing time of the Markov chain [20] and is also called as temperature. The conventional Gibbs sampling detector does not include the $\alpha$ parameter in its sample process, and thus can be viewed as a special case when $\alpha=1$. A larger temperature speeds up the mixing and aims to reduce the higher moments of the number of iterations when finding the correct solution. However, as stated in [10], the stalling problem persists even with large $\alpha$.

The MGS detector utilizes a mixing of: a) Conventional Gibbs sampling (i.e., $\alpha=1$ ); and $\mathbf{b}$ ) the infinite temperature version of (8) (i.e., $\alpha=\infty$ ), resulting in a random and uniform sample from all the possibilities, called a noisy or random solution in this paper. In this way, the MGS follows a sampling distribution given by:

$$
p\left(\hat{s}_{1}, \ldots, \hat{s}_{2 K} \mid \mathbf{y}, \mathbf{H}\right) \sim(1-q) \psi\left(\alpha_{1}\right)+q \psi\left(\alpha_{2}\right)
$$


and

$$
\psi(\alpha)=\exp \left(-\frac{\|\mathbf{y}-\mathbf{H} \hat{\mathbf{s}}\|^{2}}{\alpha^{2} \sigma^{2}}\right)
$$

where $q$ denotes the mixing ratio. The MGS detector of [10] considers the $\alpha_{1}=1, \alpha_{2}=\infty$ combination, which results in a near-ML performance, overcoming the stalling problem of the GS, being also a simple implementation choice. On the other hand, in highorder modulation, such as 64-QAM and 256-QAM, the noisy solution interferes in the algorithm's convergence, since there are a large number of symbols in the constellation and a simple random solution in this signal space has a high possibility of being far from the real solution, which causes the algorithm to require more iterations for convergence. In this sense, the proposed $d$-sMGS detector acts to mitigate this harmful effect.

Regarding the mixing ratio parameter $q$, in [10] an analysis in low order QAM constellations is carried out and its suitable value choice is presented as the inverse of the number of dimensions in the system, i.e., $q=\frac{1}{2 K}$, which is also employed in the proposed detector during our numerical simulations.

In the MGS algorithm, an initial solution $\hat{\mathbf{s}}^{(t=0)}$ is considered for the estimated symbols vector, where $t$ represents the current iteration. Indeed, the initial solution may be chosen either by a random symbols vector or as the output of a linear low-complexity detector, such as zero forcing (ZF) or MMSE. The index $i$, in addition to the position of the vector $\hat{\mathbf{s}}$, also denotes the coordinate referring to the MGS algorithm, where $i=1,2, \ldots, 2 K$. Therefore, each iteration requires $2 K$ coordinate updating. At each iteration, updating the $2 K$ coordinates is performed by sampling the distributions given by:

$$
\hat{s}_{i}^{(t)} \sim p\left(\hat{s}_{i} \mid \hat{s}_{1}^{(t)}, \ldots, \hat{s}_{i-1}^{(t)}, \hat{s}_{i+1}^{(t-1)}, \ldots, \hat{s}_{2 K}^{(t-1)}, \mathbf{y}, \mathbf{H}\right) .
$$

One can notice that by (11) each updated coordinate is fed, in the same iteration, to the next coordinate.

The probability of the $i$-th symbol assuming the value $a_{j} \in \mathbb{A}, \forall j=1, \ldots,|\mathbb{A}|$ can be written as:

$$
p\left(\hat{s}_{i}=a_{j} \mid \hat{\mathbf{s}}_{i-1}, \mathbf{y}, \mathbf{H}\right)=\frac{\exp \left(-\frac{\left\|\mathbf{y}-\mathbf{H} \hat{\mathbf{s}}_{i, j}\right\|^{2}}{\alpha^{2} \sigma^{2}}\right)}{\sum_{l=1}^{|\mathbb{A}|} \exp \left(-\frac{\left\|\mathbf{y}-\mathbf{H} \hat{\mathbf{s}}_{i, l}\right\|^{2}}{\alpha^{2} \sigma^{2}}\right)},
$$

where the cardinality of set $\mathbb{A}$ is expressed as $|\mathbb{A}|$, while $\hat{\mathbf{s}}_{i, j}$ denotes the vector $\hat{\mathbf{s}}^{(t)}$ with its $i$-th position changed to the symbol $a_{j}$.
The sampling process based on (12) can lead to a numerical limitation due to the exponential function. In this sense, such implementation was carried out through a logarithmic intermediate step, as:

$$
\begin{aligned}
& \log \left(p\left(\hat{s}_{i}=a_{j} \mid \hat{\mathbf{s}}_{i-1}, \mathbf{y}, \mathbf{H}\right)\right)= \\
& =f(i, j)-\left[f_{0}^{\text {ord }}+\log \left(1+\sum_{m=1}^{|\mathbb{A}|-1} \exp \left(f_{m}^{\text {ord }}-f_{0}^{\text {ord }}\right)\right)\right] \\
& =g(i, j)
\end{aligned}
$$

where $f(i, j)=-\frac{\left\|\mathbf{y}-\mathbf{H} \hat{\mathbf{s}}_{i, j}\right\|^{2}}{\alpha^{2} \sigma^{2}}$ and $f_{i}^{\text {ord }}$ is $i$-th position of $\mathbf{f}$ in descending order, for $i=1, \ldots,|\mathbb{A}|$. A practical and computationally efficient evaluation of MGS target Function is summarized in the Algorithm 1.

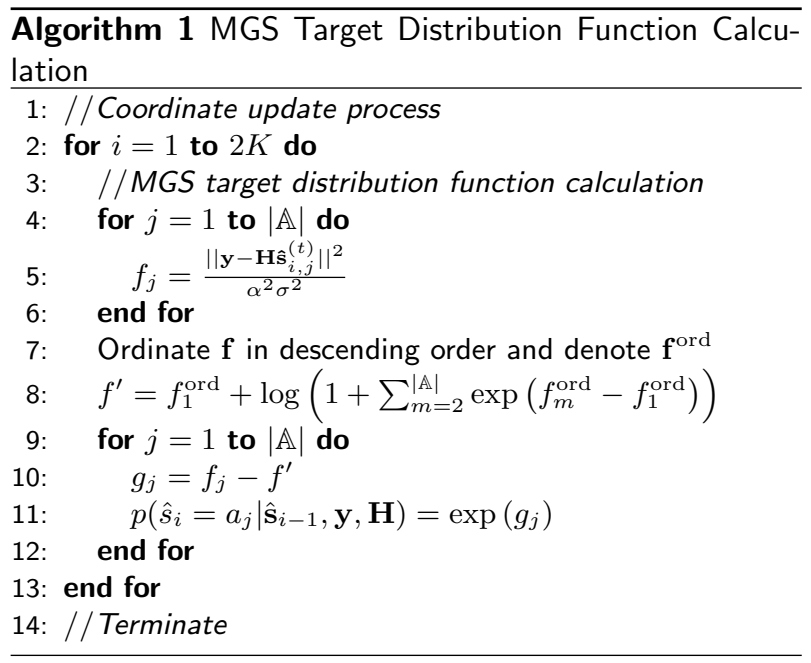

The MGS algorithm ends after a certain amount of iterations, and the vector of estimated symbols is chosen as the vector that presented the lowest ML cost, considering all iterations. In the next subsections, the additional strategy of multiple restarts (MR) [10] and the stopping criteria for the iterations and the restarts are addressed.

\subsection{Multiple restarts}

In medium QAM order modulations, such as 16-QAM, the mixing strategy of MGS is unable to achieve nearoptimal performance [21] in a reasonable number of iterations, while MR procedure, as proposed in [10] has demonstrated promising results, leading the MGS-MR under 16-QAM to near-optimal performance.

In the aMGS and $d$-sMGS detectors the MR strategy is also incorporated, namely aMGS-MR and $d$-sMGSMR detectors. Thus, the Algorithms 2 and 3 run either 
a maximal number of restarts $R_{\max }$ times or it is limited by a stopping criterion and the lowest cost found considering all restarts is the final solution. As discussed in Section 6, the MR strategy can improve the convergence of the algorithm compared to the same number of iterations in a single execution, resulting in a better performance-complexity tradeoff.

\subsection{Stopping criterion}

Given that the mixing strategy provides the local minimum escaping feature, the evolution of the cost function values across iterations becomes unpredictable and the optimal solution can be found before the maximum number of iterations $\mathcal{I}$ has been reached [14]. In this sense, an efficient stopping criterion is paramount in reducing the complexity of the MGS detector.

Similarly, the decision to set a restart in the algorithm requires a criterion definition, since the optimal solution may already have been found, not requiring an extra execution of the algorithm. Hence, MR strategy must be balanced aiming to achieve a better performance-complexity tradeoff.

Stopping criteria have been proposed in the literature. For instance, in [10], the stopping criterion is based on the difference between the best ML cost found so far and the noise variance. Moreover, the QAM constellation size could be taken into account. The main idea in [10] is to stop the detection iterations if a maximum number of iterations $\mathcal{I}$ is attained or if the iteration in stalling mode is larger than a maximum of $\Theta_{s}$ iterations.

Assume the estimated symbol vector, in the $t$-th iteration, is $\hat{\mathbf{s}}^{(t)}$. The quality metric of $\hat{\mathbf{s}}^{(t)}$ is defined as

$$
\phi\left(\hat{\mathbf{s}}^{(t)}\right)=\frac{\left\|\mathbf{y}-\mathbf{H} \hat{\mathbf{s}}^{(t)}\right\|^{2}-N \sigma^{2}}{\sqrt{N} \sigma^{2}}
$$

Hence, the stalling limit for iterations, $\Theta_{s}$, is given by

$$
\Theta_{s}\left(\phi\left(\hat{\mathbf{s}}^{(t)}\right)\right)=c_{s} \cdot e^{\phi\left(\hat{\mathbf{s}}^{(t)}\right)}
$$

where $c_{s}$ is a constant depending upon the $M$-QAM constellation size, which increases with $M$. Although (15) is suitable as a stopping criterion, a minimum number of iterations $c_{\text {min }}$ must be defined to ensure the quality of symbol detection. Therefore, $\Theta_{s}$ can be rewritten as

$$
\begin{aligned}
\Theta_{s}\left(\phi\left(\hat{\mathbf{s}}^{(t)}\right)\right) & =\left\lceil\max \left(c_{\min }, c_{s} \cdot e^{\phi\left(\hat{\mathbf{s}}^{(t)}\right)}\right)\right\rceil \\
\text { with } \quad c_{s} & =c_{1} \log _{2}(M)
\end{aligned}
$$

where $c_{1}$ is a tunning constant which defines the allowed number of iterations in stalling mode.
For the MR strategy, the criterion set the allowable number of restarts $\Theta_{r}$, which also is based on quality metric $\phi\left(\hat{\mathbf{s}}^{(t)}\right)$ :

$$
\begin{aligned}
\Theta_{r}\left(\phi\left(\hat{\mathbf{s}}^{(t)}\right)\right) & =\left\lceil\max \left(0, c_{r} \cdot \phi\left(\hat{\mathbf{s}}^{(t)}\right)\right)\right\rceil+1 \\
\text { whith } \quad c_{r} & =c_{2} \log _{2}(M),
\end{aligned}
$$

and $c_{2}$ is the tuning constant adjusting the maximum number of restarts.

At the end of each restart, $\Theta_{r}$ is computed and checked if the actual number of repetitions is less than $\Theta_{r}$. If yes, go to another run of the algorithm; else, output the solution vector with the minimum cost so far as the final solution.

For the aMGS and $d$-sMGS detectors presented below, aMGS and $d$-sMGS, we also assume the stop criteria described in this subsection.

\section{Reducing the Impact of Noisy Solution}

Originally, the mixture between the target distribution function solution and the random solution, proposed by MGS detector of [10], attempted to escape local minima that degrade system performance. In fact, this procedure showed to significantly improves the performance, specially in low-order modulation scenarios, as 4 or 16-QAM. On the other hand, in high-order modulation systems, the large number of symbols causes the random solution to degrade the convergence of the algorithm since it is based on a coordinate update process which requires the global solution, thus one or more positions that consider a random solution (probably erroneous and far from the real solution) interfere in the convergence in the other positions and, consequently, in the global one. This condition is aggravated in high-dimension problems, i.e., combining high-order modulations and number of antennas, which is the case of interest in this work.

In this sense, two approaches that tries to alleviate the harmful impact of the noisy solution are described below. Fig. 2 summarizes the coordinate update process on the aMGS and $d$-sMGS detectors. The strategy of multiple samples in mitigating the noisy solution also runs the risk of nullifying this solution if many samples are employed, this can happen since a mean among many terms from a r.v. with probabilities $q$ and $(1-q)$ - with $q<<(1-q)$ - tends to be an average value in which the term with probability $q$ is nullified. In this sense, the noisy solution would be ineffective and the condition of stalling problem could happen, since the mixing of the MGS is a strategy to specifically tackle it. 


\subsection{Approach \#1: Averaged MGS LS-MIMO detector} The aMGS proposed in [14] is addressed herein and is based on the following improvements:

1 Averaged Multiple Sampling on each coordinate: differently from the single sampling strategy [10], the aMGS employs an average between $L_{e}$ number of samples at each coordinate during the update process. By employing an averaged calculation, an intermediate (averaged) point between the target function symbol and the random symbol is more likely to be chosen, instead of a pure random symbol. As a result, the benefit of local minima escape is maintained, whereas the negative impact on the algorithm's convergence is smoothed.

2 Target Function Simplification: to reduce the computational complexity related to target function calculation of (12), the aMGS adopts a minimum ML cost approach. This simplification performs less mathematical operations, since the ||y - Hôs $\|$ computation is already performed in (12). Thus, the aMGS target function, in the $t$-th iteration is evaluated as:

$$
\xi\left(\hat{\mathbf{s}}_{i}^{(t)}, \mathbf{y}, \mathbf{H}\right)=\underset{j \in\{1, \ldots,|\mathbb{A}|\}}{\arg \min }\left\|\mathbf{y}-\mathbf{H} \hat{\mathbf{s}}_{i, j}\right\|,
$$

where $\hat{\mathbf{s}}_{i}^{(t)}$ denotes the updated estimated symbol vector until the $(i-1)$ position at the $t$ th iteration, whereas the other remaining $i,(i+$ $1), \ldots, 2 \mathrm{~K}$ positions assume the values from the previous iteration, i.e.,

$$
\hat{\mathbf{s}}_{i}^{(t)}=\left[\hat{s}_{1}^{(t)}, \ldots, \hat{s}_{i-1}^{(t)}, \hat{s}_{i}^{(t-1)}, \ldots, \hat{s}_{2 K}^{(t-1)}\right]^{T}
$$

Compared to (12), the calculation of (18) performs less operations while achieves same BER performance [14].

4.1.1 MS in coordinate update process

The coordinate update process of aMGS is defined by:

$$
\hat{s}_{i}^{(t)}=\frac{1}{L_{\mathrm{e}}} \sum_{m=1}^{L_{\mathrm{e}}} \rho_{m, i}\left(\hat{\mathbf{s}}_{i}^{(t)}, \mathbf{y}, \mathbf{H}\right),
$$

where $L_{e}$ is the number of samples (realizations), and the random variable (r.v.) $\rho_{m, i}$ is a mixture of two r.v. with weight given by the mixing ratio $q$, defined by:

$$
\rho_{m, i}\left(\hat{\mathbf{s}}_{i}^{(t)}, \mathbf{y}, \mathbf{H}\right) \sim(1-q) \cdot \xi\left(\hat{\mathbf{s}}_{i}^{(t)}, \mathbf{y}, \mathbf{H}\right)+q \cdot \psi(\infty)
$$

It is important to note that, being (18) a deterministic function, during the $L_{e}$ realizations on each coordinate, (18) is calculated only once, when $m=1$. After that, each $m$ realization has the computational cost of generating a random number (relative to the mixing ratio).

At the end of algorithm iterations, the vector with the lowest cost is assumed the best global solution. Due to the mean operation, a slicer for $M$-QAM constellation is needed at the end of the detection procedure. Thus,

$$
\hat{\mathbf{s}}_{\text {best }}=\operatorname{slicer}\left(\hat{\mathbf{s}}_{\mathrm{f}-\text { best }}\right) \text {, }
$$

where $\hat{\mathbf{s}}_{\mathbf{f} \text {-best }}$ is the "floating-best" solution which represents the estimated vector related to the best global cost attained after $\mathcal{I}$ iterations, and $\hat{\mathbf{s}}_{\text {best }}$ is the final estimated symbol vector. A pseudocode for the aMGS is described in Algorithm 2.

\subsection{Approach \#2: Simplified MGS with Neighborhood Limitation LS-MIMO detector}

We propose an different approach which is based on a neighborhood limitation of distance $d$ in the random solution and is named $d$-sMGS LS-MIMO detector. The term simplified refers to the simplified target function of Eq. 18, which is also employed in this scheme.

The proposed $d$-sMGS detector acts in the symbol constellation performing a NL, with distance $d$ in relation to the symbol estimated in the previous iteration, when sorting the random symbol. This procedure showed to significantly improves the convergence when a modulation of high-order is considered, as disposed in section 6 , and presents the lowest per-symbol complexity among MGS and aMGS, since it considers the simplified target function (overcoming the MGS in mathematical operations) and performs a single sample (overcoming the multiple sampling aMGS), as showed in section 5 .

\subsubsection{NL in coordinate update process}

The $d$-sMGS coordinate update process is based on a mixture between the simplified target function, Eq. 18 , and a limited random solution. Thus, the estimated symbol in the $t$-iteration at the $i$-th coordinate is given by:

$$
\hat{s}_{i}^{(t)}=\chi_{i}\left(\hat{\mathbf{s}}_{i}^{(t)}, \mathbf{y}, \mathbf{H}\right),
$$

where $\chi_{i}(\cdot)$ is the mixed r.v. with weight $q$, defined by:

$$
\chi_{i}\left(\hat{\mathbf{s}}_{i}^{(t)}, \mathbf{y}, \mathbf{H}\right) \sim(1-q) \cdot \xi\left(\hat{\mathbf{s}}_{i}^{(t)}, \mathbf{y}, \mathbf{H}\right)+q \cdot v\left(\hat{s}_{i}^{(t-1)}, d\right)
$$




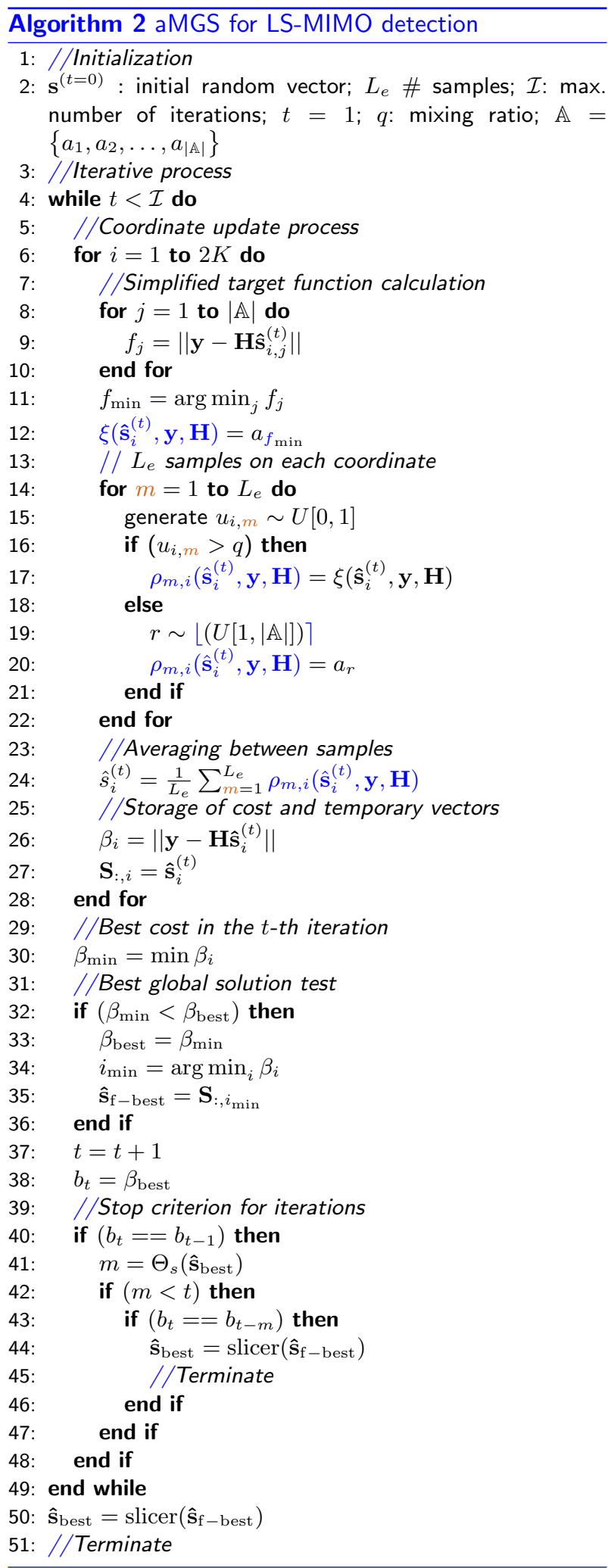

the r.v. $v\left(\hat{s}_{i}^{(t-1)}, d\right)$ denotes an uniform sorted symbol in the constellation neighborhood of $\hat{s}_{i}^{(t-1)}$, with distance $d$.

In this algorithm, the neighborhood of the current solution $\hat{s}_{i}^{(t-1)}$ is defined as

$$
\mathcal{N}\left(\hat{s}_{i}^{(t-1)}, d\right)=\left\{s^{\prime} \in \mathbb{A} \mid \kappa_{d}\left(\hat{s}_{i}^{(t-1)}, s^{\prime}\right) \leq d\right\}
$$

where $\kappa_{d}$ is the symbol distance function in the real-valued constellation considered, for example, let $\mathbb{A}=\{-7,-5,-3,-1,+1,+3,+5,+7\}, \hat{s}_{i}^{(t-1)}=-3$ and $s^{\prime}=+1$, then the symbol distance function results in $\kappa_{d}\left(\hat{s}_{i}^{(t-1)}, s^{\prime}\right)=2$.

Thus, the r.v. $v\left(\hat{s}_{i}^{(t-1)}, d\right)$ samples from a discrete uniform distribution on the set $\mathcal{N}\left(\hat{s}_{i}^{(t-1)}, d\right)=$ $\left\{n_{1}, \ldots, n_{|\mathcal{N}|}\right\}$.

A pseudocode for the proposed $d$-sMGS is described in Algorithm 3. The multiple restarts additional strategy is omitted, since it simply restarts the algorithm with another initial solution.

\section{Computational Complexity}

The computational complexity is described in terms of real number of operations (rops), in which one rop denotes the computational complexity of the real mathematical operations: addition, subtraction, multiplication or division. For the exponential and logarithmic functions, an approximation through Taylor Series with 18 terms has been considered to calculate the computational complexity. Table 1 describes the per-symbol computational complexity $\left(\mathcal{C}_{T}\right)$ involved in each step of $d$-sMGS algorithm. Additionally, the total per-symbol complexity of the aMGS and the conventional MGS has been evaluated. The per-symbol complexity of the initial solution is denoted by $\mathcal{C}_{I}$, which is adopted in this work as the output of an MMSE detector, which has also its total complexity described in the Table 1 [22]. From Table 1, one can notice that the $d$-sMGS algorithm, aMGS and MGS algorithms have the same asymptotic per-symbol complexity order of $\mathcal{O}\left(K^{2}\right)$, although the conventional MGS algorithm may require an additional complexity dependent on constellation size due to the exponential function, which is represented by the cardinality $|\mathbb{A}|$. On the other hand, the additional complexity due to the averaged strategy of the aMGS represents a negligible impact, since it requires only $\left(2 L_{e}+2\right)$ rops per iteration, whereas such additional complexity is not dependent on the problem size. The proposed $d$-sMGS algorithm combines advantages of both by using a single sample such as the MGS and the simplified aMGS 


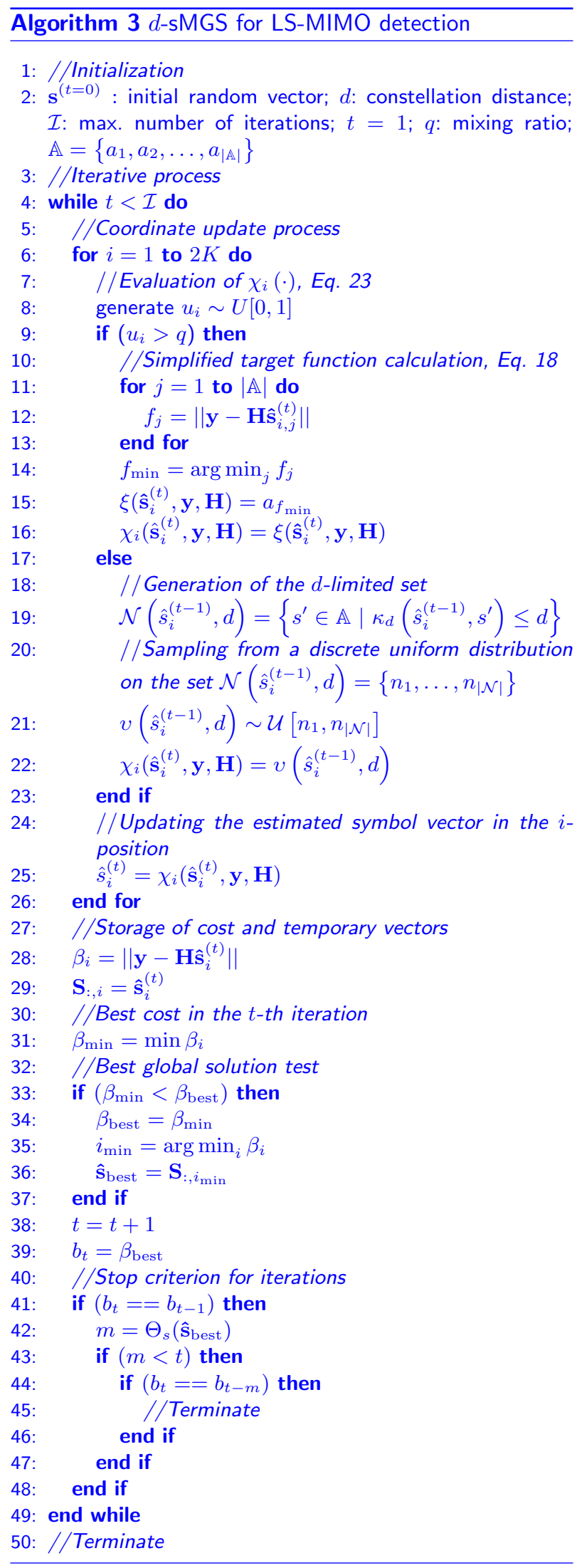

target function. The complexity increment given by the neighborhood constraint is considered negligible, since the symbol is already previously estimated and such procedure represents only a random sampling in a restricted vector.

From Table 1, it may be noted that the proposed $d$-sMGS has its per-symbol complexity independent of the parameter $d$, so the use of larger neighborhoods in the random symbol generation has no impact on complexity. With respect to the per-symbol complexity of the initial solution, $\mathcal{C}_{I}$, in this work we adopted the output of an MMSE detector, which has also its total complexity described in the Table 1.

It is important to emphasise that the complexity of the $d$-sMGS, aMGS and MGS algorithms is defined by the number of iterations, which is controlled by the stopping criterion $\Theta_{s}$, with the upper limit $\mathcal{I}$. Similarly, the amount of restarts is controlled by $\Theta_{r}$, with an upper limit $R_{\max }$. In terms of complexity, the MR procedure can be interpreted as an extra amount of iterations necessary for each new restart. In this sense, an $\mathcal{I}_{\text {eff }}$ is considered in Table 1, which denotes the total amount of iterations (including all restarts) performed at each symbol period. Since Monte-Carlo method is employed in simulations, in section 6 a mean value of $\mathcal{I}_{\text {eff }}$ considering all realisations is evaluated and is called effective number of iterations (ENI):

$$
\mathrm{ENI}=\frac{1}{T} \sum_{i=1}^{T} \mathcal{I}_{\mathrm{eff}, i}
$$

where $T$ denotes the total number of realisations (symbol periods) during the Monte-Carlo method simulation and $\mathcal{I}_{\text {eff }, i}$ denotes the $\mathcal{I}_{\text {eff }}$ in the $i$-realisation.

\subsection{Quality metric}

Due to the large number of parameters involved in the presented LS-MIMO detectors, a simple performancecomplexity tradeoff metric is considered [14], which aims to establish a fair comparison analysis between different detection strategies:

$$
\chi\left(\mathrm{BER}, \mathcal{C}_{T}\right)=-\frac{10 \log _{10}(\mathrm{BER})}{10^{-8} \cdot \mathcal{C}_{T}}=-\frac{\mathrm{BER}_{\mathrm{dB}}}{10^{-8} \cdot \mathcal{C}_{T}}
$$

where $\mathrm{BER}_{\mathrm{dB}}$ denotes the bit error rate in $\mathrm{dB}$. Higher values of $\chi(\cdot)$ imply more efficient and effective LSMIMO detector.

\section{Numerical results and discussion}

In this section the uncoded BER performance related to the $d$-sMGS algorithm for LS-MIMO detection is 
evaluated through Monte Carlo simulations. The simulations are performed for a large-scale MIMO operating in multiplexing mode and assuming that a perfect channel state information is available at the receiver side. Table 2 summarizes the main system and channel parameter values deployed in this section.

As proposed in [10], the mixing ratio parameter is adopted as the inverse of the number of dimensions in the system, i.e., $q=\frac{1}{2 K}$. For the stopping criterion parameters, we have adopted $c_{1}=10, c_{2}=1.0$, and $c_{\min }=10[14]$.

This numerical simulation section has been divided into two main parts: in subsection 6.1 the mixing ratio $q$ and number of samples $L_{e}$ parameters of the aMGS detector are discussed, as it denotes a technique that also aims at reducing the impact of the noisy solution; in subsection 6.2 , we present numerical results of performance and computational complexity of the proposed $d$-sMGS detector against the aMGS and MGS techniques, addressed in this work.

\section{1 aMGS parameters discussion}

The aMGS-MR BER performance for different mixing ratios $q=\{1 / 2 K, 1 / 3 K, 1 / 4 K\}$, considering $R_{\max }=$ $\{1,5,10\}$, is presented in Fig. 3 for each fixed $L_{e} \in$ $\{1,2,4,8\}$ samples scenario [14]. The number of users is equal to $K=96$ while $N=128 \mathrm{BS}$ antennas ( $\beta=0.75)$. The system is operating under mediumhigh SNR, $\gamma_{\mathrm{dB}}=25 \mathrm{~dB}$. First, it is evident that the choice of different mixing ratio values impact both performance and complexity (represented by the ENI quantity at convergence). In addition, one can notice that the large amount of $L_{e}=8$ samples becomes harmful to the algorithm, once convergence is achieved with larger ENI. Among the other results, the best performance-complexity tradeoff is presented with $L_{e}=2$ samples and $q=1 / 4 K$, which results in: $\left.\chi\right|_{L_{e}=2}=44.89 ;$ against $\left.\chi\right|_{L_{e}=4}=37.85$ with 4 samples and $q=1 / 2 K$; and $\left.\chi\right|_{L_{e}=1}=39.66$ with 1 sample and $q=1 / 4 K$. A detailed analysis of the aMGS performance/complexity gain in relation to the mixing ratio and the number of samples can be found in [14].

It can also be concluded that with increasing number of samples $L_{e}$, the curve represented by $q=1 / 2 K$ has its convergence improved, resulting in less complexity. That is, when the impact of the noisy solution is reduced, the choice of $q=1 / 2 K$ is presented as the best performance-complexity tradeoff. In this sense, the value $q=1 / 2 K$ is adopted for the proposed detector $d$-sMGS.

Through the analysis performed in [14], the parameter values summarized in Table 3 have been adopted for the aMGS in the reminder of this work. For the MGS-R, the following parameters have been adopted: $q=1 / 2 K, \mathcal{I}=8 K \sqrt{M}, R_{\max }=50, c_{1}=10$ and $c_{2}=0.5[10]$.

In Fig. 4 the convergence of the aMGS algorithm adopting best $q$ values, from Table 3 , is analysed against the average rops complexity, with $96 \times 128$ antennas and 64-QAM [14]. For comparison purpose, a single sampling result using the optimal mixing ratio value as proposed in [10], i.e., $q=1 / 2 K$ (curve [E]) is also included. One can notice that a less number of samples has shown to be beneficial in this LS-MIMO scenario, since the single sample case presented the best performance combined to the lowest asymptotic complexity, followed by the two $\left(L_{e}=2\right)$ and fourfold $\left(L_{e}=4\right)$ sampling case. Nevertheless, due to a slightly convergence gain observed with $L_{e}=2$ samples, the tradeoff metric for $L_{e}=1$ is found to be $\left.\chi\right|_{L_{e}=1}=39.83$ against $\left.\chi\right|_{L_{e}=2}=44.22$ with $L_{e}=2$ samples. A detailed analysis of the aMGS performance/complexity gain in relation to the mixing ratio and the number of samples can be found in [14]. An indepth analysis of the performance-complexity tradeoff of aMGS can be found in [14].

\subsection{Analysis on the proposed $d$-sMGS}

First of all, we focus on finding the maximum number of iterations $\mathcal{I}$ aiming at maximizing tradeoff performance $x$ complexity. In the literature, the quantity $\mathcal{I}=8 K \sqrt{M}$ adopted in [10] is quite reasonable since it takes into account the number of active users and the modulation order. In this sense, Figure 5 shows the performance convergence of the proposed algorithm with the increase of the maximum number of iterations. We considered $K=N=16$ antennas in 64QAM with NL distance $d=\{1,2,3\}$ and used the parameter $a$ to denote the maximum number of iterations, so that $\mathcal{I}=a K \sqrt{M}=128 a$. It can be clearly seen that the increase in the NL distance is not beneficial to the algorithm's performance, which is easily explained by the fact that, with increasing $d$, the neighborhood of the random solution increases, approaching the condition of unrestricted solution in the constellation, retaking its negative impact on the algorithm's convergence. Thus, observing the 1-sMGS curve, it can be seen that its convergence is reached with $a$ equal to 8 , which coincides with the result adopted in [10]. Therefore, this value $\mathcal{I}=8 K \sqrt{M}$ will be adopted for the proposed $d$-sMGS detector in the reminder of this work.

The Fig. 6 shows the SNR vs. performance/computational complexity of the addressed detectors. A high system loading, i.e., $\beta \approx 0.9$, in 64-QAM modulation is adopted with: a) $K=58, N=64$ and b) $K=87$, $N=96$ antennas. The parameters used for the MGSMR and aMGS-MR detectors follow in their respective 
works: for the MGS-MR $\mathcal{I}=8 K \sqrt{M}$ and $R_{\max }=50$ [10]; for the aMGS-MR $\mathcal{I}=3000, R_{\max }=5$ and the choice of the mixing ratio value is given according to the best option criterion published by the author [14]. One can notice in Fig. 6. a that both proposed detectors presented significant performance gain in the region of high SNR in relation to the other detectors, equivalent to approximately one decade against the second best performance detector aMGS-MR with $L_{e}=8$ samples. Differently from that observed previously, the increase in the NL distance did not cause a loss of performance, since the 2-sMGS detector resulted in a marginally similar performance to the 1-sMGS. Thus, it denotes a tendency that the increase of the NL distance can be beneficial in scenarios with greater number of antennas, such as LS-MIMO. Related to the computational complexity, it can be observed that the complexity of the 1-sMGS, 2-sMGS and aMGS detectors with $L_{e}=2,4$ and 8 samples are marginally equivalent, although the aMGS with 8 samples presented the least number of rops (excluding the linear MMSE detector). Considering that both $d$-sMGS and aMGS have marginally the same complexity per iteration, it is shown that the strategy of multiple samples converged with fewer iterations, on the other hand, with inferior performance to that reached by $d$-sMGS.

With increasing antenna numbers, Fig. 6.b, it is reiterated the hypothesis that the increase of the NL distance results in a performance gain. One can notice a significant performance gain in the 4 -sample aMGS detector, surpassing the result with $L_{e}=8$, which corroborates the hypothesis that a smaller restriction in the noisy solution becomes beneficial with the increase in the number of antennas. In fact, in the region of high $\mathrm{SNR}, \gamma_{\mathrm{dB}}=25 \mathrm{~dB}$, it can be seen that the 2-sMGS and aMGS with $L_{e}=4$ achieve similar performance, although in the medium SNR region $\left(\gamma_{\mathrm{dB}}=23 \mathrm{~dB}\right)$, the proposed $d$-sMGS still appear superior. With respect to the complexity in terms of rops, it is noticed that the 2-sMGS-MR and aMGS-MR detectors with $L_{e}=4$ and 8 samples presented a marginally equal complexity in $\gamma_{\mathrm{dB}}=25 \mathrm{~dB}$; however, the least complexity is again reached by the aMGS, specially in medium SNR region $\left(\gamma_{\mathrm{dB}}=[21,23] \mathrm{dB}\right)$. Therefore, it can be concluded that the proposed $d$-sMGS detection technique presented the best performance in both scenarios, and the smaller restriction of neighborhood with $d=2$ was a more interesting choice with increasing number of antennas, in addition, there was no significant increase of complexity compared to the multiple sample detector aMGS, in other words, the complexity of the 2-sMGS detector was marginally equal to the lowest complexity techniques: aMGS with $L_{e}=4$ and 8 samples.

A system loading analysis against BER and rops complexity is depicted in Fig. 7 under $\gamma_{\mathrm{dB}}=25 \mathrm{~dB}$. It may be first noted that at high loading, i.e., $\beta \approx 0.9$, the proposed detection scheme showed a significant gain in performance over the aMGS. In the other regions, there is no clearly outstanding technique, however, a lower restriction in the noisy solution demonstrated better results, which are represented by the 2sMGS overpassing the 1-sMGS and aMGS with $L_{e}=1$ or 2 in front of the $L_{e}=4$ and 8 samples. In relation to the computational complexity with $N=64$ antennas (Fig. 7.b), one can notice that in the mediumhigh loading region $(\beta \geq 0.75)$, the proposed $d$-sMGS strategy presented less complexity both with respect to multiple sampling aMGS and conventional MGS. In the medium-low system loading results $(\beta \leq 0.5)$, multiple sampling schemes presented lower computational complexity. Therefore, one can highlight the superiority of the proposed strategy in both performance and complexity in medium-high loading configurations, demonstrating the potential of this strategy when the LS-MIMO system operates under high loading crowded scenarios. This can be explained as the number of mobile users increases, approaching the full-loading system condition $\beta \rightarrow 1$, the set of possible symbol combinations becomes larger, such that the noisy solution from the mixture has its negative effect aggravated, affecting the algorithm's convergence; whereas the NL strategy is able to mitigate this effect, having a beneficial effect on the convergence which results in improvement in performance and complexity reduction.

With the increasing number of antennas at $N=128$, the system loading analysis reflects a clear superiority of the 2-sMGS detector in high loading configurations, both in performance and in complexity. This performance behavior corroborates the hypotheses raised in Fig. 6 regarding performance improvement with increasing NL distance. On the other hand, in mediumlow loading, the complexity of 2-sMGS was shown to be greater than aMGS and 1-sMGS, equating only to the conventional MGS-MR.

\section{Conclusions}

A neighborhood limited $d$-sMGS detector for largescale MIMO systems has been proposed based on the neighborhood constraint of the noisy solution at a distance of $d$.

The proposed LS-MIMO $d$-sMGS detection scheme demonstrated the ability to mitigate the impact caused by the noisy solution from the mixture, which is aggravated and can become harmful when the full system loading condition is present or when a high order modulation is implemented.

The modifications in the MGS technique proposed here have demonstrated effectiveness in achieving convergence improvements in the detection algorithm, 
which resulted in significant gains in performance and complexity compared to both the multiple sampling aMGS technique as well as the conventional MGS. These advantages are especially obtained when the system loading is high and there are a large number of antennas, condition favorable to LS-MIMO. Moreover, with increasing the number of dimensions, i.e., increasing number of antennas and/or modulation order, a smaller restriction of 2 -sMGS was shown to be a more interesting choice than 1-sMGS.

In addition, the NL strategy represented less complexity per iteration compared to aMGS or MGS, since only one sample is calculated and the simplified objective function is considered. On the other hand, when a low system loading is considered, the NL strategy resulted in a slight increase in complexity.

\section{Abbreviations}

aMGS: averaged MGS; BS: base station; BER: bit error rate; $d$-sMGS: $d$-simplified MGS; ENI: effective number of iterations; LS-MIMO: large-scale multiple-input multiple-output; MCMC: Markov chain Monte Carlo; MMSE: minimum mean square error; MGS: mixed Gibbs sampling; MR: multiple restart; MS: multiple sampling; NL: neighborhood limitation; SNR: signal-to-noise ratio.

\section{Acknowledgements}

We gratefully acknowledge the agencies: National Council for Scientific and Technological Development (CNPq) of Brazil, the University of São Paulo (USP), the State University of Londrina (UEL), the Federal Institute of Paraná (IFPR) and the Paraná State Government.

\section{Funding}

This work has been partially supported by the CNPq of Brazil under Grants $304066 / 2015-0$, by the USP, by the UEL, by the IFPR and the Paraná State Government.

\section{Availability of data and materials}

Not available online. Please contact author for data requests.

\section{Author's contributions}

Propositon of aMGS LS-MIMO detector, which is based on multiple sampling during coordinate update process. Simplification on target function from original MGS. Numerical analysis of mixing ratio.

\section{Competing interests}

The authors declare that they have no competing interests.

\section{Ethics approval and consent to participate}

Not applicable.

\section{Consent for publication}

Not applicable.

\section{Author details}

${ }^{1}$ Federal Institute of Paraná, Cívica Avenue 475, 85935-000 Assis Chateaubriand, Brazil. ${ }^{2}$ Electrical Engineering, Polytechnic School, University of São Paulo, Professor Luciano Gualberto Avenue 158, 05508-010 São Paulo, Brazil. ${ }^{3}$ Electrical Engineering Department, State University of Londrina, Rod. Celso Garcia Cid - PR445, 86057-970 Londrina, Brazil.

\section{References}

1. J. Hoydis, S. ten Brink and M. Debbah, "Massive MIMO in the UL/DL of cellular networks: how many antennas do we need?," in IEEE Journal on Selected Areas in Communications, vol. 31, no. 2, pp. 160-171, February 2013.

2. F. Rusek, D. Persson, B. K. Lau, E. G. Larsson, T. L. Marzetta, O. Edfors and F. Tufvesson, "Scaling up MIMO: opportunities and challenges with very large arrays," in IEEE Signal Processing Magazine, vol. 30, no. 1, pp. 40-60, January 2013.
3. K. V. Vardhan, S. K. Mohammed, A. Chockalingam and B. S. Rajan, "A low-complexity detector for large MIMO systems and multicarrier CDMA systems," IEEE Journal on Selected Areas in Communications, vol. 26, no. 3, pp. 473-485, April 2008.

4. N. Srinidhi, S. K. Mohammed, A. Chockalingam and B. Sundar Rajan, "Low-complexity near-ML decoding of large non-orthogonal STBCs using reactive tabu search," 2009 IEEE International Symposium on Information Theory, Seoul, 2009, pp. 1993-1997.

5. P. Som, T. Datta, A. Chockalingam and B. S. Rajan, "Improved large-MIMO detection based on damped belief propagation," 2010 IEEE Information Theory Workshop on Information Theory (ITW 2010, Cairo), Cairo, 2010, pp. 1-5

6. M. Suneel, P. Som, A. Chockalingam and B. Sundar Rajan, "Belief propagation based decoding of large non-orthogonal STBCs," 2009 IEEE International Symposium on Information Theory, Seoul, 2009, pp. 2003-2007.

7. C. Tang, C. Liu, L. Yuan and Z. Xing, "High precision low complexity matrix inversion based on newton iteration for data detection in the massive MIMO," IEEE Communications Letters, vol. 20, no. 3, pp. 490-493, March 2016.

8. A. Thanos and V. Paliouras, "Hardware trade-offs for massive MIMO uplink detection based on Newton iteration method," 2017 6th International Conference on Modern Circuits and Systems Technologies (MOCAST), Thessaloniki, 2017, pp. 1-4

9. L. Martino, V. Elvira, and G. Camps-Valls, "The recycling gibbs sampler for efficient learning," Digital Signal Processing, vol. 74, pp. 1-13, 2018.

10. T. Datta, N. A. Kumar, A. Chockalingam and B. S. Rajan, "A novel Monte-Carlo-sampling-based receiver for large-scale uplink multiuser MIMO systems," IEEE Transactions on Vehicular Technology vol. 62, no. 7, pp. 3019-3038, Sept. 2013.

11. J. Choi, "An MCMC-MIMO detector as a stochastic linear system solver using successive overrelexation," IEEE Transactions on Wireless Communications, vol. 15, no. 2, pp. 1445-1455, Feb. 2016.

12. Rong Chen, J. S. Liu and Xiaodong Wang, "Convergence analyses and comparisons of Markov chain Monte Carlo algorithms in digital communications," IEEE Transactions on Signal Processing, vol. 50, no. 2, pp. 255-270, Feb. 2002.

13. B. Farhang-Boroujeny, Haidong Zhu and Zhenning Shi, "Markov chain Monte Carlo algorithms for CDMA and MIMO communication systems," IEEE Transactions on Signal Processing, vol. 54, no. 5, pp. 1896-1909, May 2006.

14. A. M. Mussi, T. Abrão, "Multiple restarts mixed Gibbs sampling detector for large-scale antenna systems", IET Signal Processing, Nov. 2018. DOI: 10.1049/iet-spr.2018.5206.

15. B. Hassibi, M. Hansen, A. G. Dimakis, H. A. J. Alshamary and W. Xu, "Optimized Markov chain Monte Carlo for signal detection in MIMO systems: an analysis of the stationary distribution and mixing time," IEEE Transactions on Signal Processing, vol. 62, no. 17, pp. 4436-4450, Sept. 2014

16. Y. Yang, H. Peng, D. Zhang and X. Dai, "Markov chain Monte Carlo-based separation of paired carrier multiple access signals," IEEE Communications Letters, vol. 20, no. 11, pp. 2209-2212, Nov. 2016.

17. M. Mandloi and V. Bhatia, "Layered Gibbs sampling algorithm for near-optimal detection in large-MIMO systems," 2017 IEEE Wireless Communications and Networking Conference (WCNC), San Francisco, CA, 2017, pp. 1-6.

18. C. Gao, J. Xu, X. Tao and Z. Qin, "An improved mixed Gibbs sampling algorithm based on multiple random parallel Markov chains for massive MIMO systems," 2016 IEEE 27th Annual International Symposium on Personal, Indoor, and Mobile Radio Communications (PIMRC), Valencia, 2016, pp. 1-5.

19. A. Chockalingam and B. Rajan, Large MIMO Systems. New York, NY, USA: Cambridge Univ. Press, 2014.

20. M. Hansen, B. Hassibi, A. G. Dimakis and W. Xu, "Near-optimal detection in MIMO systems using Gibbs sampling," GLOBECOM 2009 - 2009 IEEE Global Telecommunications Conference, Honolulu, HI, 2009, pp. 1-6.

21. M. O. Damen, H. El Gamal and G. Caire, "On maximum-likelihood detection and the search for the closest lattice point," IEEE Transactions on Information Theory, vol. 49, no. 10, pp. 2389-2402, 


$$
\text { Oct. } 2003 .
$$

22. T. Liu, "Some results for the fast MMSE-SIC detection in spatially multiplexed MIMO systems," IEEE Transactions on Wireless Communications, vol. 8, no. 11, pp. 5443-5448, Nov 2009.

\section{Figures}

Figure 1 Single-cell uplink LS-MIMO communication system with $K$ single-antenna unit equipments (UEs) and a base-station (BS) with $N$ receive antennas.

Tables 
Figure 2 A brief description of the coordinate update process on the aMGS and the proposed $d$-sMGS detectors.

Figure 3 BER performance convergence of different mixing ratios, $q$, of aMGS in medium number of antennas scenario $(K=96$, $N=128)$ at $\gamma_{\mathrm{dB}}=25 \mathrm{~dB}, 64-\mathrm{QAM}, R_{\max }=\{1,5,10\}, \mathcal{I}=6000$ and different $L_{e}$ samples: a) $\left.L_{e}=1, \mathrm{~b}\right) L_{e}=2$, c) $L_{e}=4$ and d) $L_{e}=8$. [14]

Table 1 Per-symbol Computational Complexity of aMGS, conventional MGS and MMSE Algorithms.

\begin{tabular}{|c|c|c|}
\hline Procedure & Step & Complexity \\
\hline \multicolumn{3}{|c|}{$d$-MGS - Algorithm 3} \\
\hline Target function calculation & lines $11-16$ & $16 K N-4 N+|\mathbb{A}|(16 N+2)$ \\
\hline Generation of the $d$-limited set & line 19 & negligible \\
\hline Cost computation at each coordinate & line 28 & $20 N$ \\
\hline$\Theta_{s}, \quad$ Eq. $(16)$ & line 41 & $\frac{24}{K}$ \\
\hline \multicolumn{3}{|c|}{$\mathcal{C}_{T}=\mathcal{C}_{I}+\mathcal{I}_{\text {eff }}\left[16 K N+16 N+|\mathbb{A}|(16 N+2)+\frac{24}{K}\right]$} \\
\hline \multicolumn{3}{|c|}{ aMGS - Algorithm 2} \\
\hline Target function calculation & lines $8-12$ & $16 K N-4 N+|\mathbb{A}|(16 N+2)$ \\
\hline Averaging between samples & line 24 & $2 L_{e}+2$ \\
\hline Cost computation at each coordinate & line 26 & $20 N$ \\
\hline$\Theta_{s}, \quad$ Eq. (16) & line 41 & $\frac{24}{K}$ \\
\hline Total per-symbol complexity: & \multicolumn{2}{|c|}{$\mathcal{C}_{T}=\mathcal{C}_{I}+\mathcal{I}_{\text {eff }}\left[16 K N+16 N+|\mathbb{A}|(16 N+2)+\left(2 L_{e}+2\right)+\frac{24}{K}\right]$} \\
\hline \multicolumn{3}{|c|}{ MGS - Target distribution function calculation on Algorithm 1} \\
\hline Target distribution function calculation & lines $4-6$ & $16 K N-4 N+|\mathbb{A}|(16 N+12)$ \\
\hline Evaluation of each symbol probability & lines $8-12$ & $1238|\mathbb{A}|$ \\
\hline Cost computation of estimated vector & & $\frac{10 N}{K}$ \\
\hline$\Theta_{s}, \quad$ Eq. (16) & & $\frac{24}{K}$ \\
\hline Total per-symbol complexity: & $\mathcal{C}_{T}=\mathcal{C}_{I}+\mathcal{I}_{\text {eff }}$ & {$\left[16 K N-4 N+|\mathbb{A}|(16 N+1450)+\frac{10 N+24}{K}\right]$} \\
\hline \multicolumn{3}{|c|}{ MMSE Algorithm } \\
\hline Total per-symbol complexity: & \multicolumn{2}{|c|}{$\mathcal{C}_{T}=\left(\frac{1}{6}\right) K^{2}+\left(\frac{3}{2}\right) N K+\left(\frac{3}{2}\right) N+\left(\frac{5}{6}\right)$} \\
\hline
\end{tabular}


Figure 4 BER performance and complexity vs. convergence for the aMGS algorithm considering 64-QAM modulation, best mixing ratio $q$ (curves $[\mathrm{A}]$ to $[\mathrm{D}]$ ) from Table 3 and optimal value as proposed in [10], i.e., $q=1 / 2 K$ and $L_{e}=1$ (curve [E]) [14].

Table 2 LS-MIMO system and channel parameters.

\begin{tabular}{lc}
\hline Parameter & Value \\
\hline \hline \multicolumn{2}{c}{$L S$-MIMO System } \\
\hline Link direction & Uplink (UL) \\
\# Rx antennas (BS) & $N \in\{64,128\}$ \\
\# Tx antennas (MTs) & $K \in\{48,96\}$ \\
(single user-antenna) & $\beta=\frac{K}{N} \in[0.3125,0.90625]$ \\
System loading & $64-\mathrm{QAM}$ \\
Modulation order & $\gamma_{\mathrm{dB}} \in[0,25] \mathrm{dB}$ \\
SNR ranges & Channel \\
\hline \multicolumn{2}{c}{ Flat Rayleigh } \\
\hline Channel type & Perfectly known at receiver \\
\hline
\end{tabular}
Specific detector parameters

Max. number of iterations $\mathcal{I}=8 K \sqrt{M}$

Max. number of restarts $\quad R_{\max }=20$

NL distance $\quad d \in\{1,2,3\}$

Mixing ratio $\quad q=\frac{1}{2 K}$

Stop criterion parameters $\quad c_{1}=10 ; c_{2}=1 ; c_{\min }=10$

Table 3 Best parameters for aMGS-MR detector presented in [14]

\begin{tabular}{r|r|cccc}
\hline & \multicolumn{5}{|c}{ \# aMGS samples, $L_{e}$} \\
Parameter & BS antennas & $\mathbf{1}$ & $\mathbf{2}$ & $\mathbf{4}$ & $\mathbf{8}$ \\
\hline \hline Mixing & $N<=64:$ & $\frac{1}{4 K}$ & $\frac{1}{4 K}$ & $\frac{1}{3 K}$ & $\frac{1}{2 K}$ \\
ratio, $q$ & $N>64:$ & $\frac{1}{4 K}$ & $\frac{1}{4 K}$ & $\frac{1}{2 K}$ & $\frac{1}{2 K}$ \\
\hline Max. \# Iterations, $I$ & \multicolumn{4}{|c}{3000} \\
\hline \multicolumn{2}{r|}{ Max. Restarts, $R_{\max }$} & \multicolumn{4}{|c}{5} \\
\hline Iterations stop criterion, $c_{1}$ & \multicolumn{3}{|c}{10} \\
Restarts stop criterion, $c_{2}$ & \multicolumn{4}{|c}{}
\end{tabular}

Figure 5 Performance convergence against the maximum number of iterations $\mathcal{I}$ of the proposed $d$-sMGS detector, with $16 \times 16$ antennas in 64-QAM modulation among different NL distance values. The number of iterations is related to the parameter $a$, were $\mathcal{I}=a K \sqrt{M}$.
Figure 6 SNR vs. BER performance/Average number of rops in $d$-sMGS-MR detector against aMGS-MR approach and MGS-MR. Parameters: $K=58, N=64,64-\mathrm{QAM}, \beta \approx 0.9$. 
Figure 7 Performance and complexity versus system loading, considering $\gamma_{\mathrm{dB}}=25 \mathrm{~dB}, 64$-QAM: a) Performance for $N=64$; $\mathbf{b}$ )

Performance for $N=128$ antennas; c) Average $\operatorname{rop}$ complexity for $N=64$; d) Average $\operatorname{rop}$ complexity for $N=128$. 
Appendix E - Full paper under R1 review in the journal "EURASIP Journal on Advances in Signal Processing", January 2019209

Figure 2
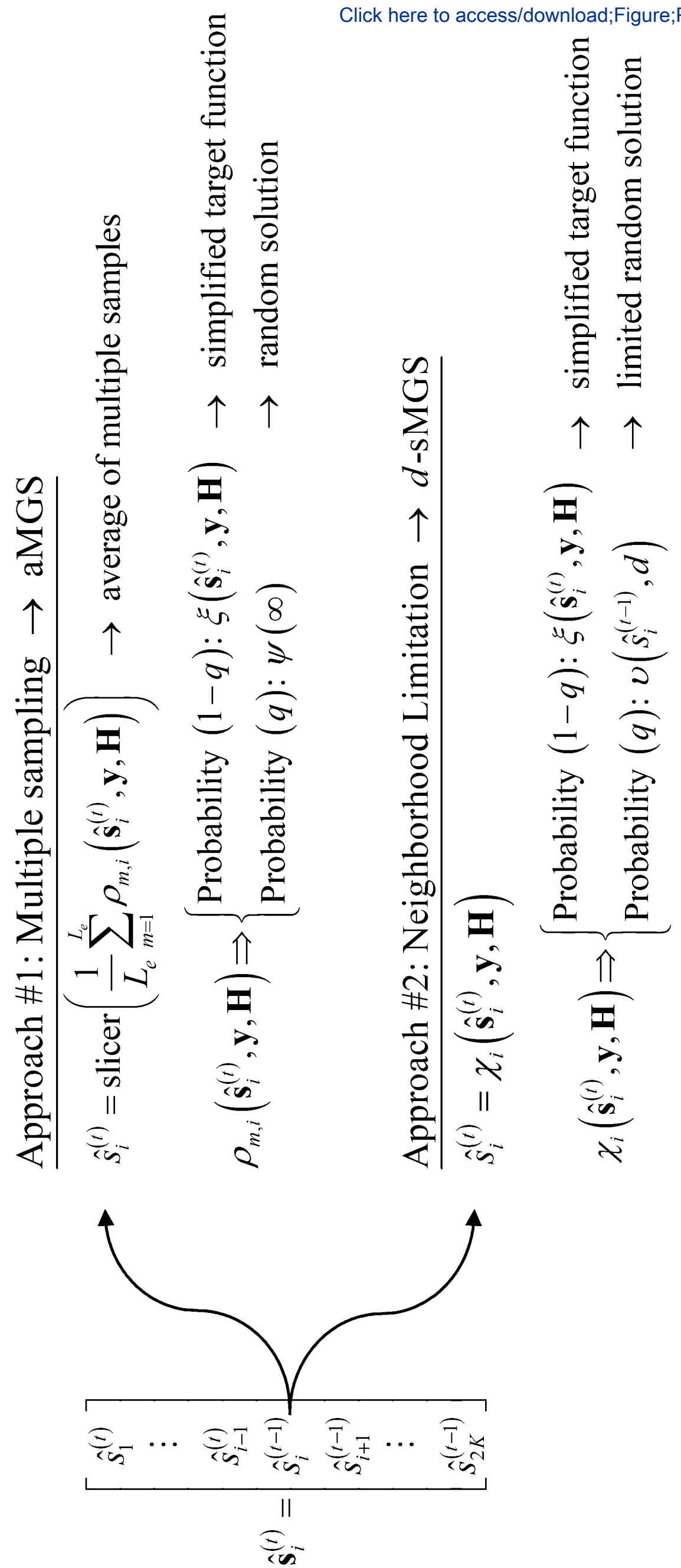


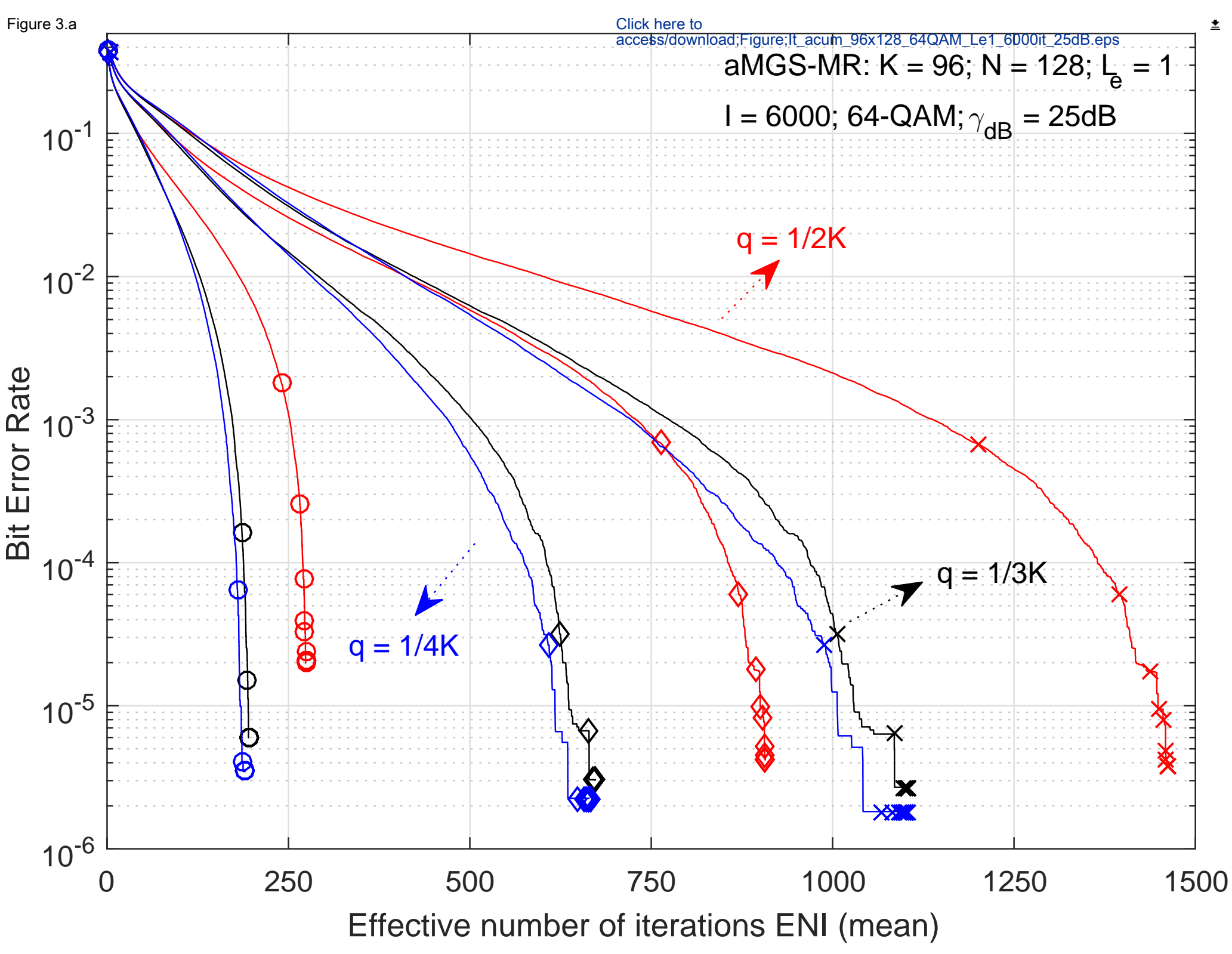




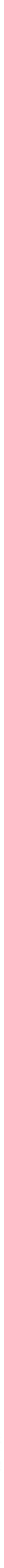




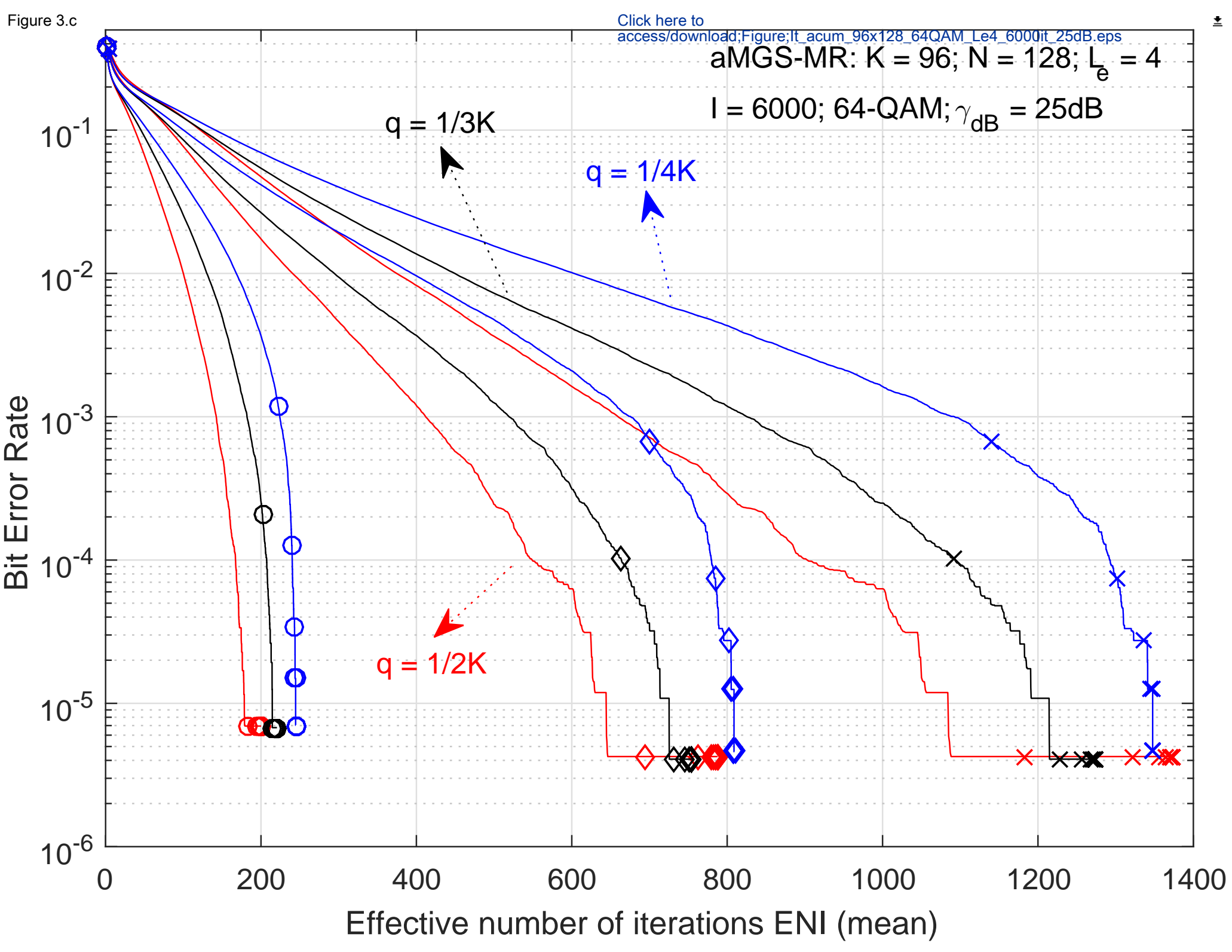




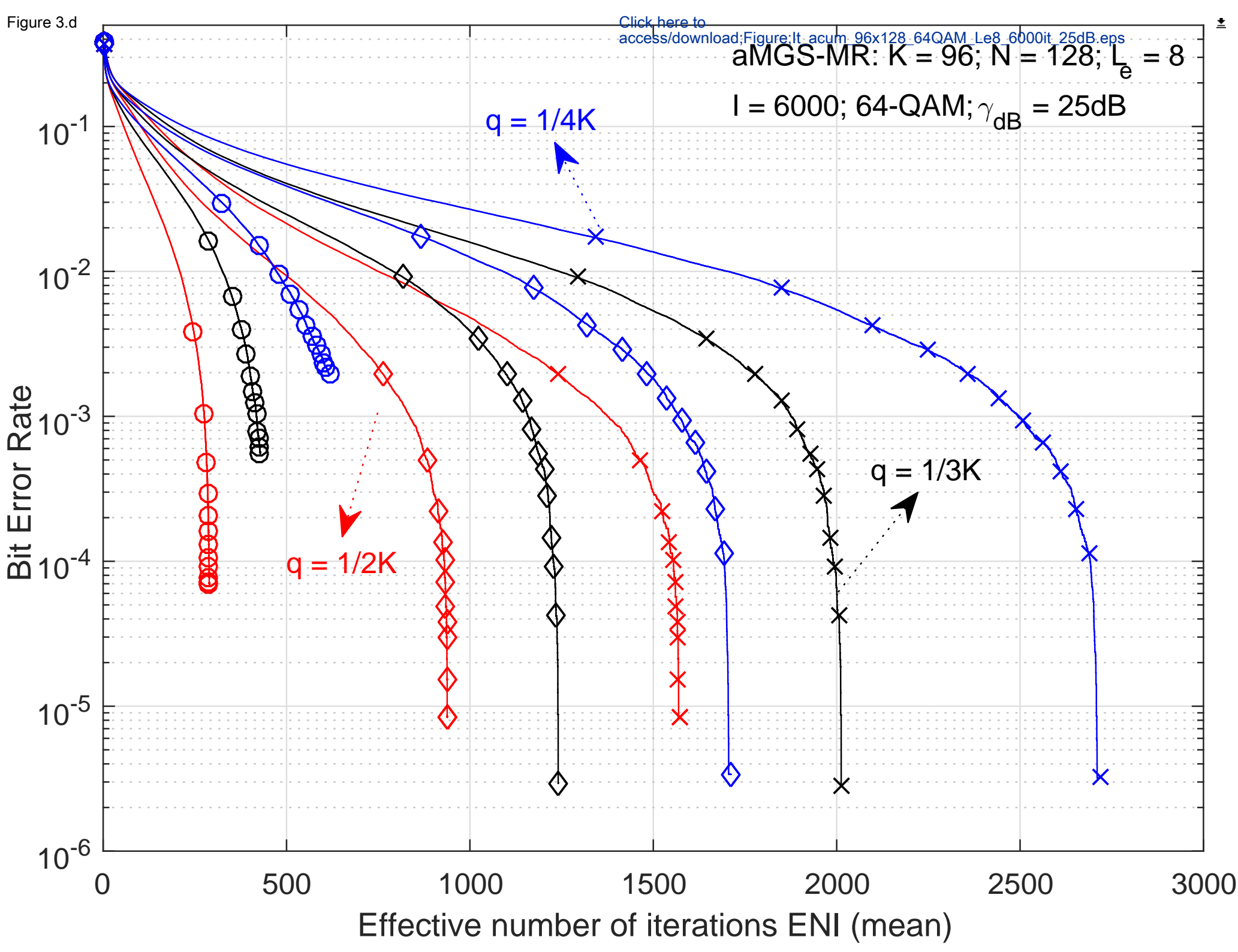




\begin{tabular}{|c|c|c|}
\hline $\begin{array}{l}-R_{\text {max }}=1 ; q=1 / 2 \mathrm{~K} \\
\checkmark-R_{\text {max }}=5 ; q=1 / 2 \mathrm{~K} \\
x-R_{\text {max }}=10 ; q=1 / 2 \mathrm{~K}\end{array}$ & $\begin{array}{l}-R_{\text {max }}=1 ; q=1 / 3 \mathrm{~K} \\
\checkmark-R_{\text {max }}=5 ; q=1 / 3 \mathrm{~K} \\
x-R_{\text {max }}=10 ; q=1 / 3 \mathrm{~K}\end{array}$ & $\begin{array}{l}-\mathrm{O} \mathrm{R}_{\max }=1 ; \mathrm{q}=1 / 4 \mathrm{~K} \\
\checkmark-\mathrm{R}_{\max }=5 ; \mathrm{q}=1 / 4 \mathrm{~K} \\
x-\mathrm{R}_{\text {max }}=10 ; \mathrm{q}=1 / 4 \mathrm{~K}\end{array}$ \\
\hline
\end{tabular}




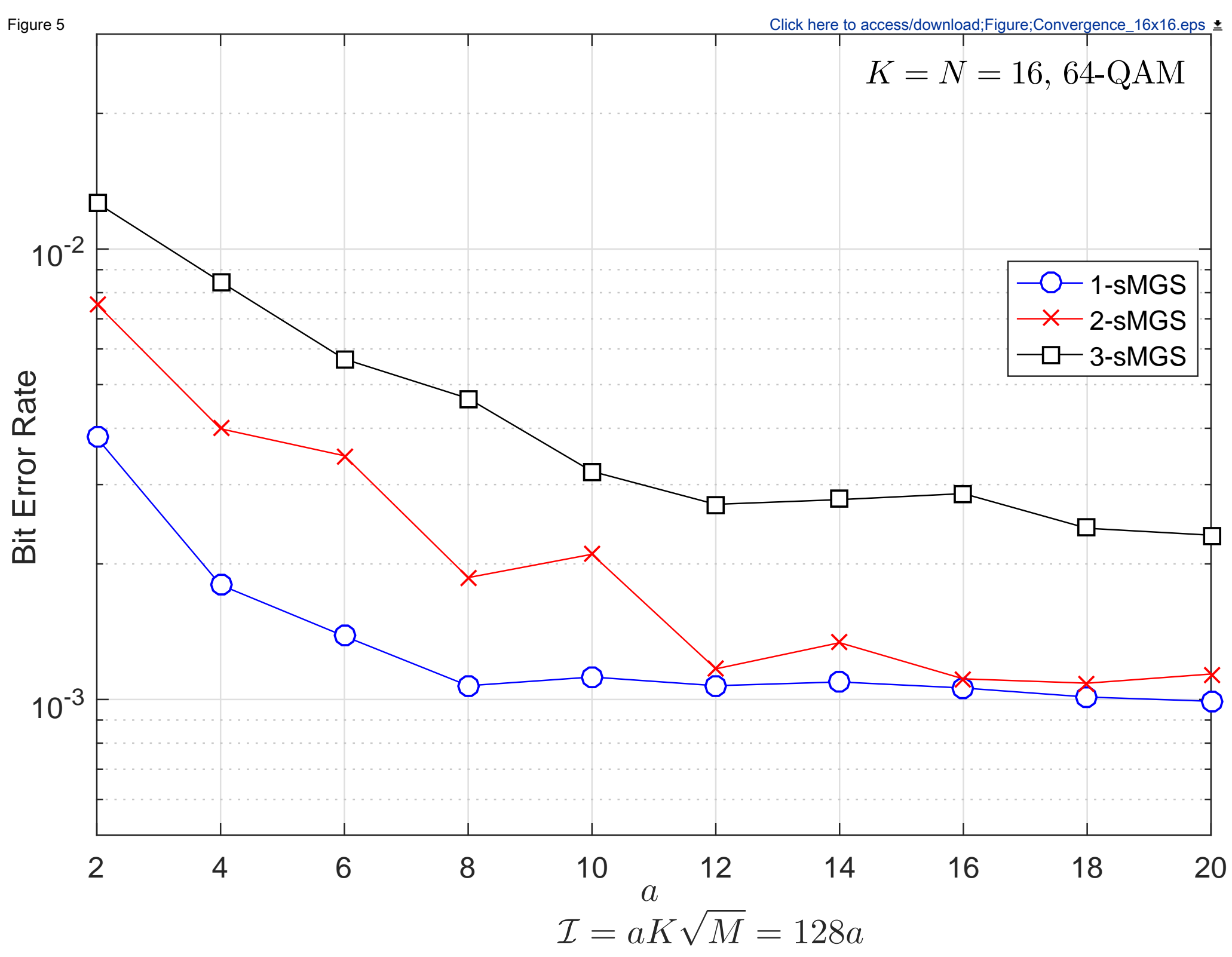




$$
\begin{aligned}
& \checkmark[\text { [A] MMSE } \\
& -\square-[B] \text { MGS-MR; } q=1 / 2 \mathrm{~K} \\
& \nabla-[C] \text { aMGS-MR } L_{e}=1 ; q=1 / 4 \mathrm{~K} \\
& \checkmark[D]_{\text {aMGS-MR L }}=2 ; q=1 / 4 \mathrm{~K} \\
& \text { [E] aMGS-MR } L_{e}=4 ; \mathrm{q}=1 / 3 \mathrm{~K}
\end{aligned}
$$

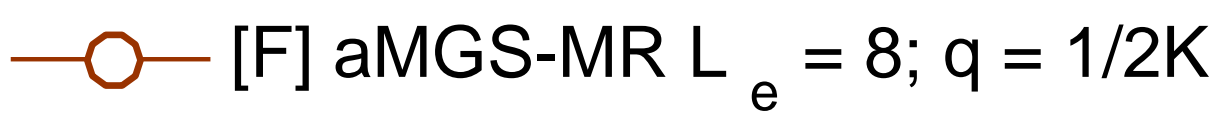

$$
\begin{aligned}
& \longrightarrow \text { [G] 1-sMGS; } q=1 / 2 \mathrm{~K} \text { (proposed) } \\
& \text { * }[H] 2 \text { 2-sMGS; } q=1 / 2 \mathrm{~K} \text { (proposed) }
\end{aligned}
$$

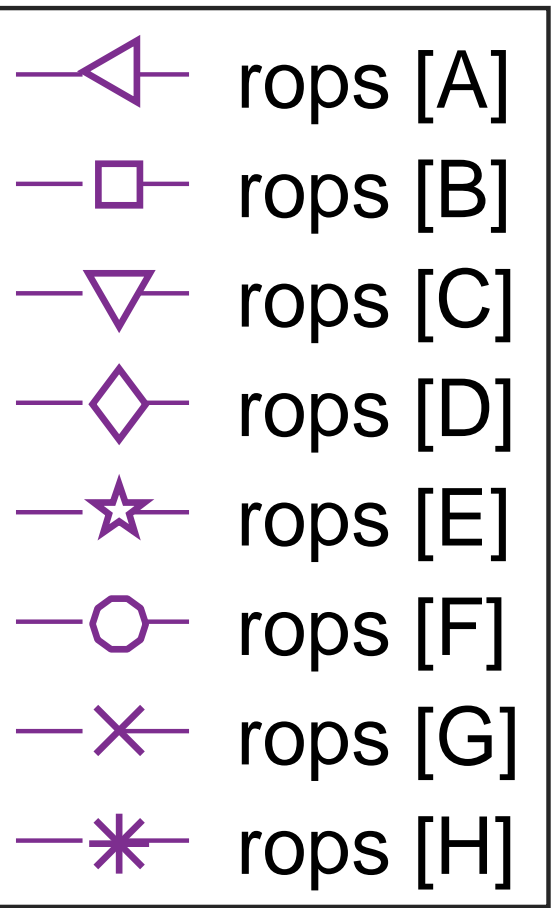




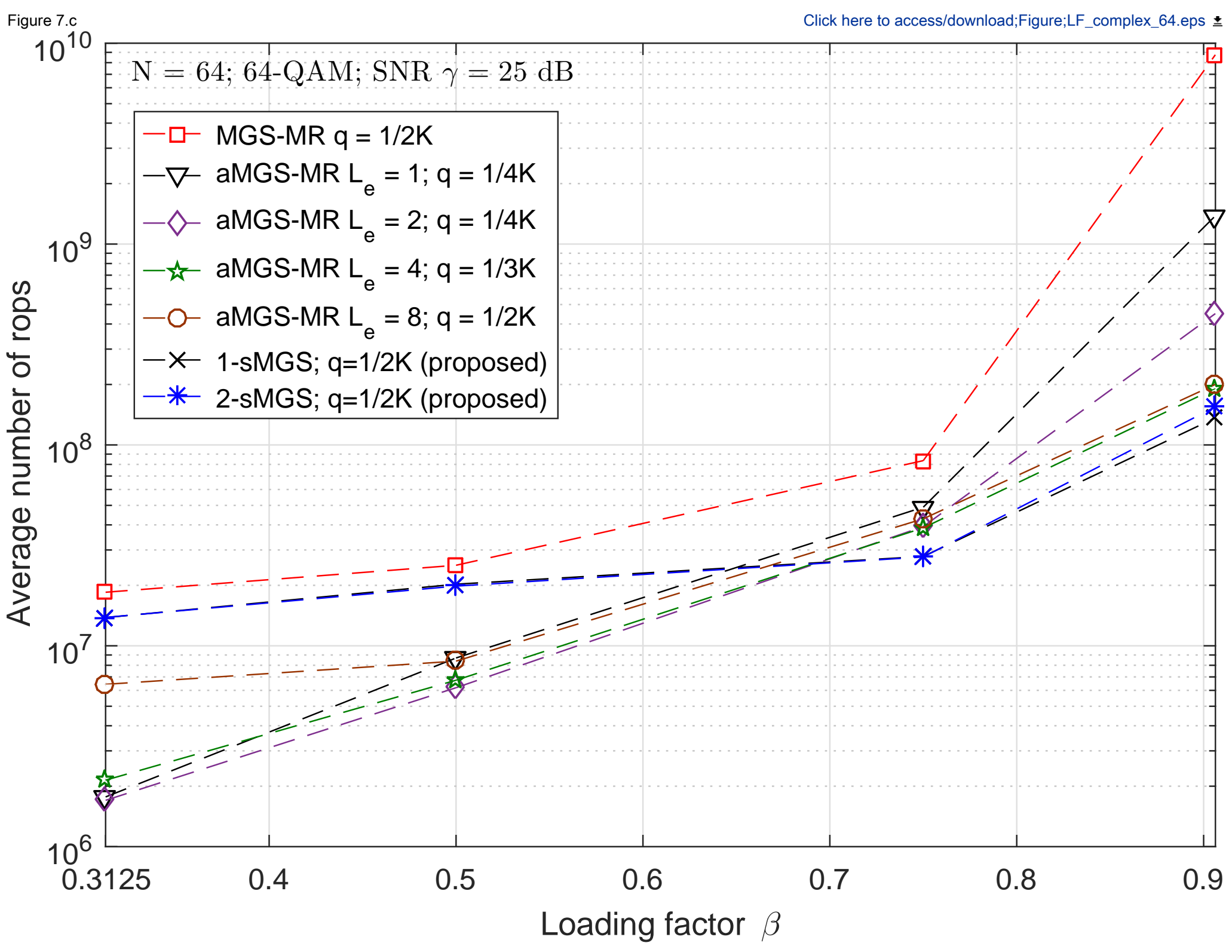




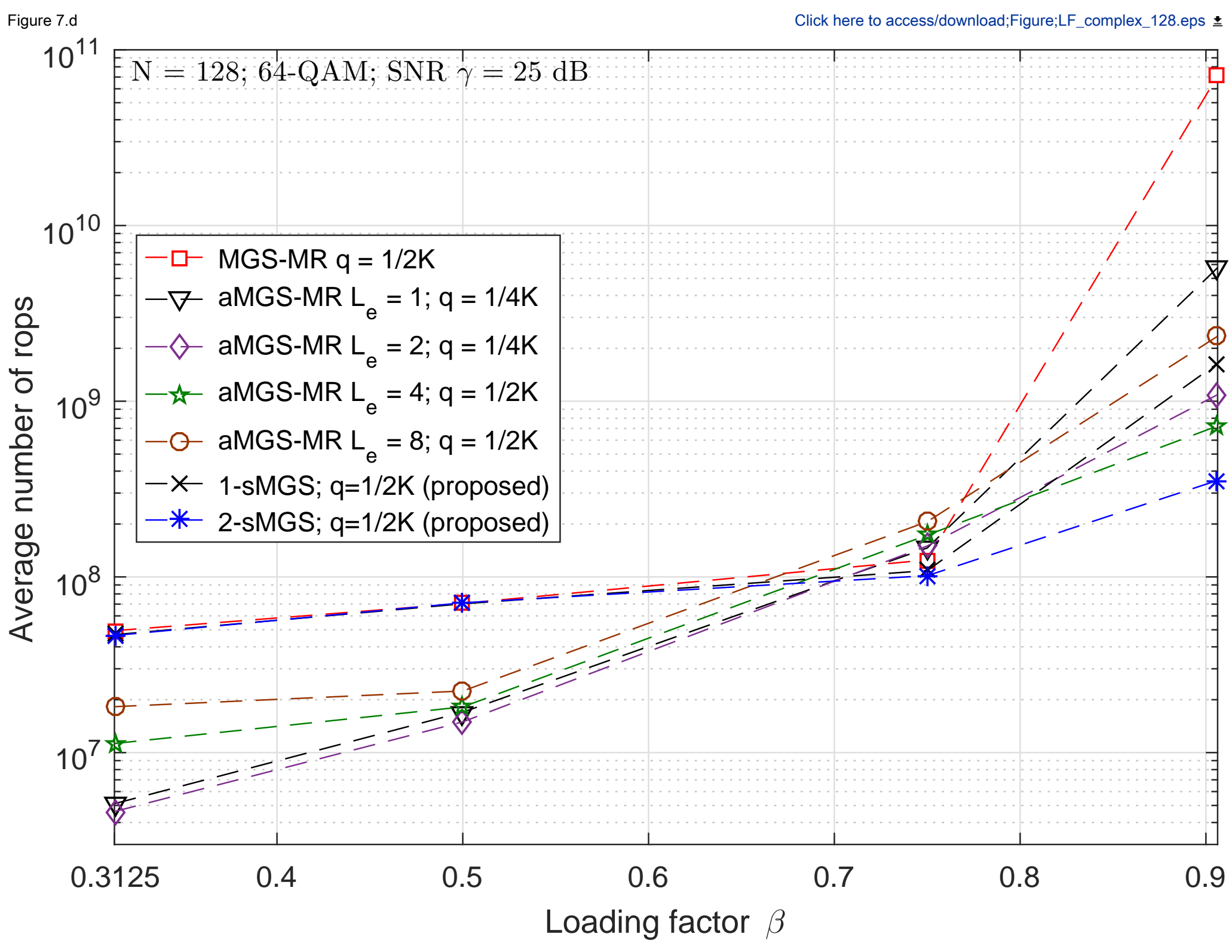

